\title{
Transient Analysis of Flexible Rotors with Nonlinear Bearings, Dampers and External Forces
}

\author{
A Dissertation \\ Presented to \\ the Faculty of the School of Engineering and Applied Science \\ University of Virginia \\ In Partial Fulfillment
of the requirements for the Degree
Doctor of Philosophy (Mechanical and Aerospace Engineering)
}

by

Jianming Cao

March 2012 


\section{APPROVAL SHEET}

This dissertation is submitted in partial fulfillment of the requirements for the degree of

Doctor of Philosophy (Mechanical and Aerospace Engineering)

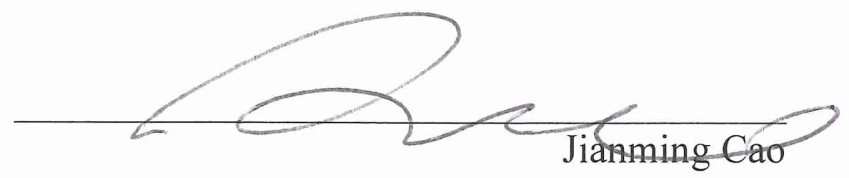

This dissertation has been read and approved by the Examining Committee:
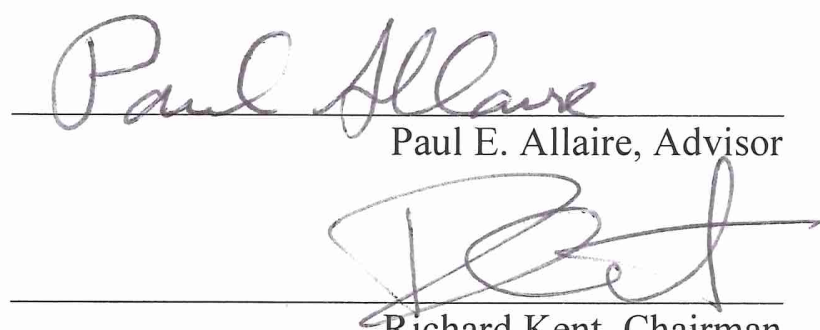

Richard Kent, Chairman
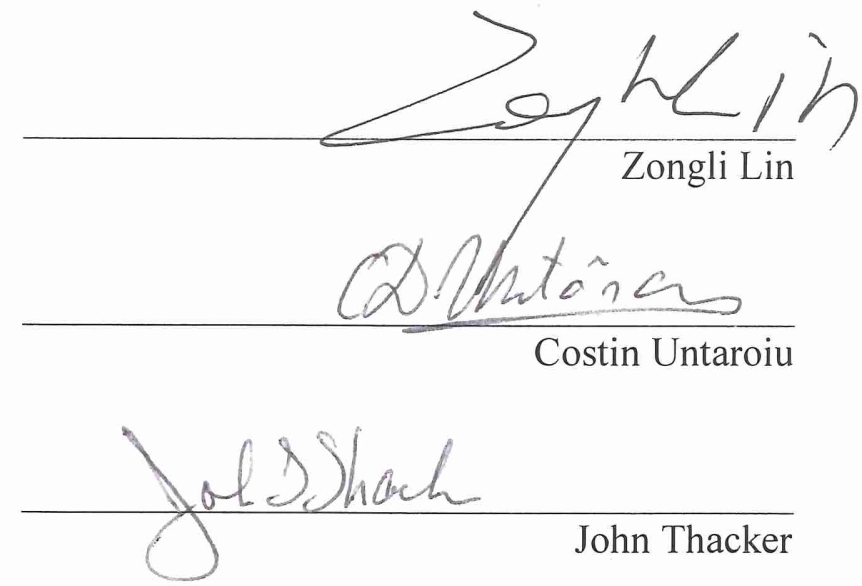

Accepted for the School of Engineering and Applied Science:

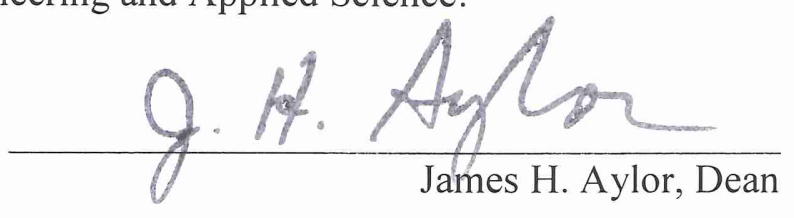

March 2012 


\begin{abstract}
General rotor-bearing systems often display nonlinear behavior due to hydrodynamic effects and external nonlinear forces. Linear methods based on finite element methods are the most common methods for performing rotor dynamic analyses. Nonlinear bearing/damping forces are often linearized into equivalent stiffness and damping coefficients. This method often works well for weakly nonlinear systems, but has limits for strongly nonlinear systems. To more accurately describe rotor behavior, transient analysis and nonlinear models are often used. Analytical solutions for Jeffcott rotors with nonlinear springs or numerical solutions for complex rotors with plain journal bearings have been developed for nonlinear rotor dynamic analyses. However, systems with complicated bearings/dampers and coupled lateral and torsional analyses of rotor-bearing systems have not been involved.
\end{abstract}

This dissertation provides time transient methods for solving nonlinear dynamic behavior of flexible rotor-bearing systems including gyroscopic effects, complicated bearings, squeeze film dampers, and external nonlinear forces/torques. The rotor is modeled as linear; and the supporting components, including bearings and dampers, are modeled as nonlinear. The nonlinear bearing forces, which depend on instantaneous nodal displacements and velocities, are calculated at each time step through nonlinear bearing/damper models. To describe the coupled motion of shaft, bearing pads/pivots and squeeze film dampers, a method of assembling both the linear rotor and the nonlinear 
components is developed. An implicit Runge-Kutta method to solve coupled lateral and torsional analysis of rotor-bearing systems is approached.

The developed methods are verified against published data. Vibration amplitudes of a linear system under different rotational speeds from transient analysis are exactly same as from linear analysis based on steady state. The tiling pad bearing full dynamic coefficients obtained via transient analysis and small perturbation method (for verification purpose only) at different rotational speeds match those from THPAD (maximum difference of $6 \%$ on stiffness). Comparisons to previous torsional analysis are made to verify the nonlinear transient torsional solver.

The transient analyses are applied to a 3-disk rotor supported with nonlinear short plain journal bearings and nonlinear short squeeze film dampers first, and then to an industrial 8-stage back-to-back compressor supported with nonlinear tilting pad bearings and nonlinear finite length squeeze film dampers under severe unbalance conditions. Coupled lateral and torsional transient analyses of the 3-disk rotor with nonlinear supporting system including acceleration effects are solved directly using an implicit $4^{\text {th }}$ order Runge-Kutta method. The effects of support flexibility in series of nonlinear bearings and nonlinear squeeze film dampers are examined. Squeeze film dampers introduced to the system increase dynamic stability of the system under high range rotational speed, decrease the bearing forces, and avoid the possible touch between the shaft and the bearings under severe unbalance conditions. Different nonlinear behavior, such as subharmonic, super-harmonic and torus are shown in transient analyses. 


\section{Acknowledgements}

There are so many people who have helped me during my research and my life in ROMAC, University of Virginia.

I would like to thank my advisor, Prof. Paul Allaire, for his excellent guidance and support throughout this research. It has been wonderful experience learning from him over the years. His knowledge, expertise, and insight help me continue my research and reach my goals.

I would also like to thank my committee, Prof. Zongli Lin, Prof. Kent Richard, Prof. John Thacker and Prof. Costin Untaroiu, for their support, input and encouragement on the work of my research.

I would also like to thank Prof. Pradip N. Sheth, who encouraged me to join the graduate program. It was pleasure learning and working with him.

I would like to thank my colleagues in the ROMAC: Dr. Amir Younan, Dr. Jawad Chaudhry, Dr. Matthew Wagner, Dr. Tim Dimond, Dr. Wei Jiang, Feng He, Jason Kaplan and all of the other students for our discussions and for their support.

Most importantly I would like to thank my wife Lihong and my parents for their love and support through this process. I would also like to thank my son Daniel (Yo-Yo) for bring happiness into my life while playing with him. 


\section{Contents}

Abstract

Acknowledgements

Nomenclature $\quad$ Xxv

1 Background and Statement of the Problem 1

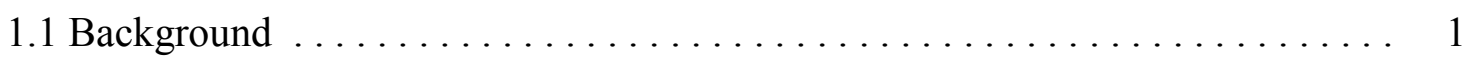

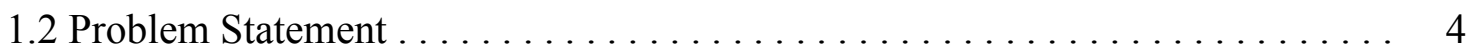

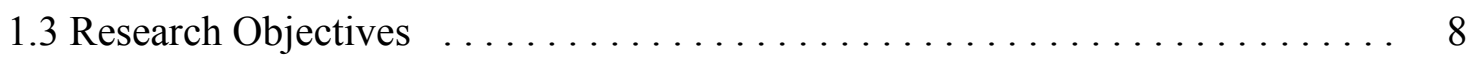

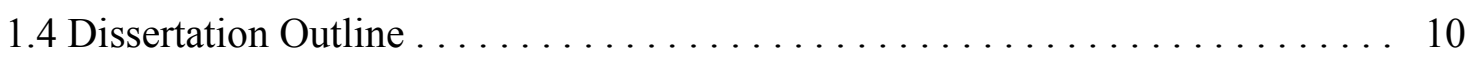

2 Lecture Reviews of Nonlinear Rotor Dynamics: Modeling, Assembling and

\begin{tabular}{l|l} 
Transient Analyses & 12
\end{tabular}

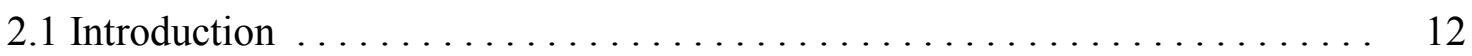

2.2 Nonlinear Phenomenon in Rotor Dynamics $\ldots \ldots \ldots \ldots \ldots \ldots \ldots \ldots$

2.3 Rotor Dynamic System Modeling and Model Formulation $\ldots \ldots \ldots \ldots$ 
2.3.1 Governing Equations of the Rotor Dynamic System . . . . . . . . . 16

2.3.2 Modeling and Assembling of Rotor Dynamic System . . . . . . . . . . 18

2.4 Methods of Nonlinear Rotor Dynamic Analysis $\ldots \ldots \ldots \ldots \ldots \ldots$

2.5 Nonlinear Modeling and Nonlinear Forces Calculation $\ldots \ldots \ldots \ldots \ldots$

2.6 Nonlinear Time Transient Analysis in Rotor Dynamics . . . . . . . . . . 26

2.6.1 Stability and Periodic Response of Nonlinear System . . . . . . . . . . 27

2.6.2 Direct Method in Nonlinear Time Transient Analysis . . . . . . . . . . 27

2.6 .3 Modal Method and Nonlinear Model Reduction ................ 29

2.7 Nonlinear Transient Analysis Methods Summary . . . . . . . . . . . . . 33

3 Nonlinear Flexible Rotor-Bearing Systems: Modeling, Assembling and Solution 34

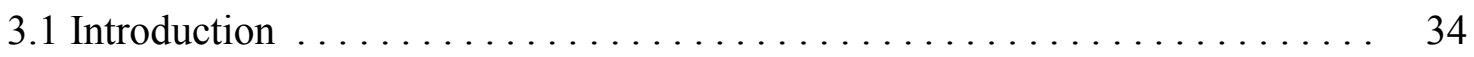

3.2 Structure Modeling and Assembling $\ldots \ldots \ldots \ldots \ldots \ldots \ldots \ldots \ldots$

3.2.1 Dynamic Model of Linear Rotor . . . . . . . . . . . . . . . . 35

3.2.2 Method of Assembling Rotor Dynamic System ............. 37

3.3 Linear Applied Loads . . . . . . . . . . . . . . . . . . . . . 47

3.4 Nonlinear Applied Loads . . . . . . . . . . . . . . . . . . . . . . 49

3.4.1 Short Plain Journal Bearings and Short Squeeze Film Dampers . . . . . . 50 
3.4.2 Tilting Pad Bearings and Finite Length Squeeze Film Dampers . . . . . . . 52

3.4.3 Nonlinear Torsional Forces and Moments . . . . . . . . . . . . . . 60

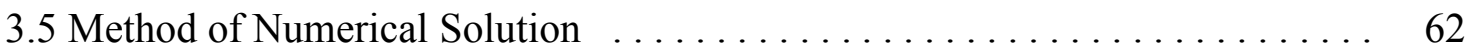

3.5.1 Integration Method for Constant Running Speed . . . . . . . . . . . 64

3.5.2 Integration Method for Non-Constant Running Speed . . . . . . . . . 65

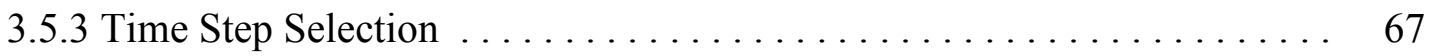

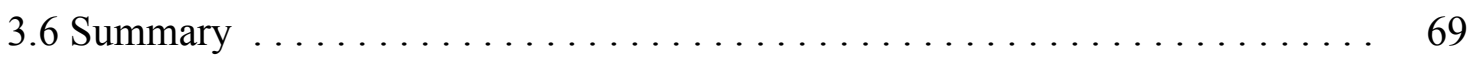

4 Flexible Three-Disk Rotor with Nonlinear Short Journal Bearings and Nonlinear
Squeeze Film Dampers

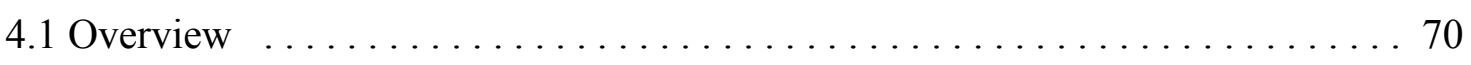

4.2 Three-Disk Flexible Rotor Model $\ldots \ldots \ldots \ldots \ldots \ldots \ldots \ldots \ldots$

4.3 Validation of Linear Transient Rotor dynamic Analysis . . . . . . . . . 73

4.3 .1 Linear Bearing Model . . . . . . . . . . . . . . . . . 73

4.3.2 Unbalance Forced Response $\ldots \ldots \ldots \ldots \ldots \ldots \ldots \ldots \ldots$

4.4 Three-Disks Rotor with Nonlinear Short Plain Journal Bearings . . . . . . . . . 8 81

4.4.1 Pressure Profile of Short Plain Journal Bearing and Nonlinear Force

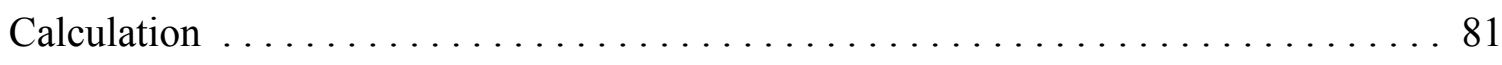

4.4.2 Transient Analyses of Rotor with Nonlinear Short Plain Journal Bearings. . 86 
4.5 Three-Disk Rotor with Nonlinear Journal Bearings and Squeeze Film Dampers. 98

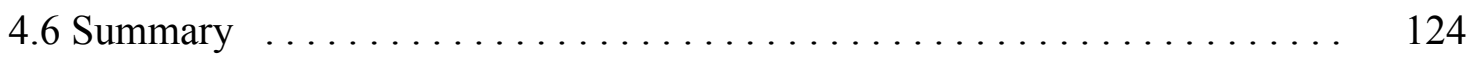

5 Rigid and Flexible Rotor with Nonlinear Tilting Pad Bearings and Nonlinear

Finite Length Squeeze Film Dampers

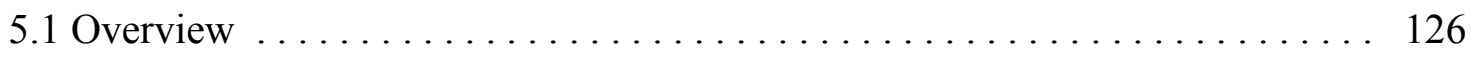

5.2 Rigid Rotor with Nonlinear Tilting Pad Bearings . . . . . . . . . . . . . 127

5.2.1 Pressure Profile of Tilting Pad Bearing and Nonlinear Bearing Force

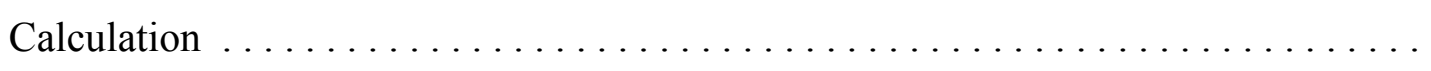

5.2.2 Nonlinear Transient Response of Rigid Rotor Supported with Nonlinear

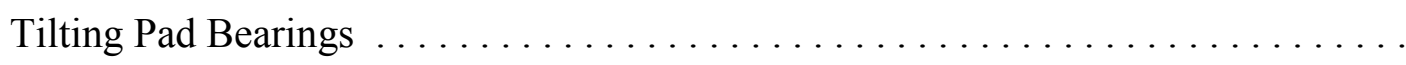

136

5.2.2.1 Validate of Bearing Force Calculation of Nonlinear Tilting Pad

Bearings

138

5.2.2.2 Nonlinear Transient Analysis of the Rigid Rotor on Two 4-Pad Tilting Pad

Bearings under Severe Unbalance $\ldots \ldots \ldots \ldots \ldots \ldots \ldots \ldots \ldots \ldots \ldots \ldots$

5.3 8-Stage Back to Back Compressor Model $\ldots \ldots \ldots \ldots \ldots \ldots \ldots \ldots$

5.3 .1 Linear Rotor Model $\quad \ldots \ldots \ldots \ldots \ldots \ldots \ldots \ldots \ldots \ldots \ldots \ldots \ldots$

5.3.2 Nonlinear Tilting Pad Bearing Model $\ldots \ldots \ldots \ldots \ldots \ldots \ldots \ldots$

5.4 Transient Analysis of 8-Stage Compressor with Nonlinear Tilting Pad

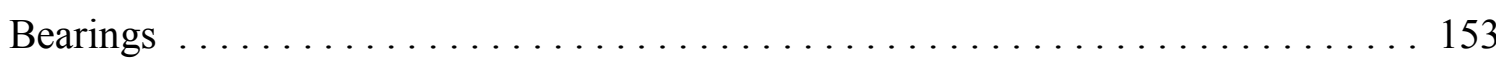


5.4.1 Critical Speed Map of the Rotor-Bearing System . . . . . . . . . . . . . 154

5.4.2 Transient Analysis of Rotor with Nonlinear Tilting Pad Bearings . . . . . 155

5.5 Transient Analysis of Rotor with Nonlinear Tilting Pad Bearings and Nonlinear

Finite Length Squeeze Film Dampers $\ldots \ldots \ldots \ldots \ldots \ldots \ldots \ldots \ldots \ldots$

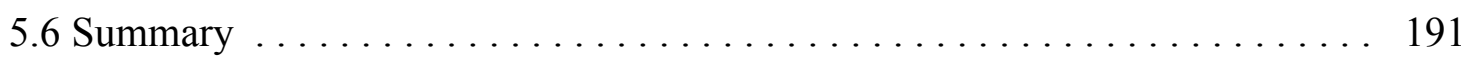

6 Transient Analysis of Non-Constant Rotational Speed and External Torsional

Load Vibration

194

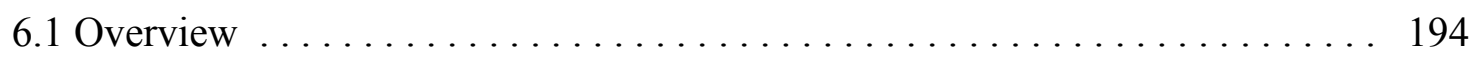

6.2 Flexible Rotor under Non-Constant Rotational Speed . . . . . . . . . . . . . . 195

6.2.1 Equations of Motion and Numerical Solver $\ldots \ldots \ldots \ldots \ldots$

6.2.2 Response of Flexible Rotor under Non-Constant Rotational Speed . . . . . 197

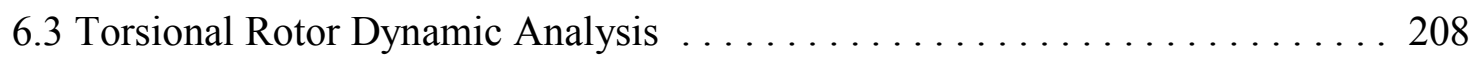

6.3.1 Motion Equations and External Torsional Forces . . . . . . . . . . . 208

6.3.2 Startup Transient Motion and Torsional Analysis . . . . . . . . . . . . 209

6.4 Coupled Torsional and Lateral Transient Analysis of the 3-Disk Rotor Model. . 215

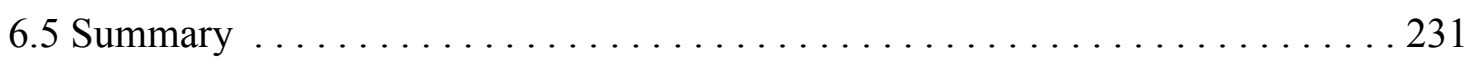

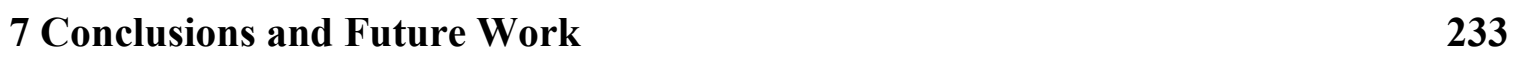

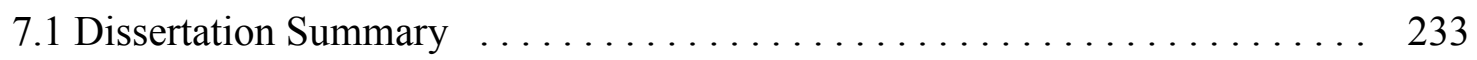


7.1 .1 Modeling and Assembling Methods $\ldots \ldots \ldots \ldots \ldots \ldots \ldots$

7.1.2 Applied Forces/Torques Calculation $\ldots \ldots \ldots \ldots \ldots \ldots \ldots$

7.1 .3 Direct Integration Solver $\ldots \ldots \ldots \ldots \ldots \ldots \ldots \ldots \ldots \ldots \ldots$

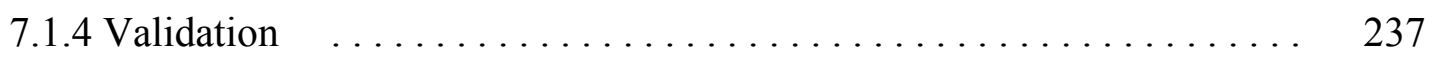

7.1.5 Conclusions on Application and Performance of Transient . . . . . . . 238

7.1 .6 Computational Times $\ldots \ldots \ldots \ldots \ldots \ldots \ldots \ldots \ldots \ldots \ldots$

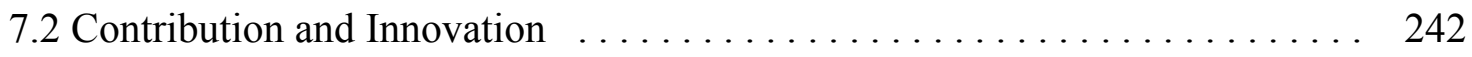

7.3 Discussion and Future Work $\ldots \ldots \ldots \ldots \ldots \ldots \ldots \ldots \ldots \ldots$

$\begin{array}{ll}\text { References } & 246\end{array}$

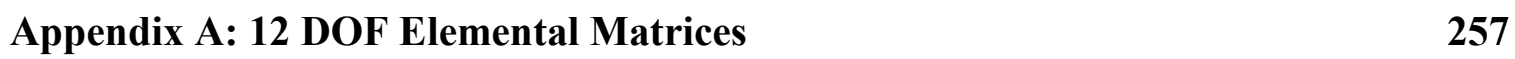

Appendix B: Assembling of Linear Rotor and Nonlinear Bearings/Dampers 263 


\section{List of Figures}

Figure 1.1 A Photograph of a Typical Steam Turbine $\ldots \ldots \ldots \ldots \ldots \ldots .2$

Figure 2.1 Vibrations during Acceleration and Deceleration $\ldots \ldots \ldots \ldots \ldots .15$

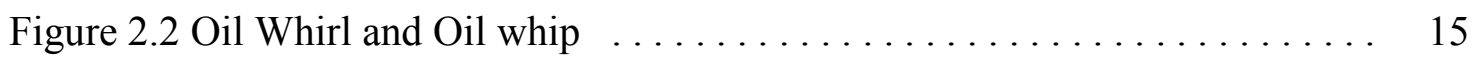

Figure 2.3 Chaotic Motions from Aircraft Gas Turbine $\ldots \ldots \ldots \ldots \ldots \ldots$

Figure 3.1 12-DOF Element and Coordinates for Modeling Linear Rotor ...... 35

Figure 3.2 Assembly of Finite Element Shaft Matrix into Global Matrix . ... . . . 39

Figure 3.3 Rotor Supported with Bearings and Dampers ............... 40

Figure 3.4 Gravitational Force in $\mathrm{XZ}$ and $\mathrm{YZ}$ planes $\ldots \ldots \ldots \ldots \ldots \ldots$

Figure 3.5 Cross Section of a Plain Journal Bearing in Fixed Coordinate ....... 51

Figure 3.6 Cross Section and Coordinate of a Tilting Pad Bearing $\ldots \ldots \ldots \ldots 53$

Figure 3.7 Cross Section and Coordinate of a Finite Length Damper . ....... 58

Figure 3.8 Typical Torque Speed Curve of Synchronous Motor $\ldots \ldots \ldots \ldots \ldots 2$

Figure 3.9 Flow Chart of the Rotor System Nonlinear Transient Solution . . . . . 63 
Figure 4.1 Twenty-Five Nodes Three-Disk Flexible Rotor Model . . . . . . . . 72

Figure 4.2 Cross Section of Bearing with Damper Outside $\ldots \ldots \ldots \ldots \ldots \ldots$

Figure 4.3 Steady State Force Response at Bearings Location (Node 4) . . . . . . . 77

Figure 4.4 Transient Force Response at Bearing Location (Node \#4) . . . . . . . . . 77

Figure 4.5 Force Response (FFT) at Bearing Location (Node \#4) . . . . . . . . . 78

Figure 4.6 Rotor Static Deformation under Gravity Loading . . . . . . . . . . . 78

Figure 4.7 Rotor Orbits at 6,000 rpm with Gravitational Loading and Unbalances . . 79

Figure 4.8 Pressure Profile and Pressure Surface for $\bar{X}=0.20, \bar{Y}=-0.1, \dot{\bar{X}}=\dot{\bar{Y}}=0 \ldots 83$

Figure 4.9 Pressure Profile and Pressure Surface for $\bar{X}=0.5, \bar{Y}=-0.7, \dot{\bar{X}}=\dot{\bar{Y}}=0 \ldots \ldots 4$

Figure 4.10 Pressure Profile and Pressure Surface for $\bar{X}=0, \bar{Y}=-0.5, \dot{\bar{X}}=0.5, \dot{\bar{Y}}=0$. . 85

Figure 4.11 Undamped Critical Map of the 3-Disk Rotor $\ldots \ldots \ldots \ldots \ldots 7$

Figure 4.12 Waterfall Map of 3-Disk Rotor - Bearing at Node 4, Horizontal

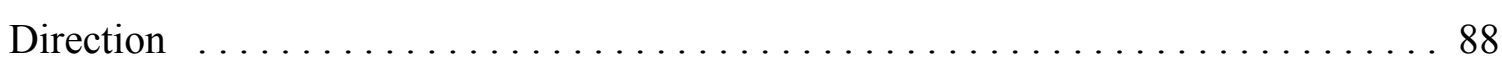

Figure 4.13 Waterfall Map of 3-Disk Rotor - Bearing at Node 22, Vertical Direction 89

Figure 4.14 Time Transient Output at Bearing (Node\#4) at 6, $000 \mathrm{rpm} \ldots \ldots$. . . . 90

Figure 4.15 Time Transient Bearing Force (Node\#4) at 6,000 rpm . . . . . . . . 90

Figure 4.16 Orbits of the Rotor at 6,000rpm at $1.0 \mathrm{sec}$, with Gravity Load and

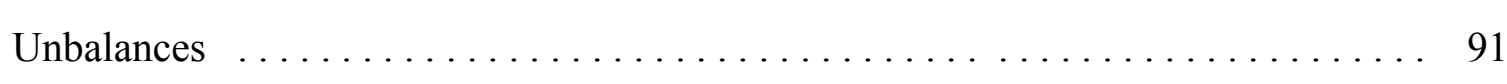


Figure 4.17 Transient Output from the Bearing (Node 4) at 12,000 rpm . . . . . 92

Figure 4.18 Orbit of Node 4 at $12,000 \mathrm{rpm}, \mathrm{t}=0.2 \sim 1 \mathrm{sec} . \ldots \ldots \ldots \ldots \ldots$

Figure 4.19 FFT of the Node\#4 Displacement at 12,000 rpm . . . . . . . . . 93

Figure 4.20 Bearing force under $12,000 \mathrm{rpm}$ from Node4 . . . . . . . . . . . . . . . 94

Figure 4.21 Orbits of Rotor at 12,000 rpm, $1.0 \mathrm{sec}$, with Gravity Loading and

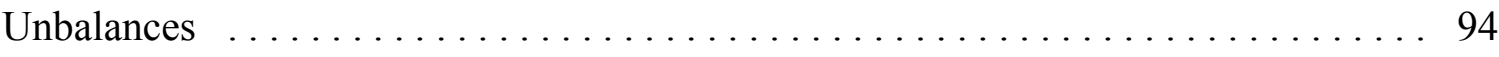

Figure 4.22 Orbit of the Center Disk (Node 13) at 16,000 rpm, $\mathrm{t}=0.1 \sim 0.5 \mathrm{sec} . \ldots 95$

Figure 4.23 FFT of the Center Disk (Node 13) at 16,000 rpm ........... 96

Figure 4.24 Output of the Center Disk at 20,000 rpm w/o Squeeze Film Damper . . 97

Figure 4.25 Output of the Center Disk at 30,000 rpm w/o Squeeze Film Damper . . 97

Figure 4.26 Shaft Center inside Bearing (Node\#4) w/o Squeeze Film Damper at 20,000

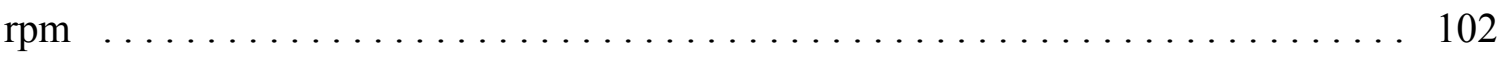

Figure 4.27 Shaft Center inside Bearing (Node\#4) with Squeeze Film Damper at 20,000

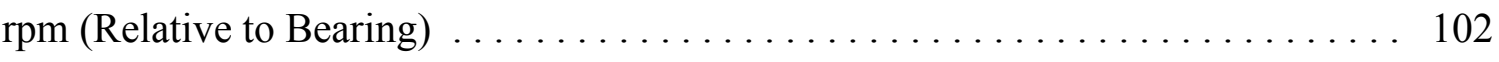

Figure 4.28 Shaft Center inside Bearing (Node\#22) with SFD outside at 20,000 rpm

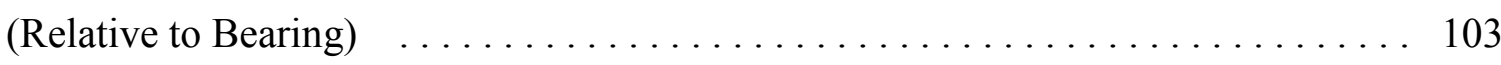

Figure 4.29 Bearing Forces (node 4) w/o Squeeze Film Damper at 20,000 rpm . . . 103

Figure 4.30 Bearing Forces (Node 4) with Squeeze Film Damper at 20,000 rpm . . 104 
Figure 4.31 Orbit of the Center Disk at 20,000 rpm w/o Damper . . . . . . . . . . . 104

Figure 4.32 FFT Results of the Center Disk at 20,000 rpm w/o Damper . . . . . . . 105

Figure 4.33 Orbit of the Center Disk at 20,000 rpm with Damper . . . . . . . . . 105

Figure 4.34 Orbit and FFT of the Center Disk at 20,000 rpm with Damper . . . . 106

Figure 4.35 Rotor Orbit with SFD at 20,000 rpm around 0.5 second with Gravity Load

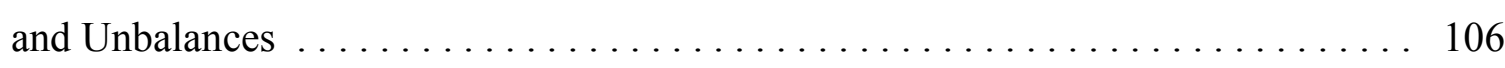

Figure 4.36 Output from Squeeze Film Damper at Node 4 at 20,000 rpm . . . . . 107

Figure 4.37 Orbit from SFD at Node\#4 under 20,000 rpm ... . . . . . . 108

Figure 4.38 FFT Results from SFD at Node\#4 under 20,000 $\mathrm{rpm} \ldots \ldots \ldots$

Figure 4.39 SFD Forces at Node\#4 under 20,000 rpm . . . . . . . . . . . . 109

Figure 4.40 Bearing Behavior inside SFD at Node 22 under 20,000 rpm . . . . . 109

Figure 4.41 Bearing Orbit inside SFD at Node 22 under $20,000 \mathrm{rpm} \ldots \ldots \ldots 110$

Figure 4.42 FFT of SFD at Node 22 under $20,000 \mathrm{rpm} \ldots \ldots \ldots \ldots$

Figure 4.43 SFD Forces at Node 22 under $20,000 \mathrm{rpm} \ldots \ldots \ldots \ldots \ldots 11$

Figure 4.44 Shaft Center inside Bearing (Node\#4) w/o Damper at 30,000 rpm . . . 112

Figure 4.45 Shaft Center inside Bearing (Node\#4) with Damper at 30,000 rpm . . 113

Figure 4.46 FFT of Shaft Center inside Bearing (Node\#4) with Damper at 30,000

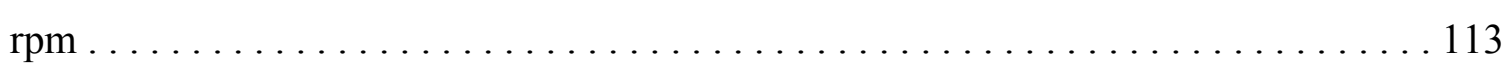


Figure 4.47 Bearing Forces (Node\#4) without Squeeze Film Damper at 30,000 rpm 114

Figure 4.48 Bearing Forces (Node\#4) with Squeeze Film Damper at 30,000 rpm. . 114

Figure 4.49 Orbit of Center Disk at 30,000 rpm without SFD . . . . . . . . 115

Figure 4.50 FFT of Center Disk at 30,000 rpm without SFD . . . . . . . . 115

Figure 4.51 Orbit of Center Disk at 30,000 rpm with SFD $\ldots \ldots \ldots \ldots \ldots$

Figure 4.52 FFT of Center Disk at 30,000 rpm with SFD $\ldots \ldots \ldots \ldots \ldots$

Figure 4.53 Orbits of the Rotor with SFD at 30,000 rpm around 0.1 second with Gravity

Loading and Unbalances $\ldots \ldots \ldots \ldots \ldots \ldots \ldots \ldots \ldots \ldots \ldots \ldots \ldots \ldots$

Figure 4.54 Bearing Center inside SFD at Node\#4 at 30,000 rpm . . . . . . . 118

Figure 4.55 Orbit of Bearing Center inside SFD at Node\#4 at 30,000 rpm . . . . 118

Figure 4.56 FFT of Bearing Center inside SFD at Node\#4 at 30,000 rpm $\ldots . . .119$

Figure 4.57 SFD Forces at Node\#4 at $30,000 \mathrm{rpm} \ldots \ldots \ldots \ldots \ldots \ldots$

Figure 4.58 Bearing Center inside SFD at Node\#22 at 30,000 rpm . . . . . 120

Figure 4.59 Orbit of Bearing Center inside SFD at Node\#22 at 30,000 rpm . . . . 120

Figure 4.60 FFT of Bearing Center inside SFD at Node\#22 at 30,000 rpm . . . . 121

Figure 4.61 SFD Forces at Node\#22 at 30,000 rpm $\ldots \ldots \ldots \ldots \ldots \ldots$

Figure 4.62 Waterfall Diagram of 3-Disk Rotor with Squeeze Film Dampers, Node 4 in

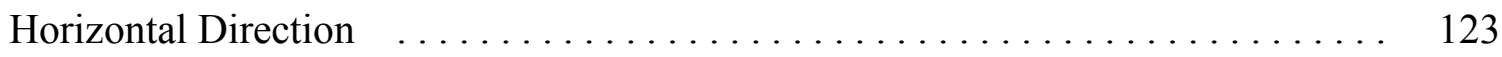


Figure 4.63 Waterfall Diagram of 3-Disk Rotor with Squeeze Film Dampers, Node 22

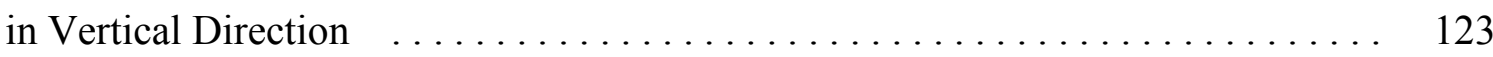

Figure 5.1 Rigid Rotor Supported on two Tilting Pad Bearings . . . . . . . . . . 128

Figure 5.2 4-Pad Tilting Pad Bearing Geometry . . . . . . . . . . . . . . . . . 129

Figure 5.3 Dimensionless Pressure Profile on Pad (Case I) . . . . . . . . . . 132

Figure 5.4 Dimensional Pressure Profile on Pad (Case I) . . . . . . . . . . . 132

Figure 5.5 Dimensionless Pressure Profile on Pad (Case II) $\ldots \ldots \ldots \ldots \ldots \ldots$

Figure 5.6 Dimensional Pressure Profile on Pad (Case II) $\ldots \ldots \ldots \ldots \ldots \ldots$

Figure 5.7 Dimensionless Pressure Profile on Pad\#4 (Case III) . . . . . . . . . . 134

Figure 5.8 Dimensionless Pressure Profile on Pad\#4 (Case IV) $\ldots . \ldots \ldots \ldots .134$

Figure 5.9 Dimensionless Pressure Profile on Pad\#4 (Case V) $\ldots \ldots \ldots \ldots \ldots$

Figure 5.10 Dimensionless Pressure Profile on Pad\#4 (Case VI) . . . . . . . . . . 135

Figure 5.11 Displacements with Various Time Step at 8,000 rpm under Unbalance 138

Figure 5.12 Time Transient of Shaft Center at 8,000 rpm $\left(\mathrm{me}_{\mathrm{u}}=0\right)$ with Time Step

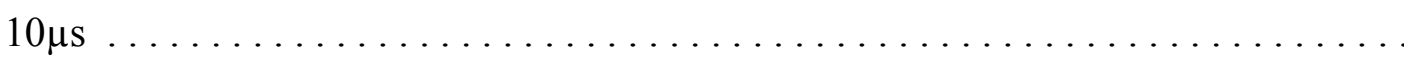

Figure 5.13 Final Steady State Pressure Profile on Pads/Pivots at 8,000 rpm $\left(\mathrm{me}_{\mathrm{u}}=0\right.$,

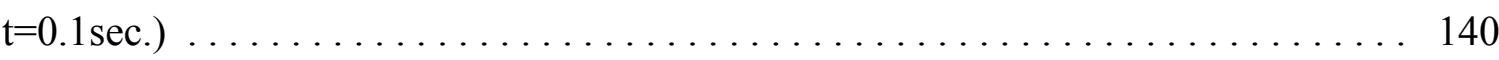


Figure 5.14 Final Steady State Shaft Center and Pads/Pivots Positions at 8,000 rpm

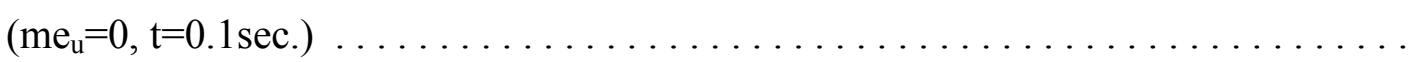

Figure 5.15 Forces on Pads at $8.000 \mathrm{rpm}\left(\mathrm{me}_{\mathrm{u}}=0\right) \ldots \ldots \ldots \ldots \ldots \ldots$

Figure 5.16 Behavior of Pad Angle at $8,000 \mathrm{rpm}_{\left(\mathrm{me}_{\mathrm{u}}=0\right)} \ldots \ldots \ldots \ldots \ldots \ldots$

Figure 5.17 Dynamic Stiffness Coefficients . . . . . . . . . . . . . . . . . 144

Figure 5.18 Dynamic Damping Coefficients . . . . . . . . . . . . . . . . . 144

Figure 5.19 Orbits of Shaft Center at 8,000rpm, different unbalances at $0.2 \mathrm{sec} . .146$

Figure $5.20 \mathrm{Pad}$ Force at $8.000 \mathrm{rpm}$, Different Unbalances . . . . . . . . . . . 147

Figure $5.21 \mathrm{Pad}$ Moment at 8,000 rpm, Different Unbalances . . . . . . . . . . 147

Figure 5.22 Pad\#4 Angle at 8,000 rpm, Different Unbalances . . . . . . . . . . . . 148

Figure 5.23 Pivot\#4 Displacement at 8,000 rpm, Different Unbalances . . . . . . . 148

Figure 5.24 Pad4 Angle Velocity at 8,000 rpm, different unbalances . . . . . . . . . 149

Figure 5.25 Pivot4 Velocity at $8,000 \mathrm{rpm}$, different unbalances . . . . . . . . . . . 149

Figure 5.26 CAD image of an STC-SH single-shaft centrifugal compressor wit horizontally split casing. Fields of application include on- and offshore oil and gas, petrochemicals, refineries, general chemistry and process engineering . . . . . 150

Figure 5.27 Finite element model of the 8-stage back-to-back compressor . . . . . 151

Figure 5.28 Undamped Critical Map of the 8-stage Compressor . . . . . . . . . . . 154 
Figure 5.29 Displacements of Bearing \#1 with Different Time Steps ........ 156

Figure 5.30 Displacements of Bearing \#2 with Different Time Steps ......... 156

Figure 5.31 Shaft Response of CASE I after 1.0 sec with Unbalance, w/o Gravity 159

Figure 5.32 Shaft Response of CASE I after 3.0 sec with Unbalance, w/o Gravity 159

Figure 5.33 Shaft Response of CASE I at 3.0 sec with Unbalance and Gravity

Loading ................................... 160

Figure 5.34 Shaft Response of CASE II at $1.0 \mathrm{sec}$ with Unbalance, w/o Gravity

Loading .................................... 160

Figure 5.35 Shaft Response of CASE II at $3.0 \mathrm{sec}$ with Unbalance, w/o Gravity

Loading $\ldots \ldots \ldots \ldots \ldots \ldots \ldots \ldots \ldots \ldots \ldots \ldots \ldots \ldots \ldots \ldots \ldots \ldots \ldots . .$.

Figure 5.36 Shaft Response of CASE II at 3.0 sec with Unbalance and Gravity

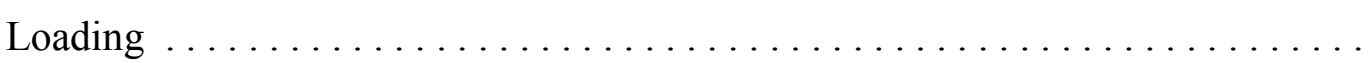

161

Figure 5.37 Bearing Forced from Node 5 of CASE I $\ldots \ldots \ldots \ldots \ldots \ldots$

Figure 5.38 Bearing Pad\#4 and Pivot\#4 Response of CASE I $\ldots \ldots \ldots \ldots \ldots 162$

Figure 5.39 Bearing Pad\#4 Moment FFT Output (CASE I) $\ldots \ldots \ldots \ldots \ldots .163$

Figure 5.40 Shaft Response CASE III (Severe Unbalance) w/o Gravity at 9.0 sec 164

Figure 5.41 Shaft Response CASE III (Severe Unbalance) with Gravity at 9.0 sec. 165

Figure 5.42 Orbit of Disk\#4 (Node\#14) of CASE III, 8.0 9.0 sec $\ldots \ldots \ldots \ldots 165$ 
Figure 5.43 Orbit of Disk\#5 (Node\#17) of CASE III, 8.0 9.0 sec $\ldots \ldots \ldots \ldots 166$

Figure 5.44 Shaft inside the Bearing (node 5) Output of CASE III, 8.0 9.0 sec 166

Figure 5.45 Bearing Forces at node 5 Output of CASE III $\ldots \ldots \ldots \ldots \ldots . \ldots 7$

Figure 5.46 Bearing Pad/Piovt\#4 Output of CASE III $\ldots \ldots \ldots \ldots \ldots \ldots \ldots 7$

Figure 5.47 FFT Output of Shaft Node 5 inside Bearing ............... 168

Figure 5.48 FFT Output of Bearing Pad\#4 Force at Node $5 \quad \ldots \ldots \ldots \ldots \ldots$

Figure 5.49 FFT Output of Bearing Pad\#4 Moment at Node $5 \quad \ldots \ldots \ldots \ldots .69$

Figure 5.50 Squeeze Film Damper with Tilting Pad Bearing Inside, RMT Inc . . 171

Figure 5.51 Pressure Profile under different Elements $\ldots \ldots \ldots \ldots \ldots \ldots \ldots .174$

Figure 5.52 Damper Forces vs. Elements $\ldots \ldots \ldots \ldots \ldots \ldots \ldots \ldots \ldots \ldots \ldots$

Figure 5.53 Shaft Orbit with Squeeze Film Dampers at $0.5 \mathrm{sec}$, 32x Unbalance. . . 176

Figure 5.54 Transient Shaft Orbit with Squeeze Film Dampers, 0 0.5 sec, 32x

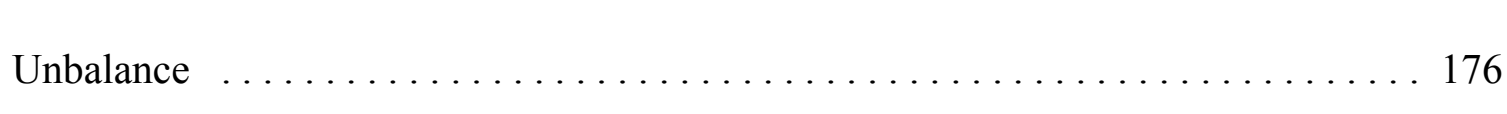

Figure 5.55 Bearing Response at Node 5 under 32x Unbalance ........... 177

Figure 5.56 Bearing Response at Node 27 under 32x Unbalance $\ldots \ldots \ldots \ldots 178$

Figure 5.57 Squeeze Film Damper Response at Node 5 under 32x Unbalance . . 178

Figure 5.58 Squeeze Film Damper Response at Node 27 under 32x Unbalance . . 179

Figure 5.59 Bearing Force at Node 5 under $32 x$ Unbalance $\ldots \ldots \ldots \ldots \ldots . . \ldots$ 
Figure 5.60 FFT of Bearing Force at Node 5 under $32 x$ Unbalance . . . . . . . . 180

Figure 5.61 Bearing Force at Node 27 under $32 x$ Unbalance . . . . . . . . . . . 181

Figure 5.62 FFT of Bearing Force at Node 27 under 32x Unbalance . . . . . . . . 181

Figure 5.63 Squeeze Film Damper Force at Node 5 under 32x Unbalance . . . . . 182

Figure 5.64 FFT of Squeeze Film Damper Force at Node 5 under 32x Unbalance 182

Figure 5.65 Squeeze Film Damper Force at Node 27 under 32x Unbalance .... 183

Figure 5.66 FFT of Squeeze Film Damper Force at Node 27 under 32x Unbalance 183

Figure 5.67 Bearing Pad/Pivot \#4 Response at Node $5 \quad \ldots \ldots \ldots \ldots \ldots \ldots$

Figure 5.68 Bearing Pad/Pivot \#4 Response at Node 27 . . . . . . . . . . . . . . 185

Figure 5.69 FFT of Bearing Pad \#4 Force at Node $5 \ldots \ldots \ldots \ldots \ldots$

Figure 5.70 FFT of Bearing Pad \#4 Force at Node $27 \ldots \ldots \ldots \ldots$

Figure 5.71 FFT of Bearing Pad \#4 Moment at Node $5 \ldots \ldots \ldots$. . . . . . 187

Figure 5.72 FFT of Bearing Pad \#4 Moment at Node $27 \ldots \ldots \ldots \ldots$. . . . . 187

Figure 5.73 FFT of Bearing Pad \#4 Tilting Angle at Node $5 \quad \ldots \ldots \ldots \ldots \ldots$

Figure 5.74 FFT of Bearing Pad \#4 Displacement at Node $5 \ldots \ldots \ldots$

Figure 5.75 FFT of Bearing Pad \#4 Tilting Angle at Node $27 \quad \ldots . \ldots \ldots \ldots . \quad 189$

Figure 5.76 FFT of Bearing Pad \#4 Displacement at Node $27 \ldots \ldots \ldots$ 
Figure 5.77 Bearing Pad/Pivot \#1 Response at Node $5 \quad \ldots \ldots \ldots \ldots \ldots \ldots$

Figure 5.78 Bearing Pad/Pivot \#2 Response at Node $5 \quad$. . . . . . . . . . . . . 190

Figure 5.79 Bearing Pad/Pivot \#3 Response at Node $5 \quad \ldots \ldots \ldots \ldots \ldots \ldots 1$

Figure 6.1 3-Disk Flexible Rotor Model with 25 Nodes . . . . . . . . . . . . . . . . 198

Figure 6.2 Transient Response of 5,950 rpm 6,000 rpm, 0 0.5 sec (BRG1, Node4)199

Figure 6.3 Transient Force Response of 6000rpm (BRG1, Node4) . . . . . . . . . 200

Figure 6.4 User Defined Speed Profile File $\ldots \ldots \ldots \ldots \ldots \ldots \ldots$

Figure 6.5 Response of Linear System (Node 13) with Linear Acceleration Rate of 700

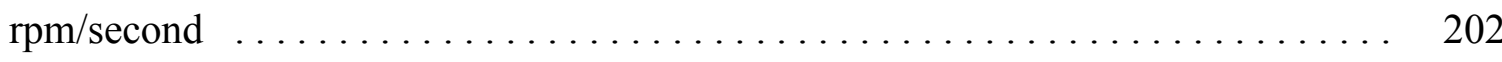

Figure 6.6 Time Transient Response at Bearing with Various Acceleration Rates 203

Figure 6.7 Time Transient Response at Center Disk with Various Acceleration

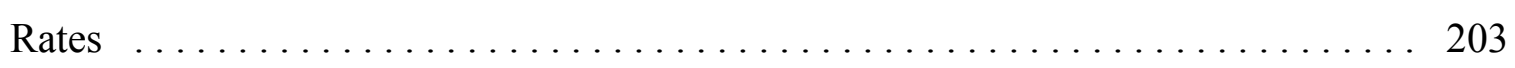

Figure 6.8 Exponential Speed Profile $\ldots \ldots \ldots \ldots \ldots \ldots \ldots \ldots \ldots$

Figure 6.9 Response of Center Disk (Node 13) with an Exponential Acceleration

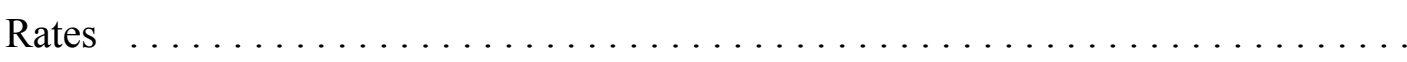

Figure 6.10 Response of the Shaft Center inside Bearing (Node 4) with an Exponential

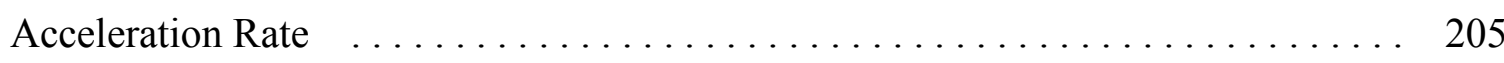

Figure 6.11 Response of the Squeeze Film Damper (Node 4) with an Exponential

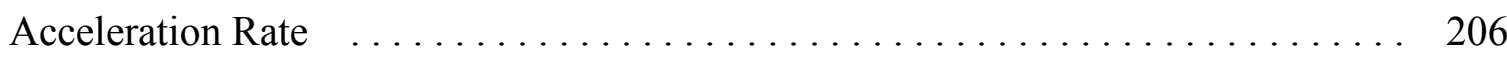


Figure 6.12 FFT of Shaft inside Bearing (Node 4) under Exponential Acceleration 206

Figure 6.13 SFD FFT Output (Node 4) with an Exponential Acceleration

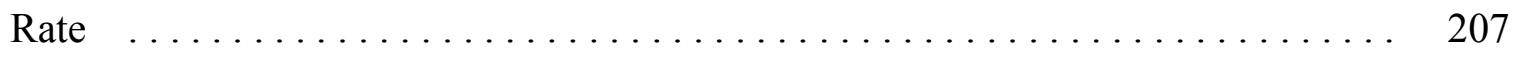

Figure 6.14 Shaft Transient Response during an Exponential Acceleration Rate under

Gravity Loading and Unbalances $\ldots \ldots \ldots \ldots \ldots \ldots \ldots \ldots \ldots \ldots \ldots \ldots$

Figure 6.15 Synchronous Motor Driven 3 Compressor System ........... 209

Figure 6.16 Torque Speed Curves $\quad \ldots \ldots \ldots \ldots \ldots \ldots \ldots \ldots \ldots \ldots .210$

Figure 6.17 Motor Speed vs. Time during Startup . . . . . . . . . . . . . . . 213

Figure 6.18 Motor Speed vs. Time during Startup from Chen $\ldots \ldots \ldots \ldots .213$

Figure 6.19 Shaft Angular Displacement vs. Time during Startup .......... 214

Figure 6.20 Shaft Vibratory Torque vs. Time $\ldots \ldots \ldots \ldots \ldots \ldots \ldots \ldots \ldots . \ldots \ldots$

Figure 6.21 Shaft Vibratory Torque vs. Time by Chen $\ldots \ldots \ldots \ldots \ldots \ldots .215$

Figure 6.22 Frequency Interface Diagram of a Synchronous Motor and the Three-disk

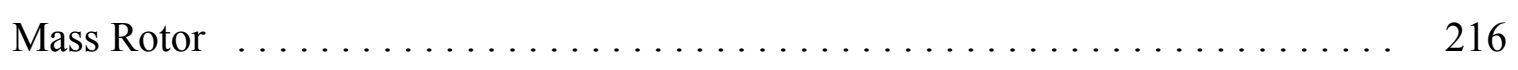

Figure 6.23 Motor Speed vs. Time during Startup, Coupled Analysis of a Synchronous

Motor and the Three-disk Mass Rotor, Node\#1 $\ldots \ldots \ldots \ldots \ldots \ldots \ldots . . \ldots . \ldots . \ldots$

Figure 6.24 Motor Speed vs. Time during Startup, Coupled Analysis of a Synchronous Motor and the Three-disk Mass Rotor, Node\#13 .................. 219 
Figure 6.25 Vibratory Torque vs. Time, Coupled Analysis of a Synchronous Motor and the Three-disk Mass Rotor, Element \#13

Figure 6.26 Nodal \#13 Displacement, Coupled Analysis of a Synchronous Motor and

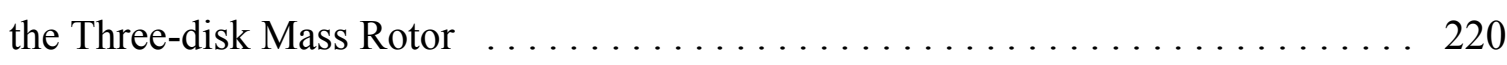

Figure 6.27 Speed vs. Time of 5X Motor Torque, Coupled Analysis of a Synchronous Motor and the Three-disk Mass Rotor, Node $13 \ldots \ldots \ldots \ldots \ldots$. . . . . . . . 220

Figure 6.28 Nodal Torque vs. Time of 5X Motor Torque, Coupled Analysis of a Synchronous Motor and the Three-disk Mass Rotor (Element 13) $\quad \ldots \ldots \ldots \ldots$.

Figure 6.29 Nodal \#13 Displacement, Coupled Analysis of a Synchronous Motor and

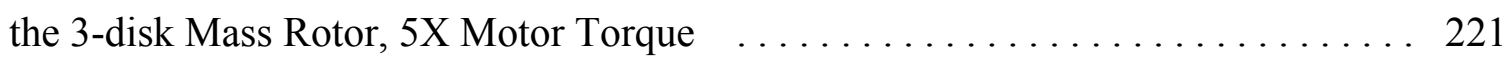

Figure 6.30 Speed vs. Time of 50X Motor Torque, Node 13 of a Synchronous Motor

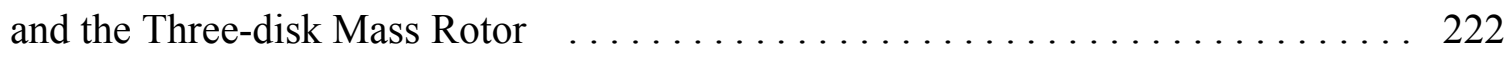

Figure 6.31 Nodal Torque vs. Time of 50X Motor Torque, Element 13 of a Synchronous Motor and the Three-disk Mass Rotor $\ldots \ldots \ldots \ldots \ldots \ldots \ldots 22$

Figure 6.32 Nodal \#13 Displacement, Coupled Analysis of a Synchronous Motor and the Three-disk Mass Rotor, 50X Motor Torque $\quad \ldots \ldots \ldots \ldots \ldots \ldots \ldots$

Figure 6.33 Speed vs. Time of Startup for Nonlinear System during Startup of a Synchronous Motor and the Three-disk Mass Rotor, Node $13 \ldots \ldots \ldots$

Figure 6.34 Nodal Torque vs. Time at Center Disk (Node 13) during Startup of a Synchronous Motor and the Three-disk Mass Rotor $\quad \ldots \ldots \ldots \ldots \ldots \ldots$ 
Figure 6.35 Displacement of Bearing inside SFD (at Node 4) during Startup of a

Synchronous Motor and the 3 -disk Mass Rotor $\ldots \ldots \ldots \ldots \ldots \ldots$

Figure 6.36 Displacement and FFT of Shaft Center inside Bearing (Node 4) during

Startup of a Synchronous Motor and the 3 -disk Mass Rotor $\ldots \ldots \ldots \ldots \ldots$

Figure 6.37 Displacement and FFT of Shaft Center inside Bearing (Node 4) during Startup of a Synchronous Motor and the 3 -disk Mass Rotor ..............

Figure 6.38 Bearing Forces and FFT at Node 4 during Startup of a Synchronous Motor

and the 3-disk Mass Rotor 228

Figure 6.39 Displacement and FFT of Bearing inside SFD (at Node 4) during Startup of a Synchronous Motor and the 3 -disk Mass Rotor . . . . . . . . . . . . . 229

Figure 6.40 SFD (at Node 4) Force and FFT during Startup of a Synchronous Motor

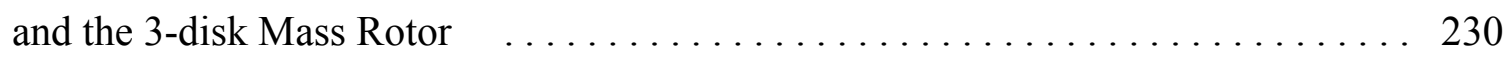

Figure 7.1 Compare of direct solver and linear modal reduction . . . . . . . . 245 


\section{List of Tables}

Table 4.1 Three-Disk Flexible Rotor Model $\ldots \ldots \ldots \ldots \ldots \ldots \ldots \ldots .73$

Table 4.2 Bearing Dynamic Coefficients as Function of Speed .......... 75

Table 4.3 Response Comparison between Linear and Transient at Bearings . . . . 80

Table 4.4 Bearing Information of 3-Disk Rotor $\ldots \ldots \ldots \ldots \ldots \ldots \ldots \ldots .82$

Table 4.5 Bearing Forces under Different Cases $\ldots \ldots \ldots \ldots \ldots \ldots \ldots \ldots 2$

Table 4.6 Squeeze Film Damper Information of the 3-disk Rotor $\ldots \ldots \ldots \ldots 100$

Table 4.7 Comparison of with and w/o SFD at $20,000 \mathrm{rpm} \ldots \ldots \ldots \ldots \ldots \quad 100$

Table 4.8 Comparison of with and w/o SFD at 30,000 $\mathrm{rpm} \ldots \ldots \ldots \ldots \ldots .112$

Table 4.9 Computing Time Comparison ..................... 125

Table 5.1 4-Pad Tilting Pad Bearing Information ................. 129

Table 5.2 Dimensionless Cases for Tilting pad Bearing $\ldots \ldots \ldots \ldots \ldots \ldots . \ldots 131$

Table 5.3 Tilting pad Bearing Forces under Different Dimensionless Cases ... 136

Table 5.4 Dynamic Stiffness and Damping $\ldots \ldots \ldots \ldots \ldots \ldots \ldots \ldots \ldots \ldots$ 
Table 5.5 Eight Stage Back to Back Compressor Data $\ldots \ldots \ldots \ldots \ldots \ldots$

Table 5.6 Finite Length Squeeze Film Damper Information . . . . . . . . . . . 172

Table 5.7 Computing Time Comparison $\ldots \ldots \ldots \ldots \ldots \ldots \ldots \ldots$

Table 6.1 Model Effective Inertia and Stiffness $\ldots \ldots \ldots \ldots \ldots \ldots$

Table 6.2 Critical Speeds Compare $\ldots \ldots \ldots \ldots \ldots \ldots \ldots \ldots \ldots \ldots \ldots$

Table 6.3 Resonance Speed of Different Accelerations $\ldots \ldots \ldots \ldots \ldots \ldots$

Table 6.4 Computing Time Comparison $\ldots \ldots \ldots \ldots \ldots \ldots \ldots \ldots 232$

Table 7.1 Computational Times of Different Conditions . . . . . . . . . . 241 


\section{Nomenclature}

$\begin{array}{ll}A & \text { State Space A Matrix } \\ B & \text { State Space B Matrix } \\ B_{1}, B_{2} & \text { Weight Factor of Axial Pressure } \\ C & \text { Damping Matrix } \\ C_{s} & \text { Structural Damping Matrix } \\ C_{b r g} & \text { Bearing Damping Matrix } \\ C_{b} & \text { Bearing Radial Clearance } \\ C_{d} & \text { Damper Radial Clearance } \\ C_{i j} & \text { Dynamic Bearing Damping Coefficients }(i, j=x, y, \delta, \gamma) \\ C_{p} & \text { Pad Clearance } \\ D & \text { Mouring/Damper Journal Diameter } \\ E & \text { Young's Modulus } \\ f_{L} & \end{array}$




\begin{tabular}{|c|c|}
\hline$F$ & Force Vector \\
\hline$F_{\text {bow }}$ & Shaft Bow Force \\
\hline$F_{\text {bow }, x}, F_{\text {bow }, y}, F_{\text {bow }, z}$ & Shaft Bow Force in Horizontal, Vertical and Axial Directions \\
\hline$F_{b r g}, F_{b, x}, F_{b, y}$ & Bearing Forces \\
\hline$F_{d, x}, F_{d, y}$ & Finite Length Squeeze Film Damper Forces \\
\hline$F_{g}$ & Gravitational Force Vector \\
\hline$F_{g, x}, F_{g, y}, F_{g, z}$ & Gravitational Force \\
\hline$F_{n}$ & Nonlinear Force \\
\hline$F_{p}$ & Pad force \\
\hline$F_{s d}$ & Squeeze Film Samper Force \\
\hline$F_{u}$ & Unbalance Force \\
\hline$F_{u, x}, F_{u, y}, F_{u, z}$ & Unbalance Force in Horizontal, Vertical and Axial Directions \\
\hline$g_{x}, g_{y}$ & Gravitational Constants \\
\hline$G$ & Gyroscopic Matrix \\
\hline$h$ & Fluid Film Thickness \\
\hline$I_{p a d}$ & Pad Moment of Inertia \\
\hline
\end{tabular}




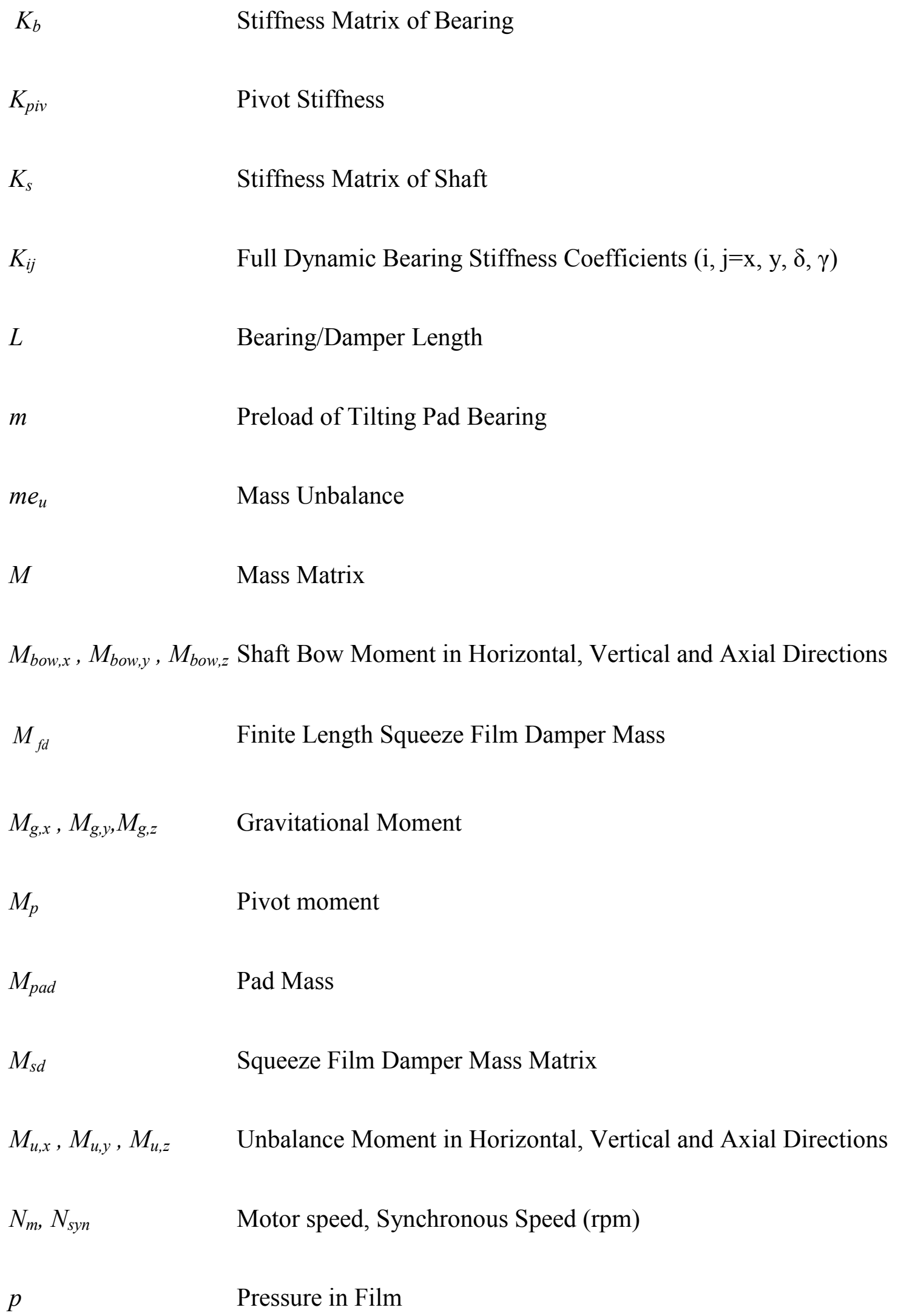




$$
p_{\theta}
$$$$
\bar{p}, \bar{p}_{\theta}
$$$$
t, \bar{t}
$$

$T_{i}, T_{\text {avg }}, T_{o s c}$

$$
u, \dot{u}, \ddot{u}
$$$$
\text { U }
$$$$
U_{0}, U_{l}
$$$$
V_{l}
$$$$
x, y, z
$$$$
x_{f d}, y_{f d}
$$$$
x_{s d}, y_{s d}
$$$$
X, Y, \dot{X}, \dot{Y}
$$$$
\bar{X}, \bar{Y}, \dot{\bar{X}}, \dot{\bar{Y}}
$$$$
\gamma, \delta
$$$$
\bar{\gamma}, \bar{\Delta}
$$$$
\Delta t
$$$$
\varepsilon
$$

Pressure in Circumferential Direction

Dimensionless Pressure in Film

Time, Dimensionless Time

External Torque, Average Torque, Oscillating Torque

Displacement, Velocity and Acceleration Vectors

State Space Displacement Vector

Relative Velocity between Sliders

Squeeze Velocity between Sliders

Direction in Horizontal, Vertical and Axial Direction

Finite Length Squeeze Film Damper Displacements

Squeeze Film Damper Displacements

Bearing/Damper Displacement and Velocity

Dimensionless Bearing/Damper Displacement and Velocity

Tilting Pad Bearing Pad and Pivot Displacements

Dimensionless Tilting Pad Bearing Pad and Pivot Displacements

Time Step

Eccentricity 
Local Coordinates Direction along Slider

Local Rotational Coordinate

$\theta_{p}$

Pivot Location

$\theta_{x}, \theta_{y}, \theta_{z}$

Rotation in Horizontal, Vertical and Axial direction

$\mu$

Lubricant Dynamic Viscosity

$\phi_{\text {bow }}$

Shaft Bow Phase Angle

$\phi_{\text {exc }}$

Excitation Phase of Plusating Torque

$\phi_{u}$

Unbalance Phase Angle

$\varphi, \dot{\varphi}, \ddot{\varphi}$

Angular Displacement, Velocity and Acceleration of Rotor

$\omega$

Shaft rotational Speed inside Bearing

$\omega_{\text {exc }}$

Excitation Frequency of Plusating Torque

$\omega_{i}$

$i^{\text {th }}$ Mode Critical Speed

$\Omega, \dot{\Omega}$

Rotational Speed and Angular Acceleration of Rotor 


\section{Chapter 1}

\section{Background and Statement of the Problem}

\subsection{Background}

Rotating machinery, such as turbines, pumps, compressors, generators, and jet engines, is widely used in diverse industrial fields. Many industrial rotating machines have a flexible multiple-stage shaft/rotor, to which numbers of built on parts (disks, blades, impellor etc.) are attached. A complex rotor-bearing system is shown in Fig. 1.1. The failure of rotating machinery due to large vibration results in enormous repair cost and, more importantly, serves failure puts human life in danger. Proper prediction of the dynamic behavior for flexible rotors, which bend when being operated at high speed, is essential.

Based on the finite element method, the linear theory is commonly used in the standard analysis of a rotor-bearing system, which includes critical speeds, stability, and unbalance forced response. Typical finite element models for linear rotors are:

- 2-D models using beam elements, lumped mass and moment of inertia for any component attached to the shaft

- 3-D models using solid elements 


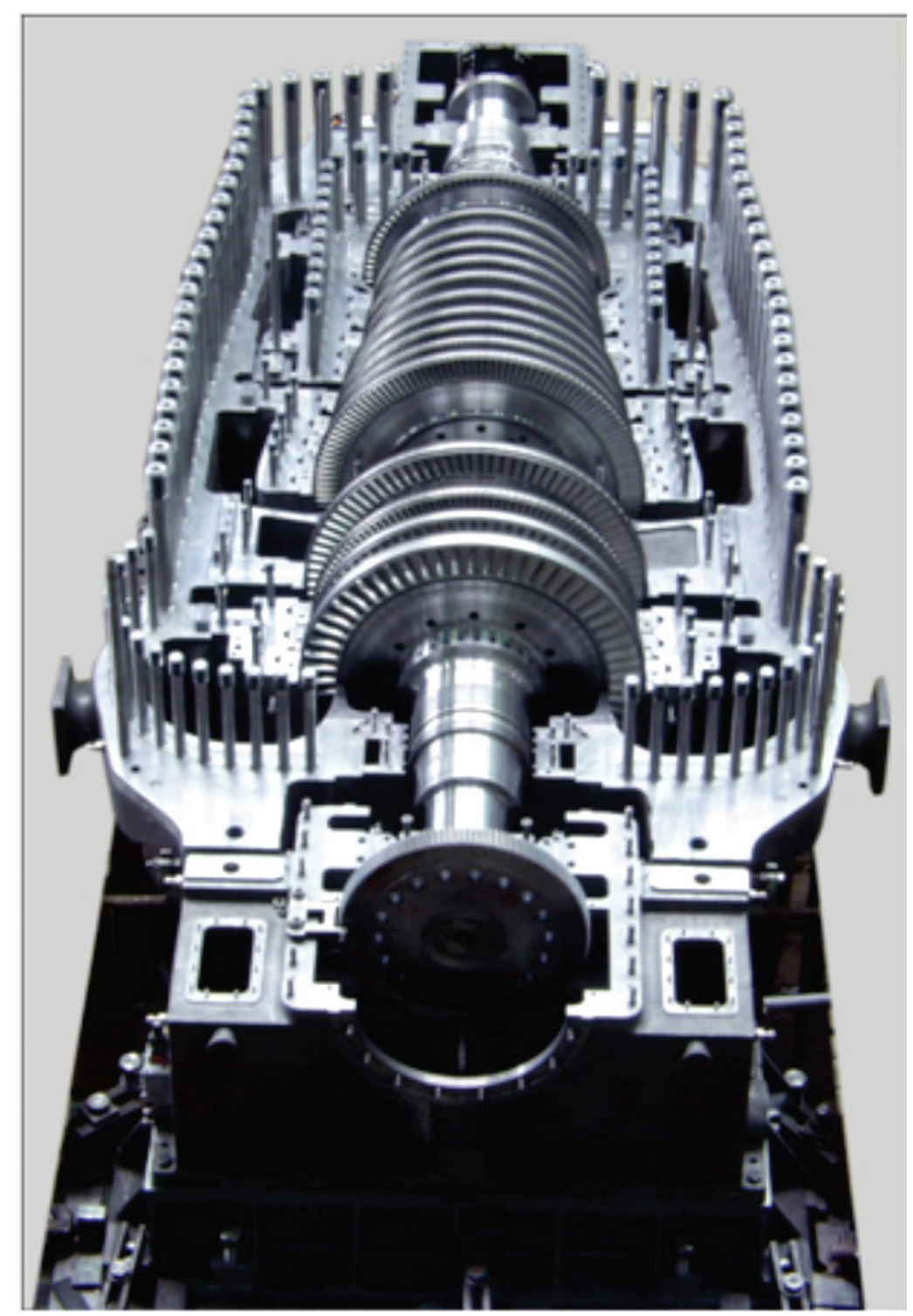

Figure 1.1 A photograph of a typical steam turbine

Based on the linear theory, the nonlinear bearing/damper forces are usually perturbed into two orthogonal directions and linearized. Then the equivalent stiffness and damping coefficients, which can be used in the integrated rotor vibration analysis, are calculated with two assumptions:

- Existence of steady state or equilibrium position

- Shaft vibration with small amplitude (10\% 20\% of bearing clearance) 
However, the dynamic behavior of a rotor-bearing system is related to the characteristics of the nonlinear forces in the case of large amplitude motion, which come from fluid bearings, squeeze film dampers, seals, and other support sources. Due to the hydrodynamic effects, a rotor system often displays nonlinear behavior. The nonlinear behavior has often been observed in experiments and verified by analytical methods. Also the nonlinear phenomena appears during different operating conditions, such as start up, shut down and going through critical speeds. External forces due to blade loss or sudden loading are treated as nonlinear procedures as well.

For a specific linear rotor-bearing system, the stability depends on the characteristic equation of the system with the assumption of a single frequency response (either synchronous or non-synchronous). If the real part of an eigenvalue from the characteristic equation is positive, the system is unstable at that running speed. However, the nonlinear system often has time dependent parameters, and normally no characteristic equation exists. Transient analysis methods are often applied to the nonlinear rotor dynamic system. The nonlinear transient analysis provides more complex motions, such as subharmonic response, super-harmonic response and chaotic response, than the linear analysis when the system is unstable.

The increasing demand for efficient machinery results in more sophisticated designs. This may make industrial rotors more complex. Therefore, the advanced capability to analyze the nonlinear rotor dynamic behavior needs to be developed. Modeling of nonlinear rotor requires careful consideration for asymmetric stiffness, non-proportional damping, frequency dependent gyroscopic effects, and nonlinear bearing forces. Also an 
effective nonlinear model reduction method for the large rotor-bearing system is necessary for accuracy and efficiency.

\subsection{Problem Statement}

The linear analysis is often taken when a rotor dynamic system is expected to behave linearly. However, that assumption is not always satisfied. Large shaft vibration inside bearing occurs (more than $20 \%$ of bearing clearance) when a rotor under severe unbalance condition. Also the squeeze film damper can only be linearized under certain conditions, such as bearing housing only whirls around center area of the damper and only small whirling vibration exists inside the damper. Also a rotor under variable rotational speed or acceleration during startup, shut down or sudden loading cannot be analyzed by the linear method as well.

Due to the small shaft vibration compared to the rotor geometry, the rotor, which includes the shaft and numbers of built on components, is considered as linear in this dissertation. The bearing/damper stiffness and damping effects, which are the rotor's nodal instantaneous displacements and velocities dependent, are considered as nonlinear and are described by the bearing/damper forces. The time transient method is chosen in this dissertation based on the following reasons:

- General rotor-bearing systems often display nonlinear behavior due to the hydrodynamic effects and the external nonlinear forces

- Current linear theory has limits in its ability to describe, explain and predicate the nonlinear dynamic phenomena observed in rotating machines 
- A nonlinear transient solution may be used to study, design and model industrial rotor-bearing systems including the determination of the bearing support characteristic to optimize the system performance

Early nonlinear transient analyses used 2 degrees of freedom (DOF) rigid rotor or Jeffcott rotor with simple nonlinear bearing models to obtain the analytical solutions. The finite element method based complicated rotor-bearing models have been developed for transient analysis in recent years. However, when a complicated rotor-bearing system was applied, to avoid complex eigenvalue \& eigevector problem, often gyroscopic effects were neglected and proportional shaft internal damping was assumed during the nonlinear transient analysis. In those rotor-bearing models, a short plain journal bearing model was applied to calculate the nonlinear effects. For the system including complicated bearings/dampers, instead of calculating the nonlinear forces directly, the equivalent stiffness and damping coefficients at each time step were often calculated by some researchers in transient analysis.

Transient analysis of flexible rotor dynamic system with complicated nonlinear bearings/dampers based on the finite element method and the nonlinear force calculation has not been approached. In general, only one type of nonlinear component was involved, either fluid bearing or squeeze film damper, in transient rotor dynamic analysis. The coupled transient analysis of a flexible rotor with different nonlinear components, say both nonlinear fluid bearing and nonlinear squeeze film damper, has not been developed. Separated lateral, torsional or axial transient analyses have been published. The coupled lateral, torsional and axial transient analysis of the flexible rotor-bearing system, however, has not been involved. 
In this research, based on the finite element method, a coupled lateral and torsional transient analysis method for the flexible rotor dynamic system including gyroscopic effects and complicated bearings/dampers is developed. The coupled behavior of the flexible rotor and different types of nonlinear components including bearings and dampers is presented. All effects from the nonlinear parts are treated as the nonlinear forces, which are the function of instantaneous displacements and velocities of the rotor, the bearing pads/pivot and the bearing housings.

To obtain the coupled lateral, torsional and axial motion of the linear rotor with nonlinear bearings/dampers, a 12 DOF element is needed. Also to describe the behavior of bearing pads/pivots and squeeze film dampers, additional nodes have to be introduced into the system. Thus a method of modeling and assembling both the linear rotor and the nonlinear bearings/dampers has to be developed. The vector equations of motion to be solved in this dissertation are:

$$
M \ddot{u}+(C+\Omega G) \dot{u}+(K+\dot{\Omega} G) u=F_{u}(\Omega, \dot{\Omega}, t)+F_{b o w}(\Omega, \dot{\Omega}, t)+F_{g}+F_{n}(u, \dot{u}, t)
$$

The nonlinear bearing/damper forces depend on instantaneous displacements and velocities of the rotor and the bearing housing at different time. Currently, there are few analytical formulas for the fluid film bearing force unless the short bearing model $(\mathrm{L} / \mathrm{D} \leq 0.5)$ is assumed. To calculate the forces of different types of bearings/dampers at specific displacement and velocity, the linearized Reynolds equation, which is derived from the continuity equation and the momentum equations, need to be solved to obtain the pressure distribution over the fluid film. The nonlinear forces are then calculated by integrating the pressure profile over the fluid film area. In rotor dynamics, the pressure in 
a bearing is up to $10^{9} \mathrm{~Pa}$, the fluid film thickness, however, is around $10^{-6} \mathrm{~m}$ (bearing clearance is approximate $0.1 \% \sim 0.15 \%$ of shaft diameter), that may result in a numerical challenge of solving the Reynolds equation.

To solve the equations of motion, a numerical integration method has to be chosen. For coupled lateral and torsional analysis, the system may be linear or nonlinear; and the rotational speed may be constant or variable. For the non-constant rotational speed problem, an implicit method of solving the equations of motion has to be developed. The Runge-Kutta method is widely used in structural dynamics to solve the ordinary differential equations, especially in engineering applications requiring high accuracy. In this dissertation, the classic explicit $4^{\text {th }}$ order Runge-Kutta method is used to solve the constant rotational speed problem. To solve the non-constant rotational speed problem or the coupled torsional and lateral problem, an implicit Runge-Kutta method has to be developed. The time step selected for direct integration is a critical parameter in a successfully numerical integration procedure. The use of a large time step will cause the loss of detail in the integral being evaluated and will cause convergence problems in the nonlinear rotor solution. On the other hand, too small a time step will produce a solution which is computationally inefficient.

For the linear system with constant rotational speed, model reduction methods are often applied to the rotor dynamic system for stability analysis and unbalance forced response. Compared to those model reduction methods applied to the linear systems, there are fewer publications successfully applied to the nonlinear systems due to the time dependent parameters including bearing stiffness and damping coefficients, variable rotational speed related gyroscopic effects and external forces/torques. The existence of 
those time dependent parameters results in the difficulty of calculating the modal basis accurately. Due to the complexity of the model reduction method for the nonlinear rotor dynamic system, the model reduction method is not studied in detail in this dissertation.

\subsection{Research Objectives}

The goal of this research is to develop a coupled lateral and torsional time transient solution for the flexible rotor with nonlinear fluid film bearings/dampers for both constant rotational speed and non-constant rotational speed under external nonlinear forces. The rotor dynamic system includes gyroscopic effects and different types of nonlinear components. The coupled lateral and torsional time transient solution is approached and the coupled behavior of the flexible rotor and different types of nonlinear components is presented by calculating the nonlinear forces at each time step. Based on this research statement, the objectives of this dissertation are as follows:

1. Develop a finite element based modeling and assembling method and apply the method to the linear flexible rotors with nonlinear bearings, nonlinear dampers and nonlinear external forces.

2. Select and apply appropriate models for calculating the pressure profile of different types of bearings and dampers taking into consideration both the fluid film and the bearing/damper structure.

3. Develop a method for solving the coupled dynamic behavior of the flexible rotor with both nonlinear bearings and nonlinear squeeze film dampers.

4. Develop a method for solving the coupled lateral and torsional transient analysis of flexible rotor with different types of nonlinear bearings/dampers and external nonlinear 
forces. For coupled lateral and torsional analysis, an implicit method has to be develpoed to solve the equations of motion.

To approach those objectives, different nonlinear components are considered in this dissertation: short plain journal bearing, short squeeze film damper, tilting pad bearing, finite length squeeze film damper, and external torques from synchronous motor and loads. Two flexible rotor models are presented in this dissertation: a 3-disk rotor model and an 8-stage compressor model. The following transient analyses are approached in this dissertation to study the nonlinear behavior in rotor dynamics:

1. Time transient analysis of the 3-disk rotor model supported with nonlinear short journal bearings.

2. Time transient analysis of the 3-disk rotor model supported with nonlinear short journal bearings and nonlinear short squeeze film dampers under high range speed.

3. Tilting pad bearing pressure profile and nonlinear force calculation based on a 1-D finite element method.

4. Transient analyses of the rigid rotor with tilting pad bearings to study the bearing pads and pivot effects.

5. Transient analyses of the 8-stage compressor supported with nonlinear 4-pad tilting pad bearings under severe unbalance condition.

6. Transient analyses of the 8-stage compressor supported with nonlinear 4-pad tilting pad bearings and nonlinear finite length squeeze film dampers under severe unbalance condition. 
7. Transient analyses of the 3-disk rotor with linear or nonlinear bearings under angular acceleration.

8. Coupled lateral and torsional transient analyses of the 3-disk rotor with linear or nonlinear bearings with external nonlinear forces from synchronous motor and loads.

\subsection{Dissertation Outline}

In Chapter 2, a lecture review of nonlinear analysis in rotor dynamics is shown. The review is focused on nonlinear phenomenon of rotor dynamics, linear/nonlinear system modeling and assembling, nonlinear bearing/damper force calculation, and methods of solving nonlinear transient analysis problem in rotor dynamics. In Chapter 3, development of solving the equations of motion with nonlinear terms in global coordinates is shown. The chapter includes modeling and assembling methods of a linear rotor with nonlinear supporting systems, nonlinear force calculation of different types of supporting systems, and direct time transient solvers. A direct $4^{\text {th }}$ order Runge-Kutta method and discussion of time step length are shown in the chapter as well. Chapter 4 shows the nonlinear transient analyses of a 3-disk rotor supported with nonlinear short plain journal bearings and nonlinear short squeeze film dampers. Nonlinear squeeze film dampers are applied to the system to increase stability of the system under high range rotational speed. Nonlinear response of coupled nonlinear bearings and nonlinear squeeze film dampers is shown. Chapter 5 shows the nonlinear transient analyses of a rigid rotor supported with nonlinear 4-pad tilting pad bearings to evaluate the dynamic effects of the bearing pads/pivots. Then the nonlinear transient analyses of an 8-stage back to back industrial compressor model supported with nonlinear 4-pad tilting pad bearings and 
nonlinear finite length squeeze film dampers is presented. Coupled motion of the rotor, the bearing pads/pivots and the damper are approached under severe unbalance conditions. Chapter 6 shows the transient analyses of the rotor-bearing system under nonconstant rotational speed first. Then the coupled lateral and torsional analyses of the 3disk rotor with nonlinear bearings and nonlinear dampers driven by a synchronous motor are approached. The acceleration effects to lateral and torsional amplitudes are presented in those analyses. Chapter 7 gives a summary of the dissertation along with future projects that are possible due to the results of this dissertation. 


\section{Chapter 2}

\section{Lecture Review of Nonlinear Rotor Dynamics:}

\section{Modeling, Assembling and Transient Analyses}

\subsection{Introduction}

Dynamic behavior of a rotor-bearing system depends on both the flexible multiple stage shaft/rotor, to which numbers of built on parts (disks, blades, impellor etc.) are attached, and the characteristics of nonlinear forces, which could come from bearings, squeeze film dampers, seals, and other support sources. Due to hydrodynamic effects, a general rotor system often displays nonlinear behavior, which has been observed in experiments $[1,2,3,4]$.

Based on the finite element (FE) method, the linear theory is commonly used in the standard analysis of a rotor-bearing system, which includes critical speed map calculation, stability analysis, and unbalance forced response [5]. Based on the assumption that the rotor runs around its equilibrium position with small shaft vibration inside bearing (typically $10 \% \sim 20 \%$ of bearing clearance), the rotor dynamic system is often modeled as linear based on the FE method. In those models, 2-D beam elements or 3-D solid 
elements are typically used. The nonlinear forces are linearized and perturbed into equivalent coefficients, which include full dynamic coefficients or 2 by 2 stiffness and damping matrices [6].

In some cases, such as startup, shutdown, going through critical speeds or severe unbalance forces, a time transient analysis of the rotor-bearing system is often applied to obtain dynamic behavior of the system. To more accuracy describe the rotor behavior, the nonlinear rotor dynamic models had been developed to analyze and explain the nonlinear phenomena observed in experiments. Modeling of a nonlinear rotor-bearing system requires careful consideration for asymmetric stiffness, non-proportional damping, frequency dependent gyroscopic effects, and nonlinear bearing forces. Also an effective nonlinear model reduction method for the large rotor-bearing system is often necessary considering accuracy and efficiency.

The lecture review in this dissertation involves following fields:

- Nonlinear phenomenon in rotor dynamics

- Rotor dynamic system modeling and model formulation

- Nonlinear modeling and nonlinear forces calculation

- Direct methods and modal methods in time transient analysis

\subsection{Nonlinear Phenomenon in Rotor Dynamics}

It is well know that a general rotor-bearing system often displays nonlinear behavior due to hydrodynamic effects, which are from fluid bearings, squeeze film dampers, seals and other interconnect components. The nonlinear behavior, such as sub-harmonic 
response, super-harmonic response and chaotic motion under high operating speed, has been observed in experiments $[1,2,3]$.

The nonlinear vibration in rotor dynamics was first observed in the 1950's. Yamaoto [1, 7] shows various kinds of non-synchronous vibrations from a rotor in 1954. In his experiment, the test rig includes a long shaft, an adjustable disc on the shaft and two ball bearings to support the shaft. He included that most of those non-synchronous vibrations occur because of the irregularity of ball bearings and the flexibility of bearing pedestals. The sub-harmonic response was reported by Ehrich [3] on an aircraft gas turbine engine in 1966. He concluded that the half running speed sub-harmonic motion was due to nonlinear forces from the squeeze film dampers. In 1974, Bently [2] observed the existence of the sub-harmonic whirling motion at vertical direction of the plain journal bearings from his experiments. In his experiments, a simple horizontal rigid rotor supported by two fluid plain journal bearings was used. He concluded that the nonlinear response was from the bearing clearance. Furthermore, based on Bently's experiential results, Muszynska $[8,9,10]$ proposed a mathematical model for the rotor-bearing system to analyze the self-excited vibrations, which are known as "fluid whirl" and "fluid whip". For the fluid plain journal bearing, the sub-synchronous vibration occurs with the running speed beyond the "Instability Threshold". According to Bently [2] and Muszynska [9], the instability occurs when the ratio of the critical speed of the system and the running speed $\left(\omega_{1} / \Omega\right)$ is around 0.47 , here $\omega_{1}$ is the $1^{\text {st }}$ bending critical speed and $\Omega$ is the running speed, which matches Ehrich's observation on the jet engine [3]. Aperiordic whirling motion or chaotic motion was reported by Childs [4] on the High Pressure Oxygen Turbo Pump (HPOTP) of the Space Shuttle Main Engine (SSEM), and 
by Ehrich [11] on an aircraft gas turbine. Numbers of typical nonlinear response in rotor dynamics from experiments are shown in Figs. 2.1 2.3.

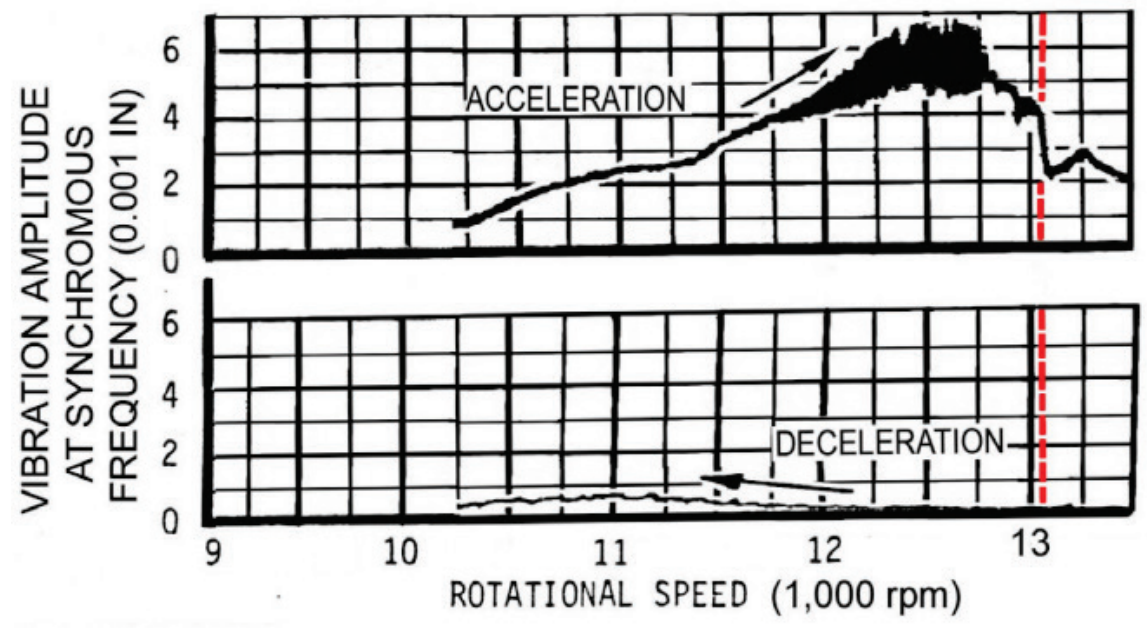

Figure 2.1 Vibrations during Acceleration and Deceleration [11]

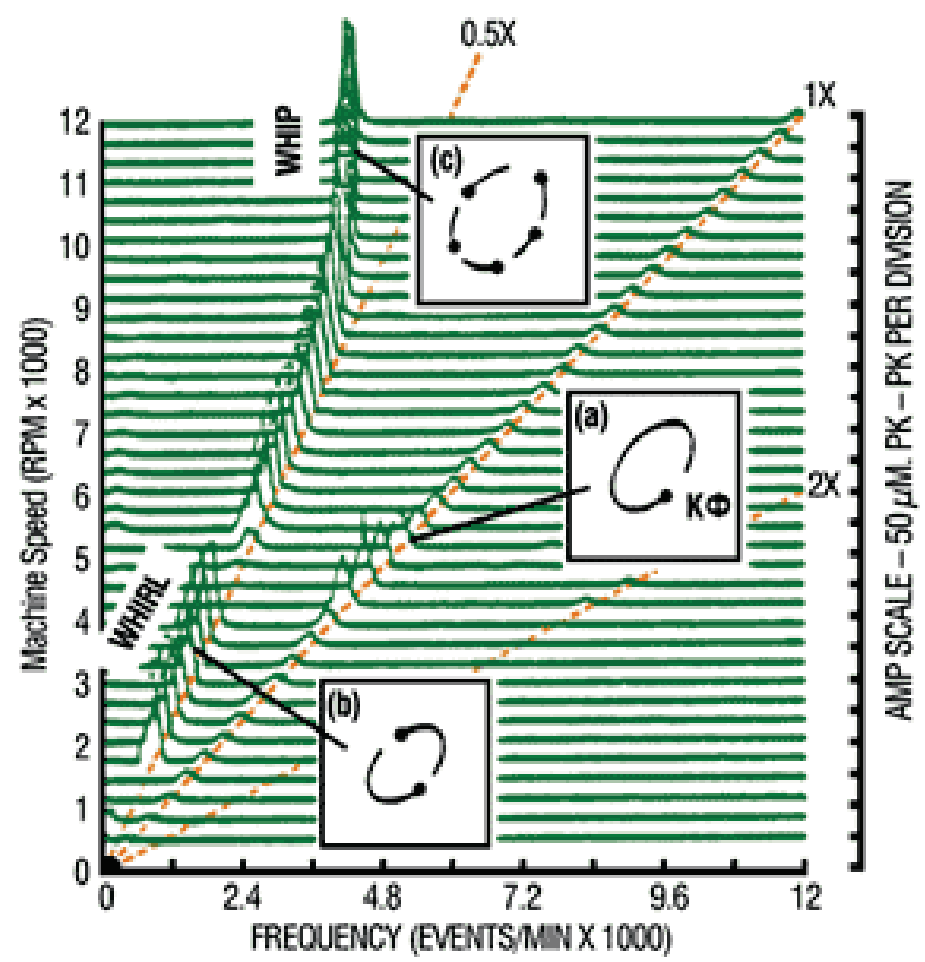

Figure 2.2 Developing of Oil Whirl and Oil whip [12] 


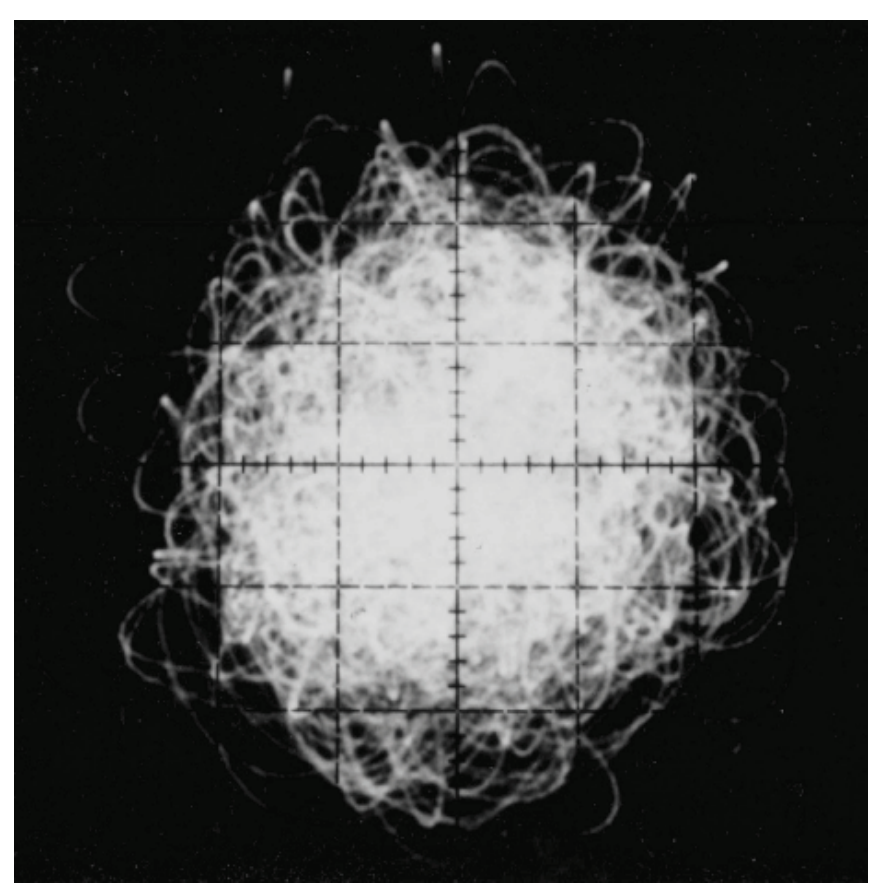

Figure 2.3 Chaotic Motions from Aircraft Gas Turbine [11]

\subsection{Rotor Dynamic System Modeling and Model Formulation}

Typically a rotor including a shaft and components attached is modeled as linear using the FE method. 2-D models using beam elements, or 3-D models using solid elements, have been developed in rotor dynamics. The supporting system, which includes bearings, seals, and squeeze film damper, is often modeled with 1) linearized stiffness and damping coefficients or 2) nonlinear forces, which depend on the instantaneous position and velocity of the system.

\subsubsection{Governing Equations of Rotor Dynamic System}

The governing equation of motion for a general rotor-bearing system, with a constant rotational speed, $\Omega$, is [13]: 


$$
M \ddot{u}(t)+\left(C_{s}+C_{b r g}+\Omega G\right) \dot{u}(t)+\left(K_{s}+K_{b}\right) u(t)=F(\dot{u}, u, \Omega, t)
$$

where $u$ is the system displacement vector to be solved, $M$ is the mass/inertia matrix, $C_{s}$ is the structural damping matrix, $C_{b r g}$ is the linearized bearing damping matrix, $G$ is the skew-symmetric gyroscopic matrix, $K_{s}$ is the structural stiffness matrix derived from strain energy, $K_{b}$ is the linearized bearing dynamic stiffness matrix, and $F$ is the force vector. The force vector contains all forces/moments, such as synchronous excitations due to the unbalance and the shaft bow, constant gravitational and/or static loads, applied forces due to blades loss and/or sudden loading, and all nonlinear interconnection forces.

The governing equation of motion of a variable operating speed system is [13]:

$$
M \ddot{u}(t)+[C+\dot{\varphi} G] \dot{u}(t)+[K+\ddot{\varphi} G] u(t)=\dot{\varphi}^{2} F_{1}(\varphi)+\ddot{\varphi} F_{2}(\varphi)+F_{3}(\dot{u}, u, \varphi, \dot{\varphi}, \ddot{\varphi}, t)
$$

where $\varphi, \dot{\varphi}$, and $\ddot{\varphi}$ are angular displacement, angular velocity, and angular acceleration/deceleration of the rotor dynamic system. All the linearized damping and stiffness terms are in the damping matrix $C$ and stiffness matrix $K$, and all the other interconnection nonlinear forces are included in $F_{3} . F_{1}$ and $F_{2}$ are function of $(\varphi, \dot{\varphi})$ and $(\varphi, \ddot{\varphi})$, respectively. The unbalance forces, $F_{1}$ and $F_{2}$, at the station with unbalance are:

$$
\left\{\begin{array}{c}
F_{u, x} \\
F_{u, y}
\end{array}\right\}=\dot{\varphi}^{2}\left\{\begin{array}{l}
m e_{u} \cos \varphi \\
m e_{u} \sin \varphi
\end{array}\right\}+\ddot{\varphi}\left\{\begin{array}{c}
m e_{u} \sin \varphi \\
-m e_{u} \cos \varphi
\end{array}\right\}
$$

For a system with constant rotational speed $(\dot{\varphi}=\Omega, \ddot{\varphi}=0)$, the transient response can be obtained by numerical integration algorithms directly in physical coordinates or by modal expansion using a linear transformation in modal coordinates. 


\subsubsection{Modeling and Assembling of Rotor Dynamic System}

A detailed history of rotor dynamics was described by Allaire [14] and Nelson [15]. Rotor dynamics has gone through more than 100 years since Rankine (1869). He incorrectly included that a rotor can only worked stably below its first critical speed, and this conclusion discouraged the development of rotor dynamics for almost 50 years. In 1919, Jeffcott [16] added damping to the rotor system and concluded that operation above the first critical speed was possible and stable, which conclusion was firstly given by Foppl in 1895 using an undamped rotor. With the subsequent series of accidents, however, people found that the rotor system would appear a strong vibration and led to instability caused by internal damping, oil whip and oil whirl, cross-coupling stiffness in bearings and seals, and gyroscopic effects. Two degrees of freedom (DOF) rigid rotor and Jeffcott rotor are still wildly used in rotor dynamics due to its simplistic formation and assumption to analyze the rotor system and those effects, especially are applied for the dynamic analysis of bearings and squeeze film dampers. However, with development and requirement of rotor dynamic analysis of more complex systems, such as large scale turbines, compressors and jet engines, the rigid rotor or Jeffcott rotor model would not accurate enough to model those systems, so more methods of modeling the complex rotor system were developed. Also difficulties of deriving analytical solution for complex rotor systems led to development of computational models, with development of computer in the 1960s and 1970s. Two methods developed of modeling rotor dynamic systems were the Transfer Matrix (TM) method and the well known Finite Element (FE) method.

The TM method, which needs smaller memory compare to the FE method, divided structural elements into linear arrangement $[17,18]$. This method could be used when the 
total system can be broken into a sequence of subsystems. However, this method has limitation of processing nonlinear dynamic problem. An improved TM method was developed to analyze a complex rotor-bearing system, in which the nonlinear forces were given in closed-form [19].

The FE beam model of rotor systems, which includes stiffness/damping crossing coupling, gyroscopic effects and nonlinear bearings, were developed using BernoulliEuler [20] or Timoshenko beam theory [21, 22] and taking advantage of the axis symmetry of rotor-bearing systems $[23,24]$. In this method, attached parts were modeled as lamped masses and moments of inertia; support parts such as bearings, dampers and flexible support were modeled as either linear by calculating equivalent coefficients or nonlinear by solving the Reynolds equation [25]. Although attached components are modeled as rigid, in general they have high natural frequencies far beyond the range of the operating speed and the flexible shaft's first or second critical speeds. Compare to the TM method, there is higher computational cost of using the FE method. However, the standard FE analysis couples the behavior of the flexible shaft, built on parts and the support system into a massive multidimensional model. The beam element model is widely used these days for rotor dynamic analyses. Modified beam elements have been applied for more complex geometries, such as hollow section, tapered beam section [26] and conical section [27].

To account more effects of the complex rotor dynamic system, which include the behavior of built on components, 3-D solid elements models have been developed in rotor dynamics $[28,29,30]$. However, those 3 -D models can easily reach a high order DOF in a gas turbine or a jet engine [31]. If there are multiple disks with blades, DOF of 
the system model can grow rapidly. Thus, the computational problems of without adequate computing memory or extremely computing time cost enforce researchers to find a way to reduce size of those complex systems. 3-D solid models are seldom applied to transient analyses nowadays.

\subsection{Methods of Nonlinear Rotor Dynamic Analysis}

For nonlinear transient analysis, despite the method of reducing model size, a more effective algorithm to calculate the nonlinear forces and to solve the equations of motion at the same time is needed. The unstable phenomenon caused by fluid bearings was first found by Newkirk in 1924 [32]. In 1965, J.W. Lund [6] analyzed the stability of a flexible rotor system, and proposed a linear oil film force model expressed by eight stiffness and damping coefficients. This method is widely used for stability analysis of rotor dynamic systems under steady state. The result calculated by this method, however, could not match various dynamic behavior caused by nonlinear effects. With the development of computer technology, there are a variety of algorithms to analyze and solve different dynamics problems $[7,8,33]$. Published results have shown that a rotorbearing system had many nonlinear phenomena, such as multi-frequency, quasi-periodic and chaotic vibration, which can't be explained by the linear theory $[34,35,36]$.

To study and analyze nonlinear phenomena observed in rotor dynamics, two main methods have been presented: one is analytical method to obtain close-form solution, which is applied to simple rotors with simplified nonlinear bearing models; another is numerical solution, which is applied to complex rotor-bearing systems. The FE method is 
the most common way to solve rotor dynamic problem of complex rotor-bearing systems in recent years.

The Jeffcott rotor, a 2 DOF model with all masses concentrated onto disk, supported by simplified nonlinear bearing model was commonly used for analytical solutions. A power series solution with low-order terms to approximate nonlinear characteristics was used on the system with weak nonlinearity to calculate harmonic and sub-harmonic response by Agrawal and Evan-Iwanowski [37], Yamamoto and Ishida [38]. A harmonic balance technique using a discrete Fourier transform, which was originally used by Chio and Noah [39], was developed by Kim and Noah [34, 40] to obtain synchronous and subsynchronous motions (super \& sub harmonics) on a Jeffcott rotor with bearing clearance. According to those papers, quasi-periodic motion and chaotic motion occur with increasing cross-coupling stiffness coefficients in those rotor models. Assuming small nonlinearity from nonsymmetrical bearing clearance, Child [41, 42] used a perturbation technique to study the occurrence of sub-harmonic behavior of a Jeffcott rotor. He explained the $1 / 2$ speed and the $1 / 3$ speed whirling motions in the rotor which subjected to the periodic normal-loss or normal-tight radial stiffness variation. An analytical solution for high order sub-harmonic response (up to ninth order) of a high speed Jeffcott rotor with bearing clearance was published by Ehrich [43]. In his model, the bearing stiffness coefficients kept same when the shaft's position was in the center area of the bearing and jumped to higher values when the shaft was close to the bearing's boundary. In 1991, using the same model, Ehrich [35, 44] calculated a bifurcation map of transition from second order sub-harmonic motion to chaotic motion with ratio of the running speed and the critical speed increasing. 
To study nonlinear vibrations of complex rotor systems with multi-degrees-of-freedom, various numerical and analytical methods were proposed: a harmonic balanced method and transfer matrix method by Yamauchi [17], Shiau and Jean [45], Kim and Noah [46, 47]; a trigonometric collection method by Nataraj and Nelson [48], Jean and Nelson [49]; a substructure synthesis method by Kobayashi, Saito and Yamauchi [50], Moon, Kim and Yang [51]; a FE method by more researchers $[25,52,53]$.

In addition to sub\&super harmonic whirling motions, Child [4] and Ehrich [11] reported that a nonlinear rotor system could show aperiodic whirling motion or chaotic response. An attempt to explain the occurrence of this chaotic motion in a Jeffcott rotor model was made by Day [54] using the multiple scale method. Day's analysis, however, failed to yield an adequate explanation for the periodic whirling motion because of the limitation of this method to highly nonlinear, non-analytic functions. Kim, Choi and Noah $[55,56]$ used a modified Jeffcott model to determine the onset of aperiodic whirling motion based on the bifurcation theory. Zheng [25] used a FE method to obtain the chaotic motion at a high range running speed. However, the calculated unstable threshold of the flexible rotor-bearing system was much higher than other papers.

Due to difficulties of deriving analytical solutions for complex rotor systems, numerical solutions based on the FE method have been developed in recent years $[25,52$, 53, 57, 58, 59]. Yamauchi [17] showed some nonlinear vibrations of a flexible rotor using a transform matrix method and a harmonic balance method. He compared his method with the Runge-Kutta method to show the new method's accuracy and efficiency. Using the FE method, Zheng [25] showed the nonlinear response of a multi-degree of freedom rotor with two plain journal fluid bearings. In his paper, quasi-periodic bifurcation was 
found for a group of bearing parameters. After the bifurcation point, a jump phenomenon was detected and the system appears a large number of sub-harmonic motions. As the rotating speed increases, the system undergoes torus, mode locking, torus bifurcation states, and finally goes to chaos. Compared to experimental data reported by Bently [2] and Ehrich [3], however, the calculated unstable threshold for this flexible rotor was too high (around 50,000 rpm). The transfer matrix method was used by $\mathrm{Gu}$ [60] to analyze the transient response of a large scale rotor-bearing system with ball bearings and nonlinear squeeze film dampers. Castro [61] showed a nonlinear solution to simulate the fluid induced instability during run-up and run-down due to oil whirl and oil whip of the bearing. Assuming laminar flow in short fluid bearings, he calculated nonlinear forces and then applied them to two flexible rotor models (horizontal and vertical). The instability thresholds were verified by comparing to experimental results. Chang-Jian et al. [62] calculated the long journal bearing forces and applied the nonlinear forces to the "dual flexible rotor", which actually a two disks Jeffcott rotor without considering gyroscopic effects. The system exhibited different types of periodic, quasi-periodic, and chaotic vibrations with the running speed increasing.

\subsection{Nonlinear Modeling and Nonlinear Forces Calculation}

For a fluid bearing or a squeeze film damper, the hydrodynamic force is nonlinear and is the function of the journal's displacement, velocity and acceleration. Due to oil whirl and oil whip, a plain journal bearing is often unstable under a high operating speed. To overcome this limitation, squeeze film dampers and tilting pad bearings were designed and developed. Squeeze film dampers are widely used in jet engines, in which ball 
bearings or roller bearings with few damping effects are used, to provide additional damping to systems. To eliminate the dynamic behavior of squeeze film dampers, the interaction of shaft, bearings and dampers needs to be described [52]. For tilting pad bearings, despite of the shaft motion, motions of bearing pads/pivots, as well as the assembling method of tilting pad bearings and the rotor system, has to be taken in consideration [63].

A review of journal bearings, especially for tilting pad bearings, was provided by Dimond $[64,65]$. The foundations of fluid film lubrication were established in 1886 by Reynolds [66]. He assumed that the flow could be treated as isoviscous and laminar flow and there was no pressure gradient across the fluid film. Then Reynolds' equation, which simplifies Navier-Stokes' momentum equations and the continuity equation for thin film lubrication, was derived:

$$
\frac{d}{d \eta}\left(h^{3} \frac{d p}{d \eta}\right)+\frac{d}{d z}\left(h^{3} \frac{d p}{d z}\right)=6 \mu\left[\left(U_{0}+U_{1}\right) \frac{d h}{d \eta}+2 V_{1}\right]
$$

where $\eta$ is the local coordinates direction along the slider, $h$ is the film thickness, $p$ is the developed pressure in lubricating film, $\mu$ is the lubricant dynamic viscosity, $z$ is the axial direction, $U_{0}$ and $U_{l}$ are relative velocities between sliders in $\eta$ direction, and $V_{1}$ is the squeeze velocity between sliders. According to the boundary condition, if the pressure profile could be solved, the nonlinear bearing forces could be calculated by integrating the pressure over the fluid film area.

A closed-form solution of the Reynolds equation was given by Sommerfeld [67] in 1904, and then the fluid force expression was given by Harrison [68] in 1913. Newkirk 
[32] in 1924 took the fluid film bearing into the problem of rotor dynamic stability, and then long bearings and short bearings were investigated by Robertson [69] and Cardullo [70] in 1930s. The transient analysis of a plain journal bearing, based on analytical expression of the bearing force, was presented by Badgley [71] for the long bearing, then by Kirk and Gunter [72] for the short bearing. The FE method [73, 74] was used to solve the flow problem in 1970s. Using the FE method with different boundary conditions, the linear Reynolds equation was solved to calculate the pressure profile on fix-pad bearings $[75,76]$, tilting pad bearings $[77,78]$, thrust bearings [79], and squeeze film dampers [52]. Effects of the fluid inertia, which was neglected in the Reynolds' equation, but existed in those fluid momentum equations, were approached by Ballal and Rivlin [80], San Andres and Vancea [81], Tichy and Bou-Said [82], and Kim and Szeri [83]. Two main methods, the perturbation analysis and the method of averaged inertia, were developed in those analyses.

Some applications [84] used water or other low-viscosity fluid as the bearing lubricant, which results in a turbulent lubricating flow problem (a typical Reynolds number $(R e)$ of 1000-1500). The Ng-Pan-Elrod model, which was first attempted by Boussinesq in 1877 and developed by $\mathrm{Ng}$ in 1964 [85, 86], introduced a non-constant "eddy viscosity" to modify Stokes constitutive equations for laminar flow. This method was used by Branagan $[75,76]$ and $\mathrm{He}[77]$ in solving the momentum equation of the fixed geometry journal bearing and the tilting pad bearing considering the turbulence flow via the FE method.

The Reynolds equation focused solely on the hydrodynamic flow field problem. Later research included the simultaneous solution of the energy equation, resulting in the 
thermo-hydrodynamic analysis [77]. Another refinement to the problem was to include mechanical deformation effects due to the applied pressure and the thermal growth, resulting in the thermo-elasto-hydrodynamic theory $[65,77,87,88]$.

Other than calculate those linearized equivalent coefficients after solving the pressure file, some publications focused on the nonlinear dynamic behavior of bearings and squeeze film dampers $[89,90]$. Laha et al. [91] showed the nonlinear dynamic analysis of a Jeffcott rotor under unbalance excitation. The rotor mounted on two porous oil journal bearings at the two ends. The Hopf Bifurcation Theory (HBT) was used for the stability analysis and the system undergoes Hopf bifurcation as the running speed increases. Chang-Jian et al. published a nonlinear analysis of a rigid rotor supported with nonlinear squeeze film dampers [92].

\subsection{Nonlinear Time Transient Analysis in Rotor Dynamics}

Nonlinear time transient analyses involve not only time transient dynamics of high speed rotating machinery, but also coupling of the rotating structure with the non-rotating support structure. In general, due to small shaft displacement compare to the rotor's diameter and length $(1 \mu \mathrm{m} / \mathrm{mm}$ is a typical clearance ratio for fluid film bearings), typically the rotor is modeled as linear, and the applied forces due to sudden loading, blade loss and nonlinear interconnection (bearing, damper, flexible support etc.) are modeled as nonlinear. In most applications, nonlinear interconnection forces are linearized to full dynamic coefficients or stiffness and damping matrices (2 by 2 ). In some conditions, such as startup, shutdown, going through critical speeds, and large 
bearing eccentricity due to severe unbalance forces, nonlinear time transient analyses of rotor-bearing systems were applied to obtain dynamic behavior of systems [52, 53, 93].

Traditionally, two approaches have been applied to nonlinear rotor dynamic transient analyses based on FE method: 1) direct formulation and integration of governing equations, and 2) modal formulation and integration of governing equations. In the first approach, based on geometric, mass, inertia, stiffness, and damping properties of the rotor and its supports, equations of motion were assembled in terms of nodal displacements, velocities and acceleration. Those physical equations are subsequently integrated in time domain using an explicit or an implicit iterative method, such as Wilson method, Newmark method and Runge-Kutta method [33, 73, 94]. In the second approach, modal formulation of equations of motion was derived by reducing DOF of the system and keeping high accuracy of original full model at the same time. In general, a model reduction method needs to be carefully chosen for the nonlinear system $[25,53$, 95]. At each step, the modal method solved the transient problem in modal coordinates and then transferred modal displacement and velocity vectors to physical vectors through a transfer matrix, so that nonlinear forces were calculated based on those vectors in physical coordinates.

\subsubsection{Stability and Periodic Response of Nonlinear System}

For a Linear Time Invariant (LTI) system, the system stability is determined by characteristic equations, from which eigenvalues and eigenvectors are calculated. The imaginary part of an eigenvalue gives a natural frequency or critical speed of the system, and the real part of an eigenvalue indicates stability of the system: negative number 
means stabile [14]. For the Nonlinear Time Variant (NTV) system, however, characteristic equations are time dependent. Instead of calculating eigenvalues and eigenvectors directly, Floquet theory or Floquet multiplier [96] is often used for stability analysis of the periodic solution. If all Floquet multipliers are located inside the unit circle, the system is stable. The shooting method [25], which is a method for solving a boundary value problem by reducing the problem to the solution of an initial value problem, is often used to solve Floquet multipliers. Some other terms, such as Poincáre map, bifurcation (branching), plane map, trajectory map, and power spectrum (Fourier transform), are often used in nonlinear periodic response analyses [96].

\subsubsection{Direct Method in Nonlinear Time Transient Analysis}

Direct method is more applied for low dimensional nonlinear rotor systems. Due to diversity of nonlinear differential equations of rotor dynamic systems, different methods are applied. Classical methods, which approach the periodic solution of the weakly nonlinear dynamic system, include the harmonic balance method and the small perturbation method [17, 45, 46, 97]. Those analytical methods showed super \& sub harmonic motions based on the Jeffcott rotor model with bearing clearance. Numerical methods are effective to solve dynamic equations of low dimensional nonlinear rotorbearing systems. Based on the shooting method and the Floquet stability theory, the nonlinear numerical method was proposed to approach the unbalance periodic solution and to approach the stability analysis of the rotor-bearing system [89]. In those solutions, 2-DOF rigid rotors or Jeffcott rotors were used, and instability due to oil whirl and /or oil whip under various rotational speeds were shown. 
Direct integration method based on the FE method was used by Kirk [72] and Shen [98] on a single multi-mass rotor. Choy [99] used direct integration to solve the motion of a simple three-mass rotor system. He abandoned the direct integration and turned to a modal formulation for the general problem due to high computational costs. Adams [100] preformed a transient vibration analysis of a complex rotor-bearing system with highly nonlinear effects from journal bearings using direct method. The failure mode analysis due to large vibration amplitudes from the rotor, such as high imbalance, critical speed operation, and journal bearing dynamic instability, was presented with comparing with experiment. In of those cited studies, gyroscopic effects were neglected and proportional damping was assumed.

A continuous beam model for a symmetric simple rotor-bearing system with nonlinear bearing forces was developed by Jing [58] and dynamic response were calculated using the Newmark- $\beta$ method. A nonlinear model of a symmetric rotor with nonlinear bearings and a nonlinear seal was proposed for a steam turbine system by Li [101]. Instead of calculating nonlinear forces directly according to the shaft displacement and velocity, stiffness and damping coefficients were calculated at each step through analytical equations. The unsteady bearing oil-film force model was applied and the Runge-Kutta method was used to solve equations of motion.

\subsubsection{Modal Method and Nonlinear Model Reduction}

For nonlinear transient analysis of a large order complex rotor-bearing system, the numerical solution requires to solve differential equations with high DOF, which is computationally expensive in terms of time and storage. Very often nonlinear equations 
of motion are linearized at an operating point and the DOF of the system is reduced using a model reduction method, and then the reduced model is solved for the transient response. Using of the model reduction is based on the fact that "in many situations the large numbers of DOF of freedom in the complex system are dictated by their topology, or rapid changes in system properties, rather than by the expected complexity of the response" [102]. This fact has been recognized, and techniques for reducing the DOF have been proposed for a variety of engineering and mechanics problems.

From reviews of model reduction method by Noor [102] and Wagner [103], the most common methods used for linear rotors are Guyan Reduction [74, 104], Model Analysis $[95,59]$, and Component Mode Synthesis $[105,106]$. The main difficulties presented in rotor dynamic systems are the inclusion of non-symmetric stiffness, non-proportional damping and skew-symmetric frequency dependent gyroscopic effects [5]. Beyond those difficulties, how to deal with nonlinear forces in a rotor dynamic system is a challenge for a successful model reduction of a nonlinear system.

Steady state dynamic analysis of the nonlinear rotor-bearing system, which includes journal bearings and squeezes film dampers, was addressed using different methods. Those methods include the harmonic balance method [39], the component mode synthesis (CMS) [107], and the shooting method [47]. The Floquet theory was applied to calculate stability, unbalance forced response, and bifurcation of the periodic response of the nonlinear rotor system with periodic response.

Guyan reduction [74], also is known as static reduction, reduces the system order by designating the DOF into masters and slavers. The slave coordinates are assumed to have 
low inertia, which can be ignored, so it is dictated by the motion of masters and the stiffness matrix. In this way the dimension of the FE model was reduced directly to the DOF of the masters. Guyan reduction method has been proposed to reduce the DOF of several linear flexible rotor-bearing systems for standard rotor dynamic analyses [103].

Component mode synthesis (CMS) $[74,108]$ is a method by which a multi-degree of freedom (MDOF) system is represents by dividing the system into some smaller substructures. Each substructure is analyzed and reduced individually and then those substructures are assembled into a full system model for a dynamic analysis. To apply the CMS method, the weak coupling between substructures should be satisfied. Nelson [107] first applied the CMS method for reduction of a large order rotor dynamic system and used this method to calculate system eigenvalues and the stability of a rotor. He $[105,106]$ extended the CMS procedure to calculate the periodic transient response of a nonlinear rotor system. Sundararajan and Noah [47] developed the fixed-interface CMS procedure to calculate the response of a MDOF system with strongly local nonlinearities. The DOF associated with non-linear elements were retained in physical coordinates, while all others, whose number far exceeds the number of non-linear DOF, were transformed to modal coordinates and were reduced using the real mode CMS. Apiwattanalunggarn [109] developed a nonlinear extension of the fixed interface CMS method. Nonlinear normal modes are calculated and then applied to a large scale structural system based on the FE method. The undamped model truncation error was studied by Li and Gunter using the CMS method on a dual rotor system engine [110]. They suggested that the selection of modes to be kept should be based on an upper frequency -- 4 to 5 times of the rotor's operating speed. 
Galerkin reduction method, a projection-based order reduction, has been widely used in structural dynamics $[111,112,113]$. The Galerkin method is a very effective approach to reduce the dimension of rotor-bearing systems [114]. Matthies and Meyer [115] applied a nonlinear Galerkin method for a finite element model of a horizontal axis wind turbine. A nonlinear post-processed Galerkin method based on the model truncation and the calculation of Approximate Inertial Manifolds (AIM) were proposed. The numerical analysis showed that the post-processed method was valid in dealing with the dimension reduction of the rotor-bearing system with highly accuracy. Fuchs [116] applied the Galerkin method to the order reduction of a real-time diesel engine model. Both linear Galerkin method and nonlinear Galerkin approximations were approached by projecting the full model onto a lower dimensional sub-space.

According to local nonlinearities of flexible rotor dynamic systems, an order reduction and the corresponding integral method for the dynamic transient response was proposed by Zheng [25]. The FE method based on variational inequality was used to solve the Reynolds boundary problem for the fluid bearing at each time step. Another model truncation method, named residual flexibility truncation, which used both the model truncation and the Guyan reduction, was presented by Cook et al [74, 104, 108] first and then developed by Hassenpflug [53, 117] for nonlinear lateral analysis of a rotor under acceleration, and by Orsey [93] for nonlinear torsional analysis of the flexible rotor. After the model truncation, the transfer matrix between global coordinates and modal coordinates was divided into masters and slaves for the Guyan reduction to obtain a square transfer matrix. Then the reduced model in modal coordinates is transferred back to global coordinates. In this way, "unimportant" information in matrices was removed. 


\subsection{Nonlinear Transient Analysis Methods Summary}

Early nonlinear transient analyses used the 2 DOF rigid rotors or Jeffcott rotors with simple nonlinear bearing models to obtain analytical solutions. Flexible rotor-bearing models based on FE method have been developed for transient analyses in recent years. In general, when a flexible rotor was applied, to avoid complex eigenvalue \& eigevector problems, often gyroscopic effects were neglected and a proportional damping was assumed for nonlinear transient analyses.

The short plain journal bearing model was often used to calculate the nonlinear effect. The flexible rotor system with more complicated bearings/dampers has not been involved. Instead of the calculating nonlinear forces of specific positions and velocities at each time step, the small perturbation method to calculate bearing/damper stiffness and damping coefficients was used by some researchers in the nonlinear transient analysis.

In general, the lateral analysis was approached for constant running speed and the torsional analysis was applied for variable rotational speed case. Coupled lateral, torsional and axial analysis has not been involved.

Few nonlinear model reduction methods were applied to the flexible nonlinear rotor dynamic system. Guyan reduction, CMS, and model truncation (Galerkin method) were applied to some nonlinear rotor dynamic systems by neglecting gyroscopic effects, assuming proportional damping and solving constant rotational speed problem. 


\section{Chapter 3}

\section{Nonlinear Flexible Rotor-Bearing Systems:}

\section{Modeling, Assembling and Solution}

\subsection{Introduction}

The method of modeling and assembling a flexible rotor with different types of nonlinear bearing forces/moments is described in this chapter first. Then the time transient solver for the nonlinear equations of motion using the $4^{\text {th }}$ order Runge-Kutta method is introduced. A flexible rotor with built on components is modeled as linear. The supporting systems, including fluid bearings and squeeze film dampers, and the external torsional forces from motor are treated as nonlinear.

In this dissertation, three distinct steps are considered in the nonlinear transient rotor dynamic analysis:

1) The dynamic structural model, including mass, damping, gyroscopic, and stiffness matrices of the linear rotor with multi-degrees of freedom. 
2) The linear and nonlinear applied loads, including shaft bow forces, unbalance forces, constant gravitational forces, nonlinear bearings/dampers forces, and external nonlinear torsional forces/torques.

3) The assembly method of the system and the integration method used for the equations of motion describing behavior of the system.

\subsection{Structure Modeling and Assembling}

\subsubsection{Dynamic Model of Linear Rotor}

To calculate the coupled lateral, torsional and axial analysis, an element with 12 degrees of freedom (DOF) is developed to model the linear shaft of the complex rotor system based on the finite element (FE) method. Built on components, such blades, discs and impellers, are modeled as lumped masses and moments of inertia. The global coordinates and a 12 DOF element used for shaft modeling are shown in Fig. 3.1.

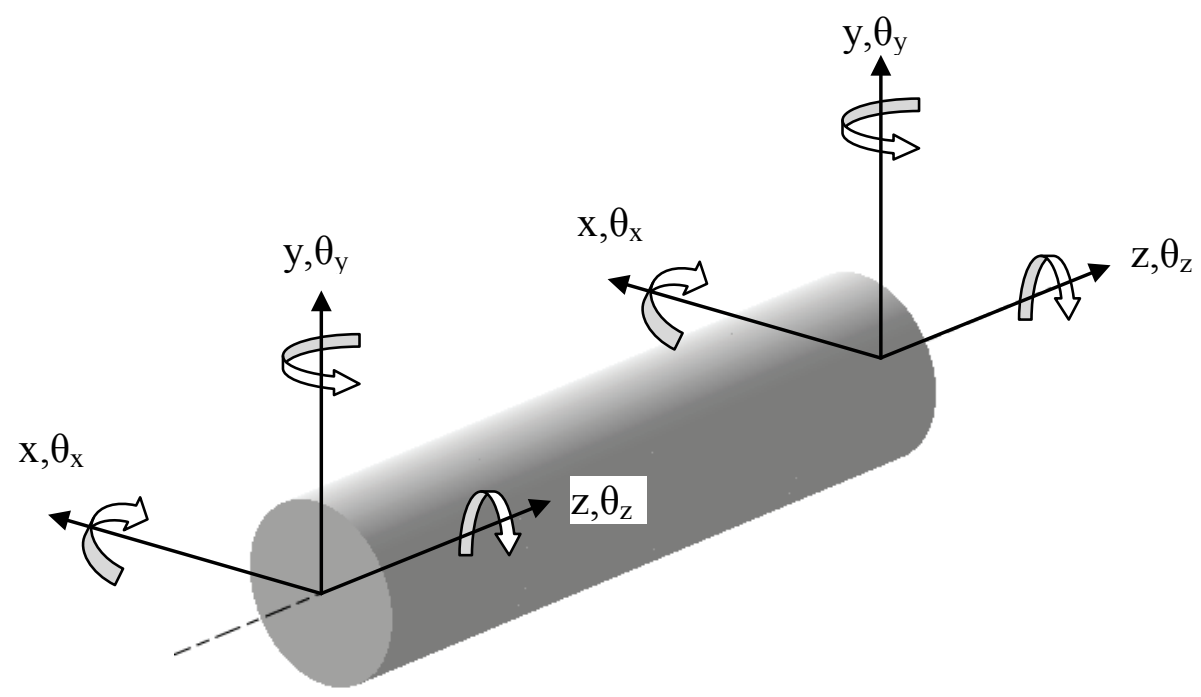

Figure 3.1 12-DOF Element and Coordinates for Modeling Linear Rotor 
There are some restrictions on the use of this linear model for a time transient analysis, which are listed as following:

1) Right hand coordinate system is assumed and applied individually to the rotor and the supporting system.

2) For the linear rotor model, the motion of each node has 6 DOF. The displacement vector of each node is $\left(z, x, y, \theta_{z}, \theta_{x}, \theta_{y}\right)$. An additional node with 2 DOF $\left(x_{s d}, y_{s d}\right)$ is added for each squeeze film damper (SFD). For tilting pad bearing (TPB), one node with 2 DOF $(\delta, \gamma)$ for each pad/pivot is added. An additional node with 2 DOF $\left(x_{f d}, y_{f d}\right)$ is added for each finite length squeeze film damper (FLD).

3) The shaft is assumed to be axial symmetric and modeled with a Timoshenko beam element for lateral directions $\left(x, y, \theta_{x}, \theta_{y}\right)$ and a linear element for axial and torsional directions $\left(z, \theta_{z}\right)$. The built on components, such blades, discs and impellers, are modeled as lumped masses plus polar and transverse moments of inertia.

4) Proportional internal structural damping is considered in this dissertation. Gyroscopic effects, which are function of rotational speed and acceleration, are taken into consideration.

5) Bearings or squeeze film dampers can be treated either linear or nonlinear components. If they are treated as linear, linearized stiffness and damping coefficients of the bearings or/and the squeeze film dampers are used. If they are treated as nonlinear, stiffness \& damping effects from the bearings or/and the squeeze film dampers are described by the nonlinear bearing/damper forces. 
The governing equations of motion for the linear rotor with additional nodes for nonlinear components and nonlinear forces are shown in Eq. (3.2.1). In the equation, the stiffness matrix $K$ and the damping matrix $C$ are from linear components only.

$$
M \ddot{u}+(C+\Omega G) \dot{u}+(K+\dot{\Omega} G) u=F_{u}(t, \Omega, \dot{\Omega})+F_{b o w}(t, \Omega, \dot{\Omega})+F_{g}+F_{n}(u, \dot{u}, t)
$$

The displacement vector includes shaft, short squeeze film dampers, pads/pivot of tilting pad bearings and finite length squeeze film dampers is:

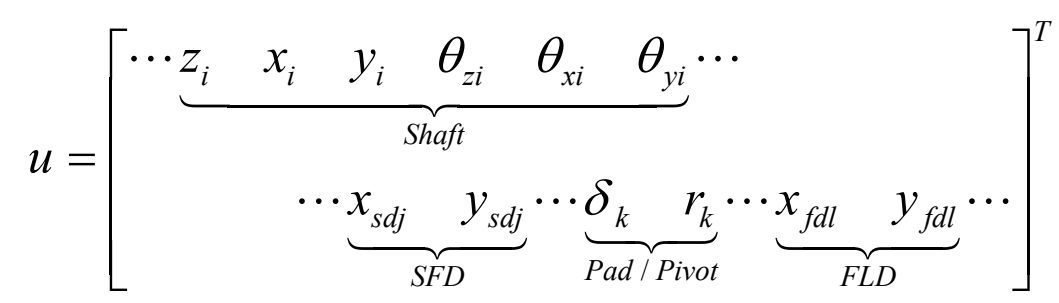

Linear FE shape function is used for mass, damping and stiffness matrices in axial and torsional directions. Based on the full Timoshenko beam differential equations (including shear deformation, rotary inertia, and gyroscopic terms), the potential and kinetic energy formulations are substituted into the Lagrange's equation to obtain the element mass, stiffness, and gyroscopic matrices in lateral direction. Generating of nodal mass, stiffness, and gyroscopic matrices of a linear rotor is shown in Appendix A.

\subsubsection{Method of Assembling Rotor Dynamic System}

The entire rotor dynamic system is separated into linear and nonlinear subsystems first. Each subsystem is modeled separately and then assembled together. Assembling of the entire system is accomplished layer by layer. The first layer is the linear rotor including shaft and built on components; the second layer is the linear supporting components including linear bearings, dampers, couplings and gearboxes; the third layers is any 
nonlinear supporting system, including nonlinear bearings, nonlinear dampers and nonlinear external forces/torques. Steps of assembling the rotor-bearing system are listed as following:

1) Generate the linear shaft and built-on disk matrices of each element using the combined Timoshenko element ( $x, y, \theta_{x}$ and $\theta_{y}$ directions) and linear mass-spring element ( $z$ and $\theta_{z}$ directions).

2) Assemble the linear rotor including the shaft and all built-on components to generate global matrices.

3) Adding linear bearing, coupling, gearbox and damper into global matrices if linear supporting components are included; adding an additional 2 DOF node for each damper.

4) Adding nonlinear components into the system. If at the same location there are both a linear bearing and a nonlinear bearing, the linear one will be replaced by the nonlinear bearing (the linear stiffness \& damping coefficients are replaced by the nonlinear forces).

\section{Assembly of linear rotor}

Assembly of the finite element rotor into global mass, damping, gyroscopic, and stiffness matrices is explained in details by Chen and Gunter [13] as shown in Fig. 3.2. Here a similar method is used, but the difference is that for each element, a 12 by 12 matrix instead of an 8 by 8 matrix [13] is applied due to the 12 DOF of each element.

\section{Adding linear components}

The linear bearings, couplings and gearboxes are modeled with equivalent stiffness and damping coefficients, which are two 6 by 6 matrices if the component connects to the 
ground or 12 by 12 matrices if the component connects two different nodes. If the rotational speed is constant, the coefficient matrices are added into the global stiffness matrix and the global damping matrices according to locations of those linear components in the system. If the rotational speed varies with time, the stiffness and damping coefficients won't be added into the global stiffness/damping matrices directly. Those coefficients are calculated based upon the current rotation speed using a linear interpolation method. Then the component forces are calculated from those coefficients, as well as the current nodal displacement and velocity in the time transient solver. This will be discussed further in Section 3.5.

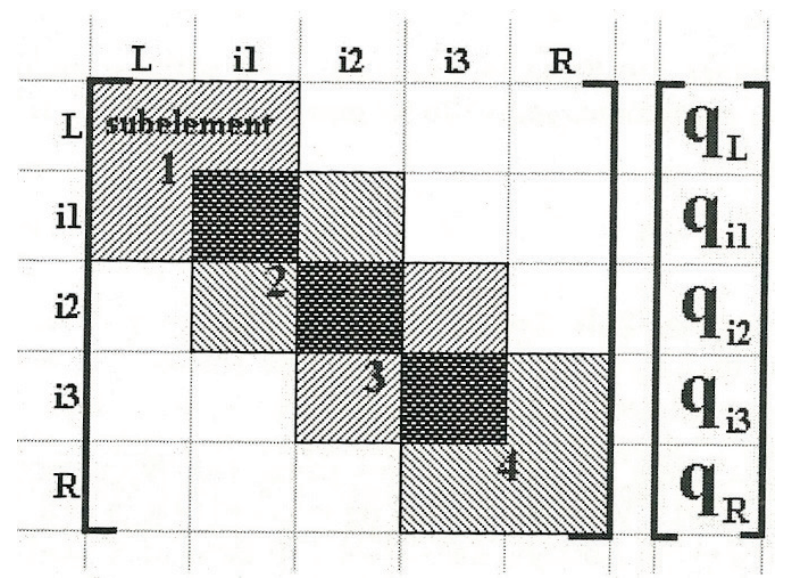

Figure 3.2 Assembly of Finite Element Shaft Matrix into Global Matrix [13]

\section{Adding nonlinear components}

In this dissertation, four nonlinear components including short journal bearings $(\mathrm{L} / \mathrm{D} \leq 0.5)$, short squeeze film dampers $(\mathrm{L} / \mathrm{D} \leq 0.5)$, tilting pad bearings, and finite length dampers, are taken into consideration. Nonlinear models will be added into the assembly method when needed. A general support component model is shown in Fig. 3.3. The rotor is supported with two bearings and there is a squeeze film damper on each bearing. The 
method of adding nonlinear components is discussed case by case. Because linear bearings have been added into the system during last step, it is possible that a linear bearing has been added to the position of a nonlinear bearing. If this condition occurs, the linear bearing will be removed firstly and the nonlinear bearing will be added into. The procedure of replacement of a linear bearing with a nonlinear bearing at same location is listed in Appendix B.

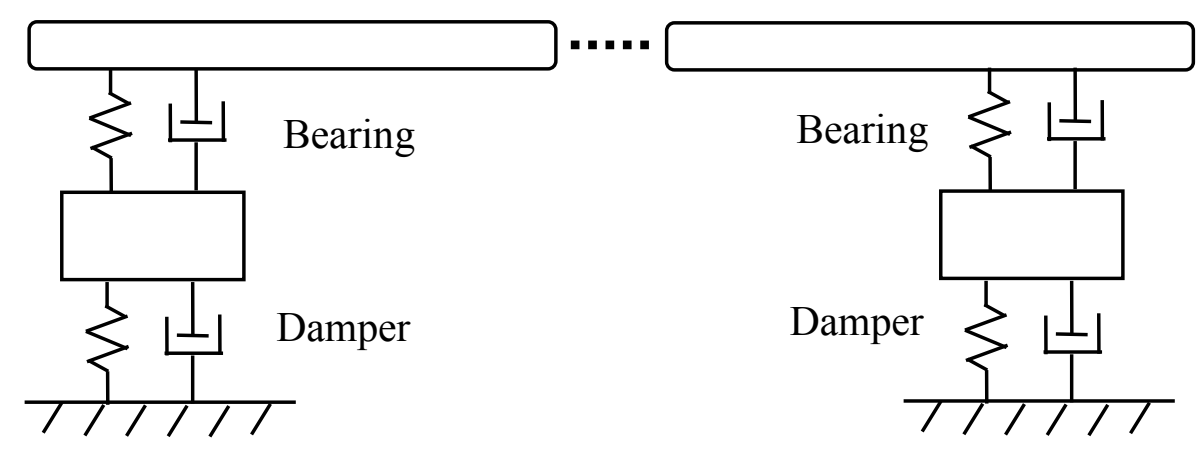

Figure 3.3 Rotor Supported with Bearings and Dampers

\section{Nonlinear short journal bearing only}

In this case, no additional node is needed during the assembly, the nonlinear bearing force $\left(F_{b r g}\right)$ is added to the force vector according to the nonlinear bearing location. Eq. (3.2.3) shows this method of assembling a nonlinear plain journal bearing, which connect to shaft at the $\mathrm{i}^{\text {th }}$ node.

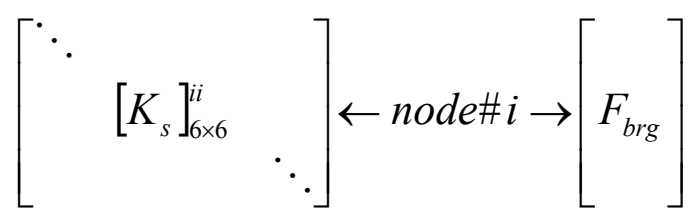

where $\left[K_{s}\right]^{i i}$ is the linear shaft stiffness coefficients of node $i$, and $F_{b r g}$ is the nonlinear bearing forces. 
A similar method applies to the bearing located between two nodes, node $\# i$ and node $\# j$, for example. The opposite nonlinear bearing forces of the bearing at node $\# i$ and $\# j$ are calculated at each time step. The force vector is changed by adding the nonlinear bearing forces to the bearing locations, shown in Eq. (2.3.4).

$$
\left[\begin{array}{ccccc}
\ddots & & & & \\
& {\left[K_{s}\right]^{i i}} & \ldots & {\left[K_{s}\right]^{j j}} & \\
\vdots & \ddots & \vdots & \\
& {\left[K_{s}\right]^{j i}} & \ldots & {\left[K_{s}\right]^{j j}} & \\
& & & & \ddots
\end{array}\right] \leftarrow \text { node\# } i \rightarrow\left[\begin{array}{c}
\vdots \\
F_{\text {brg }} \\
\vdots \\
-F_{\text {brg }} \\
\vdots
\end{array}\right]
$$

where $\left[K_{s}\right]^{i i},\left[K_{s}\right]^{j i},\left[K_{s}\right]^{j i},\left[K_{s}\right]^{j j}$ are the linear shaft stiffness and damping coefficients of node $i \& j$, and $F_{b r g}$ is the nonlinear bearing forces.

\section{Nonlinear short journal bearing and nonlinear squeeze film damper}

In this case, to describe behavior of the damper, one additional node for each damper is needed. The bearing inside each damper can move in both horizontal direction and vertical direction, so a two-degree of freedom node for each damper is added. If the total number of dampers is $S$, the assembled global mass matrix is shown in Eq. (3.2.5), and the global mass matrix size expands from $6 \mathrm{~N} * 6 \mathrm{~N}$ to $(6 \mathrm{~N}+2 \mathrm{~S}) *(6 \mathrm{~N}+2 \mathrm{~S})$ as well.

$$
\left[\begin{array}{ccc}
{[M]_{6 N^{*} 6 N}} & & {[0]} \\
{[0]} & {\left[\begin{array}{ccc}
M_{s d}^{1} & & \\
& \ddots & \\
& & M_{s d}^{s}
\end{array}\right]_{2 S^{*} 2 S}}
\end{array}\right]
$$

where $[M]$ is the linear shaft mass matrix, $M_{s d}^{1} \cdots M_{s d}^{1}$ are masses inside the nonlinear squeeze film dampers respectively. 
To balance the dimension of the mass matrix, the global gyroscopic matrix expands to the same size of the mass matrix by adding zeros. The global stiffness and the global damping matrices expand to the same size of the global mass matrix to balance the added squeeze film damper nodes as well. The force vector is changed by adding the nonlinear bearing force and the nonlinear damper force to the bearing node and the damper node respectively. Also the NEGATIVE bearing force has to add to the damper's node, because the bearing force applies to both the shaft inside the bearing (positive) and the bearing inside the damper (negative). A similar method applies to the global damping matrix as well. Eq. (3.2.6) shows the method of assembling the rotor, a nonlinear short journal bearing and a nonlinear short squeeze film damper, for which the added node is node $\# \mathrm{j}$. Additional nodes to describe the behavior of the bearing inside the damper are shown in Fig. 3.3.

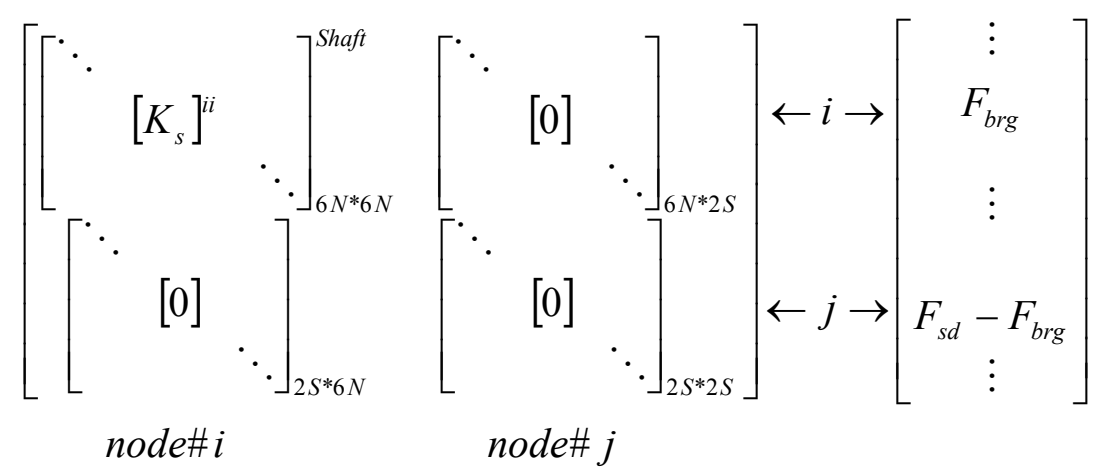

where $F_{b r g}$ is the nonlinear bearing force, $F_{s d}$ is the nonlinear squeeze film damper force.

\section{Nonlinear tilting pad bearing only}

Tilting pad bearings are more complicated than fixed pad journal bearings because the pads/pivots behavior is coupled with the shaft inside. To describe the coupled behavior of the rotor and the tilting pad bearings, additional nodes for all bearing pads and pivots are 
needed. Eq. (3.2.7) shows the assembling method of the global mass matrix, where the linear rotor has $N$ nodes and the tilting pad bearing has $R$ nodes ( $R$ pads and $R$ pivots). The total DOF of the system is $6 N+2 R$.

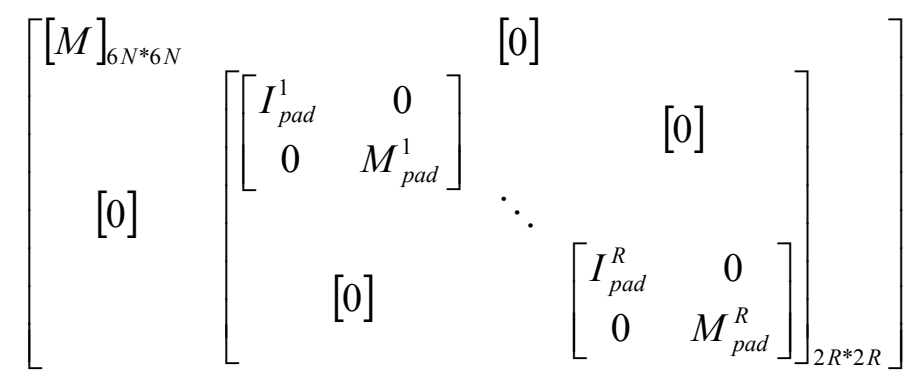

where $M_{p a d}^{1} \cdots M_{p a d}^{R}$ and $I_{p a d}^{1} \cdots I_{p a d}^{R}$ are masses and moments of inertia of all pads.

If a tilting pad bearing connects to ground directly the nonlinear bearing force $\left(F_{b r g}\right)$, the pad force $\left(F_{p}\right)$ and the pivot moment $\left(M_{p}\right)$ are added to the force vector, as shown in Eq. (3.2.8).

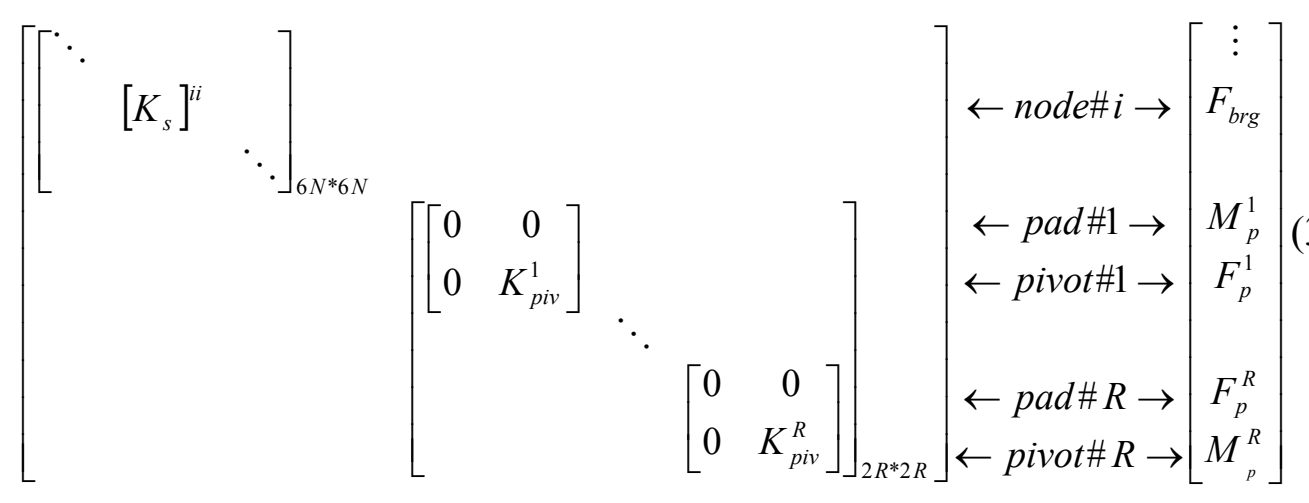

where $K_{p i v}^{1} \cdots K_{p i v}^{R}$ are the pivot stiff nesses, $M_{p}^{1} \cdots M_{p}^{R}$ are the pad moments, $F_{p}^{1} \cdots F_{p}^{R}$ are all pad force.

If the tilting pad bearing connects between two different nodes (node $\# \mathrm{i}$ and $\# \mathrm{j}$ ), the assembled stiffness matrix is shown in Eq. (3.2.9). The assembly of global damping 
matrix is similar to that used with the with stiffness matrix. The global gyroscopic matrix is expanded to same size as the global mass matrix by adding zeros since there is no gyroscopic effect from the tilting pad bearing.

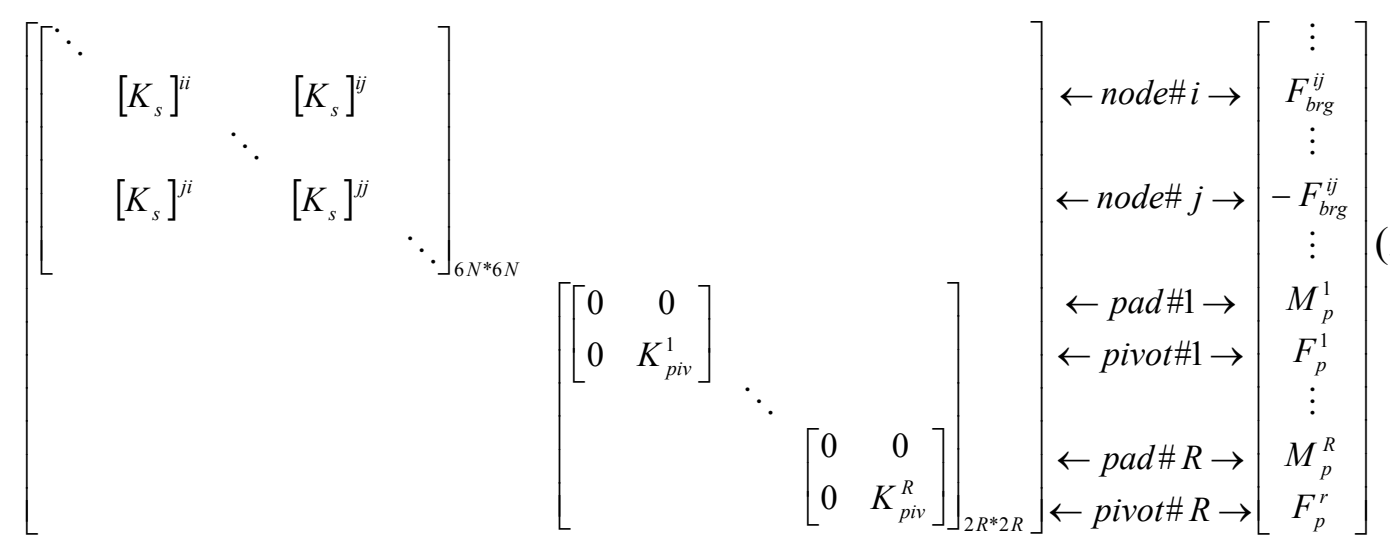

\section{Nonlinear tilting pad bearing and nonlinear finite length damper}

If both the bearing and the damper at same location are treated as nonlinear components, the mass matrix adds additional nodes for the bearing pads and pivots to describe behavior of the tilting pad bearing first, then 2-DOF nodes for all the dampers are added into the global mass matrix as shown in Eq. (3.2.10). To balance the dimension of the global mass matrix, the global gyroscopic matrix expands to the same size of the mass matrix by adding zeros to those additional nodes since there are no gyroscopic effects from either tilting pad bearings or finite length dampers.

The global stiffness matrix expands to the same dimension of the global mass matrix to balance the added nodes for the bearings and the finite length dampers. For each pivot node, the pivot stiffness is added to couple the pivot behavior into the system, as shown in Eq. (3.2.11). The force vector is changed by adding the bearing forces, the pad forces, the pivot moments, and the damper forces to the bearing nodes, the pad nodes, the pivot 
nodes and the damper nodes respectively. The NEGATIVE bearing force needs to be added to the damper's node as well, because the bearing force applies to both the shaft inside the bearing and the bearing inside the damper. The Eq. (3.2.11) shows the method of assembling the rotor, the tilting pad bearing and the finite length damper, which are located on $\mathrm{i}^{\text {th }}$ node of the shaft. The additional node for the finite length damper (node $j$ ) is shown in Fig. 3.3. A similar method applies to the global damping matrix.

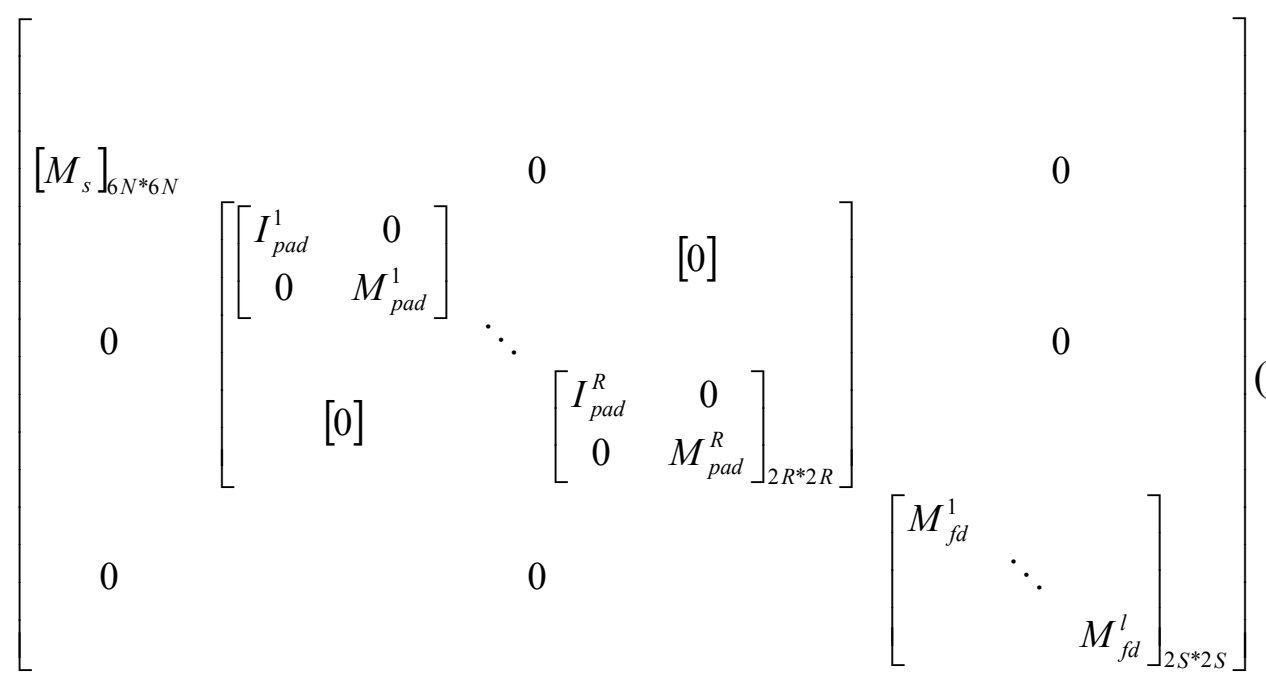

Rotor

Bearing

Damper

where $M_{p a d}^{1} \cdots M_{p a d}^{R}$ are the masses of all pads, $I_{p a d}^{1} \cdots I_{p a d}^{R}$ are the moments of inertia of all pads, $M_{f d}^{1} \cdots M_{f d}^{l}$ are the masses of all finite length squeeze film dampers. 


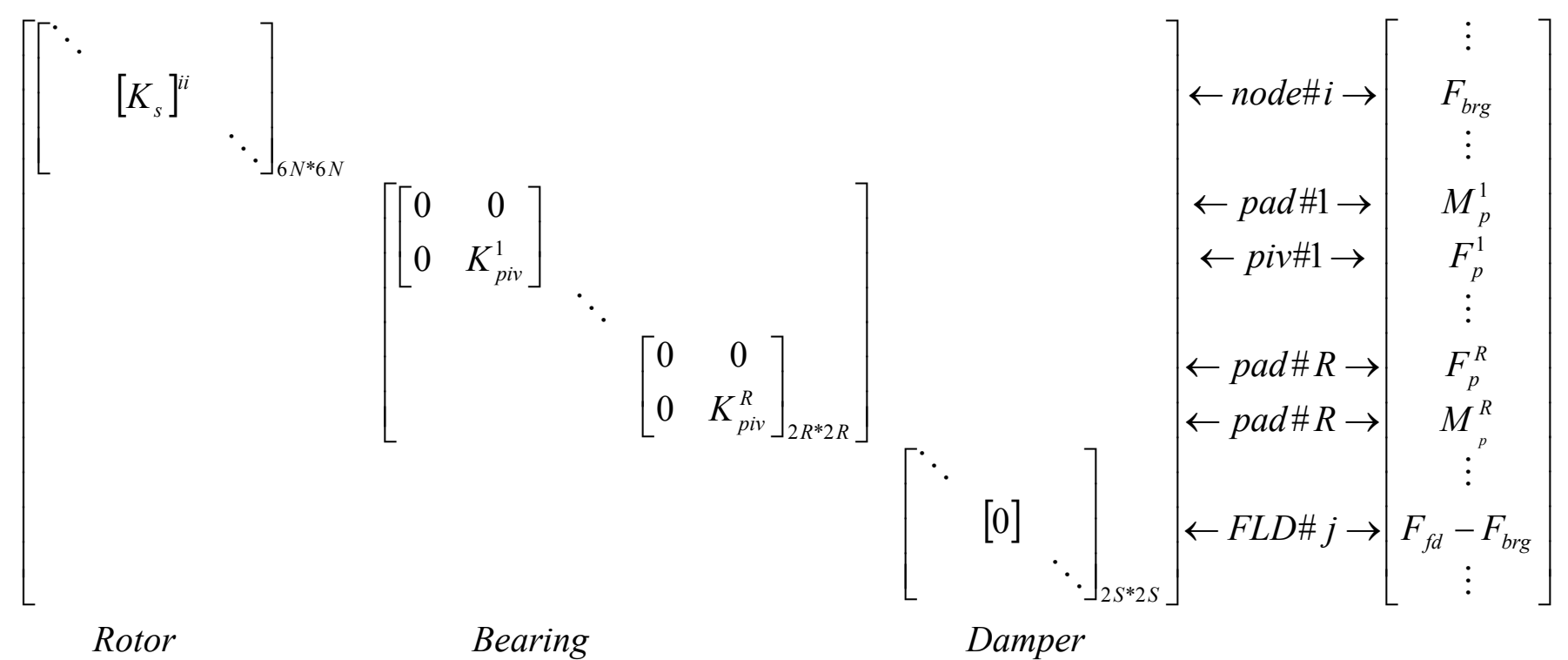

where $K_{p i v}^{1} \cdots K_{p i v}^{R}$ are the stiffness coefficients of all pivots, $M_{p}^{1} \cdots M_{p}^{R}$ are moments of all pads, $F_{p}^{1} \cdots F_{p}^{R}$ are the forces of all pads, and $F_{f d}$ is the force of the finite length squeeze film damper. 


\subsection{Linear Applied Loads}

The force vector includes the linear applied force and the nonlinear applied forces. The linear applied forces include the unbalance forces/moments due to unbalance, the shaft bow forces due to shaft static deformation, and the gravitational forces due to gravity.

There are two types of time transient analysis provided in this research. One is the rotor with a constant rotational speed, and another is the rotor with variable rotational speed. The unbalance forces are assumed to be independent and each unbalance force at unbalance position has the form:

$$
\left\{\begin{array}{l}
F_{u, z} \\
F_{u, x} \\
F_{u, y} \\
M_{u, z} \\
M_{u, x} \\
M_{u, y}
\end{array}\right\}=\left\{\begin{array}{c}
0 \\
m e_{u} \Omega^{2} \cos \left(\Omega t+\phi_{u}\right) \\
m e_{u} \Omega^{2} \sin \left(\Omega t+\phi_{u}\right) \\
0 \\
0 \\
0
\end{array}\right\}+\left\{\begin{array}{c}
0 \\
m e_{u} \dot{\Omega} \sin \left(\Omega t+\phi_{u}\right) \\
-m e_{u} \dot{\Omega} \cos \left(\Omega t+\phi_{u}\right) \\
0 \\
0 \\
0
\end{array}\right\}
$$

where $m e_{u}, \phi_{u}, \Omega$ and $\dot{\Omega}$ are specified for each rotor node point.

For a system with a constant rotational speed $\left(\Omega=\dot{\theta}_{z}, \dot{\Omega}=\ddot{\theta}_{z}=0\right)$, each unbalance force at unbalance position have the form:

$$
\left\{\begin{array}{l}
F_{u, z} \\
F_{u, x} \\
F_{u, y} \\
M_{u, z} \\
M_{u, x} \\
M_{u, y}
\end{array}\right\}=\left\{\begin{array}{c}
0 \\
m e_{u} \Omega^{2} \cos \left(\Omega t+\phi_{u}\right) \\
m e_{u} \Omega^{2} \sin \left(\Omega t+\phi_{u}\right) \\
0 \\
0 \\
0
\end{array}\right\}
$$


The shaft bow forces are related to the shaft bow amplitude and the shaft bow phase angle at each shaft node and have the form (3.3.3) in general or the form (3.3.4) when the system has constant rotational speed $\left(\dot{\Omega}=\ddot{\theta}_{z}=0\right)$.

$$
\left\{\begin{array}{l}
F_{b o w, z} \\
F_{b o w, x} \\
F_{b o w, y} \\
M_{b o w, z} \\
M_{b o w, x} \\
M_{b o w, y}
\end{array}\right\}=\left\{\begin{array}{c}
0 \\
A_{b} \Omega^{2} \cos \left(\Omega t+\phi_{b}\right) \\
A_{b} \Omega^{2} \sin \left(\Omega t+\phi_{b}\right) \\
0 \\
0 \\
0
\end{array}\right\}+\left\{\begin{array}{c}
0 \\
A_{b} \dot{\Omega} \sin \left(\Omega t+\phi_{b}\right) \\
-A_{b} \dot{\sin }\left(\Omega t+\phi_{b}\right) \\
0 \\
0 \\
0
\end{array}\right\}
$$

where $A_{b}, \phi_{b}, \Omega$ and $\dot{\Omega}$ are specified for each rotor node point.

$$
\left\{\begin{array}{c}
F_{b o w, z} \\
F_{b o w, x} \\
F_{b o w, y} \\
M_{b o w, z} \\
M_{b o w, x} \\
M_{b o w, y}
\end{array}\right\}=\left\{\begin{array}{c}
0 \\
A_{b} \Omega^{2} \cos \left(\Omega t+\phi_{b}\right) \\
A_{b} \Omega^{2} \sin \left(\Omega t+\phi_{b}\right) \\
0 \\
0 \\
0
\end{array}\right\}
$$

Gravitational forces/moments are different in $(\mathrm{X}-\mathrm{Z})$ and $(\mathrm{Y}-\mathrm{Z})$ planes due to the gravitational constants, $g_{x}$ and $g_{y}$, in the $\mathrm{x}$ and $\mathrm{y}$ directions, respectively. The coordinates of $x-z$ and $y-z$ planes are shown in Fig. 3.4.
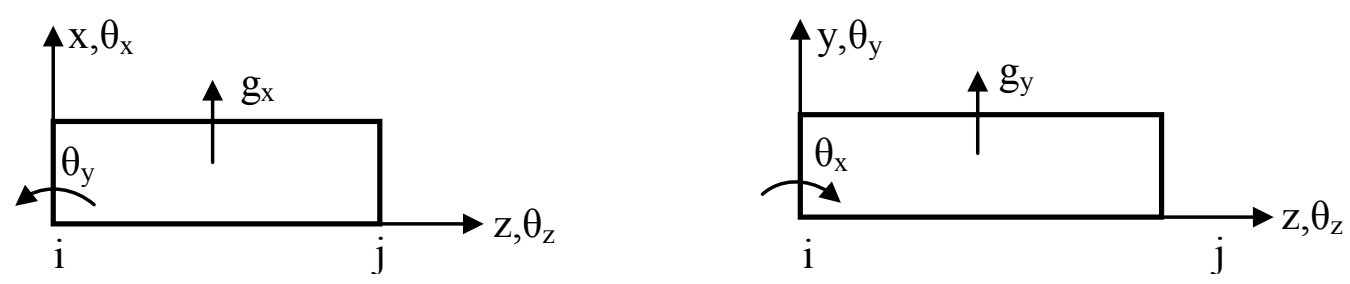

Figure 3.4 Gravitational Force in $\mathrm{XZ}$ and $\mathrm{YZ}$ planes 
According to the system coordinate used in this research as shown in Fig. 3.1, the gravitational constants in $\mathrm{x}$ direction is zero $\left(g_{x}=0\right)$. The form of gravitational force vector of one element with two nodes $(i \& j)$ as shown in Fig. 3.4, is:

$$
\left\{\begin{array}{l}
F_{g, z} \\
F_{g, x} \\
F_{g, y} \\
M_{g, z} \\
M_{g, x} \\
M_{g, y}
\end{array}\right\}=\left\{\begin{array}{c}
0 \\
\rho A g_{x} L / 2 \\
\rho A g_{y} L / 2 \\
0 \\
-\rho A g_{y} L^{2} / 12 \\
\rho A g_{x} L^{2} / 12
\end{array}\right\} ; \quad\left\{\begin{array}{c}
F_{g, z} \\
F_{g, x} \\
F_{g, y} \\
M_{g, z} \\
M_{g, x} \\
M_{g, y}
\end{array}\right\}=\left\{\begin{array}{c}
0 \\
\rho A g_{x} L / 2 \\
\rho A g_{y} L / 2 \\
0 \\
\rho A g_{y} L^{2} / 12 \\
-\rho A g_{x} L^{2} / 12
\end{array}\right\}
$$

\subsection{Nonlinear Applied Loads}

In this dissertation, the nonlinear forces/moments, which are the function of current displacements and velocities of related nodes, are obtained from the bearings, the squeeze film dampers and other external nonlinear forces/moments, such as the torsional torque of an electric motor during startup or shutdown. For the fluid bearings and dampers, the pressure profile is calculated by solving the linearized Reynolds equation, which is derived from the continuity equation and three momentum equations. Then nonlinear forces are calculated by integrating the pressure profile. The following assumption is used to derive Reynolds equation in this dissertation:

- Laminar flow everywhere in the lubricant

- Newtonian fluid

- Weight of fluid (body forces) and inertia forces are neglected

- Pressure across the lubricant is constant

- Density and viscosity of the lubricant is constant 
Based on those assumptions, the Reynolds equation of the fluid in the bearing is:

$$
\frac{\partial}{\partial x}\left(\frac{h^{3}}{6 \mu} \frac{\partial p}{\partial x}\right)+\frac{\partial}{\partial z}\left(\frac{h^{3}}{6 \mu} \frac{\partial p}{\partial z}\right)=\omega \frac{\partial h}{\partial \theta}+2 \frac{\partial h}{\partial t}
$$

\subsubsection{Short Plain Journal Bearings and Short Squeeze Film Dampers}

Using the methodology developed by Kirk and Gunter [81] for the short bearing, the pressure profile of the short plain journal bearing is calculated by solving Reynolds' Eq. (3.4.1). This equation is applied to the squeeze film dampers as well; the only difference between the bearing and the squeeze film damper is the rotating speed $(\omega)$. For the bearing, the shaft inside the bearing is rotating with running speed $\omega$ and whirling inside the bearing at the same time. For the squeeze file damper, however, only the whirl speed exists inside the damper, the bearing housing inside the squeeze film damp does not rotate, so the rotating speed $(\omega)$ for the squeeze film damper is zero.

For the short bearing $(\mathrm{L} / \mathrm{D} \leq 0.5)$, the pressure gradient along the $\mathrm{x}$ direction is far smaller than along the $\mathrm{z}$ direction. By ignoring $\partial P / \partial x$, the Reynolds equation becomes:

$$
\frac{\partial}{\partial z}\left(\frac{h^{3}}{6 \mu} \frac{\partial p}{\partial z}\right)=\omega \frac{\partial h}{\partial \theta}+2 \frac{\partial h}{\partial t}
$$

The film thickness is derived according to geometry of the bearing and the shaft inside as shown in Fig. 3.5, which is a cross section of a plain journal bearing. The film thickness, $h=|P Q|$, in fixed coordinate, is given by (3.4.3):

$$
\begin{aligned}
& h=C_{b}-X \cos \theta-Y \sin \theta \\
& \frac{\partial h}{\partial \theta}=X \sin \theta-Y \cos \theta ; \frac{\partial h}{\partial t}=-\dot{X} \cos \theta-\dot{Y} \sin \theta
\end{aligned}
$$




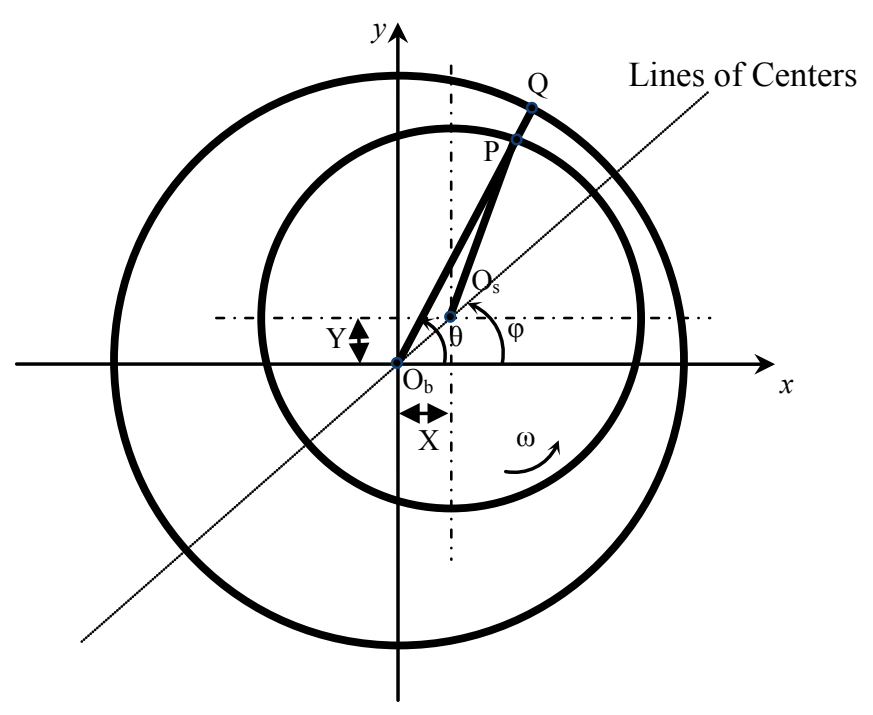

Figure 3.5 Cross Section of a Plain Journal Bearing in Fixed Coordinate

The closed-form dimensionless pressure solution for the short plain bearing is shown by Eq. (3.4.4) with open end boundary conditions $(\bar{p}(\theta, 0)=\bar{p}(\theta, L)=0)$. The nonlinear force then can be calculated by integrating the pressure over the film area in form (3.4.5). During the integration, only the positive pressures will be integrated, to take into account cavitations.

$$
\begin{aligned}
& \bar{p}= \begin{cases}3 \bar{Z}(1-\bar{Z})\left(\frac{-\bar{X} \sin \theta+\bar{Y} \cos \theta+2(\dot{\bar{X}} \cos \theta+\dot{\bar{Y}} \sin \theta)}{(1-\bar{X} \cos \theta-\bar{Y} \sin \theta)^{3}}\right) & \bar{p} \geq 0 \\
0 & \bar{p}<0\end{cases} \\
& \bar{Z}=\frac{z}{L} ; \bar{X}=\frac{X}{C_{b}} ; \bar{Y}=\frac{Y}{C_{b}} ; \dot{\bar{X}}=\frac{\dot{X}}{C_{b} \omega} ; \dot{\bar{Y}}=\frac{\dot{Y}}{C_{b} \omega} ; \bar{p}=\frac{p(\theta, z)}{\mu \omega}\left(\frac{C_{b}}{L}\right)^{2} \\
& F_{b, x}=\int_{0}^{L} \int_{0}^{2 \pi} p(\theta, z) R \cos \theta d \theta d z \\
& F_{b, y}=\int_{0}^{L} \int_{0}^{2 \pi} p(\theta, z) R \sin \theta d \theta d z
\end{aligned}
$$


For the short squeeze film damper, the bearing housing is inside and its rotational speed is zero $(\omega=0)$ because of an anti-rotation pin in the damper housing. The Reynolds equation and pressure profile for the short squeeze film damper $(\mathrm{L} / \mathrm{D} \leq 0.5)$ is:

$$
\begin{aligned}
& \frac{\partial}{\partial z}\left(\frac{h^{3}}{6 \mu} \frac{\partial p}{\partial z}\right)=2 \frac{\partial h}{\partial t} \\
& \bar{p}=\left\{\begin{array}{l}
3 \bar{Z}(1-\bar{Z})\left(\frac{2(\dot{\bar{X}} \cos \theta+\dot{\bar{Y}} \sin \theta)}{(1-\bar{X} \cos \theta-\bar{Y} \sin \theta)^{3}}\right) \quad \bar{p} \geq 0 \\
0 \quad \bar{p}<0
\end{array}\right. \\
& \bar{Z}=\frac{z}{L} ; \bar{X}=\frac{X}{C_{d}} ; \bar{Y}=\frac{Y}{C_{d}} ; \dot{\bar{X}}=\frac{\dot{X}}{C_{d}} ; \dot{\bar{Y}}=\frac{\dot{Y}}{C_{d}} ; \bar{p}=\frac{p(\theta, z)}{\mu}\left(\frac{C_{d}}{L}\right)^{2}
\end{aligned}
$$

Same method and equations, as shown in Eq. (3.4.5), are used to calculate the damper forces. An example of calculating pressure profile and nonlinear bearing force is shown in Chapter 4.

\subsubsection{Tilting Pad Bearings and Finite Length Squeeze Film Dampers}

The tilting pad bearing and the finite length squeeze film damper are put together here since both of them use the similar method solving Reynolds Eq. (3.4.1) to obtain pressure profile and then calculating forces at given position and velocity of component inside the bearing or the damper. The difference between the tilting pad bearing and the finite length bearing is the boundary condition and the rotating speed of the component inside the bearing or the damper. In this section, there is no limitation of the short bearing or damper condition $(\mathrm{L} / \mathrm{D} \leq 0.5)$. Without that limitation, the close-form of pressure profile does not exist, and the finite element method is applied for pressure profile calculation. 


\section{Tilting pad bearing}

The tilting pad bearing is more complicated than the plain journal bearing due to angular and radial displacement of the bearing pads and pivots, which change the stability of the system. Unlike the plain journal bearing, the pressure profile is related not only to the shaft displacement and velocity inside the bearing, but also to positions and velocities of all pads and pivots of the bearing. Also the behavior of pads, pivots and shaft are coupled together. Without assumption of short bearing $(\mathrm{L} / \mathrm{D} \leq 0.5)$, a 1-D finite element method is used to solve the pressure profile on each pad.

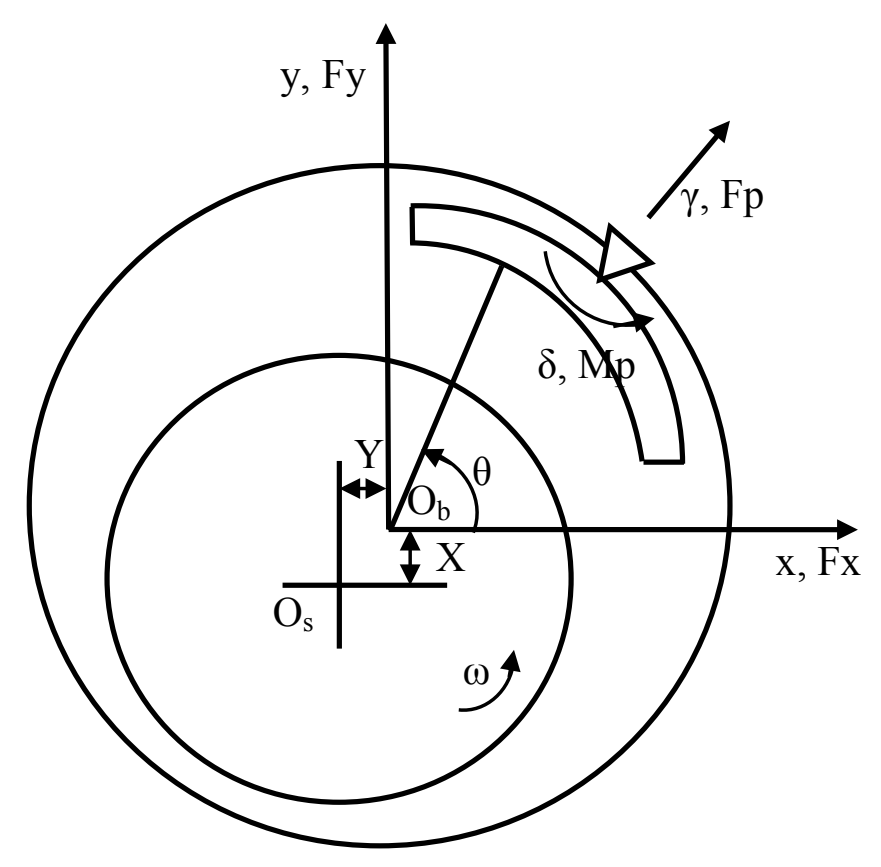

Figure 3.6 Cross Section and Coordinate of a Tilting Pad Bearing

Fig. 3.6 shows the cross section of a tilting pad bearing geometry. Normally, a tilting pad bearing includes $4 \sim 6$ pads \& pivots. Only one pad is shown in this figure for illustration purpose. Subject to the thin film lubrication approximation, the govern equation of pressure profile of a tilting pad bearing is Reynolds equation on each pad, 
which is shown in Eq. (3.4.1). Under the assumption of an incompressible Newtonian fluid and the laminar flow inside the bearing, the dimensionless form of linearized Reynolds equation is:

$$
\frac{\partial}{\partial \theta}\left(\frac{\bar{h}^{3}}{12} \frac{\partial \bar{p}}{\partial \theta}\right)+\left(\frac{D}{L}\right)^{2} \frac{\partial}{\partial \bar{z}}\left(\frac{\bar{h}^{3}}{12} \frac{\partial \bar{p}}{\partial \bar{z}}\right)=\frac{1}{2} \frac{\partial \bar{h}}{\partial \theta}+\frac{\partial \bar{h}}{\partial \bar{t}}
$$

where

$$
\begin{aligned}
& \theta=x / R ; \bar{z}=z /(L / 2) ; \bar{h}=h / C_{p} ; \bar{t}=\omega t \\
& \bar{p}=\left(C_{p}^{2} / \mu \omega R^{2}\right) p
\end{aligned}
$$

For the pad with circumferential angle from $\theta_{1}$ to $\theta_{2}$, the boundary conditions in the circumferential direction and axial direction are:

$$
\left\{\begin{array}{l}
\bar{p}\left(\theta_{1}, \bar{z}\right)=\bar{p}\left(\theta_{2}, \bar{z}\right)=0 \\
\bar{p}(\theta, \bar{z}= \pm L / 2)=0 \\
\bar{p} \geq 0
\end{array}\right.
$$

where $\theta_{1}$ is the leading edge of pad, $\theta_{2}$ is the trailing angle of pad, and $\bar{p} \geq 0$ is the cavitation condition.

The pressure profiles of all pads and pivots have to be calculated at each time step during the time transient analysis. To save computing time, an approximate 1-D finite element method along circumferential direction is applied to solve the 2-D Reynolds equation under assumption of the pressure distribution along the axial direction. The pressure distribution along the axial direction is taken of the form $[118,119]$ :

$$
\bar{p}(\theta, \bar{z})=\bar{p}_{\theta}\left(1-\bar{z}^{n}\right)
$$


For different L/D ratios, $n$ has different values. For example, Shelly and Ettles [119] gives $\mathrm{n}=2$ for eccentricity ratio $\varepsilon<0.75$ and $\mathrm{n}=2.12$ for $\varepsilon>0.75$ when $\mathrm{L} / \mathrm{D}=0.5$, which is a short bearing. Other researchers recommend $n=2$ for $\mathrm{L} / \mathrm{D}$ close to 1.0 and for a small eccentricity ratio $(\varepsilon<0.3)$.

Shelly [119] provides a method to solve the Reynolds Eq. (3.4.5) with assumption of (3.4.1). First, Put (3.4.11) into (3.4.8):

$$
\frac{d}{d \theta}\left\{\frac{\bar{h}^{3}}{12} \frac{d \bar{p}_{\theta}}{d \theta}\left(1-\bar{z}^{n}\right)\right\}+\left(\frac{D}{L}\right)^{2} \frac{\bar{h}^{3}}{12} \frac{d^{2}}{d \bar{z}^{2}}\left(\bar{p}_{\theta}\left(1-\bar{z}^{n}\right)\right)-\left(\frac{1}{2} \frac{\partial \bar{h}}{\partial \theta}+\frac{\partial \bar{h}}{\partial \bar{t}}\right)=0
$$

Eq. (3.4.12) is then integrated twice over axial direction $(z=[0, L]$ or $\bar{z}=[-1,1])$ with boundary conditions (3.4.10), so the $2^{\text {nd }}$ order partial differential Reynolds Eq. (3.4.8) can be rewritten to the following ordinary differential equation at middle of bearing ( $z=L / 2$ or $\bar{z}=0$ ). The dimensionless equation to solve the pressure profile at $\bar{z}=0$ is:

$$
\frac{d}{d \theta}\left(B_{1} \frac{\bar{h}^{3}}{12} \frac{d \bar{p}_{\theta}}{d \theta}\right)+\frac{\bar{h}^{3}}{12}\left(-3\left(\frac{D}{L}\right)^{2}\right) \bar{p}_{\theta}-B_{2}\left(\frac{1}{2} \frac{\partial \bar{h}}{\partial \theta}+\frac{\partial \bar{h}}{\partial \bar{t}}\right)=0
$$

where $\mathrm{B} 1$ and $\mathrm{B} 2$ are the weight factor with form (3.4.14):

$$
B_{1}=\left(\frac{n+4}{n+3}\right) ; \quad B_{2}=\left(\frac{n+1}{n}\right)
$$

We consider the tilting pad bearing geometry shown in Fig. 3.6. The main variables in the film thickness equation are the radial clearance of the bearing $\left(C_{b}\right)$, the radial clearance of each pad $\left(C_{p}\right)$, the current position $(X$ and $Y)$ of the shaft center inside the 
bearing, the current pad tilting angle $(\delta)$ and the current pivot position $(\gamma)$. The film thickness along the pad is:

$$
\begin{aligned}
h=C_{p}- & X \cos \theta-Y \sin \theta \\
& -\left(C_{p}-C_{b}-\gamma\right) \cos \left(\theta-\theta_{p}\right)-\delta R \sin \left(\theta-\theta_{p}\right)
\end{aligned}
$$

In dimensionless form, the equation is:

$$
\begin{aligned}
\bar{h}=1-\bar{X} & \cos \theta-\bar{Y} \sin \theta \\
& -(m-\bar{\gamma}) \cos \left(\theta-\theta_{p}\right)-\bar{\Delta} \sin \left(\theta-\theta_{p}\right)
\end{aligned}
$$

where

$$
\bar{X}=\frac{X}{C_{p}} ; \bar{Y}=\frac{Y}{C_{p}} ; m=1-\frac{C_{b}}{C_{p}} ; \bar{\gamma}=\frac{\gamma}{C_{p}} ; \bar{\Delta}=\frac{\delta R}{C_{p}}
$$

For specified nodal displacements and velocities of the shaft and the bearing, based on the finite element method, the pressure profile of each pad can be solved through (3.4.13) with boundary condition (3.4.10) [69]. Nonlinear forces are calculated by integrating pressure on each pad in fixed coordinate. Nonlinear forces and moments of each pad/pivot are listed in following forms:

$$
\begin{aligned}
& F_{b, x}=2 \int_{0}^{L / 2}\left(\sum_{\theta_{1}}^{\theta_{2}} p(\theta, z) R \cos \theta d \theta\right) d z=\frac{n}{n+1} R L \sum_{\theta_{1}}^{\theta_{2}} p_{\theta} \cos \theta d \theta \\
& F_{b, y}=2 \int_{0}^{L / 2}\left(\sum_{\theta_{1}}^{\theta_{2}} p(\theta, z) R \sin \theta d \theta\right) d z=\frac{n}{n+1} R L \sum_{\theta_{1}}^{\theta_{2}} p_{\theta} \sin \theta d \theta \\
& F_{p}=2 \int_{0}^{L / 2}\left(\sum_{\theta_{1}}^{\theta_{2}} p(\theta, z) R d \theta\right) d z=\frac{n}{n+1} R L \sum_{\theta_{1}}^{\theta_{2}} p_{\theta} \cos \left(\theta-\theta_{p}\right) d \theta
\end{aligned}
$$




$$
\begin{array}{r}
M_{p}=2 \int_{0}^{L / 2}\left(\left(\sum_{\theta_{1}}^{\theta_{2}} p(\theta, z) R d \theta\right)\left(-R \sin \left(\theta-\theta_{p}\right)\right)\right) d z \\
=-\frac{n}{n+1} R^{2} L \sum_{\theta_{1}}^{\theta_{2}} p_{\theta} \sin \left(\theta-\theta_{p}\right) d \theta
\end{array}
$$

where $\theta_{1}$ is the leading edge of pad, $\theta_{2}$ is the trailing angle of pad.

An example of 4-pad tilting pad bearing pressure profile and nonlinear forces is shown in Chapter5. Also effects of pad/pivot behavior to pressure distribution are discussed in the chapter.

\section{Finite length squeeze film damper}

As mentioned at the beginning of this section, the finite length damper only experiences the whirling speed since the inside bearing housing does not rotate. Fig. 3.7 shows the cross section of a finite length damper's geometry in a fixed coordinate. Under assumption of impressible Newtonian fluid and laminar flow, the dimensionless form of Reynolds equation is:

$$
\frac{\partial}{\partial \theta}\left(\frac{\bar{h}^{3}}{12} \frac{\partial \bar{p}}{\partial \theta}\right)+\left(\frac{D}{L}\right)^{2} \frac{\partial}{\partial \bar{z}}\left(\frac{\bar{h}^{3}}{12} \frac{\partial \bar{p}}{\partial \bar{z}}\right)=\frac{\partial \bar{h}}{\partial t}
$$

where

$$
\begin{aligned}
& \theta=x / R ; \quad \bar{z}=z /(L / 2) \\
& \bar{h}=h / C_{d} \\
& \bar{p}=\left(C_{d}^{2} / \mu R^{2}\right) p
\end{aligned}
$$




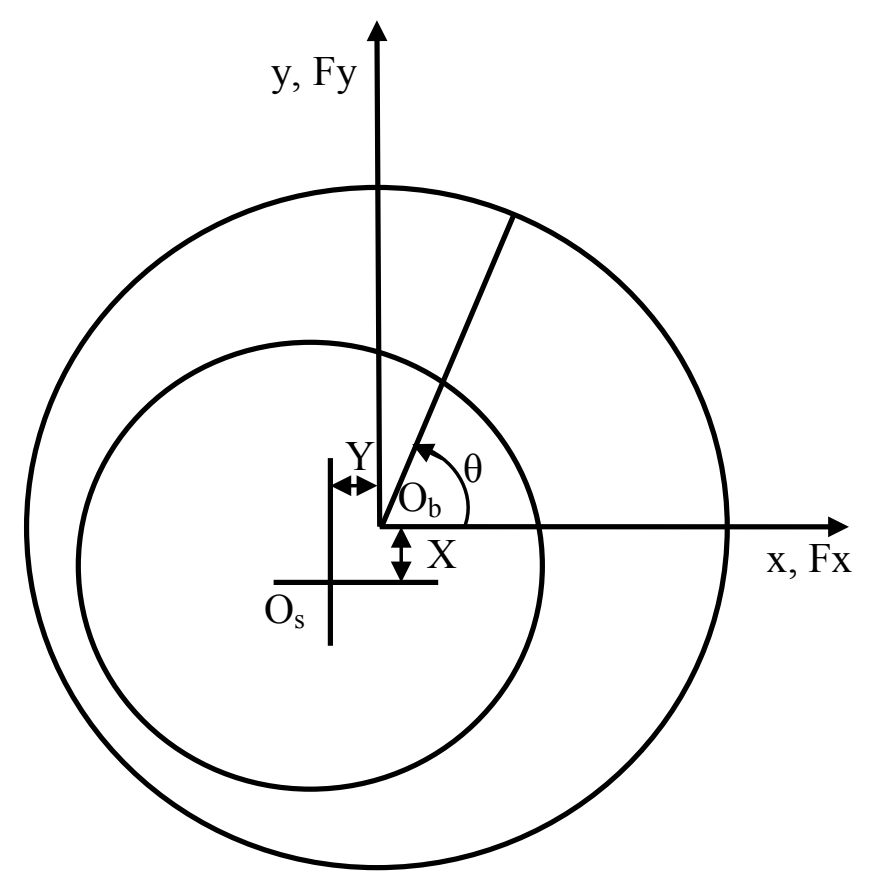

Figure 3.7 Cross Section and Coordinate of a Finite Length Damper

The boundary conditions in the circumferential direction and axial direction for the open ends damper are:

$$
\left\{\begin{array}{l}
\bar{p}(0, \bar{z})=\bar{p}(2 \pi, \bar{z}) \\
\left.\frac{\partial \bar{p}}{\partial \theta}\right|_{\theta=0 \mid}=\left.\frac{\partial \bar{p}}{\partial \theta}\right|_{\theta=2 \pi \mid} \\
\bar{p}(\theta, z= \pm L / 2)=0 \\
\bar{p} \geq 0 \quad \text { (Cavitation Condition) }
\end{array}\right.
$$

Use same assumption of the pressure distribution along the axial direction (3.4.8), the dimensionless equation to solve the pressure profile at $\bar{z}=0$ is:

$$
\frac{d}{d \theta}\left(B_{1} \frac{\bar{h}^{3}}{12} \frac{d \bar{p}_{\theta}}{d \theta}\right)+\frac{\bar{h}^{3}}{12}\left(-3\left(\frac{D}{L}\right)^{2}\right) \bar{p}_{\theta}-B_{2}\left(\frac{\partial \bar{h}}{\partial t}\right)=0
$$


The film thickness equation is function of the radial clearance of the damper $\left(C_{d}\right)$ and the current position $(X$ and $Y$ ) of the bearing housing inside the damper with the form:

$$
h=C_{d}-X \cos \theta-Y \sin \theta
$$

In dimensionless form, this equation is:

$$
\bar{h}=1-\bar{X} \cos \theta-\bar{Y} \sin \theta
$$

where

$$
\bar{X}=\frac{X}{C_{d}} ; \quad \bar{Y}=\frac{Y}{C_{d}}
$$

For specified nodal displacements and velocities of the bearing housing inside the damper, based on similar finite element method used for the tilting pad bearing, the pressure profile of the damper can be solved through (3.4.25) with boundary condition (3.4.24). Nonlinear forces are calculated by integrating the pressure profile. Nonlinear forces in horizontal and vertical directions are:

$$
\begin{aligned}
& F_{d, x}=2 \int_{0}^{L / 2}\left(\sum_{0}^{2 \pi} p(\theta, z) R \cos \theta d \theta\right) d z=\frac{n}{n+1} R L \sum_{0}^{2 \pi} p_{\theta} \cos \theta d \theta \\
& F_{d, y}=2 \int_{0}^{L / 2}\left(\sum_{0}^{2 \pi} p(\theta, z) R \sin \theta d \theta\right) d z=\frac{n}{n+1} R L \sum_{0}^{2 \pi} p_{\theta} \sin \theta d \theta
\end{aligned}
$$

An example of the finite length squeeze film damper pressure profile and nonlinear forces under different mesh densities is shown in Chapter 5. 


\subsubsection{Nonlinear Torsional Forces and Moments}

External torques or torsional forces are applied to the rotor for the torsional analysis and the coupled lateral/torsional analysis. The external torques in a rotor may be produced by a variety of different sources. Often a rotor has a motor attached at one end and a load attached at the other end, sometimes the loads are distributed among multiple impellers. Oscillations in the torque input may cause a failure of the torsional system. Three general torque models that are discussed in this dissertation are time dependent torque, speed dependent torque, and both time and speed dependent torques, such as torques produced by the synchronous motors.

A time dependent nonlinear external torque is depends only on time. If a time history of the input torque is known, then the appropriate torque is added to the node, at which the torque is acting vs. time. There are no limitations to the shape of the time dependent torque, as shown in Eq. (3.4.31).

$$
T_{i}=T(t)
$$

In practice, time history of the torque input is rarely known. More commonly, the torque is often known as a function of the current running speed of the rotor (speed dependent torque), as shown in Eq. (3.4.32). The average speed dependent torque is

determined by the angular velocity of the $\operatorname{rotor}\left(\Omega\right.$ or $\left.\dot{\theta}_{z}\right)$. The external nonlinear torque is then added to the appropriate node in the model. There are no restrictions to the shape of the speed dependent torque curve.

$$
T_{i}=T_{\text {avg }}\left(\dot{\theta}_{z}\right)
$$


In general, the driving torque, which is both time and speed dependent, at any instant during startup, includes a constant average torque component and an oscillating torque component:

$$
T_{i}=T_{\text {avg }}+T_{\text {osc }} \sin \left(\omega_{\text {exc }} t+\phi_{\text {exc }}\right)
$$

where the average torque $T_{\text {avg }}$, the oscillating torque $T_{\text {osc }}$, and the oscillating frequency $\omega_{\text {exc }}$ are functions of the running speed. During the analysis, the torque applied can be calculated by determining $T_{\text {avg }}$ and $T_{\text {osc }}$ at the current running speed. The excitation frequency of the pulsating torque for a synchronous motor during startup is equal to twice the slip frequency and is a linear function of the rotor speed:

$$
\omega_{e x c}(\mathrm{rad} / \mathrm{sec})=2 \times 2 \pi f_{s}=4 \pi f_{L}\left(1-\frac{N_{m}}{N_{s y n}}\right)
$$

where $f_{L}$ is the linear frequency ( $50 \mathrm{or} 60 \mathrm{~Hz}$ ), $N_{m}$ is the motor speed (rpm) at given time during startup, and $N_{s y n}$ is the synchronous speed (rpm) calculated by the following expression:

$$
N_{s y n}=\frac{120 f_{L}}{\text { No. of Poles }} \mathrm{rpm}
$$

The speed dependent load torque is due to the resistance of the system. The load curve for different loading conditions usually can be obtained from the equipment manufacturers. Fig. 3.8 shows a typical torque speed curve of synchronous motor including average drive, pulsating and load [67]. An example synchronous motor startup analysis was given using that torque speed curve. 


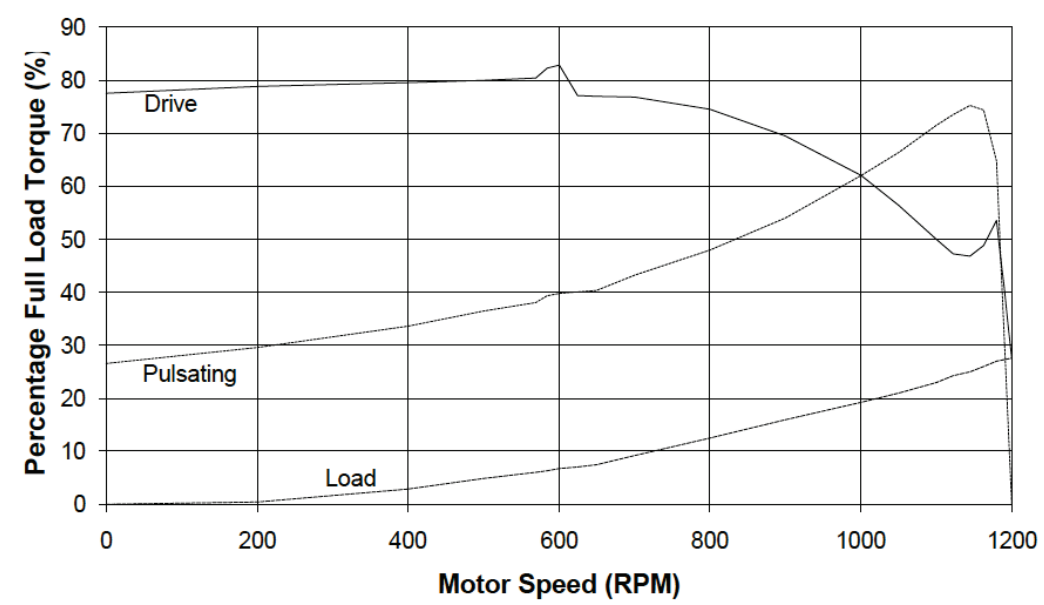

Figure 3.8 Typical Torque Speed Curve of Synchronous Motor [67]

\subsection{Method of Numerical Solution}

The direct step-by-step numerical integration calculates the transient response for a given constant rotor speed or variable speed rotor. The system can be linear or nonlinear; the running speed can be constant or non-constant. In structural dynamics, a commonly used direct integration algorithm that solves the original system of second-order differential equation is Newmark- $\beta$ method. It assumes that the average acceleration in the time interval to be average value of the discrete initial and final accelerations. Another numerical integration method, called Runge-Kutta method, solves the system of differential equations in first order form. To solve the second-order differential equations of motion using this method, the state space form of equations of motion is applied. In numerical analysis, the fourth order Rnuge-Kutta method is an important family member of implicit and explicit iterative methods for the approximation of transient solutions of ordinary differential equations, especially in some engineering applications requiring high accuracy. 
The nonlinear bearing forces and the nonlinear damper forces are functions of the shaft nodal displacements and velocities at the current time step. The implicit Newmark- $\beta$ method has been applied [25] to solve the equations of motion in rotor dynamics. To keep high solution accuracy of the nonlinear complicated system, such as with tilting pad bearings involved in the system, the $4^{\text {th }}$ order Runge-Kutta method is selected in this dissertation for both calculation of the nonlinear forces and integration of the whole system. The classic explicit fourth-order Runge-Kutta method is applied to nonlinear transient analysis of rotor with constant running speed. A modified implicit Runge-Kutta method is applied to solve the non-constant rotational speed rotor dynamic problem. Fig. 3.9 gives a flowchart illustrating the transient analysis of the rotor system.

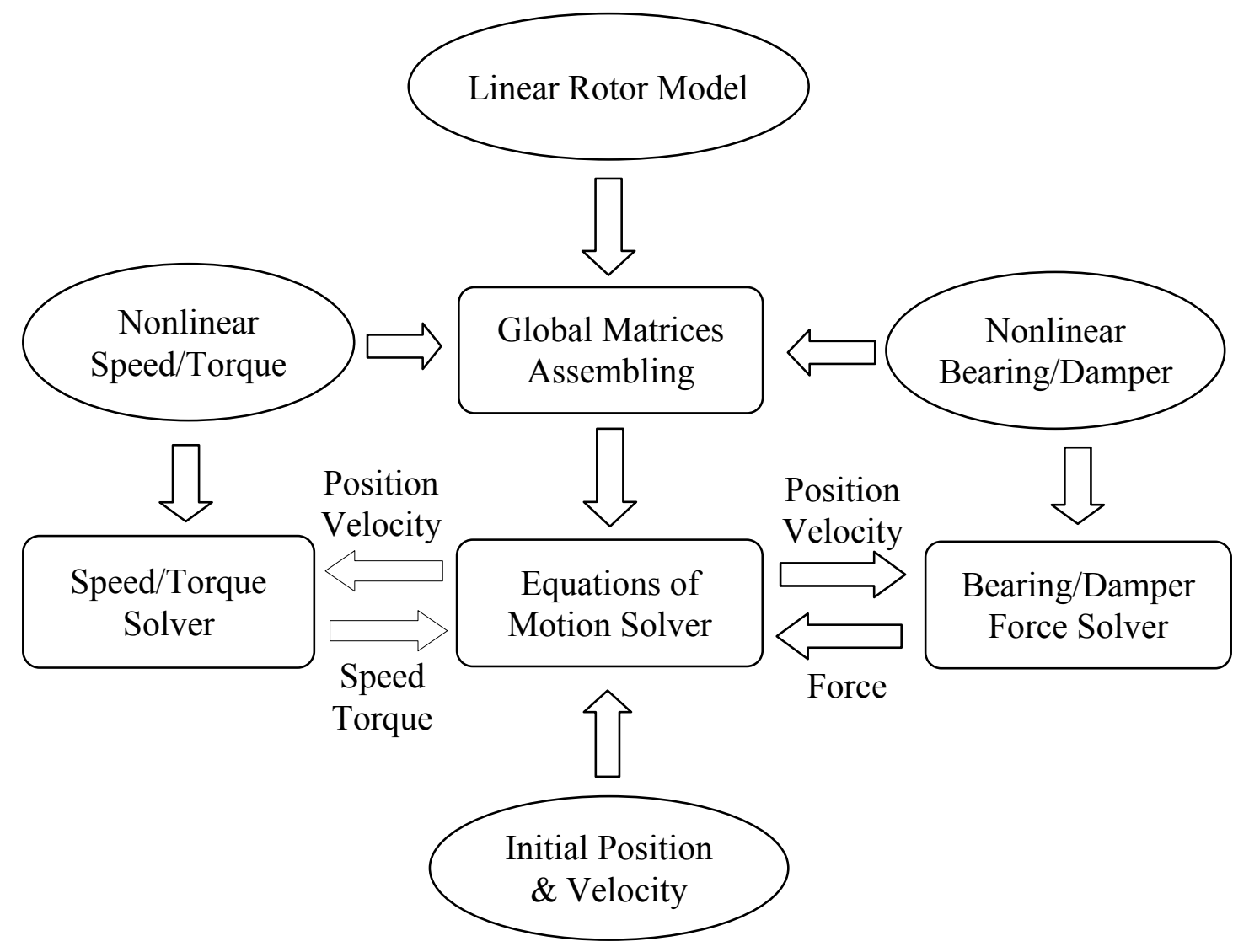

Figure 3.9 Flow Chart of the Rotor System Nonlinear Transient Solution 


\subsubsection{Integration Method for Constant Running Speed}

When the running speed of a rotor is constant without axial force or axial torque, normally the lateral time transient analysis is carried out. The equations of motion of a rotor with constant running speed, second-order ordinary differential equation, are shown in Eq. (3.5.1).

$$
M \ddot{u}+(C+\Omega G) \dot{u}+K u=F(u, \dot{u}, \Omega, t)
$$

In the equations, all linear parts related matrices are on the left, and all forces from unbalance, bow, gravity and nonlinear bearings/dampers go to the right.

When the rotating speed $(\Omega)$ is constant, the equation of motion can be converted to the state space form (3.5.2):

$$
\begin{gathered}
{\left[\begin{array}{cc}
M & 0 \\
0 & M
\end{array}\right]\left[\begin{array}{l}
\dot{u} \\
\ddot{u}
\end{array}\right]+\left[\begin{array}{cc}
0 & -M \\
K & C+\Omega G
\end{array}\right]\left[\begin{array}{l}
u \\
\dot{u}
\end{array}\right]=\left[\begin{array}{c}
0 \\
F(u, \dot{u}, t)
\end{array}\right]} \\
\operatorname{Let} U=\left[\begin{array}{c}
u \\
\dot{u}
\end{array}\right], A=\left[\begin{array}{cc}
M & 0 \\
0 & M
\end{array}\right], B=\left[\begin{array}{cc}
0 & -M \\
K & C+\Omega G
\end{array}\right], F=\left[\begin{array}{c}
0 \\
F(u, \dot{u}, t)
\end{array}\right] . \text { The Eq. (3.5.2) has the }
\end{gathered}
$$

general state space matrix form (3.5.3):

$$
A \dot{U}+B U=F \Rightarrow \dot{U}=\left(-A^{-1} B\right) U+A^{-1} F
$$

Using this state space form (3.5.3), the second-order ordinary differential equation (ODE) has been converted to a set of first-order ODE to be solved using the explicit fourth-order Runge-Kutta method. However, the matrix size has been increased four times, from $N^{*} N$ to $2 N^{*} 2 N$, where $N$ is the total degrees of freedom of the whole system. 


\subsubsection{Integration Method for Non-Constant Running Speed}

When the running speed of a rotor is not constant (normally an external torque exists), the torsional time transient analysis or the coupled lateral and torsional time transient analysis is applied. The equations of motion of a rotor with non-constant running speed are shown in Eq. (3.5.4).

$$
M \ddot{u}+\left(C_{s}+C_{b r g}(\Omega)+\Omega G\right) \dot{u}+\left(K_{s}+K_{b}(\Omega)+\dot{\Omega} G\right) u=F(u, \dot{u}, \Omega, \dot{\Omega}, t)
$$

Compared to the equations of motion under a constant running speed, there is an additional gyroscopic effect related term, $\dot{\Omega} G$, in the Eq. (3.5.4). In the non-constant equations of motion (3.5.4), the mass matrix $(M)$, stiffness and the damping matrix from the shaft and disks $\left(K_{s}, C_{s}\right)$ are not related to the running speed; the linear bearing coefficients $\left(K_{b}, C_{b r g}\right)$ changes with different running speeds; the gyroscopic effects ( $\Omega G, \dot{\Omega} G$ ) are the function of running speed and acceleration as well. To solve the equations of motion (3.5.4) numerically, all linear and running speed independent parts related matrices are move to the left, and all speed related matrices, all forces from unbalance, bow, gravity and nonlinear bearings/dampers are moved to the right.

$$
M \ddot{u}+C_{s} \dot{u}+K_{s} u=F(u, \dot{u}, \Omega, \dot{\Omega}, t)-\left(C_{b r g}(\Omega)+\Omega G\right) \dot{u}-\left(K_{b}(\Omega)+\dot{\Omega} G\right) u
$$

For a coupled lateral and torsional analysis, the running speed $(\Omega)$ is the axial angular rotating speed $\left(\dot{\theta}_{z}\right)$, and the acceleration $(\dot{\Omega})$ is the axial angular acceleration $\left(\ddot{\theta}_{z}\right)$, that results in an implicit problem of solving the Eq. (3.5.5) numerically. The equations of motion can be converted to the state space form to be solved using the $4^{\text {th }}$ order Runge- 
Kutta method. However, the acceleration $\left(\dot{\Omega}\right.$ or $\ddot{\theta}_{z}$ ) has to be solved using a shooting method at each time point first according to the current rotor system nodal displacements and velocities:

$$
\begin{aligned}
& {\left[\begin{array}{cc}
M & 0 \\
0 & M
\end{array}\right]\left[\begin{array}{l}
\dot{u} \\
\ddot{u}
\end{array}\right]+\left[\begin{array}{cc}
0 & -M \\
K & C
\end{array}\right]\left[\begin{array}{l}
u \\
\dot{u}
\end{array}\right]=} \\
& {\left[F(u, \dot{u}, \Omega, \dot{\Omega}, t)-\left(C_{b r g}(\Omega)+\Omega G\right) \dot{u}-\left(K_{b}(\Omega)+\dot{\Omega} G\right) u\right]}
\end{aligned}
$$

This form is similar to the constant running speed case, where we let $U=\left[\begin{array}{l}u \\ \dot{u}\end{array}\right]$, $F=\left[\begin{array}{c}0 \\ F(u, \dot{u}, \Omega, \dot{\Omega}, t)\end{array}\right]+\left[\begin{array}{c}0 \\ -\Omega G \dot{u}-\dot{\Omega} G u\end{array}\right]+\left[\begin{array}{c}0 \\ -C_{b r g}(\Omega) \dot{u}-K_{b}(\Omega) u\end{array}\right] \quad, \quad A=\left[\begin{array}{cc}M & 0 \\ 0 & M\end{array}\right] \quad$ and $B=\left[\begin{array}{cc}0 & -M \\ K & C\end{array}\right]$. The Eq. (3.5.6) then has the state space form:

$$
A \dot{U}+B U=F \Rightarrow \dot{U}=\left(-A^{-1} B\right) U+A^{-1} F
$$

At each discrete time point, a fixed-point iteration method is used to solve the implicit equation (3.5.7) to obtain the initial nodal acceleration $(\dot{U})$ based upon the current nodal displacements and velocities $(U)$.

$$
\begin{aligned}
& \dot{U}_{i}^{0}=\dot{U}_{i-1}, \\
& \dot{U}_{i}^{1}=\left(-A^{-1} B\right) U_{i}+A^{-1} F\left(\dot{U}_{i}^{0}, U_{i}, t_{i}\right), \\
& \dot{U}_{i}^{2}=\left(-A^{-1} B\right) U_{i}+A^{-1} F\left(\dot{U}_{i}^{1}, U_{i}, t_{i}\right), \\
& \dot{U}_{i}^{3}=\left(-A^{-1} B\right) U_{i}+A^{-1} F\left(\dot{U}_{i}^{2}, U_{i}, t_{i}\right), \\
& \ldots \ldots \\
& \text { until }\left|\dot{U}_{i}^{k}-\dot{U}_{i}^{k-1}\right| \leq \text { error } \\
& \Rightarrow \dot{U}_{i}=\dot{U}_{i}^{k}
\end{aligned}
$$


where $\dot{U}_{i}^{0}$ is the starting acceleration value from the last time point, $\dot{U}_{i}^{k}, \mathrm{k}=0,1,2,3 \ldots$, is a sequence of values obtained using the iterative rule. The fixed-point iteration method starts from the known acceleration from the last time point and the new acceleration is calculated using Eq. (3.5.7). The loop, described in Eq. (3.5.8), will stop until the difference of two accelerations from last step and current step is less than the given error.

Using the state space form, the second-order ODE converts to a first-order form to be solved using the fourth-order Runge-Kutta method. However, the matrix size increases by four times from $N^{*} N$ to $2 N^{*} 2 N$, where $N$ is the total degrees of freedom of the whole system. Also the fixed-point interaction method at each time step is computationally inefficient, especially for nonlinear problem.

\subsubsection{Time Step Selection}

The time step selected for direct integration is a critical parameter in a successfully numerical integration procedure. The use of coarse time step will cause loss of detail in the integral being evaluated and will cause convergence problem in the nonlinear problem. On the other hand, too fine a time step will produce a solution which is computationally inefficient.

Optimization of time step or adaptive step size control has been developed for the Runge-Kutta method. With the fourth-order Runge-Kutta, the most straightforward technique by far is step doubling. This algorithm takes each step twice, once as a full step, then, independently, as two half steps, to reevaluate the next integration time step. A disadvantage to this approach, however, is that the evaluation of time size is in itself a time-consuming calculation. Further, the use of variable time steps complicate the 
subsequent analysis of the output in frequency domain, such as the discrete Fourier transformation of the output. The present algorithm in this dissertation therefore uses only a fixed time step.

In selection of integration time step, Cook [82] recommends that 2 times of maximum natural frequency should be considered to obtain stable and accurate output from analysis. Hassenpflug [53] used 3.5 times of the maximum natural frequency of the system to calculate the time step. Unfortunately, the accurate natural frequency of a nonlinear system is not absolute known because the nonlinear stiffness and damping effects change with the rotor shaft position and velocity for constant rotational speed. For various rotational speeds, only stiffness, damping and mass matrices from the shaft with built on disks are used to calculate the natural frequencies (free-free mode). However, for most problems encounter in rotor dynamics, the lower frequency modes (normally $5 \sim 10$ times of the rotational speed) are excited by the external force/torque. Also the maximum natural frequency of the system won't shift too much after adding the nonlinear bearings and the nonlinear dampers into the system.

In this dissertation, one approach is to consider 2.5 times of the maximum natural frequency of the linear system as initial selection of the time step, which nearly always provides a stable solution. If nonlinear components involved, the maximum natural frequency is calculated only from linear substructures of the system. Another approach of the time step is to find the stability threshold by decreasing time step gradually and executing very short simulations for each selected time step till stability occurs. The process is only worthwhile for either a system with large number of elements or cases requiring a long simulated time. 


\subsection{Summary}

This chapter describes principle and method of solving time transient analysis problem with nonlinear components involved. An element with 12 DOF is developed to model the linear shaft of the complex rotor system for coupled lateral and torsional analysis. To solve the nonlinear problem, the system is separated into linear and nonlinear substructures first and then modeled separately. Finally the entire system is assembled layer by layer. Nonlinear forces are calculated from current shaft position and velocity at each time step, and then applied to the equations of motion.

In this dissertation, four nonlinear components including short journal bearing $(\mathrm{L} / \mathrm{D} \leq 0.5)$, short squeeze film damper ( $\mathrm{L} / \mathrm{D} \leq 0.5)$, tilting pad bearing, and finite length damper, are taken into consideration. The external nonlinear torque is applied to force vector. To describe the coupled behavior of the linear rotor with nonlinear components, additional nodes have to be added into the system. The effects of those nonlinear components are applied to the system in terms of nonlinear forces/moments. For nonlinear bearings and dampers, nonlinear forces/moments are calculated by solving Reynolds equation.

The $4^{\text {th }}$ Runge-Kutta method is applied to solve the equations of motion numerically for constant or various rotational speed problems. To solve the various rotational speed cases, the implicit fixed-point iteration method is used to solve initial acceleration of each time point. 


\section{Chapter 4}

\section{Flexible Three-Disk Rotor with Nonlinear Short Journal Bearings and Nonlinear Squeeze Film}

\section{Dampers}

\subsection{Overview}

This chapter describes several transient analyses of a three-disk flexible rotor model supported with two short journal bearings in Section 4.1 through 4.4. A linear transient analysis is applied first to validate the transient solver by comparing forced response between the steady state method and the transient method. Then a nonlinear analysis is applied to the linear rotor with nonlinear short journal bearings. According to the results from both numerical analyses and experimental data, the rotor supported with plain journal bearings goes to unstable with increasing the rotational speed. To increase stability of the system under high rotational speed, two nonlinear squeeze film dampers are added onto the bearings. Waterfall diagrams of before and after adding the squeeze film dampers are calculated o evaluate the effects of the dampers. 
Section 4.2 discusses the rotor system and the beam element based rotor model. Section 4.3 presents the transient analysis of the linear system to validate the finite element based fourth-order Runge-Kutta solver by comparing the results based on steady state analysis. Section 4.4 demonstrates transient analyses of the rotor supported with nonlinear short journal bearings. Section 4.5 discusses the transient analyses of the rotor supported with nonlinear short journal bearings and nonlinear short squeeze film dampers together. Section 4.6 discusses the results found through the nonlinear transient analyses of the flexible 3-disk rotor.

The analyses performed in this chapter were done using a Dell Precision T3500 computer with an Intel Xeon CPU @ $2.53 \mathrm{GHz}, 12 \mathrm{~GB}$ of RAM and a 64-bit Windows OS. Matlab version 7.13 (R2011b) was used for the 64-bit OS.

\subsection{Three-Disk Flexible Rotor Model}

The three-disk axial symmetric rotor described in this chapter is shown in Fig. 4.1. The rotor has a length of 24 inches, a shaft diameter of 2 inches and a total mass of $101.35 \mathrm{lb}$ including the three attached disks. The figures illustrate the overall layout and the locations the bearing locations.

The rotor discussed in this Chapter is modeled with Timoshenko beam element. The system model, shown in Fig. 4.1, is defined by 25 nodes and 24 elements. Each node has 6 degrees of freedom for the lateral coordinates $\mathrm{x}$ and $\mathrm{y}$, the rotation about these axes $\theta_{x}$ and $\theta_{y}$, and the axial displacement $\mathrm{z}$ and rotation of the rotor $\theta_{y}$. The respective coordinate system of the rotor is described in Fig. 4.1. The bearing locations are donated with the red markers across the center of shaft in Fig. 4.1, the damper locations are same as the 
bearings but installed outside of the bearings. A side view of a simplified structure of the shaft-bearing-damper is shown in Fig. 4.2. The probe locations and the unbalance locations are indicated as blue and yellow colors, respectively. Table 4.1 describes the detailed finite element model information including element length, diameter and lumped mass/inertia. The Young's Modulus of elasticity is $30 \mathrm{E}+6 \mathrm{psi}$, the density is $0.283 \mathrm{lb} / \mathrm{m}^{3}$, and the Poisson's ratio is 0.3. Since there is no axial force and moment, to save computing time, only the lateral analyses are applied to the rotor in this chapter.

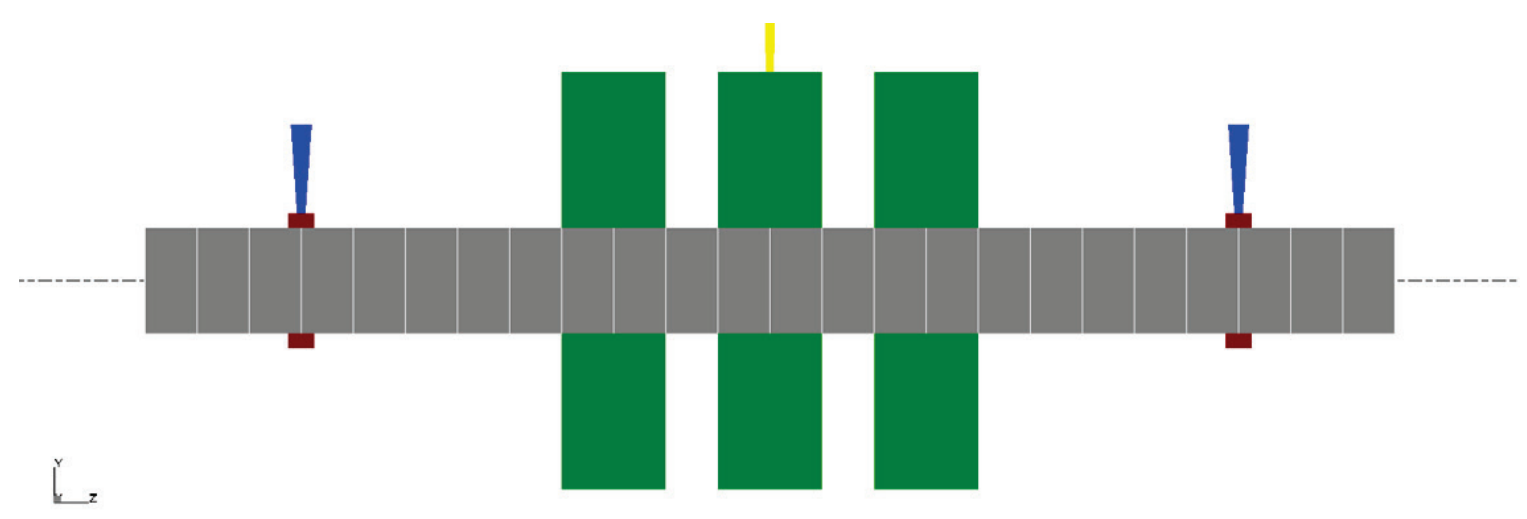

Figure 4.1 Twenty-Five Nodes Three-Disk Flexible Rotor Model

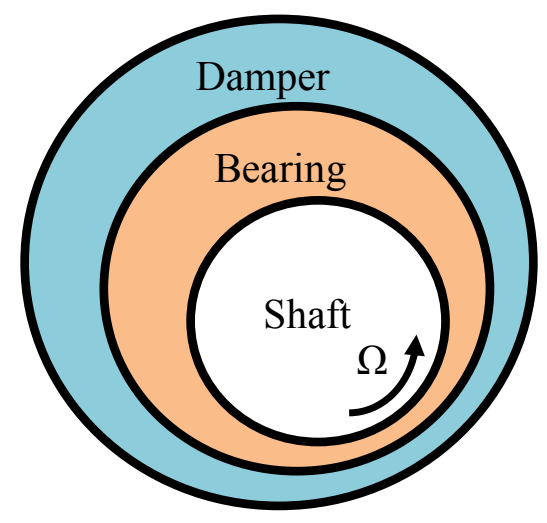

Figure 4.2 Cross Section of Bearing with Damper Outside 
Table 4.1 Three-Disk Flexible Rotor Model

\begin{tabular}{|c|c|c|c|c|c|c|}
\hline Node & L (in) & OD (in) & ID (in) & W & ${\text { It }\left(\mathrm{lbf}_{-}{ }^{2}\right)}^{2}$ & Ip $\left(\mathrm{lbf}_{-1}{ }^{2}\right)$ \\
\hline 1 & 1 & 2 & 0 & 0 & 0 & 0 \\
\hline 2 & 1 & 2 & 0 & 0 & 0 & 0 \\
\hline 3 & 1 & 2 & 0 & 0 & 0 & 0 \\
\hline 4 & 1 & 2 & 0 & 0 & 0 & 0 \\
\hline 5 & 1 & 2 & 0 & 0 & 0 & 0 \\
\hline 6 & 1 & 2 & 0 & 0 & 0 & 0 \\
\hline 7 & 1 & 2 & 0 & 0 & 0 & 0 \\
\hline 8 & 1 & 2 & 0 & 0 & 0 & 0 \\
\hline 9 & 1 & 2 & 0 & 0 & 0 & 0 \\
\hline 10 & 1 & 2 & 0 & 26.672 & 266.713 & 122.247 \\
\hline 11 & 1 & 2 & 0 & 0 & 0 & 0 \\
\hline 12 & 1 & 2 & 0 & 0 & 0 & 0 \\
\hline 13 & 1 & 2 & 0 & 26.672 & 266.713 & 122.247 \\
\hline 14 & 1 & 2 & 0 & 0 & 0 & 0 \\
\hline 15 & 1 & 2 & 0 & 0 & 0 & 0 \\
\hline 16 & 1 & 2 & 0 & 26.672 & 266.713 & 122.247 \\
\hline 17 & 1 & 2 & 0 & 0 & 0 & 0 \\
\hline 18 & 1 & 2 & 0 & 0 & 0 & 0 \\
\hline 19 & 1 & 2 & 0 & 0 & 0 & 0 \\
\hline 20 & 1 & 2 & 0 & 0 & 0 & 0 \\
\hline 21 & 1 & 2 & 0 & 0 & 0 & 0 \\
\hline 22 & 1 & 2 & 0 & 0 & 0 & 0 \\
\hline 23 & 1 & 2 & 0 & 0 & 0 & 0 \\
\hline 24 & 1 & 2 & 0 & 0 & 0 & 0 \\
\hline 25 & 0 & 2 & 0 & 0 & 0 & 0 \\
\hline & & & & & & \\
\hline
\end{tabular}

\subsection{Validation of Linear Transient Rotor dynamic Analysis}

The accuracy of the transient solution of any model is of extreme importance to engineers when they are designing a new mechanical system or modeling an existing one. The predicted behavior that is obtained by transient analysis in a computer program has to give the design engineer confidence in its accuracy and enable that person to make justified design decisions with the acquired results. Although all model results should 
never be blindly accepted to be $100 \%$ accurate, they are often used as a basis in all engineering methods to build a new structure or model an existing one. It is better when they are experimentally validated with test results. The modeling of structure using finite elements has been a norm in the industry for years now and the precision of the results have gotten closer and closer to the actual behavior of the structure that is being modeled.

\subsubsection{Linear Bearing Model}

Before the nonlinear analysis, a transient response of a linear rotor-bearing system is applied to compare the unbalance forced response based on a steady state analysis. For a stable linear system, the transient forced response should be exactly same as steady state results, after the steady state has been established. From the published example [122], the 3-disk rotor is supported by two identical 5-pad tilting pad bearings. The synchronously reduced dynamic coefficients from 2,000 to $16,000 \mathrm{rpm}$ listed in Table 4.2. In this dissertation, those coefficients are used to validate the transient solver only. To excite different modes, three unbalances placed at different phase angles are located on three disks. Some other rotor key parameters are following:

- Steel shaft with the length of 24 inches, the diameter of 2 inches

- Three disks at 9 inch, 12 inches and 15 inches from left to right on shaft

- Steel disk: 8 inch diameter, 2 inches thickness, with mass of $26.672 \mathrm{lbm}$ and polar inertia of $226.713 \mathrm{lbm}-\mathrm{in}^{2}$

- Two plain journal bearings ( $\mathrm{L}=1$ in; $\mathrm{D}=2$ in) at 3 inches \& 21 inches with radial clearance of 3 mils 
- Two SFDs ( $\mathrm{L}=1$ in; $\mathrm{D}=3$ in) located outside of the two bearings with radial clearance 6 mils

- Three unbalances placed on disks $\left(0.5\right.$ oz-in $0^{\circ}, 1$ oz-in $90^{\circ}, 0.5$ oz-in $\left.180^{\circ}\right)$

- 2 Probes, at $0^{\circ}$ and $90^{\circ}$, at each bearing location

- Timoshenko beam element, 25 shaft nodes +2 SFD nodes, total $104(100+4)$ degrees of freedom

Table 4.2 Bearing Dynamic Coefficients as Function of Speed [122]

\begin{tabular}{|c|c|c|c|c|}
\hline Speed $(\mathrm{rpm})$ & $\mathrm{K}_{\mathrm{xx}}(\mathrm{lbf} / \mathrm{in})$ & $\mathrm{K}_{\mathrm{xy}}(\mathrm{lbf} / \mathrm{in})$ & $\mathrm{K}_{\mathrm{yx}}(\mathrm{lbf} / \mathrm{in})$ & $\mathrm{K}_{\mathrm{yy}}(\mathrm{lbf} / \mathrm{in})$ \\
\hline 2000 & 38601.54 & 36.10938 & 81.86719 & 52588.27 \\
\hline 4000 & 57810.68 & -127.9844 & 102.3594 & 68011.77 \\
\hline 6000 & 75565.91 & -274.1094 & 258.4062 & 83794.3 \\
\hline 8000 & 91934.54 & -483.4531 & 474.75 & 99067.23 \\
\hline 10000 & 105787.4 & -755.2969 & 752.5625 & 112254. \\
\hline 12000 & 117891.2 & -1094.219 & 1088.594 & 123904.6 \\
\hline 14000 & 128550.6 & -1496.406 & 1487.719 & 134217. \\
\hline 16000 & 139211.9 & -1951.906 & 1962.719 & 144593. \\
\hline
\end{tabular}

\begin{tabular}{|c|c|c|c|c|}
\hline Speed $(\mathrm{rpm})$ & $\mathrm{C}_{\mathrm{xx}}(\mathrm{lbf}-\mathrm{s} / \mathrm{in})$ & $\mathrm{C}_{\mathrm{xy}}(\mathrm{lbf}-\mathrm{s} / \mathrm{in})$ & $\mathrm{C}_{\mathrm{yx}}(\mathrm{lbf}-\mathrm{s} / \mathrm{in})$ & $\mathrm{C}_{\mathrm{yy}}(\mathrm{lbf}-\mathrm{s} / \mathrm{in})$ \\
\hline 2000 & 544.5408 & 0 & 0 & 496.1638 \\
\hline 4000 & 449.5349 & 0 & 0 & 474.8226 \\
\hline 6000 & 391.7378 & 0 & 0 & 404.688 \\
\hline 8000 & 350.412 & 0 & 0 & 358.4907 \\
\hline 10000 & 315.4584 & 0 & 0 & 321.0936 \\
\hline 12000 & 286.6295 & 0.755979 & -1.00606 & 290.8449 \\
\hline 14000 & 262.4451 & 0 & 0 & 265.7449 \\
\hline 16000 & 243.596 & 0 & 0 & 246.2656 \\
\hline
\end{tabular}




\subsubsection{Unbalance Forced Response}

The unbalance forced response is performed to predict the response of the rotor due to the unbalances placed on the rotor. To predict the response of the rotor, the unbalances are applied at locations with large mass, such as disks, to excite the modes under the rotational speed. The linear theory is based on the steady state assumption, which means the vibration frequency, either synchronized with the rotational speed or nonsynchronized, is given to solve the amplitude and phase angle of the forced response. The time transient method numerically solves the equations of motion directly, with given initial conditions, without the assumption of steady state or known response frequency. The amplitude and the phase angle are calculated from a time dependent output, and the frequency of vibration is calculated from a Fourier transformation of time domain output.

Fig. 4.3 shows the steady state output at bearing location (node \#4) from MatlabRotor [123]. The time transient output at the same location is shown in Fig. 4.4. The initial nodal displacement vector $(u)$ is the static displacements of all nodes, which are calculated from the shaft stiffness matrix, the bearing stiffness coefficients and the gravitational forces vector. The initial nodal velocity vector $(\dot{u})$ is zero in this example. During the transient analysis, the time step is $4.0 \times 10^{-6}$ second. For the transient analysis of 0.25 second, the total computing time is 41.8 seconds. The time transient output at bearing location (node \#4) under the rotational speed of 6,000 rpm is shown in Fig. 4.4. The figure provides time transient displacements in the $\mathrm{x}$ and $\mathrm{y}$ direction, displacement orbit of the node, and velocity orbit of the node. The system goes into steady-state after $\mathrm{t}>0.025$ second, as shown in Fig. 4.4. The fast Fourier transformation results of the output in frequency domain are shown in Fig. 4.5. There is only one peak, which is same as the 
rotational speed $(6,000 \mathrm{rpm})$, thus the response is a purely synchronous response. Fig. 4.6 shows the static deformation of rotor due to gravity only. Fig. 4.7 shows orbits of all nodes around 0.25 second due to both gravitational loading and unbalances. Due to gravitational force, the average displacement in vertical direction is less than zero.

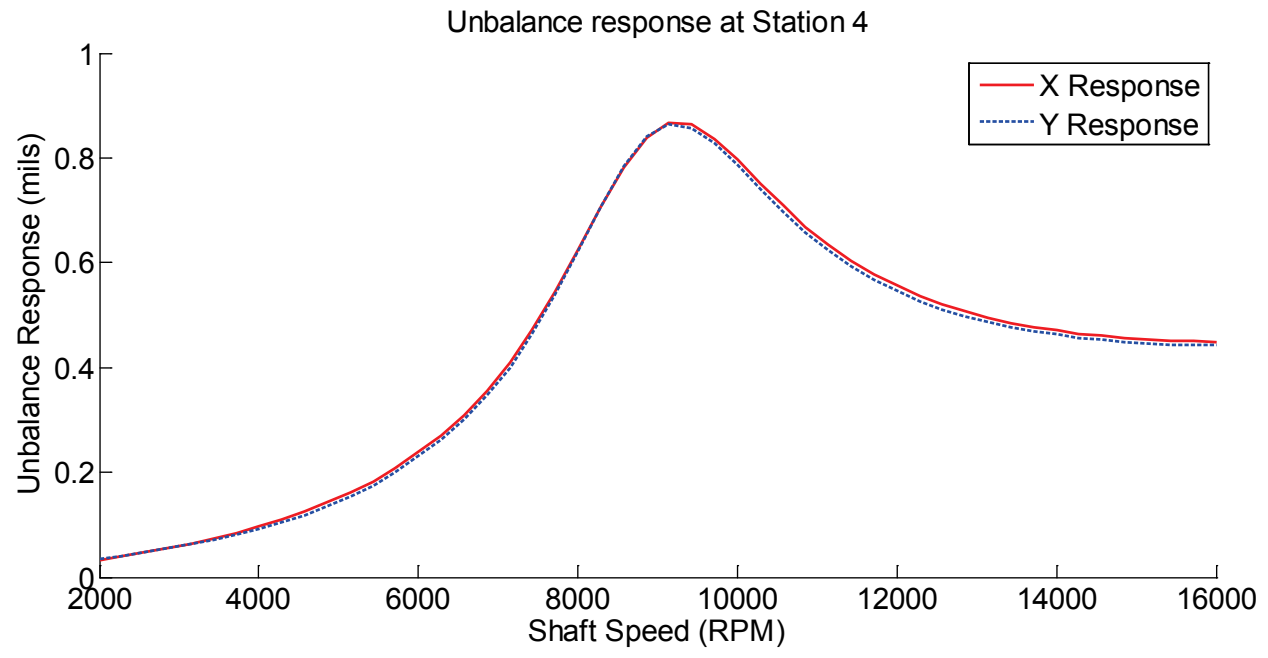

Figure 4.3 Steady State Forced Response at Bearings Location (Node 4)
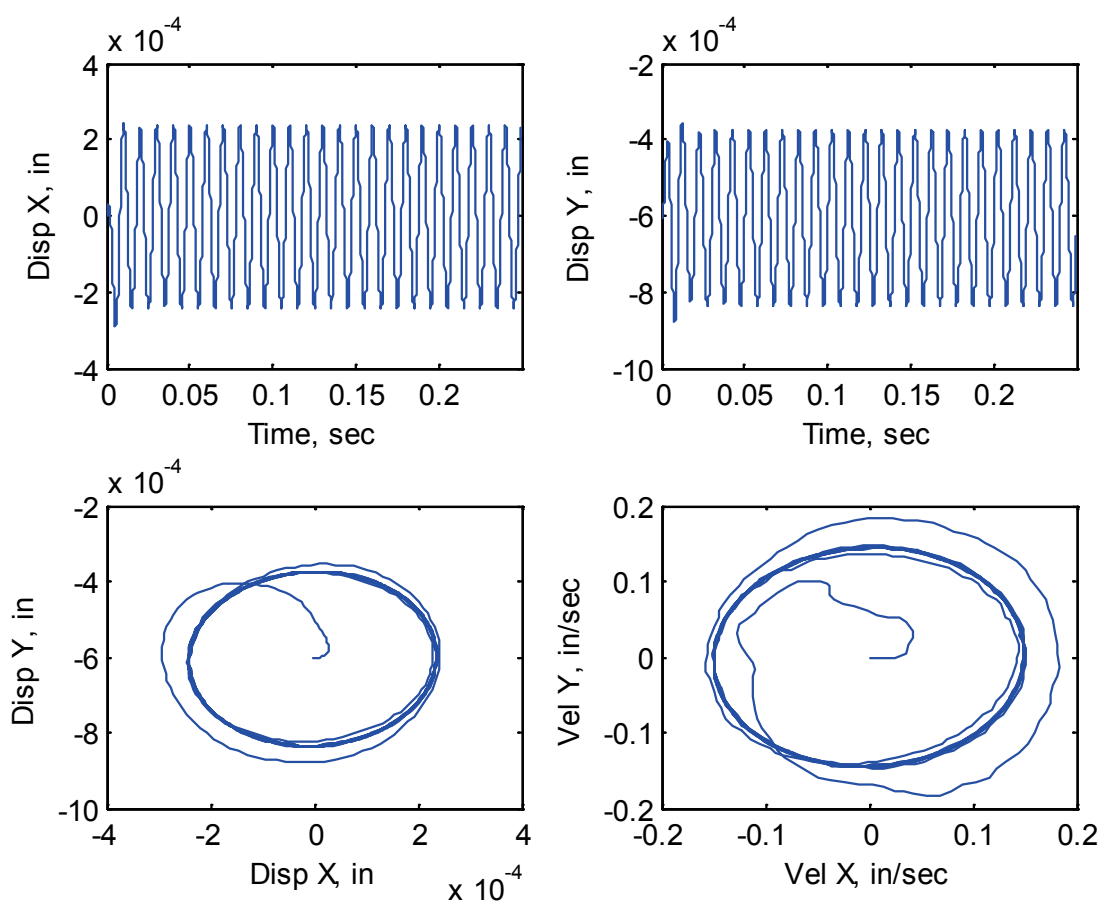

Figure 4.4 Transient Forced Response at Bearing Location (Node \#4) 

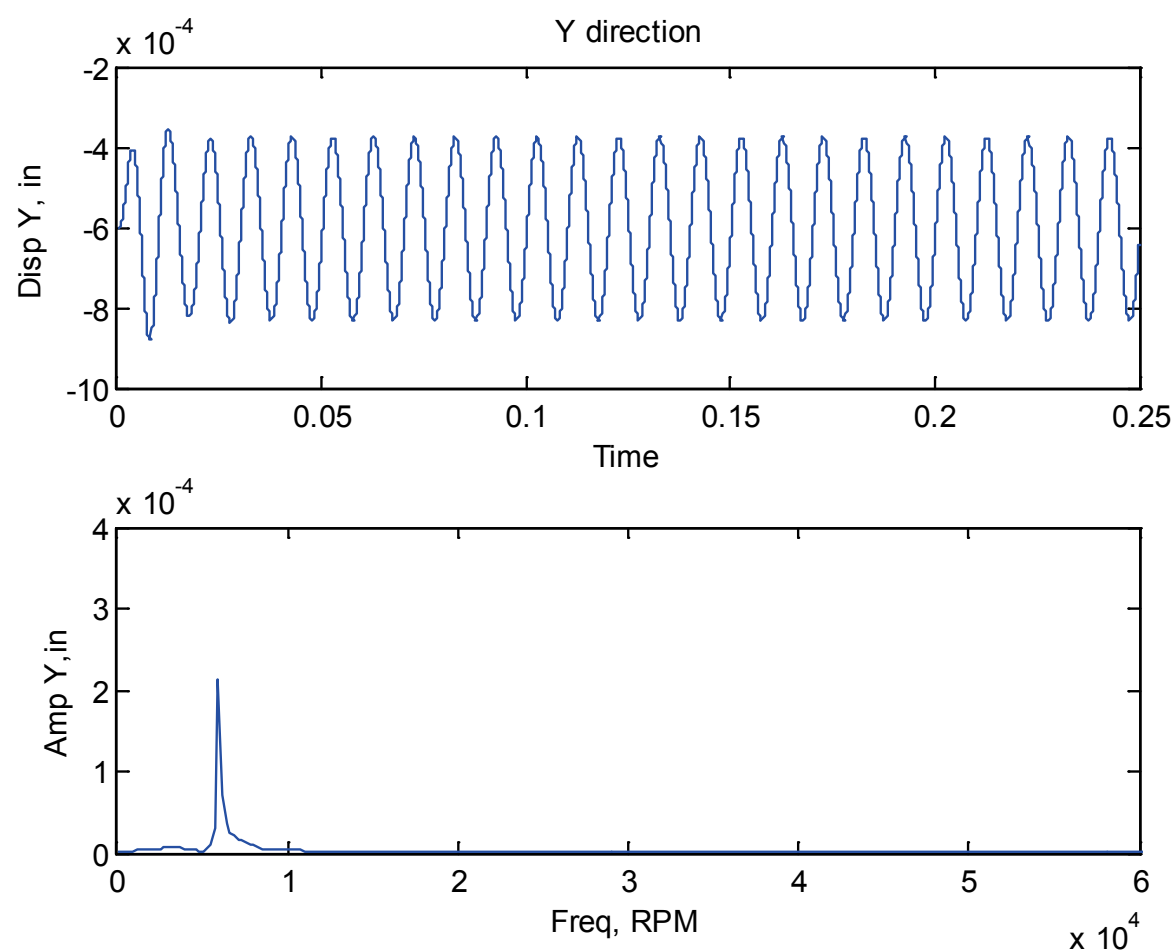

Figure 4.5 Forced Response (FFT) at Bearing Location (Node \#4)

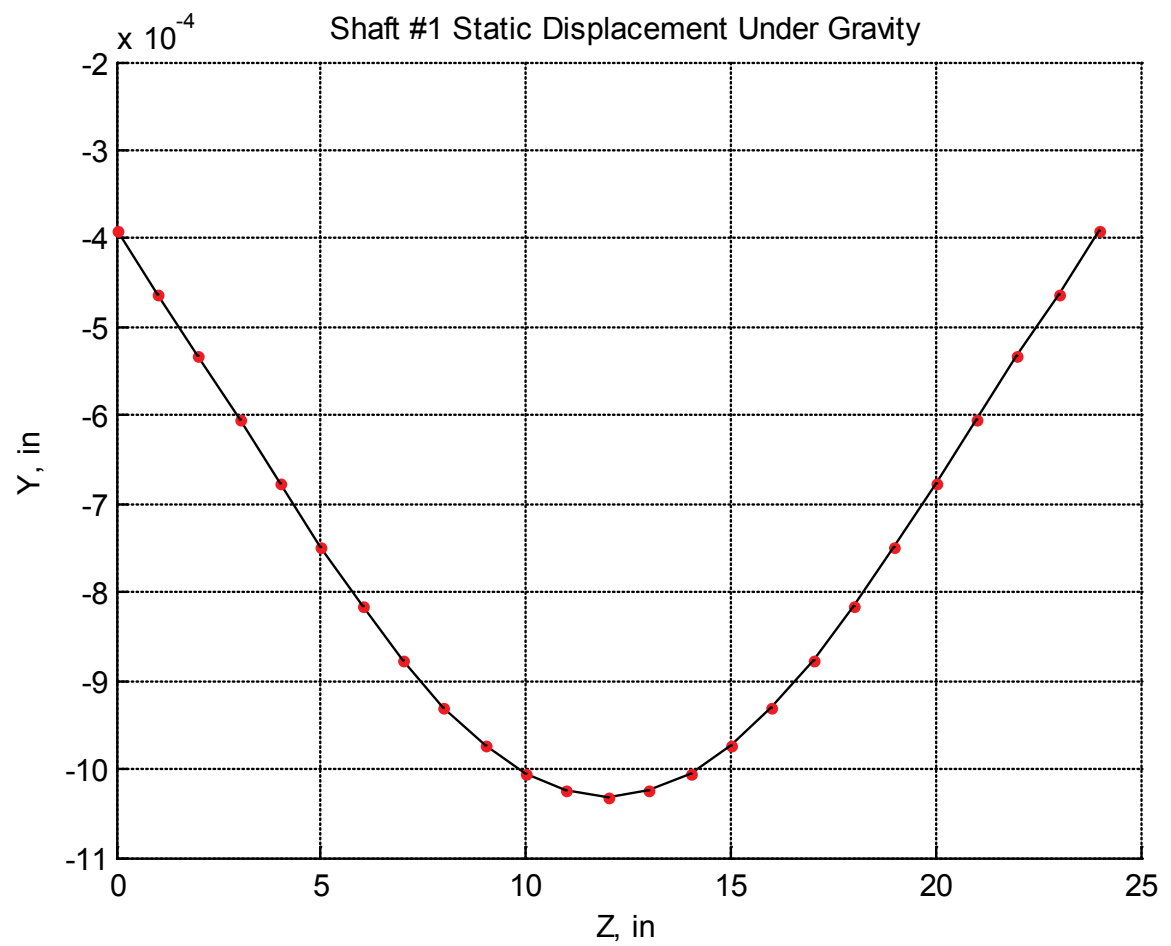

Figure 4.6 Rotor Static Deformation under Gravity Loading 


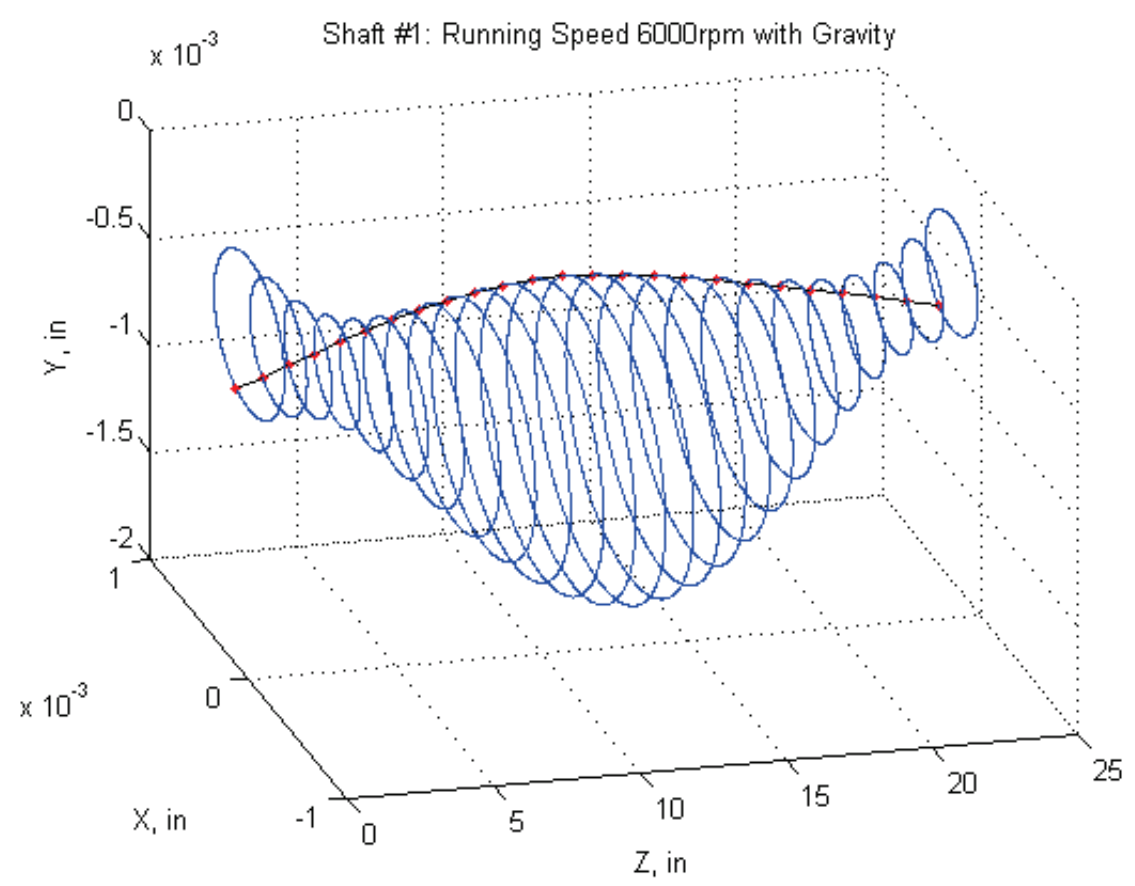

Figure 4.7 Rotor Orbits at 6,000 rpm with Gravitational Loading and Unbalances

The computational comparison of amplitudes at different speeds between steady state analyses and transient analyses for the linear system is listed in Table 4.3. In the Table, the output data are from the bearing location at node \#4. The amplitudes of each rotational speed are calculated from time transient response after the steady state is established, as shown in Fig. 4.4. From the comparison of MatlabRotor and RotorTran (transient code), the forced response difference in amplitudes is less than $0.2 \%$ than for the linear system. The transient result, however, gives more details of nodal displacements due to gravity in linear analysis and there is no initial assumption of synchronous response. The response frequency is calculated through a fast Fourier transformation as shown in Fig. 4.5.

A steady state based linear method using the finite element method is the most common method for performing rotor-dynamic analysis. The nonlinear bearing forces are 
usually linearized into equivalent stiffness and damping coefficients. This method works well for the weakly nonlinear systems, but has some limits for the strongly nonlinear systems. To more accurately describe the rotor behavior, often transient analyses and nonlinear models are used.

Table 4.3 Response Comparison between Linear and Transient at Bearings

\begin{tabular}{|c|c|c|c|}
\hline Speed (rpm) & $\begin{array}{c}\text { MatlabRotor } \\
\text { Amp. X (mil) }\end{array}$ & $\begin{array}{c}\text { RotorTran } \\
\text { Amp. X (mil) }\end{array}$ & Diff. \% \\
\hline 2,000 & 0.0334 & 0.0334 & 0.00 \\
\hline 4,000 & 0.0978 & 0.0978 & 0.00 \\
\hline 6,000 & 0.2381 & 0.2381 & 0.00 \\
\hline 8,000 & 0.6236 & 0.6225 & 0.18 \\
\hline 10,000 & 0.7964 & 0.7961 & 0.04 \\
\hline 12,000 & 0.5559 & 0.5555 & 0.07 \\
\hline 14,000 & 0.4712 & 0.4711 & 0.02 \\
\hline 16,000 & 0.4496 & 0.4495 & 0.02 \\
\hline
\end{tabular}

\begin{tabular}{|c|c|c|c|}
\hline Speed (rpm) & $\begin{array}{c}\text { MatlabRotor } \\
\text { Amp. Y (mil) }\end{array}$ & $\begin{array}{c}\text { RotorTran } \\
\text { Amp. Y (mil) }\end{array}$ & Diff. \% \\
\hline 2,000 & 0.0347 & 0.0347 & 0.00 \\
\hline 4,000 & 0.0917 & 0.0917 & 0.00 \\
\hline 6,000 & 0.2301 & 0.2301 & 0.00 \\
\hline 8,000 & 0.6210 & 0.6210 & 0.00 \\
\hline 10,000 & 0.7861 & 0.7858 & 0.04 \\
\hline 12,000 & 0.5455 & 0.5453 & 0.04 \\
\hline 14,000 & 0.4631 & 0.4628 & 0.06 \\
\hline 16,000 & 0.4428 & 0.4423 & 0.11 \\
\hline
\end{tabular}




\subsection{Three-Disk Rotor with Nonlinear Short Plain Journal}

\section{Bearings}

This section describes the unbalance forced response of the three-disk rotor with nonlinear bearings. Unlike the bearing used in Section 4.3 , the bearing is treated as a nonlinear component. The bearings used in this Chapter are the short plain journal bearings as shown in Table 4.4. For a given displacement and velocity of the shaft node inside the bearing, the pressure profile is calculated first by solving the Reynolds equation with the assumption of laminar flow of fluid film, constant density and viscosity of fluid. The method is presented in Section 3.3. Then the bearing forces are calculated by integrating the pressure profile on the fluid area, and finally applied to the equations of motion. For the transient analysis, the bearing forces need to be calculated at each time step according to nodal displacements and velocities.

\subsubsection{Pressure Profile of Short Plain Journal Bearing and Nonlinear Force Calculation}

For the short bearing $(\mathrm{L} / \mathrm{D} \leq 0.5)$, the pressure gradient along the $\mathrm{x}$ direction is far smaller than along the $\mathrm{z}$ direction. By ignoring $\partial P / \partial x$, the closed-form dimensionless pressure solution for the short plain bearing is shown in Eq. (3.4.4) with the boundary conditions and the cavitation condition. The nonlinear force then can be calculated by integrating the pressure over the film area (only positive pressures will be integrated to take into account cavitation). 
The bearing information used in this chapter is listed in Table 4.4. The dimensionless pressure profiles, calculated by Eq. (3.4.4), under various cases are presented in Figs. 4.8 4.10. A 3-dimensional plot of the "unwrapped" pressure profile is shown for each case. The values of the dimensionless displacements and velocities are given at the top left corner of the figures. An end view of the section at the bearing midspan is given with the pressure profile represented.

The bearing forces are calculated by integrating the pressure profile using Eq. (3.4.5). The result of the bearing forces with different example cases as Figs. 4.8 4.10 at the rotational speed $\Omega$ of $8,000 \mathrm{rpm}$ is listed in Table 4.5 . The dimensionless pressure profile and forces are same as calculated by Kirk [81].

Table 4.4 Bearing Information of 3-Disk Rotor

\begin{tabular}{l|l|l}
\hline Type: Short plain journal bearing \\
\hline Property & Value & Unit \\
\hline Length & 1 & in \\
\hline Diameter & 2 & in \\
\hline Bearing Clearance & 0.003 & in \\
\hline Viscosity & $5.8 \mathrm{E}-6$ & lbf-s/in \\
\hline
\end{tabular}

Table 4.5 Bearing Forces under Different Cases

\begin{tabular}{l|r|r}
\hline$\Omega=8,000 \mathrm{rpm}$ & Fx, lbf & Fy, lbf \\
\hline Case I: $\quad \bar{X}=0.20, \bar{Y}=-0.1, \dot{\bar{X}}=\dot{\bar{Y}}=0$ & 19.02 & 104.97 \\
\hline Case II: $\quad \bar{X}=0.5, \bar{Y}=-0.7, \dot{\bar{X}}=\dot{\bar{Y}}=0$ & -1183.4 & 6389.1 \\
\hline Case III: $\bar{X}=0, \bar{Y}=-0.5, \dot{\bar{X}}=0.5, \dot{\bar{Y}}=0$ & -326.42 & 239.29 \\
\hline
\end{tabular}



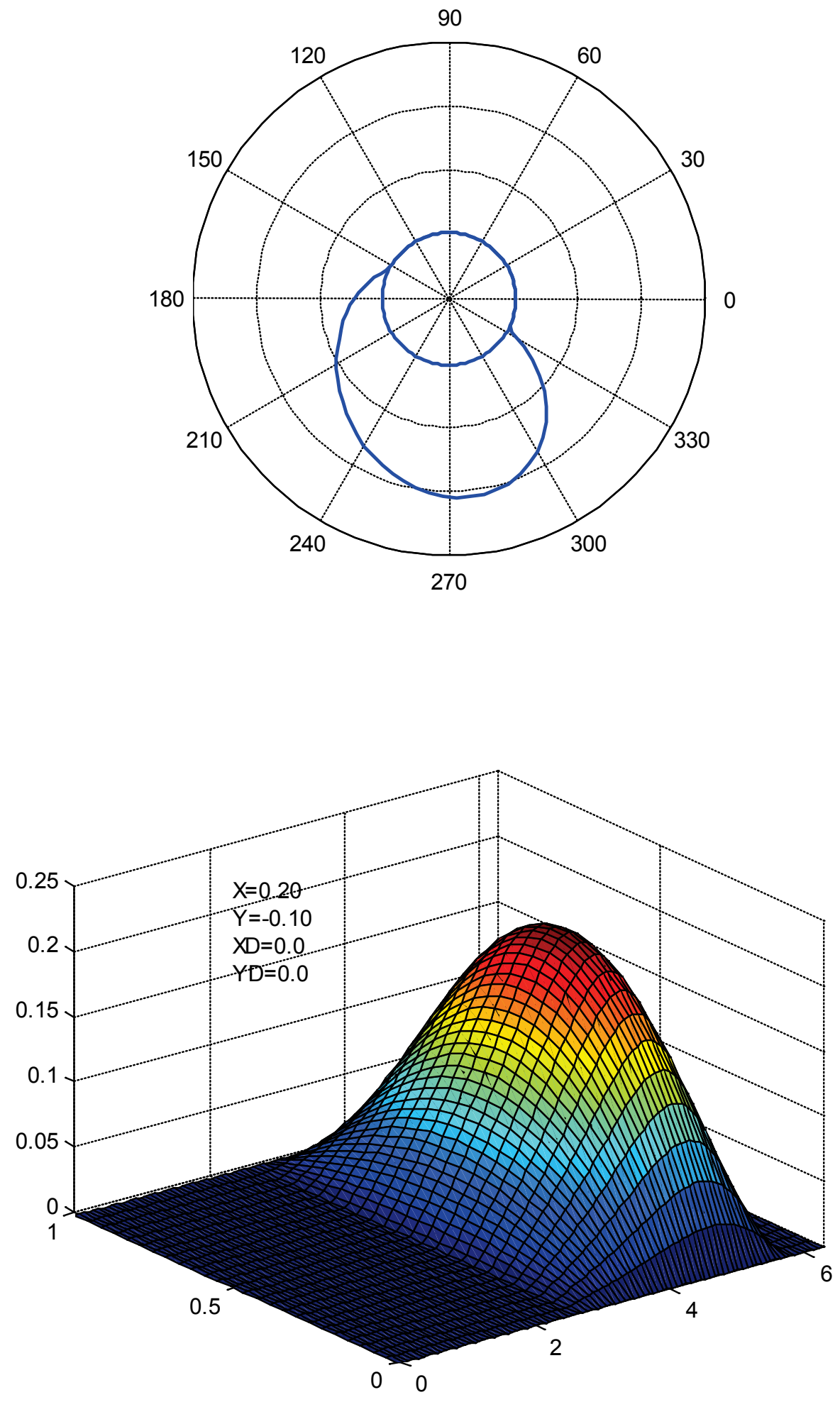

Figure 4.8 Pressure Profile and Pressure Surface for $\bar{X}=0.20, \bar{Y}=-0.1, \dot{\bar{X}}=\dot{\bar{Y}}=0$ 

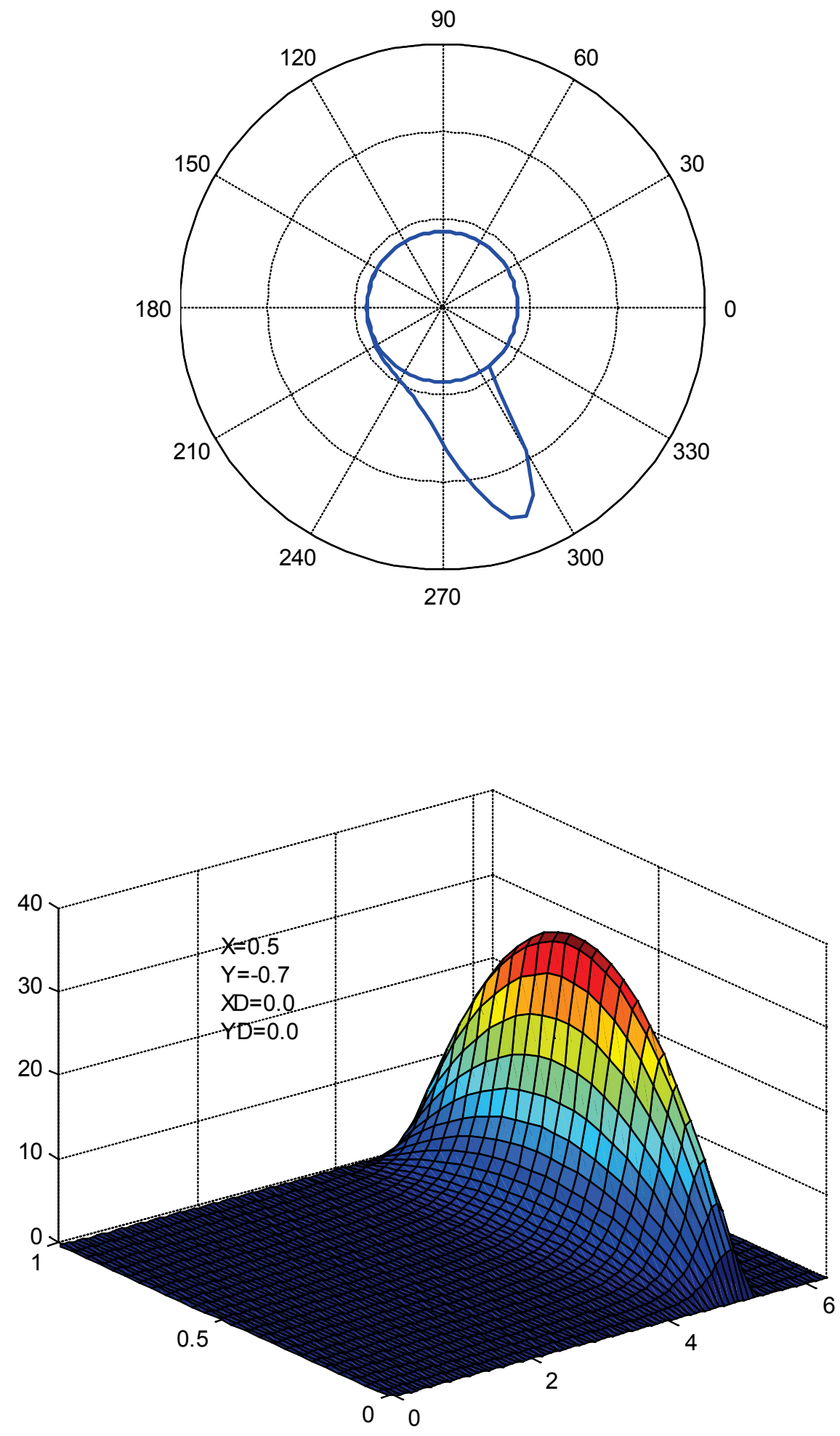

Figure 4.9 Pressure Profile and Pressure Surface for $\bar{X}=0.5, \bar{Y}=-0.7, \dot{\bar{X}}=\dot{\bar{Y}}=0$ 

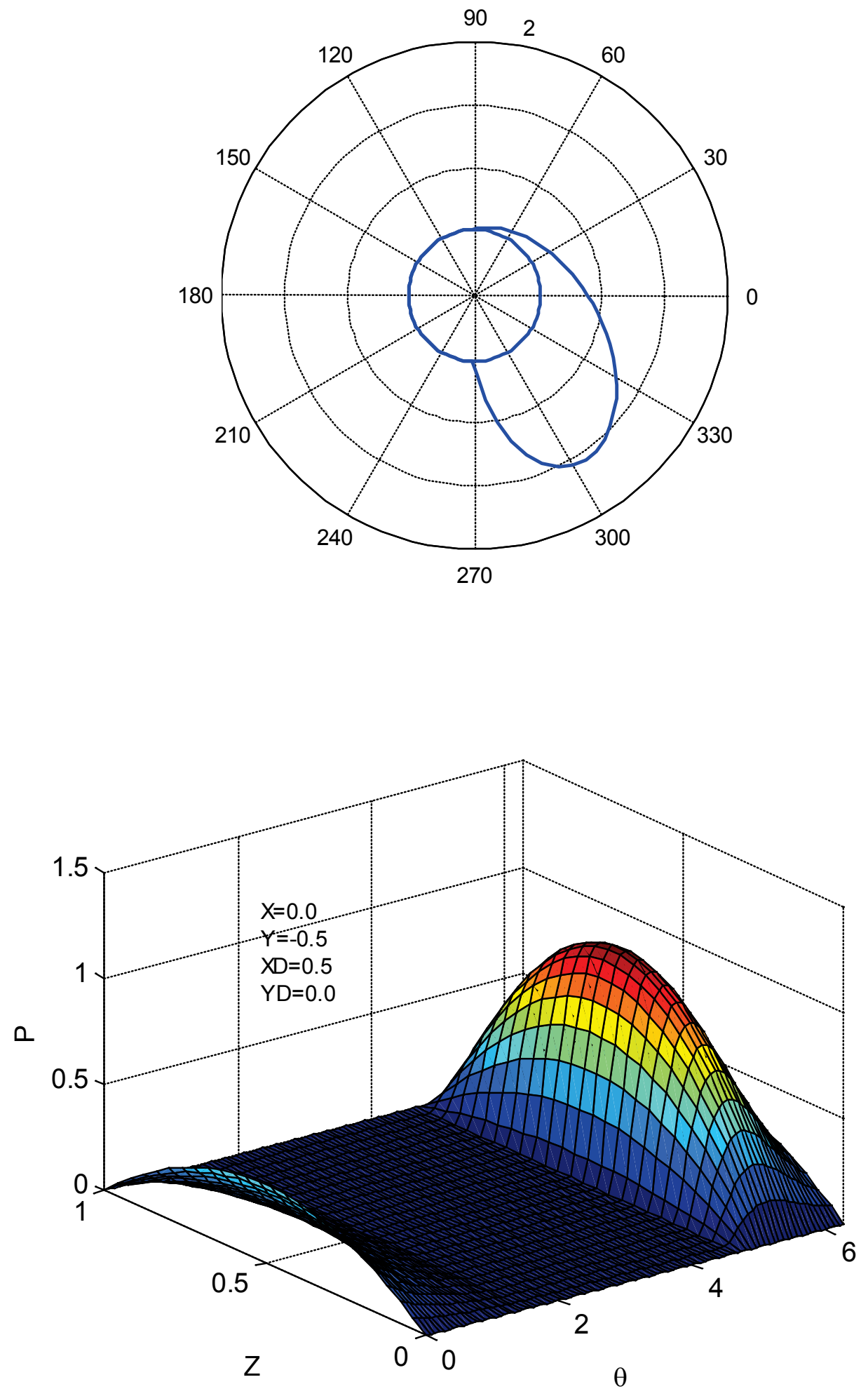

Figure 4.10 Pressure Profile and Pressure Surface for $\bar{X}=0, \bar{Y}=-0.5, \dot{\bar{X}}=0.5, \dot{\bar{Y}}=0$ 


\subsubsection{Transient Analyses of Rotor with Nonlinear Short Plain Journal}

\section{Bearings}

Using the Timoshenko beam based finite element method and the fourth-order RungeKutta integration method, the time transient response is calculated for the three-disk rotor supported with nonlinear short plain journal bearings. Due to the hydrodynamic effects, named "oil whirl" and "oil whip", in the plain journal bearings, the general rotor system often displays the nonlinear behavior with increased the rotational speed. The nonlinear behavior has been observed in experiments [2] and verified by analytical methods $[8,9]$. As to prior research, the sub-harmonic response appears when the rotational speed is around two times of the first bending critical speed of the system.

To understand and explain the sub-harmonic behavior of this rotor-bearing system, the undamped critical speed map, Fig. 4.11, was calculated first by solving natural frequencies of undamped system with possible stiffness ranges for the bearings. In the figure, the horizontal axis is the bearing stiffness from $1 \times 10^{3} \mathrm{lb} /$ in to $1 \times 10^{7} \mathrm{lb} / \mathrm{in}$, which covers the possible stiffness for the plain journal bearings. The vertical axis is the critical speeds with unit of rpm. In the figure, the first eight modes, beginning from the first bending mode, are given.

Waterfall diagrams of the of the rotor supported with two nonlinear plain journal bearings are shown in Figs. 4.12 and 4.13 with a step time $(\Delta t)$ of $4 \times 10^{-6}$ second and the total span time (or interval of integration) of $0 \sim 1.0$ second. The time transient output is from the horizontal direction of the bearing (node\#4) and the vertical direction of the bearing (node\#21). The three red lines $0.5 \mathrm{X}, 1 \mathrm{X}$ and $2 \mathrm{X}$ in figures indicate sub-harmonic, 


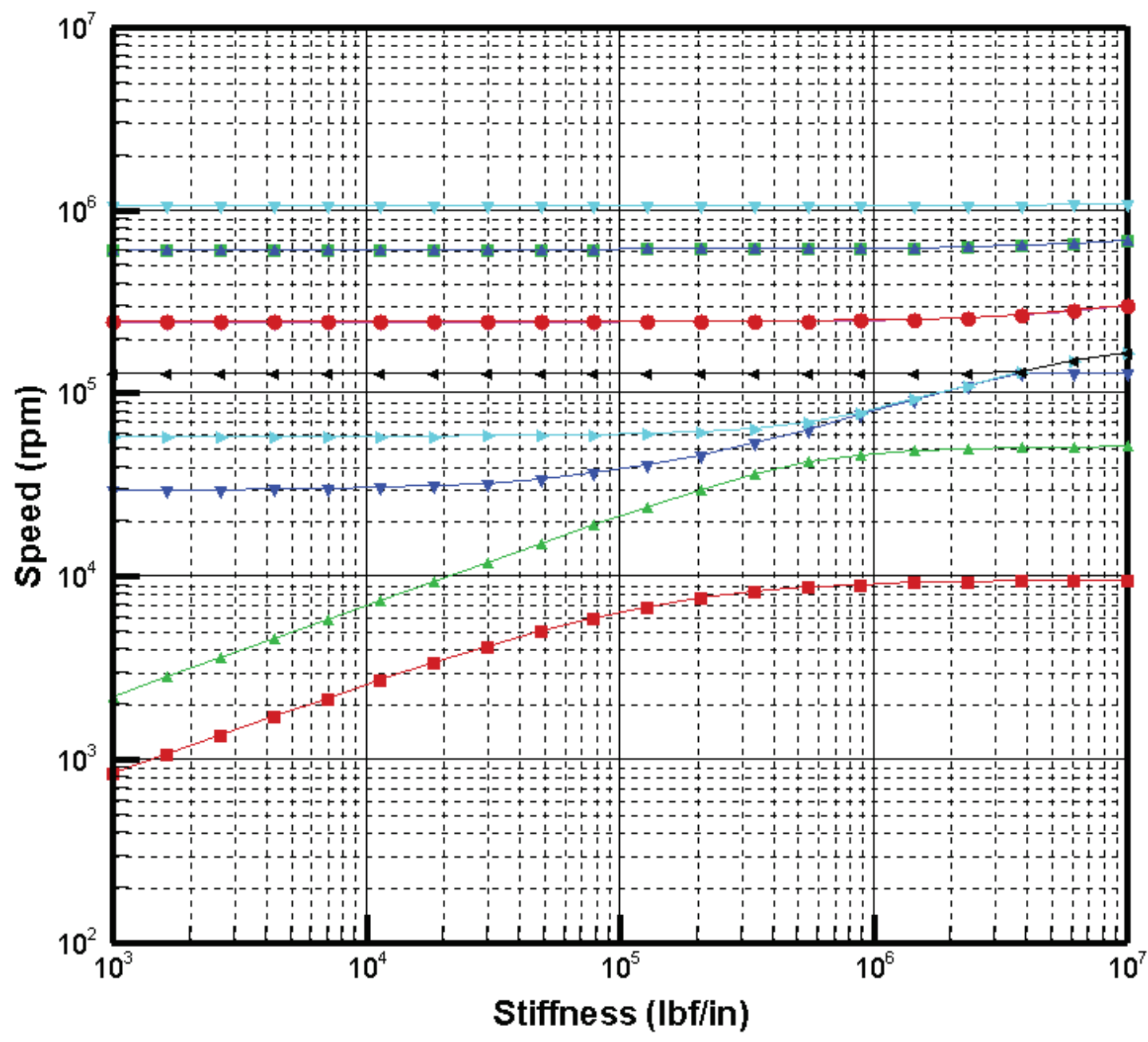

Figure 4.11 Undamped Critical Map of the 3-Disk Rotor

synchronous, and super-harmonic response, respectively. To make the sub-harmonic peaks clearer in the figure, two different methods (3D and 2D) of drawing waterfall diagrams are shown in the Fig. 4.12 and Fig. 4.13 respectively. According to experimental results by Bently [2] and Ehrich [3], the sub-harmonic appears when the 1st bending natural frequency is around 0.5 of the rotational speed due to "oil whirl". From the two waterfall diagrams, the sub-harmonic response appears when the rotational speed is close to $12,000 \mathrm{rpm}$, so the first bending critical speed for this system is around 6,000 rpm. In the critical speed map, Fig. 4.11, the bearing stiffness corresponding to the first 
bending mode of $6,000 \mathrm{rpm}$ is around $0.9 \times 10^{5} \mathrm{lbf} / \mathrm{in}$, which is a reasonable stiffness for the fluid plain journal bearings. The ratio of the sub-harmonic frequency and rotational speed is less than 0.5 due to "oil whip" when the rotational speed keeps increasing $[2,3]$. From the waterfall diagrams, Figs. $4.12 \& 4.13$, the sub-harmonic frequency goes below the $0.5 \mathrm{X}$ line to the lower frequencies at higher rotational speed $(\Omega>20,000 \mathrm{rpm})$.

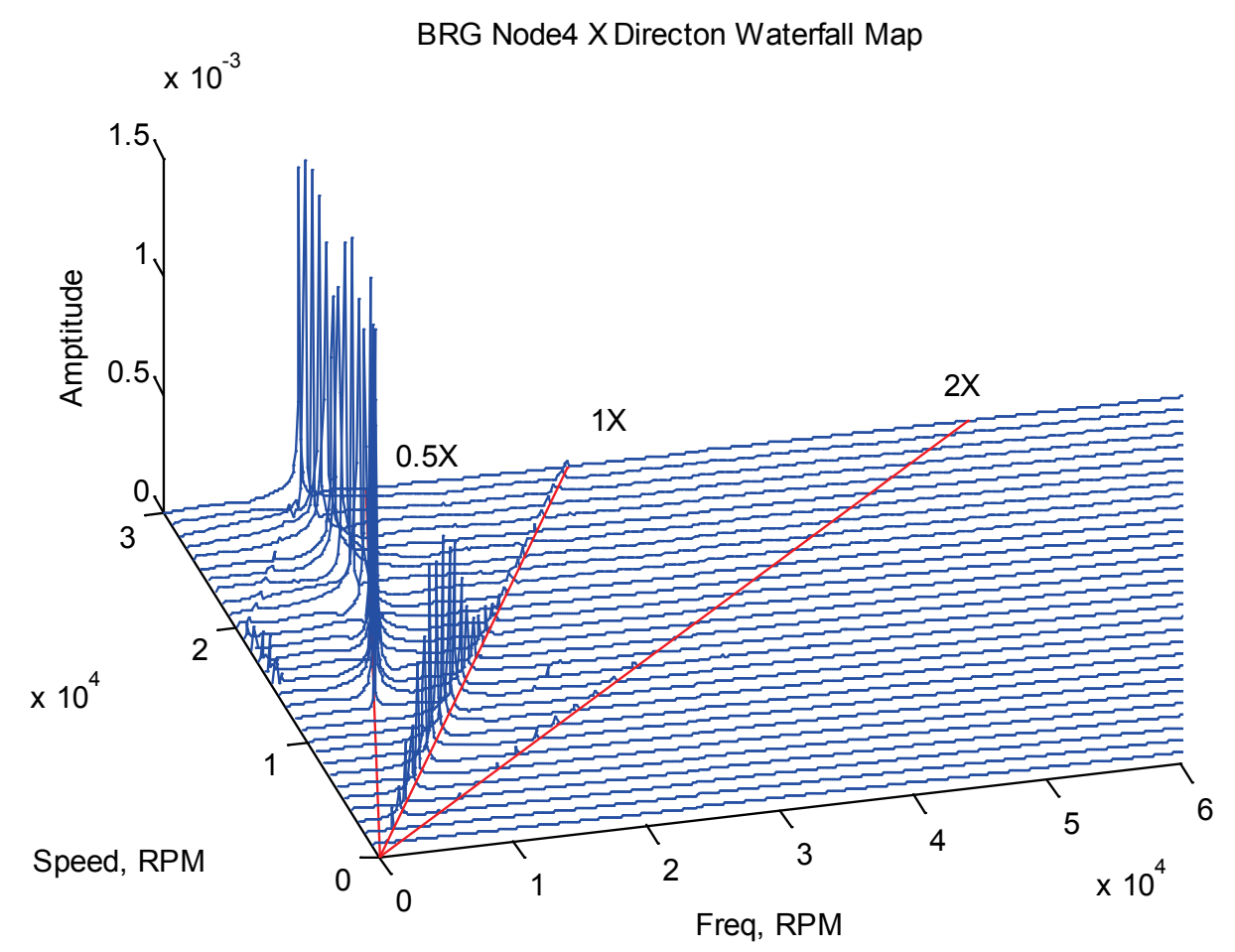

Figure 4.12 Waterfall Diagram of 3-Disk Rotor, Bearing at Node 4, Horizontal Direction 


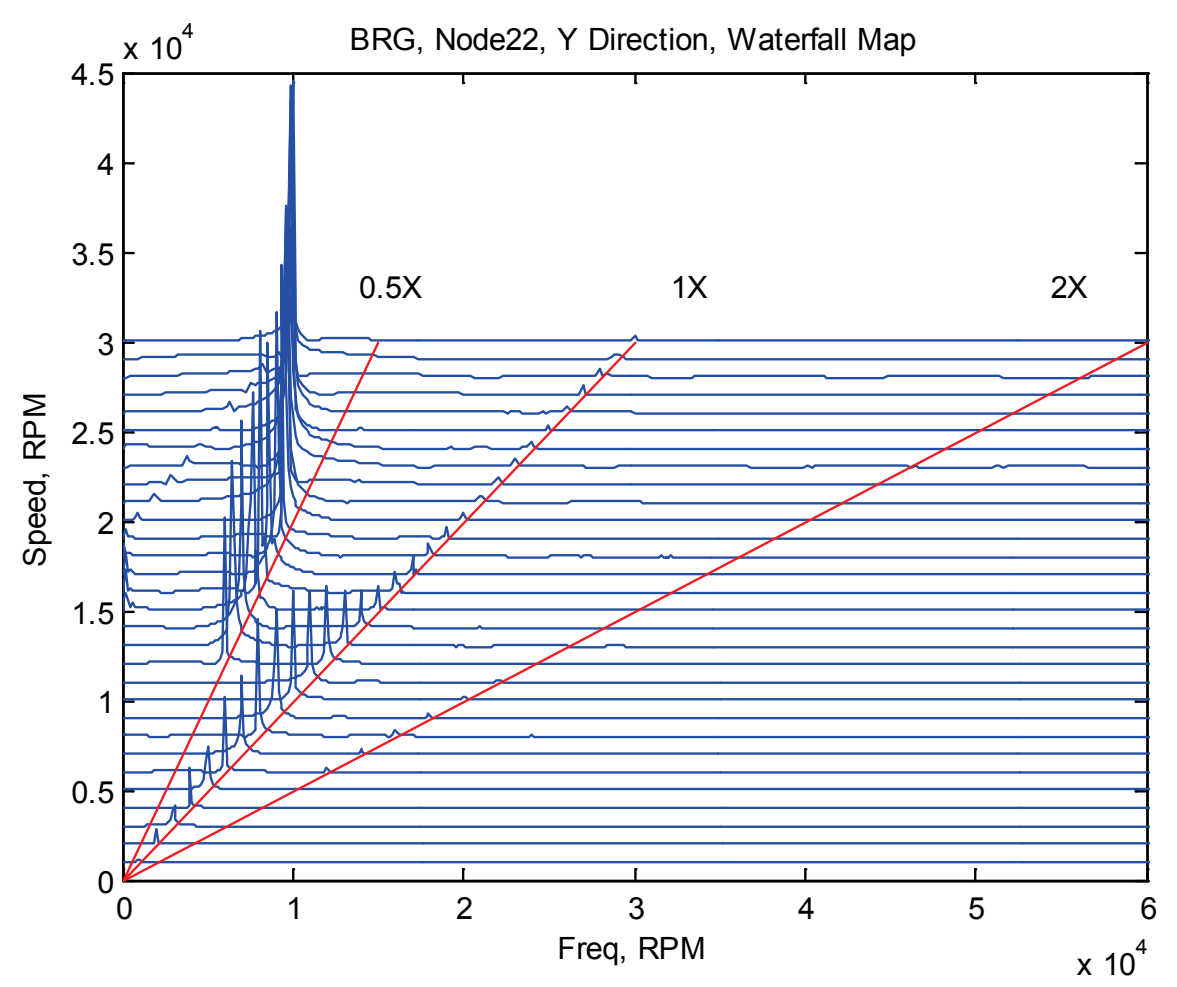

Figure 4.13 Waterfall Diagram of 3-Disk Rotor, Bearing at Node 22, Vertical Direction

According to the waterfall diagrams, the rotor is stable at the lower rotational speed $(\Omega<12,000 \mathrm{rpm})$. The transient response of the node inside the bearing at $6,000 \mathrm{rpm}$ is given in Fig. 4.14. The lower right figure shows the shaft center position and the bearing clearance, which is denoted with a circle in red. The bearing forces on horizontal $(X)$ and vertical $(Y)$ directions under at 6,000 rpm are shown in Fig. 4.15. Due to the rotor gravity load on negative $y$ direction, the average bearing force at vertical direction is half of the rotor weight $(50.51 \mathrm{bf})$. In the $X$ direction, however, the average bearing force is nearly zero. Since the rotor is stable at this rotating speed and the response is synchronous. The orbit of each node is a closed single round as shown in Fig. 4.16. In the figure, each blue orbit indicates several cycles of the node around time of 1.0 second, and the shaft center at 1.0 second is indicated with the black line with red points on it. 

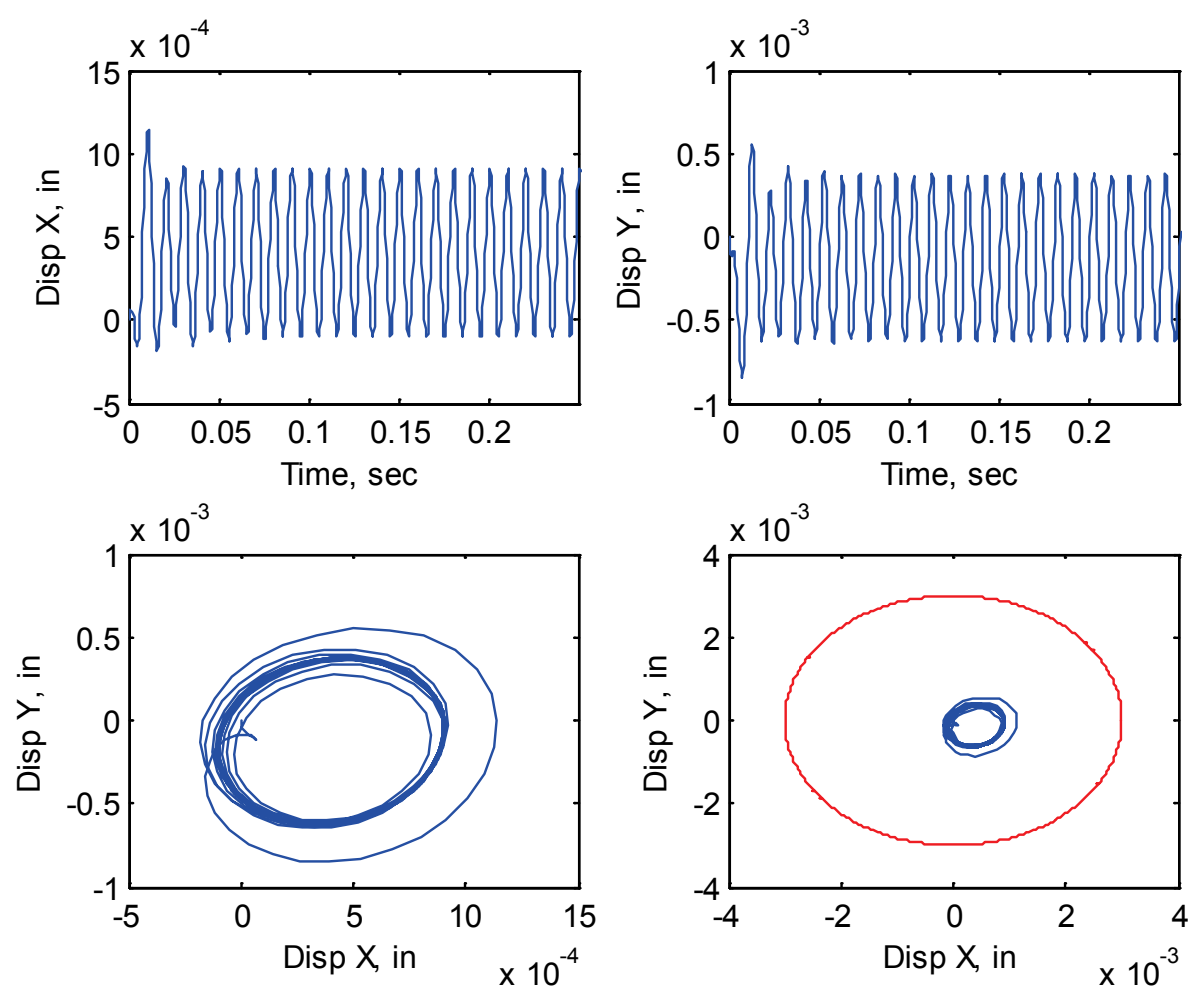

Figure 4.14 Time Transient Output at Bearing (Node\#4) at 6,000 rpm
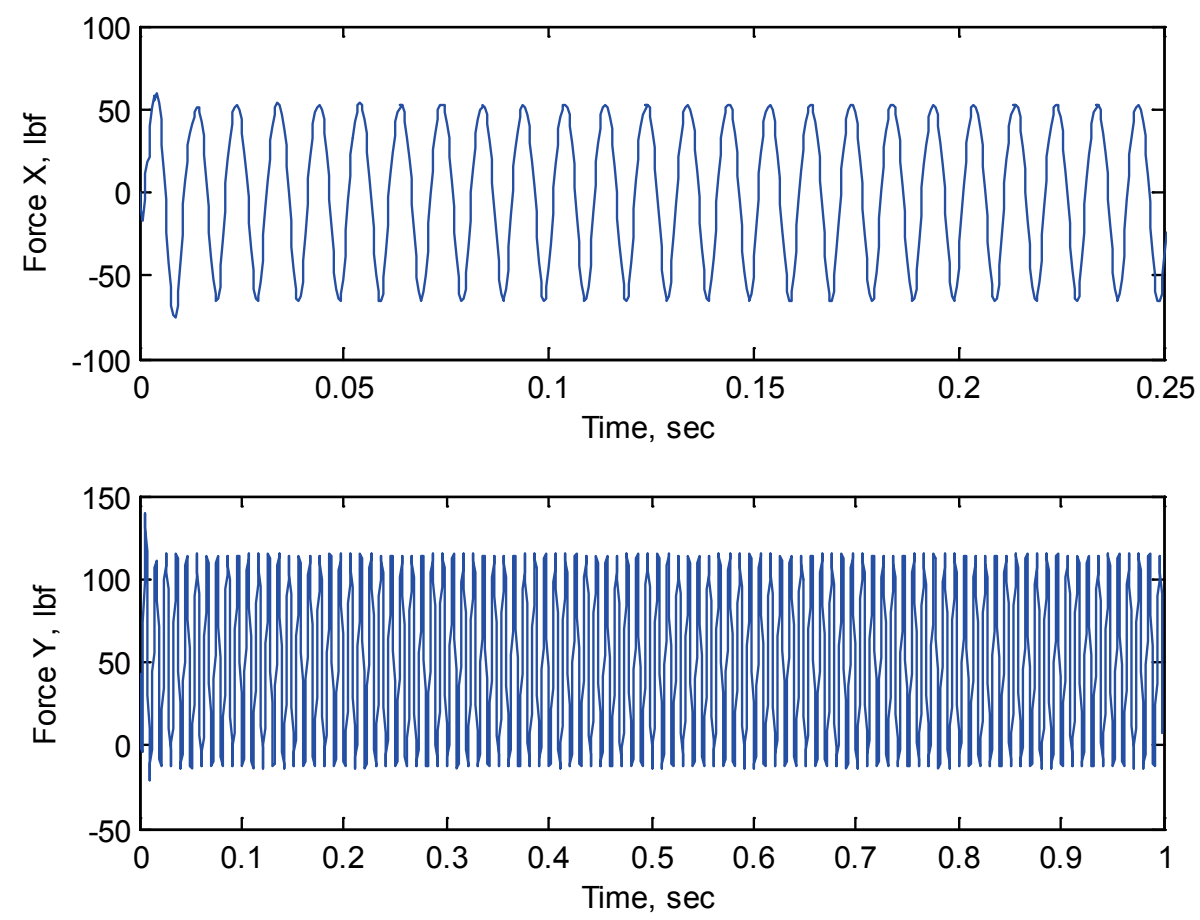

Figure 4.15 Time Transient Bearing Force (Node\#4) at 6,000 rpm 


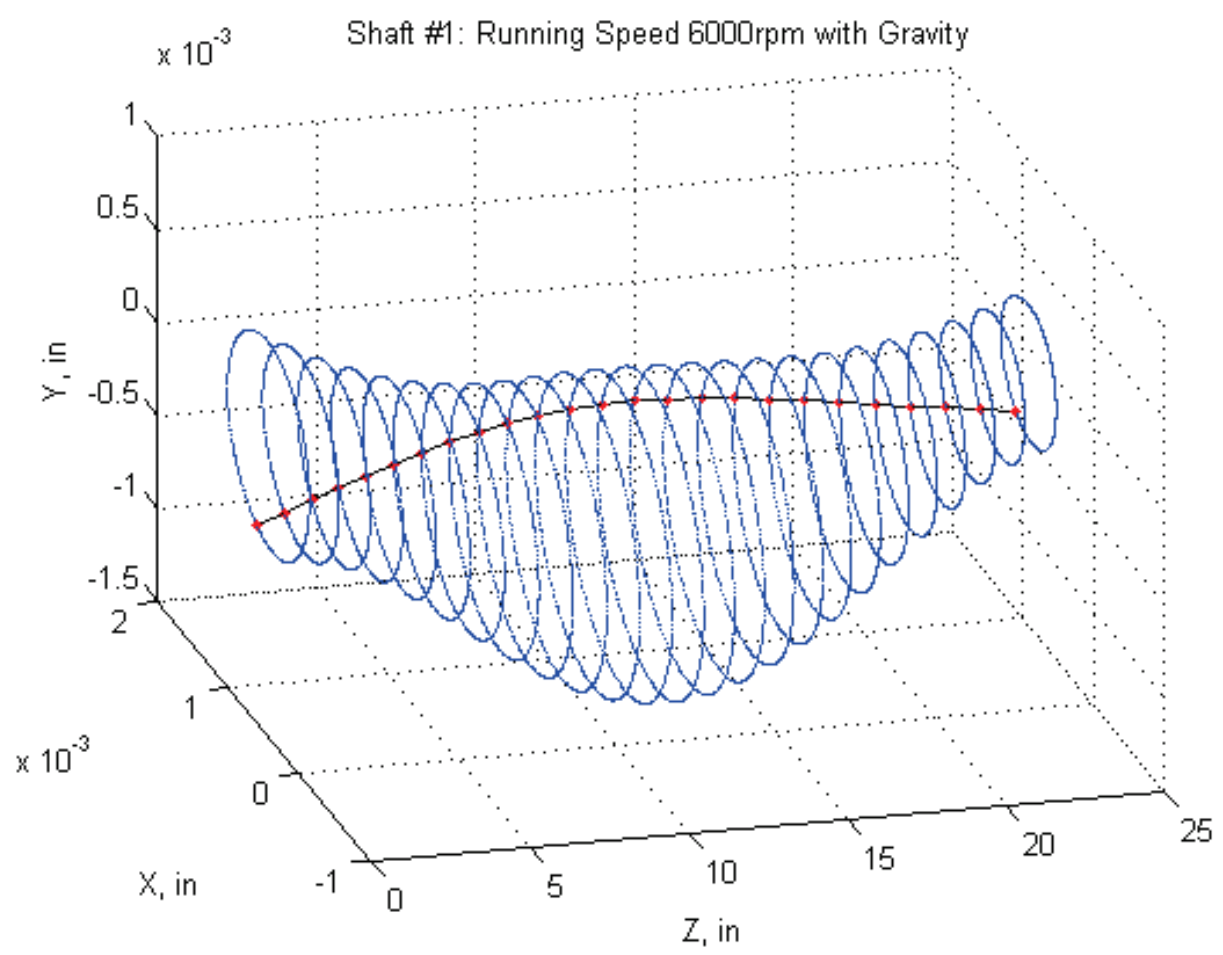

Figure 4.16 Orbits of the Rotor at 6,000 rpm, $1.0 \mathrm{sec}$, with Gravity Load and Unbalances

When the operating speed goes higher, the unbalance forced response of the rotor supported with two nonlinear plain journal bearings becomes unstable and the nonlinear behavior appears. A typical sub-harmonic response from the bearing output under 12,000 rpm, as shown in Fig. $4.17\left(\Delta \mathrm{t}=4 \times 10^{-6}\right.$ sec. $)$, is detected in both $x$ and $y$ displacements. The orbit of the displacements is a closed curve after 0.2 second, Fig. 4.18 , but it is a doubled circle because of combination of both the synchronized frequency and the subsynchronized frequency, which is clearer in the frequency domain. The fast Fourier transformation of the shaft center inside the bearing at node 4 is given in Fig. 4.19. In the frequency domain, there is a sub-harmonic frequency of $6,000 \mathrm{rpm}$ excited by the nonlinear bearing forces, which is shown in Fig. 4.20. At the operating speed of 12,000 rpm, the whole rotor-bearing system displays sub-harmonic response. Orbits of all nodes, 
at $\mathrm{t}=1.0$ second, are shown in Fig. 4.21. The shaft center at 1.0 second is indicated by the black line with red points on it. From the shape of the curve, the rotor is mostly excited with the first bending mode.
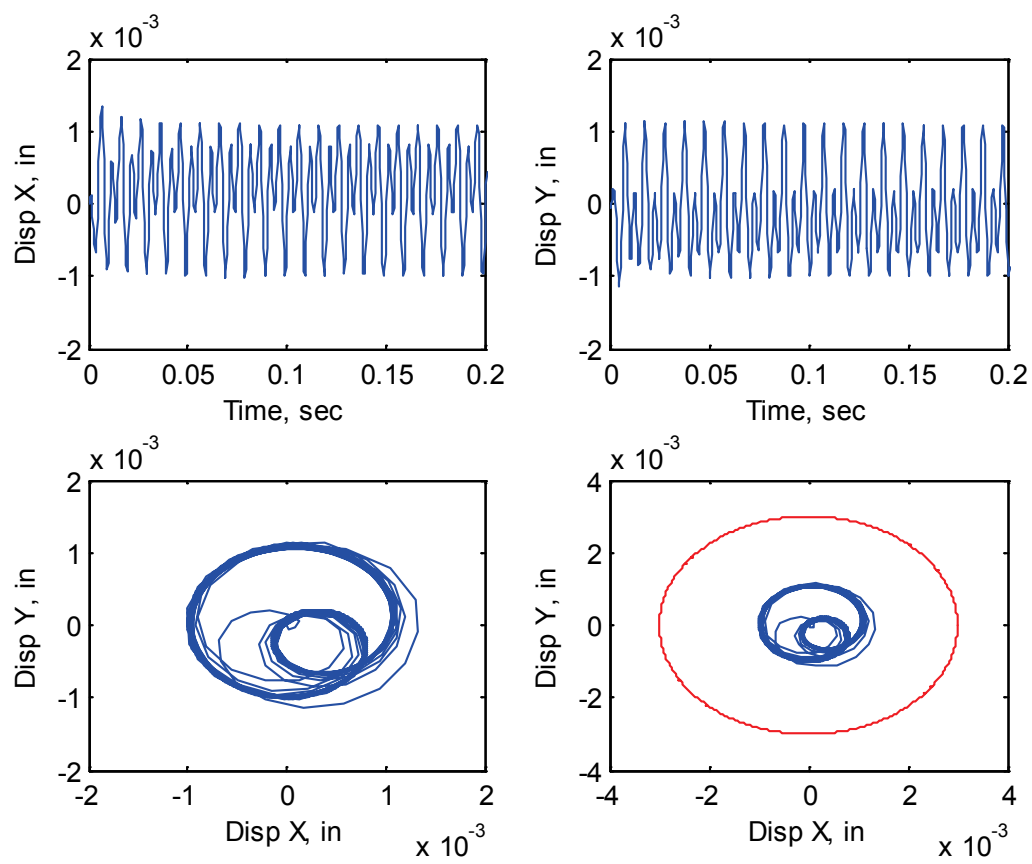

Figure 4.17 Transient Output from the Bearing (Node 4) at 12,000 rpm

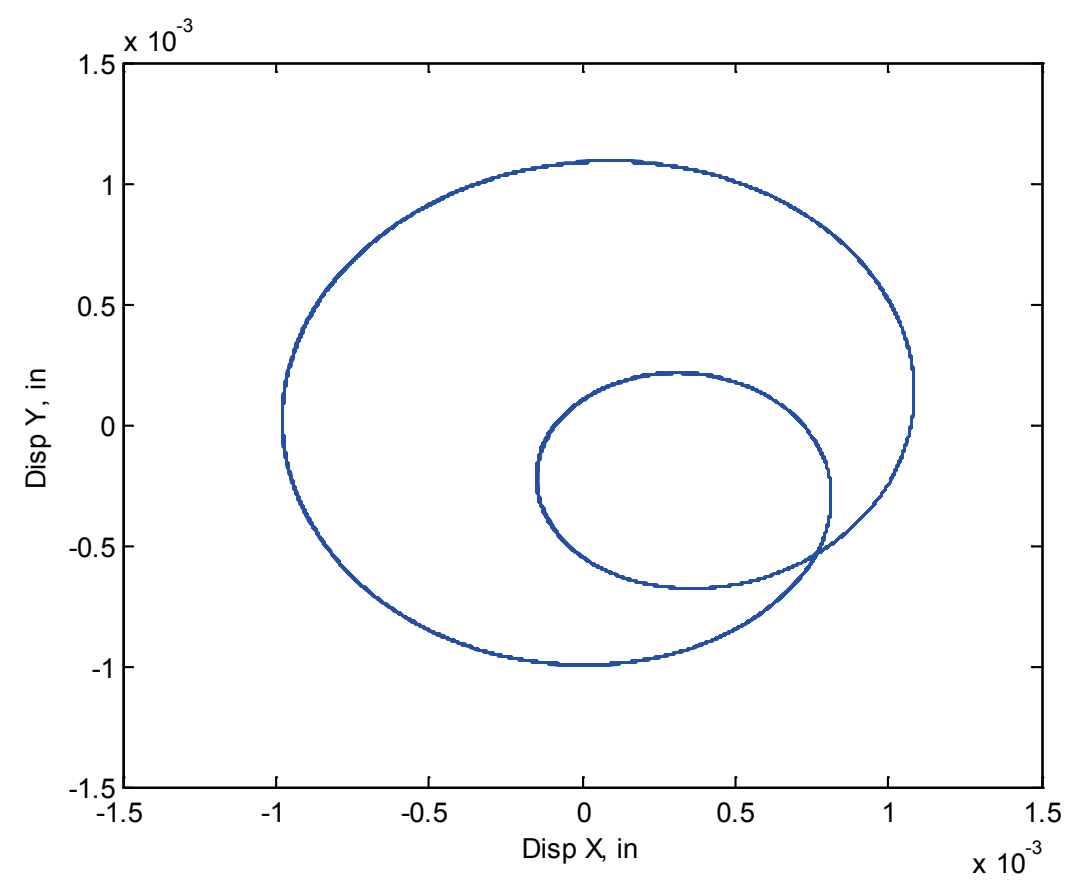

Figure 4.18 Orbit of Node 4 at $12,000 \mathrm{rpm}, \mathrm{t}=0.2 \sim 1 \mathrm{sec}$. 

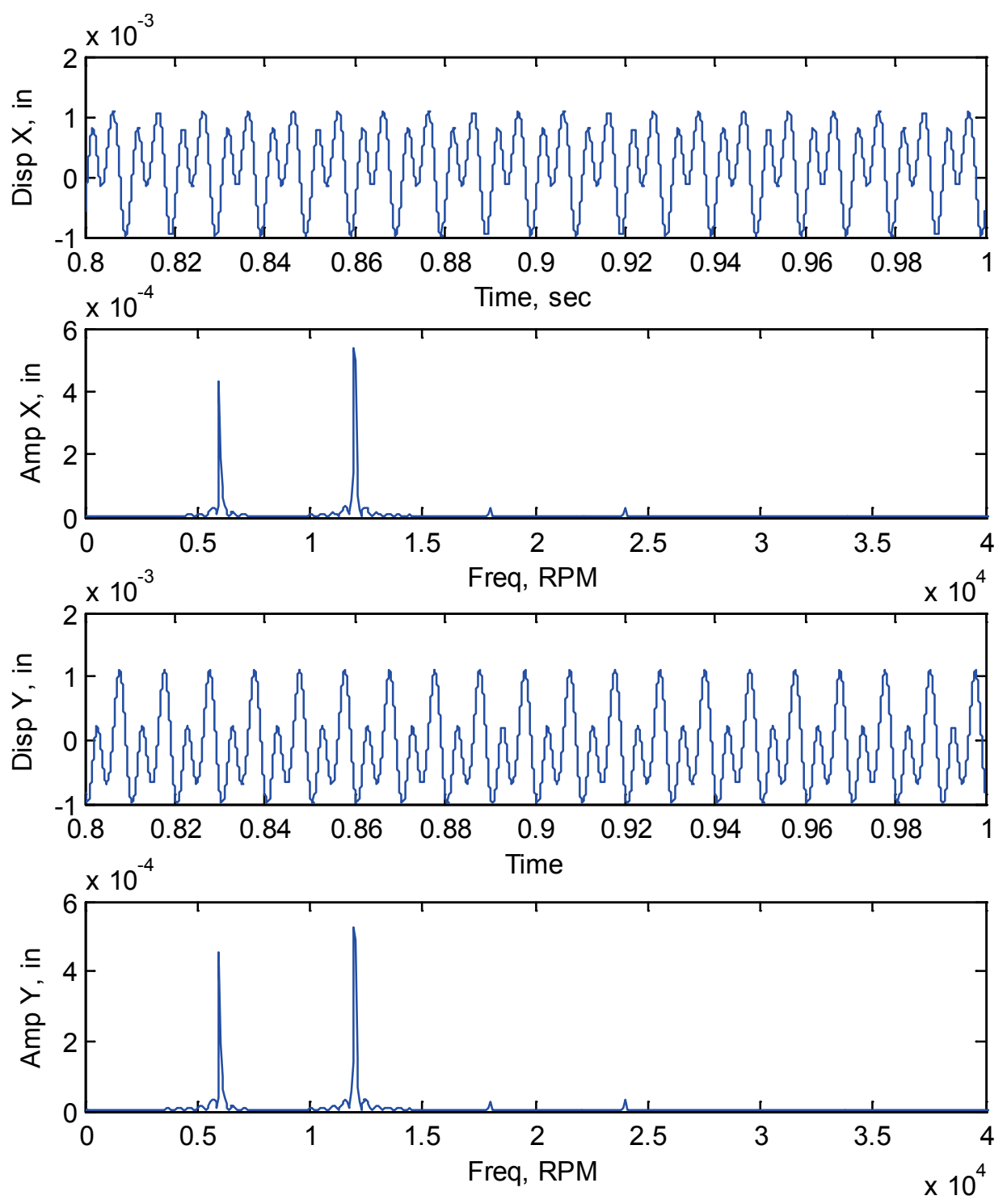

Figure 4.19 FFT of the Node\#4 Displacement at 12,000 rpm 

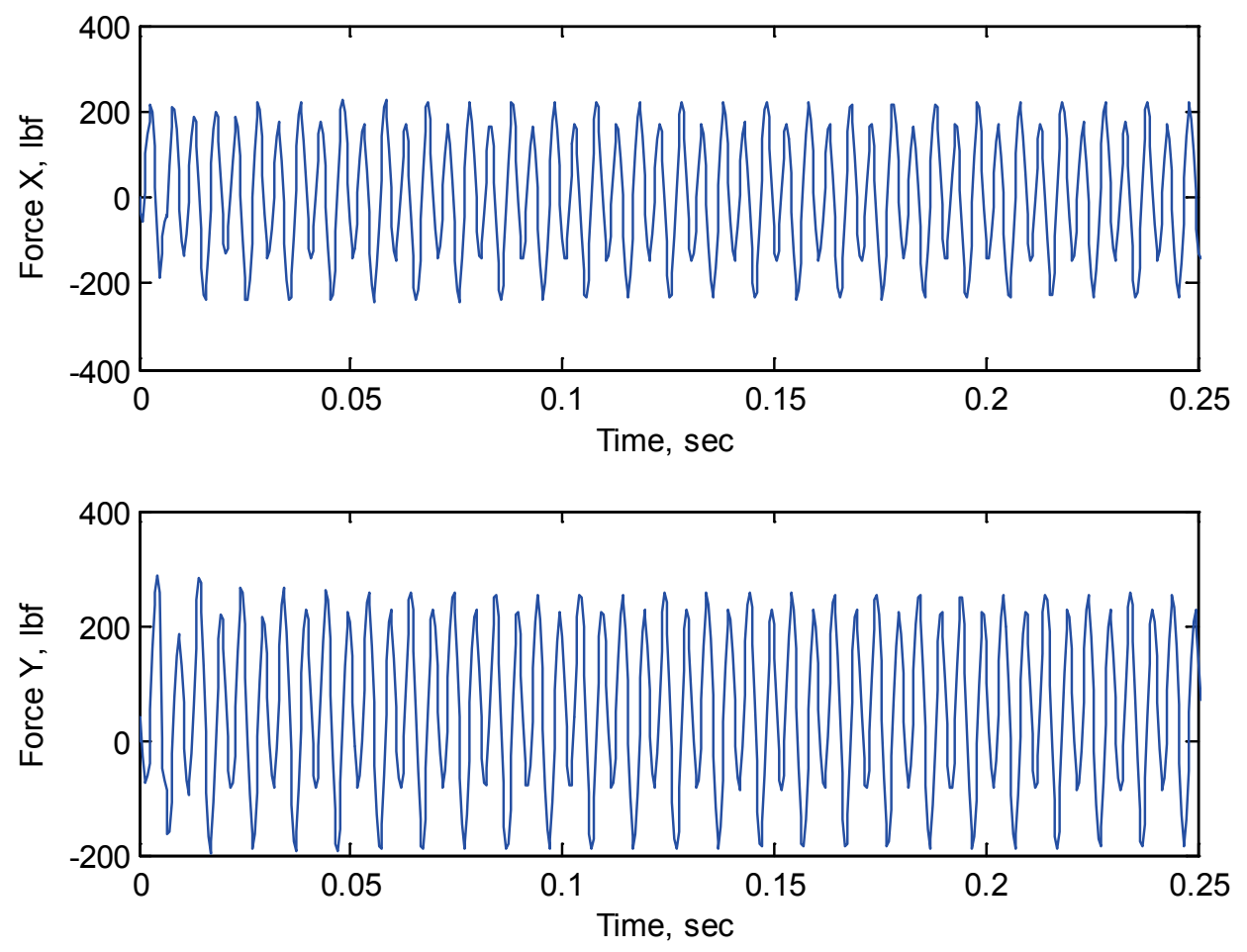

Figure 4.20 Bearing Force under 12,000 rpm from Node4

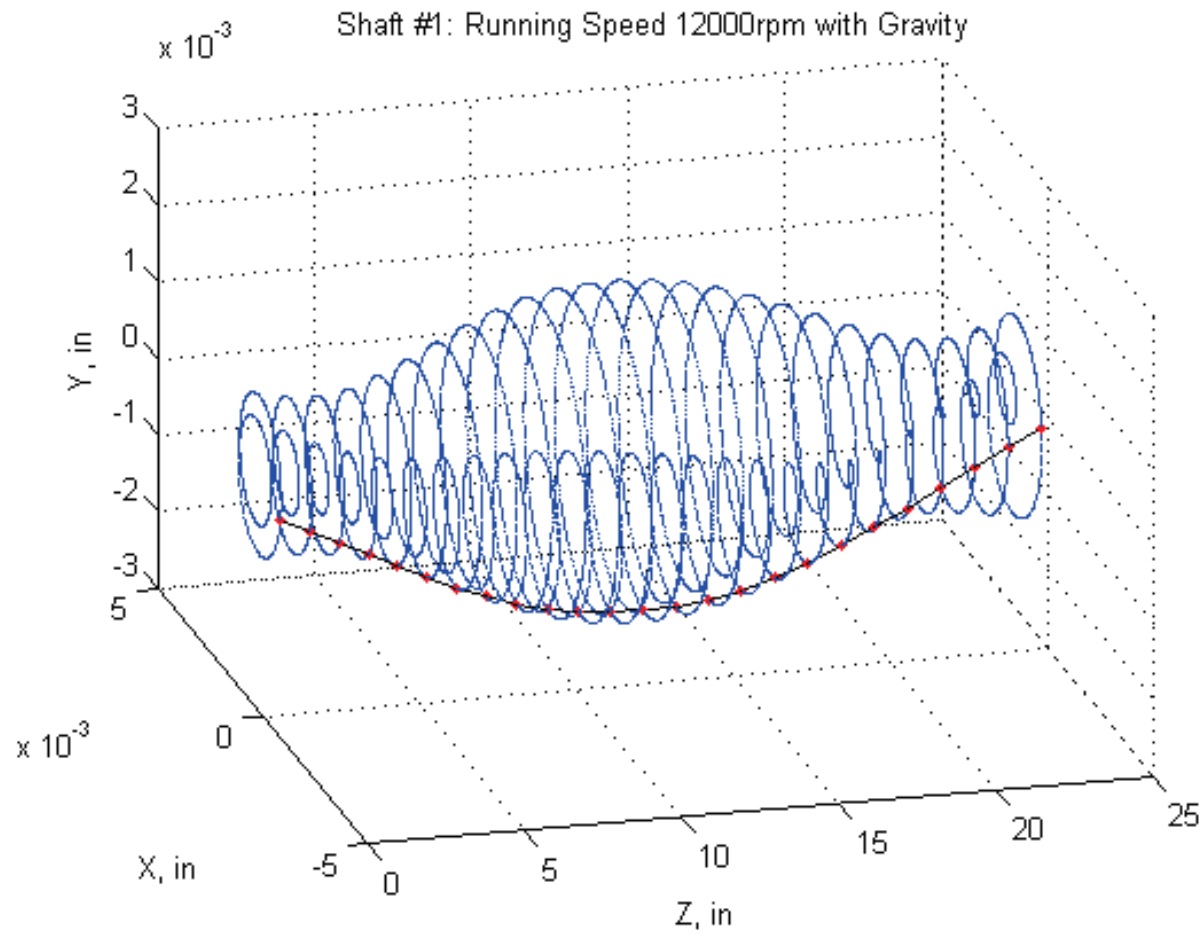

Figure 4.21 Orbits of Rotor at 12,000 rpm, $1.0 \mathrm{sec}$, with Gravity Load and Unbalances 
At the higher operating speed, more nonlinear behavior, such as a torus, is detected at $\Omega=16,000 \mathrm{rpm}$. From the time transient response of the center disk located at node 13 , shown as Fig. 4.22, the orbit repeats in a closed ring. The frequency domain of the time transient result is shown in Fig. 4.23, there are two peaks: one is the synchronous operating speed at $16,000 \mathrm{rpm}$; another is close to one half of the rotational speed of $8,000 \mathrm{rpm}$. Due to the torus response in time domain, the ration of the two frequencies is an irrational number.

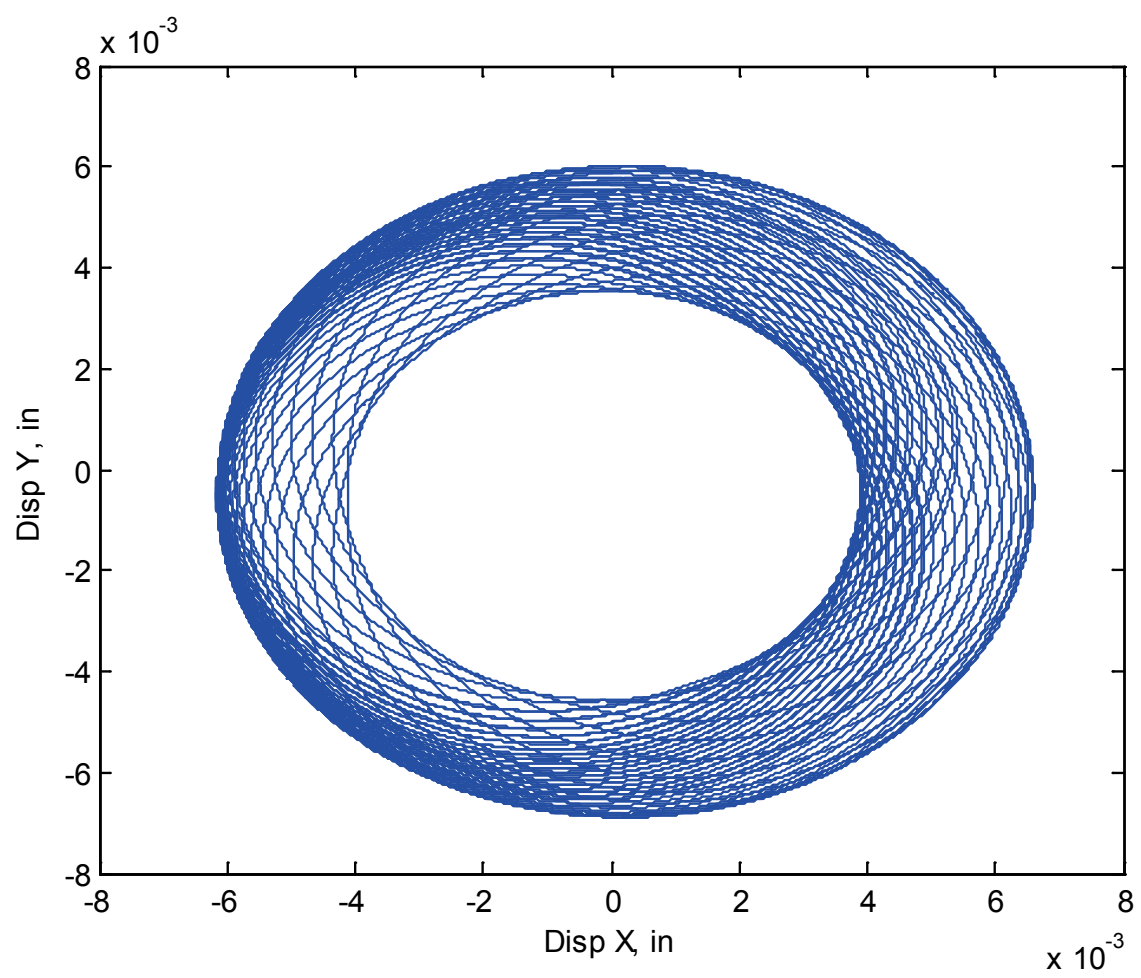

Figure 4.22 Orbit of the Center Disk (Node 13) at $16,000 \mathrm{rpm}, \mathrm{t}=0.1 \sim 0.5$ second 

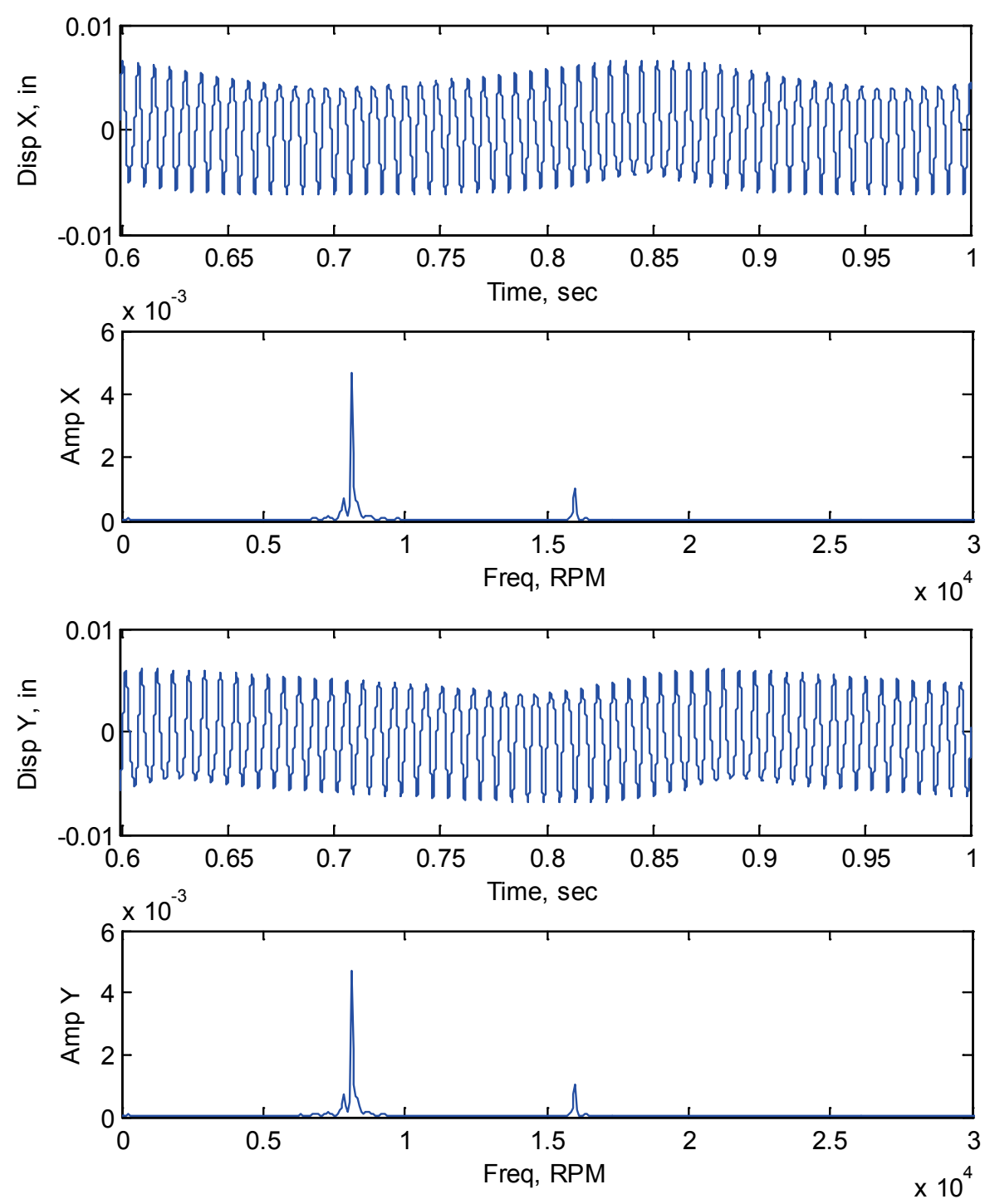

Figure 4.23 FFT of the Center Disk (Node 13) at 16,000 rpm

With the operating speed increases to a higher level $(>20,000 \mathrm{rpm})$, the maximum displacement of the whole rotor reaches the limitation of the transient code (RotorTran), which is $5 \%$ of the rotor diameter, then the code stops computing. Fig. 4.24 and Fig. 4.25 $\left(\Delta \mathrm{t}=4 \times 10^{-6}\right.$ sec.) show the time transient shaft displacements at the center disk (node 13). From the figures, the displacements in both $x$ direction and $y$ direction increase with time. 

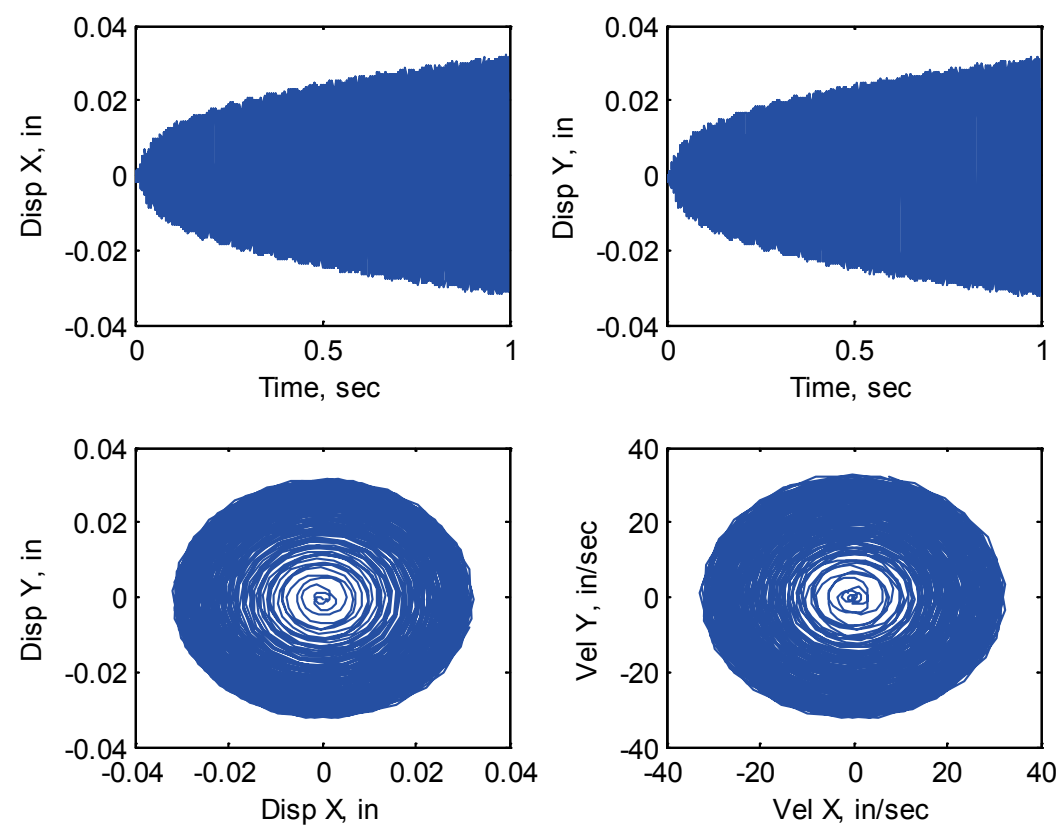

Figure 4.24 Output of the Center Disk at 20,000 rpm w/o Squeeze Film Damper
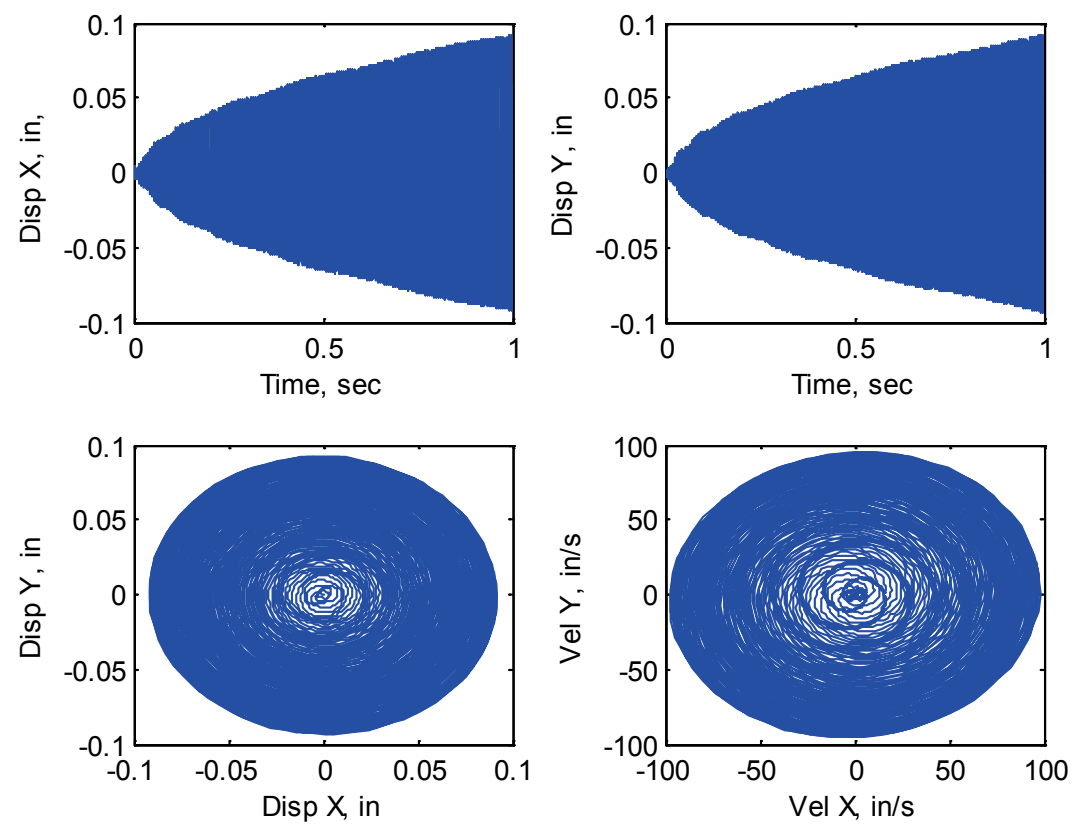

Figure 4.25 Output of the Center Disk at 30,000 rpm w/o Squeeze Film Damper 


\subsection{Three-Disk Rotor with Nonlinear Journal Bearings and}

\section{Squeeze Film Dampers}

The squeeze film dampers are used to 1) reduce bearing stiffness, 2) add system damping, 3) shift critical speeds, and/or 4) increase the dynamic stability of the rotorbearing system. One of the key features introduced by a squeeze film damper is the introduction of support flexibility and damping in the bearing/support structure, that will lower transmitted forces and longer bearing life, particularly for machinery that are designed to operate at super critical speeds.

The centering spring, sometimes called the squirrel cage, is part of the conventional design of most squeeze film dampers. The main role of the centering spring is to ensure a central position of the mass in the damper. From the modeling standpoint, the centering spring often is considered as a stiffness element and helps to ensure a central orbital response of the shaft [69]. In squeeze film dampers with new designs, the centering spring is often removed to achieve shorter rotors, because the central spring geometry takes almost the same length as the damper itself.

The three-disk rotor model supported with two plain journal bearings is unstable under high rotational speed as previously shown in Figs. $4.24 \& 4.25$, which showed the time transient output of the center disk located at node 13 with the rotational speed of 20,000 rpm \& 30,000 rpm. The dynamic vibration amplitudes in both $\mathrm{x}$ and $\mathrm{y}$ directions keep increasing till reach the limitation of the code. In those unstable cases, the nonlinear squeeze film dampers will be added onto the bearing to decrease the vibration amplitudes 
and to increase the stability of the system. Using of squeeze film dampers is usual on jet engines with ball/roller bearings and high speed compressors or stream turbines.

In this section, the nonlinear squeeze film damper analysis is introduced into the rotorbearing system to increase the dynamic stability of the system. The transient analysis was conducted to study the response of the three-disk rotor model supported with two plain journal bearings and two squeeze film dampers without any centering spring as shown in Figs. 4.1\&4.2. Also the transient analysis evaluates the response of the rotors without the initial assumption of a circular orbit. The nonlinear forces of the bearings and the dampers are obtained through the integration of the pressure distribution are applied to the system directly.

The pressure profile and the nonlinear force of a squeeze film damper are calculated using Eq. (3.4.7\&3.4.5). To describe the behavior of the bearing housing, which locates inside the damper, an additional node for each damper is added into the equations of motion as described in Chapter 3. The dimension and parameters of the damper are listed in Table 4.6. The same lubricant is used for both the bearings and the dampers. The fluid viscosity is the fluid temperature dependent. For the short bearings and dampers $(\mathrm{L} / \mathrm{D} \leq 0.5)$, same fluid viscosities are applied by assuming same fluid temperature inside the bearings and the dampers. The damper force applies to the bearing housing and calculated based on the position and velocity of the bearing housing. The bearing forces apply to the shaft inside the bearings and calculated based on the displacements and velocities of both the shaft center inside the bearing and the bearing housing inside the damper. 
Table 4.6 Squeeze Film Damper Information of the 3-disk Rotor

\begin{tabular}{l|l|l}
\hline \multicolumn{3}{l}{ Type: Short squeeze film damper } \\
\hline Property & Value & Unit \\
\hline Bearing Weight & 5 & lbf \\
\hline Length & 1 & in \\
\hline Diameter & 3 & in \\
\hline Clearance & 0.006 & in \\
\hline Viscosity & $5.8 \mathrm{E}-6$ & $\mathrm{lbf}-\mathrm{s} / \mathrm{in}^{2}$ \\
\hline
\end{tabular}

The rotor behavior, before and after adding two squeeze film dampers, is compared at first with the rotational speed of $20,000 \mathrm{rpm}\left(\Delta \mathrm{t}=4 \times 10^{-6}\right.$ second $)$. The output from the bearing output (node \#4) without damper installed is shown in Fig. 4.26; the output after adding the squeeze film dampers is shown in Figs. $4.27 \& 4.28$. If there is no squeeze film damper on the bearing, the bearing connects to the ground directly in this model, so the output from the bearing is relatively to the ground. If there is a squeeze film damper on the bearing, however, the bearing connects to the damper, and the damper connects to the ground. The behavior of the shaft center inside the bearing and at the center disk before and after adding the squeeze film dampers is listed in Table 4.7.

Table 4.7 Comparison of with and w/o SFD at 20,000 rpm

\begin{tabular}{l|c|c|c}
\hline Displacement, mil & $\begin{array}{c}\text { Shaft Center } \\
\text { w/o SFD }\end{array}$ & $\begin{array}{c}\text { Shaft Center } \\
\text { with SFD }\end{array}$ & $\begin{array}{c}\text { Bearing Center } \\
\text { with SFD }\end{array}$ \\
\hline Node 4, peak-to-peak & 3.0 & $1.5^{*}$ & 3.2 \\
\hline Node 22, peak-to-peak & 3.0 & $0.5^{*}$ & 0.35 \\
\hline Node13, peak-to-peak & $>80$ & 2.2 & - \\
\hline
\end{tabular}

*Relative displacement between the shaft center and the bearing

\begin{tabular}{l|c|c|c|c|c|c}
\hline \multirow{2}{*}{ Force (p-p), lbf } & \multicolumn{2}{|c|}{ Brg Only } & \multicolumn{4}{c}{ Brg + SFD } \\
\cline { 2 - 7 } & $\mathrm{Brg} \mathrm{F}_{\mathrm{x}}$ & $\mathrm{Brg} \mathrm{F}_{\mathrm{y}}$ & $\mathrm{Brg} \mathrm{F}_{\mathrm{x}}$ & $\mathrm{Brg} \mathrm{F}_{\mathrm{y}}$ & $\mathrm{SFD} \mathrm{F}_{\mathrm{x}}$ & $\mathrm{SFD} \mathrm{F}_{\mathrm{y}}$ \\
\hline Node 4 & 5,000 & 5,000 & 469 & 481 & 490 & 500 \\
\hline node 22 & 5,000 & 5,000 & 98 & 118 & 94 & 122 \\
\hline
\end{tabular}


Before adding the damper, the shaft orbit at the bearing (Node\#4) is about $50 \%$ of the bearing clearance or $1.5 \mathrm{mil}$. After adding the damper, the shaft orbit inside the bearing (Node\#4) is about $25 \%$ of the bearing clearance or $0.75 \mathrm{mil}$. Another change is about the bearing forces, without the squeeze film damper, the bearing forces at node 4 is around $2,500 \mathrm{lb}$ on both $\mathrm{x}$ and $\mathrm{y}$ direction and there are lots of sharp peaks, which means the bearing forces is not smooth as shown in Fig. 4.29. After adding squeeze film dampers, the bearing forces are only $250 \mathrm{lb}$ as shown in Fig. 4.30. Also the bearing forces are smoother than those without the dampers on the bearings. The reason is that the damper provides lower stiffness and additional damping that tends to reduce the amplitude of oscillations in the system. The displacement of the center disk (node 13) and the fast Fourier transformation (FFT) before and after adding the squeeze film dampers are shown in Figs. 4.31 4.34. To make the figure clearer, only those data of $0 \sim 0.15$ second time span are shown, because the nonlinear forces are periodic after 0.1 second. Without the squeeze film dampers, the displacement keeps increasing $(0.02$ in at $0.5 \mathrm{~s})$ with a strong sub-harmonic frequency at 9,510 rpm. After adding the dampers, the sub-harmonic frequency at 6,800 rpm (Fig. 4.34), which is due to "oil whirl" and "oil whip" of the bearing, is also exists but is shifted to a lower frequency. The maximum displacement, however, is stabilized at $1.1 \mathrm{mil}$ (p-p) only. The whole shaft orbits under 20,000 rpm with two squeeze film dampers are shown in Fig. 4.35. Although the rotor is symmetric, the nodal displacements are not symmetric due to the unequal three unbalances on three disks with different phase angles $\left(0^{\circ}, 90^{\circ}\right.$ and $\left.180^{\circ}\right)$. From the Fig. 4.35, each nodal orbit has double circles, which means the motion of the whole is still a sub-harmonic response. 

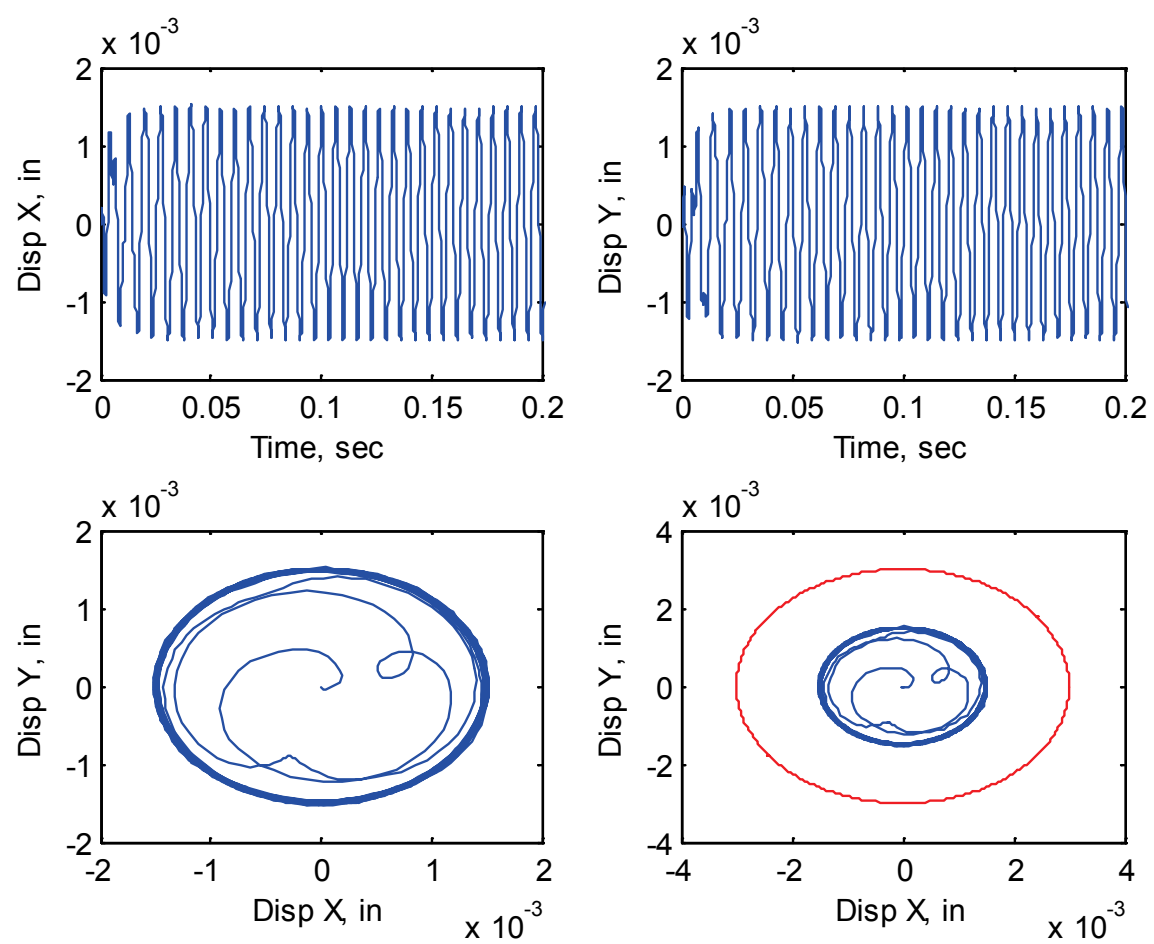

Figure 4.26 Shaft Center inside Bearing (Node\#4) without SFD at 20,000 rpm
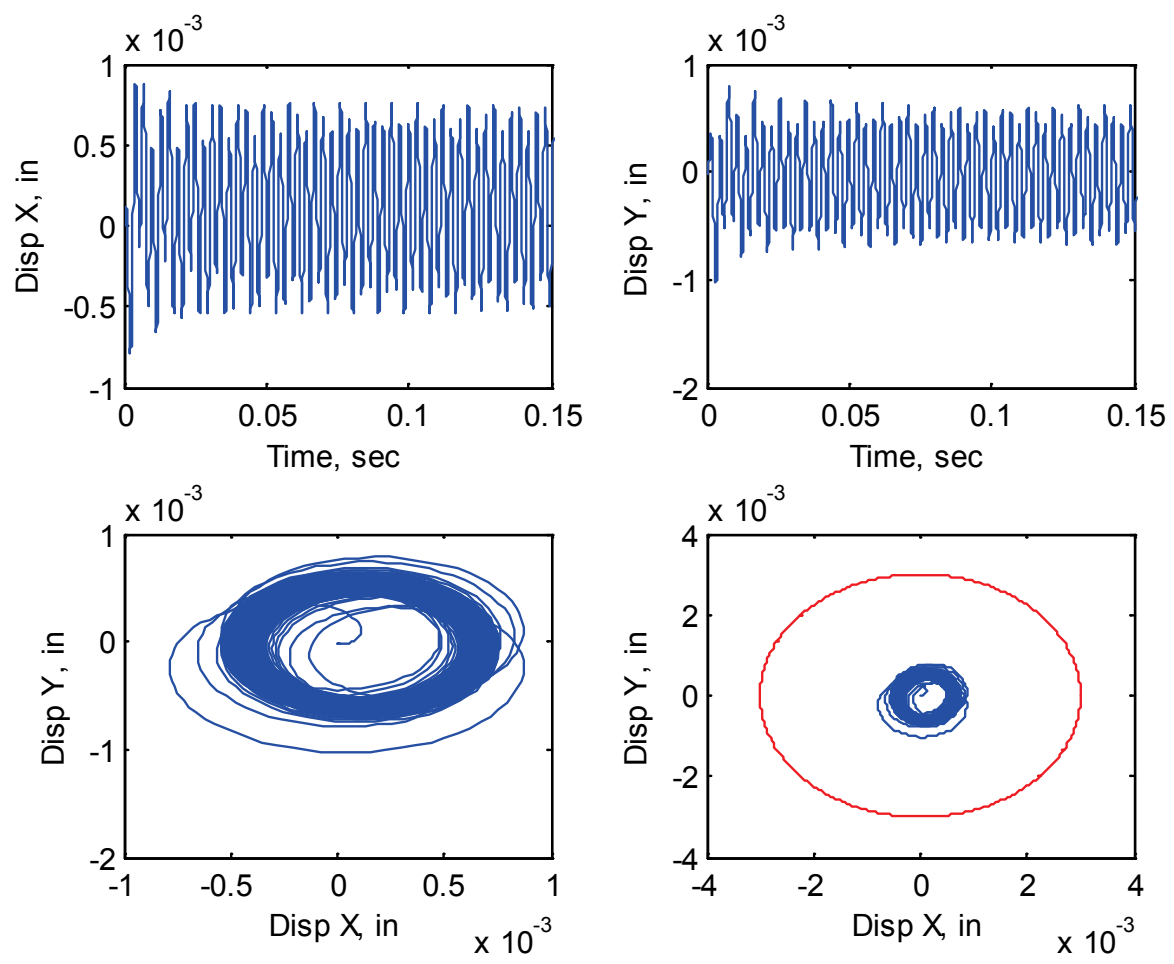

Figure 4.27 Shaft Center inside Bearing (Node\#4) with SFD outside at 20,000 rpm (Relative to Bearing) 


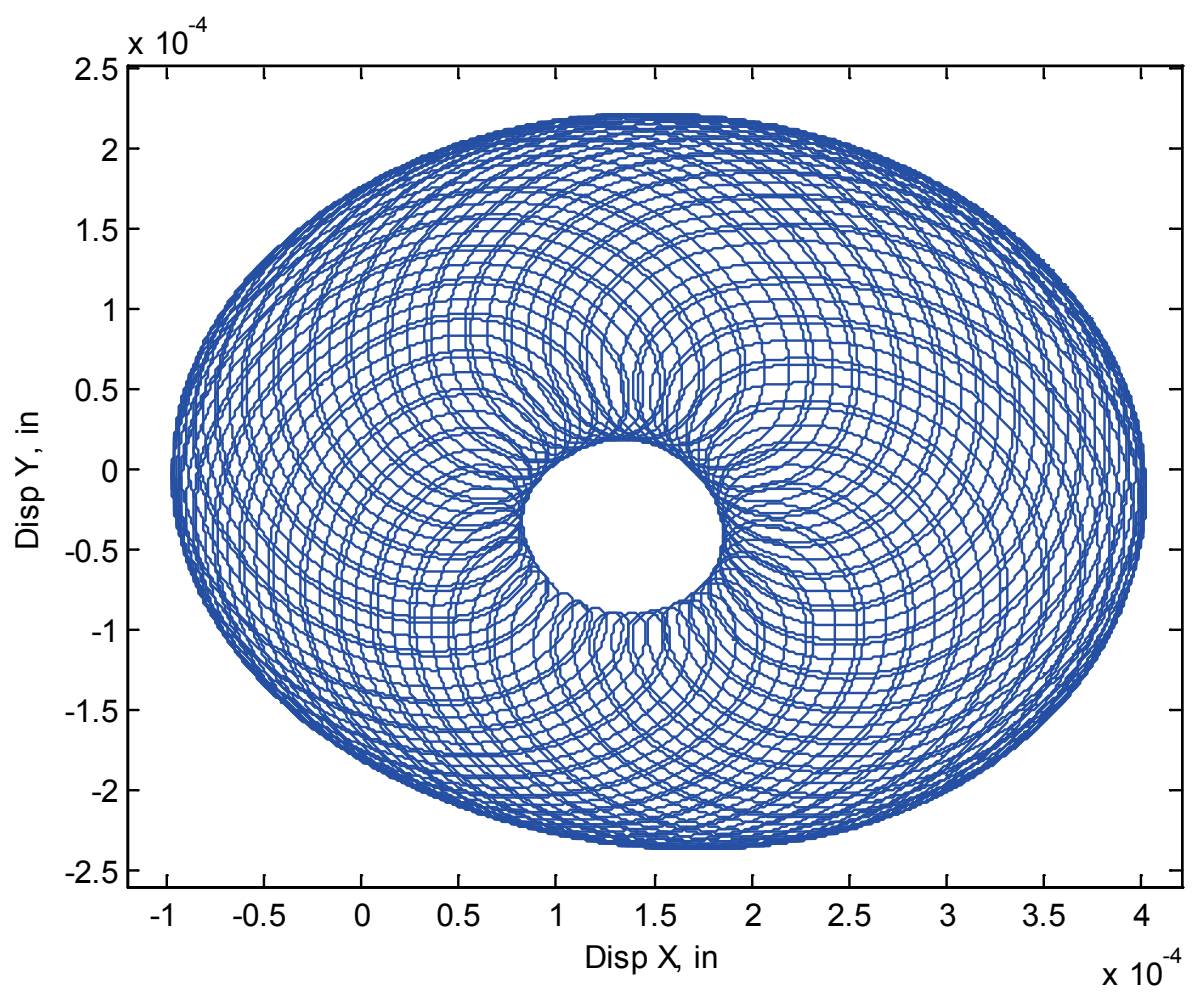

Figure 4.28 Shaft Center inside Bearing (Node\#22) with SFD outside at 20,000 rpm (Relative to Bearing)
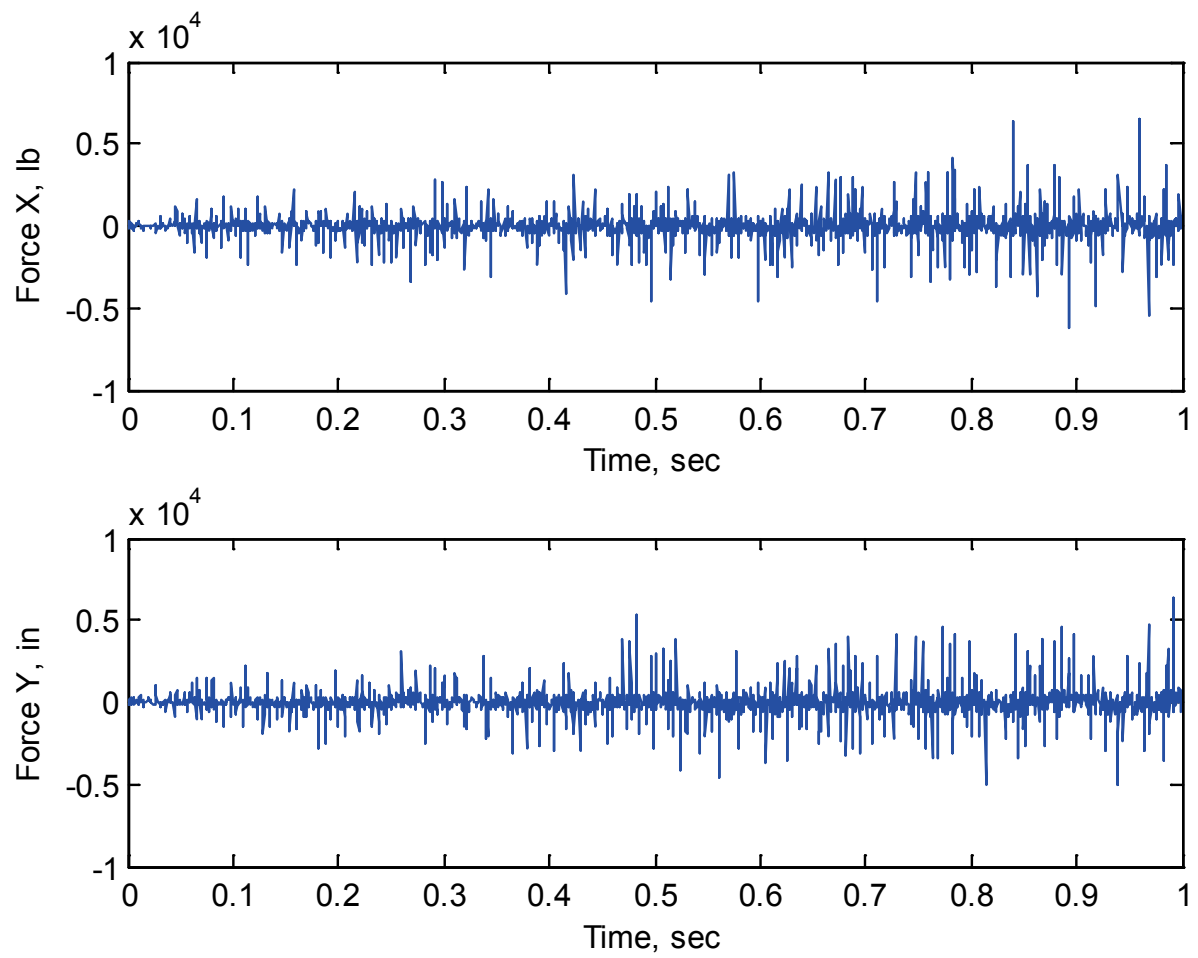

Figure 4.29 Bearing Forces (node 4) w/o Squeeze Film Damper at 20,000 rpm 

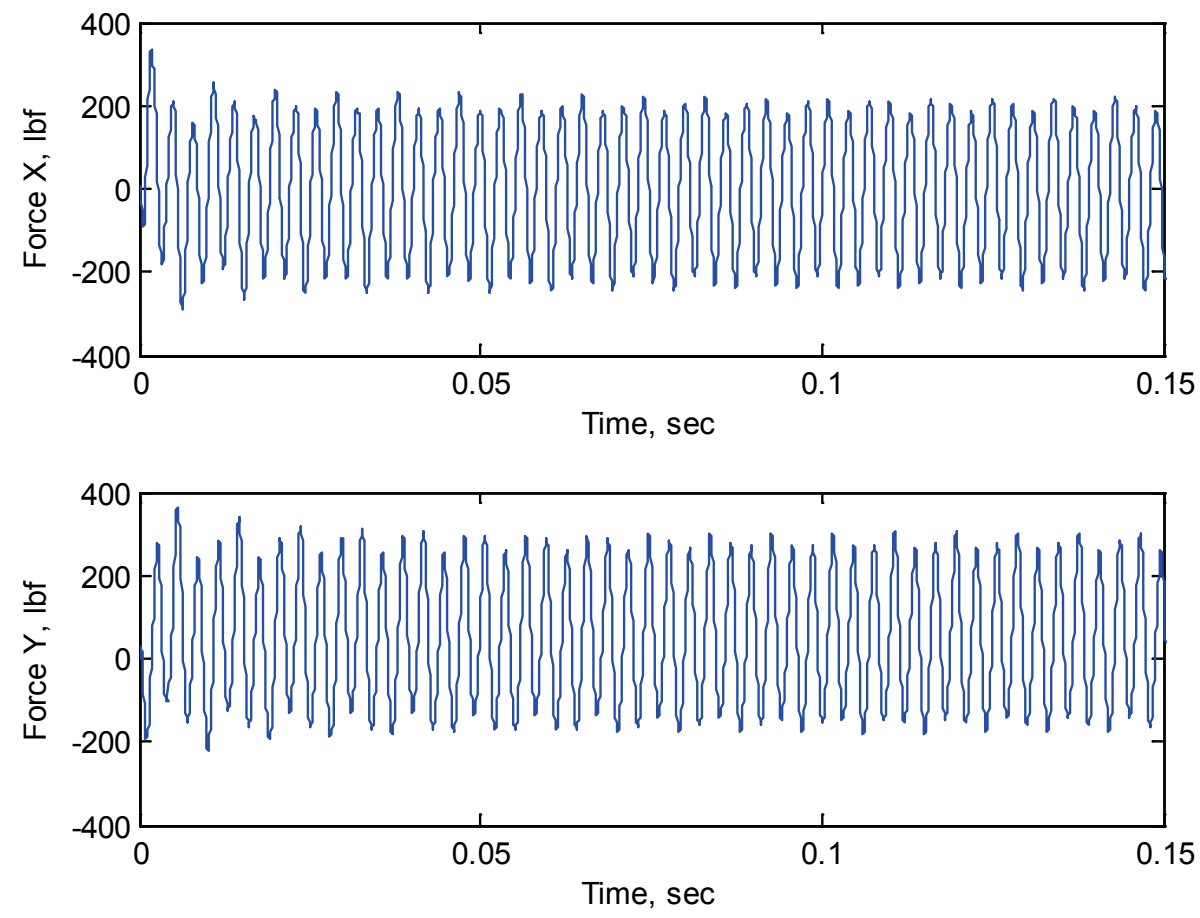

Figure 4.30 Bearing Forces (Node 4) with Squeeze Film Damper at 20,000 rpm

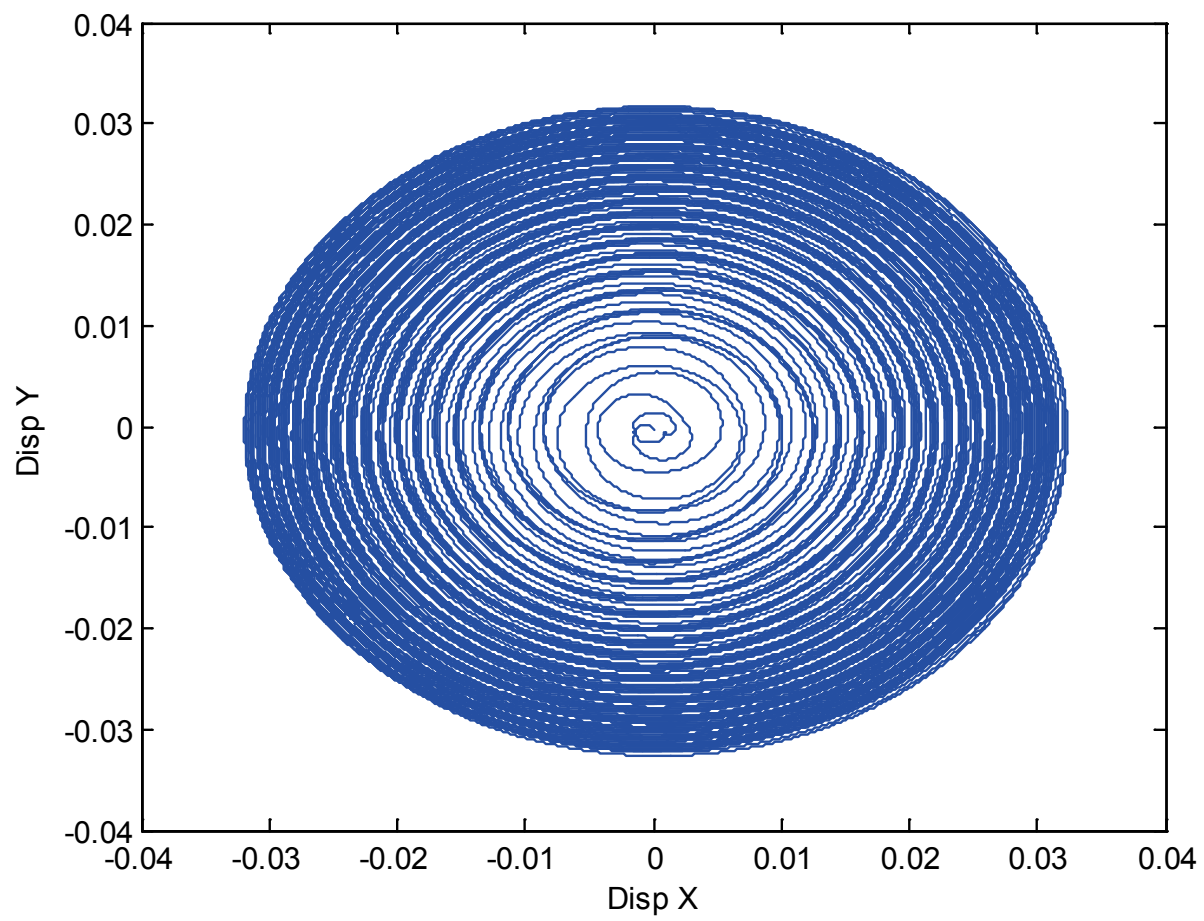

Figure 4.31 Orbit of the Center Disk at 20,000 rpm w/o Damper 

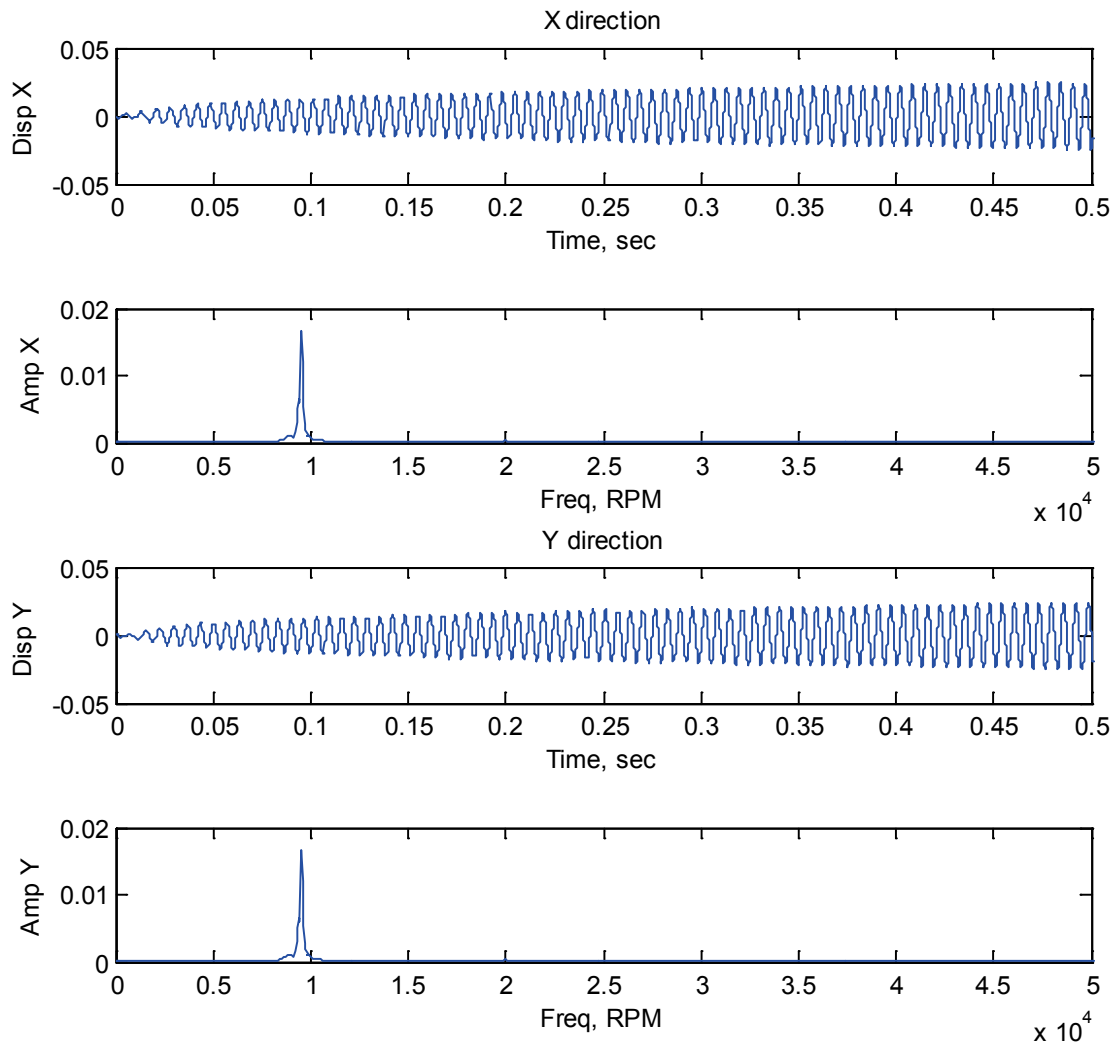

Figure 4.32 FFT Results of the Center Disk at 20,000 rpm w/o Damper

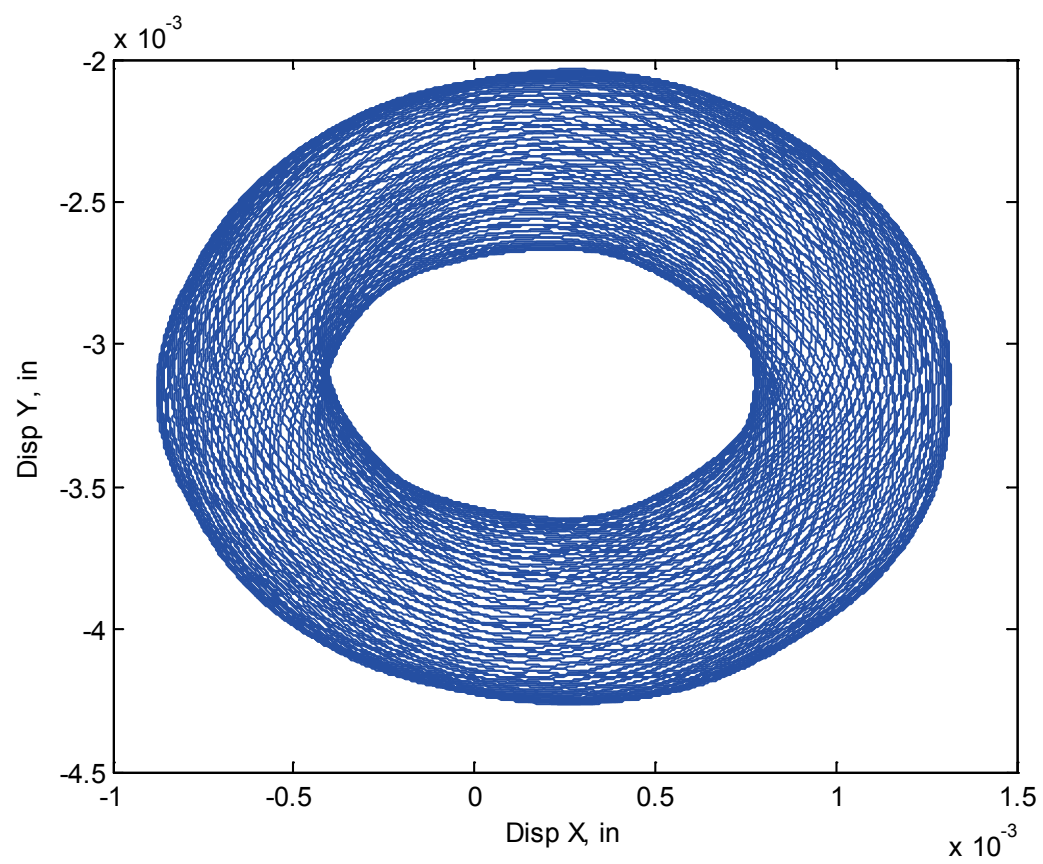

Figure 4.33 Orbit of the Center Disk at 20,000 rpm with Damper 

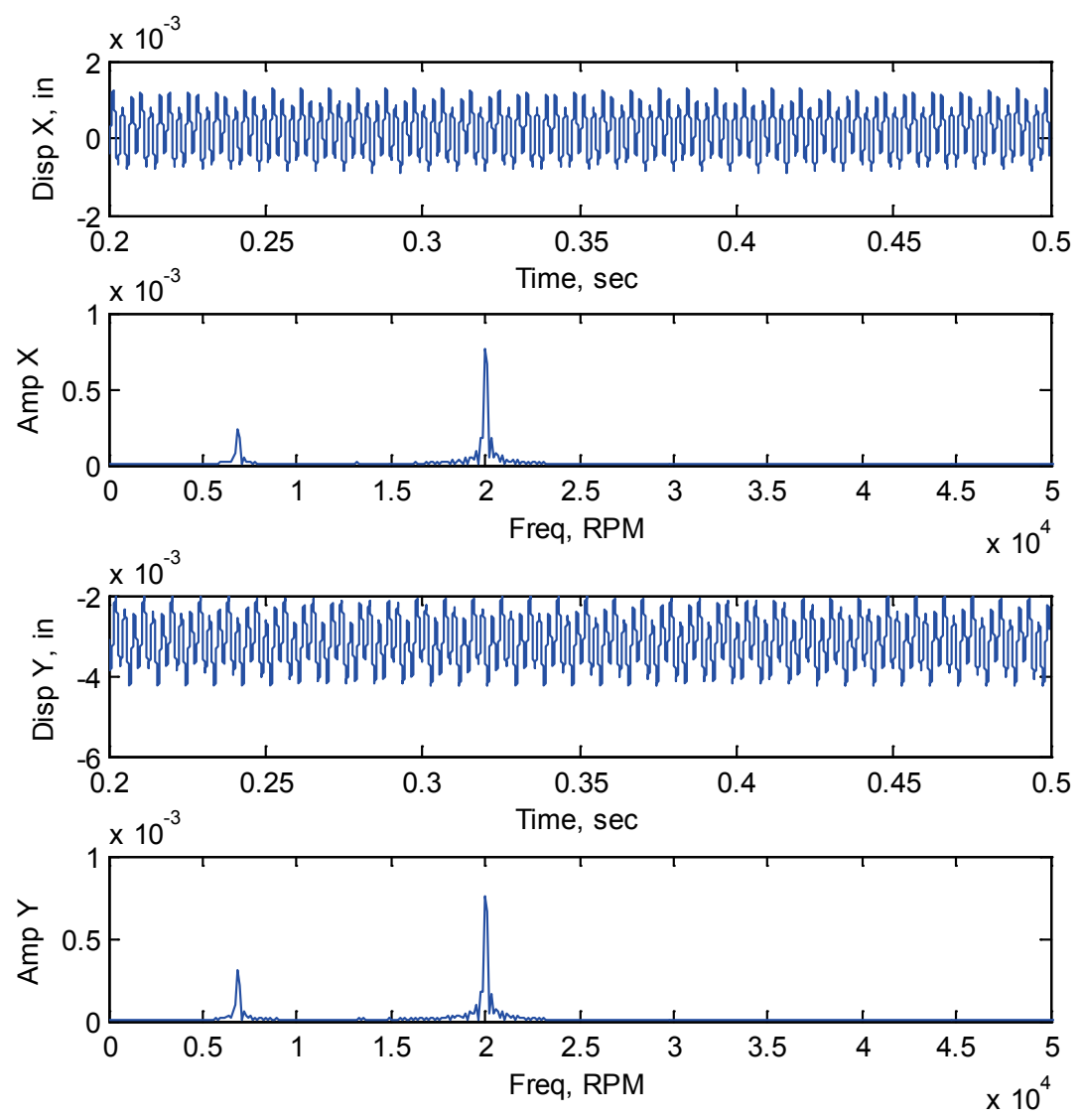

Figure 4.34 Orbit and FFT of the Center Disk at 20,000 rpm with Damper

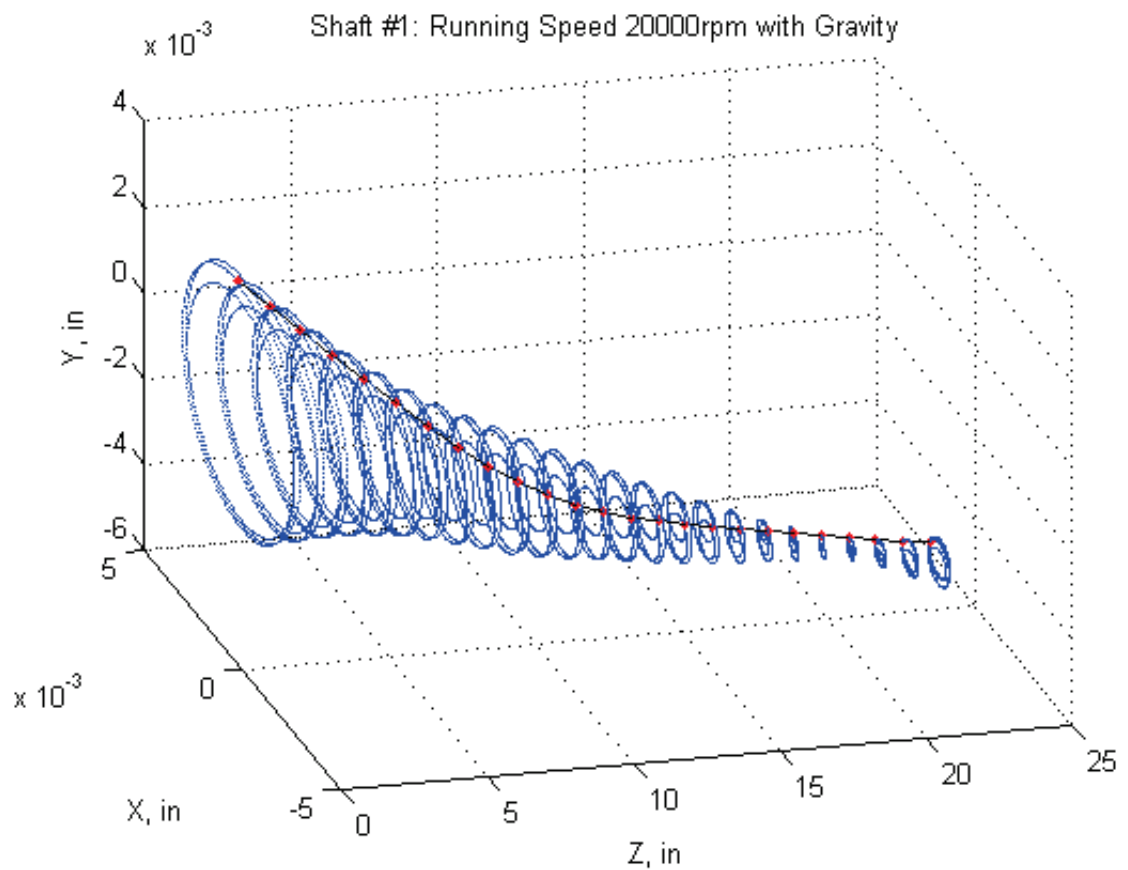

Figure 4.35 Rotor Orbit with SFD at 20,000 rpm around 0.5second with Gravity Load and Unbalances 
The bearing displacement inside the squeeze film damper at node 4 is shown in Fig. 4.36, and the orbit, the frequency domain information and the damper forces after 0.2 second are shown in Figs. 4.37 4.39. In those figures, there are two peaks at 20,000 rpm, which is same as the rotational speed, and 6,800 rpm, which is a sub-harmonic frequency. Another squeeze film damper on the right, at node 22, shows strong nonlinear behavior, which is different from the damper on the left. The orbit and displacements in both the time and frequency domains are shown in Figs. 4.40 4.43. The FFT result, Fig. 4.41, shows multi-peak frequencies, and the orbit of the bearing inside the damper shows the strong nonlinear as well. The reason of strong nonlinear behavior is that the bearing inside the damper is whirling at about $80 \%$ of the damper clearance (large eccentricity) in the negative vertical direction as shown in Fig. 4.41.
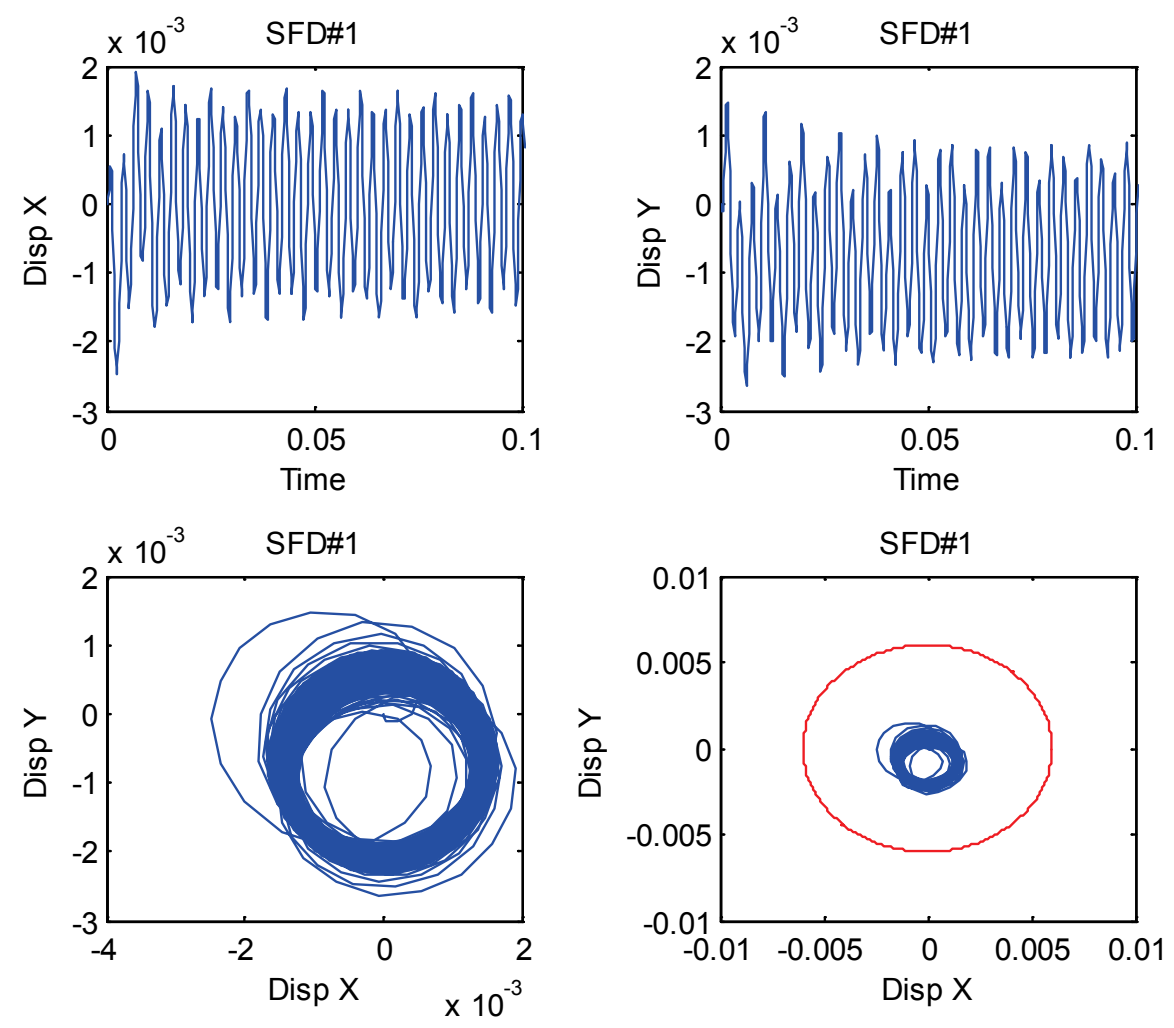

Figure 4.36 Output from Squeeze Film Damper at Node 4 at 20,000 rpm 


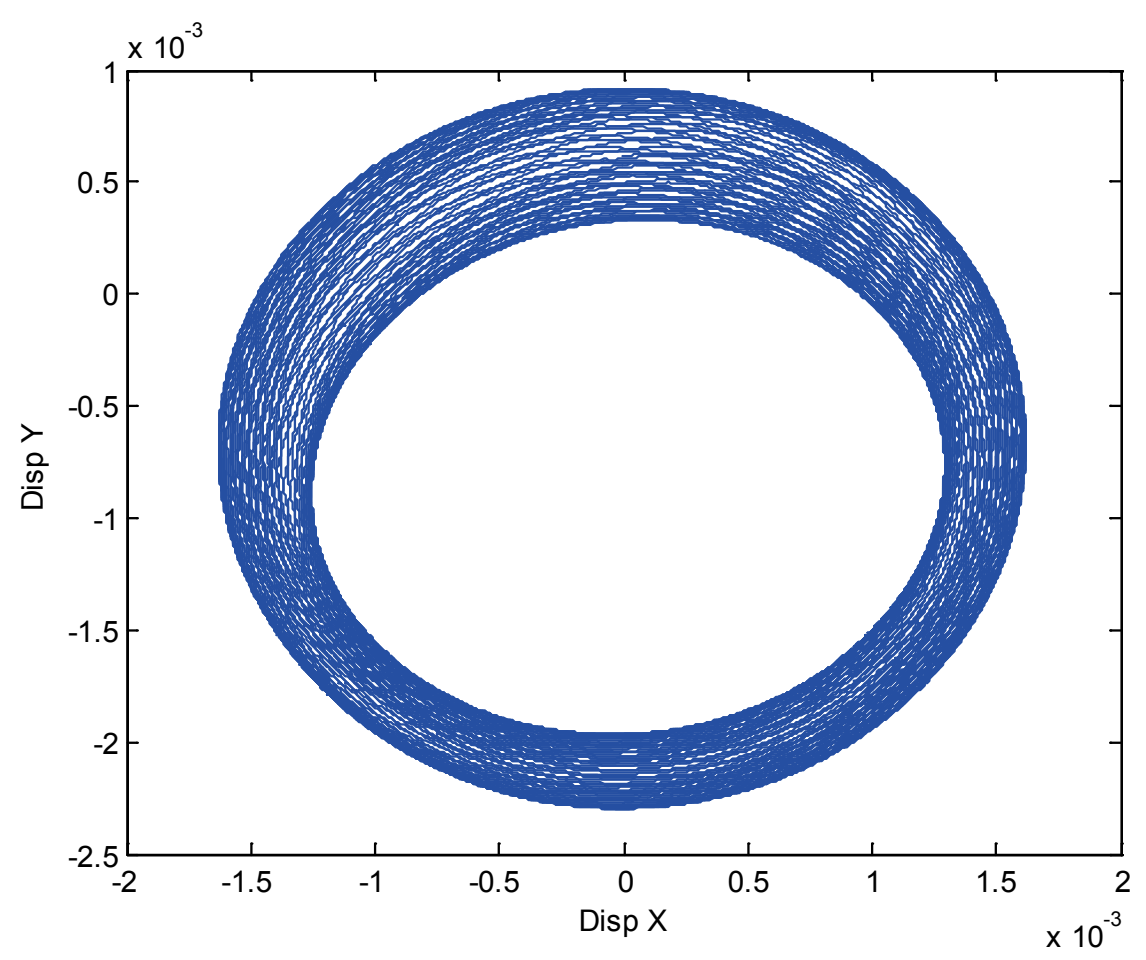

Figure 4.37 Orbit from SFD at Node\#4 under 20,000 rpm
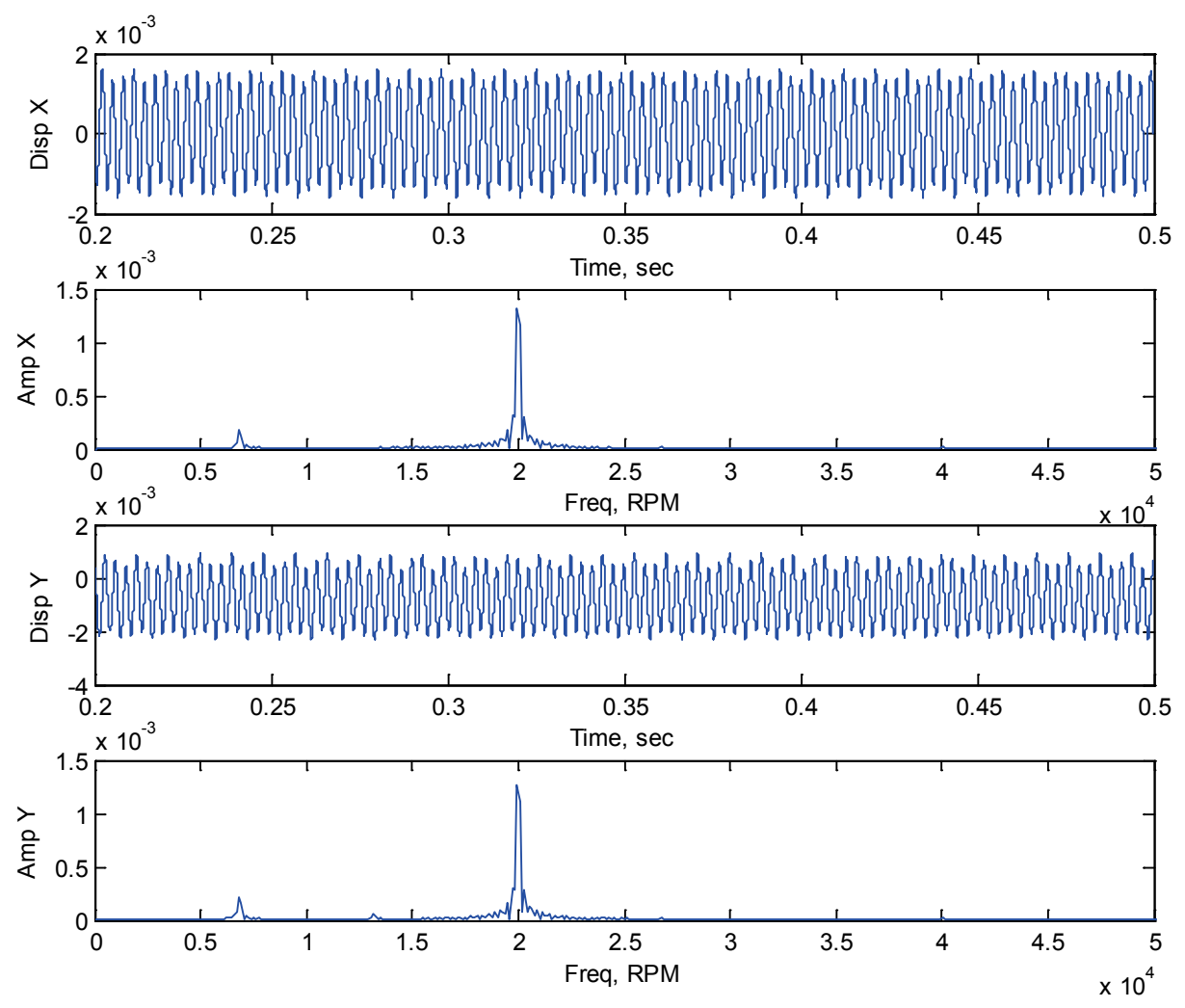

Figure 4.38 FFT Results from SFD at Node\#4 under 20,000 rpm 

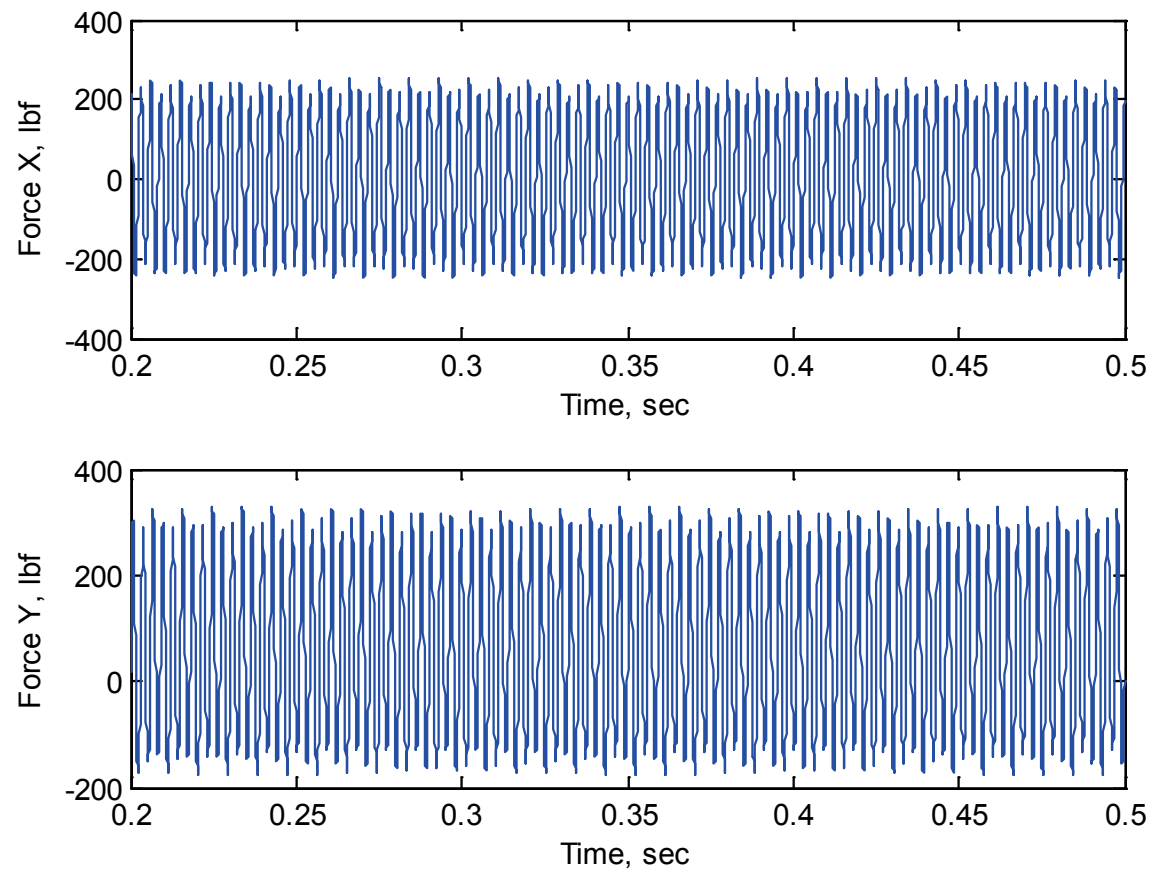

Figure 4.39 SFD Forces at Node\#4 under 20,000 rpm
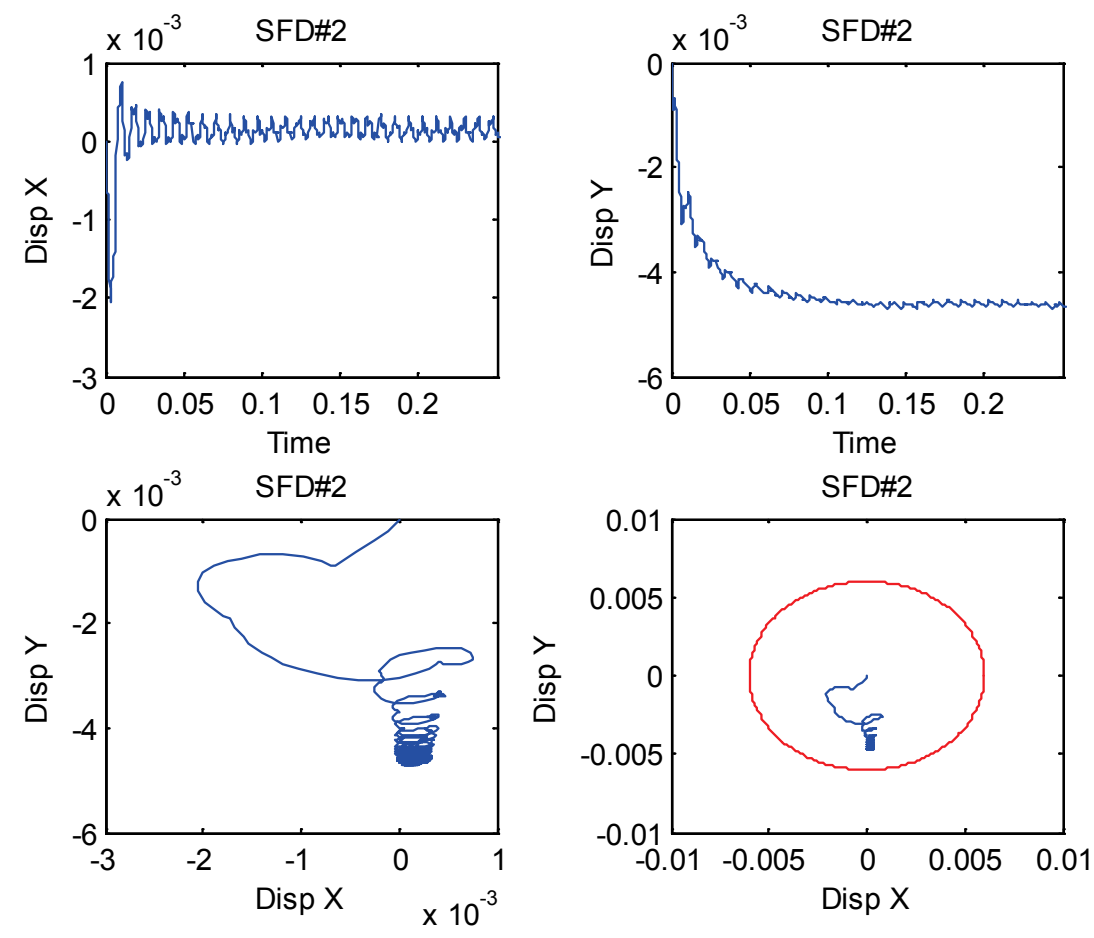

Figure 4.40 Bearing Behavior inside SFD at Node 22 under 20,000 rpm 


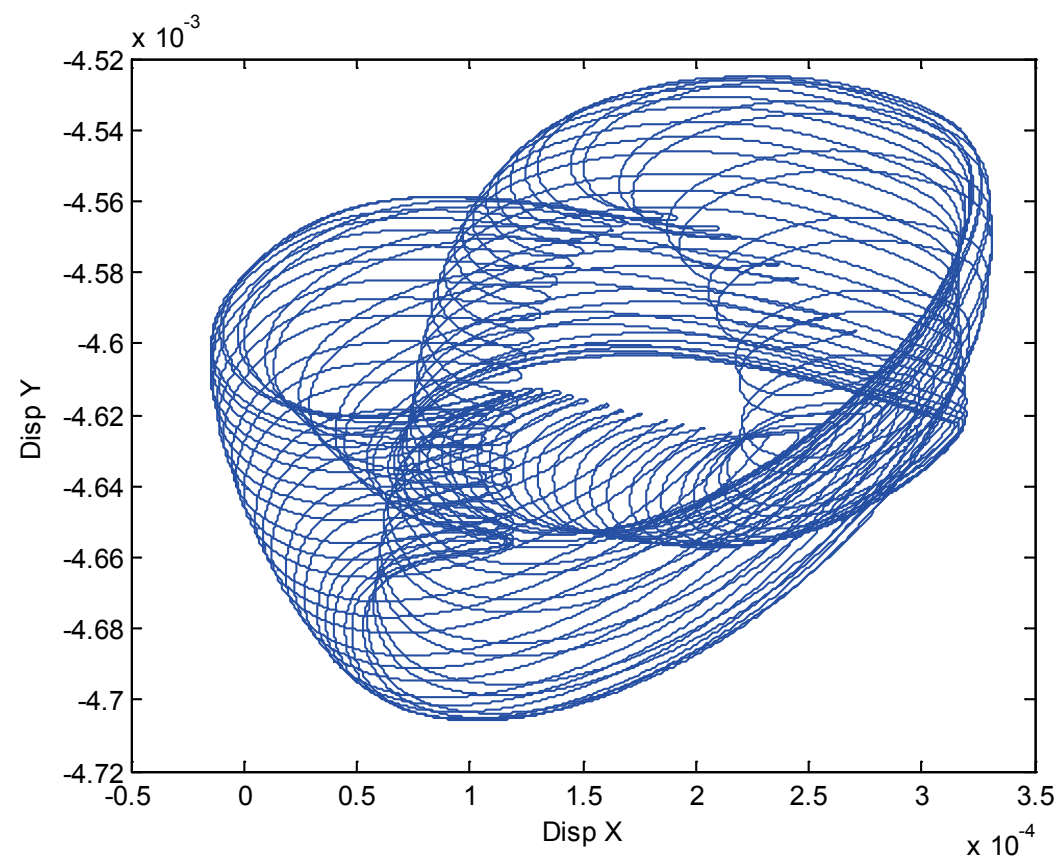

Figure 4.41 Bearing Orbit inside SFD at Node 22 under 20,000 rpm
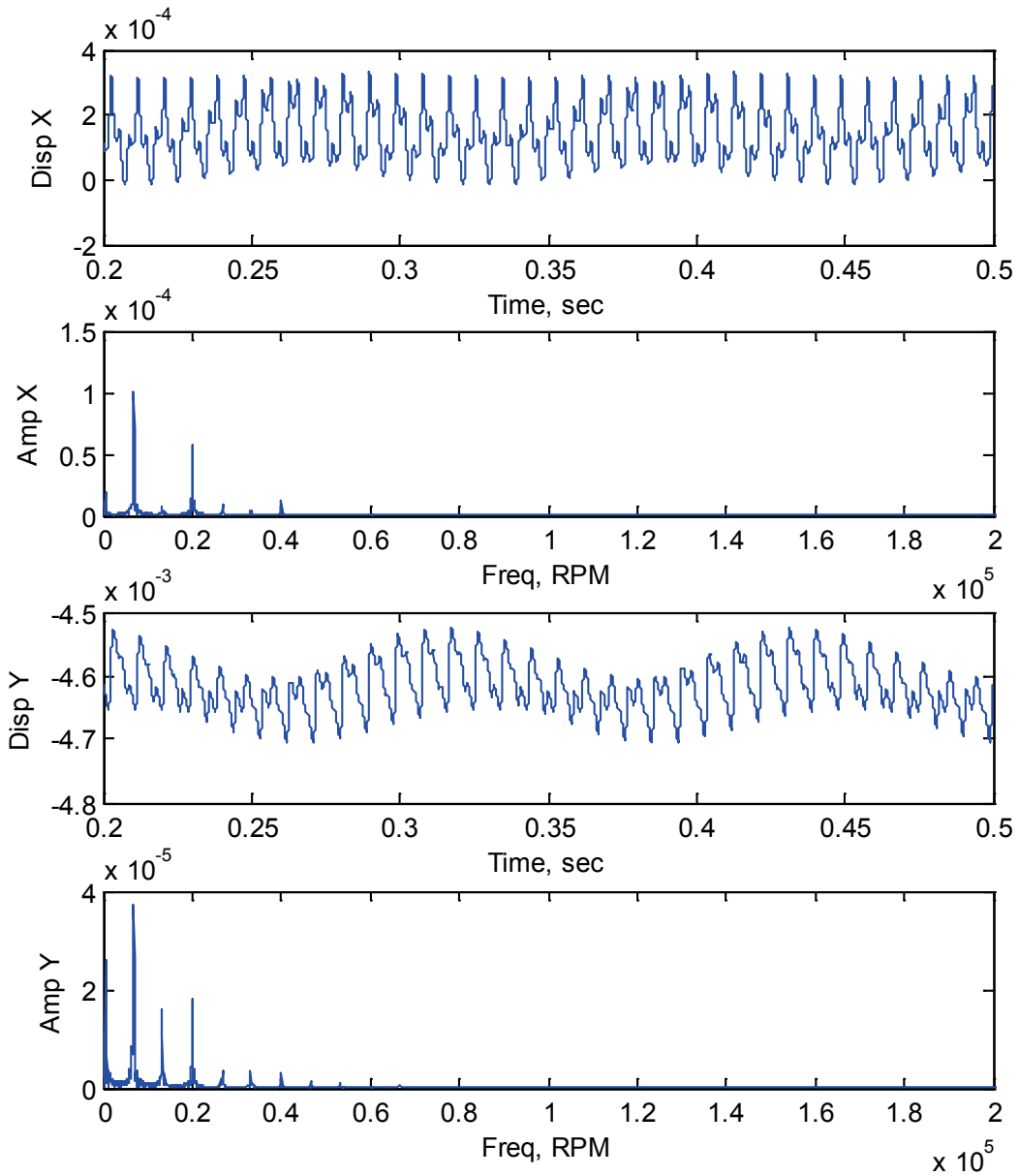

Figure 4.42 FFT of SFD at Node 22 under 20,000 rpm 

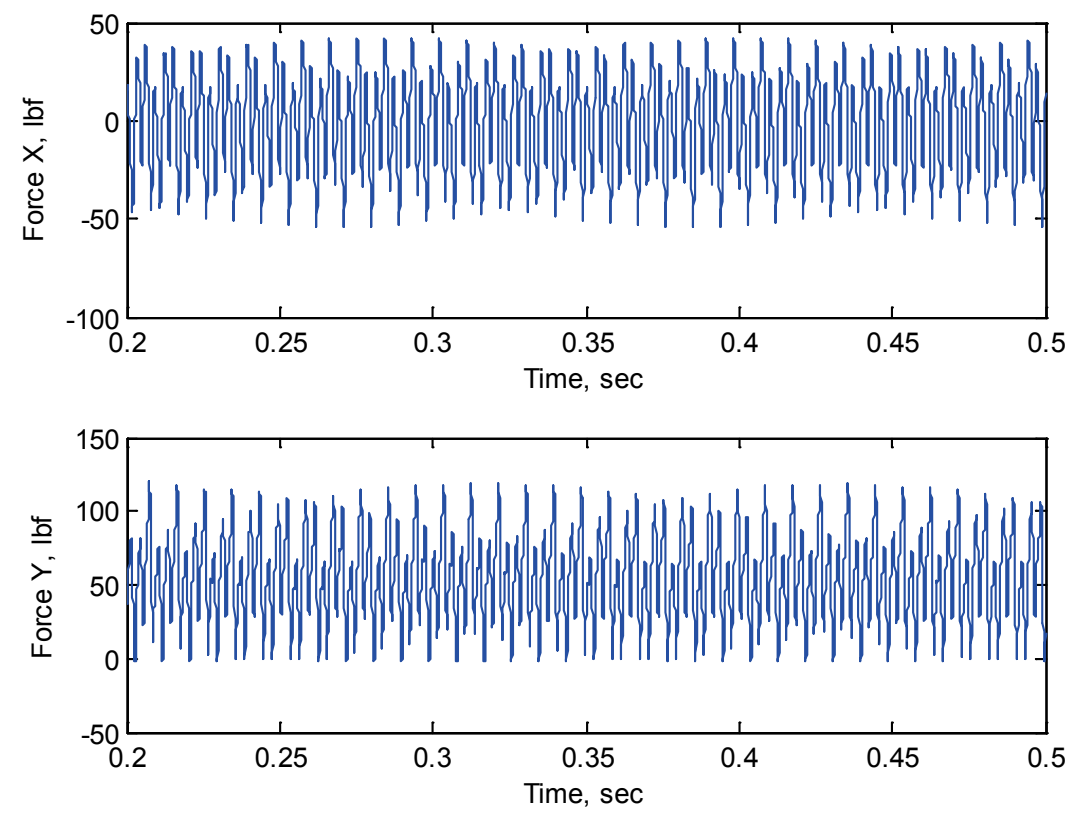

Figure 4.43 SFD Forces at Node 22 under 20,000 rpm

The results of the rotor with and without two squeeze film dampers at 30,000 rpm are shown in Figs. 4.44 4.52. The response comparison before and after adding the squeeze film damper is listed in Table 4.8. Adding the squeeze film dampers increases the stability of the rotor which is running at 30,000 rpm. At 30,000 rpm, the response of the rotor is nearly synchronous with the rotational speed, as shown in FFT analysis (Figs. 4.46\&4.52). Also the shaft center inside the bearing, Fig. 4.45, is whirling around the center area of the bearing clearance with the small eccentricity of $20 \%$. The shaft center behavior is compared in Figs. 4.44 and 4.45. The bearing forces are compared in Figs. 4.47 and 4.48. The maximum displacement from the center disk is compared in Figs. 4.49 4.52.

The shaft orbit, which is supported with nonlinear short plain journal bearings and nonlinear short squeeze film dampers, is shown in Fig. 4.53 (0.1 second, 30,000 rpm). The shaft deformation is indicated with the black line with red points on it. As discussed 
before, the system is under synchronous response with closed single orbit after the transient response dies out. The increased stability is from the behavior of squeeze film dampers.

Table 4.8 Comparison of with and w/o SFD at 30,000 rpm

\begin{tabular}{l|c|c|c}
\hline Displacement, mil & $\begin{array}{c}\text { Shaft Center } \\
\text { w/o SFD }\end{array}$ & $\begin{array}{c}\text { Shaft Center } \\
\text { with SFD }\end{array}$ & $\begin{array}{c}\text { Bearing Center } \\
\text { with SFD }\end{array}$ \\
\hline Node 4, peak-to-peak & 3.0 & $1.3^{*}$ & 3.2 \\
\hline Node 22, peak-to-peak & 3.0 & $0.6^{*}$ & 0.35 \\
\hline Node13, peak-to-peak & $>80$ & 1.8 & - \\
\hline
\end{tabular}

*Relative displacement between the shaft center and the bearing

\begin{tabular}{l|c|c|c|c|c|c}
\hline \multirow{2}{*}{ Force, lbf } & \multicolumn{2}{|c|}{ Brg Only } & \multicolumn{4}{c}{ Brg + SFD } \\
\cline { 2 - 7 } & Brg F F $_{\mathrm{x}}$ & $\mathrm{Brg} \mathrm{F}_{\mathrm{y}}$ & $\mathrm{Brg} \mathrm{F}_{\mathrm{x}}$ & $\mathrm{Brg} \mathrm{F}_{\mathrm{y}}$ & $\mathrm{SFD} \mathrm{F}_{\mathrm{x}}$ & SFD F $_{\mathrm{y}}$ \\
\hline Node 4 & 5,000 & 5,000 & 734 & 761 & 763 & 793 \\
\hline node 22 & 5,000 & 5,000 & 298 & 316 & 282 & 300 \\
\hline
\end{tabular}
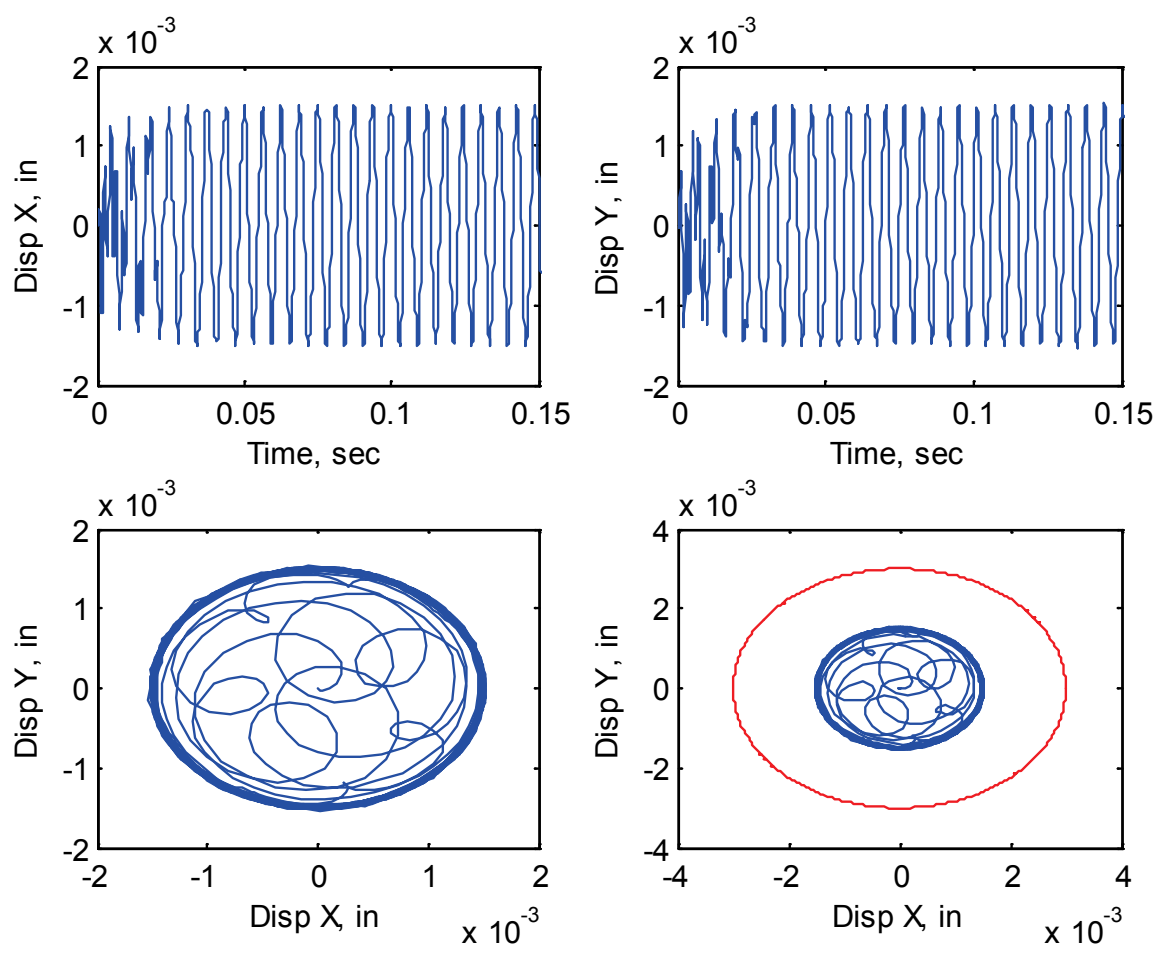

Figure 4.44 Shaft Center inside Bearing (node\#4) w/o Damper at 30,000 rpm 

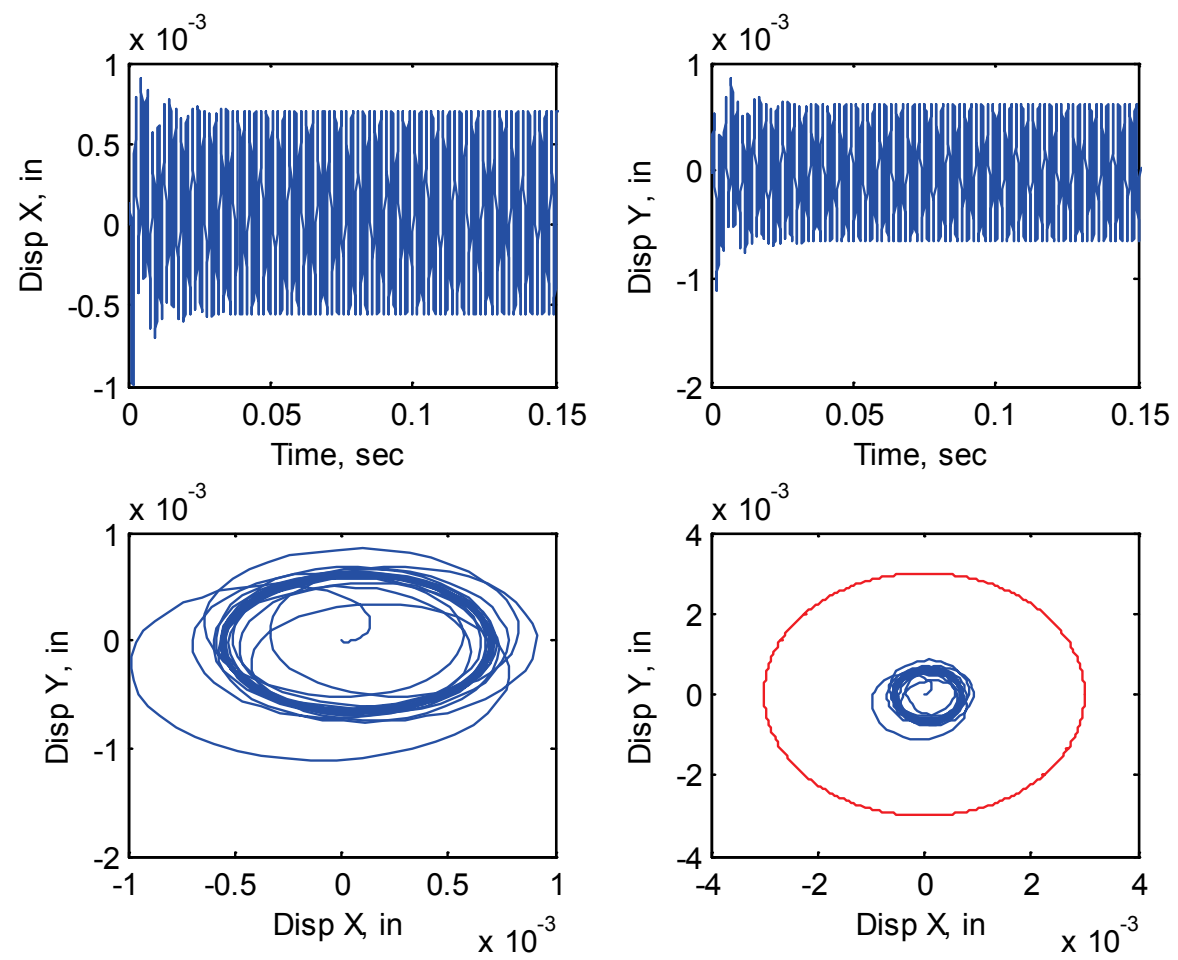

Figure 4.45 Shaft Center inside Bearing (Node\#4) with Damper at 30,000 rpm
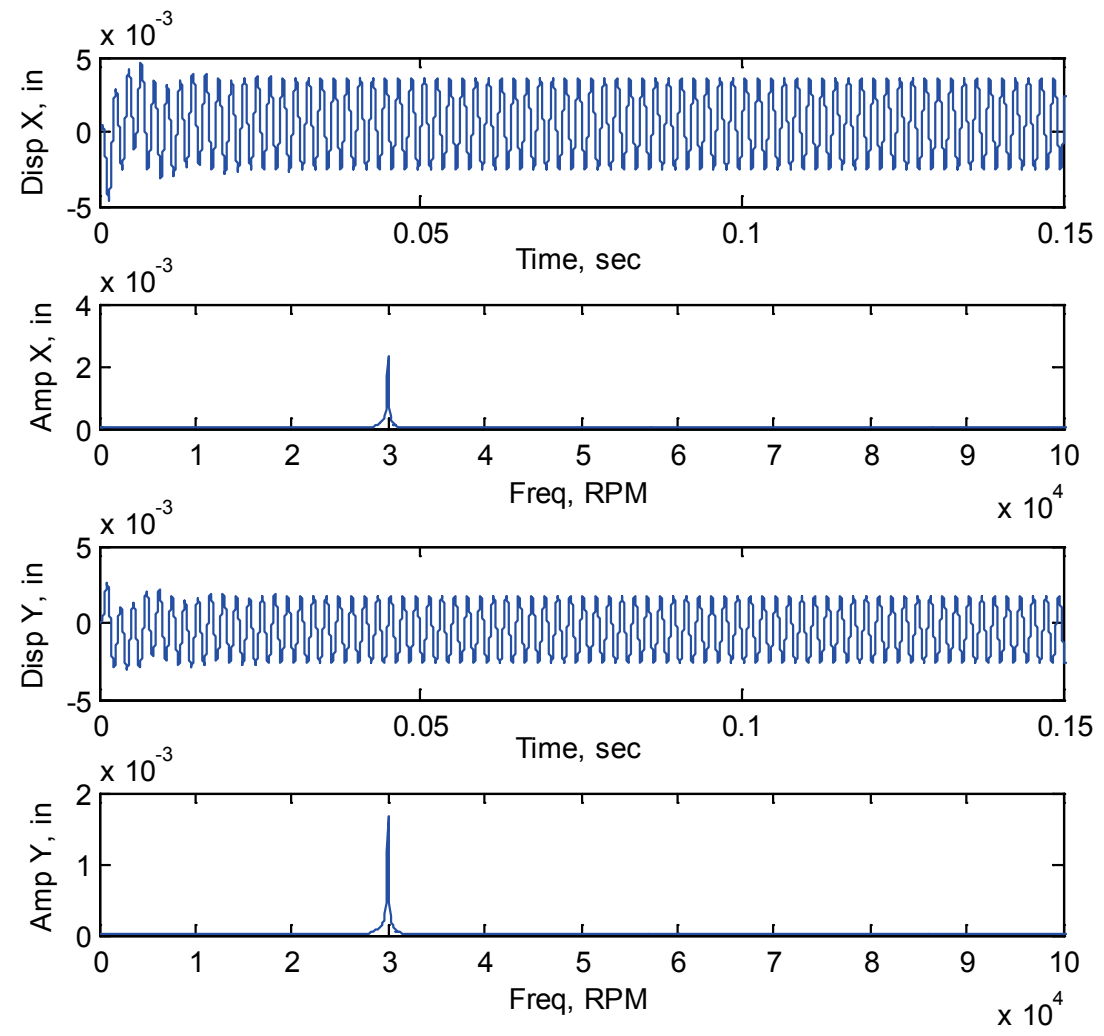

Figure 4.46 FFT of Shaft Center inside Bearing (Node\#4) with Damper at 30,000 rpm 

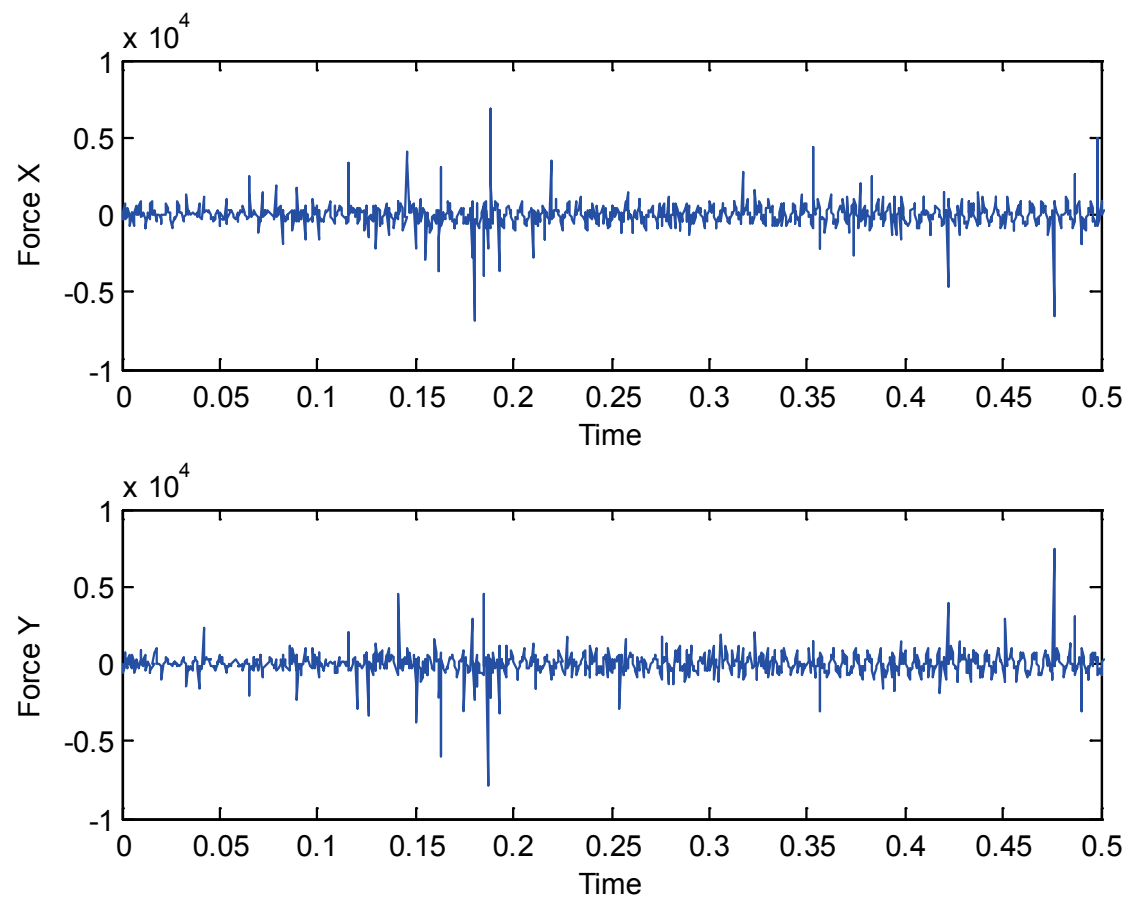

Figure 4.47 Bearing Forces (Node\#4) without Squeeze Film Damper at 30,000 rpm
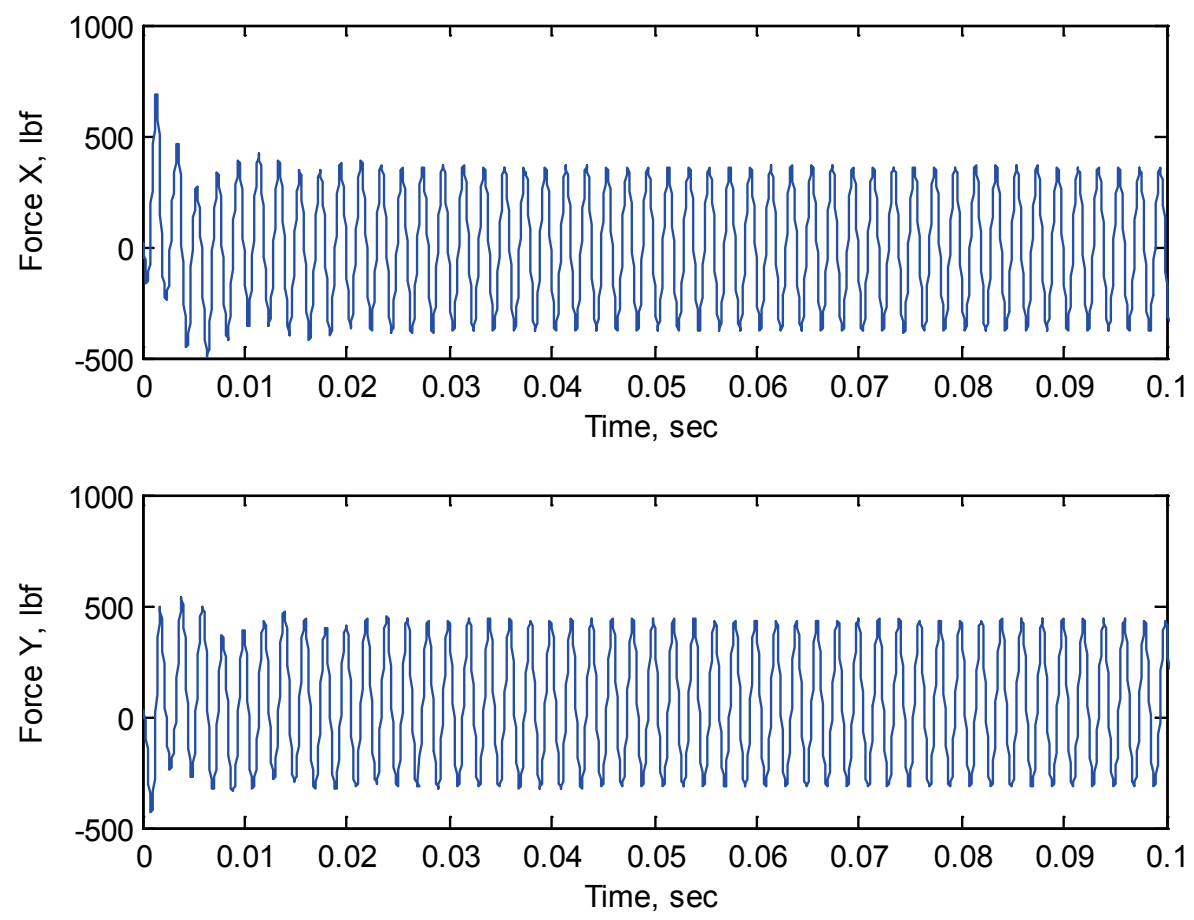

Figure 4.48 Bearing Forces (Node\#4) with Squeeze Film Damper at 30,000 rpm 


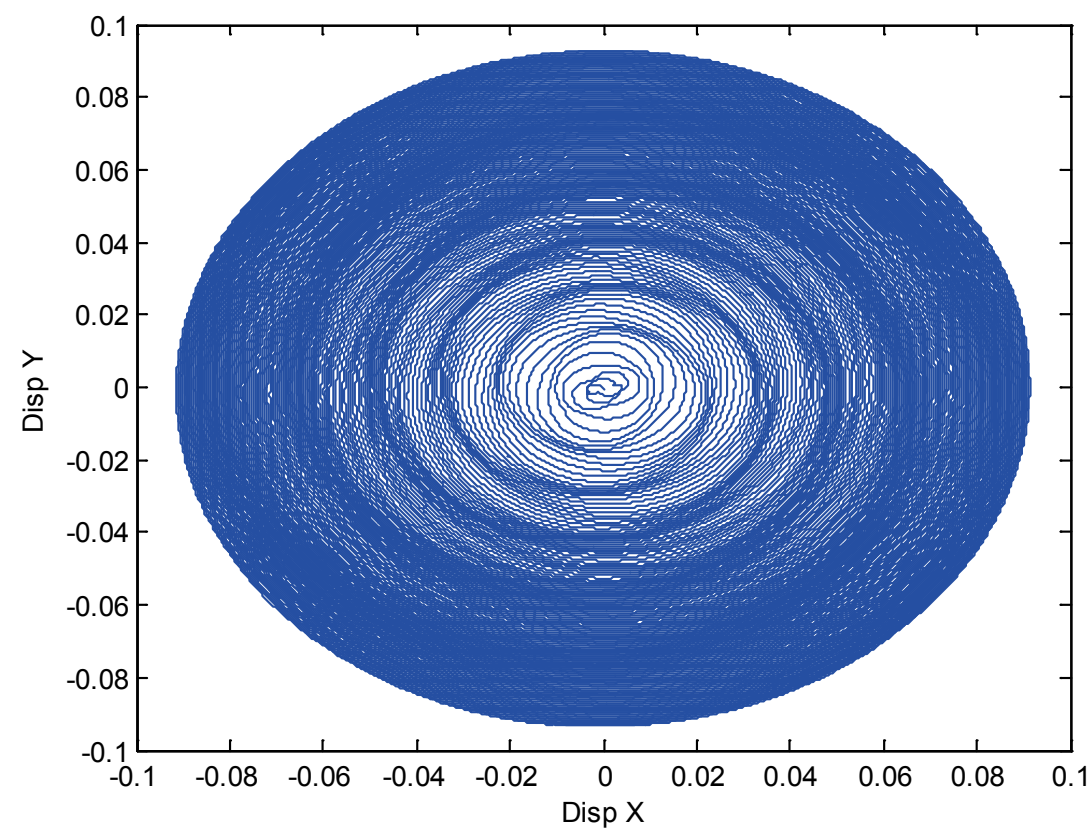

Figure 4.49 Orbit of Center Disk at 30,000 rpm without SFD
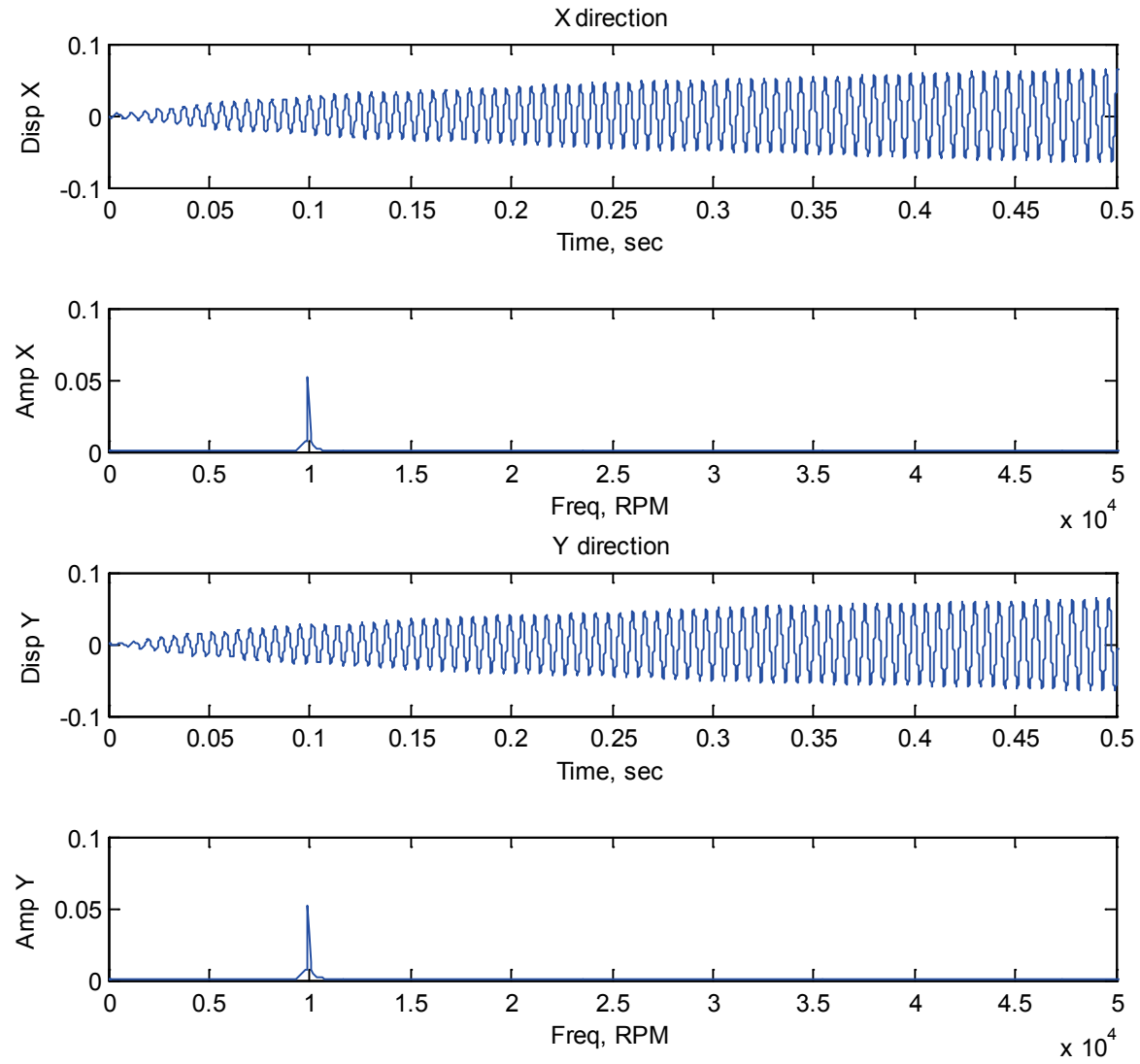

Figure 4.50 FFT of Center Disk at 30,000 rpm without SFD 


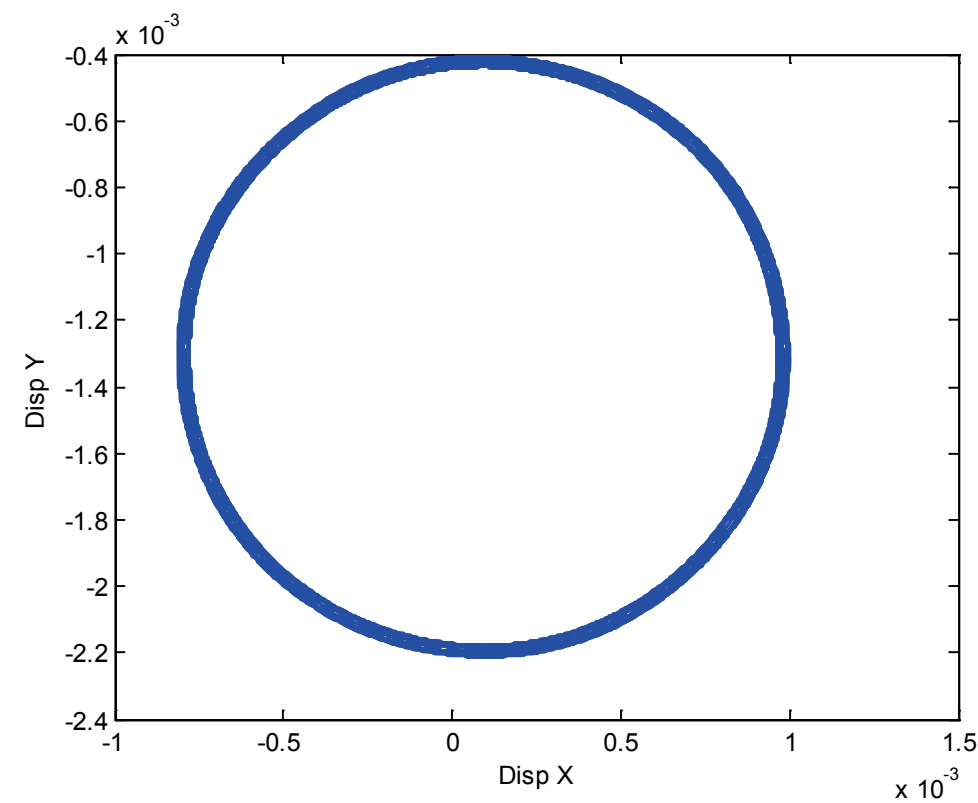

Figure 4.51 Orbit of Center Disk at 30,000 rpm with SFD
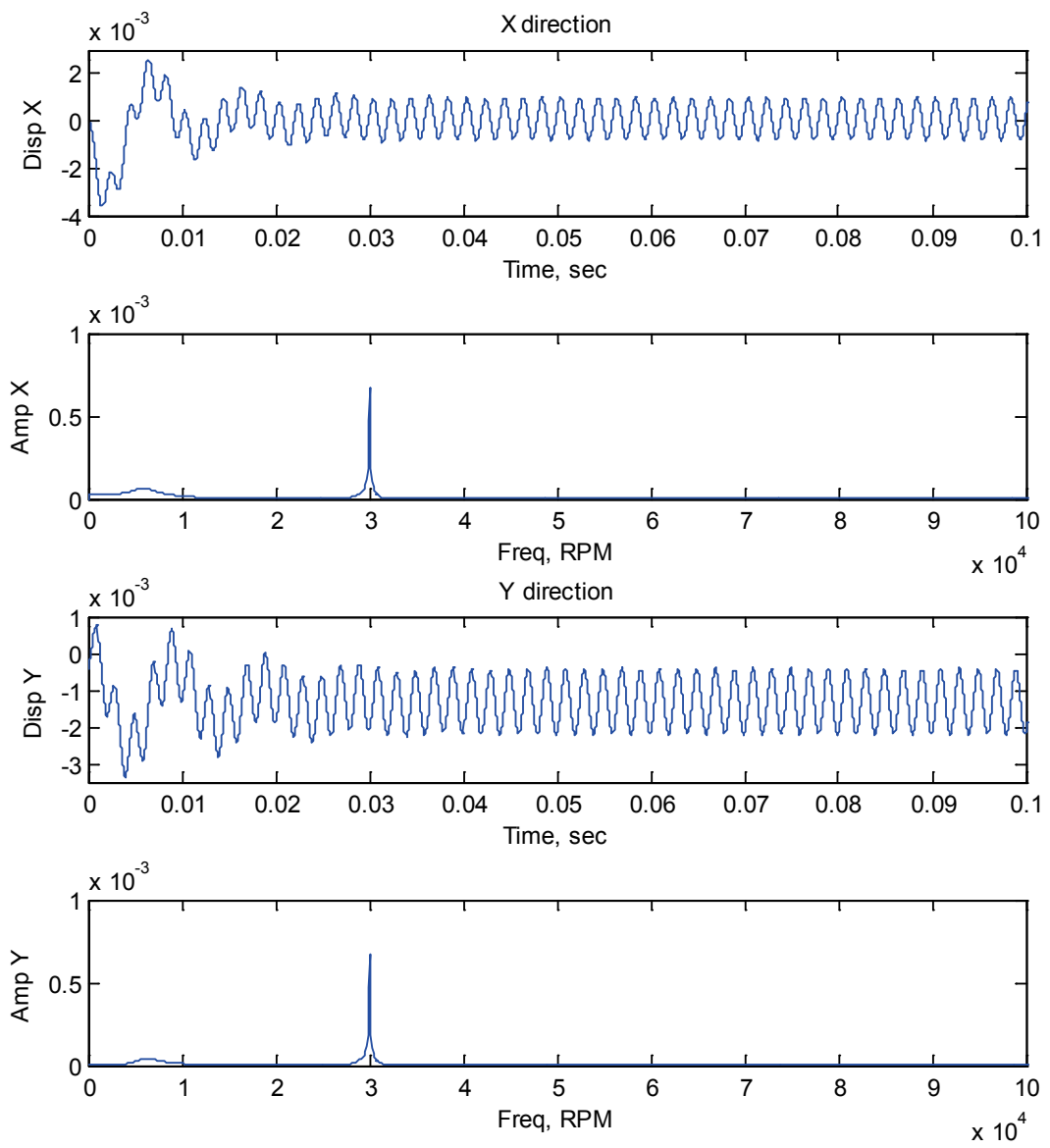

Figure 4.52 FFT of Center Disk at 30,000 rpm with SFD 


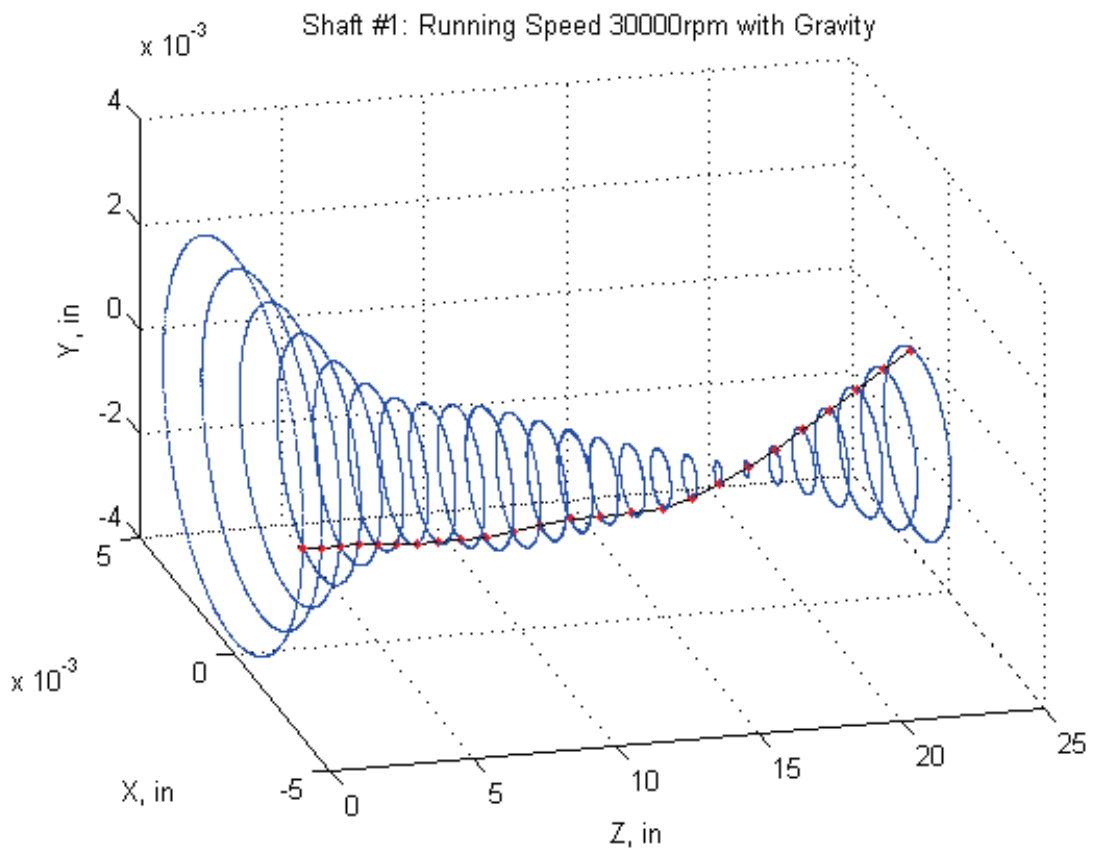

Figure 4.53 Orbits of the Rotor with SFD at 30,000 rpm around 0.1 second with Gravity Loading and Unbalances

The time and frequency response from the two dampers are shown in Figs. 4.54 4.61. The time transient output, the bearing housing inside the damper is whirling a nearly the center area of the damper clearance. From the FFT result, there is only one peak of $30,000 \mathrm{rpm}$, which indicates the synchronous response of the bearings inside the dampers.

For this nonlinear rotor-bearing-damper system, the strong nonlinear behavior, includes sub-harmonic, torus and super-harmonic as shown in Figs. 4.36 4.43, is detected at 20,000 rpm. When the operating speed increases to $30,000 \mathrm{rpm}$, however, only the weakly nonlinear behavior is detected from the response of the shaft, the bearings and the dampers. If the damper forces are able to center the orbital response of the bearing housing, which is inside the squeeze film damper, near to the geometric center of the damper, the rotor response will have a weakly nonlinear behavior. Some strong nonlinear behavior will appear if the damper forces are not able to center the 
orbital response near to the geometric center of the damper. Under the case of 30,000 rpm, both the bearing and dampers are whirling with small eccentricity in the lower central area of the clearances, which results in a stable synchronous response.
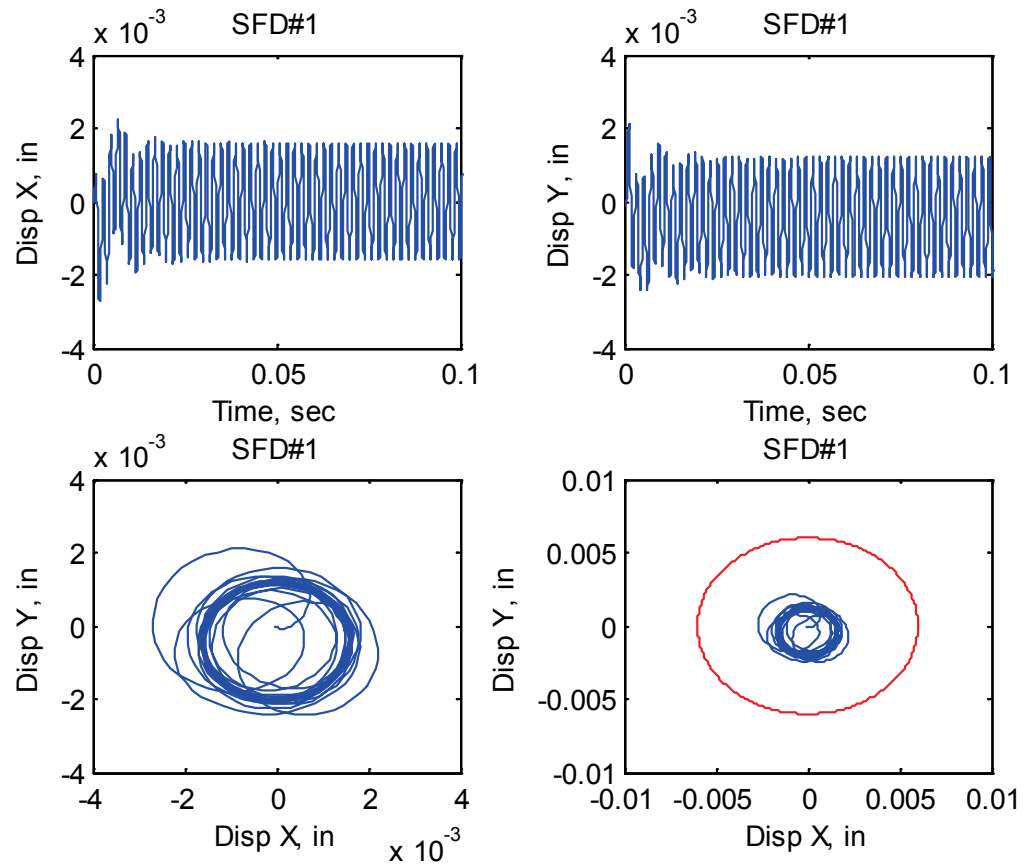

Figure 4.54 Bearing Center inside SFD at Node\#4 at 30,000 rpm

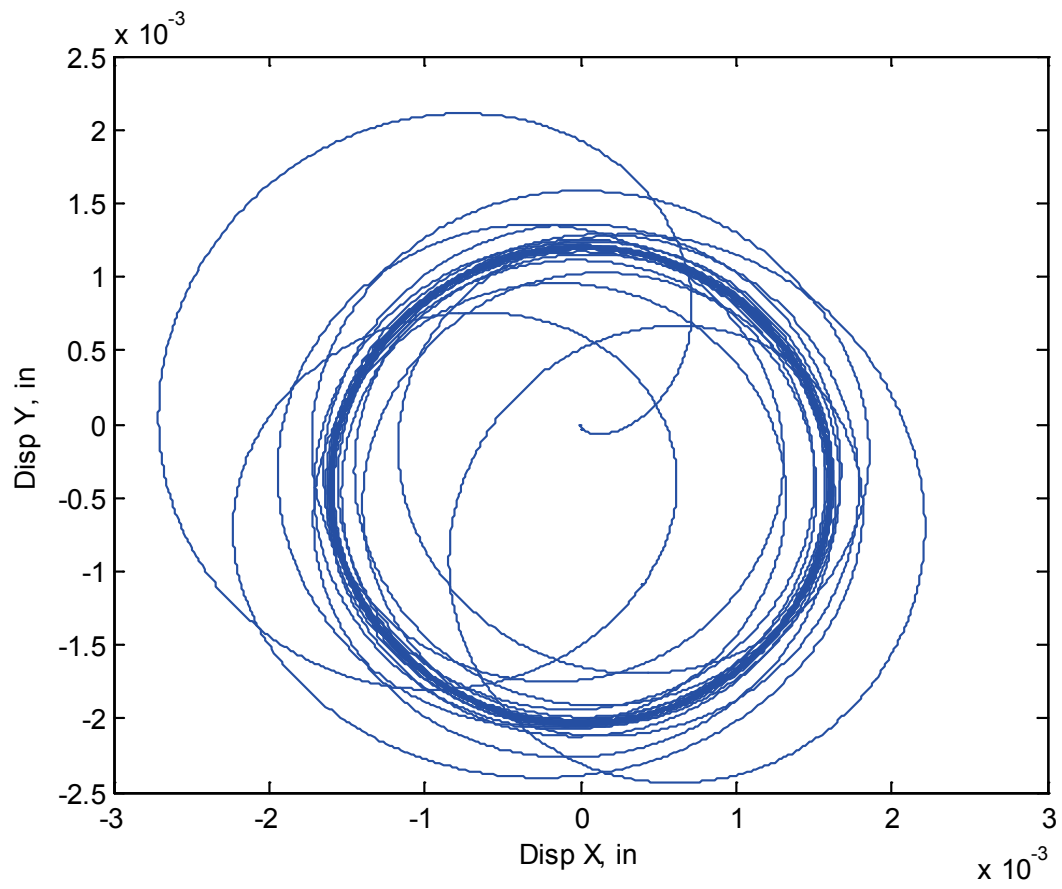

Figure 4.55 Orbit of Bearing Center inside SFD at Node\#4 at 30,000 rpm 

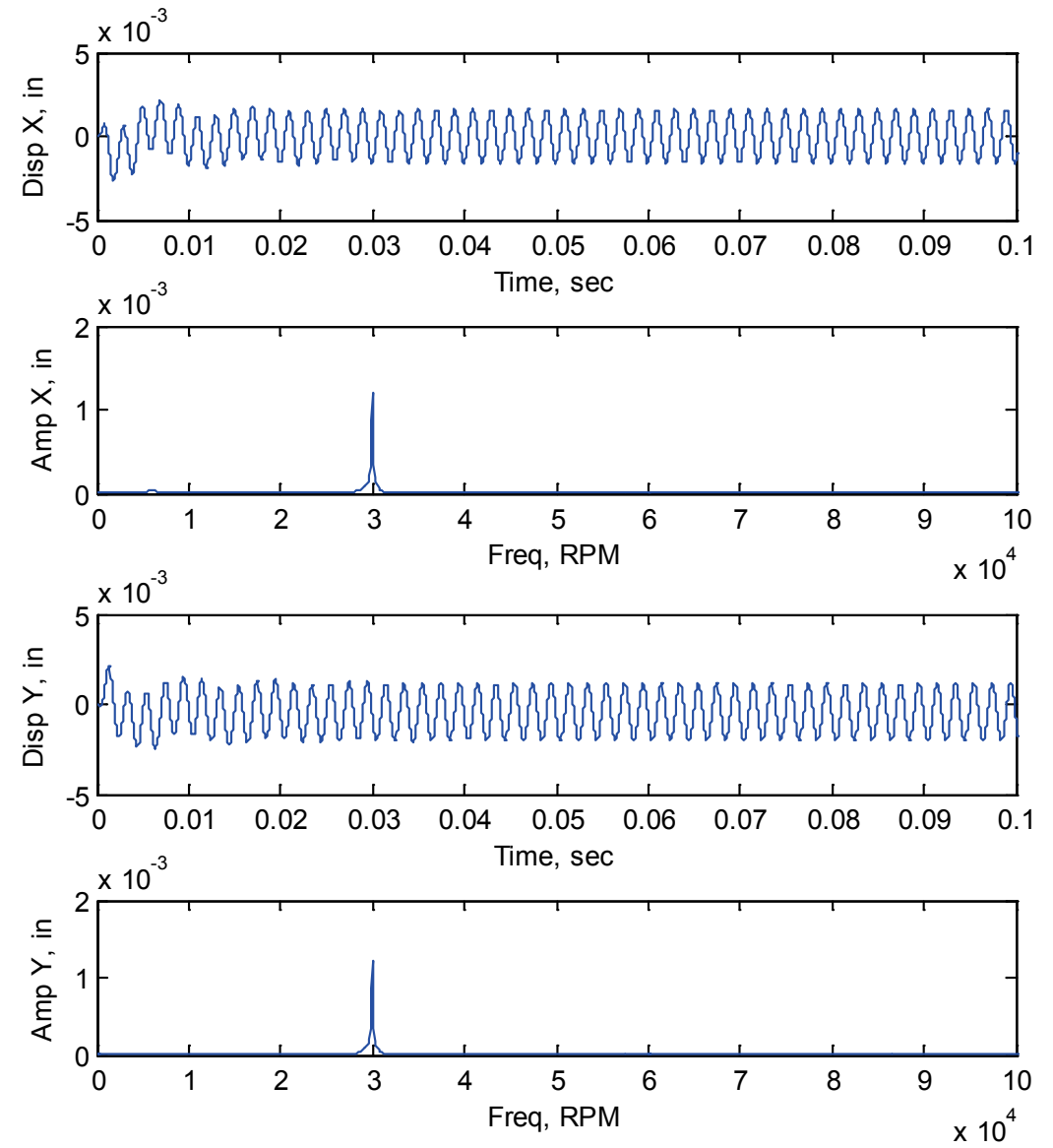

Figure 4.56 FFT of Bearing Center inside SFD at Node\#4 at 30,000 rpm
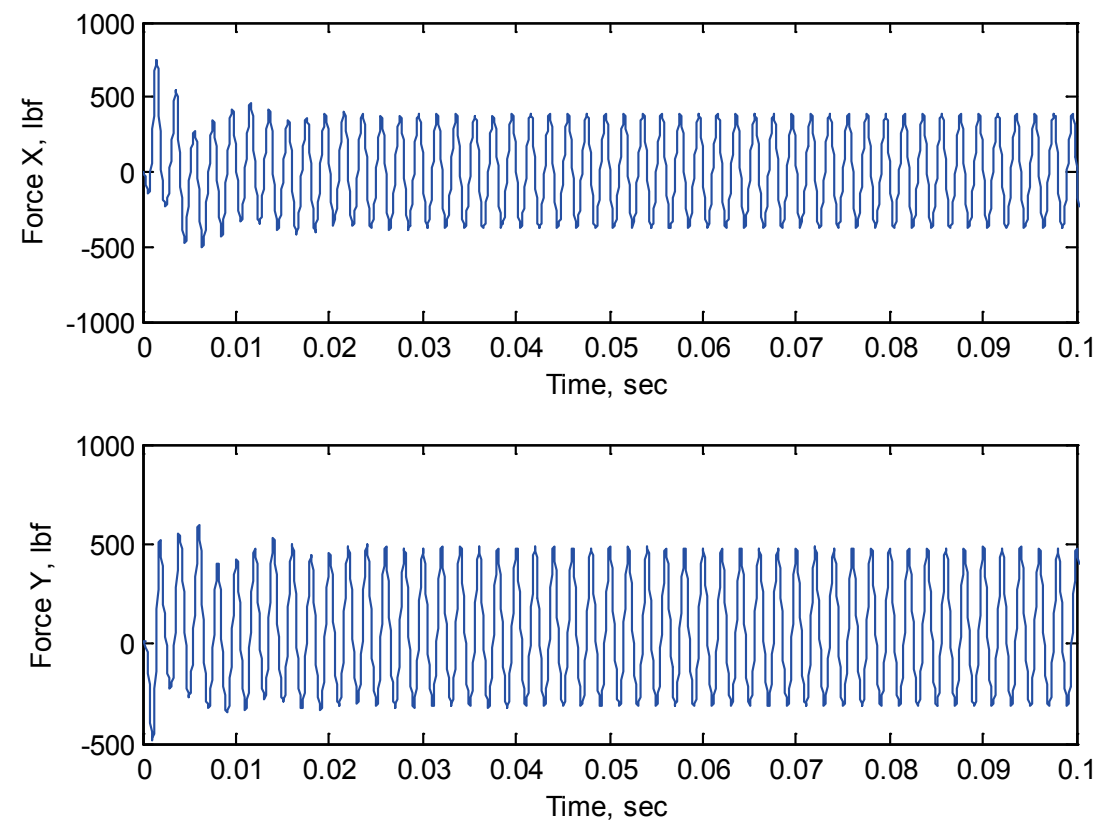

Figure 4.57 SFD Forces at Node\#4 at 30,000 rpm 

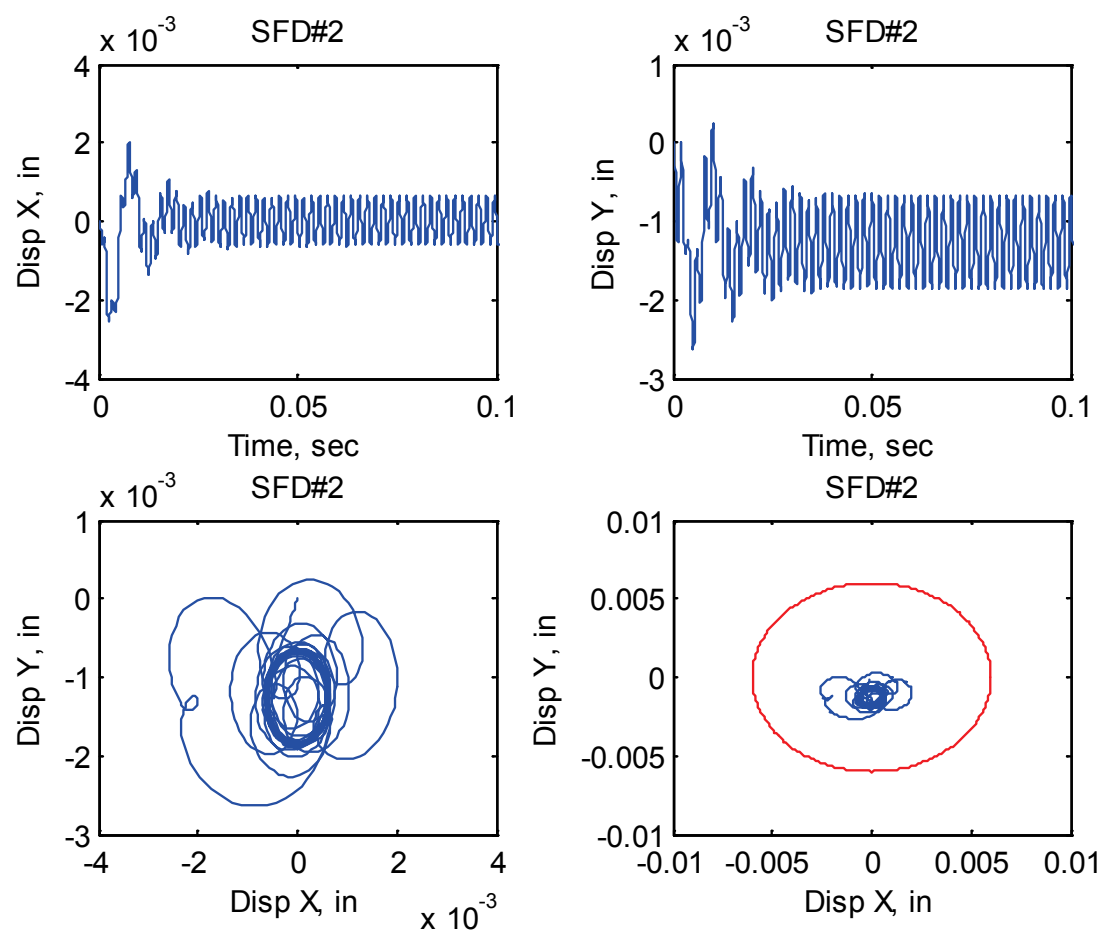

Figure 4.58 Bearing Center inside SFD at Node\#22 at 30,000 rpm

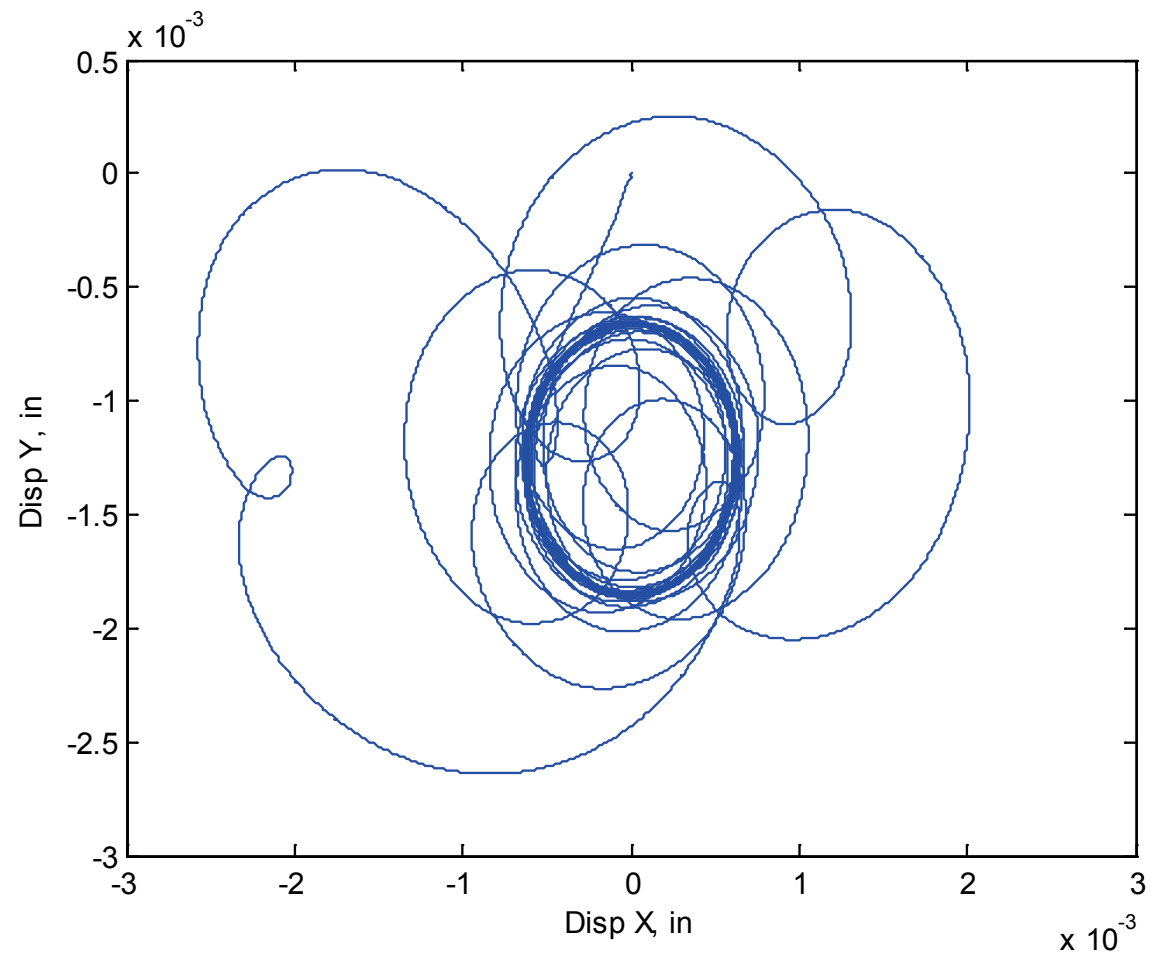

Figure 4.59 Orbit of Bearing Center inside SFD at Node\#22 at 30,000 rpm 

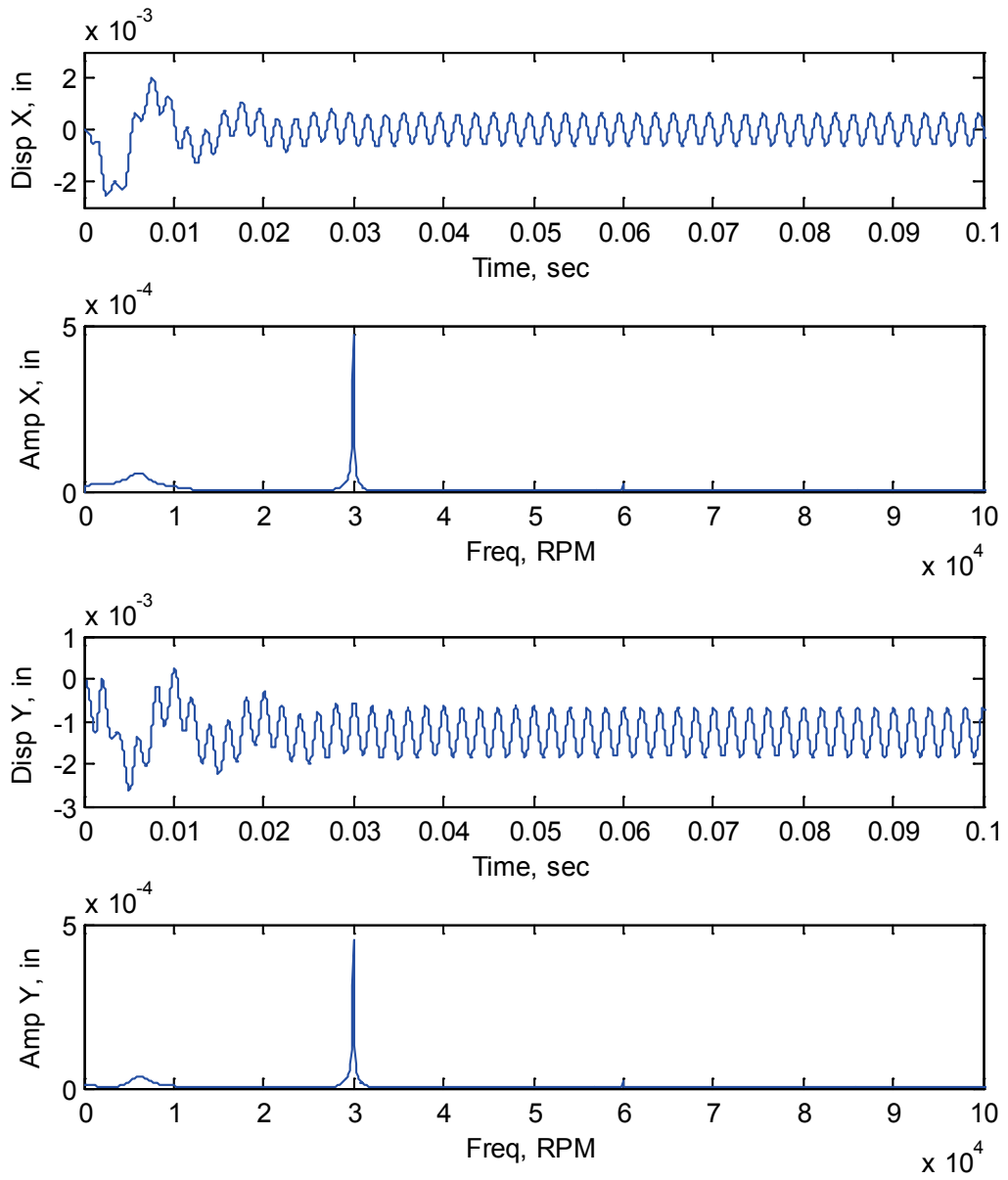

Figure 4.60 FFT of Bearing Center inside SFD at Node\#22 at 30,000 rpm
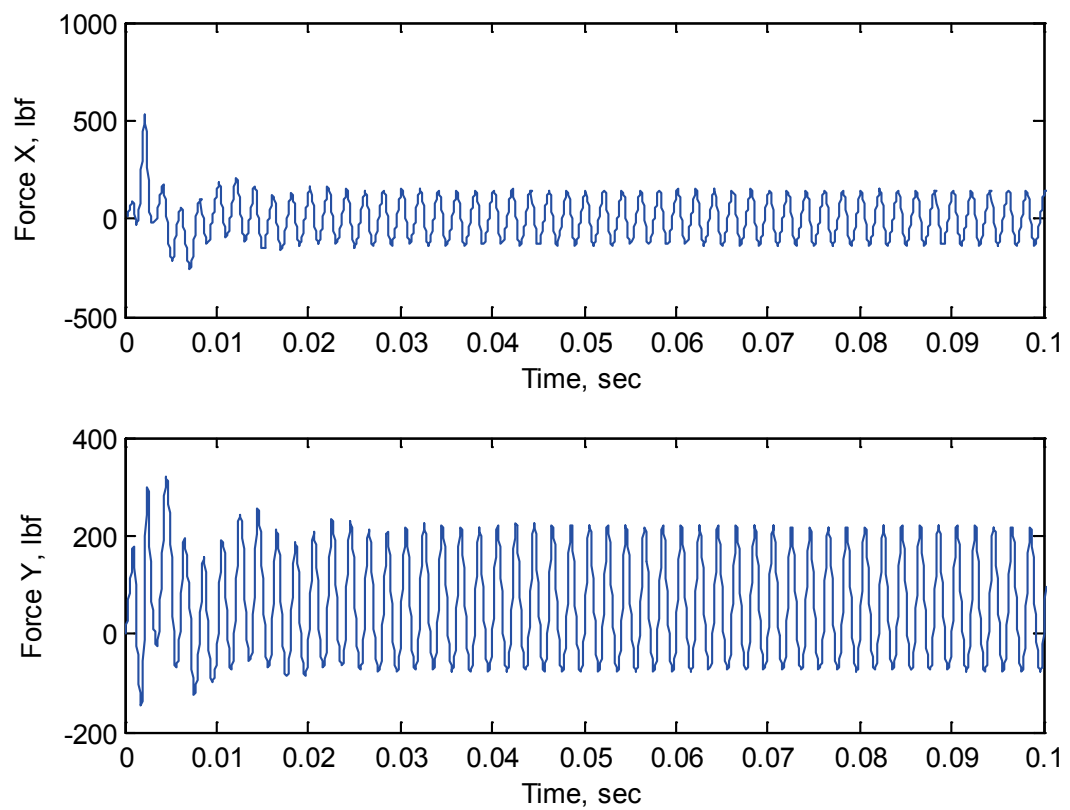

Figure 4.61 SFD Forces at Node\#22 at 30,000 rpm 
Finally waterfall diagrams of the 3-disk rotor with both nonlinear short plain journal bearings and nonlinear squeeze film dampers are shown in Figs. 4.62\&4.63. Compare to those waterfall diagrams (Figs. $4.12 \& 4.13$ ) of this rotor with nonlinear short journal bearing only, there is no sub-harmonic response at high running speeds after two short squeeze film dampers were added. Between running speed of 11,000 rpm 20,000 rpm, sub-harmonic response is shown due to the motions of both bearings and dampers. At this range of running speed, the bearing housing whirls at the bottom area of the squeeze film damper and strong nonlinear behavior appears. There two sub-harmonic frequencies: one is lower than half running speed, and another is between half running speed and the running speed. When the running speed is great than $21,000 \mathrm{rpm}$, only synchronous response is shown from the two waterfall diagrams from node\#4 and node\#22. When the running speed is lower than $10,000 \mathrm{rpm}$, only synchronous response is exist either with or without the squeeze film dampers, so no squeeze film damper is need at low range running speed. Based on those waterfall diagrams (Figs. 4.12\&4.13, Figs. 4.61\&4.62) of before and after adding two squeeze film dampers, the following suggestions are given for this 3-disk rotor:

1) No squeeze film damper is needed if the running speed is lower than $10,000 \mathrm{rpm}$ $(\Omega<10,000 \mathrm{rpm})$

2) Squeeze film damper with center spring is recommended if the running speed is between $10,000 \mathrm{rpm}$ and $21,000 \mathrm{rpm}(10,000 \mathrm{rpm}<\Omega<21,000 \mathrm{rpm})$.

3) Squeeze film damper without center spring is recommended if the running speed is great than $21,000 \mathrm{rpm}(\Omega>21,000 \mathrm{rpm})$ 


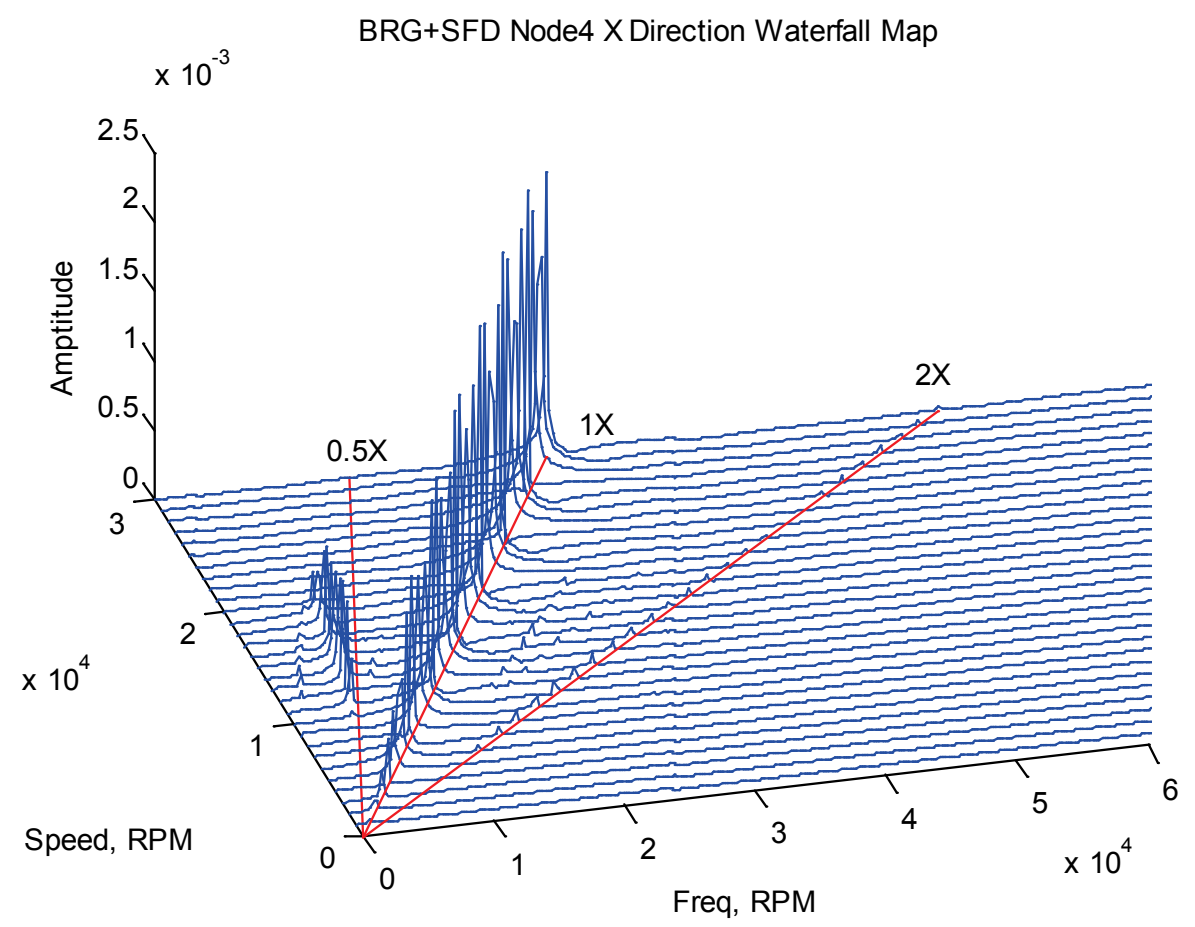

Figure 4.62 Waterfall Diagram of 3-Disk Rotor with Squeeze Film Dampers, Node 4 in Horizontal Direction

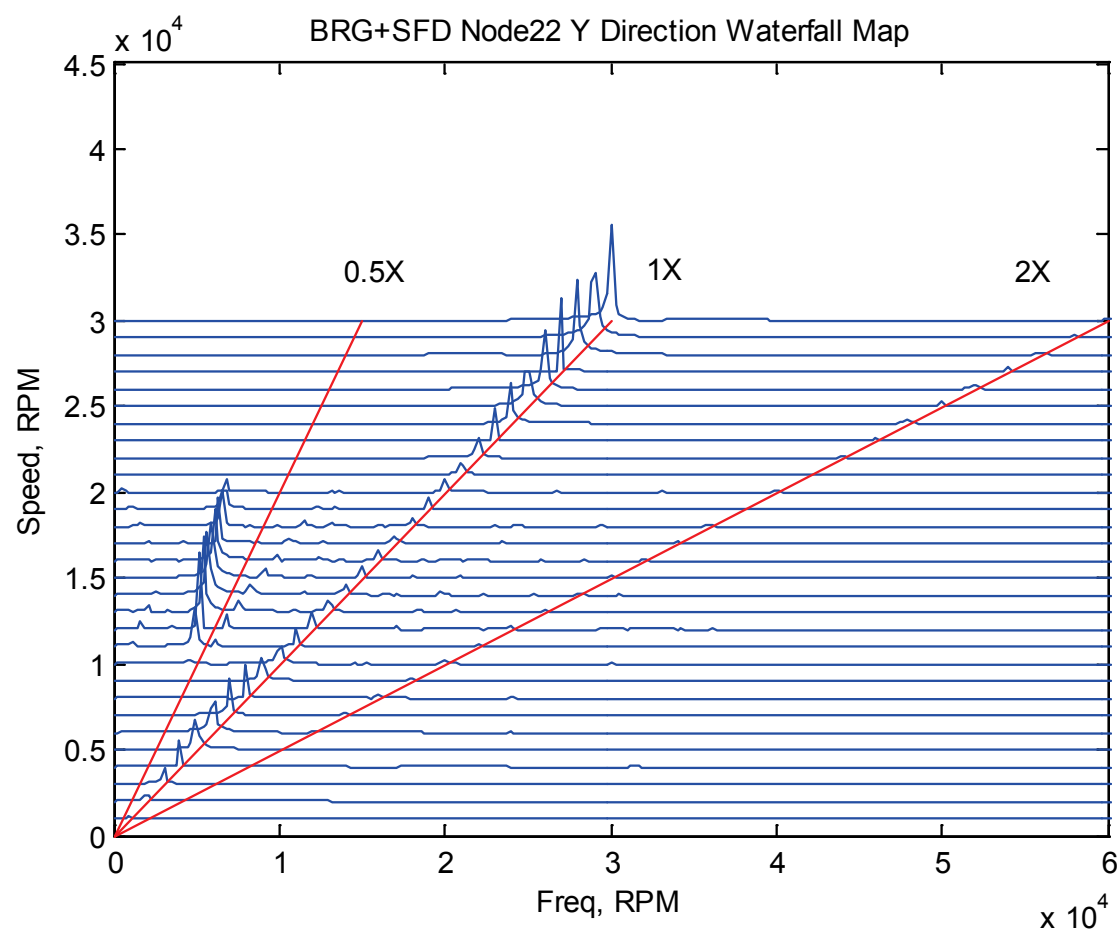

Figure 4.63 Waterfall Diagram of 3-Disk Rotor with Squeeze Film Dampers, Node 22 in Vertical Direction 


\subsection{Summary}

The chapter presented the transient analyses of a three-disk rotor model supported with linear bearing, nonlinear bearings, and nonlinear squeeze film dampers without a centering spring. The verification of the transient solver was done by comparing the response between steady state analysis and transient analysis on a stable linear rotorbearing system. The results show a good agreement with the computational comparison showing a maximum percentage difference of $0.12 \%$. The waterfall diagrams generated from the nonlinear analysis also showed a good correlation with the experimental behavior of a rotor on fluid film bearings due to oil whirl and oil whip. Detailed subharmonic and torus response is detected during the time transient analyses. The rotor supported with fluid journal bearings goes unstable with increased rotational speed. Adding the squeeze film dampers increases the stability of the system and decreases the vibration amplitude at the same time. If the damper forces provide the movement of the bearing housing near the center area of the damper, the rotor has a weakly nonlinear response; if the bearing housing inside the damper whirls far away from the center area of the damper, some strong nonlinear behavior, such as sub-harmonic, torus and superharmonic, appears.

The transient method presented is able to evaluate non-central bearing and damper orbit problems, which are needed in the analysis of the dampers. At rotational speed of $20,000 \mathrm{rpm}$, the orbital response of the rotor is not centered in the geometric center of the squeeze film dampers, and strong nonlinear response is detected. At rotational speed of $30,000 \mathrm{rpm}$, the damper forces are able to center the orbital response near to the 
geometric center of the damper without the need for a centering spring. In this case only synchronized response is detected.

Computing time for different cases are listed here with same time step $\Delta \mathrm{t}=4 \times 10^{-6} \mathrm{sec}$, and same time span: $0 \sim 0.1 \mathrm{sec}$, for the three-disk rotor with 25 nodes. For the linear bearing case, the computing time is $82.54 \mathrm{sec}$; for the nonlinear bearing only case, the computing time is $124.22 \mathrm{sec}$.; and for the nonlinear bearing with the nonlinear squeeze film damper case, the computing time is $159.66 \mathrm{sec}$. For nonlinear analysis, $40 \%$ of extra computational time is used for the nonlinear force calculation. The computational time of different cases are listed in Table 4.9. The file size is for the displacements of all nodes.

Table 4.9 Computing Time Comparison

\begin{tabular}{l|c|c|c}
\hline 3-Disk Model, $0 \sim 0.1 \mathrm{sec} ., \Delta \mathrm{t}=4 \times 10^{-6}$ & sec., Lateral Analyses \\
\hline & DOF & Time, sec & File Size, KB \\
\hline Linear Bearing & 100 & 82.54 & 58,645 \\
\hline Nonlinear Bearing & 100 & 124.22 & 58,645 \\
\hline Nonlinear Bearing + SFD & 104 & 159.66 & 60,208 \\
\hline
\end{tabular}




\section{Chapter 5}

\section{Rigid and Flexible Rotors with Nonlinear Tilting}

\section{Pad Bearings and Nonlinear Finite Length}

\section{Squeeze Film Dampers}

\subsection{Overview}

This chapter describes the transient analyses of both a rigid and linear flexible rotor models supported with nonlinear tilting pad bearings and nonlinear finite length squeeze film dampers. The transient analysis of a rigid rotor supported with tilting pad bearings is applied first to validate the force calculation method for the tilting pad bearings. The flexible rotor model is an eight-stage back to back compressor supported with two 4-pad tilting pad bearings. To increase stability under severe unbalance conditions, the finite length squeeze film dampers are added to outside of the tilting pad bearings. Unlike the plain journal bearing, displacements and velocities of all pads and pivots inside the tilting pad bearings have to be taken into consideration. The nonlinear analyses are applied to the 8-stage compressor to show the behavior of the rotor and the tilting pad bearings under different unbalances. To investigate the stability of the system under more severe 
unbalance conditions, two finite length nonlinear squeeze film dampers, which installed outside of the bearings, are introduced to the system.

The following sections, Section 5.2 shows the transient analysis of a rigid rotor supported with a 4-pad tilting pad bearings. Section 5.3 discusses the flexible 8 -stage compressor system and the finite element beam based linear rotor model in details. Section 5.4 presents the transient analyses of the 8-stage compressor supported with two nonlinear tilting pad bearings. Section 5.5 presents the transient analyses of the 8 -stage rotor system supported with two nonlinear tilting pad bearings and two nonlinear finite length squeeze film dampers under severe unbalance conditions. Section 5.6 discusses the results found through the various nonlinear transient analyses.

The analyses performed in this chapter were done using a Dell Precision T3500 computer with an Intel Xeon CPU@2.53 GHz, 12GB of RAM and a 64-bit Windows OS. Matlab version 7.13 (R2011b) was used for the 64-bit OS.

\subsection{Rigid Rotor with Nonlinear Tilting Pad Bearings}

The transient analysis of a rigid rotor supported on two 4-pad tilting pad bearings, as shown in Figure 5.1, is applied first. The tilting pad bearing geometry, given in Table 5.1, is used in the transient analysis. The tilting pad bearing is treated as a nonlinear component and the bearing forces/moments at each time step are calculated from instantaneous displacements and velocities of the bearing pads, the bearing pivots, and the shaft center inside the bearing. According to the instantaneous displacements and velocities, the pressure profile of each pad is calculated at first by solving the Reynolds equation. The pressure calculation is based on the finite element method with assumption 
of laminar flow in the lubricant, constant lubricant density and constant lubricant viscosity as described in Chapter 3. The bearing forces are then calculated by integrating the pressure profile over each pad, and are finally applied to the force vector of the equations of motion at each step. For transient analysis, the bearing profiles of all pads and the bearing forces need to be calculated at each time step.

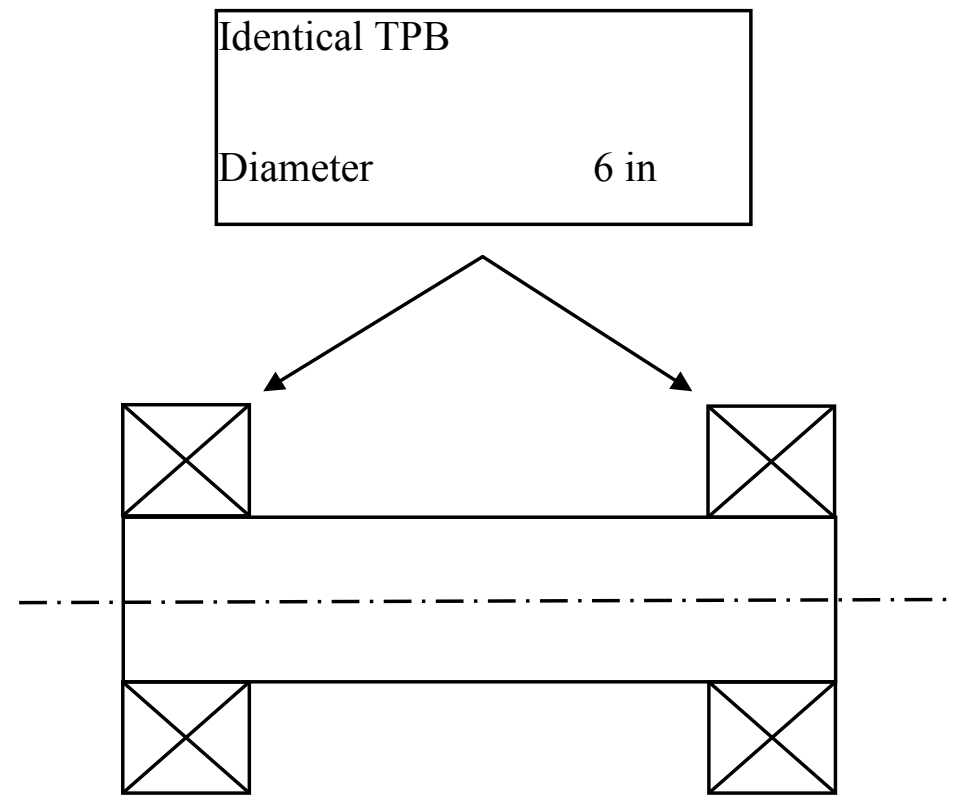

Figure 5.1 Rigid Rotor Supported on two Tilting Pad Bearings

\subsubsection{Pressure Profiles of Tilting Pad Bearing and Nonlinear Bearing}

\section{Force Calculation}

Each of the two identical tilting pad bearings has 4 pads with load between pads (LBP). Each bearing was assumed to have the load of half rotor weight, 1500lbf. This represents the "bounce" mode motion of the rotor only. The bearing was designed with outer diameter of 8 inch, inner diameter of 6 inch, pad clearance of 0.006 inch, bearing clearance of 0.006 inch, pivot offset of $50 \%$ and axial length of 6 inch. The tilting pad 
geometric parameters are listed in Table 5.1 and the bearing geometry is given in Fig. 5.2. In the figure, the pivots are denoted with black arrows at $45^{\circ}, 135^{\circ}, 225^{\circ}$, and $315^{\circ}$.

Table 5.1 4-Pad Tilting Pad Bearing Information

\begin{tabular}{l|l|l}
\hline \multicolumn{3}{l}{ Type: Tilting Pad Bearing } \\
\hline No. of pads: 4, Load Between Pads (LBP) \\
\hline Property & Value & Unit \\
\hline Pad Angle & 75 & deg \\
\hline Bearing Length & 6 & in \\
\hline Journal Diameter & 6 & in \\
\hline Pad Outer Diameter & 8 & in \\
\hline Bearing Assembled Clearance & 0.006 & in \\
\hline Pad/Machined Clearance & 0.006 & in \\
\hline Load in x direction & 0 & lbf \\
\hline Load in y direction & -1500 & lbf \\
\hline Preload & 0 & - \\
\hline Pivot offset & $50 \%$ & - \\
\hline Pivot Stiffness & $5.00 \mathrm{E}+6$ & lbf/in \\
\hline Oil Viscosity & $1.54 \mathrm{E}-6$ & lbf-s/in \\
\hline & &
\end{tabular}

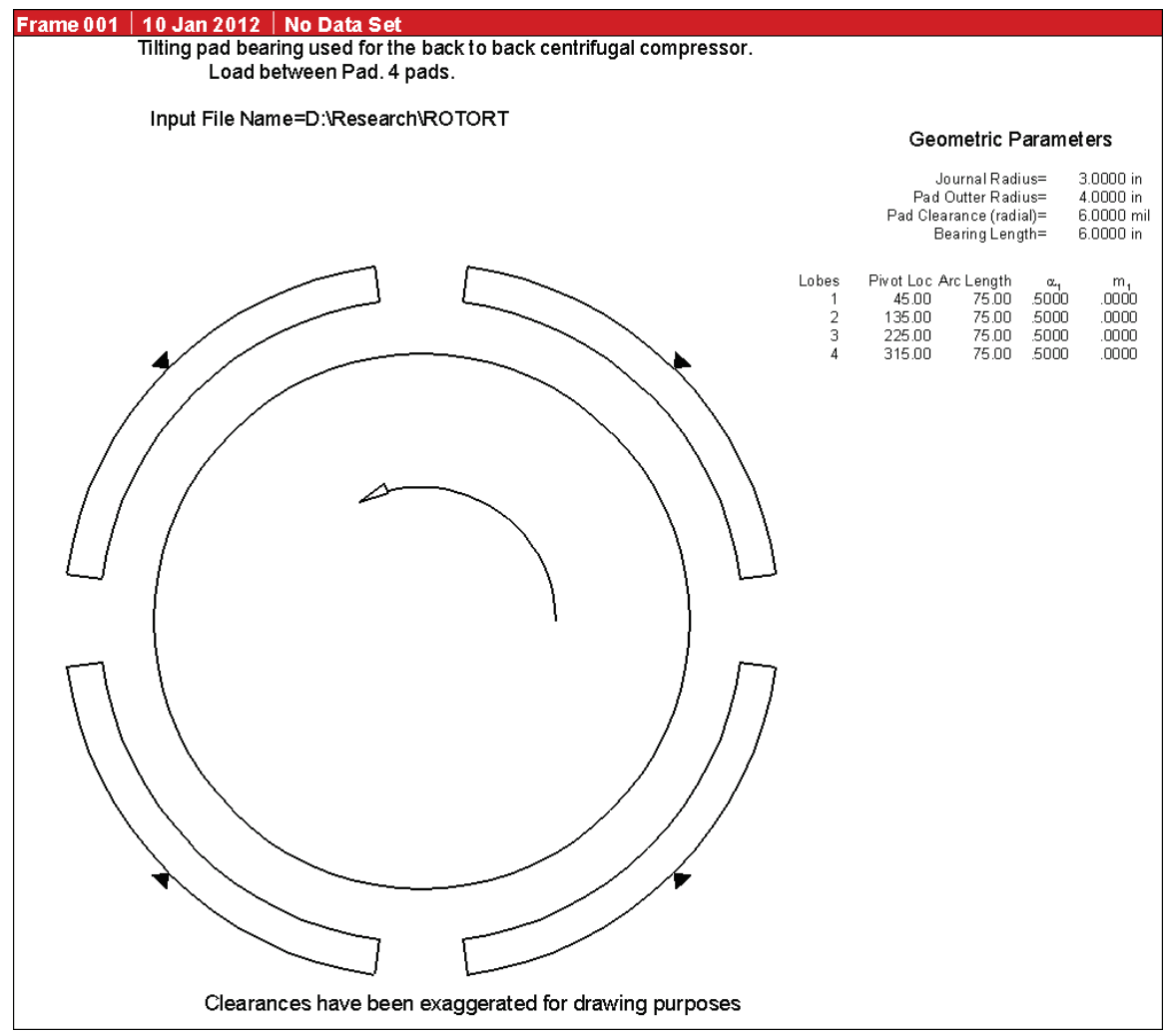

Figure 5.2 4-Pad Tilting Pad Bearing Geometry 
As discussed in Chapter 3, the axial pressure distribution is assumed with Eq. (3.4.11), and the weighting factors $B 1 \& B 2$ in Eq. (3.4.13\&3.4.14) are calculated with $n=2.25$ for this bearing with $\mathrm{L} / \mathrm{D}=1.0$ [119]. A different value of $n$ may be used for different $\mathrm{L} / \mathrm{D}$ ratio [119] if desired. The dimensionless pressure profile of each pad is then calculated by solving Eq. (3.4.13) based on the finite element method with 30 circumferential elements per pad.

The pressure profile and the nonlinear force of each pad are affected by the motion of the shaft center node inside the bearing, the bearing pads and the bearing pivots. Effects of the shaft motion inside the short journal bearing has been shown in Chapter 4; in this Section, more detailed effects from the bearing pad and pivot are given. Different cases are designed, as shown in Table 5.2, to evaluate the effects from the behavior of bearing pads and pivots.

The pressure profiles of various cases with the rotational speed $\Omega$ of $8,000 \mathrm{rpm}$ are presented in Figs. 5.3 5.10. The values of the displacements and velocities are given at the top left corner of the figures. The bearing forces are calculated by integrating pressure profile using Eq. (3.4.18 21).

The results of the bearing forces under different cases are listed in Table 5.3, which shows that the behavior of bearing pads and pivots affects the pressure profiles, and then changes the bearing forces. According to the results of different cases, the behavior of the bearing pads, the bearing pivots and the shaft is coupled. The bearing pads/pivots behavior is taken into consideration by adding nodes of the bearing pads and pivots during the nonlinear transient analysis. 
Table 5.2 Different Dimensionless Cases for Tilting pad Bearing

4-pad, LBP, $\Omega=8,000 \mathrm{rpm}, 30$ elements per pad

Case I: Shaft Displacement

$\bar{X}=0.2, \bar{Y}=-0.1, \dot{\bar{X}}=\dot{\bar{Y}}=0, \bar{\gamma}=\bar{\delta}=\dot{\bar{\gamma}}=\dot{\bar{\delta}}=0$

Case II: Shaft Velocity

$\bar{X}=0.2, \bar{Y}=-0.1, \dot{\bar{X}}=-0.05, \dot{\bar{Y}}=0.05, \bar{\gamma}=\bar{\delta}=\dot{\bar{\gamma}}=\dot{\bar{\delta}}=0$

\begin{tabular}{l|l}
\hline Case III: Pivot Displacement & $\bar{\gamma}=0$ \\
$\bar{X}=0.2, \bar{Y}=-0.1, \dot{\bar{X}}=\dot{\bar{Y}}=0, \bar{\delta}=\dot{\bar{\delta}}=\dot{\bar{\gamma}}=0$ & $\bar{\gamma}=-0.05$ \\
& $\bar{\gamma}=+0.05$ \\
\hline Case VI: Pivot Velocity & $\dot{\bar{\gamma}}=0$ \\
$\bar{X}=0.2, \bar{Y}=-0.1, \dot{\bar{X}}=\dot{\bar{Y}}=0, \bar{\delta}=\dot{\bar{\delta}}=\bar{\gamma}=0$ & $\dot{\bar{\gamma}}=-0.05$ \\
$\dot{\bar{\gamma}}=+0.05$ \\
\hline Case V: Pad Angle Displacement & $\bar{\delta}=0$ \\
$\bar{X}=0.2, \bar{Y}=-0.1, \dot{\bar{X}}=\dot{\bar{Y}}=0, \dot{\bar{\delta}}=\bar{\gamma}=\dot{\bar{\gamma}}=0$ & $\bar{\delta}=-0.1$ \\
\hline Case VI: Pad Angle Velocity & $\bar{\delta}=+0.1$ \\
$\bar{X}=0.2, \bar{Y}=-0.1, \dot{\bar{X}}=\dot{\bar{Y}}=0, \bar{\delta}=\bar{\gamma}=\dot{\bar{\gamma}}=0$ & $\dot{\bar{\delta}}=0$ \\
\hline$\dot{\bar{\delta}}=-0.1$ \\
$\dot{\bar{\delta}}=+0.1$ \\
\hline
\end{tabular}




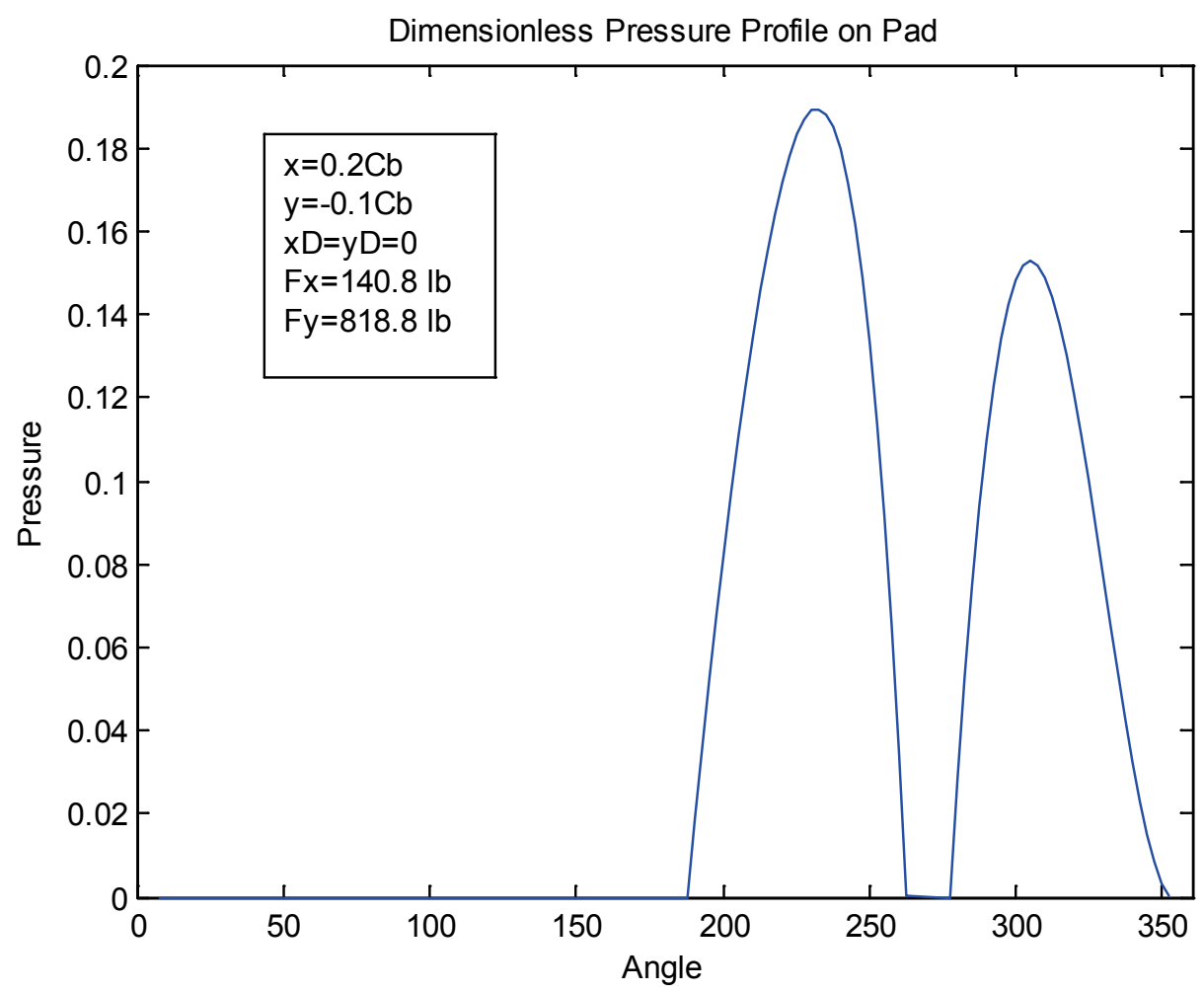

Figure 5.3 Dimensionless Pressure Profile on Pad (Case I)

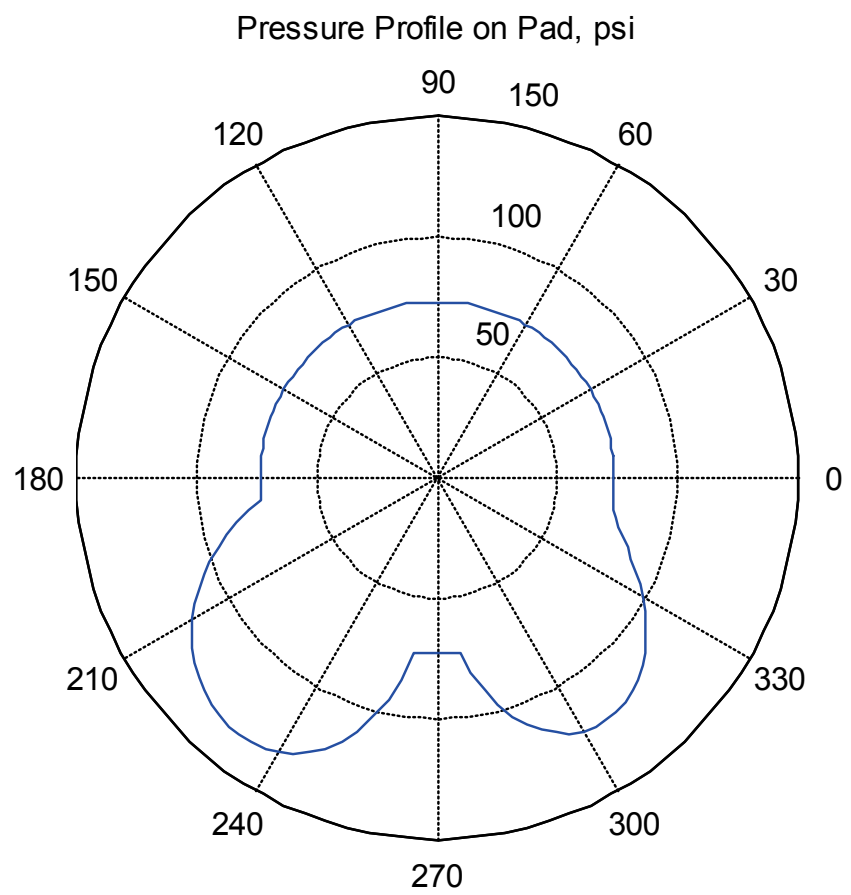

Figure 5.4 Dimensional Pressure Profile on Pad (Case I) 


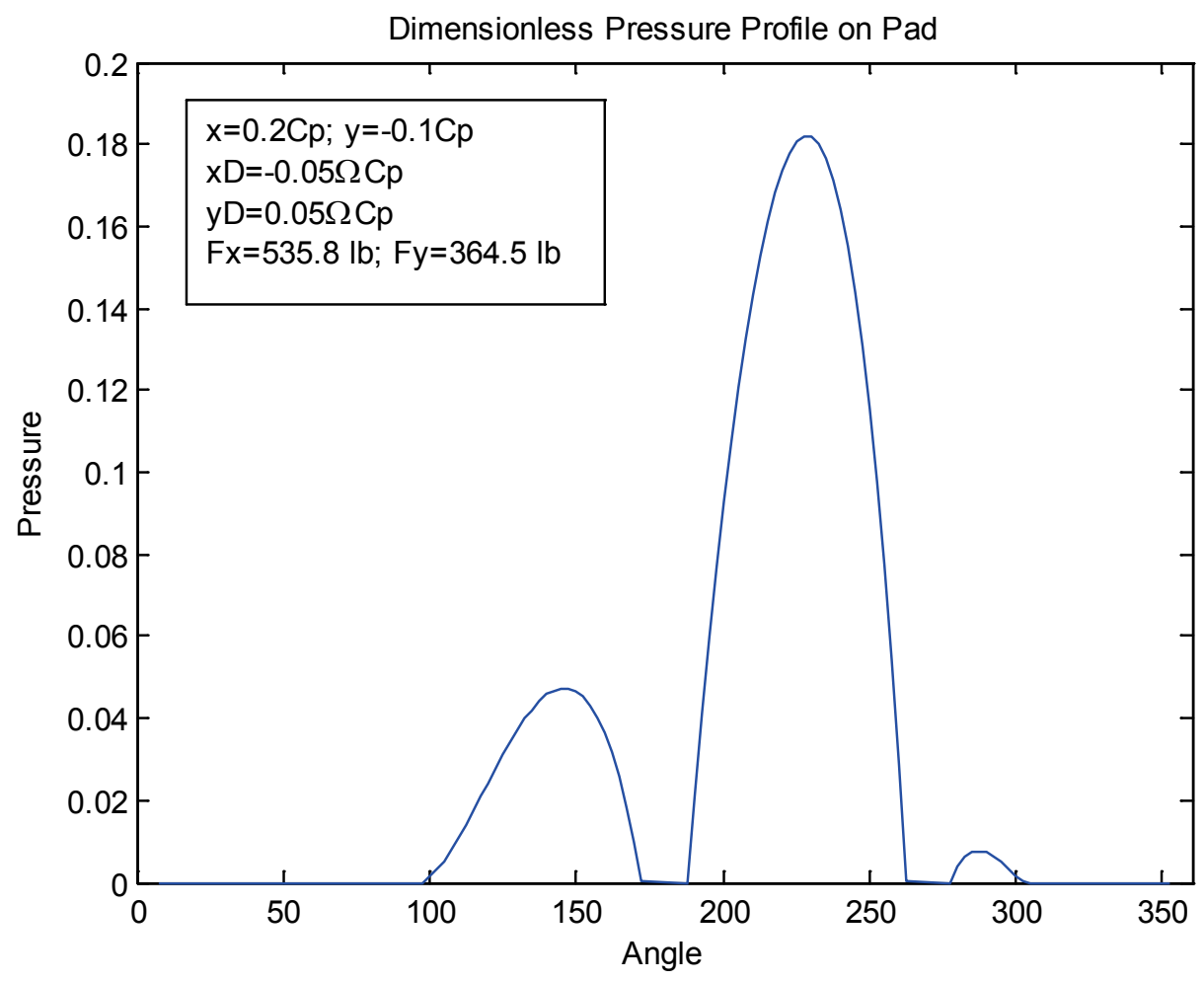

Figure 5.5 Dimensionless Pressure Profile on Pad (Case II)

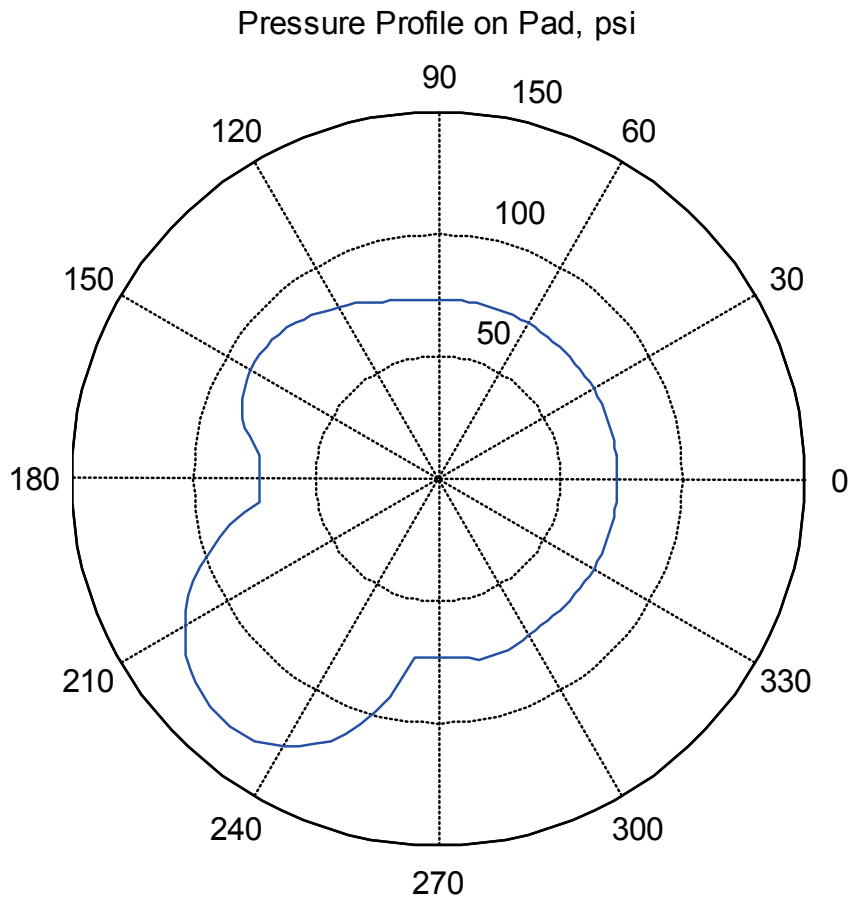

Figure 5.6 Dimensional Pressure Profile on Pad (Case II) 


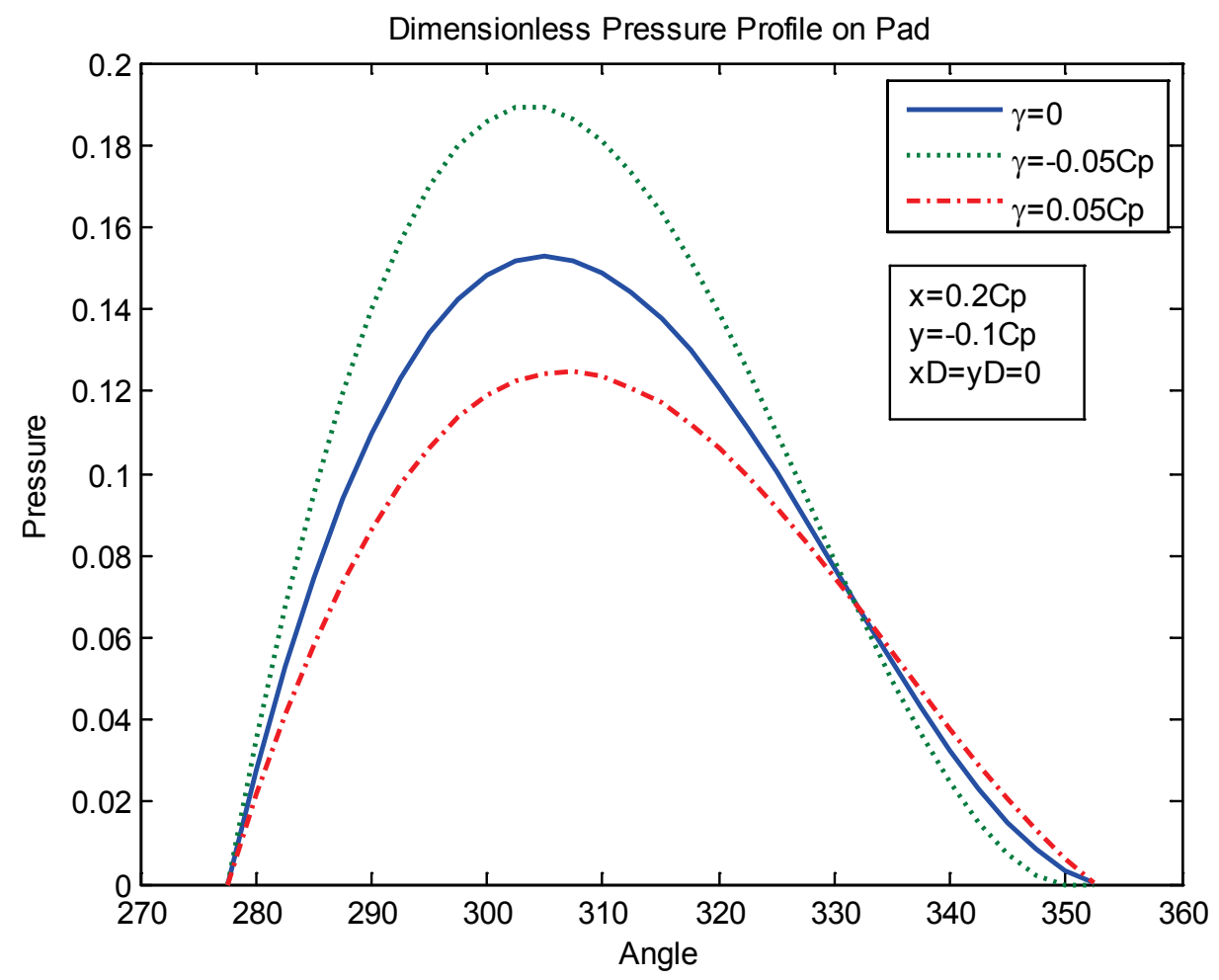

Figure 5.7 Dimensionless Pressure Profile on Pad\#4 (Case III)

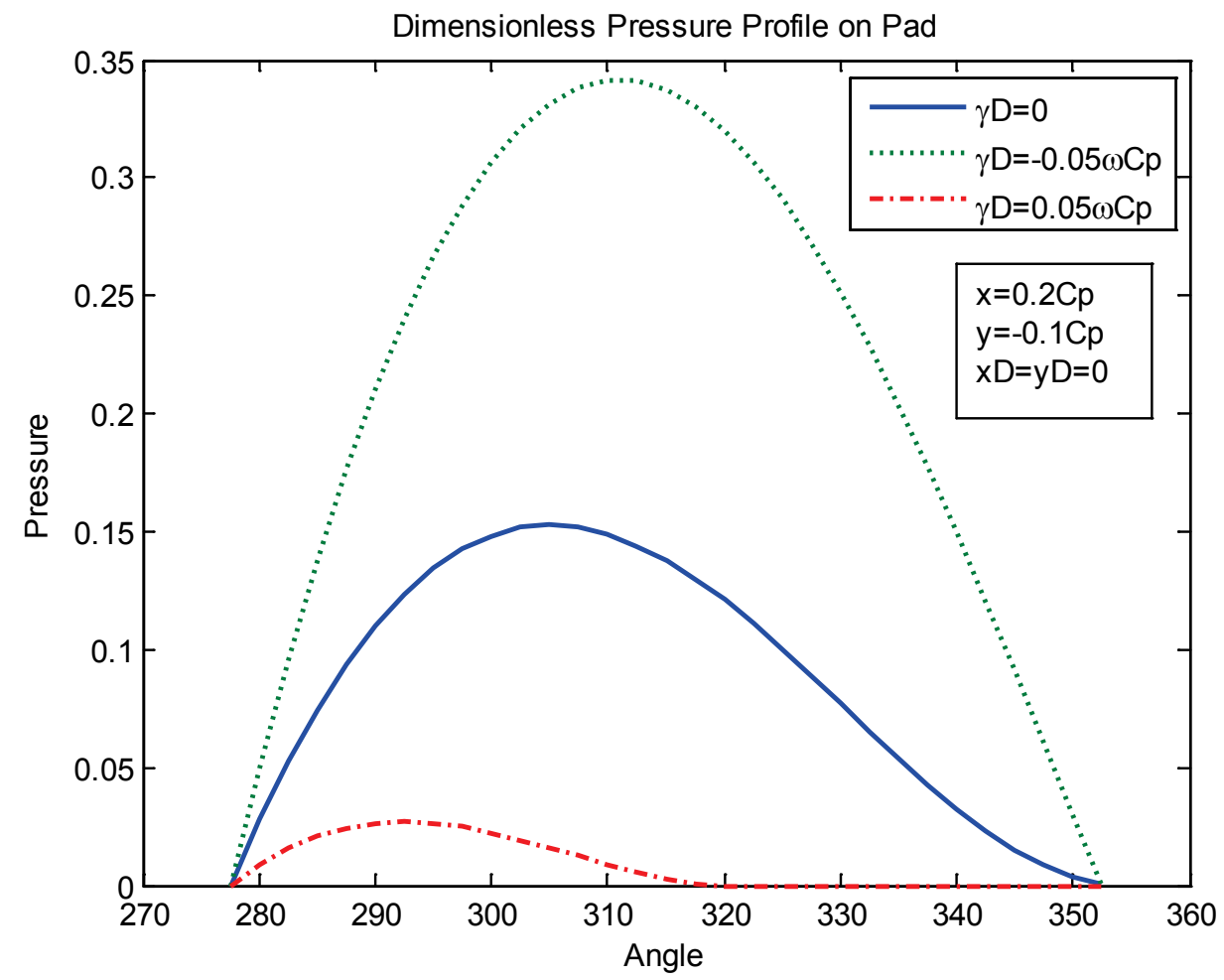

Figure 5.8 Dimensionless Pressure Profile on Pad\#4 (Case IV) 


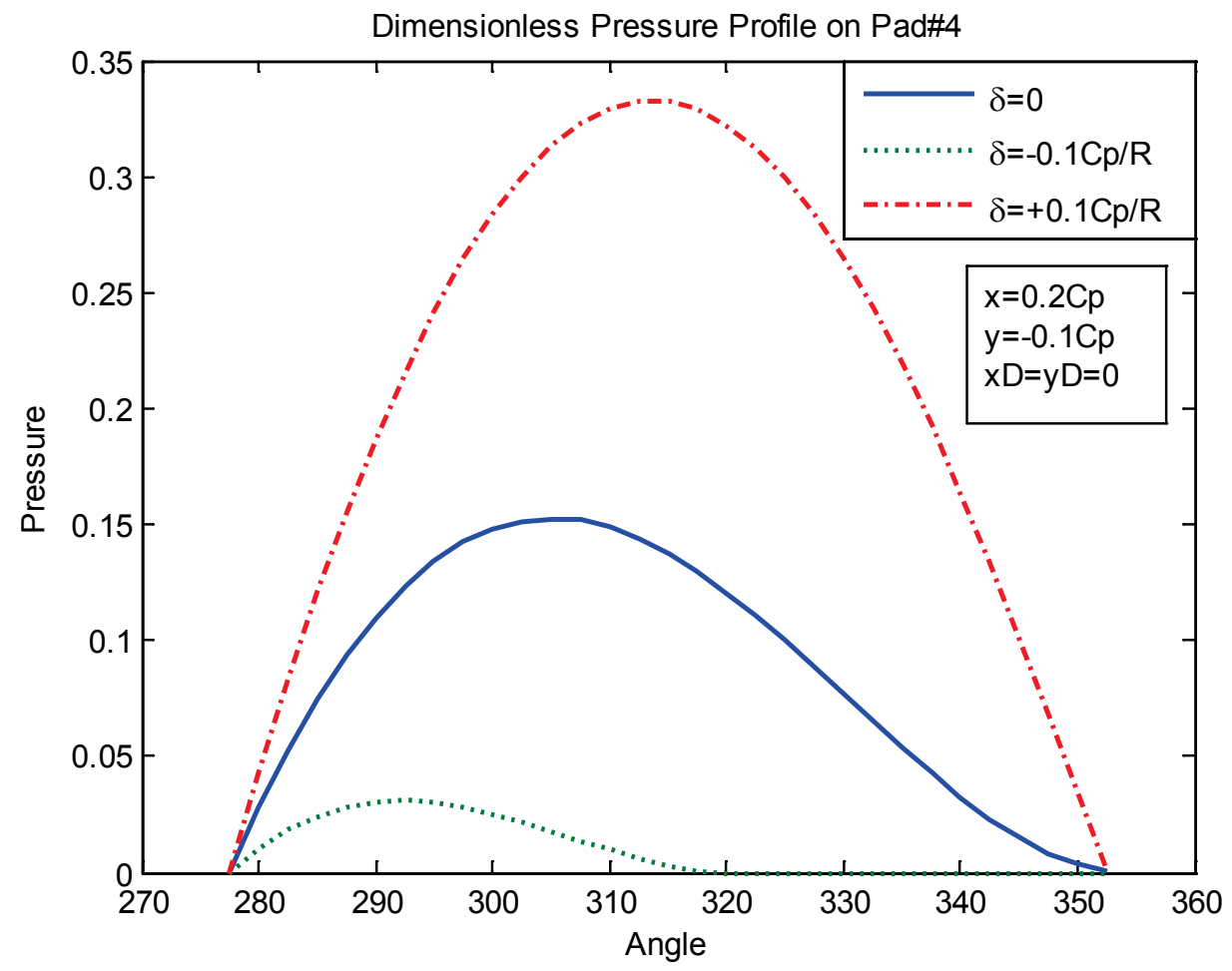

Figure 5.9 Dimensionless Pressure Profile on Pad\#4 (Case V)

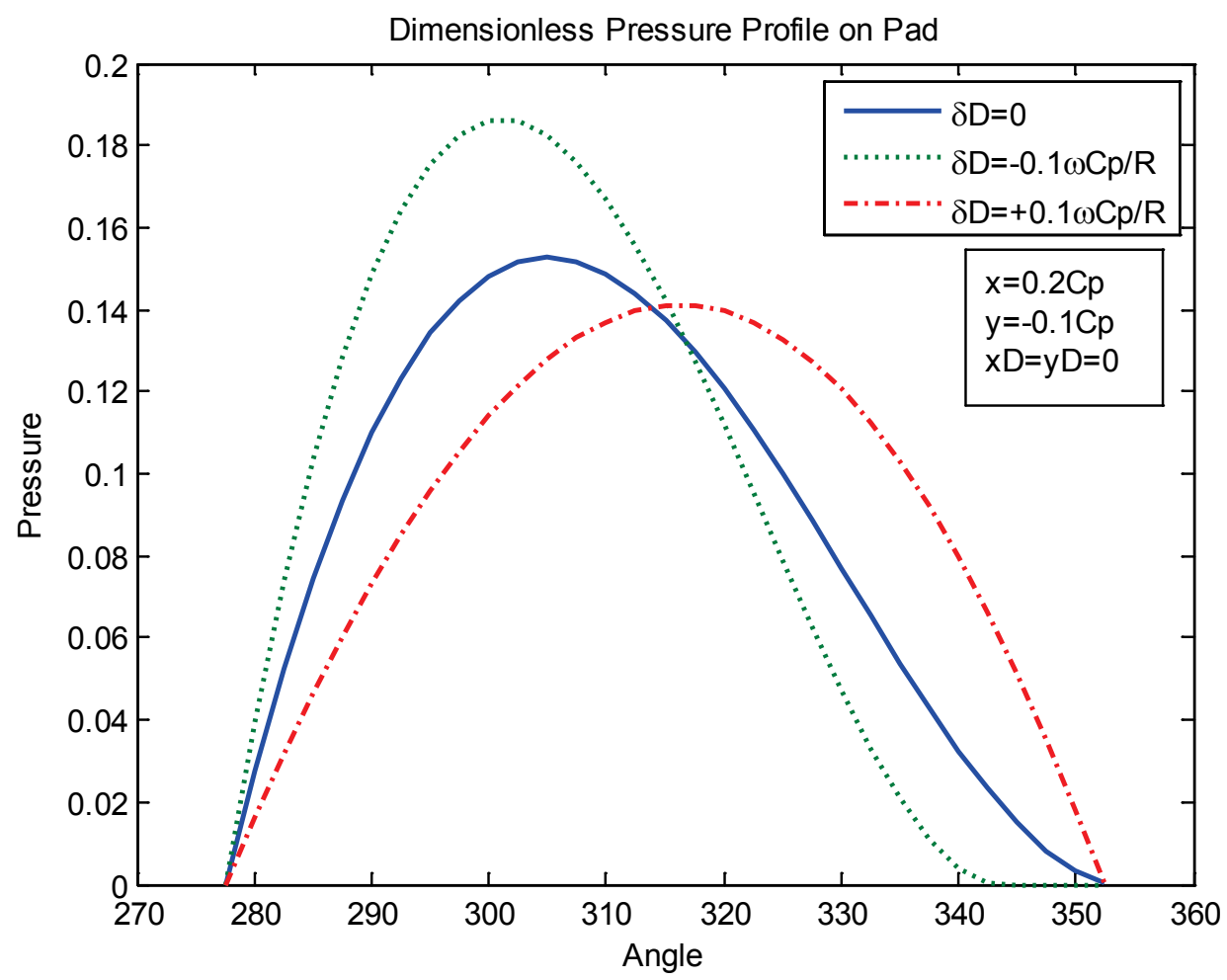

Figure 5.10 Dimensionless Pressure Profile on Pad\#4 (Case VI) 
Table 5.3 Tilting pad Bearing Forces under Different Dimensionless Cases

\begin{tabular}{|c|c|c|c|}
\hline \multicolumn{2}{|c|}{ 4-pad, LBP, $\Omega=8,000 \mathrm{rpm}, 30$ elements per pad } & Fx, lbf & Fy, lbf \\
\hline \multicolumn{2}{|l|}{$\begin{array}{ll}\text { Case I: } & \bar{X}=0.2, \bar{Y}=-0.1 \\
& \dot{\bar{X}}=\dot{\bar{Y}}=\bar{\gamma}=\bar{\delta}=\dot{\bar{\gamma}}=\dot{\bar{\delta}}=0\end{array}$} & 140.8 & 818.8 \\
\hline \multicolumn{2}{|c|}{ Case II: $\begin{aligned} & \bar{X}=0.2, \bar{Y}=-0.1, \dot{\bar{X}}=-0.05, \dot{\bar{Y}}=0.05 \\
& \bar{\gamma}=\bar{\delta}=\dot{\bar{\gamma}}=\dot{\bar{\delta}}=0\end{aligned}$} & 535.8 & 364.5 \\
\hline Case III: $\begin{aligned} \bar{X} & =0.2, \bar{Y}=-0.1 \\
& \dot{\bar{X}}=\dot{\bar{Y}}=0, \bar{\delta}=\dot{\bar{\delta}}=\dot{\bar{\gamma}}=0\end{aligned}$ & $\begin{array}{l}\bar{\gamma}=0 \\
\bar{\gamma}=-0.05 \\
\bar{\gamma}=+0.05\end{array}$ & $\begin{array}{l}140.8 \\
101.3 \\
172.9\end{array}$ & $\begin{array}{l}818.8 \\
890.5 \\
762.1\end{array}$ \\
\hline $\begin{aligned} & \text { Case VI: } \bar{X}=0.2, \bar{Y}=-0.1 \\
& \dot{X}=\dot{Y}=0, \bar{\delta}=\dot{\bar{\delta}}=\bar{\gamma}=0\end{aligned}$ & $\begin{array}{l}\dot{\bar{\gamma}}=0 \\
\dot{\bar{\gamma}}=-0.05 \\
\dot{\bar{\gamma}}=+0.05\end{array}$ & $\begin{array}{l}140.8 \\
-336.9 \\
406.7\end{array}$ & $\begin{array}{l}818.8 \\
1282.4 \\
509.0\end{array}$ \\
\hline $\begin{aligned} \text { Case V: } & \bar{X}=0.2, \bar{Y}=-0.1 \\
\dot{\bar{X}} & =\dot{\bar{Y}}=0, \dot{\bar{\delta}}=\bar{\gamma}=\dot{\bar{\gamma}}=0\end{aligned}$ & $\begin{array}{l}\bar{\delta}=0 \\
\bar{\delta}=-0.1 \\
\bar{\delta}=+0.1\end{array}$ & $\begin{array}{l}140.8 \\
403.9 \\
-338.8\end{array}$ & $\begin{array}{l}818.8 \\
515.4 \\
1252.4\end{array}$ \\
\hline $\begin{aligned} \text { Case VI: } & \bar{X}=0.2, \bar{Y}=-0.1 \\
& \dot{\bar{X}}=\dot{\bar{Y}}=0, \bar{\delta}=\bar{\gamma}=\dot{\bar{\gamma}}=0\end{aligned}$ & $\begin{array}{l}\dot{\bar{\delta}}=0 \\
\dot{\bar{\delta}}=-0.1 \\
\dot{\bar{\delta}}=+0.1\end{array}$ & $\begin{array}{l}140.8 \\
152.7 \\
91.1\end{array}$ & $\begin{array}{l}818.8 \\
826.8 \\
767.3\end{array}$ \\
\hline
\end{tabular}

\subsubsection{Nonlinear Transient Response of Rigid Rotor Supported with Nonlinear Tilting Pad Bearings}

The transient analysis was conducted to study the response of the rigid rotor supported on two 4-pad tiling pad bearings, as shown in Fig. 5.1. The axial symmetric rigid rotor has a total mass of $3000 \mathrm{lbf}$ (load of half rotor weight, 1500lbf, to each bearing). The unbalance is placed at the center of the shaft. The equations of motion of a rigid rotor supported with 4-pad tilting pad bearings are shown in Eq. (5.2.1). The bearing forces, as indicated in last section, are the function of the displacements and velocities of the shaft center, the bearing pads, and the bearing pivots. 


$$
\begin{aligned}
& m_{r i g} \ddot{x}=F_{b, x}(x, y, \delta, \gamma, \dot{x}, \dot{y}, \dot{\delta}, \dot{\gamma})+m e_{u} \Omega^{2} \cos (\Omega t) \\
& m_{r i g} \ddot{y}=F_{b, y}(x, y, \delta, \gamma, \dot{x}, \dot{y}, \dot{\delta}, \dot{\gamma})+m e_{u} \Omega^{2} \sin (\Omega t)-W \\
& I_{\text {pad }_{i}} \ddot{\delta}_{i}=M_{p_{i}}(x, y, \delta, \gamma, \dot{x}, \dot{y}, \dot{\delta}, \dot{\gamma}), i=1,2,3,4 \\
& m_{\text {piv }_{i}} \ddot{\gamma}_{i}+k_{p i v_{i}} \gamma_{i}=F_{p_{i}}(x, y, \delta, \gamma, \dot{x}, \dot{y}, \dot{\delta}, \dot{\gamma}), i=1,2,3,4
\end{aligned}
$$

where $m_{r i g}$ is the half mass of the rigid rotor, $F_{b, x}$ and $F_{b, y}$ are the bearing forces in horizontal and vertical directions, $W$ is the half weight of the rigid rotor, $I_{p a d}$ is the moment of inertia of each pad, $m_{p i v}$ is the mass of each pad, $k_{p i v}$ is the pivot stiffness, $M_{p}$ and $F_{p}$ are the pad moment and pad force, respectively.

For the evaluation of the unbalance forced response, the maximum acceptable unbalance level is based on the following formula, as required by API 671:

$$
m e_{u}=\frac{8 W}{N}
$$

where $W$ is the weight in $l b$ and $N$ is the rotational speed in rpm. For this rigid rotor with two 4-pad tilting pad bearing example, the first unbalance is $1.5 \mathrm{oz}$-in from Eq. (5.5.2).

Using the $4^{\text {th }}$ order Runge-Kutta method with initial displacements and velocities of all zeros, the equations of motion (5.2.1) can be solved step by step. During the calculation, the time step has to be chosen carefully. The use of large time step will cause the loss of detail in the integral being evaluated and will cause convergence problems in the nonlinear rotor solution. On the other hand, too small a time step will produce a solution which is computationally inefficient. The displacement comparation of various time steps at a constant rotational speed of $8,000 \mathrm{rpm}$ with unbalance of $1.5 \mathrm{oz}$-in is shown in Fig. 5.11. In the figure, the time steps are $5 \mu \mathrm{s}, 10 \mu \mathrm{s}$ and $20 \mu \mathrm{s}$. 

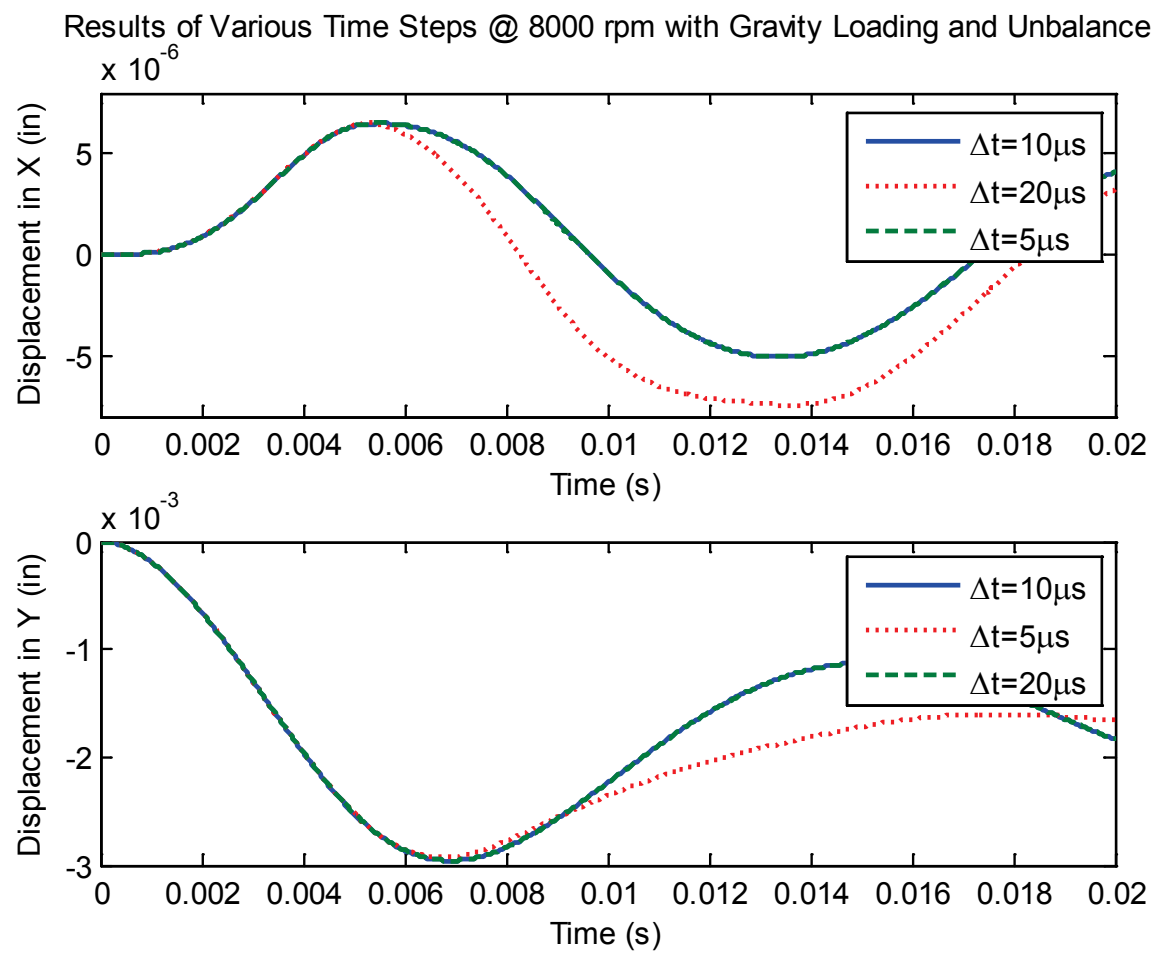

Figure 5.11 Displacements with Various Time Step at 8,000 rpm under Unbalance

\subsubsection{Validate of Bearing Force Calculation of Nonlinear Tilting Pad Bearings}

In the following transient analysis of this Section, the time step, $\Delta t$, is taken as $1 \times 10^{-5}$ second. If the system is stable and there is no unbalance force, the rotor will reach the steady state or equilibrium position gradually. Fig. 5.12 shows the transient procedure of reaching the equilibrium positions of the shaft and the bearing pads/pivots at a constant running speed of $8,000 \mathrm{rpm}$. After 0.08 seconds, the shaft displacement is constant in both horizontal direction and vertical direction, as shown in upper two figures of Fig. 5.12. The orbit of the shaft center converges to a spot gradually, as shown in lower figure of Fig. 5.12. The pressure profile at steady state is presented in Fig. 5.13. The shaft center and the bearing pads/pivots locations after the system reaches the steady state is shown in Fig. 5.14. The shaft center is indicated with a big blue point; the red circle indicates the 
bearing clearance; the fine blue lines indicate positions/angles of all pads and pivots. From the pad forces in Fig. 5.15, and the displacement of pads (pad angle) in Fig. 5.16, the two upper pads are unloaded (zero force) pads after the system reaches the steady state due to the rotor weight. However, there are small forces/moments applied to the pad \#2 during transient procedure, which push it moves to a constant angle. That motion is indicated by the green lines in Figs. 5.15\&5.16. The equilibrium position of the rotor center is located at the central area of the bearing clearance, and is lower than the geometric center due to the rotor weight. Due to the symmetric geometry of the bearing (LBP) and self adaptive pads, the equilibrium position in the horizontal direction is almost zero, but in the vertical direction the position is -2 mils.
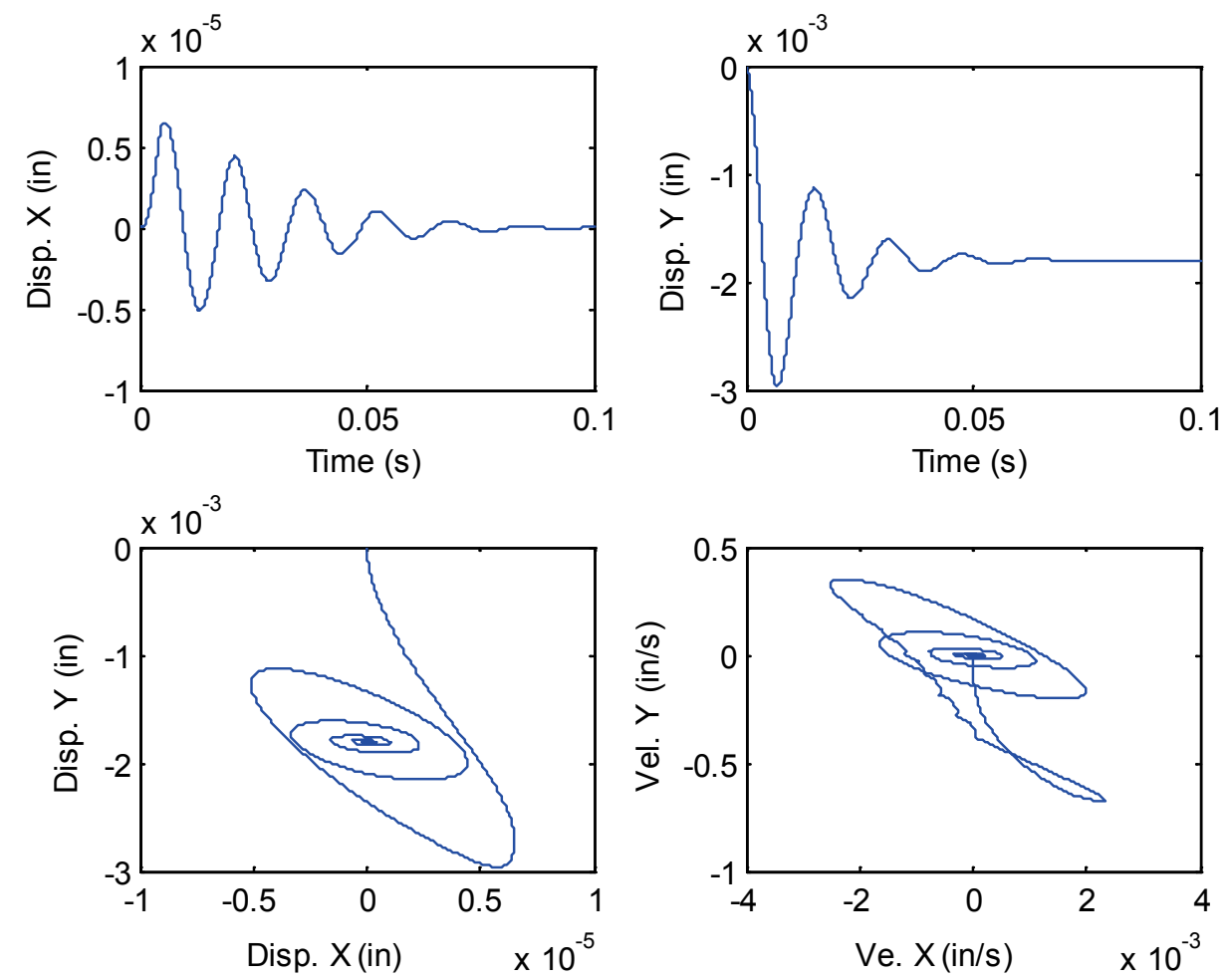

Figure 5.12 Time Transient of Shaft Center at 8,000 rpm $\left(\mathrm{me}_{\mathrm{u}}=0\right)$ with Time Step $10 \mu \mathrm{s}$ 
Pressure Profile on Pad, psi at $0.1 \mathrm{~s}$

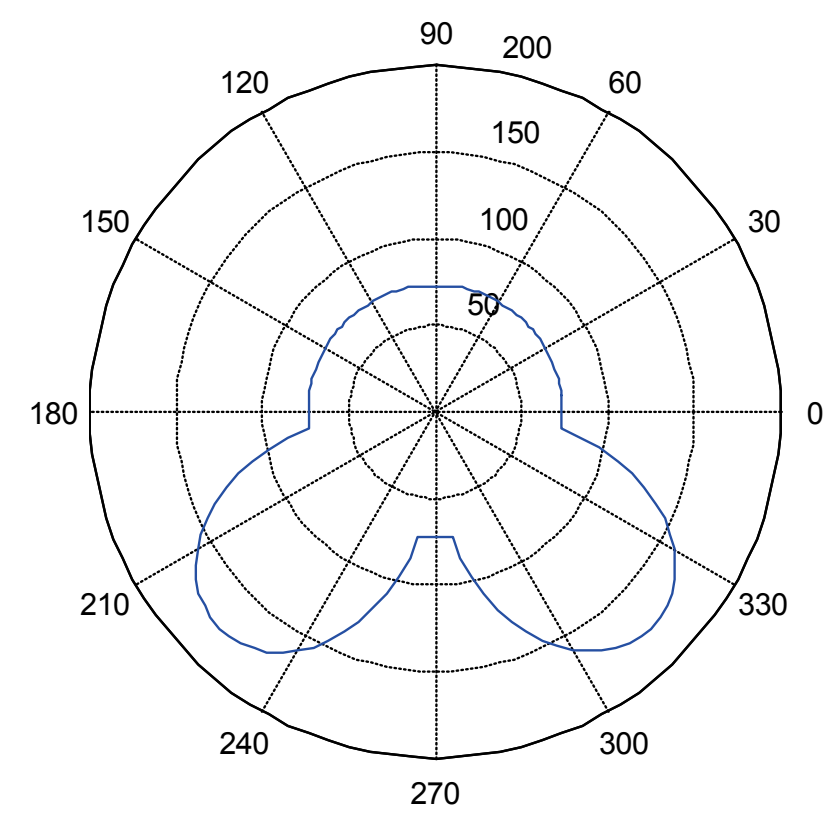

Figure 5.13 Final Steady State Pressure Profile on Pads at $8,000 \mathrm{rpm}\left(\mathrm{me}_{\mathrm{u}}=0, \mathrm{t}=0.1 \mathrm{sec}\right.$.)

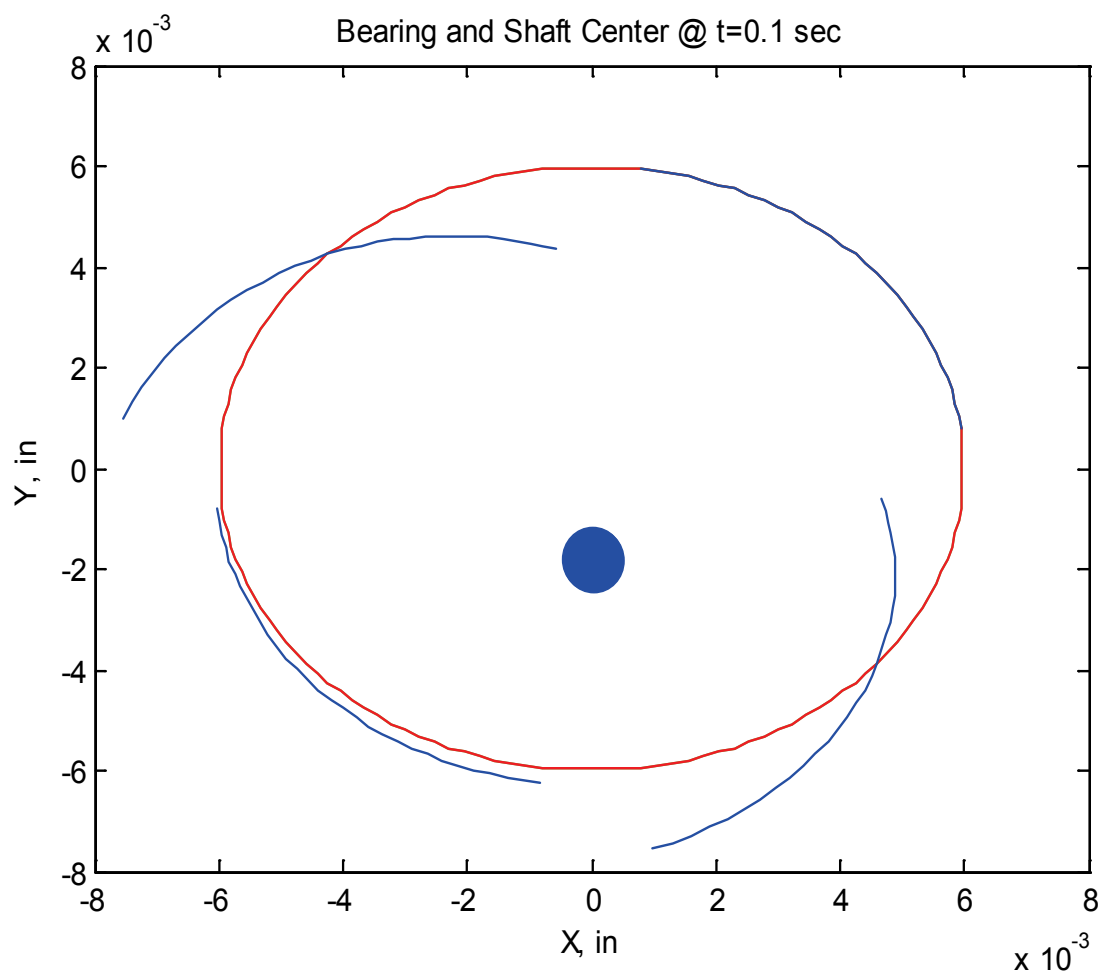

Figure 5.14 Final Steady State Shaft Center and Pads/Pivots Positions at 8,000 rpm

$$
\left(\mathrm{me}_{\mathrm{u}}=0, \mathrm{t}=0.1 \mathrm{sec} .\right)
$$




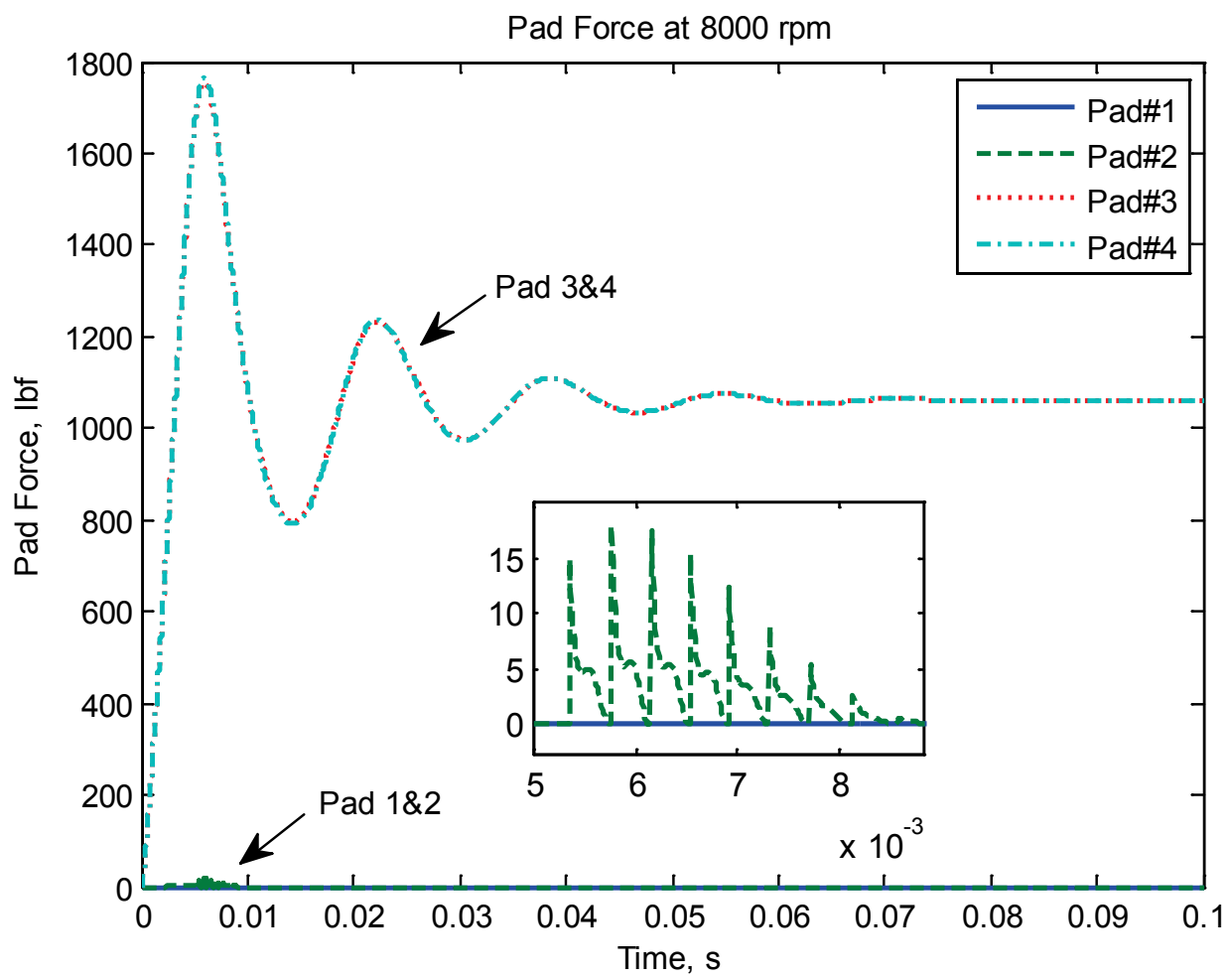

Figure 5.15 Forces on Pads at 8,000 rpm $\left(\mathrm{me}_{\mathrm{u}}=0\right)$

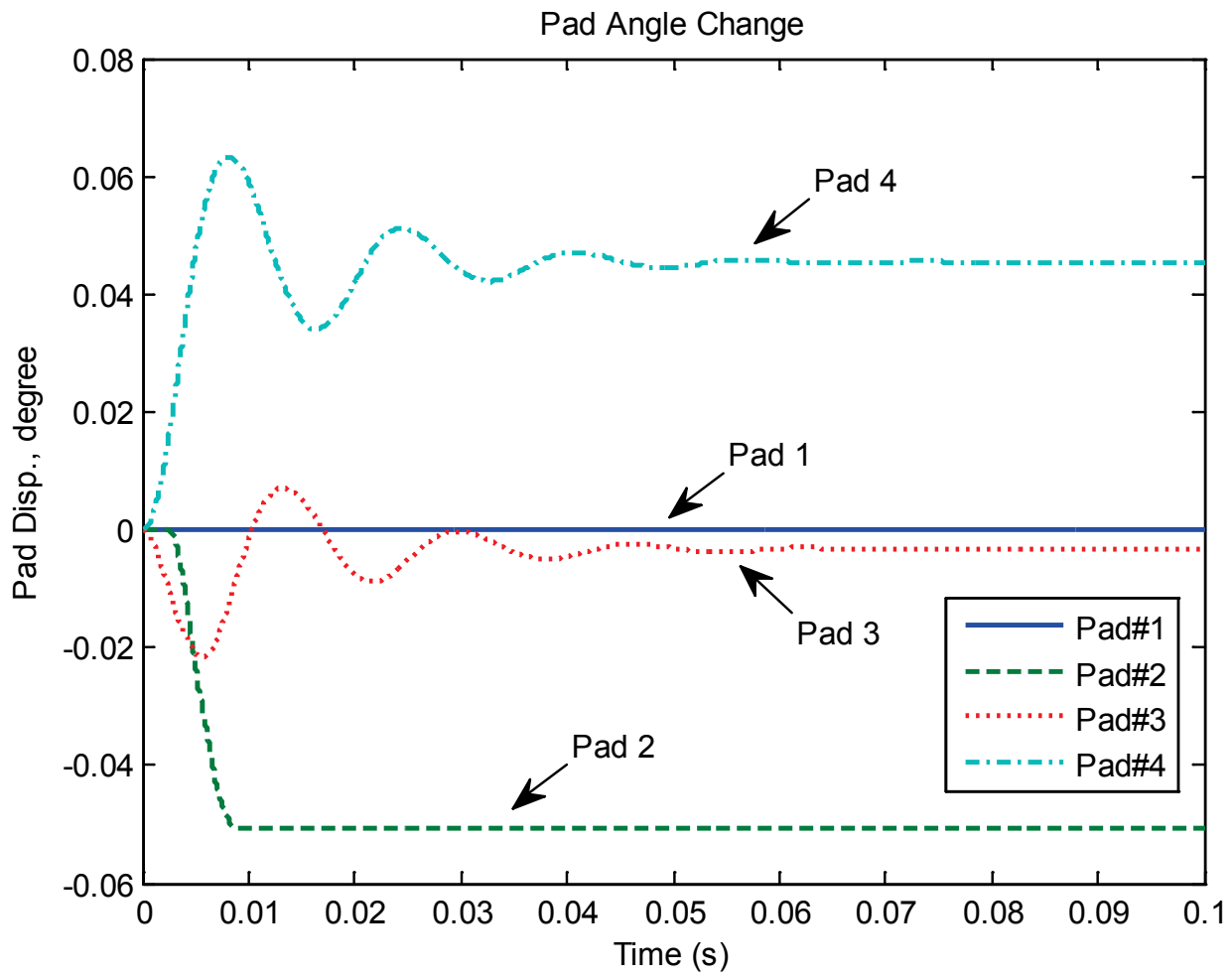

Figure 5.16 Behavior of Pad Angle at 8,000 rpm $\left(\mathrm{me}_{\mathrm{u}}=0\right)$ 
Once the system steady state is determined, the full dynamic stiffness and damping coefficients of the tilting pad bearing can be calculated using small perturbation method as shown in Eq. (5.2.3 5.2.5) if desired.

$$
K=\frac{\Delta F}{\Delta u} ; \quad C=\frac{\Delta F}{\Delta \dot{u}}
$$

$\left[\begin{array}{c}\Delta F_{b, x} \\ \Delta F_{b, y} \\ \Delta M_{p_{1}} \\ \vdots \\ \Delta M_{p_{4}} \\ \Delta F_{p_{1}} \\ \vdots \\ \Delta F_{p_{4}}\end{array}\right]=\left[\begin{array}{cccccccc}K_{x x} & K_{x y} & K_{x \delta_{1}} & \ldots & K_{x \delta_{4}} & K_{x \gamma_{1}} & \cdots & K_{x \gamma_{4}} \\ K_{y x} & K_{y y} & K_{y \delta_{1}} & \ldots & K_{y \delta_{4}} & K_{y \gamma_{1}} & \ldots & K_{y \gamma_{4}} \\ K_{\delta_{1} x} & K_{\delta_{1} y} & K_{\delta_{1} \delta_{1}} & \ldots & K_{\delta_{1} \delta_{4}} & K_{\delta_{1} \gamma_{1}} & \ldots & K_{\delta_{1} \gamma_{4}} \\ \vdots & \vdots & \vdots & \ddots & \vdots & \vdots & \vdots & \vdots \\ K_{\delta_{4} x} & K_{\delta_{4} y} & K_{\delta_{4} \delta_{1}} & \ldots & K_{\delta_{4} \delta_{4}} & K_{\delta_{4} \gamma_{1}} & \ldots & K_{\delta_{4} \gamma_{4}} \\ K_{\gamma_{1} x} & K_{\gamma_{1} y} & K_{\gamma_{1} \delta_{1}} & \ldots & K_{\gamma_{1} \delta_{4}} & K_{\gamma_{1} \gamma_{1}} & \ldots & K_{\gamma_{1} \gamma_{4}} \\ \vdots & \vdots & \vdots & \cdots & \vdots & \vdots & \ddots & \vdots \\ K_{\gamma_{4} x} & K_{\gamma_{4} y} & K_{\gamma_{4} \delta_{1}} & \cdots & K_{\gamma_{4} \delta_{4}} & K_{\delta_{4} x} & \cdots & K_{\gamma_{4} \gamma_{4}}\end{array}\right]\left[\begin{array}{c}\Delta x \\ \Delta y \\ \Delta \delta_{1} \\ \Delta \delta_{4} \\ \Delta \gamma_{1} \\ \vdots \\ \Delta \gamma_{4}\end{array}\right]$

$$
\left[\begin{array}{c}
\Delta F_{b, x} \\
\Delta F_{b, y} \\
\Delta M_{p_{1}} \\
\vdots \\
\Delta M_{p_{4}} \\
\Delta F_{p_{1}} \\
\vdots \\
\Delta F_{p_{4}}
\end{array}\right]=\left[\begin{array}{cccccccc}
C_{x x} & C_{x y} & C_{x \delta_{1}} & \ldots & C_{x \delta_{4}} & C_{x \gamma_{1}} & \ldots & C_{x \gamma_{4}} \\
C_{y x} & C_{y y} & C_{y \delta_{1}} & \ldots & C_{y \delta_{4}} & C_{y \gamma_{1}} & \ldots & C_{y \gamma_{4}} \\
C_{\delta_{1} x} & C_{\delta_{1} y} & C_{\delta_{1} \delta_{1}} & \ldots & C_{\delta_{1} \delta_{4}} & C_{\delta_{1} \gamma_{1}} & \ldots & C_{\delta_{1} \gamma_{4}} \\
\vdots & \vdots & \vdots & \ddots & \vdots & \vdots & \vdots & \vdots \\
C_{\delta_{4} x} & C_{\delta_{4} y} & C_{\delta_{4} \delta_{1}} & \ldots & C_{\delta_{4} \delta_{4}} & C_{\delta_{4} \gamma_{1}} & \ldots & C_{\delta_{4} \gamma_{4}} \\
C_{\gamma_{1} x} & C_{\gamma_{1} y} & C_{\gamma_{1} \delta_{1}} & \ldots & C_{\gamma_{1} \delta_{4}} & C_{\gamma_{1} \gamma_{1}} & \ldots & C_{\gamma_{1} \gamma_{4}} \\
\vdots & \vdots & \vdots & \ldots & \vdots & \vdots & \ddots & \vdots \\
C_{\gamma_{4} x} & C_{\gamma_{4} y} & C_{\gamma_{4} \delta_{1}} & \cdots & C_{\gamma_{4} \delta_{4}} & C_{\delta_{4} x} & \cdots & C_{\gamma_{4} \gamma_{4}}
\end{array}\right]\left[\begin{array}{c}
\Delta \dot{x} \\
\Delta \dot{y} \\
\Delta \dot{\delta}_{1} \\
\vdots \\
\dot{\delta}_{4} \\
\Delta \dot{\gamma}_{1} \\
\vdots \\
\dot{\gamma}_{4}
\end{array}\right]
$$

The calculated dynamic stiffness and damping coefficients $\left(K_{x x}\right.$ and $\left.C_{x x}\right)$ at different rotational speeds are listed in Table 5.4 and compared in Figs. 5.17\&5.18. The calculated stiffness and damping coefficients are compared with THPAD in the table. The maximum relative difference between THPAD and RotorTran is $6.67 \%$ for the stiffness coefficients $\left(K_{x x}\right)$ and $12.08 \%$ for the damping coefficients $\left(C_{x x}\right)$. In those calculations, 
RotorTran has the same curve with THPAD but has higher calculated coefficients than THPAD. One possible reason is that THPAD takes into consideration of the pad mechanical deformation and the thermal equation, which results variable lubricant density and viscosity. Another possible reason is that different perturbation methods applied to calculate full dynamic coefficients. To save calculating time, in this dissertation, constant lubricant density and viscosity are used to solve the Reynolds equation. Also the difference between RotorTran and THPAD increases with rotational speed increasing, especially for the calculated damping coefficients. Using of different perturbation methods may result in those differences.

Table 5.4 Dynamic Stiffness and Damping

\begin{tabular}{l|l|l|l|l|l|l}
\hline $\begin{array}{l}\text { Speed, } \\
\text { rpm }\end{array}$ & $\begin{array}{l}\text { THPAD } \\
\text { Kxx, lbf/in }\end{array}$ & $\begin{array}{l}\text { RotorTran } \\
\text { Kxx, lbf/in }\end{array}$ & $\begin{array}{l}\text { Diff. } \\
\%\end{array}$ & $\begin{array}{l}\text { THPAD } \\
\text { Cxx, lbf/in-s }\end{array}$ & $\begin{array}{l}\text { RotorTran } \\
\text { Cxx, lbf/in-s }\end{array}$ & $\begin{array}{l}\text { Diff. } \\
\%\end{array}$ \\
\hline 1,000 & $1.0594 \mathrm{E} 6$ & $1.1167 \mathrm{E} 6$ & 5.13 & 15944.00 & 16305.25 & 2.22 \\
\hline 2,000 & 850891.02 & 889495.8 & 4.34 & 7576.41 & 7804.31 & 2.92 \\
\hline 3,000 & 755288.54 & 788561.9 & 4.22 & 5093.95 & 5289.96 & 3.71 \\
\hline 4,000 & 698457.86 & 729386.7 & 4.24 & 3926.67 & 4109.53 & 4.45 \\
\hline 5,000 & 660132.63 & 690148.6 & 4.35 & 3254.24 & 3432.32 & 5.19 \\
\hline 6,000 & 632314.46 & 662260.2 & 4.52 & 2819.58 & 2997.30 & 5.93 \\
\hline 7,000 & 611443.74 & 641546.9 & 4.69 & 2517.69 & 2696.83 & 6.64 \\
\hline 8,000 & 594867.08 & 625696.6 & 4.93 & 2295.82 & 2478.55 & 7.37 \\
\hline 9,000 & 581654.96 & 613308.7 & 5.16 & 2126.97 & 2314.01 & 8.08 \\
\hline 10,000 & 570857.81 & 603478.4 & 5.41 & 1994.39 & 2186.40 & 8.78 \\
\hline 11,000 & 561908.62 & 595591.1 & 5.66 & 1887.74 & 2085.19 & 9.47 \\
\hline 12,000 & 554387.27 & 589213.4 & 5.91 & 1800.24 & 2003.44 & 10.14 \\
\hline 13,000 & 548005.09 & 584029.5 & 6.17 & 1727.22 & 1936.39 & 10.80 \\
\hline 14,000 & 542533.15 & 579794.6 & 6.43 & 1665.41 & 1880.71 & 11.45 \\
\hline 15,000 & 537932.95 & 576362.4 & 6.67 & 1612.48 & 1833.93 & 12.08 \\
\hline
\end{tabular}




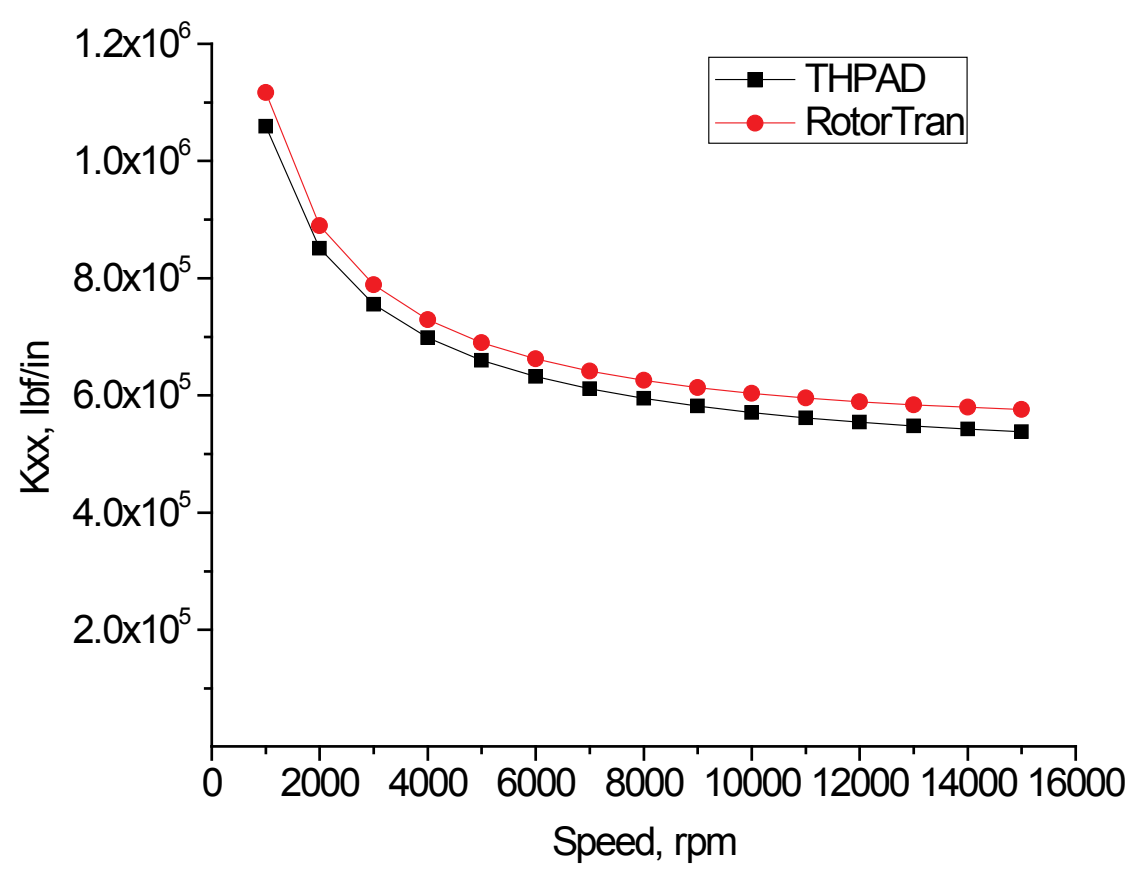

Figure 5.17 Dynamic Stiffness Coefficients

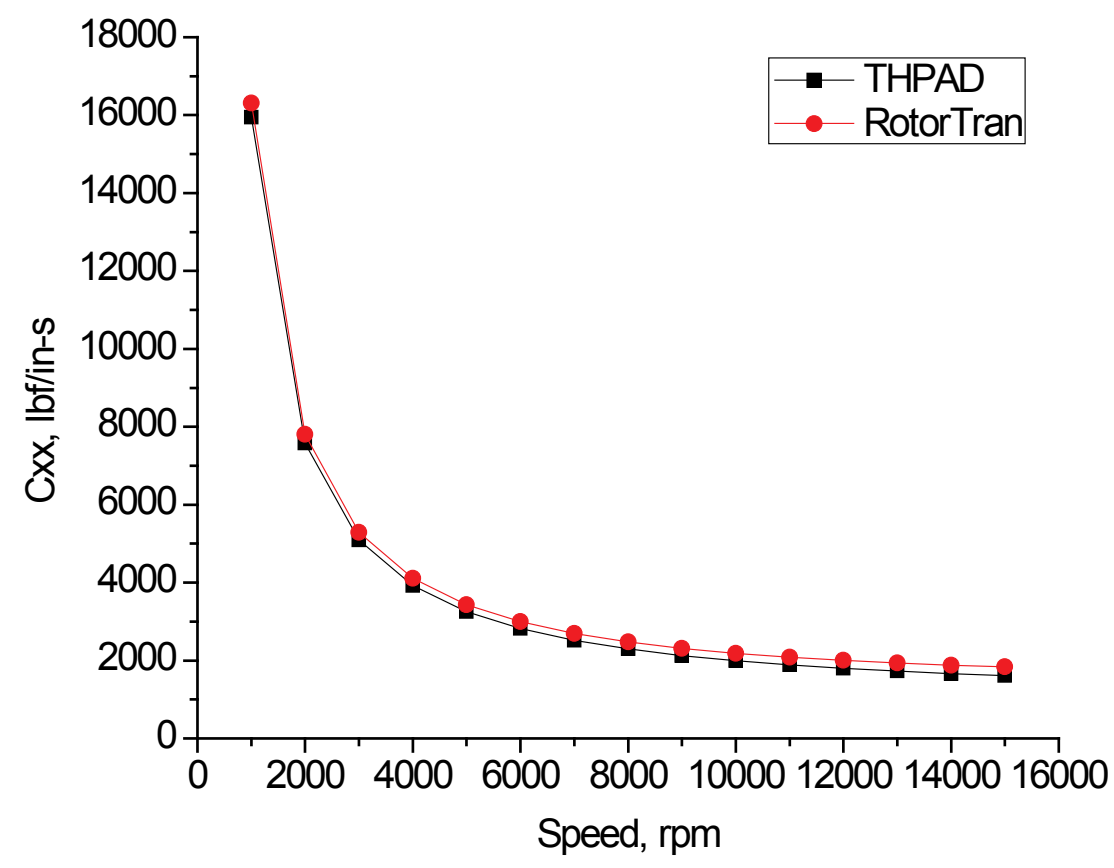

Figure 5.18 Dynamic Damping Coefficients 


\subsubsection{Nonlinear Transient Analysis of the Rigid Rotor on Two 4-Pad Tilting Pad Bearings under Severe Unbalance}

Another analysis is to simulate the transient response of the rotor under severe unbalance condition. At $\mathrm{t}=0.2$ second, the unbalance suddenly increases from $1.5 \mathrm{oz}$-in, which is the maximum acceptable unbalance level according to API (Eq. 5.2.2), to $20 \mathrm{oz}$ in to simulate the rotor with suddenly severe condition.

Under the severe unbalance case, the shaft center jumped from one stable orbit to another orbit, which is still a stable and synchronous response. The results of instantaneously changing unbalance at $\mathrm{t}=0.2$ second are shown in Figs. 5.19 5.25. The shaft center orbit in time domain is shown in Fig. 5.19. Before 0.2 second $(t<0.2$ sec.), under the first unbalance of $1.5 \mathrm{oz}$-in, the shaft center amplitude is 0.15 mil (peak to peak). At $\mathrm{t}=0.2$ second, a suddenly severe unbalance of $20 \mathrm{oz}$-in is applied to the system. After the transient response dies out at $\mathrm{t}=0.3$ second, the peak-peak amplitude of the shaft center vibration is 1.8 mils, which is about 12 times larger than the first unbalance condition. Two orbits, before and after $\mathrm{t}=0.2$ second, are indicated by two arrows in Fig. 5.19. Due to the rotor weight of $1500 \mathrm{lb}$, the upper two pads are still load-free under the severe conditions, which are indicated from the pad forces, Fig. 5.20, and the pad moments, Fig. 5.21. However, there are small forces/moments applied to the pad \#2 before $\mathrm{t}=0.05$ second, which pushes the pad \#2 to a constant angle. The pad \#4 moment, indicated by the cyan color in Fig. 5.21, has a sudden change during the transient response at the time when the severe unbalance is applied ( $t=0.2$ sec.). The suddenly large change of the pad moment may result in touching between the pad and the shaft, which is discussed in Section 5.3\&5.4. Under the severe unbalance condition, the 
response of shaft center is still synchronous and the shaft whirls at a large orbit from Fig. 5.19. The pad moments, as shown in Fig. 5.21, however, are nonlinear. To show the results more clearly, the pad moment outputs between 0.36 0.4 second is presented in bottom right area of Fig. 5.21. The bearing pad/pivot \#4 displacements and velocities are presented in Figs. 5.22\&5.25, which indicate the tilting motion of the pad \#4 in time domain.
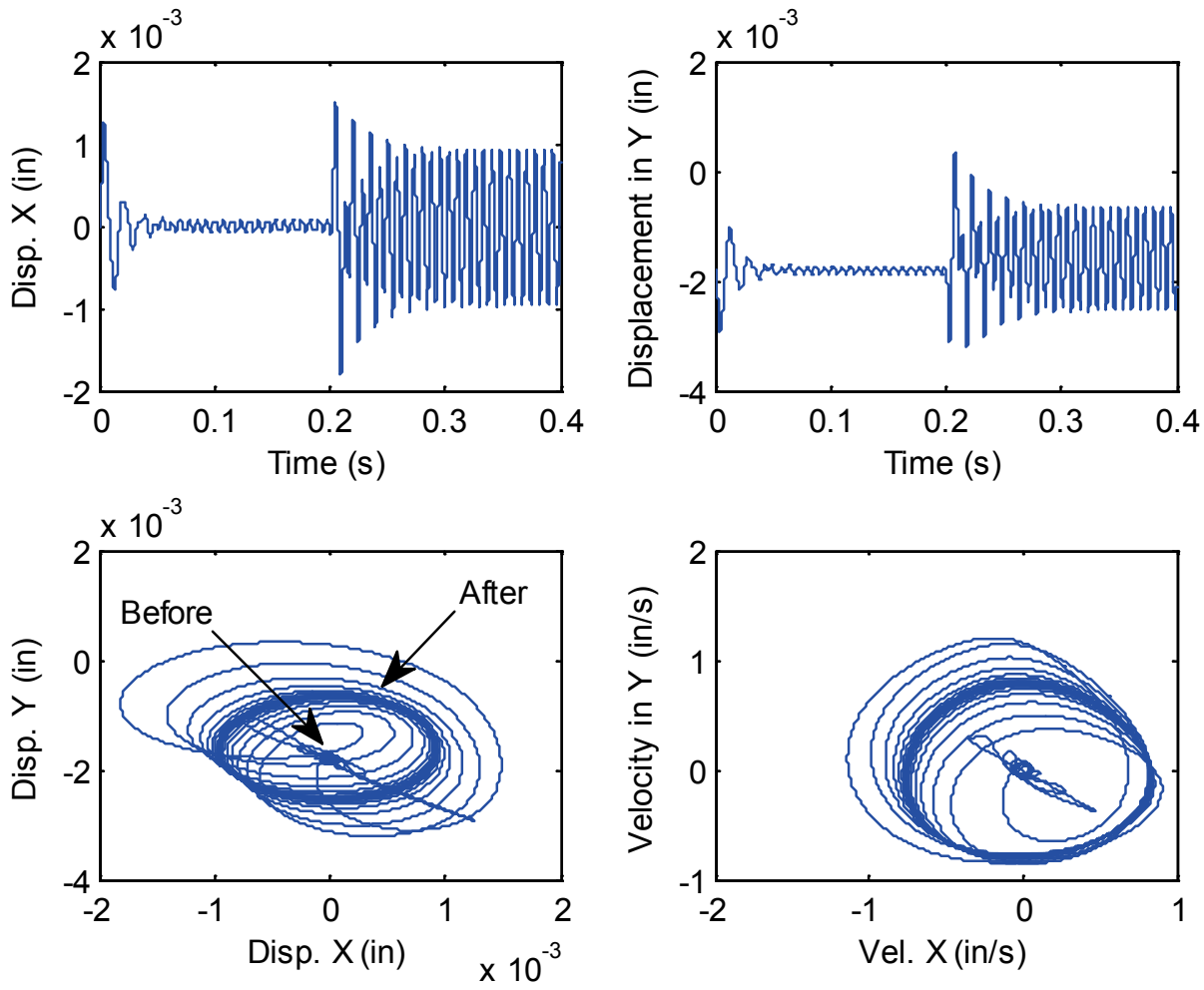

Figure 5.19 Orbits of Shaft Center at 8,000 rpm, different unbalances at $0.2 \mathrm{sec}$. 


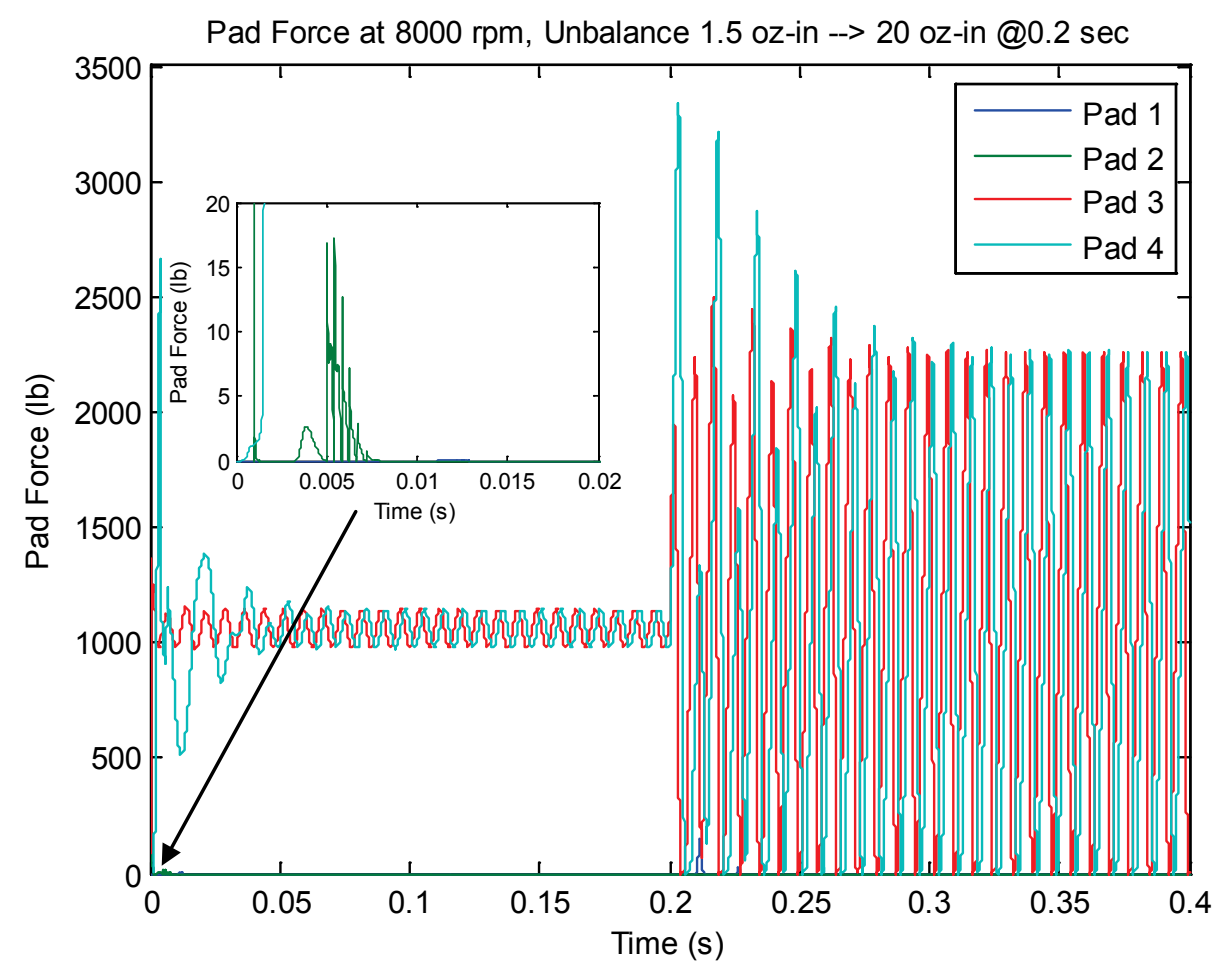

Figure 5.20 Pad Force at 8,000 rpm, Different Unbalances

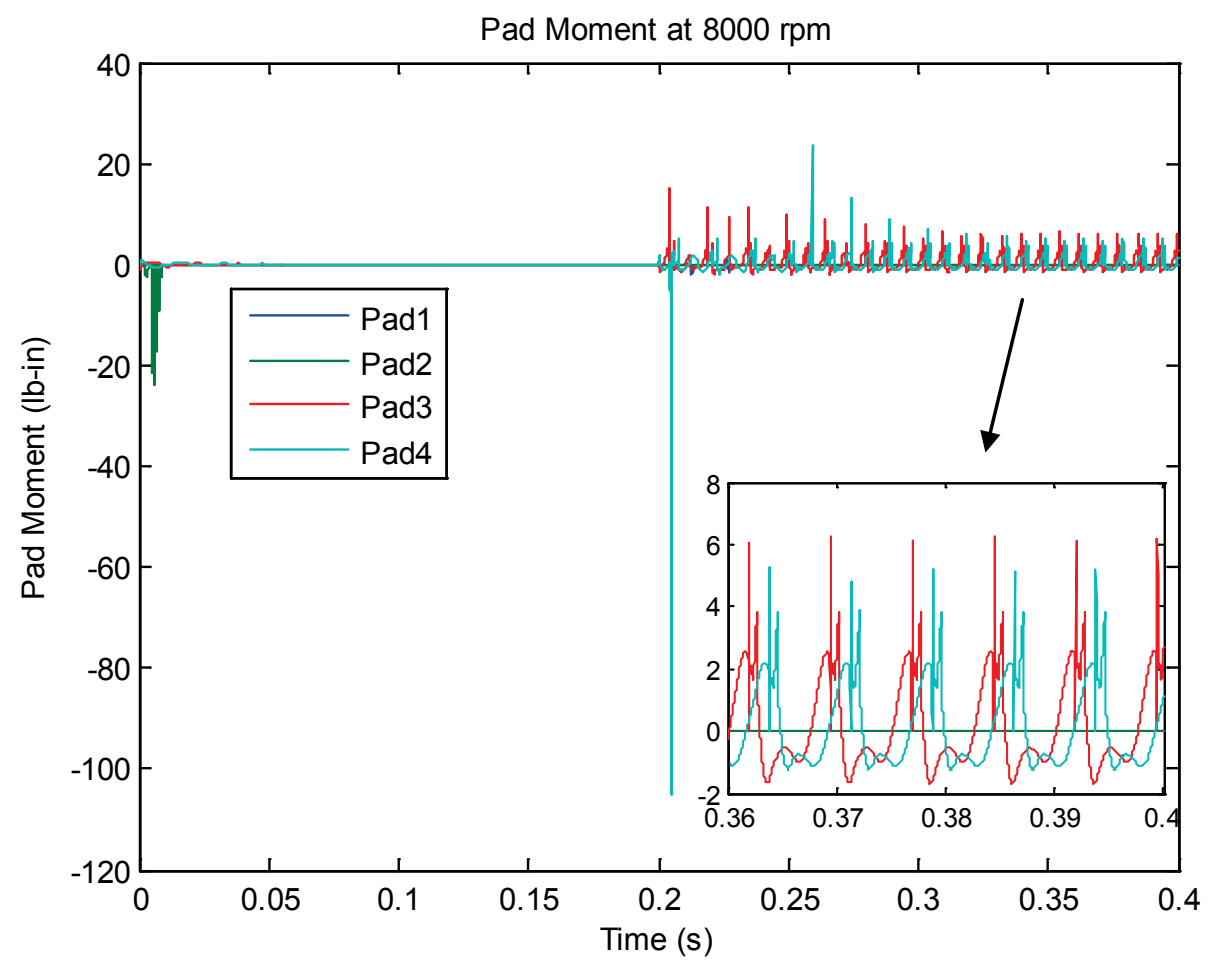

Figure 5.21 Pad Moment at 8,000 rpm, Different Unbalances 


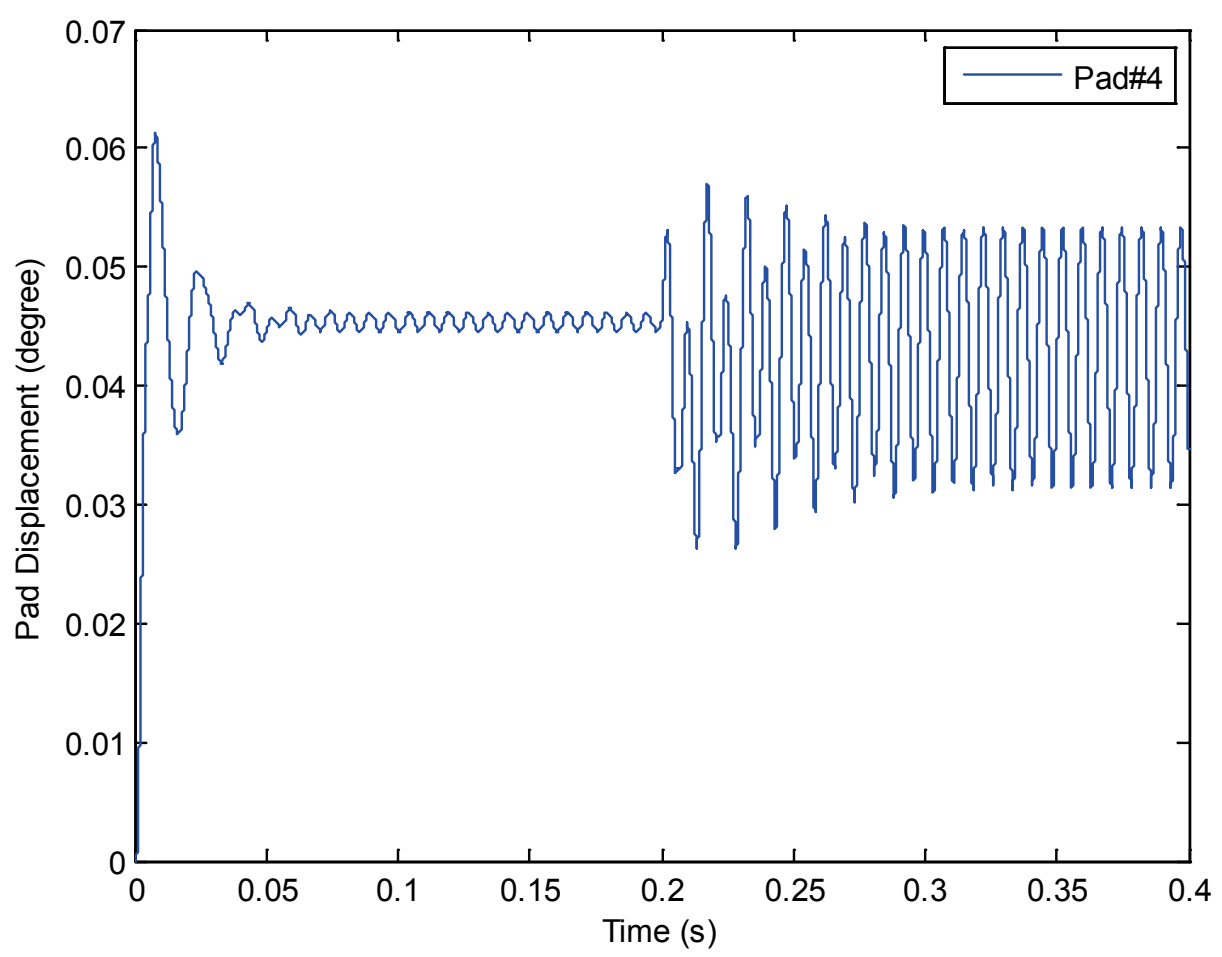

Figure 5.22 Pad \#4 Angle at 8,000 rpm, Different Unbalances

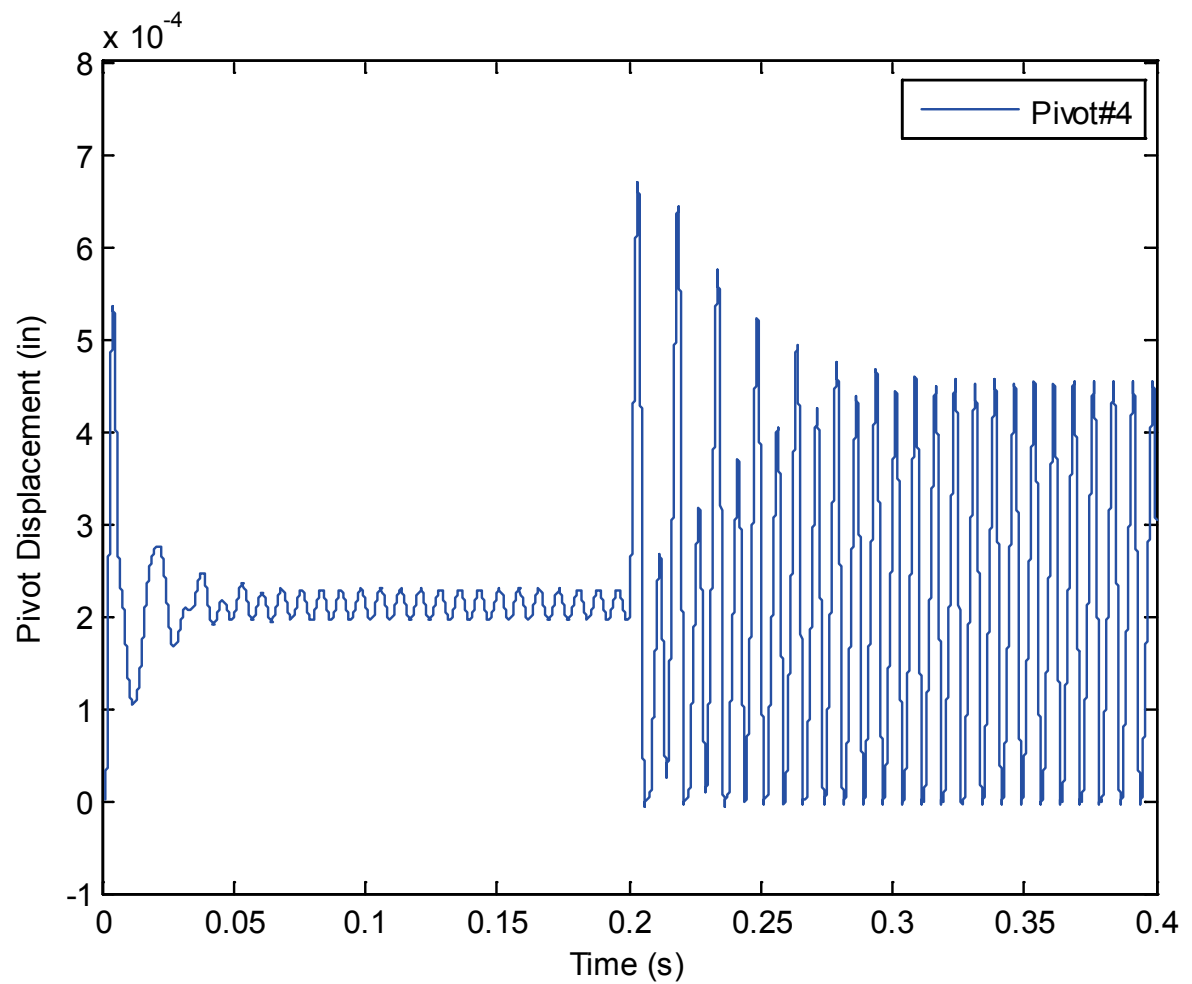

Figure 5.23 Pivot \#4 Displacement at 8,000 rpm, Different Unbalances 


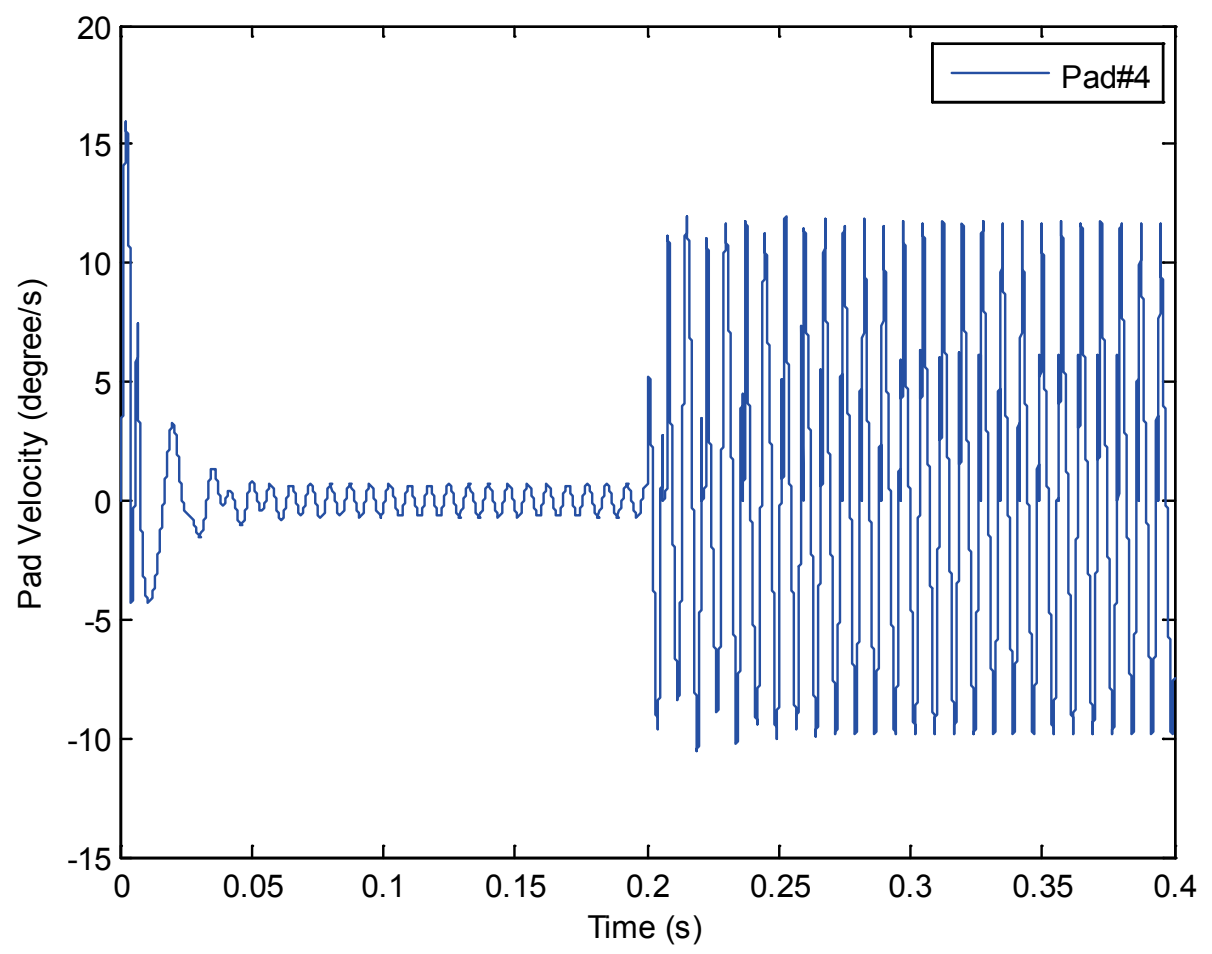

Figure 5.24 Pad \#4 Angle Velocity at 8,000 rpm, different unbalances

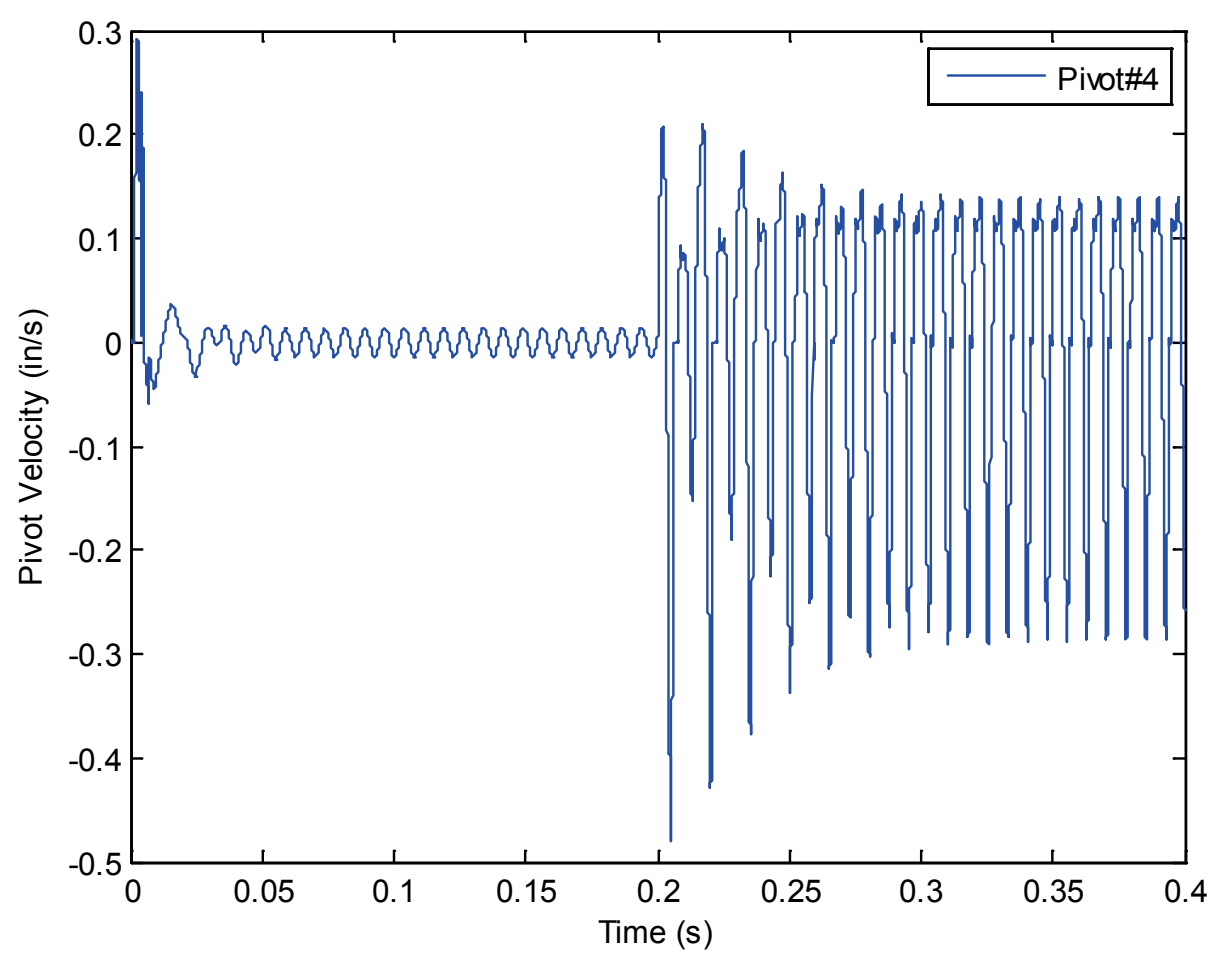

Figure 5.25 Pivot \#4 Velocity at 8,000 rpm, different unbalances 


\subsection{8-Stage Back to Back Compressor Rotor Model}

The 8-stage back-to-back centrifugal compressor is typically used for natural gas reinjection at an offshore drilling site. Fig. 5.26 shows a CAD image of a Siemens STC-SH single-shaft centrifugal compressor (www.siemens.com), which is similar to the eightstage back to back centrifugal compressor model in Fig. 5.27, but has seven stages only. Those figures illustrate the overall layout and the locations of the compressor stages and the bearings. The modeled rotor, based on beam element, has a length of $116.5 \mathrm{inch}$ and a total mass of 2,853.3 lb including the attached disks. The system mass center is near the mid span. The two bearing locations are indicated with the red color in Fig. 5.27.

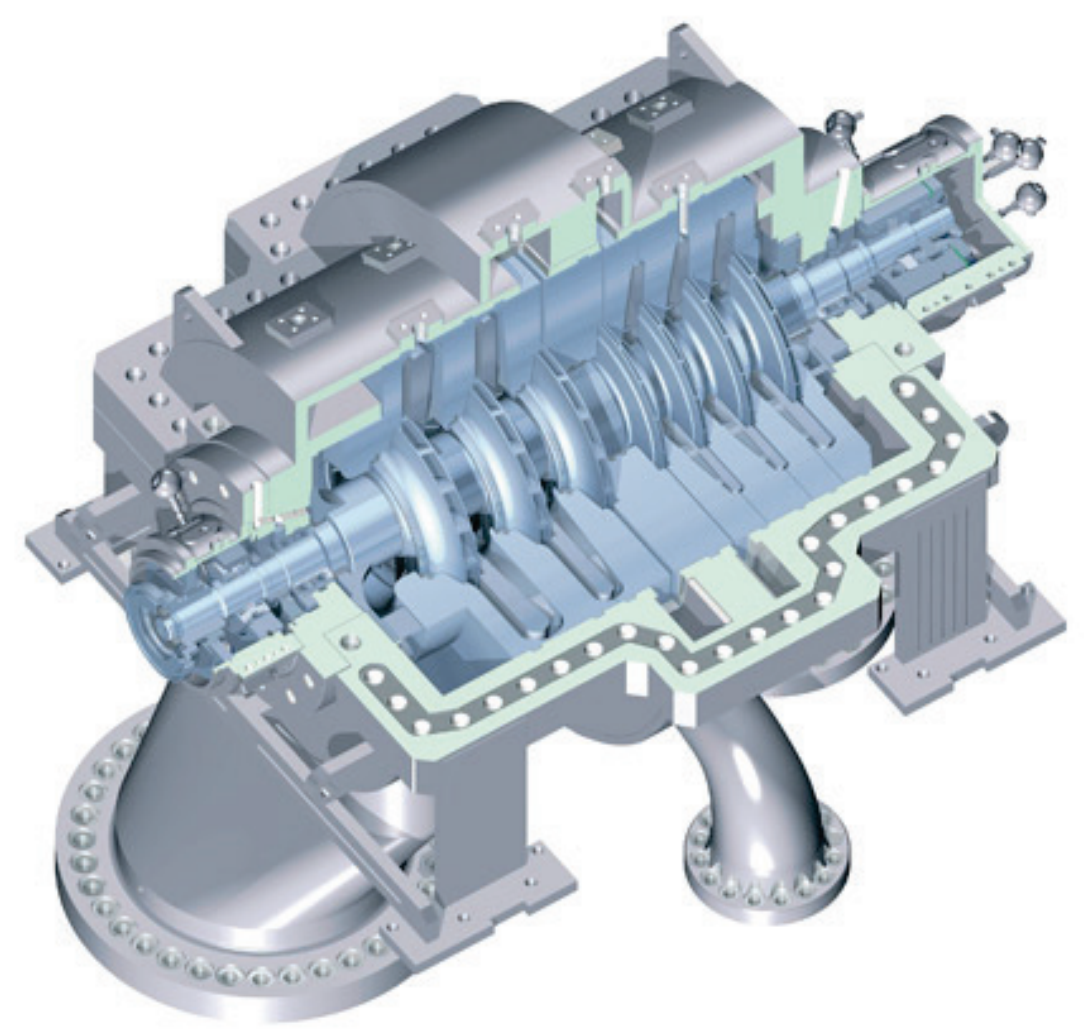

Figure 5.26 CAD image of an STC-SH single-shaft centrifugal compressor with horizontally split casing. Fields of application include on- and offshore oil and gas, petrochemicals, refineries, general chemistry and process engineering, www.siemens.com 


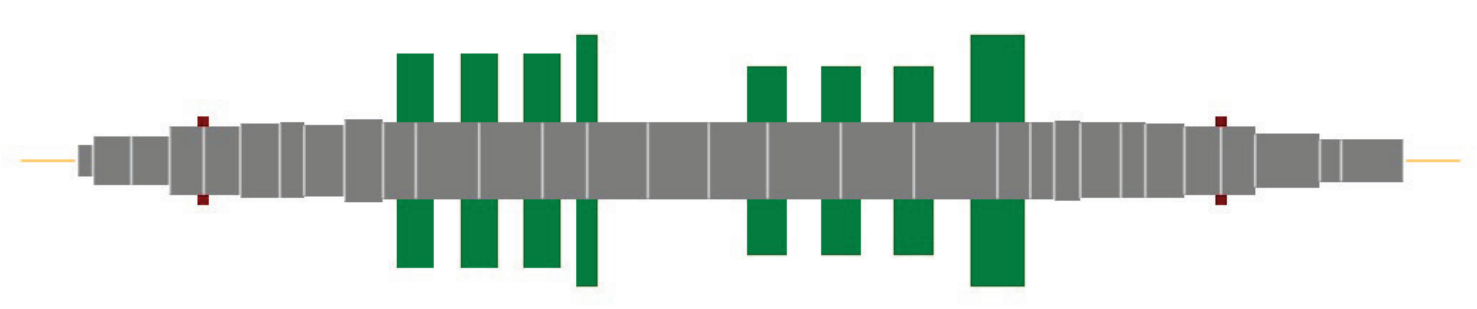

Figure 5.27 Finite element model of the 8 -stage back-to-back compressor

\subsubsection{Linear Rotor Model}

The geometric dimensions (diameters and lengths) are given in Table 5.5 [123]. The centrifugal compressor is composed of one shaft, which is divided into nodes and elements (stations and sections), and 8 disks. Fig. 5.27 illustrates the finite element model of the compressor. The model consists of 30 at Timoshenko beam elements with 31 node points. Each node has 6 DOF for the lateral coordinates $\mathrm{x}$ and $\mathrm{y}$, the rotation about these axes $\theta_{x}$ and $\theta_{y}$, and the axial displacement $\mathrm{z}$ and the rotation of the rotor $\theta_{y}$. The respective coordinate system of the rotor is described in Fig. 3.1. Since there is no axial force and moment, to save computational time of integration the equations of motion, only the lateral analyses are applied to the model in this chapter, and 4 DOF per node is used for the transient analysis. The four lateral DOF of each node are: two displacements in the horizontal and vertical directions ( $\mathrm{x}$ and $\mathrm{y}$ ) and two rotational angles (for bending) in the horizontal and vertical directions $\left(\theta_{x}\right.$ and $\left.\theta_{y}\right)$. The rotor model including the shaft and attached disks has 124 DOF $(31 * 4)$. To conduct the nonlinear transient analysis, however, additional nodes and DOF to describe the behavior of the tilting pad bearings have to be added to the rotor dynamic system, which will be discussed in next Section 5.4. The tilting pad bearing locations denoted with the red markers across the center of shaft 
in Fig. 5.27. The finite length squeeze film damper locations are same as the bearings but they are installed outside of the bearings. A side view of a simplified structure of shaftbearing-damper has been shown in Chapter 4, Fig. 4.2.

Table 5.5 Eight-Stage Back to Back Compressor Data

\begin{tabular}{|l|l|l|l|l|l|l|l|l|}
\hline Node & L & OD & ID & E & Rho & W & It & Ip \\
\hline & in & in & in & psi & lbf/in & lb & lbf-in $^{2}$ & lbf-in $^{2}$ \\
\hline 1 & 1.36 & 2.75 & 0 & 2935000 & 0.283 & 0 & 0 & 0 \\
\hline 2 & 3.37 & 4.36 & 0 & 2935000 & 0.283 & 0 & 0 & 0 \\
\hline 3 & 3.37 & 4.35 & 0 & 2935000 & 0.283 & 0 & 0 & 0 \\
\hline 4 & 3 & 6 & 0 & 2935000 & 0.283 & 0 & 0 & 0 \\
\hline $5(\mathrm{Brg})$ & 3.2 & 6 & 0 & 2935000 & 0.283 & 0 & 0 & 0 \\
\hline 6 & 3.47 & 6.5 & 0 & 2935000 & 0.283 & 0 & 0 & 0 \\
\hline 7 & 2.14 & 6.62 & 0 & 2935000 & 0.283 & 0 & 0 & 0 \\
\hline 8 & 3.53 & 6.22 & 0 & 2935000 & 0.283 & 0 & 0 & 0 \\
\hline 9 & 3.4 & 7.18 & 0 & 2935000 & 0.283 & 0 & 0 & 0 \\
\hline 10 & 2.871 & 6.7 & 0 & 2935000 & 0.283 & 0 & 0 & 0 \\
\hline 11 & 5.561 & 6.7 & 0 & 2935000 & 0.283 & 224.15 & 5676.66 & 11298.41 \\
\hline 12 & 5.561 & 6.7 & 0 & 2935000 & 0.283 & 224.15 & 5676.66 & 11298.41 \\
\hline 13 & 3.92 & 6.7 & 0 & 2935000 & 0.283 & 224.15 & 5676.66 & 11298.41 \\
\hline 14 & 5.34 & 6.7 & 0 & 2935000 & 0.283 & 180.07 & 6003.47 & 11904.78 \\
\hline 15 & 5.34 & 6.7 & 0 & 2935000 & 0.283 & 0 & 0 & 0 \\
\hline 16 & 5.162 & 6.7 & 0 & 2935000 & 0.283 & 0 & 0 & 0 \\
\hline 17 & 6.442 & 6.7 & 0 & 2935000 & 0.283 & 177.56 & 3594.73 & 7191.72 \\
\hline 18 & 6.442 & 6.7 & 0 & 2935000 & 0.283 & 177.56 & 3594.73 & 7191.72 \\
\hline 19 & 7.331 & 6.7 & 0 & 2935000 & 0.283 & 177.56 & 3594.73 & 7191.72 \\
\hline 20 & 2.89 & 6.7 & 0 & 2935000 & 0.283 & 464.11 & 15800.4 & 30942.02 \\
\hline 21 & 2.09 & 6.7 & 0 & 2935000 & 0.283 & 0 & 0 & 0 \\
\hline 22 & 2.31 & 7 & 0 & 2935000 & 0.283 & 0 & 0 & 0 \\
\hline 23 & 3.55 & 6.62 & 0 & 2935000 & 0.283 & 0 & 0 & 0 \\
\hline 24 & 2.14 & 6.62 & 0 & 2935000 & 0.283 & 0 & 0 & 0 \\
\hline 25 & 3.45 & 6.5 & 0 & 2935000 & 0.283 & 0 & 0 & 0 \\
\hline 26 & 3.2 & 6 & 0 & 2935000 & 0.283 & 0 & 0 & 0 \\
\hline $27(\mathrm{Brg})$ & 3 & 6 & 0 & 2935000 & 0.283 & 0 & 0 & 0 \\
\hline 28 & 5.69 & 4.68 & 0 & 2935000 & 0.283 & 0 & 0 & 0 \\
\hline 29 & 1.87 & 3.74 & 0 & 2935000 & 0.283 & 0 & 0 & 0 \\
\hline 30 & 5.5 & 3.68 & 0 & 2935000 & 0.283 & 0 & 0 & 0 \\
\hline 31 & 0 & 3.68 & 0 & 2935000 & 0.283 & 0 & 0 & 0 \\
\hline & & & & & & & & \\
\hline
\end{tabular}

Table 5.5 illustrates the geometric dimension of the rotor. Each element has 10 properties: length $(L)$, outer diameter $(O D)$, inner diameter $(I D)$, elastic modulus $(E)$, 
density $(\rho)$, attached disk weight $(W)$, transverse mass moment of inertia $\left(I_{t}\right)$ and polar mass moment of inertia $\left(I_{p}\right)$ for the attached lumped mass, residual bow magnitude $\left(A_{b}\right)$ and residual bow phase $\left(\varphi_{b}\right)$ respectively. For this problem, there is no the residual shaft bow magnitude and phase, and the Poisson's ratio is 0.3. The two 4-pad tilting pad bearings are located at nodes \#5 and node \#27 (red color in Fig. 5.27).

\subsubsection{Nonlinear Tilting Pad Bearing Model}

The rotor is supported by two titling pad bearings at nodes 5 and 27 as red color indicated in Fig. 5.27. The tilting pad bearing is composed of 4 pads with a load between pad (LBP) configuration, which is same as discussed in Section 5.1 and Fig. 5.2.

\subsection{Transient Analysis of 8-Stage Compressor with Nonlinear}

\section{Tilting Pad Bearings}

This section describes the unbalance forced response of the 8-stage compressor supported by the tilting pad bearings. The bearings are treated as nonlinear. Using the displacements and velocities of the shaft center inside the bearing and the bearing pads/pivots, the pressure profile of each pad is calculated at first by solving the Reynolds equation based on the finite element method with assumption of polynomial distribution of axial pressure, laminar flow in the lubricant, constant lubricant density and constant lubricant viscosity. The bearing forces are calculated by integrating the pressure profile over the fluid area, and then applied to the force vector of the equations of motion. For transient analyses, the bearing forces need to be calculated with the instantaneous displacements and velocities of the rotor and the bearings at each time step. 


\subsubsection{Critical Speed Map of the Rotor-Bearing System}

The critical speed map identifies the frequencies at which the rotational speed of the rotor equals the undamped natural frequencies. The critical speed map of this rotorbearing system, Fig. 5.28, is calculated based on a range of bearing stiffness values. This figure provides basic information to understand the force response of the rotor and to design the bearings. In this chapter, the bearings are treated as nonlinear; the stiffness and damping coefficients from the bearings are unknown and depend on the displacements and velocities of the shaft center inside the bearing and the bearing pads/pivots at different time. Stability analysis and natural frequencies calculation based on the linear theory can not apply directly to the nonlinear rotor dynamic system. However, from the calculated range of steady state bearing stiffness, the approximate critical speeds may obtain from the undamped critical speed map.

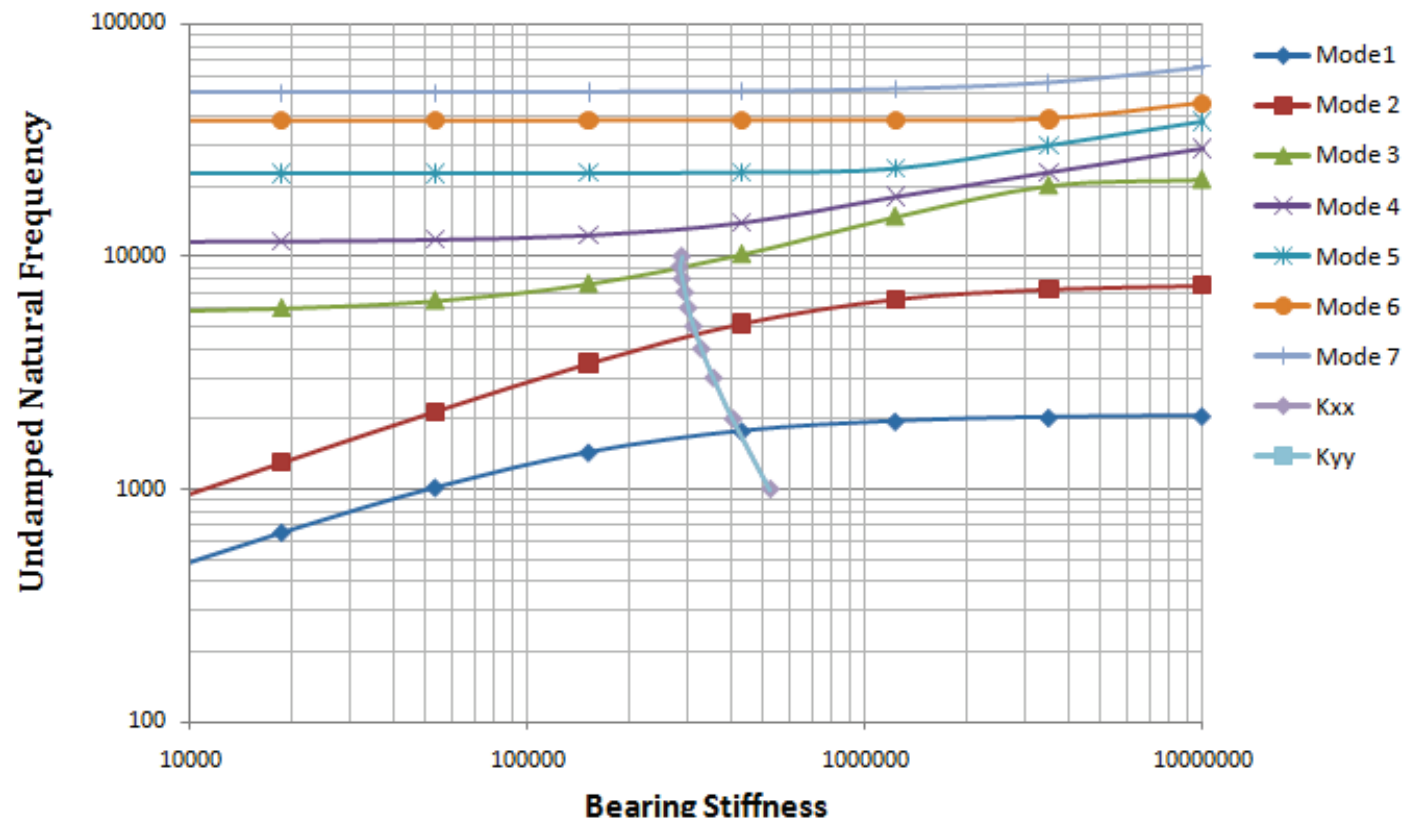

Figure 5.28 Undamped Critical Map of the 8-stage Compressor 
In the figure, the horizontal axis is the bearing stiffness from $1 \times 10^{4} \mathrm{lbf} /$ in to $1 \times 10^{7}$ lbf/in, which covers the possible stiffness range of the tilting pad bearing. The vertical axis is the critical speeds with unit of rpm. In the figure, the first seven modes begin from the first bending mode are given.

\subsubsection{Transient Analysis of Rotor with Nonlinear Tilting Pad Bearings}

The 8-stage compressor rotor has total nodes of 31 and each node has 4 DOF (x, y, $\theta_{x}$ and $\theta_{y}$ ). The total additional nodes from the two bearing are 8 and each node has 2 DOF (pad angle $\delta$ and pivot displacement $\gamma$ ). The total DOF of the system including the tilting pad bearings are now $140(31 * 4+8 * 2)$. Another important issue in the transient analysis is the time step, which depends on the maximum natural frequency of the system. For the nonlinear system, since the stiffness and damping effects from the bearings are unknown and the rotor behavior dependent, the exactly maximum natural frequency of the system cannot be calculated directly. However, for most rotor dynamic systems, the modes related to those lower natural frequencies (10 times of operating speed) mainly decide the forced response of the system. In this dissertation, the maximum natural frequency of the linear parts including the shaft and built on disks in this example, is used to calculate the time step for the integration method. The time step is calculated by considering $2.5 \sim 3$ times the maximum natural frequency of the linear system for the first try. For the 8-stage back to back industrial compressor model, the time step $(\Delta t)$ is taken as $8 \times 10^{-6}$ second, and the $4^{\text {th }}$ order Runge-Kutta method is stable at that time step. Comparison of the shaft center nodal displacements inside the two bearings calculated with different time steps of $4 \times 10^{-6} \mathrm{sec}$. and $8 \times 10^{-6} \mathrm{sec}$. are shown in Figs. 5.29\&5.30. 

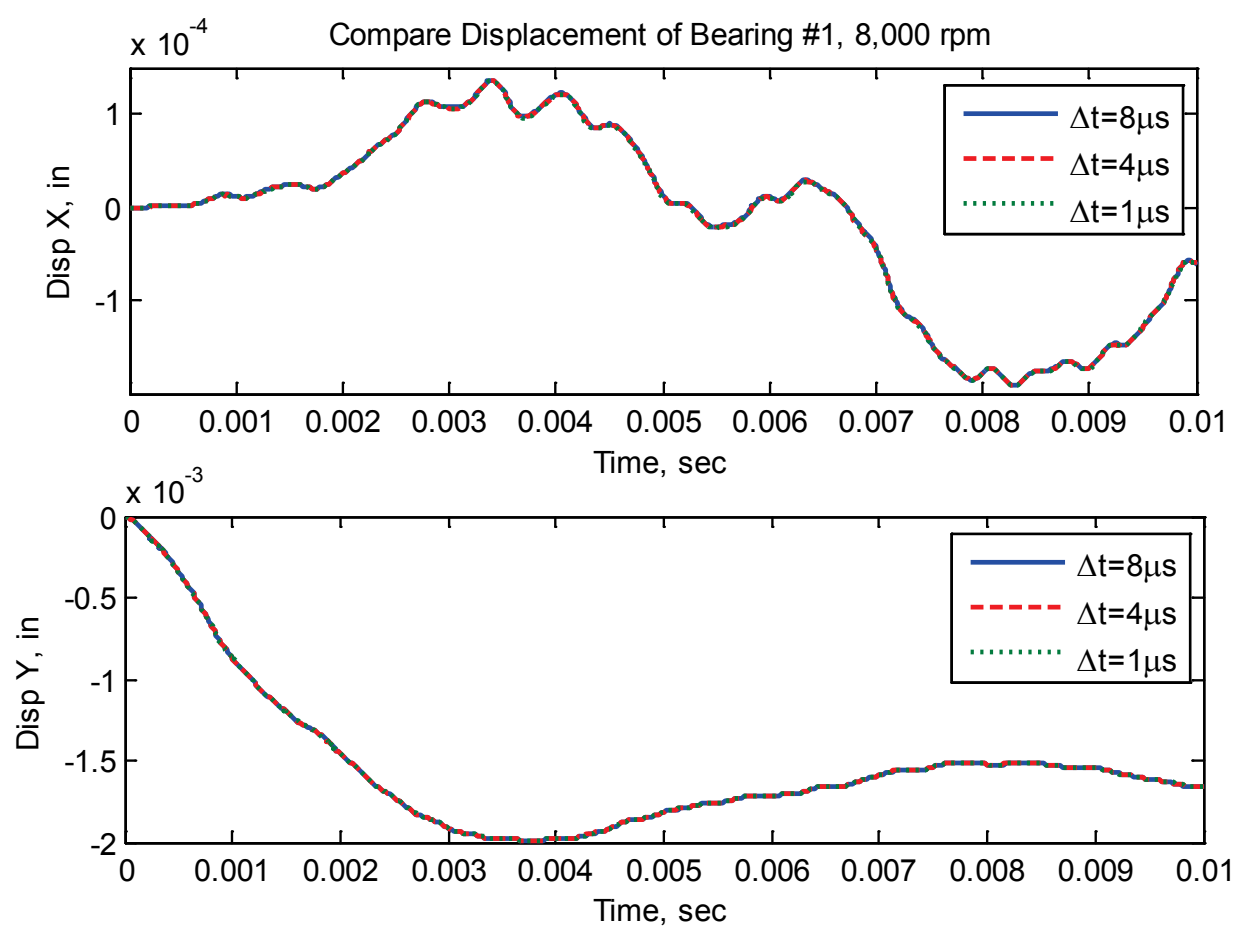

Figure 5.29 Displacements of Bearing \#1 with Different Time Steps
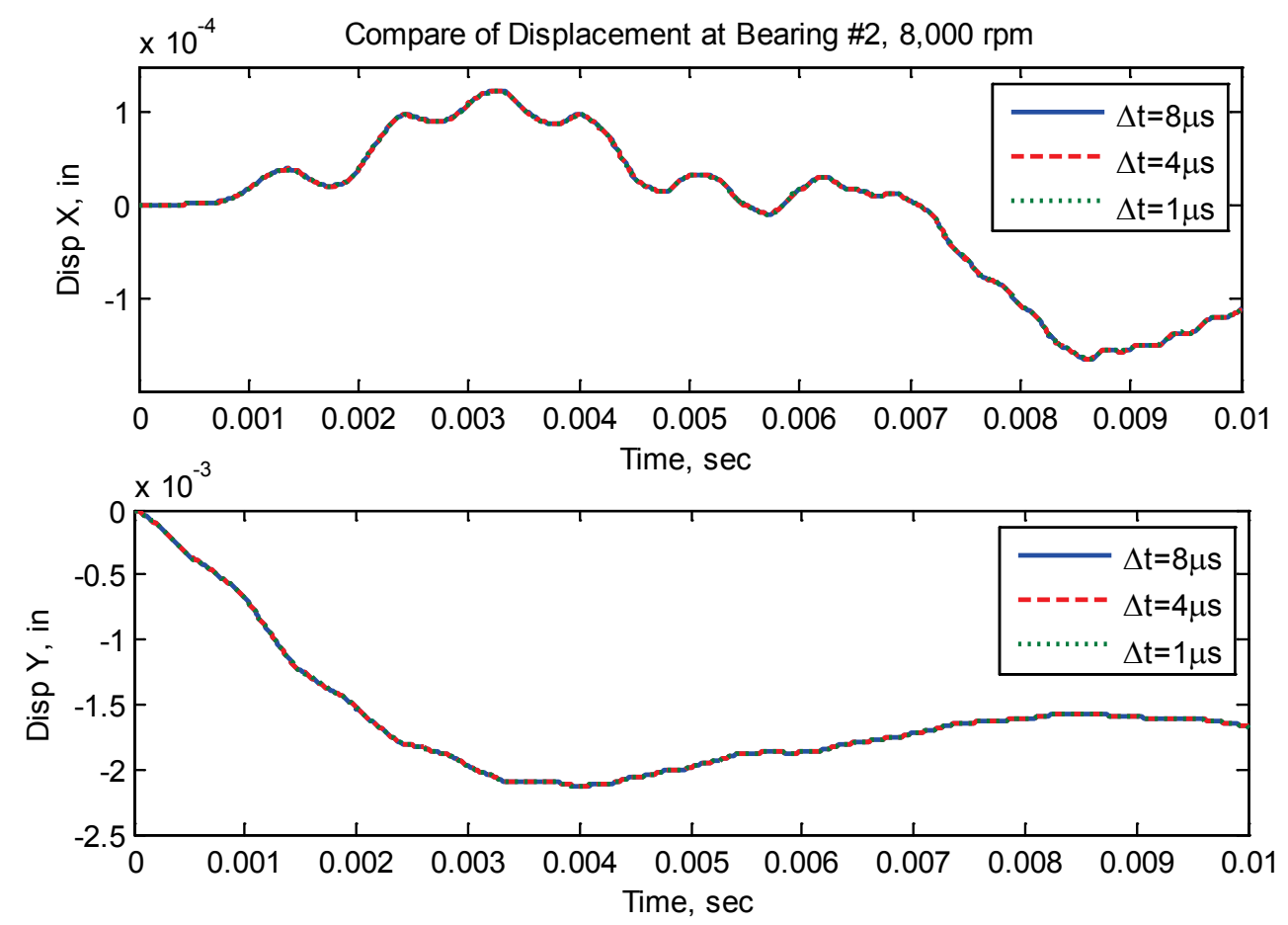

Figure 5.30 Displacements of Bearing \#2 with Different Time Steps 
Two unbalances are applied to two central disks (node $14 \& 17)$ at same phase angle $\left(0^{\circ}\right)$ to excite the $1^{\text {st }}$ bending mode. To excite the $2^{\text {nd }}$ bending mode of the system, two unbalances were added to the left disk (node 12) and the right disk (node 20) with an $180^{\circ}$ phase difference, e.g. $0^{\circ}$ and $180^{\circ}$. According to API 671 , for the evaluation of the unbalance response, the maximum acceptable unbalance level is calculated by $8 \mathrm{~W} / \Omega$, where $W$ is the total rotor weight with unit $l b f, \Omega$ is the rotor operating speed with unit rpm, the unit of unbalance calculated is oz-in. The total weight of the 8-stage compressor is $2,853.3 \mathrm{lbf}$, and the operating speed is $8,000 \mathrm{rpm}$, so the unbalance to evaluate the forces response is $2.85 \mathrm{oz}$-in, which is named as first unbalance in this Section to identify the server unbalance condition, which has large unbalance than the first one.

The $4^{\text {th }}$ order Runge-Kutta method was selected as the numerical method to calculate the time transient response. The equation of motion is represented in state space form, as shown in Eq. (3.5.2). Because of the existence of nonlinear bearings, the initial displacements of the shaft nodes inside the bearing are zero. The initial positions of the other shaft nodes are calculated according to the gravitational force vector at zero running speed. The initial velocities of all nodes are zero. From the initial conditions, the tilting pad bearing forces are calculated step by step, and are updated in the equations of motion to calculate the new position and velocity at each step. The iteration steps progress until the time is equal to the final time. The external force is the rotor unbalance captured force vector as shown in Eq. (3.3.2). The forced response is calculated at first using API evaluation level unbalance at $2.85 \mathrm{oz}$-in (first). Then the unbalance changes to a severe unbalance condition, which is 16 times of API evolution level unbalance, to obtain the time transient response after the severe unbalance condition is applied. 
To excite the $1^{\text {st }}$ bending mode, the first unbalances are added to central disks (node \#14\&\#17) with same phase angles (CASE I); to excite the $2^{\text {nd }}$ bending mode, the first unbalances are added to the left disk (node \#12) and the right disk (node \#20) with phase angles equal to $0^{\circ}$ and $180^{\circ}$ (CASE II). The time transient response of the whole rotor under 2.85 oz-in unbalance (CASE I) is shown in Figs. 5.31 5.33. Fig. 5.31 shows the shaft transient orbits at 1.0 second. At that time the rotor has not reached steady state yet and the orbits are not stable. In the figure, each blue line indicates the orbits of each node, the current shaft position is indicated by the black line with red points (current nodal positions) on it. After the transient response dies out, the system is stable and synchronous with the rotational speed. The shaft orbit of steady state at 3.0 seconds is shown in Fig. 5.32. To make the response clearer and more easily to be recognized, the deformation due to the static gravitational force vector is removed from those two figures. Fig. 5.33 shows the same orbit as in Fig. 5.32 but with the effect from the gravitational force. The similar results for the $2^{\text {nd }}$ bending mode are obtained for CASE II under the first unbalance, as shown in Figs. 5.34 5.36. Under the first unbalance of CASE I, the time domain transient response of the bearing forces and moments are shown in Figs. 5.37 5.39. The transient response dies out as the system reaches the steady-state unbalance response orbits, as shown in Figs. 5.32\&5.33. The bearing force from node \#5 under steady state condition is shown in Fig. 5.37. Although the rotor orbits show the synchronous response, the outputs of the bearing pad \#4 in the time and frequency domains, Fig. 5.38\&5.39 indicates a weak super harmonic response with low amplitude, especially from the output of pad moment. Also from Fig. 5.39, a very low frequency of $200 \mathrm{rpm}$ is shown in the pad moment response. 


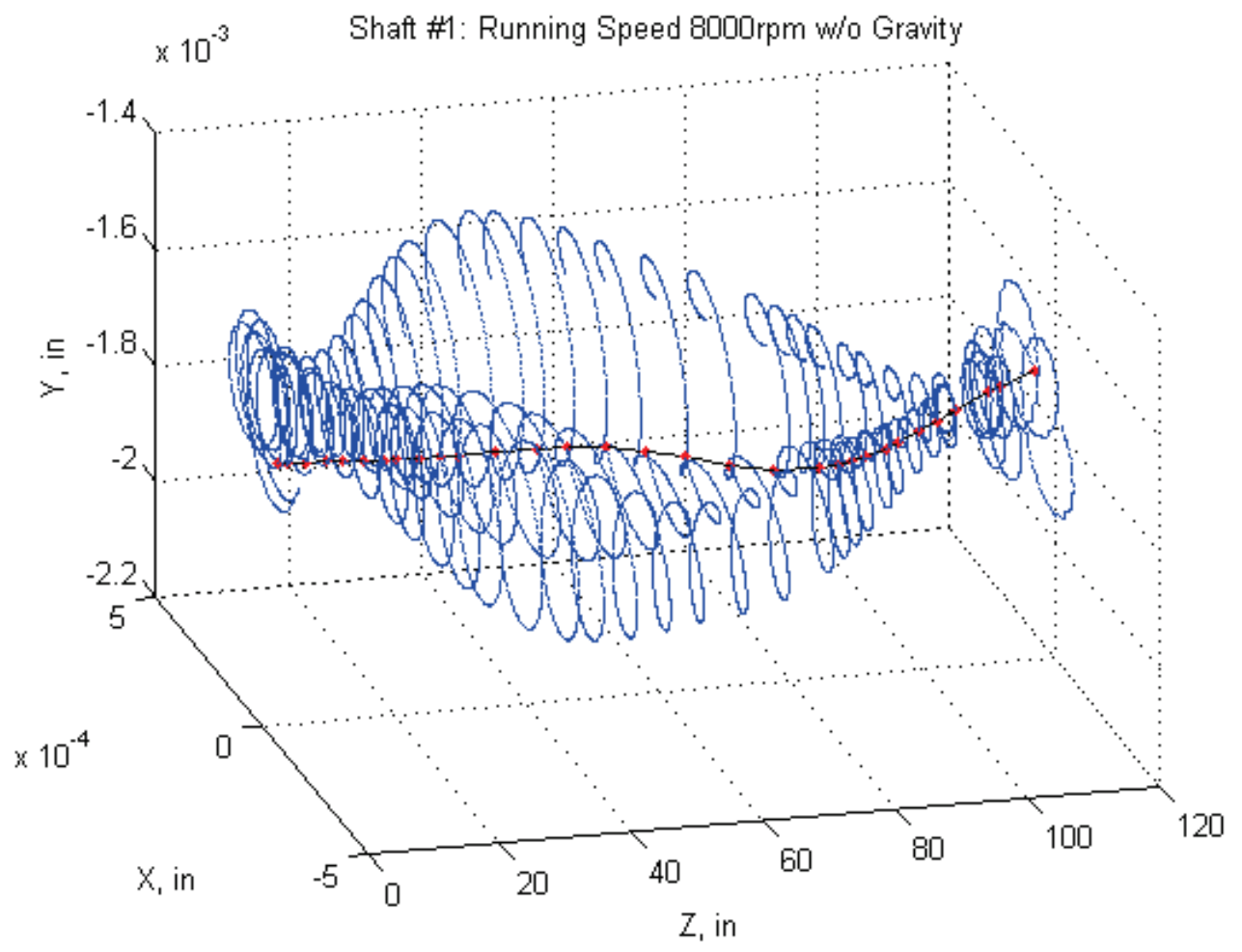

Figure 5.31 Shaft Response of CASE I after $1.0 \mathrm{sec}$ with Unbalance, w/o Gravity

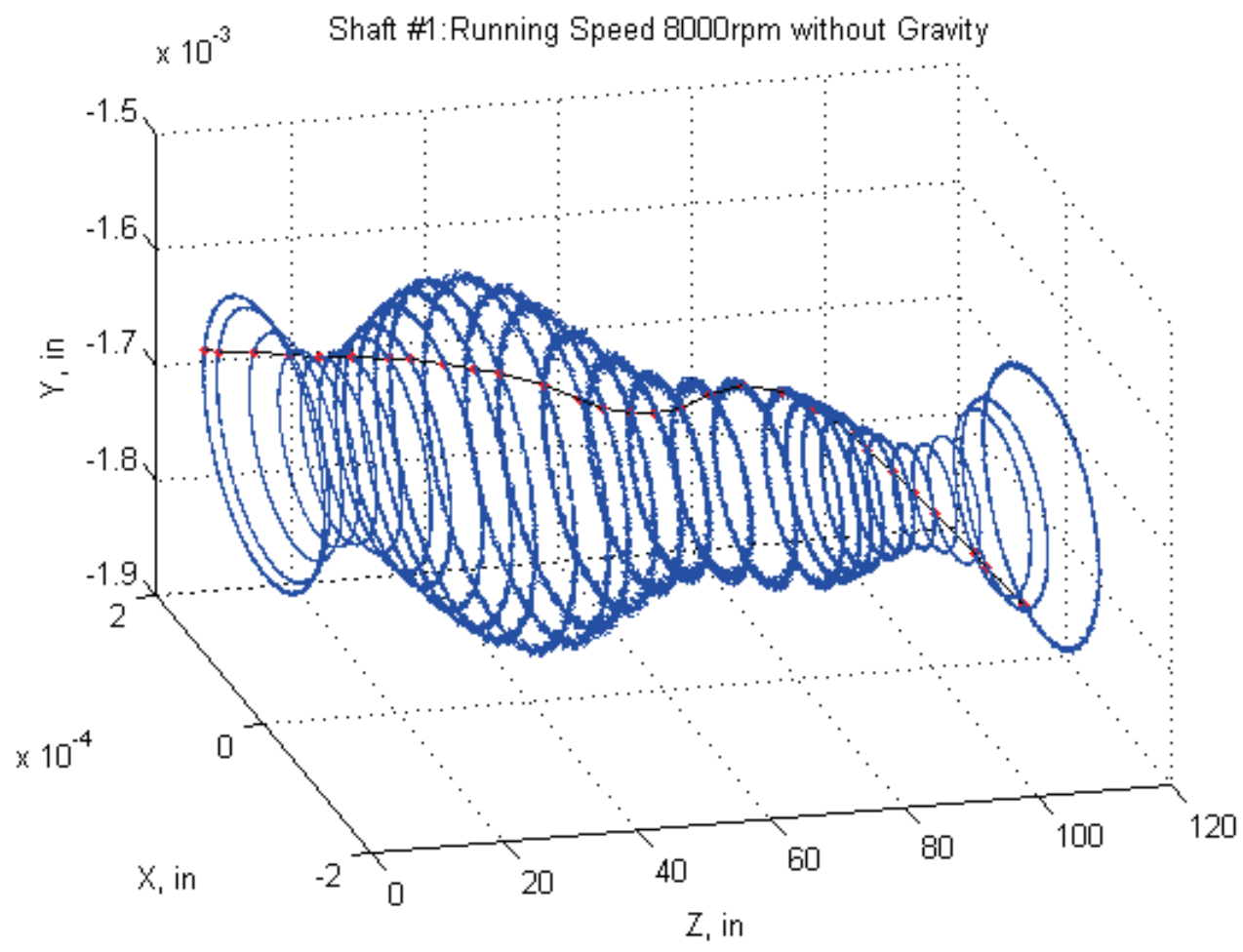

Figure 5.32 Shaft Response of CASE I after $3.0 \mathrm{sec}$ with Unbalance, w/o Gravity 


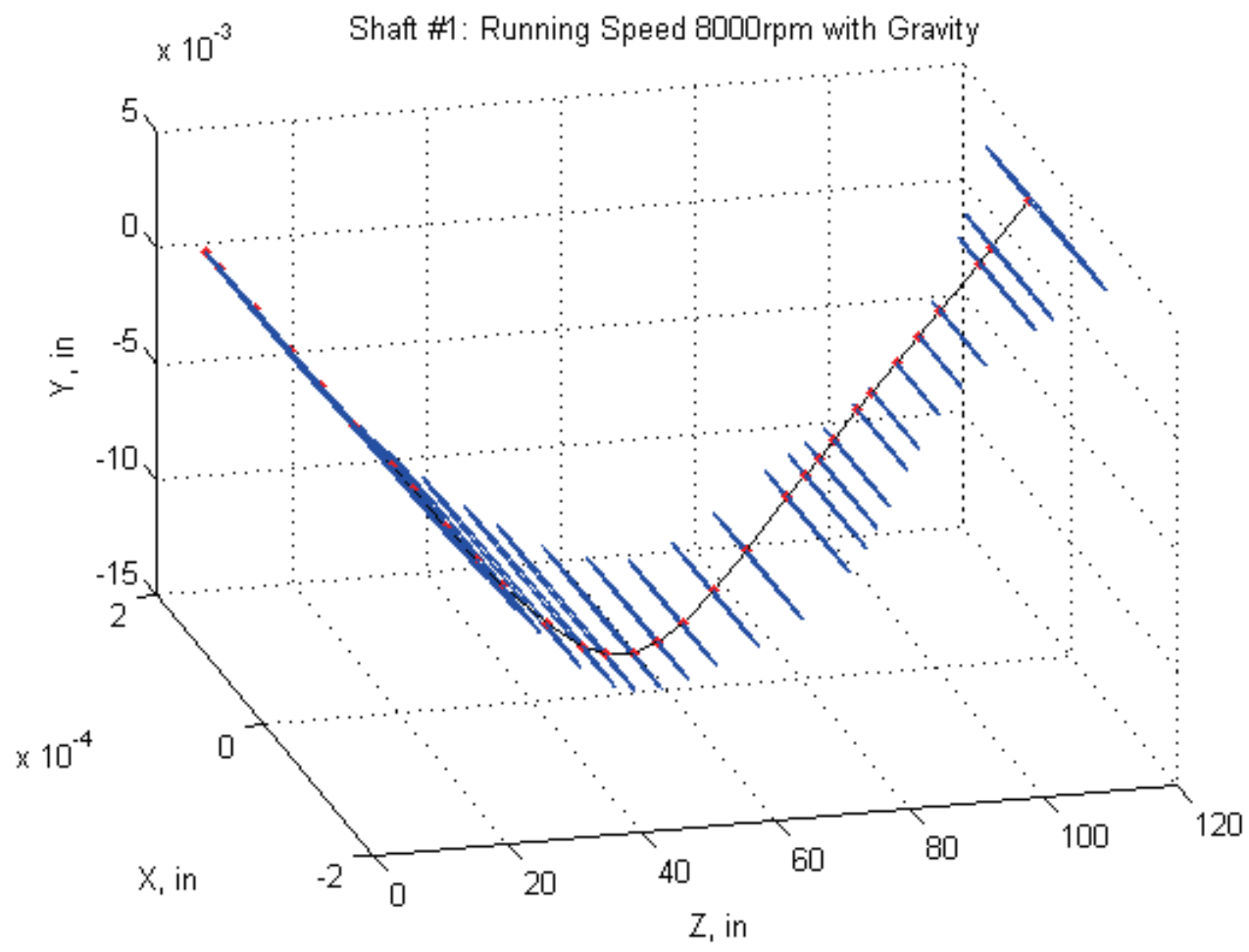

Figure 5.33 Shaft Response of CASE I at $3.0 \mathrm{sec}$ with Unbalance and Gravity Loading

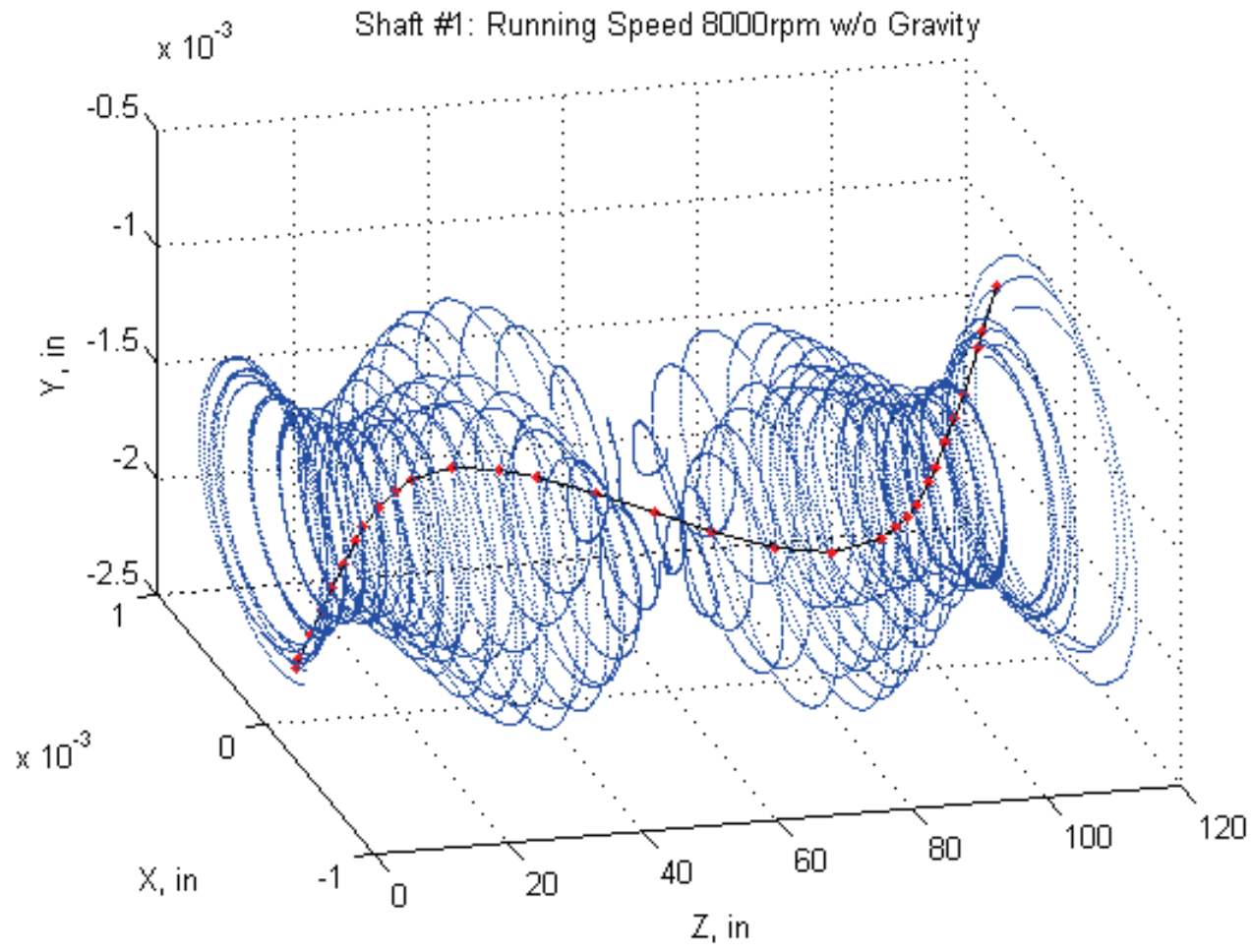

Figure 5.34 Shaft Response of CASE II at $1.0 \mathrm{sec}$ with Unbalance, w/o Gravity Loading 


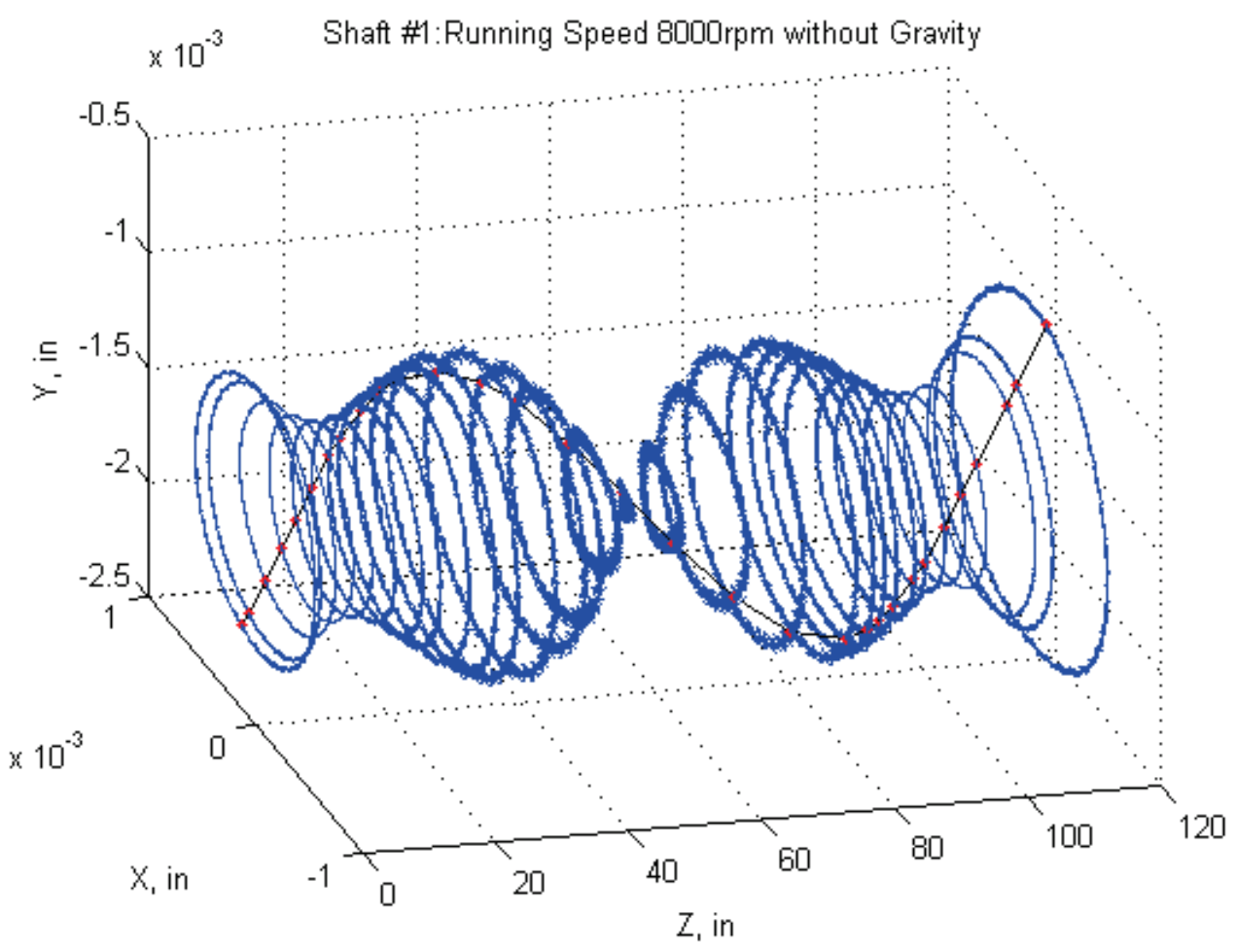

Figure 5.35 Shaft Response of CASE II at $3.0 \mathrm{sec}$ with Unbalance, w/o Gravity Loading

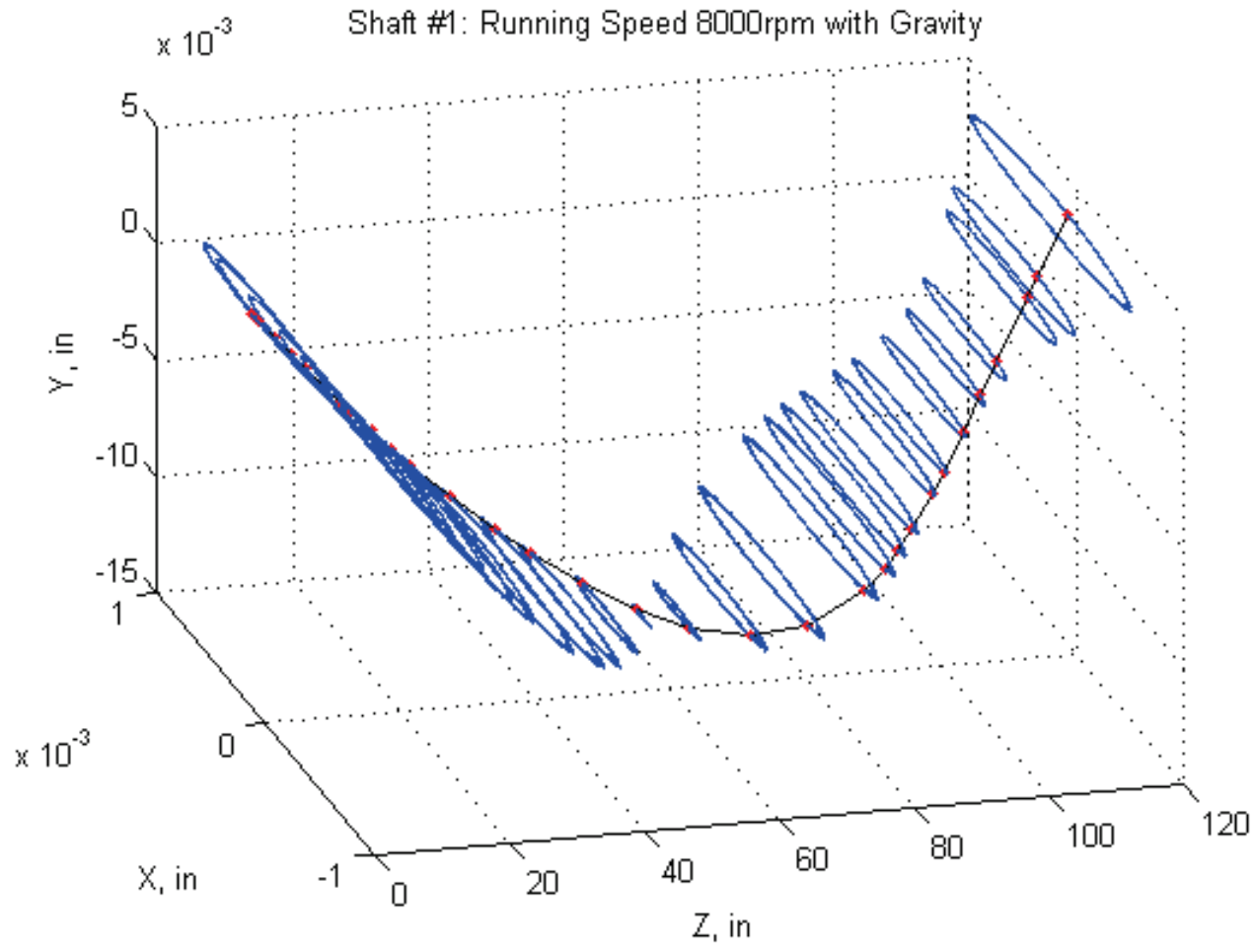

Figure 5.36 Shaft Response of CASE II at $3.0 \mathrm{sec}$ with Unbalance and Gravity Loading 

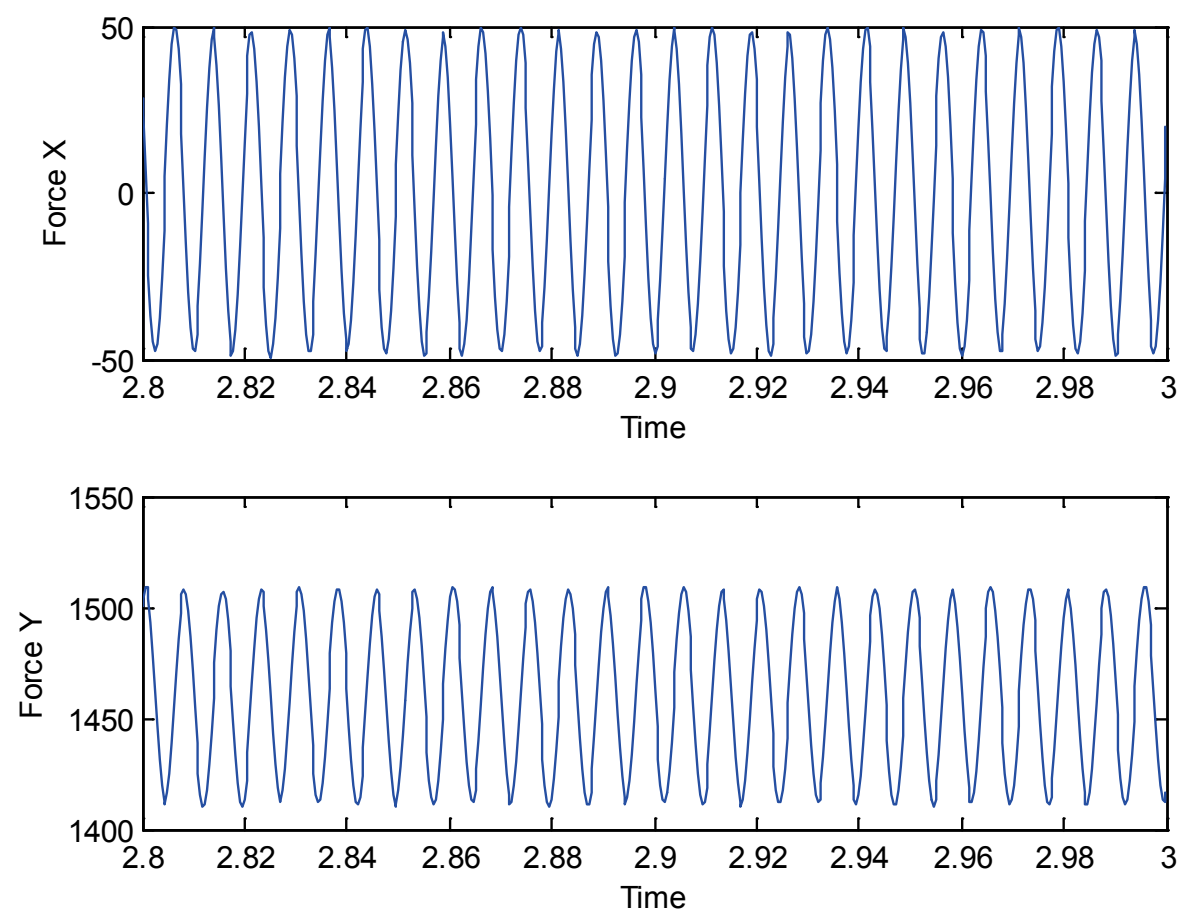

Figure 5.37 Bearing Forced from Node 5 of CASE I
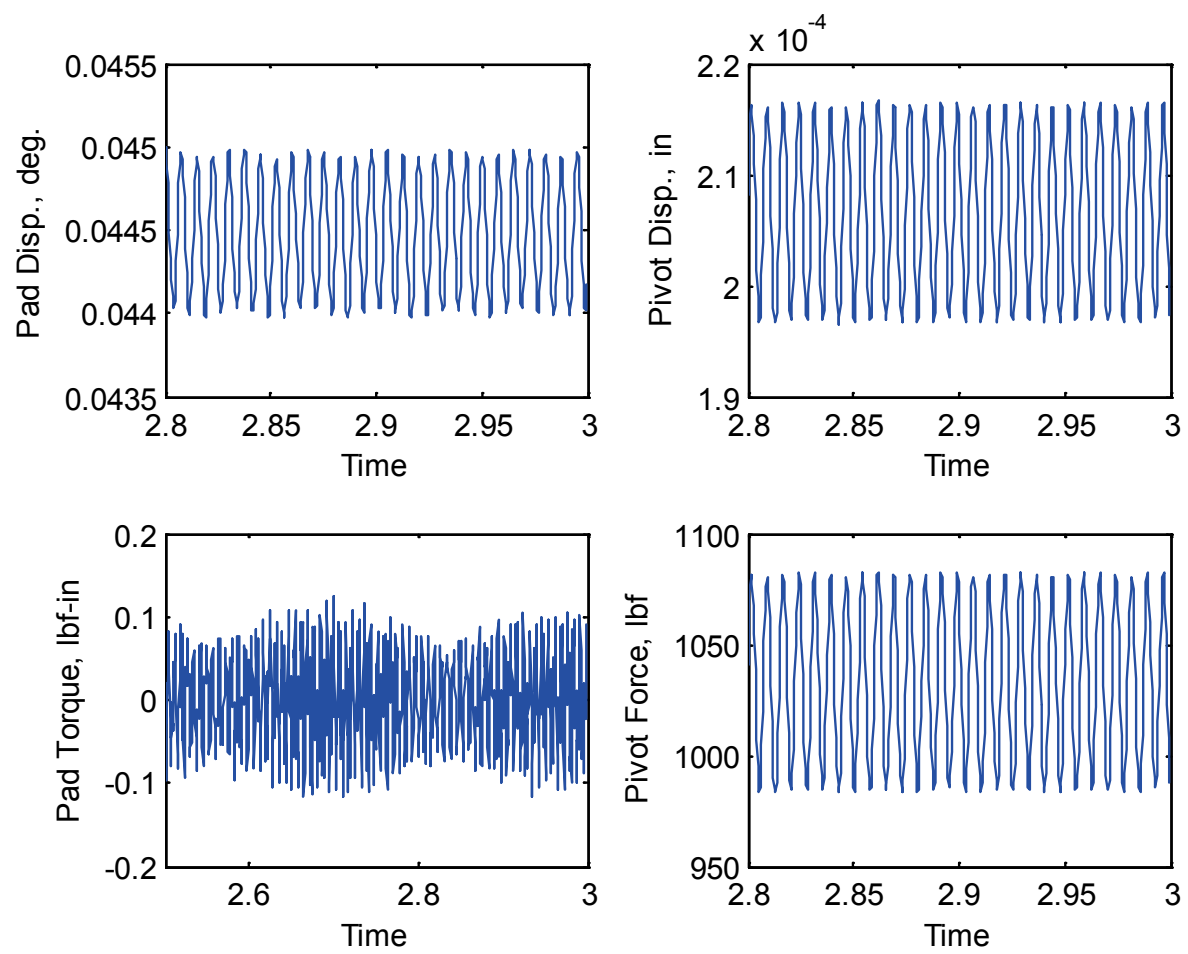

Figure 5.38 Bearing Pad \#4 and Pivot \#4 Response of CASE I 

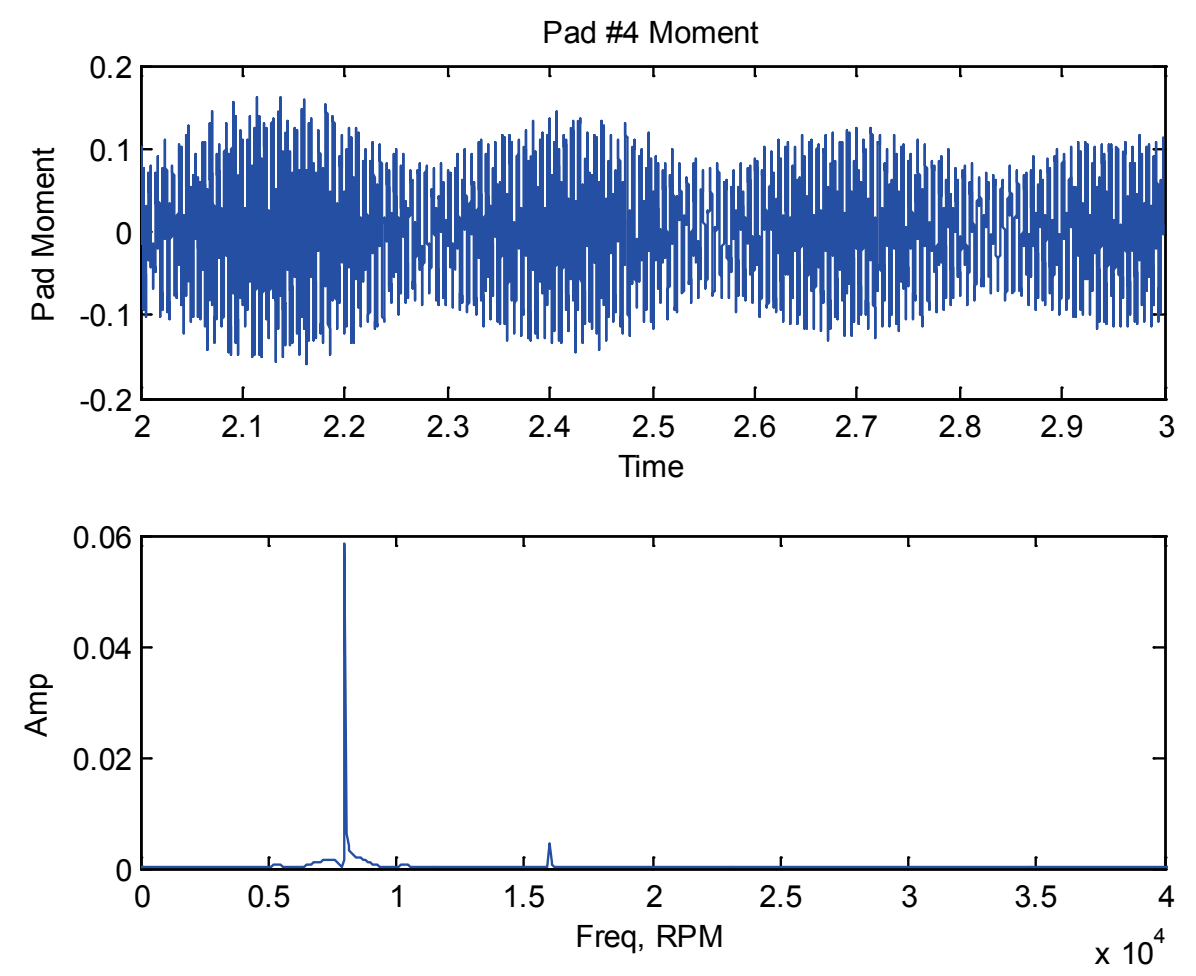

Figure 5.39 Bearing Pad\#4 Moment FFT Output (CASE I)

To simulate the severe condition of a sudden large unbalance force, the rotor runs 3.0 seconds under the first unbalance (CASE I) to obtain the steady-state, then the unbalance suddenly increases at a given time point (3.0 second). In the following calculation, the unbalance increases from 2.85 oz-inch to 45.6 oz-inch (CASE III), which is 16 times larger than the first unbalance. Due to the large sudden unbalance force, the rotor does not reach synchronous steady state response, and strong nonlinear periodic multi-circle orbits are shown. The response of the whole rotor is shown in Fig. 5.40 (without gravity) and Fig. 5.41 (with gravity).

The orbit of disk\#4 (node\#14), disk \#5 (node\#17), and node \#5, which is the shaft center inside the bearing, from 8.0 seconds to 9.0 seconds, are presented in Fig. 5.42 5.44. The bearing forces at node\#5 and the bearing pad/pivot\#4 outputs in time and 
frequency domains are shown in Figs. 5.45 5.49. Compared to the bearing response under the first unbalance, the response under the severe unbalance condition, which is 16 times of the first unbalance, has strongly nonlinear behavior, especially from the frequency domain results of the bearing pad/pivot\#4 (Figs. 5.48\&5.49). In Figs. 5.47, several peaks, including sub-harmonic and super-harmonic response, other than the harmonic peak at $8,000 \mathrm{rpm}$ are shown. Strong nonlinear behavior is shown in Figs.5.48\&5.49, which are the response of the bearing pad/pivot\#4 force and moment in frequency domain.

When the severe unbalance is suddenly applied to the system, e.g. 32 times of the first unbalance, the calculation was stopped due to touching between the shaft and the bearing pads. To obtain the system response under the larger unbalance forces, the finite length squeeze film dampers are introduced into the system to increase stability of the system. The effect of the finite length squeeze damped is discussed in Section 5.5.

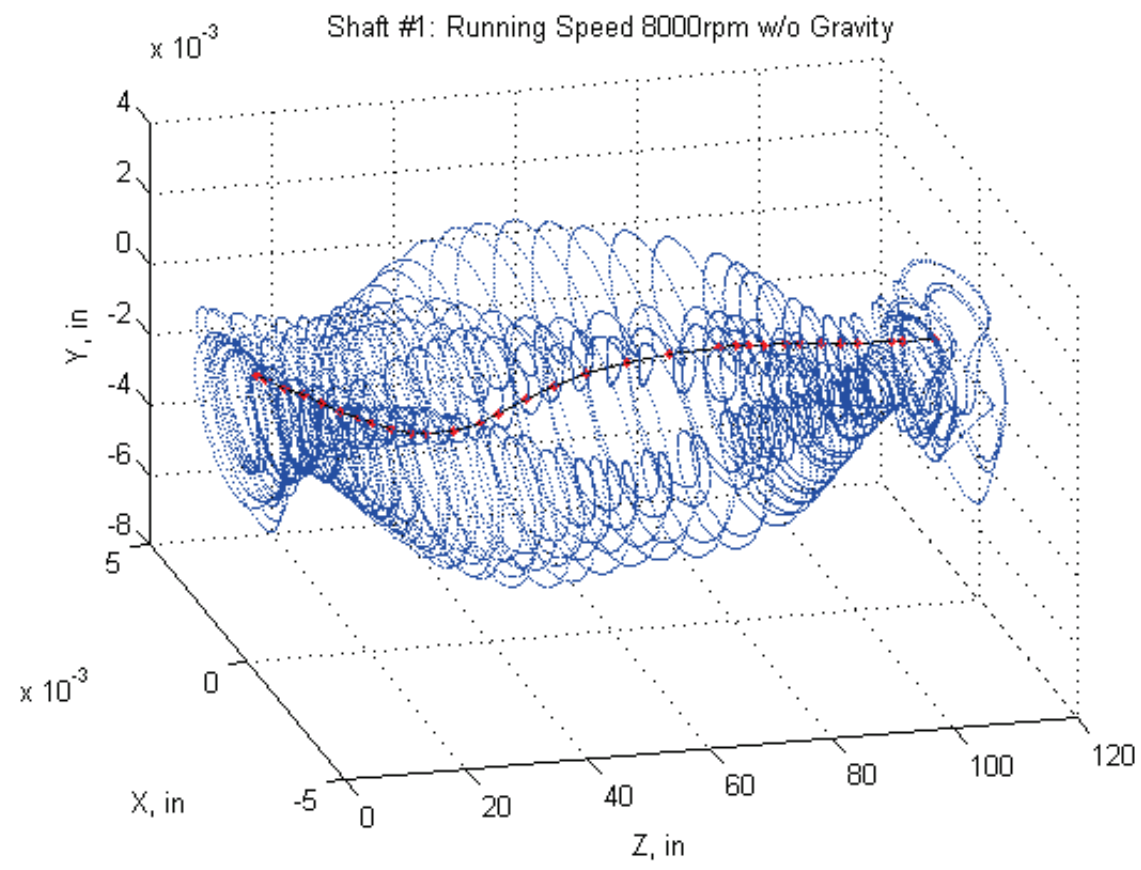

Figure 5.40 Shaft Response CASE III (Severe Unbalance) w/o Gravity at $9.0 \mathrm{sec}$ 
Shaft \#1: Running Speed 8000rpm with Gravity

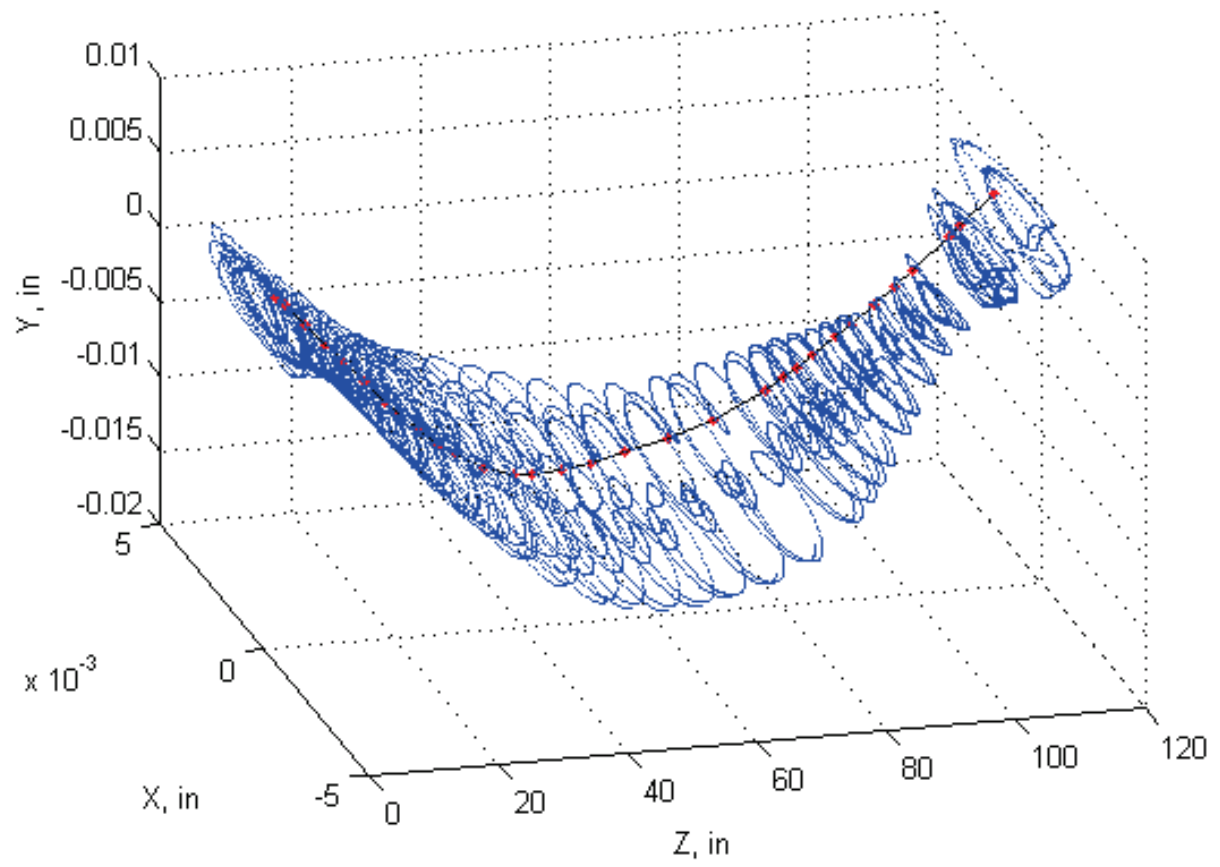

Figure 5.41 Shaft Response CASE III (Severe Unbalance) with Gravity at $9.0 \mathrm{sec}$

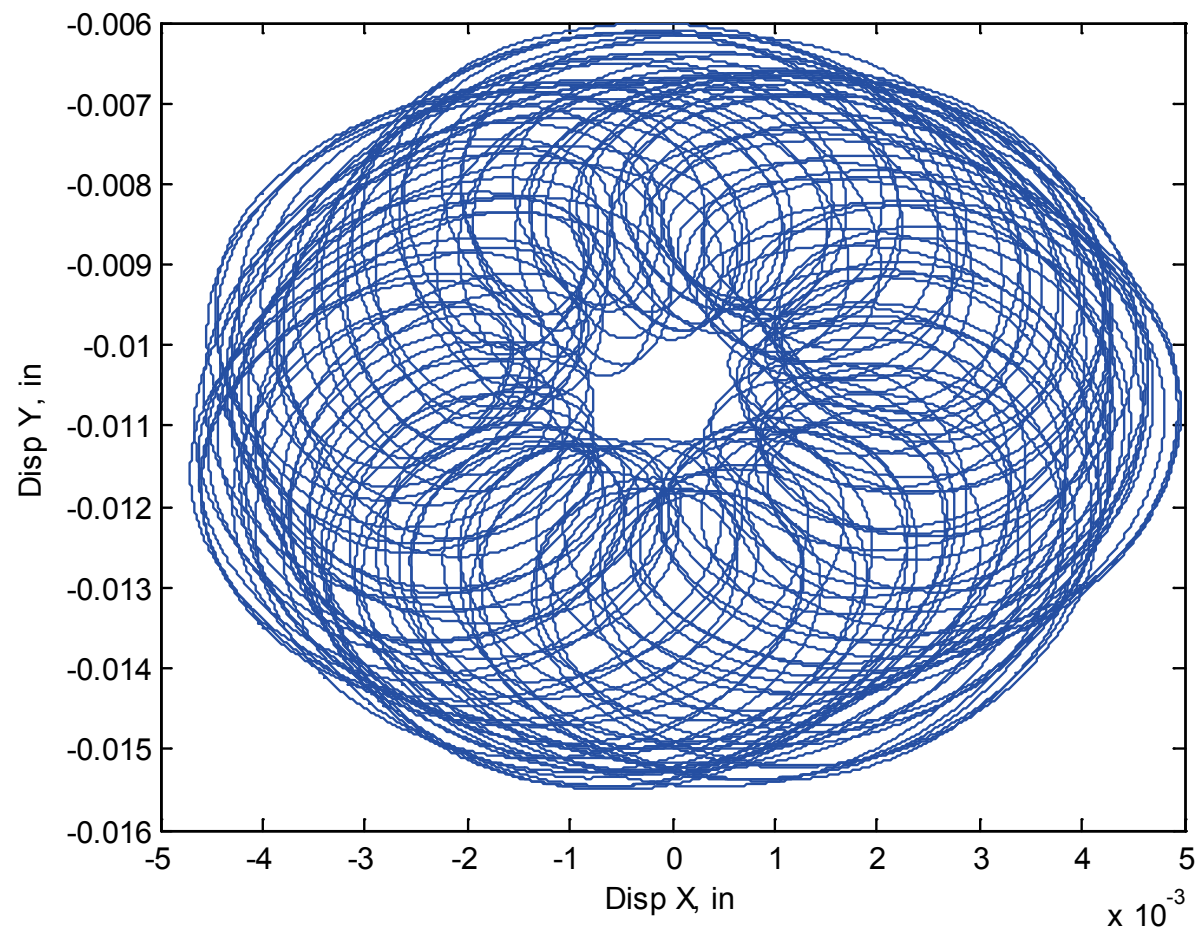

Figure 5.42 Orbit of Disk\#4 (Node\#14) of CASE III, 8.0 9.0 sec 


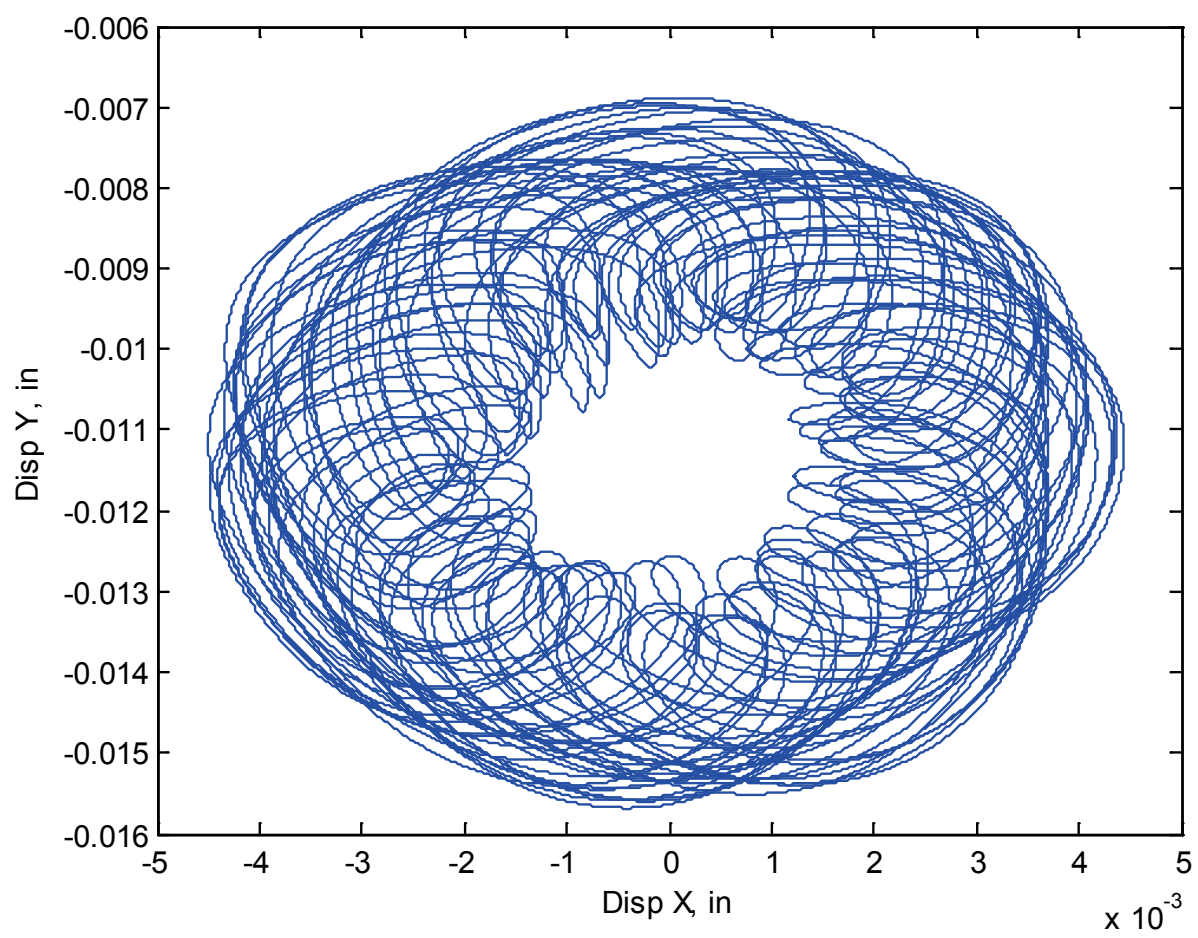

Figure 5.43 Orbit of Disk\#5 (Node\#17) of CASE III, 8.0 9.0 sec

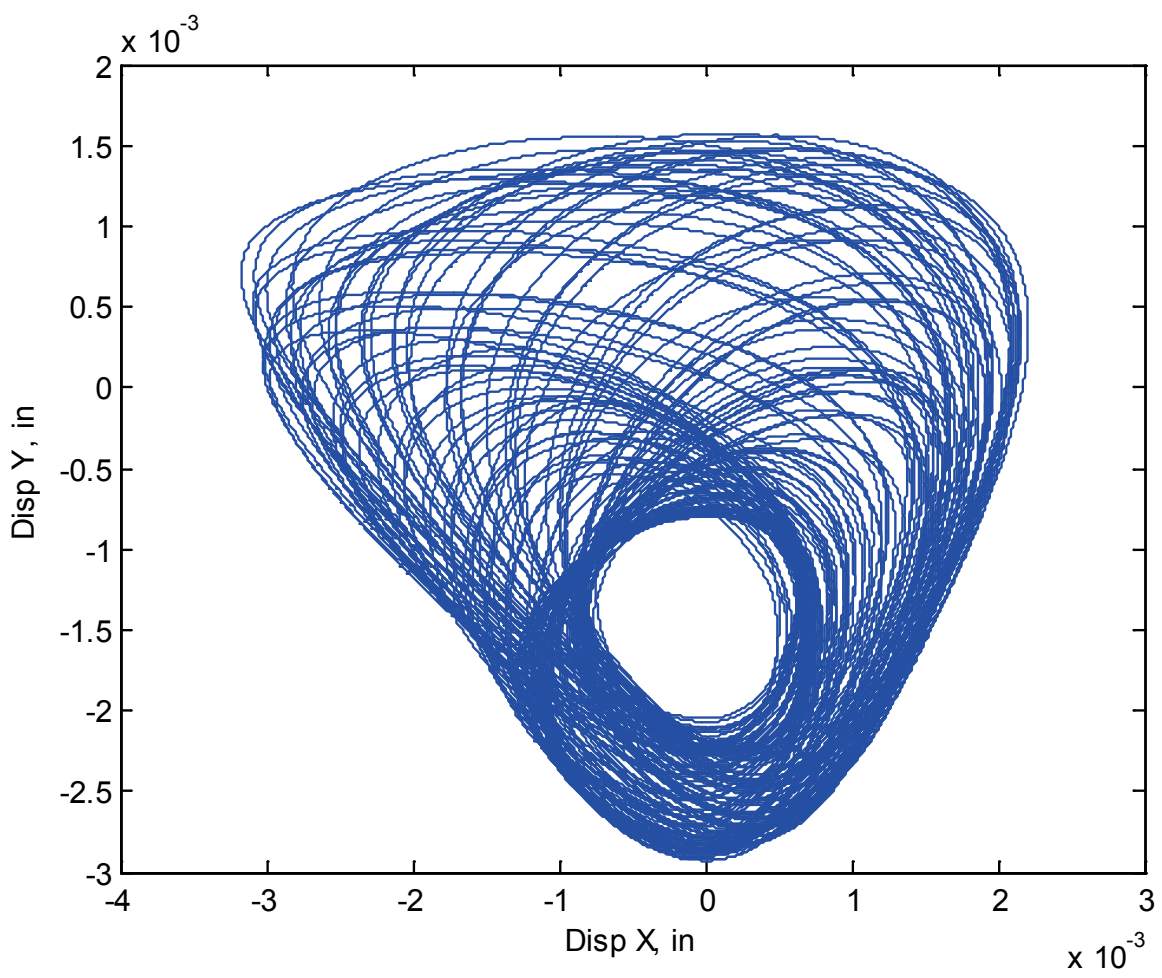

Figure 5.44 Shaft inside the Bearing (node 5) Output of CASE III, 8.0 9.0 sec 

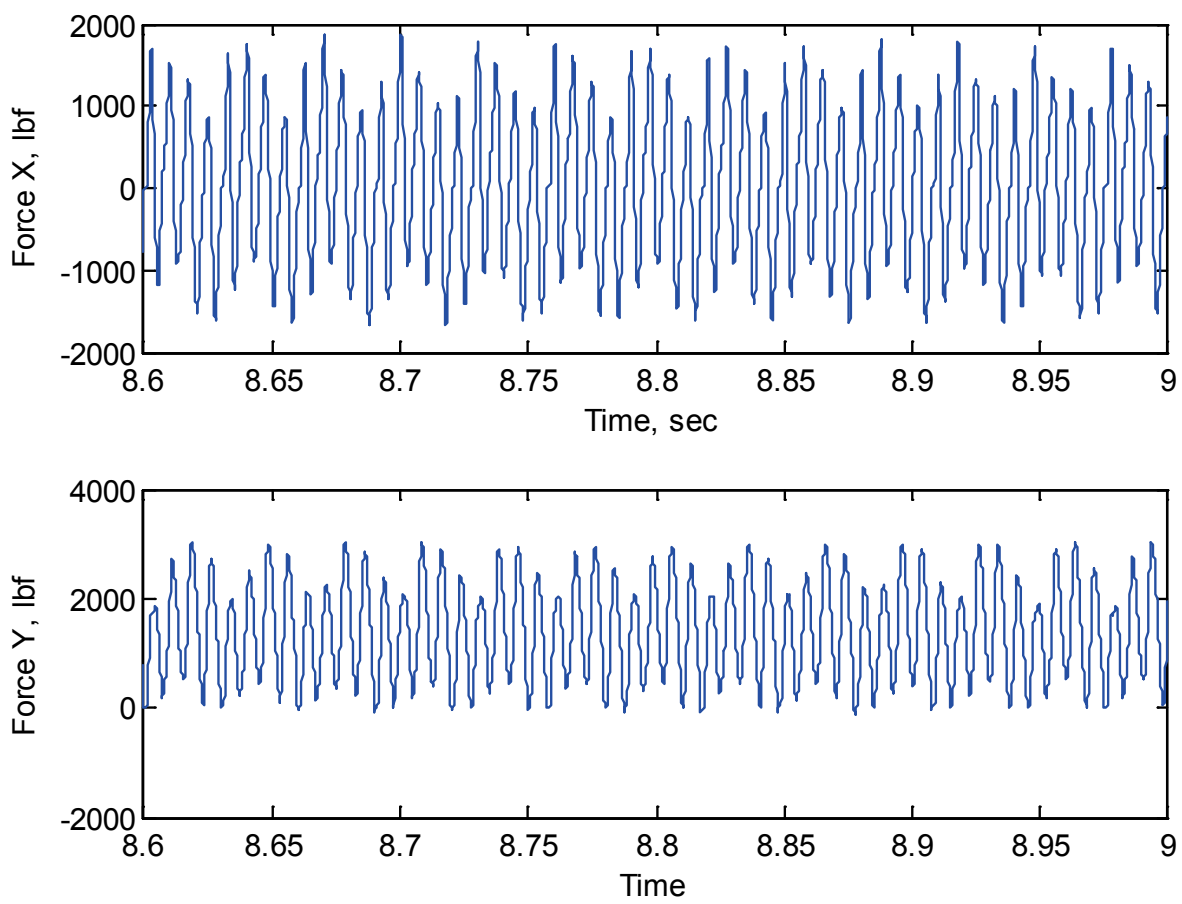

Figure 5.45 Bearing Forces at node 5 Output of CASE III
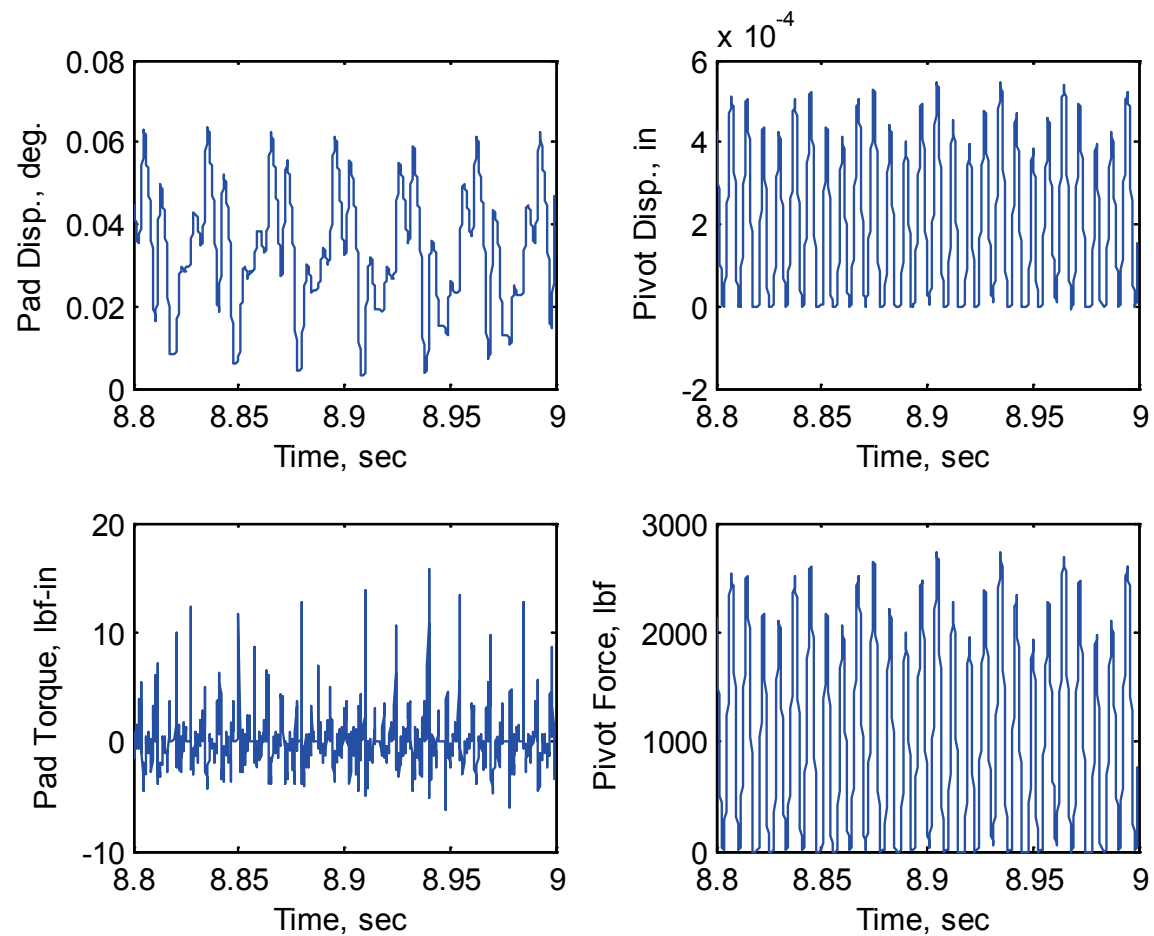

Figure 5.46 Bearing Pad/Piovt\#4 Output of CASE III 

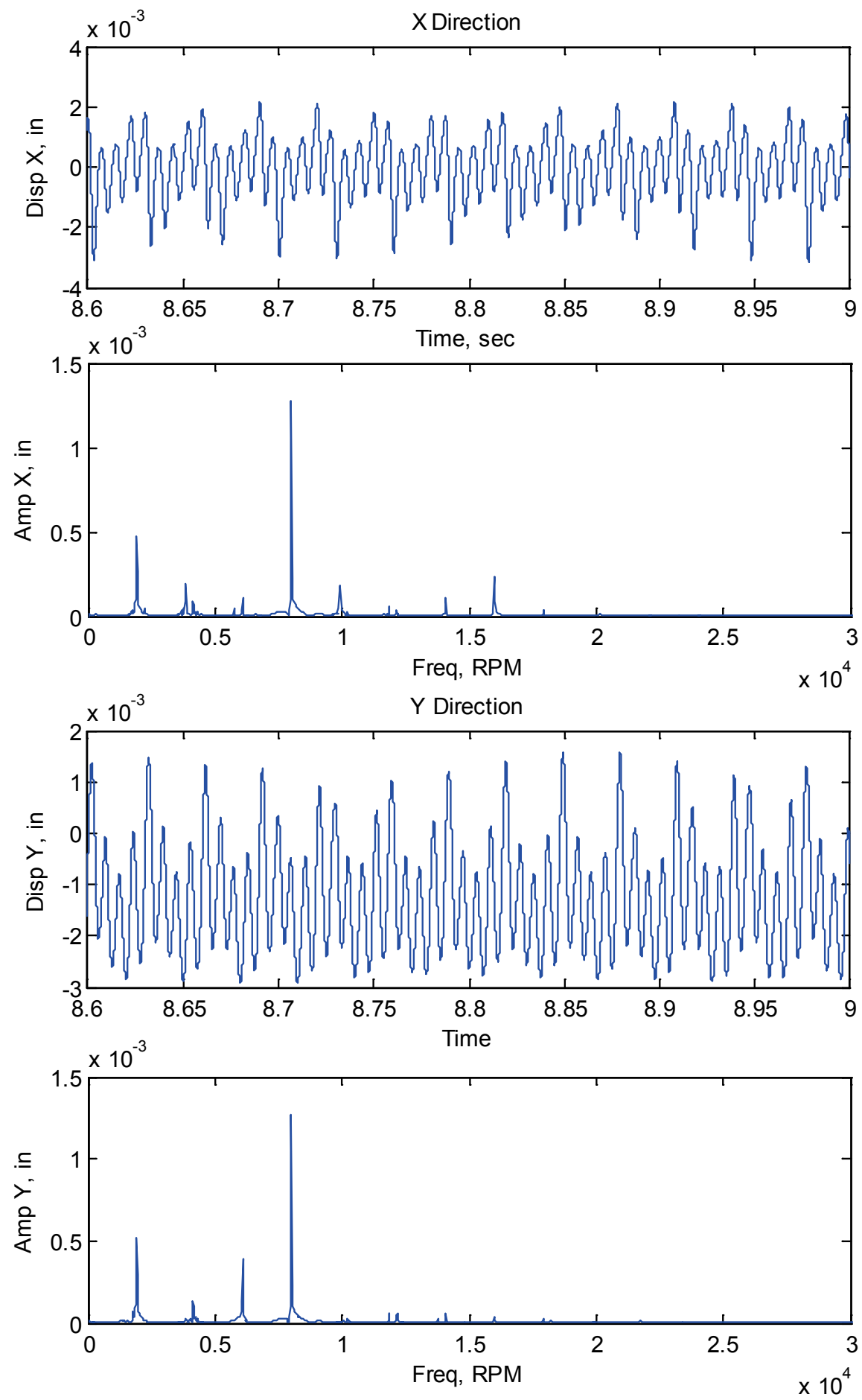

Figure 5.47 FFT Output of Shaft Node 5 inside Bearing 

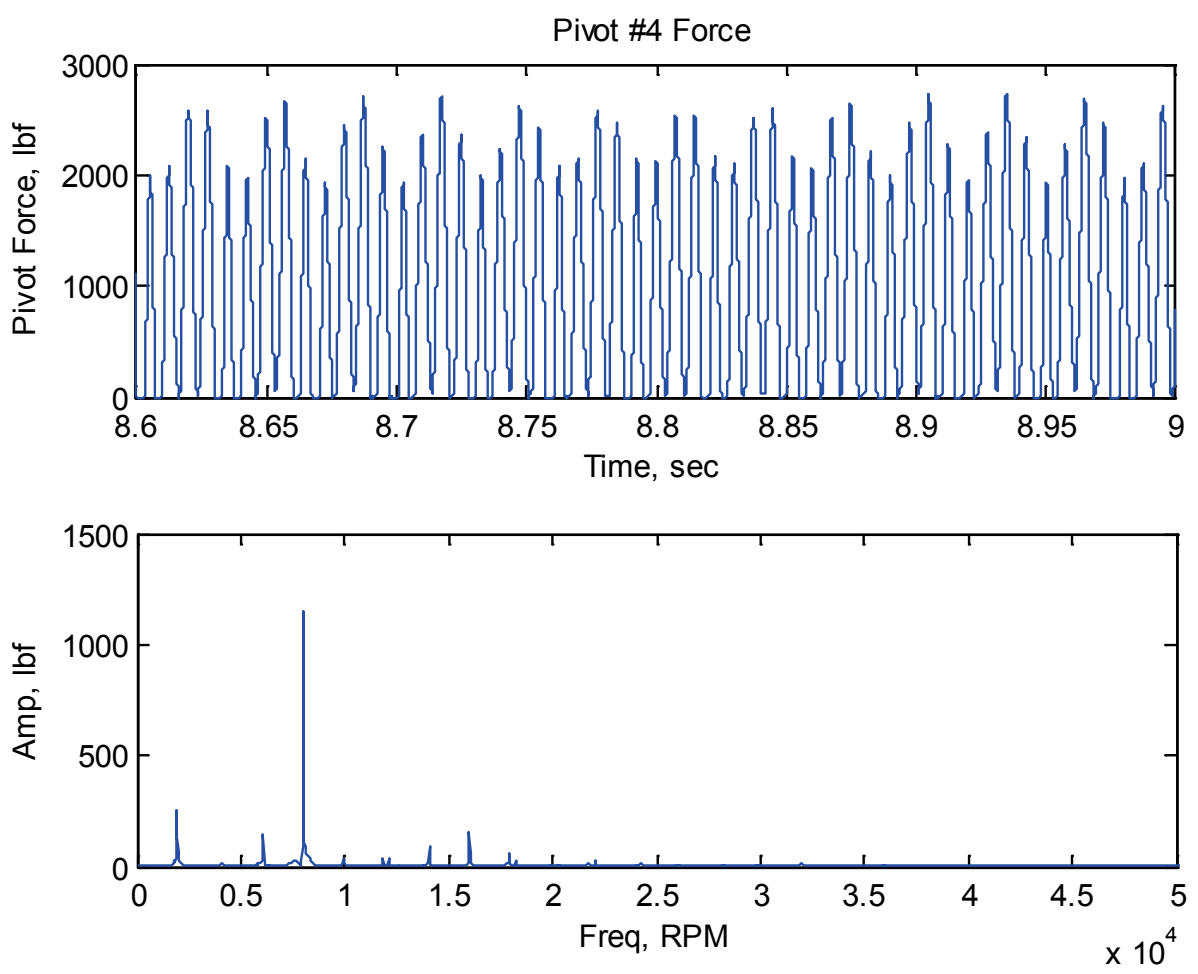

Figure 5.48 FFT Output of Bearing Pad\#4 Force at Node 5
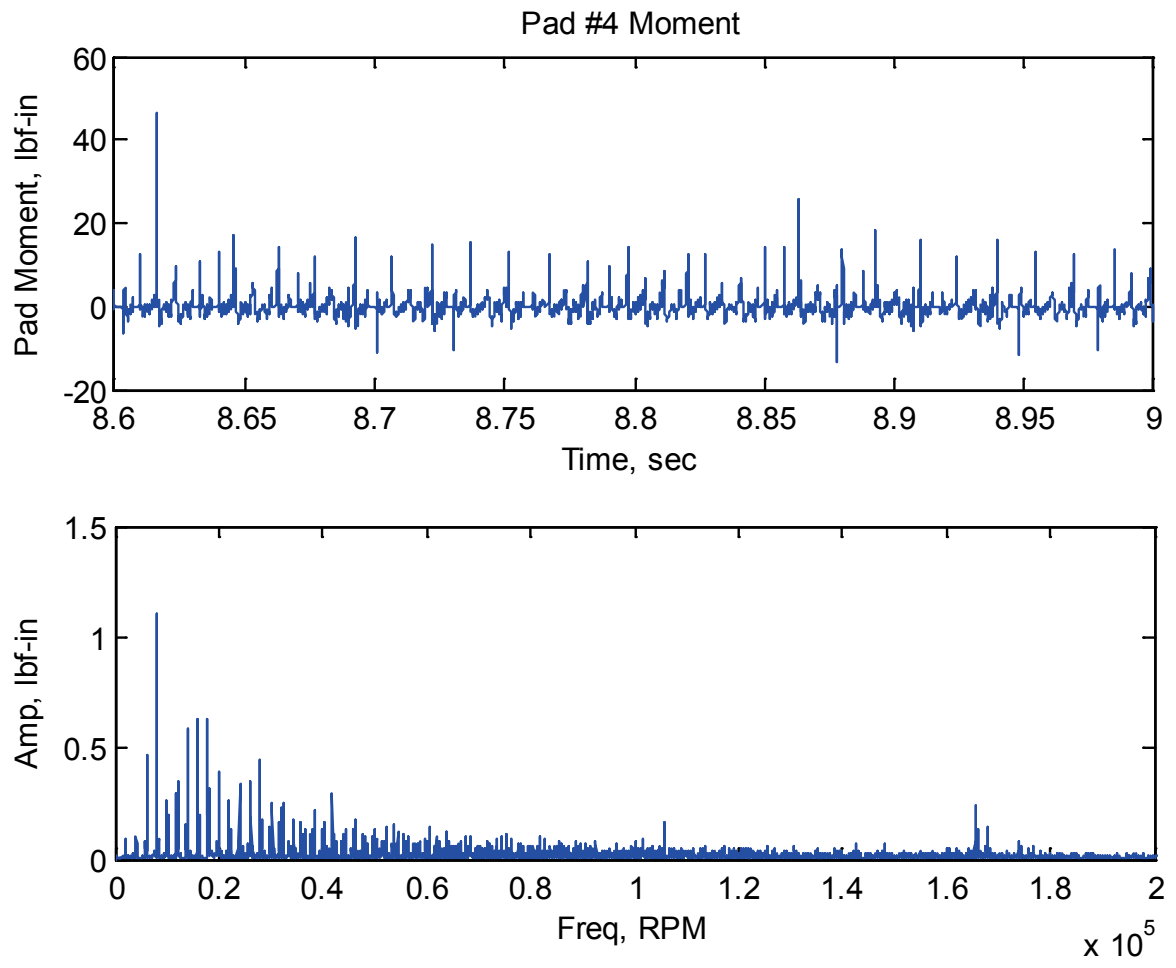

Figure 5.49 FFT Output of Bearing Pad\#4 Moment at Node 5 


\subsection{Transient Analysis of Rotor with Nonlinear Tilting Pad Bearings and Nonlinear Finite Length Squeeze Film Dampers}

In rotor dynamics, the squeeze film dampers are used to shift the critical speeds and/or to increase the damping of the rotor-bearing system. One of the key features introduced by a squeeze film damper is the introduction of support flexibility and damping in the bearing/support structure, particularly for machinery that are designed to operate at super critical speeds. The centering spring, sometimes called a squirrel cage, is part of the conventional design of most squeeze film dampers. The main role of the centering spring is to ensure a central position of the bearing housing in the damper. For the new designed squeeze film dampers, as shown in Fig. 5.50, the centering spring is often removed to achieve shorter rotors, because the central spring geometry takes almost the same length as the damper.

In this section, the nonlinear finite length squeeze film dampers, which are different with the short dampers introduced in Chapter 4, are introduced into the rotor-bearing system to increase the dynamic stability of the system. The transient analysis is conducted to study the response of an 8-stage back to back compressor model supported with 4-pad tilting pad bearings and finite length squeeze film dampers without centering spring or assumption of a circular orbit. The rotor-bearing system has been shown in Fig.

5.27. The nonlinear forces of the bearing and the damper are calculated through the integration of the pressure profiles at each time step. The pressure profiles of the bearings and the dampers are calculated by solving the Reynolds equations. The method of solving the pressure profile using the finite element method has been introduced in Chapter 3 . 

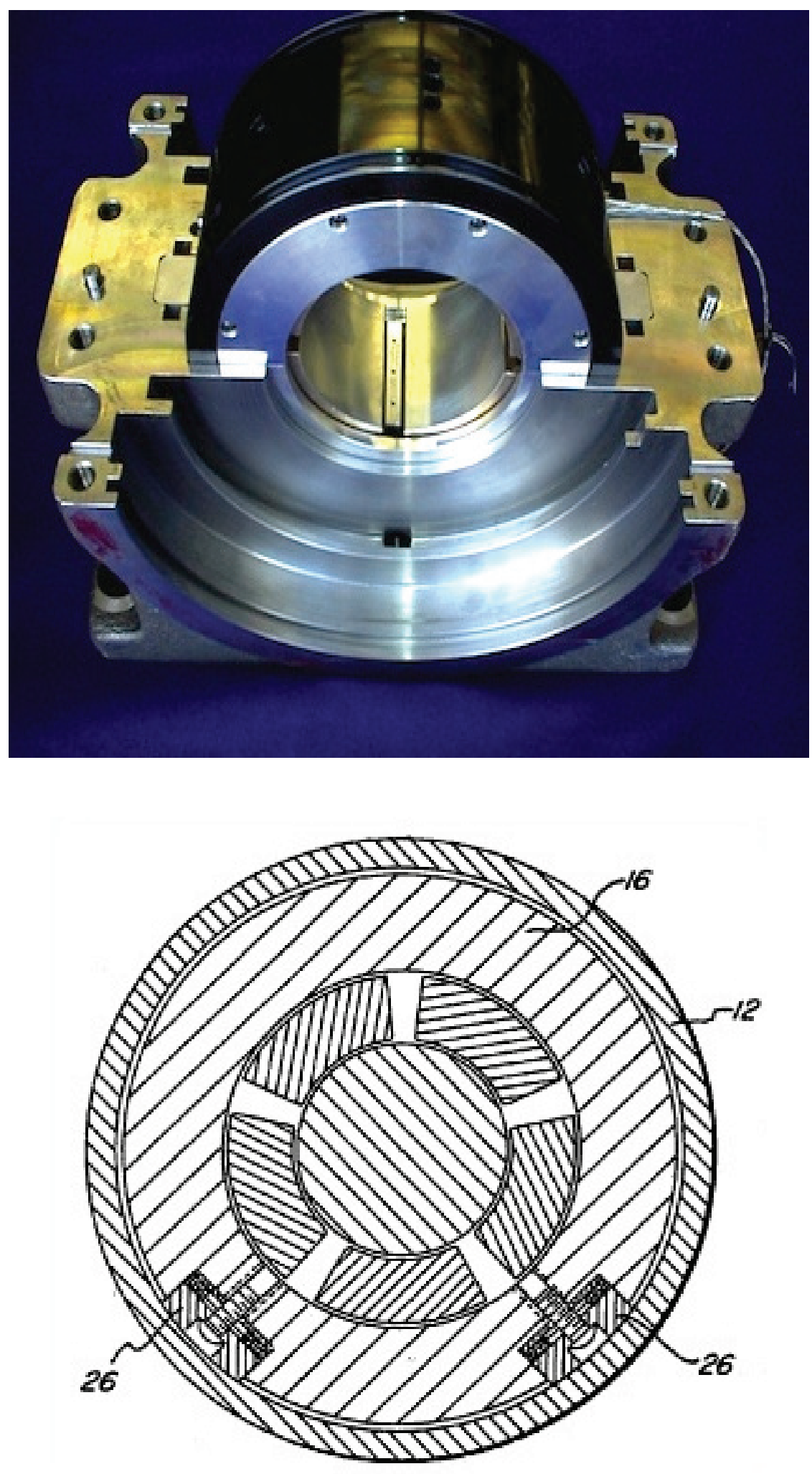

Figure 5.50 Squeeze Film Damper with Tilting Pad Bearing Inside, RMT Inc 
The bearing-damper system is similar to that in Fig. 5.50, but the bearing is a 4-pad, load between pads (LBP) tilting pad bearing. The bearing parameters are listed in Table 5.1, and the finite length squeeze film damper information is listed in Table 5.6. Normally the same lubricant is used for both the bearings and the dampers, but different viscosities, which are the function of the lubricant temperature, are applied here. For this bearing-damper system, the lubricant temperature inside the bearings is somewhat higher than that inside the dampers due to different locations (the bearing is inside the damper), length-diameter ratios ( 0.6 for the dampers vs. 1.0 for the bearings), and clearances (20 mil for the dampers vs. 6 mil for the bearings).

Table 5.6 Finite Length Squeeze Film Damper Information

\begin{tabular}{l|l|l}
\hline \multicolumn{3}{l}{ Type: Finite Length Squeeze Film Damper } \\
\hline Property & Value & Unit \\
\hline Weight(Inside Damper) & 60 & lbf \\
\hline Length & 6 & in \\
\hline Diameter & 10 & in \\
\hline Clearance & 0.02 & in \\
\hline Viscosity & $5.8 \mathrm{E}-6$ & lbf-s $/$ in $^{2}$ \\
\hline
\end{tabular}

The pressure profile and the nonlinear force of a squeeze film damper are calculated using Eq. (3.4.25, 3.4.29 and 3.4.30). The pressure profiles of the finite length squeeze film damper with different mesh densities are shown in Fig. 5.51. From the figure, the pressure at boundary $(\theta=0 \& \theta=2 \pi)$ is continues, even at the condition of lower mesh density of 10 elements in circumferential direction. With the total number of elements increases, the pressure profile going through the boundary is continues and smoother. The finite length squeeze film damper forces vs. the total elements in circumferential direction are shown in Fig. 5.52. The damper force converges when the total number of 
elements is greater than 40 . Because the nonlinear forces have to be calculated according to the instantaneous displacements and velocities at each time step, the total number of element in circumferential direction for the squeeze film damper needs to be chosen carefully. In this example of the 8-stage back to back industrial compressor model, the total number of elements to calculate the finite length damper pressure profile at each time step is 60 .

To describe the behavior of the bearing housing inside the damper, an additional node with 2 DOF ( $x$ and $y$ directions) for each damper is added into the equations of motion as described in Chapter 3. As mentioned in last section (Section 5.4), when the unbalance increases to 32 times of the first unbalance, the response cannot be calculated because of touching between the bearing pads and the shaft when there is no additional damper. In general, the squeeze film dampers introduce support flexibility and damping into the structure to lower transmitted forces and enable longer bearing life. In this section, the nonlinear finite length squeeze film dampers are introduced to avoid possible touching between the bearing and the shaft under the more severe unbalance conditions.

To calculate the nonlinear forces from both the tilting pad bearings and the finite length squeeze film dampers, 30 elements per pad and 60 elements per damper in circumferential directions are used to keep the force calculation accurate and to save computational time at the same time. The polynomial axial pressure profile power $n$ is 2.25 for the tilting pad bearings $(\mathrm{D} / \mathrm{L}=1)$ and 2.15 for the finite length squeeze film dampers $(\mathrm{D} / \mathrm{L}=0.6)$. The rotor-bearing-damper system has a total DOF of 144 . Of those DOF, $124(31 * 4)$ are from the rotor and built on lumped masses, $16(8 * 2)$ are the two bearings and $4(2 * 2)$ are from the two squeeze film dampers. 
Normalized Pressure, Element $=10$

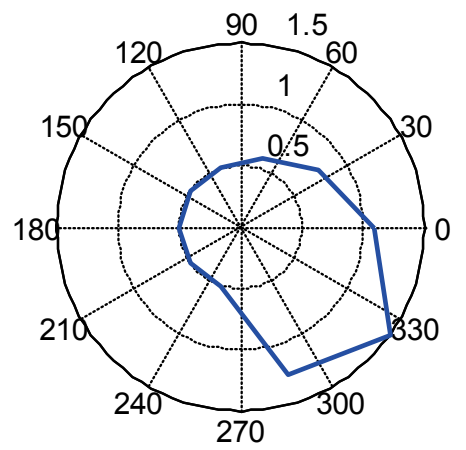

Normalized Pressure, Element $=60$

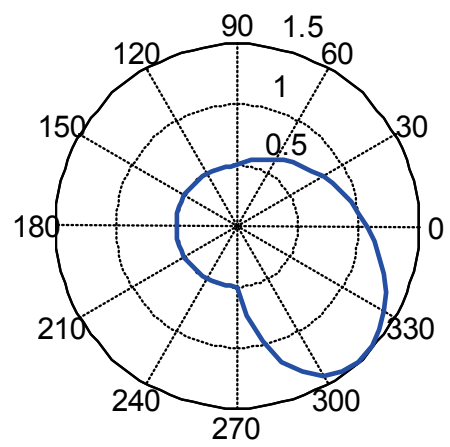

Normalized Pressure, Element $=30$

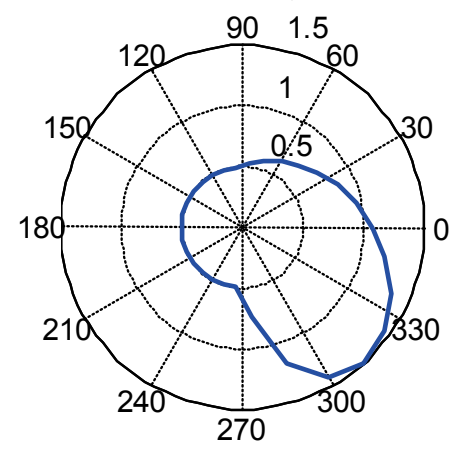

Normalized Pressure, Element $=90$

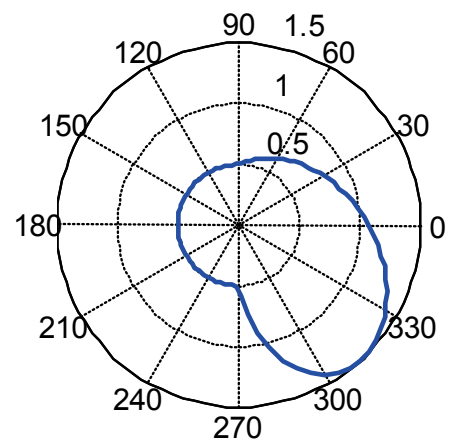

Figure 5.51 Pressure Profile with Increasing Element Density

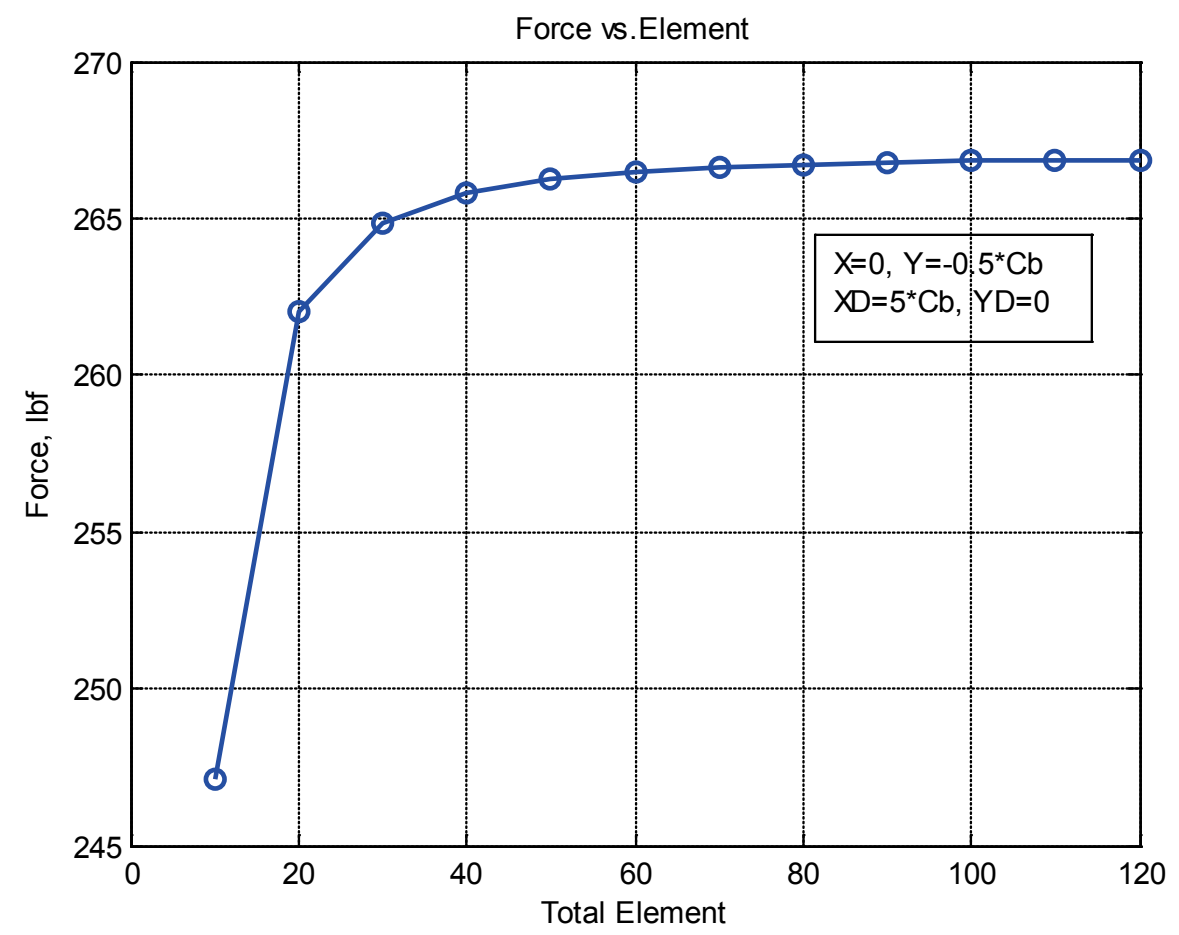

Figure 5.52 Damper Forces vs. Elements 
The rotor behavior after adding two finite length squeeze film dampers is evaluated with the constant running speed of $8,000 \mathrm{rpm}$. The server unbalances, 91.2 oz-inch (which is 32 times of the first unbalance, 2.85 oz-inch), are applied to the two central disks at node \#14 (45.6 oz-in, $0^{\circ}$ ) and node \#17 (45.6 oz-in, $0^{\circ}$ ). The integration time step, $\Delta \mathrm{t}$, which is calculated from the maximum natural frequency of the linear rotor, is same as used in Section $5.4--8 \times 10^{-6}$ second. The initial nodal displacements inside the bearings are taken as zero. The initial positions of all other shaft nodes are calculated based on the gravitational force vector under zero running speed. The initial velocities of all shaft nodes are taken as zeros. Additional nodes for the bearing pads/pivots and the squeeze film dampers are assembled to have zero displacements and zero velocities. At each time step, the bearing and damper forces need to be calculated according to the nodal displacements and velocities of the system, and then applied to the equations of motion. For the shaft-bearing-damper system, the damper force is calculated according to the instantaneous displacements and velocities of the bearing housing inside the damper. However, the bearing force is calculated according to the instantaneous displacements and velocities of both the shaft center inside the bearing and the bearing housing center inside the damper.

The shaft behavior under the $32 \mathrm{x}$ unbalance (91.2 oz-in) is shown in Figs. 5.53\&5.54. Unlike the response in Section 5.4, there is no touching among the shaft, the bearing pads and the squeeze film dampers during transient analysis. The system reaches its steady state after 0.15 second, which is 10 times faster than the rotor without the squeeze film dampers. For the rotor on the tilting pad bearings only, it takes at least 1.5 second to reach the steady state under the first unbalance with the same initial conditions. 


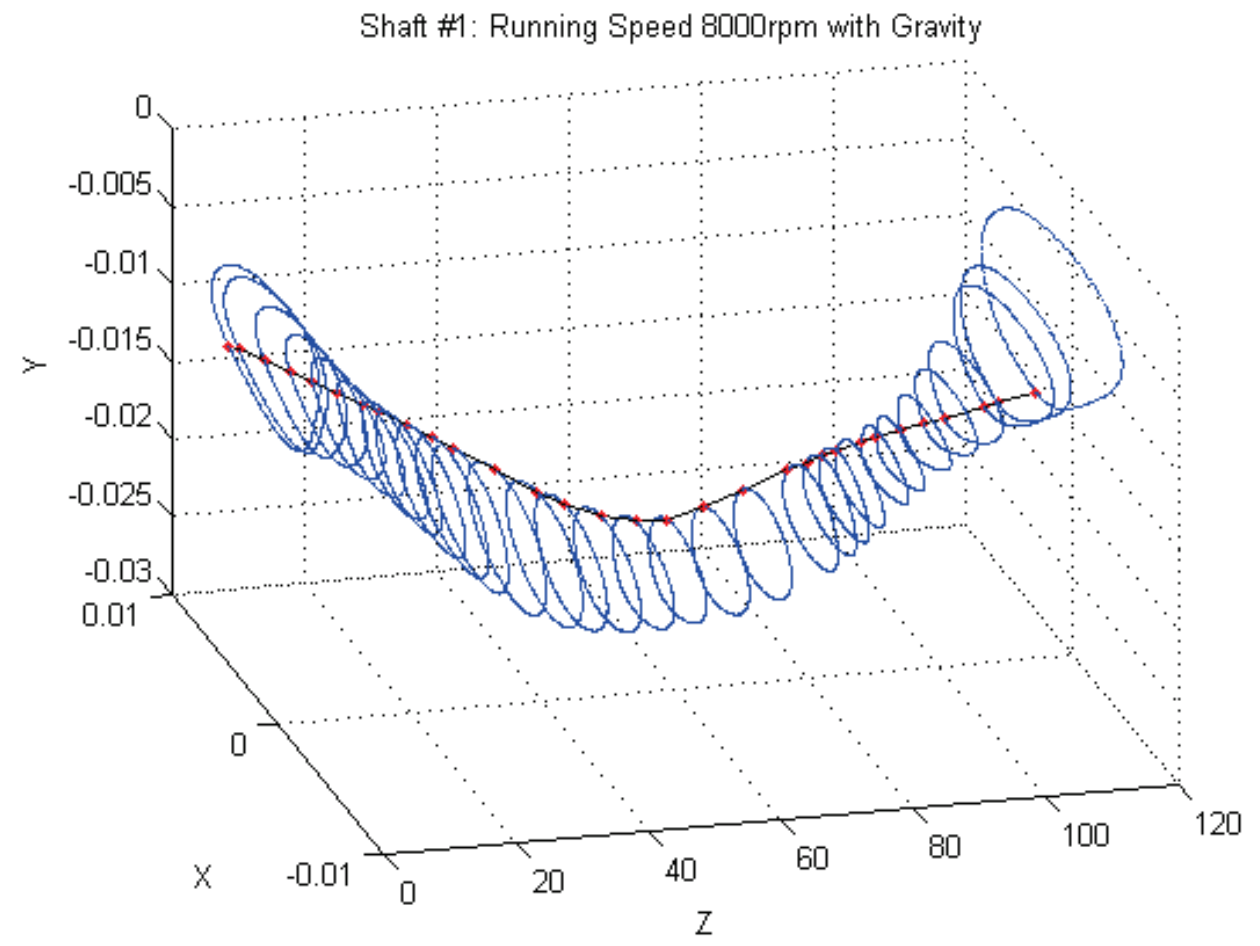

Figure 5.53 Shaft Orbit with Squeeze Film Dampers at $0.5 \mathrm{sec}, 32 \mathrm{x}$ Unbalance

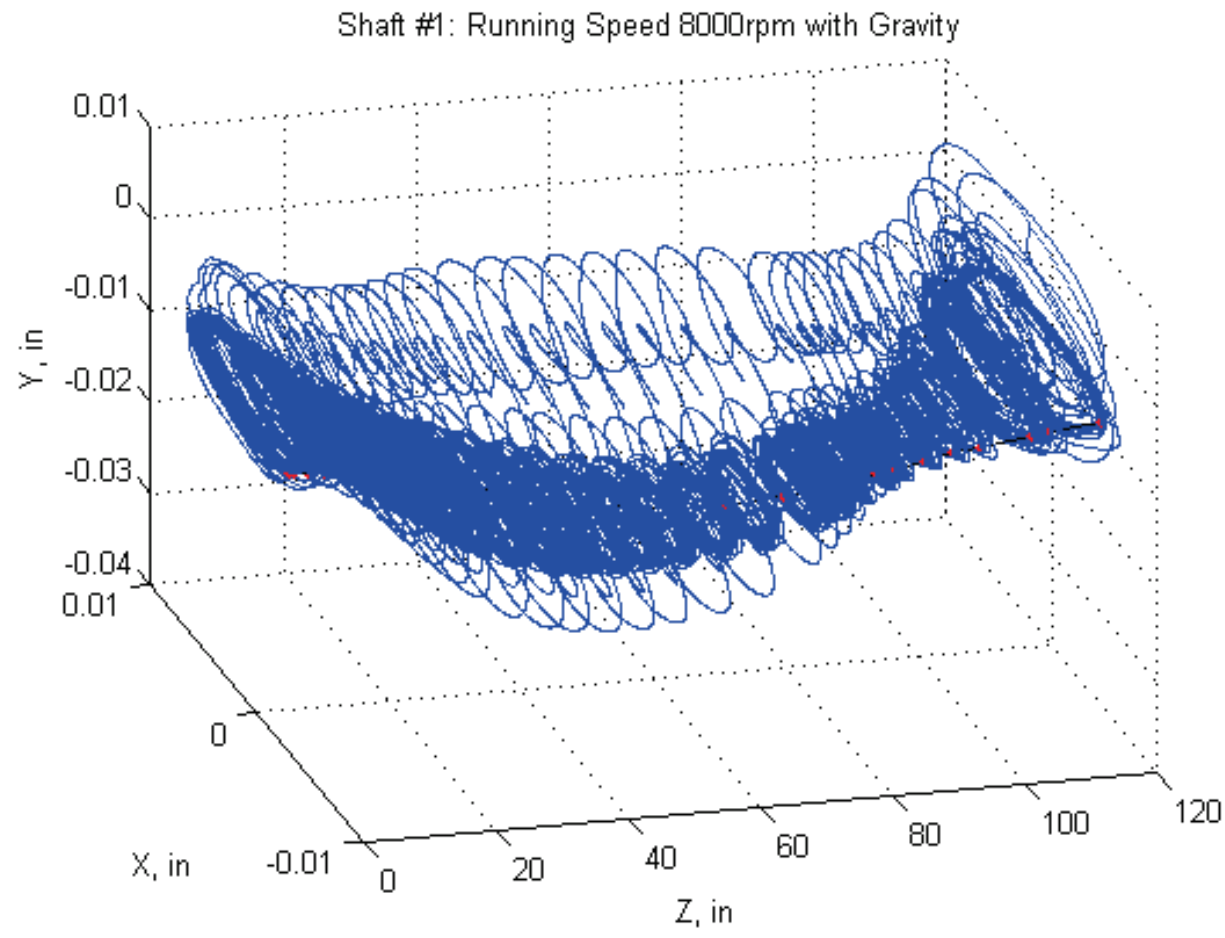

Figure 5.54 Transient Shaft Orbit with Squeeze Film Dampers, 0 0.5 sec, 32x Unbalance 
The response of shaft center inside the bearings (nodes \#5\&\#27) is presented in Figs. 5.55\&5.56. In those figures, the upper two figures are the transient response in the horizontal and vertical directions. The lower left one is the orbit of the shaft center, the same data are used in the lower right figure with the red circle indicates the bearing clearance $\left(C_{b}=6 \mathrm{mil}\right)$. The response of the bearing housings, which are inside the two squeeze film dampers, is shown in Figs. 5.57\&5.58, in which the red circle indicates the damper clearance $\left(C_{d}=20 m i l\right)$. The steady state orbits of the bearing housings are negative in the vertical direction due to the weight of the bearing housing. Since the orbit is not close the bottom of the damper, as discussed in Chapter 4, only synchronous response is shown from all shaft nodes, as shown in Fig 5.53.
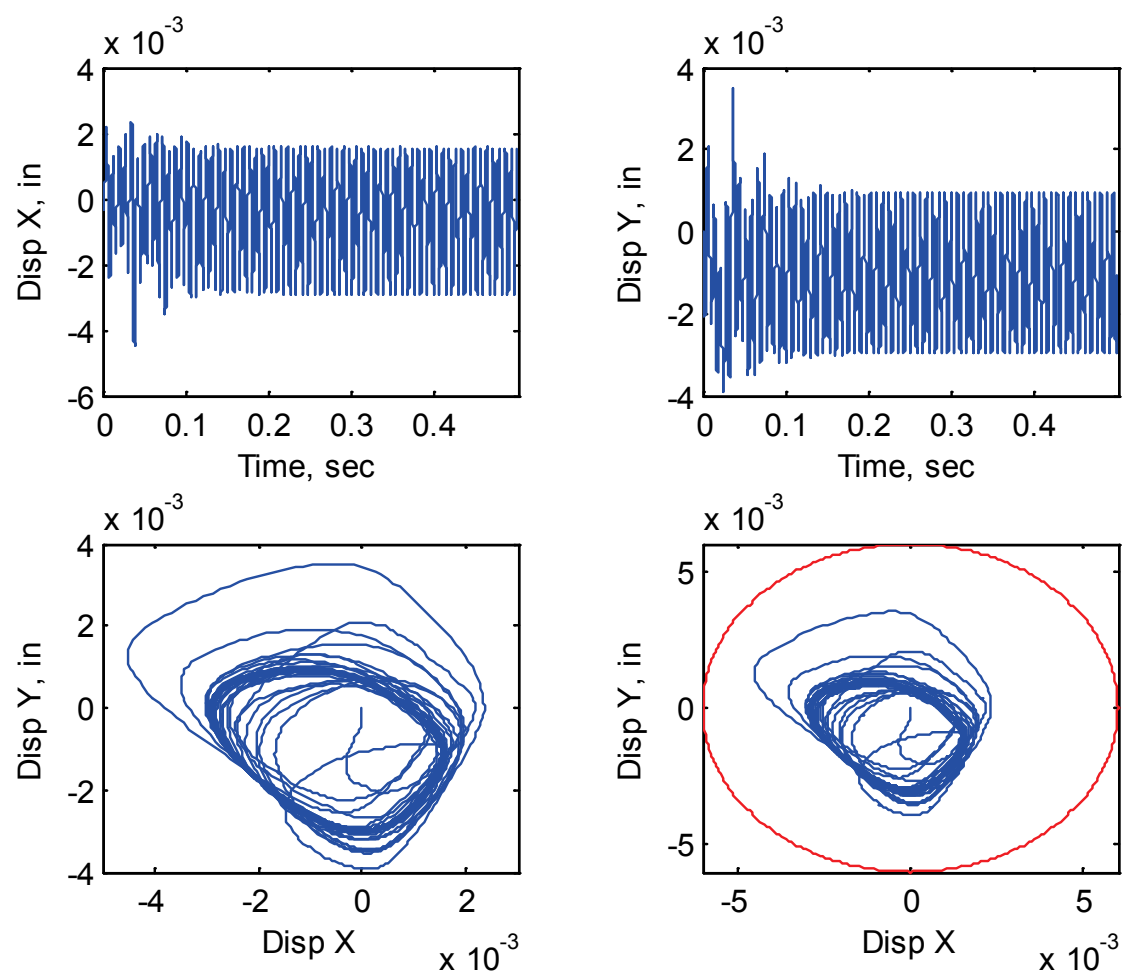

Figure 5.55 Bearing Response at Node 5 under 32x Unbalance 

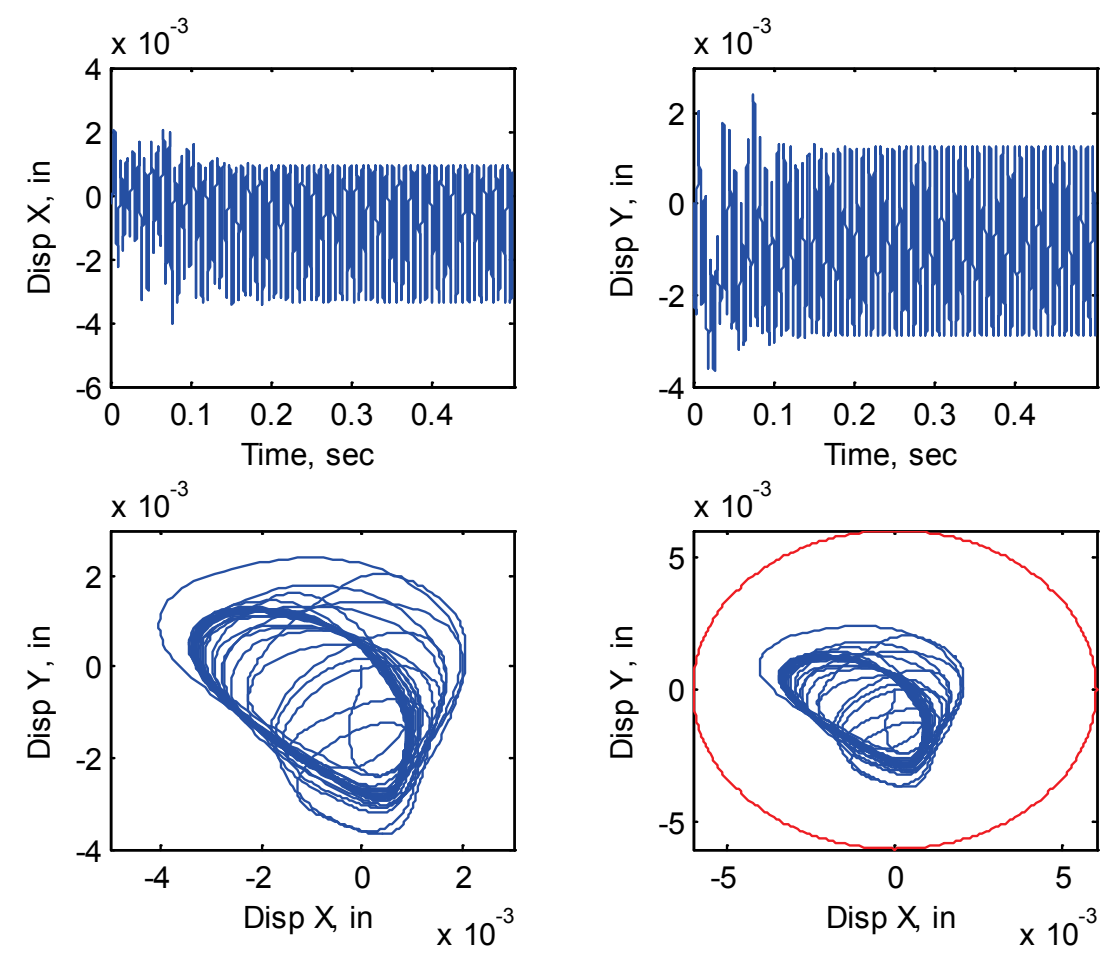

Figure 5.56 Bearing Response at Node 27 under 32x Unbalance
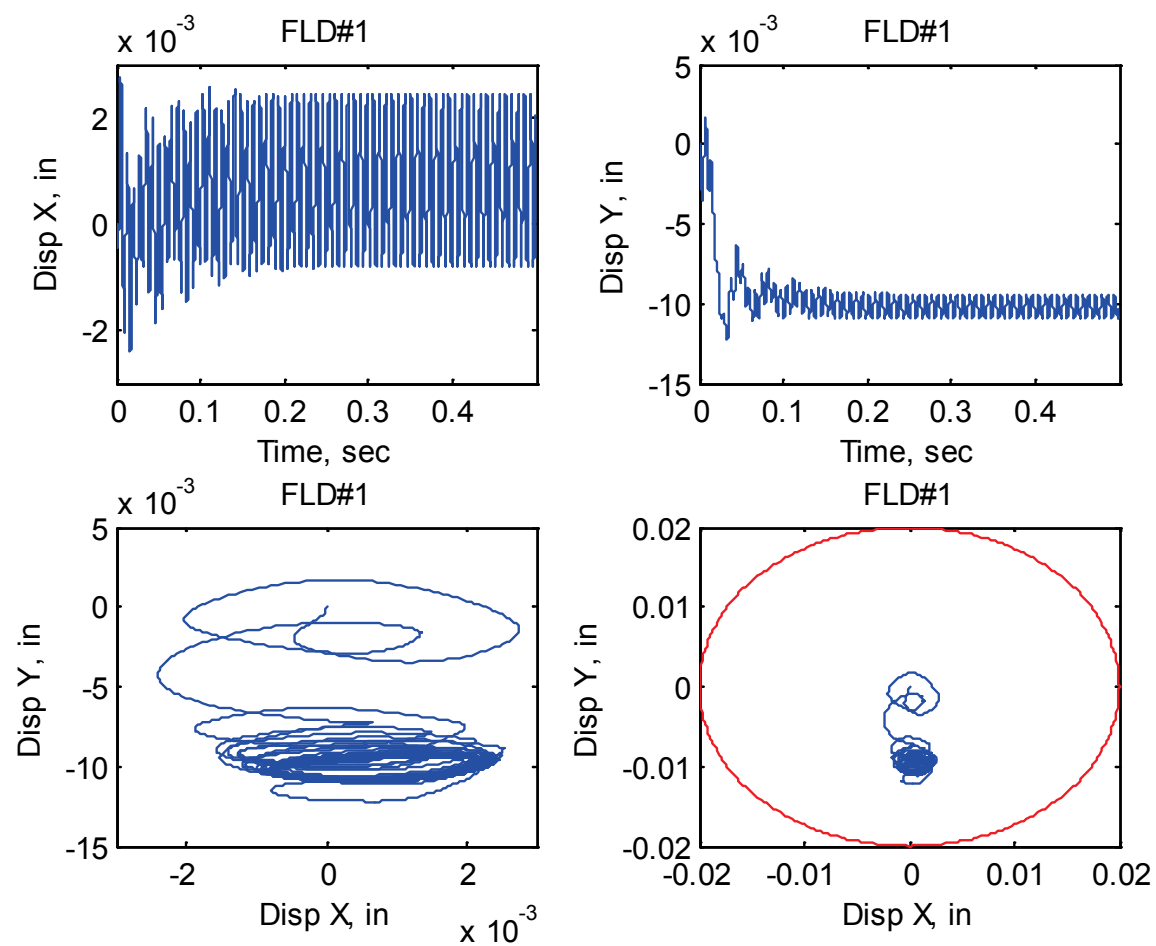

Figure 5.57 Squeeze Film Damper Response at Node 5 under 32x Unbalance 

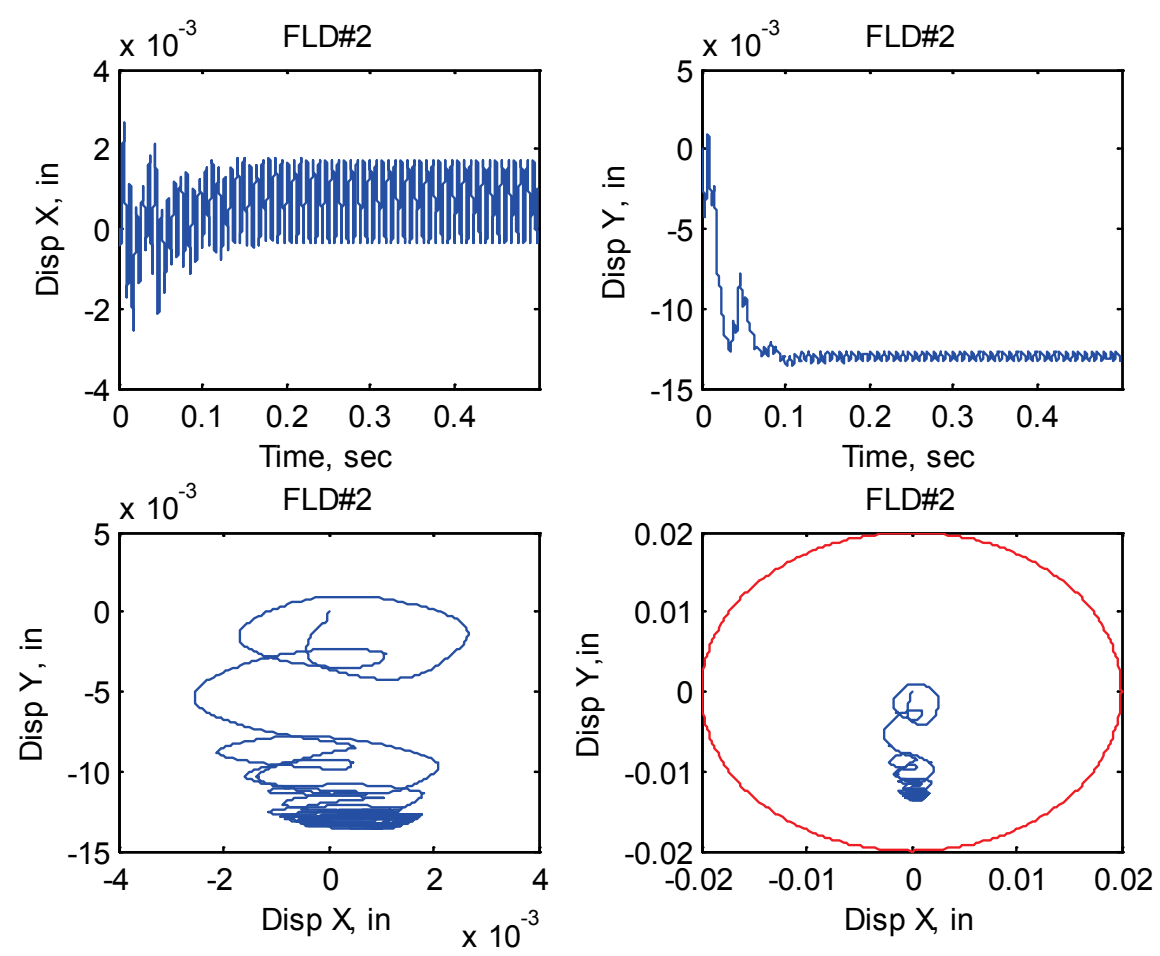

Figure 5.58 Squeeze Film Damper Response at Node 27 under 32x Unbalance

The results of the nonlinear forces at node\#5 and node\#27 from the tilting pad bearings and the finite length squeeze film dampers in time and frequency domains are shown in Figs. 5.59 5.66. Although the response of the whole shaft indicates a synchronous behavior, as shown in Fig. 5.53, the super-harmonic response is presented in the transient output of those nonlinear forces, especially from the frequency domain response. However, since the bearing housing orbits inside the two finite length squeeze film dampers is not close the bottom of the damper, as discussed in Chapter 4, only weakly nonlinear response is shown from those nonlinear forces. The super-harmonic response of the nonlinear bearing and damper forces is from the periodic unloading on the bearing pads. The procedure of periodic unloading on bearing pads/pivots will be explained in the following paragraph according to the response of pad forces and moments. 

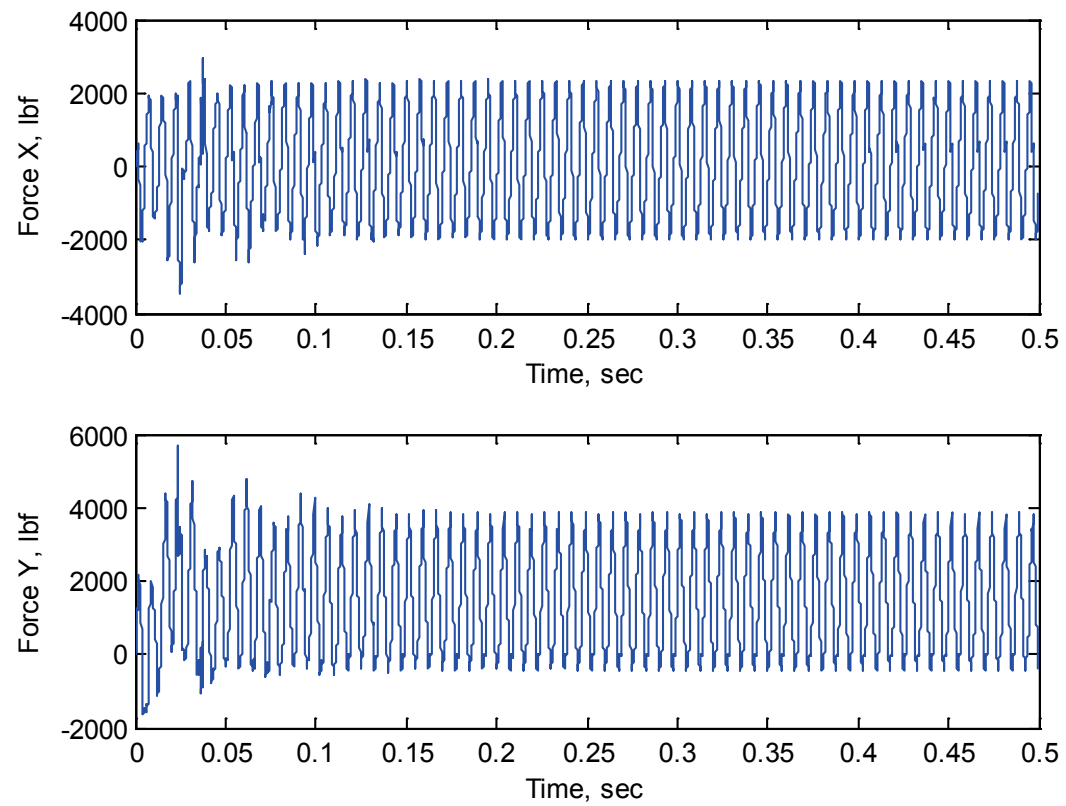

Figure 5.59 Bearing Force at Node 5 under 32x Unbalance
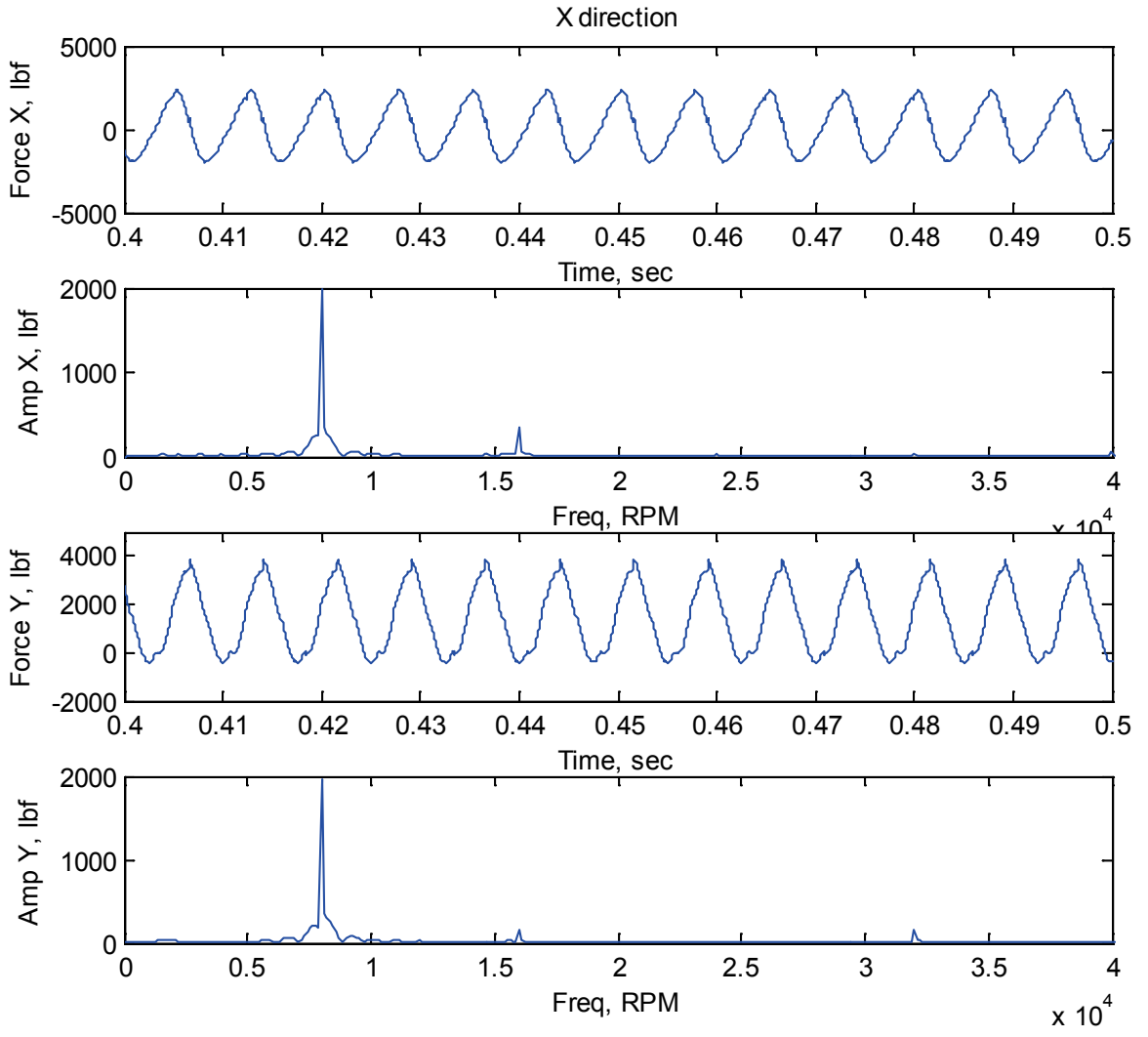

Figure 5.60 FFT of Bearing Force at Node 5 under 32x Unbalance 

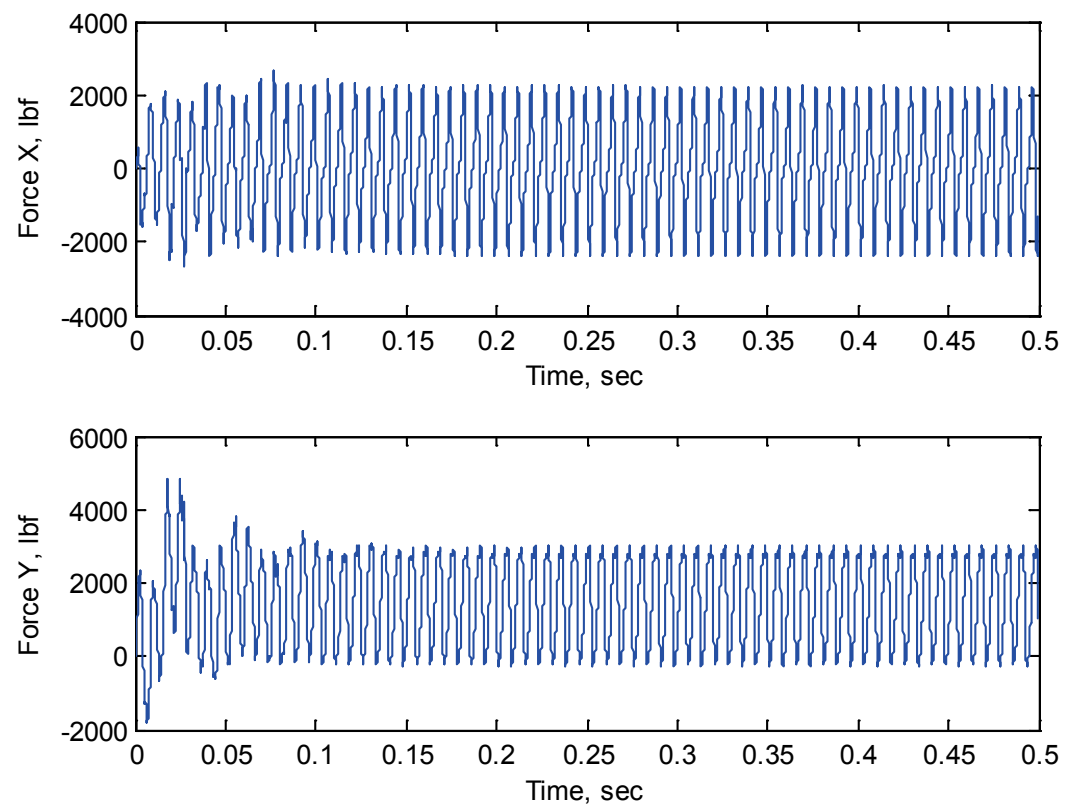

Figure 5.61 Bearing Force at Node 27 under 32x Unbalance
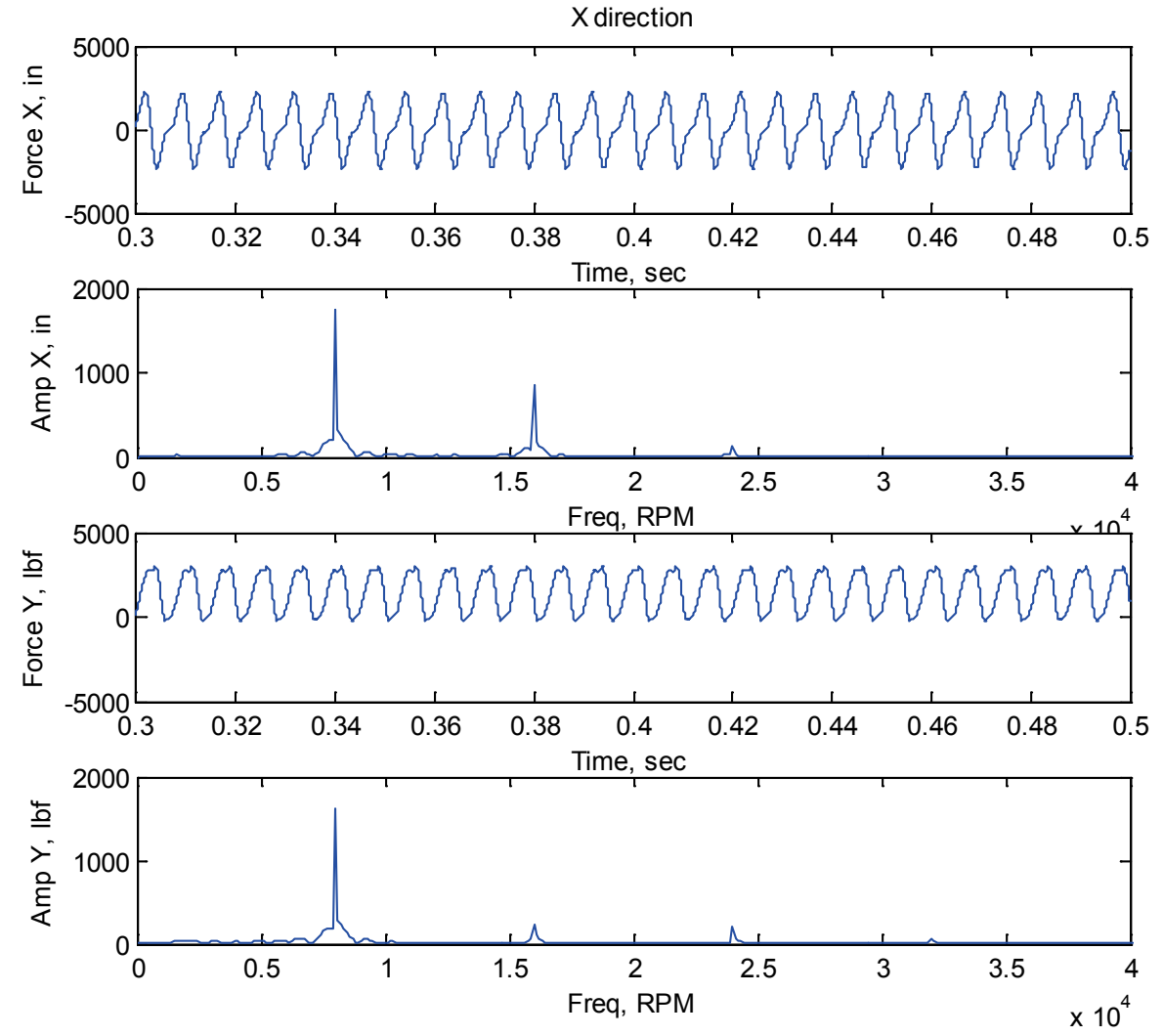

Figure 5.62 FFT of Bearing Force at Node 27 under 32x Unbalance 

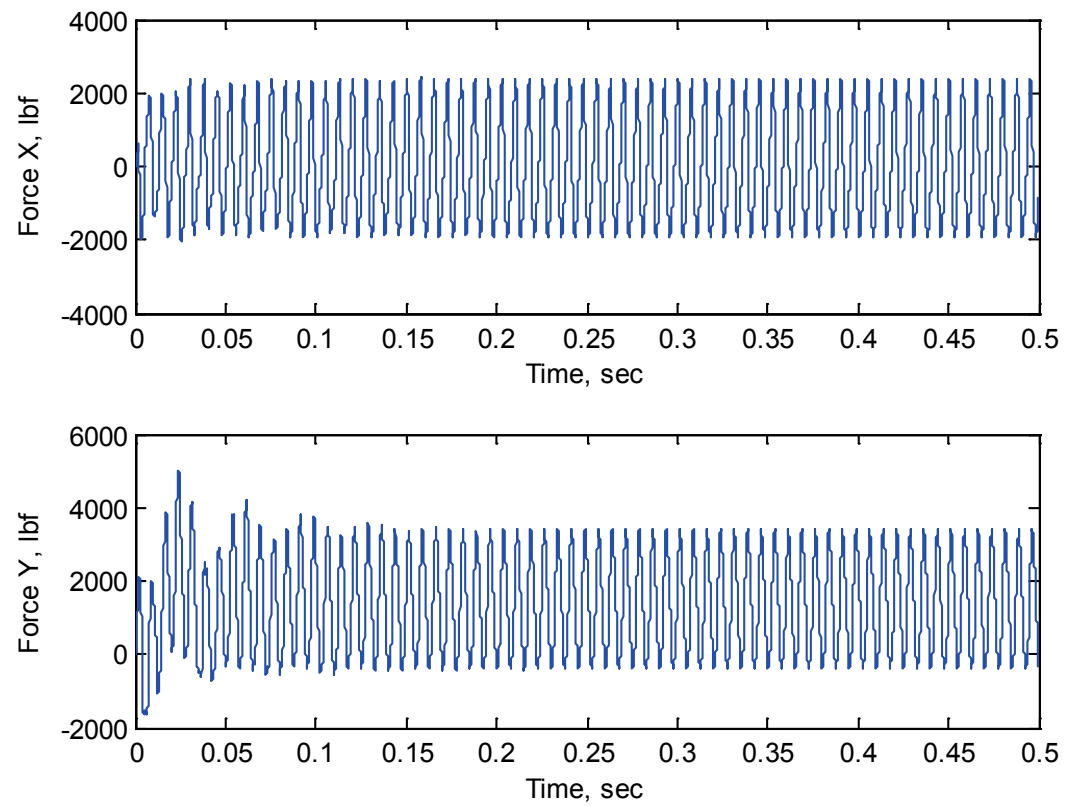

Figure 5.63 Squeeze Film Damper Force at Node 5 under 32x Unbalance
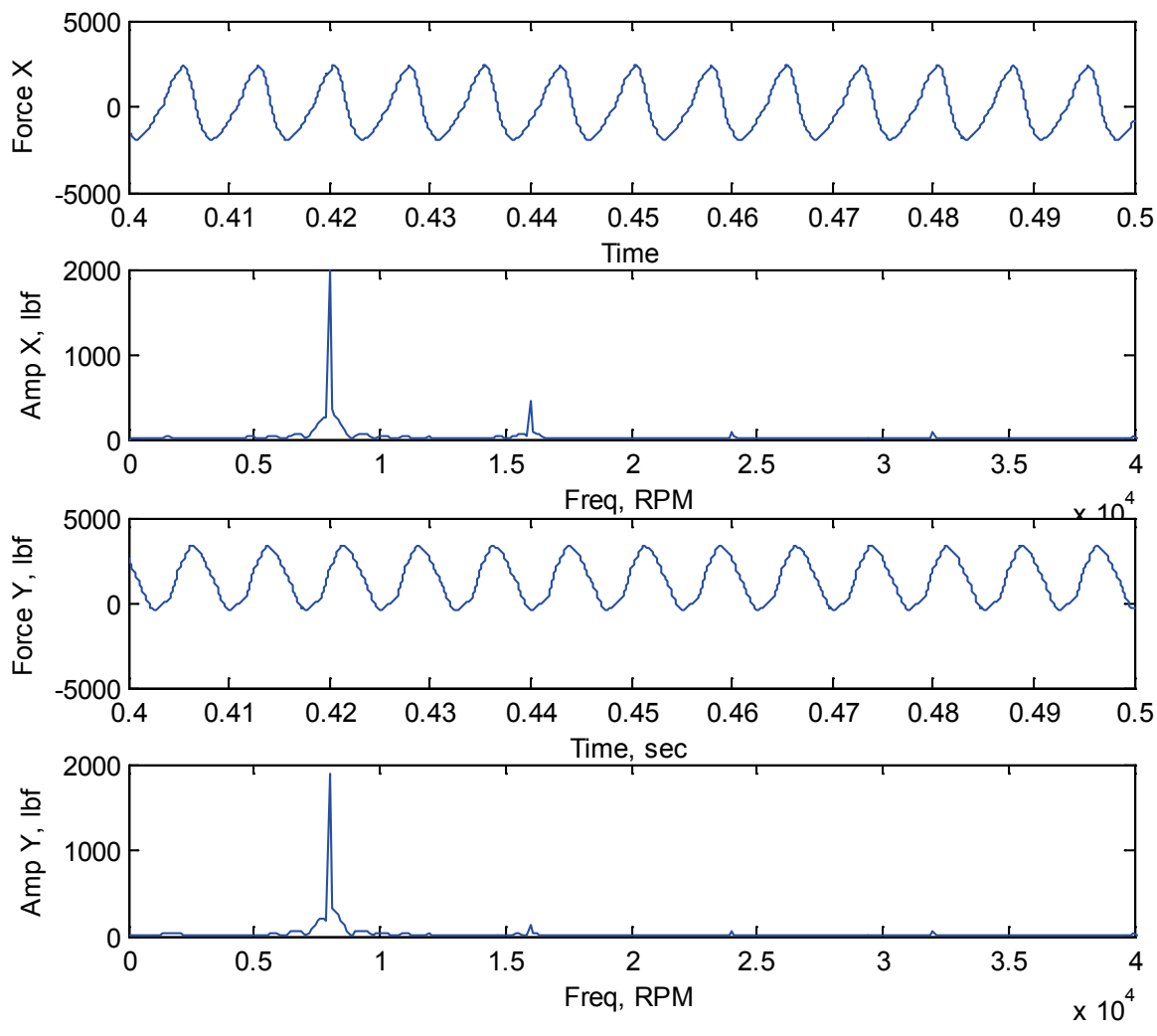

Figure 5.64 FFT of Squeeze Film Damper Force at Node 5 under 32x Unbalance 

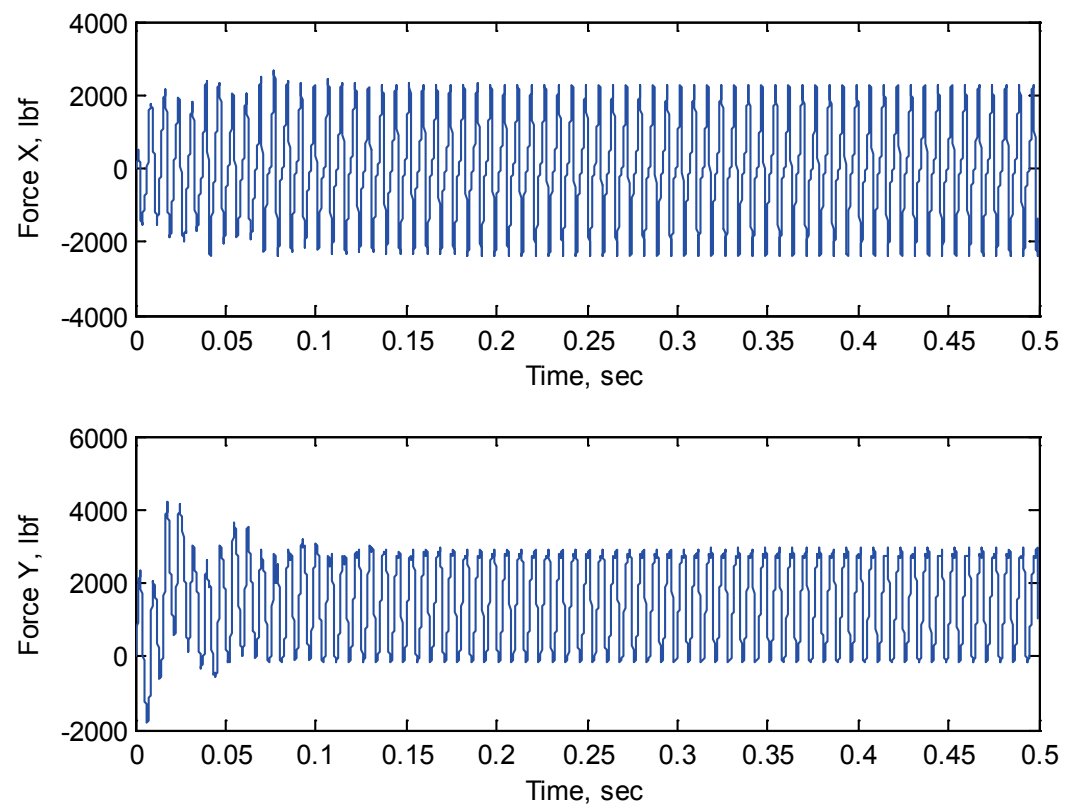

Figure 5.65 Squeeze Film Damper Force at Node 27 under 32x Unbalance
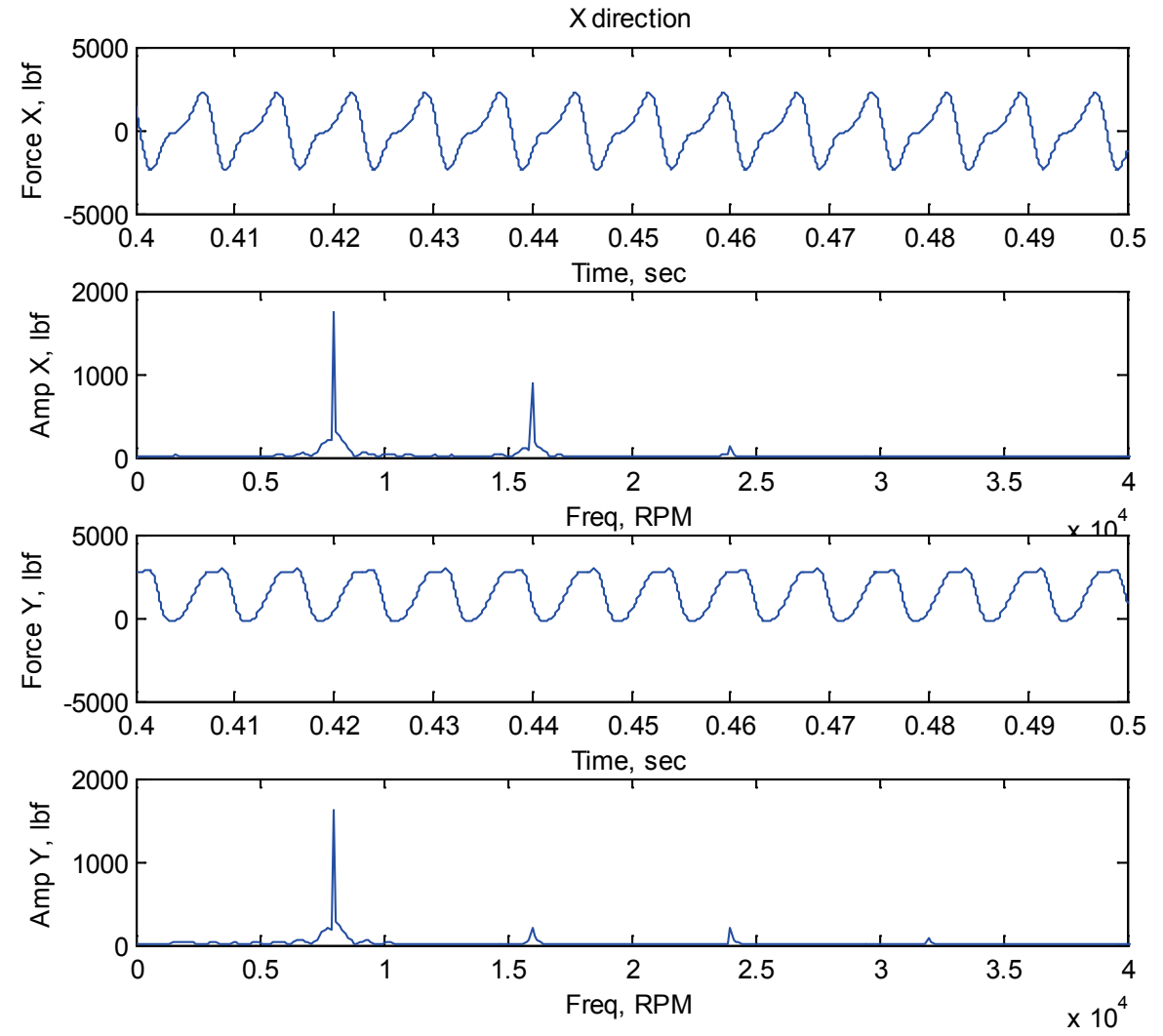

Figure 5.66 FFT of Squeeze Film Damper Force at Node 27 under 32x Unbalance 
The bearing pads and pivots response at node\#5 and node\#27 is shown in Figs. $5.67 \& 5.68$ (pad\#4). In the figures, the response of pad tilting angle is given at top left, the pivot displacement is shown in top right, and the two bottom figures are the time transient response of pad moment and force. After the time transient response dies out after 0.15 second, the pad tilting angle is 0.04 degree with a magnitude of 0.006 degree (peak to peak) at node \#5. Since no negative pressure applies to the pad, the pivot force (the right bottom figure in Figs. 5.67\&5.68), which is calculated from the pressure profile, is always positive or zero (unloaded). For the same reason, there is almost no negative pivot displacement, as shown in the left top figure in Figs. 5.67\&5.68. The super-harmonic response is obtained from the pivot force, Figs. 5.69\&5.70, due to the periodic unloading on pad during the time transient response.

Although the shaft shows a synchronous response, as shown in Fig. 5.53, the bearing pad moments have strong nonlinear response in frequency domain, as shown in Figs. 5.71 5.76. Detailed pad moment in time domain shows a periodic response with short time unload (zero value) on pad. The periodic unloaded pads result in the super-harmonic response of the tilting angles and the pivot displacements, as shown in Fig.5.73 5.76

The bearing pad/pivot (node\#5) transient response including the pad forces and moments is shown in Figs. 5.77 5.79 and Fig. 5.67. Under smaller unbalance (1X or 16X) without the finite length squeeze film dampers, the upper two bearing pads/pivots (pad\#1\&2) are unloaded after the system has periodic response. From those figures, however, after adding the finite length film dampers, there is no unloaded pad due to server unbalance (32X). The two lower pads (\#3\&4) provide larger forces than the upper pads (\#1\&2) due to the rotor weight. 

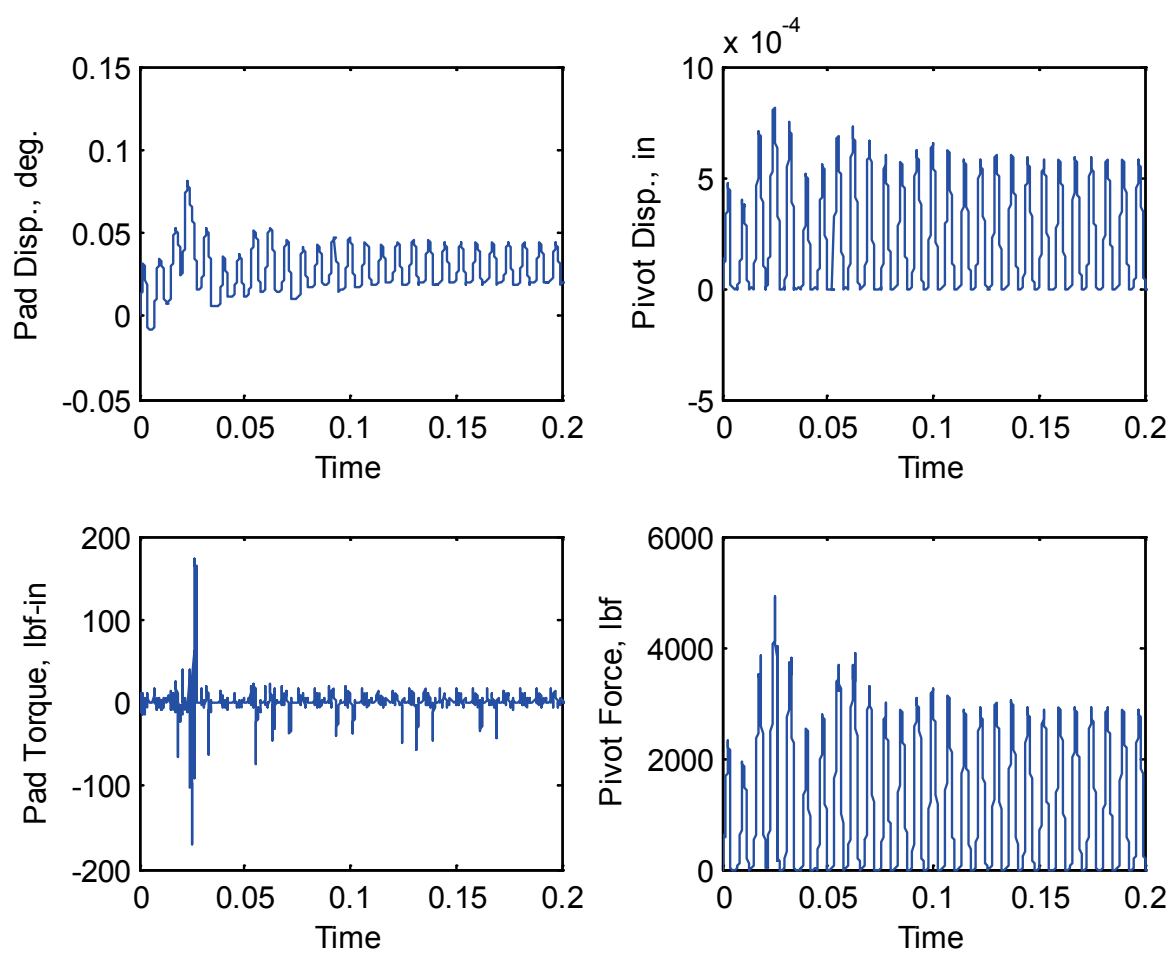

Figure 5.67 Bearing Pad/Pivot \#4 Response at Node 5
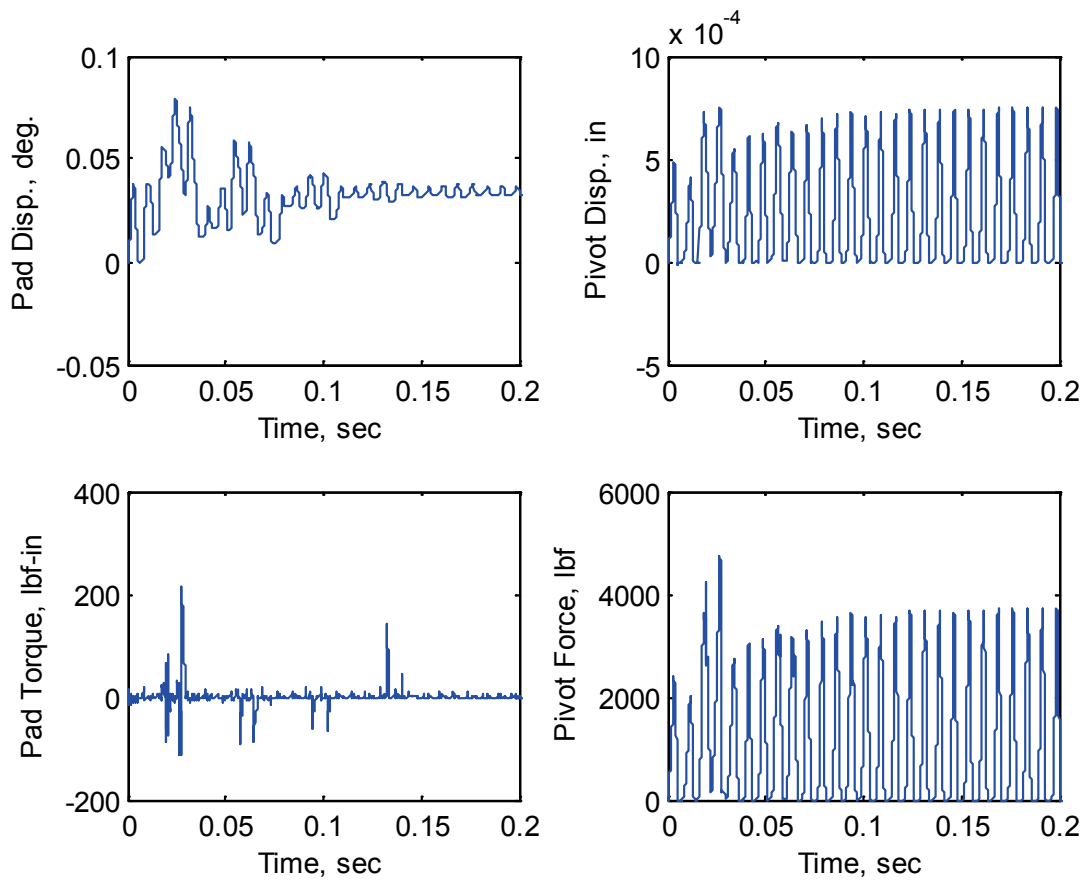

Figure 5.68 Bearing Pad/Pivot\#4 Response at Node 27 
Pivot \#4 Force
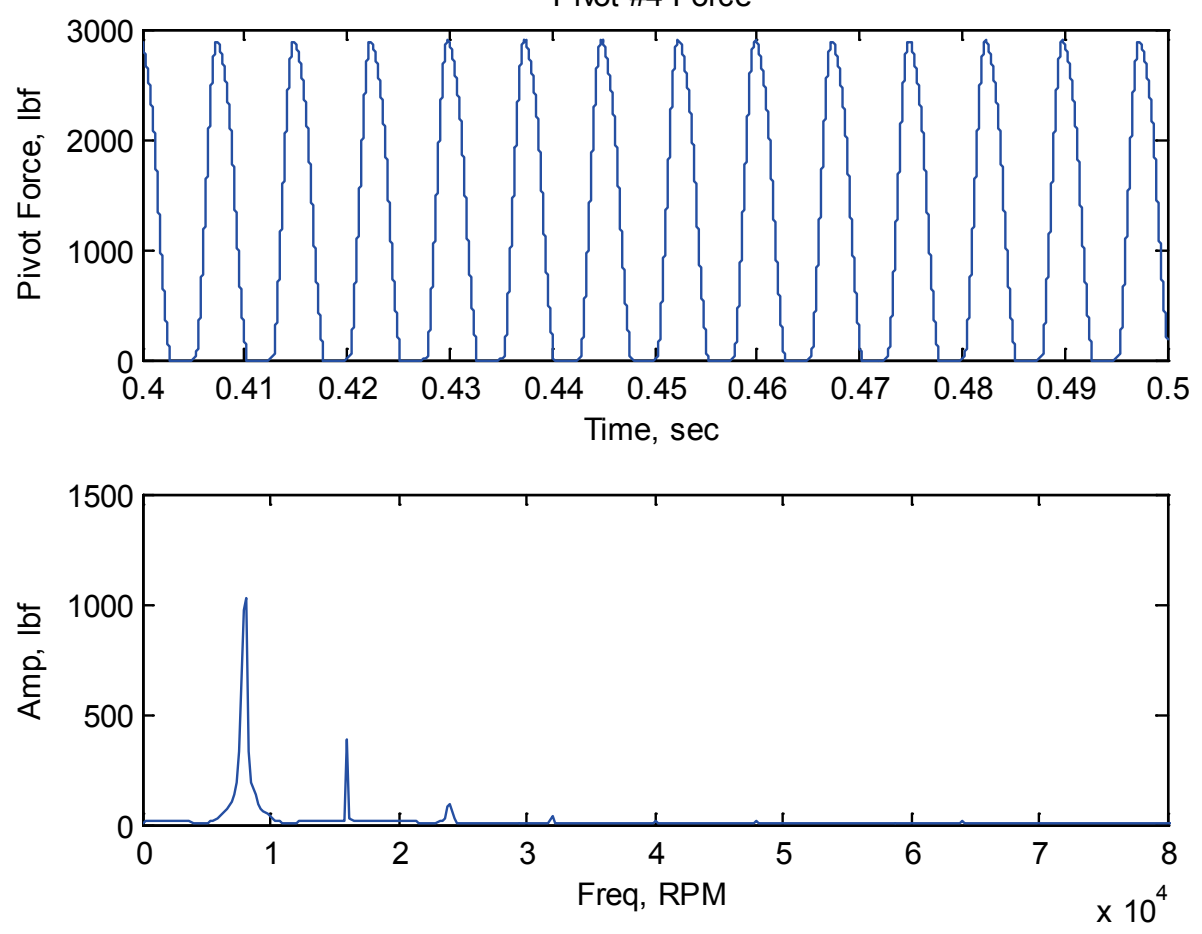

Figure 5.69 FFT of Bearing Pad \#4 Force at Node 5
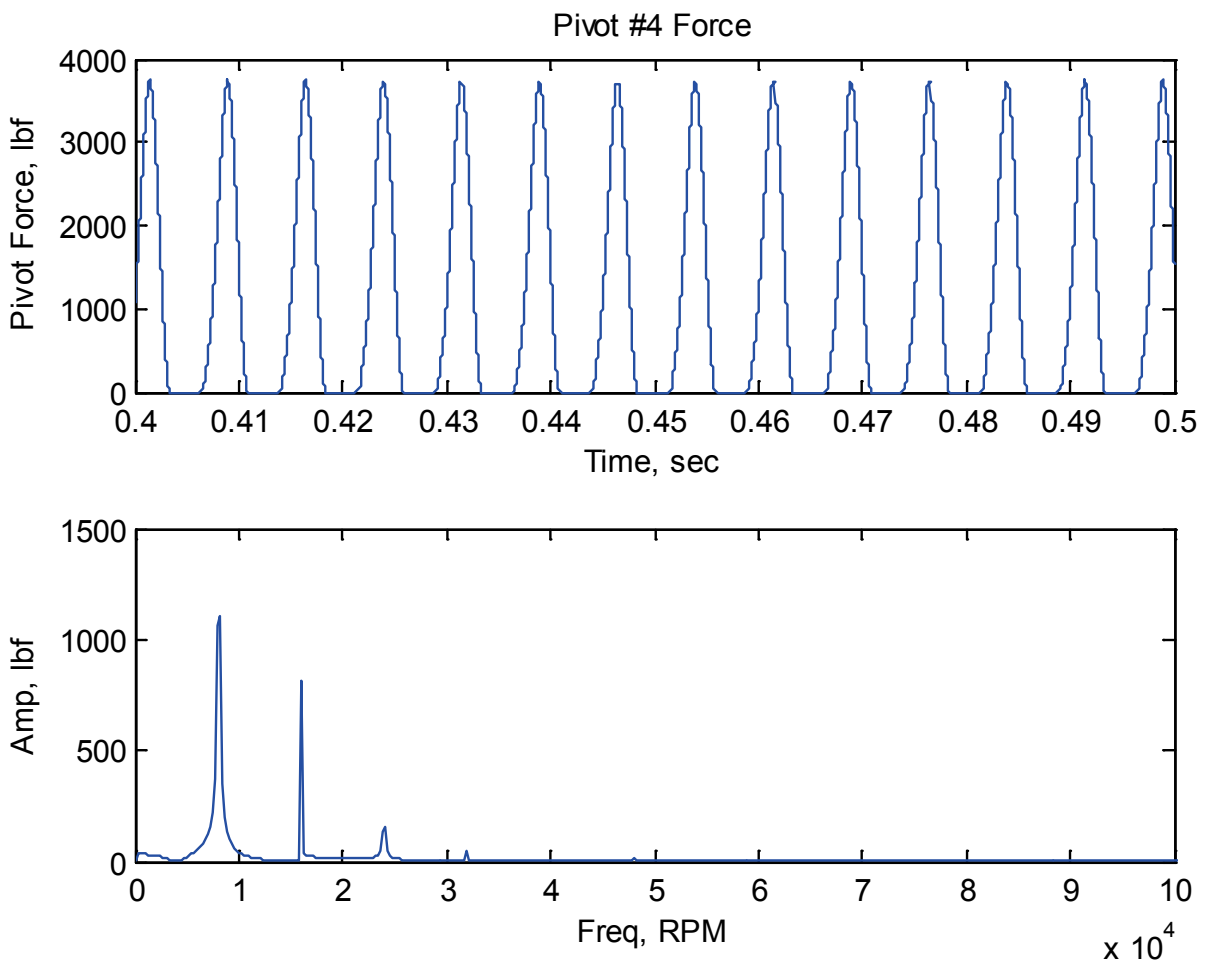

Figure 5.70 FFT of Bearing Pad \#4 Force at Node 27 

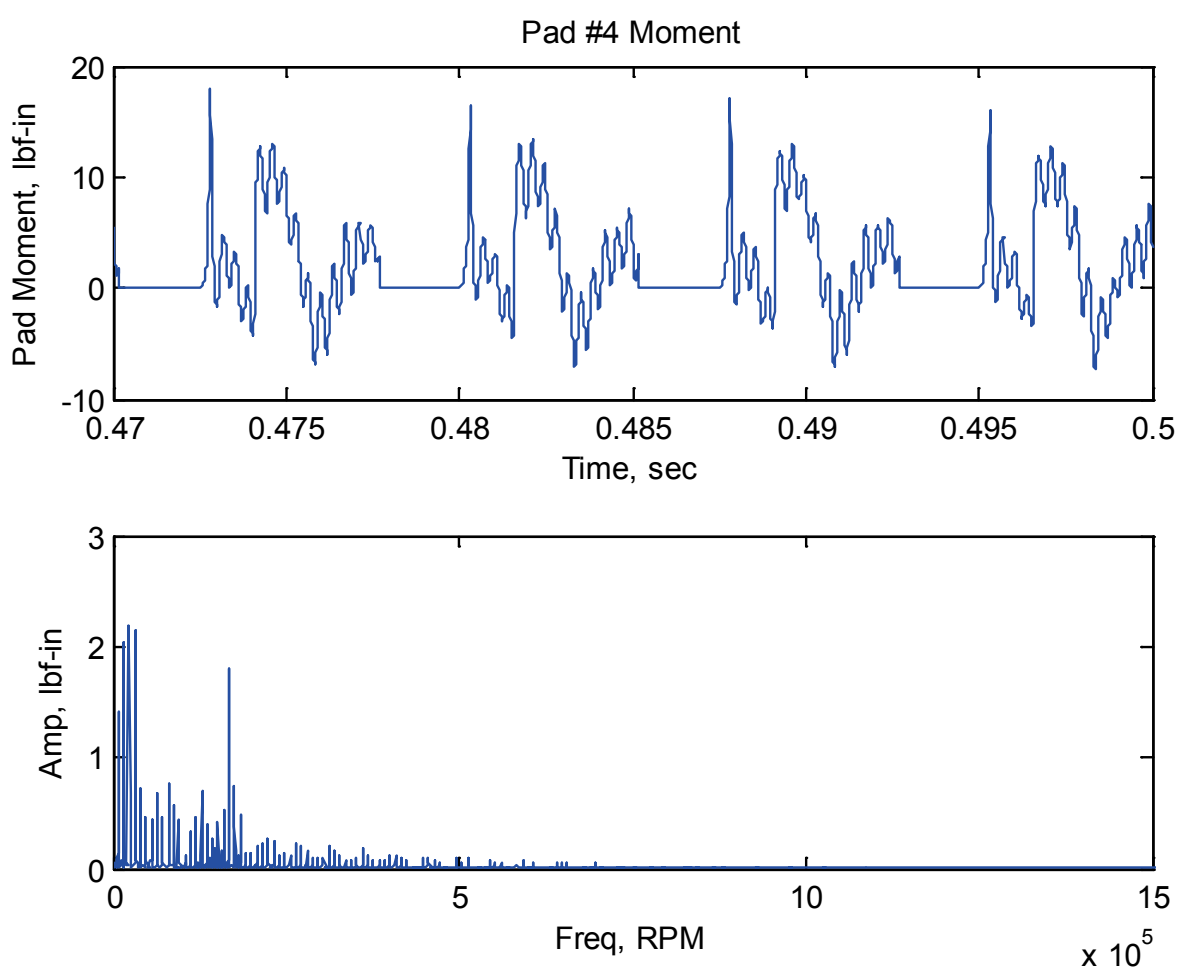

Figure 5.71 FFT of Bearing Pad \#4 Moment at Node 5
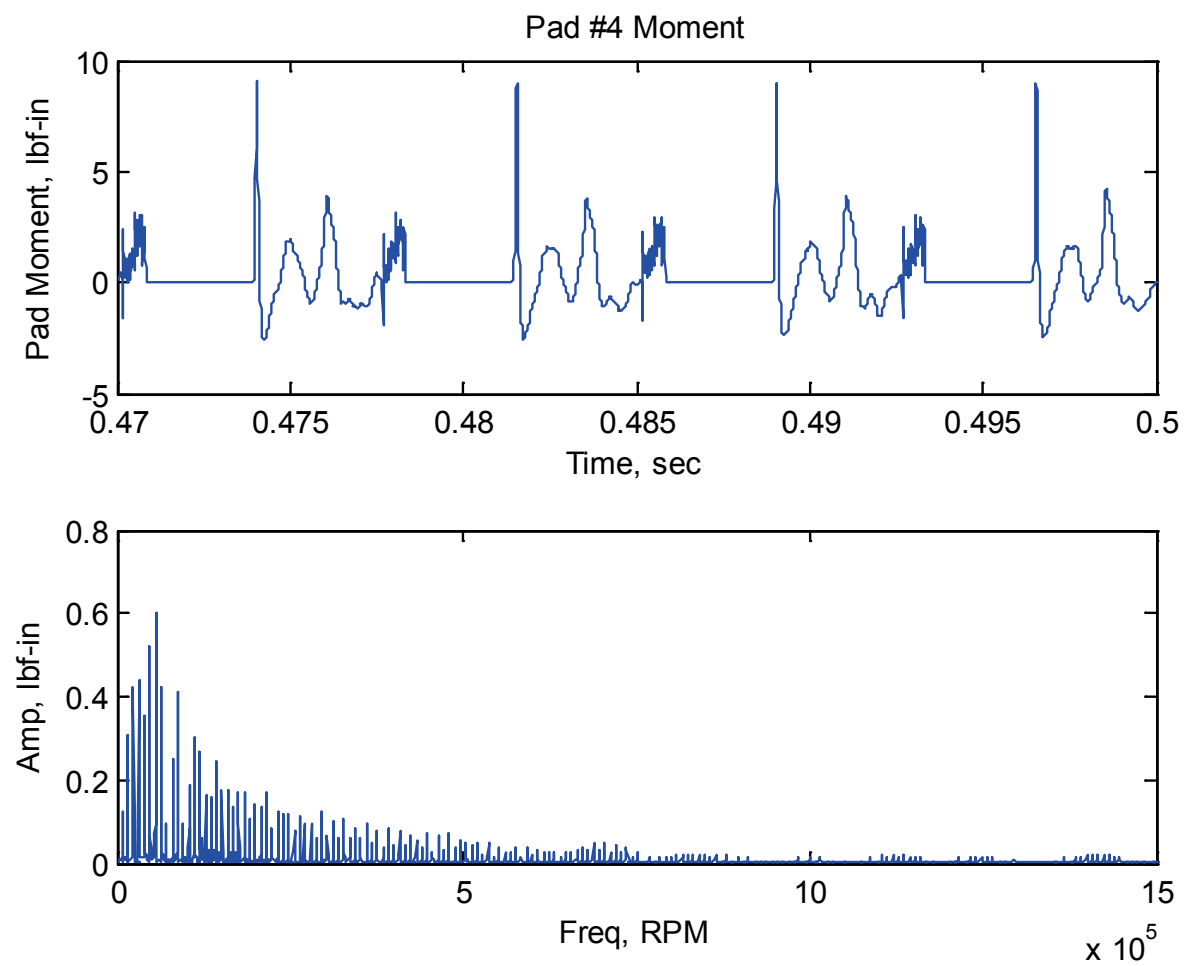

Figure 5.72 FFT of Bearing Pad \#4 Moment at Node 27 
Pad\#4 Tilting Angle
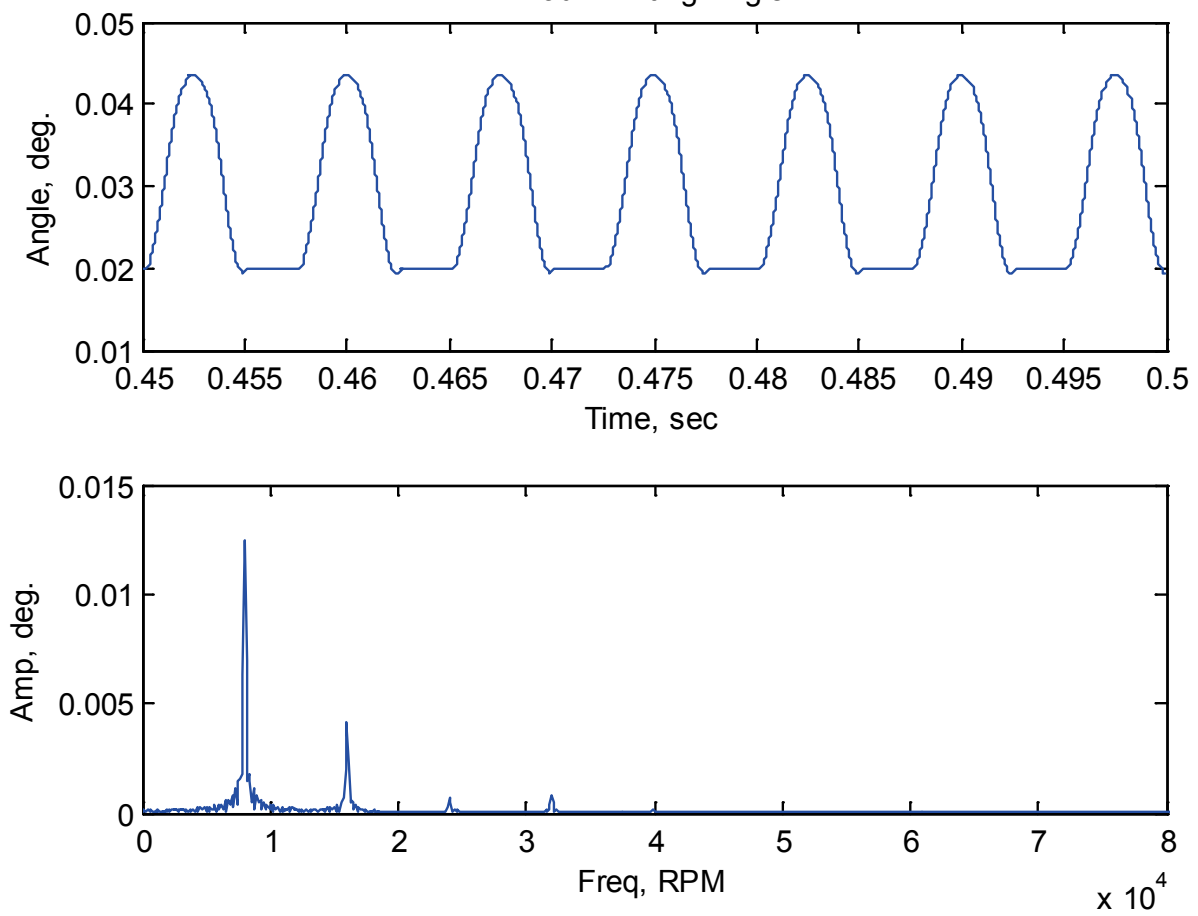

Figure 5.73 FFT of Bearing Pad \#4 Tilting Angle at Node 5
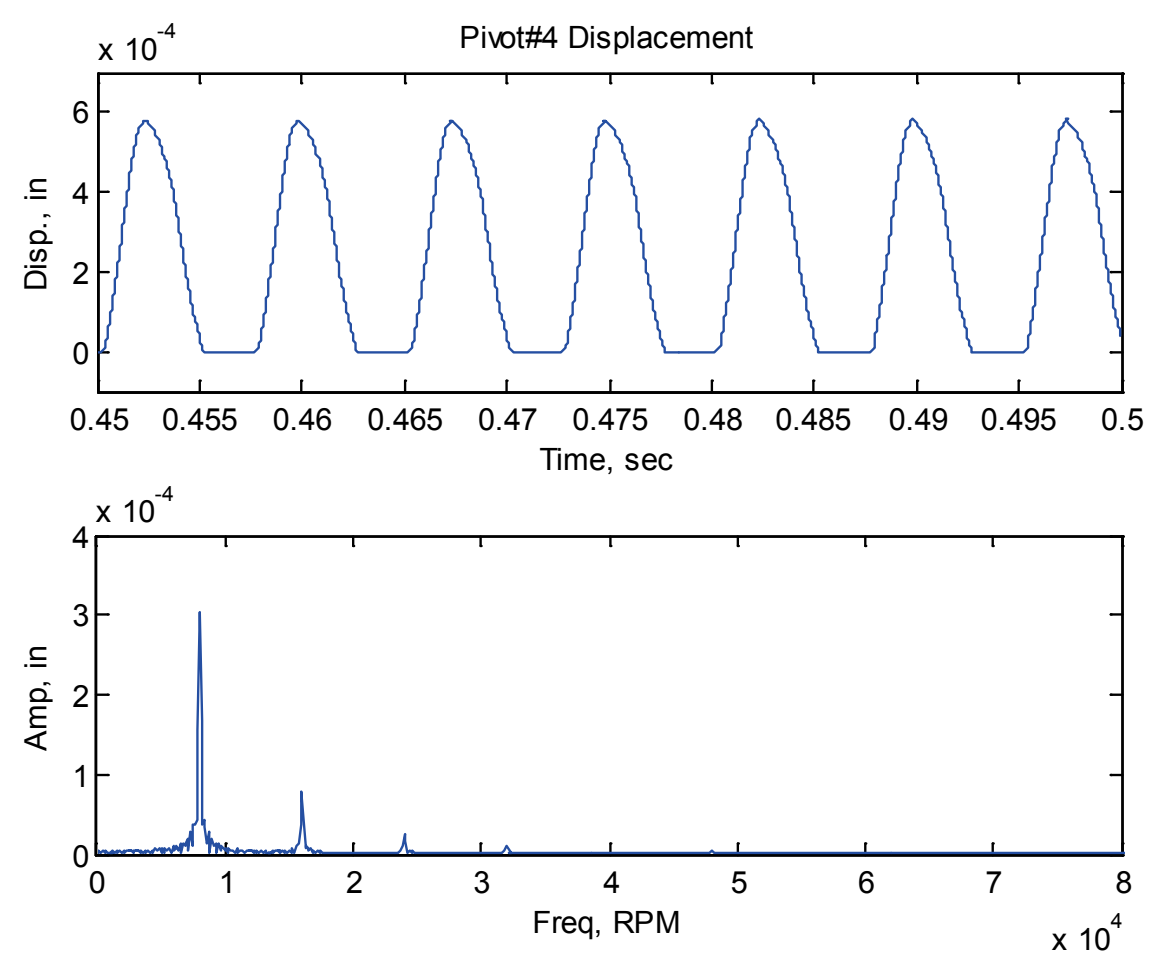

Figure 5.74 FFT of Bearing Pad \#4 Displacement at Node 5 

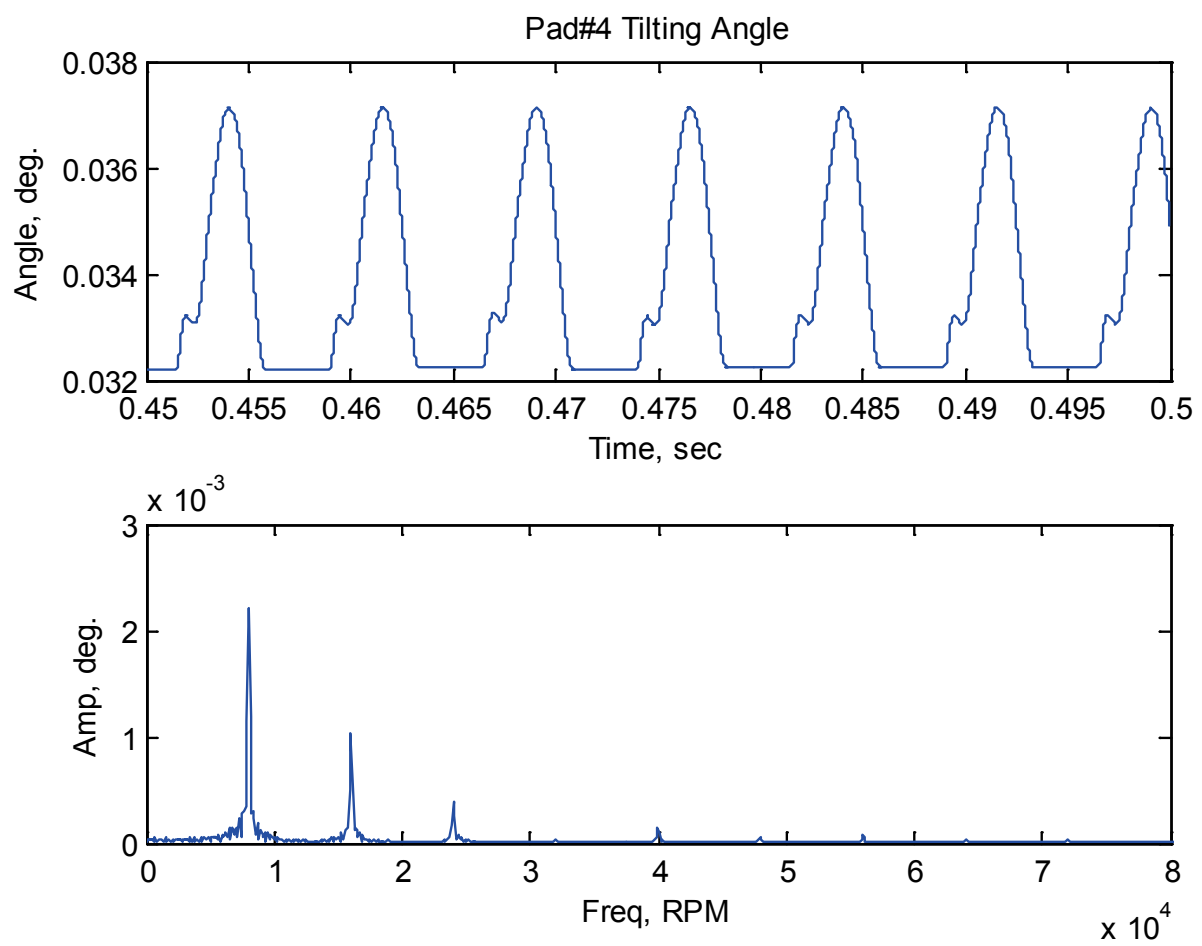

Figure 5.75 FFT of Bearing Pad \#4 Tilting Angle at Node 27
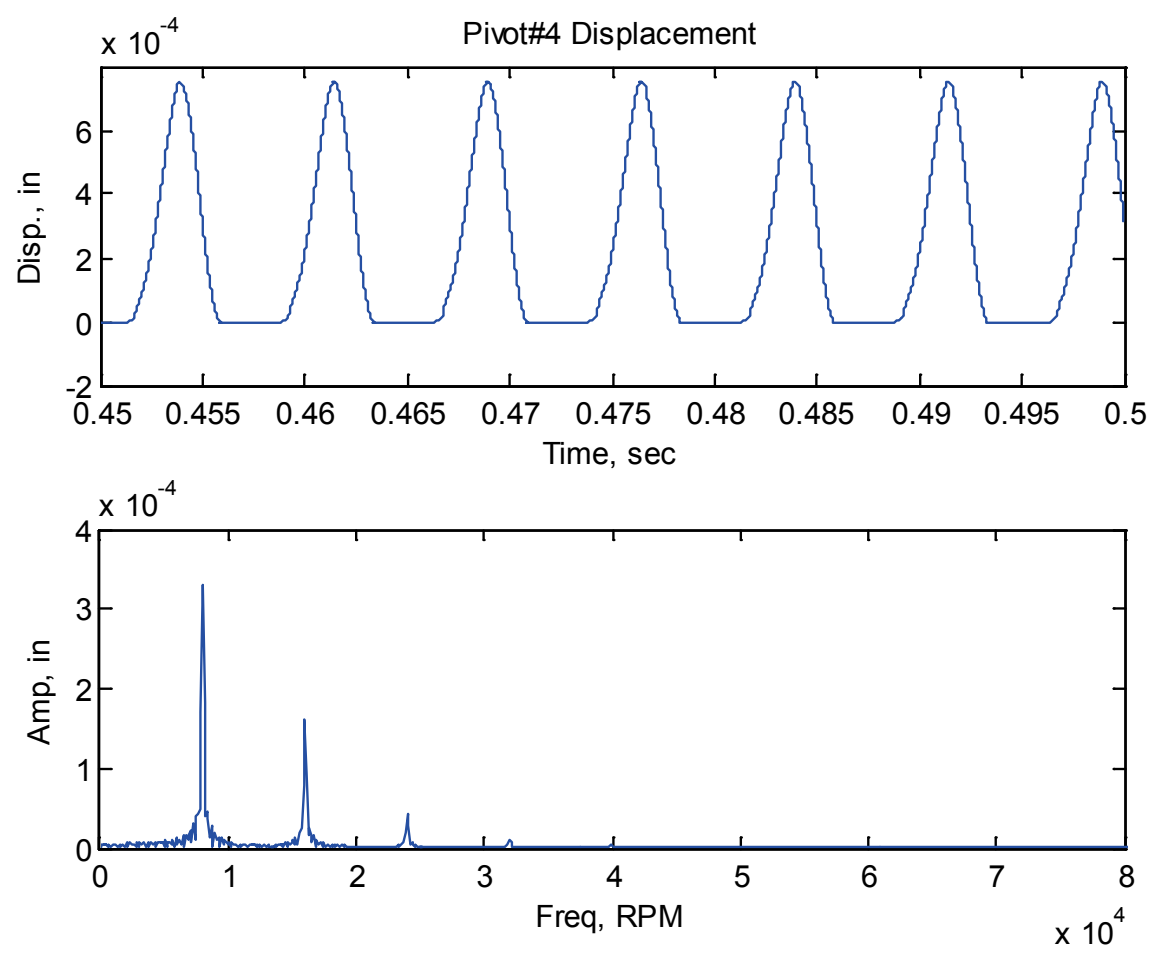

Figure 5.76 FFT of Bearing Pad \#4 Displacement at Node 27 

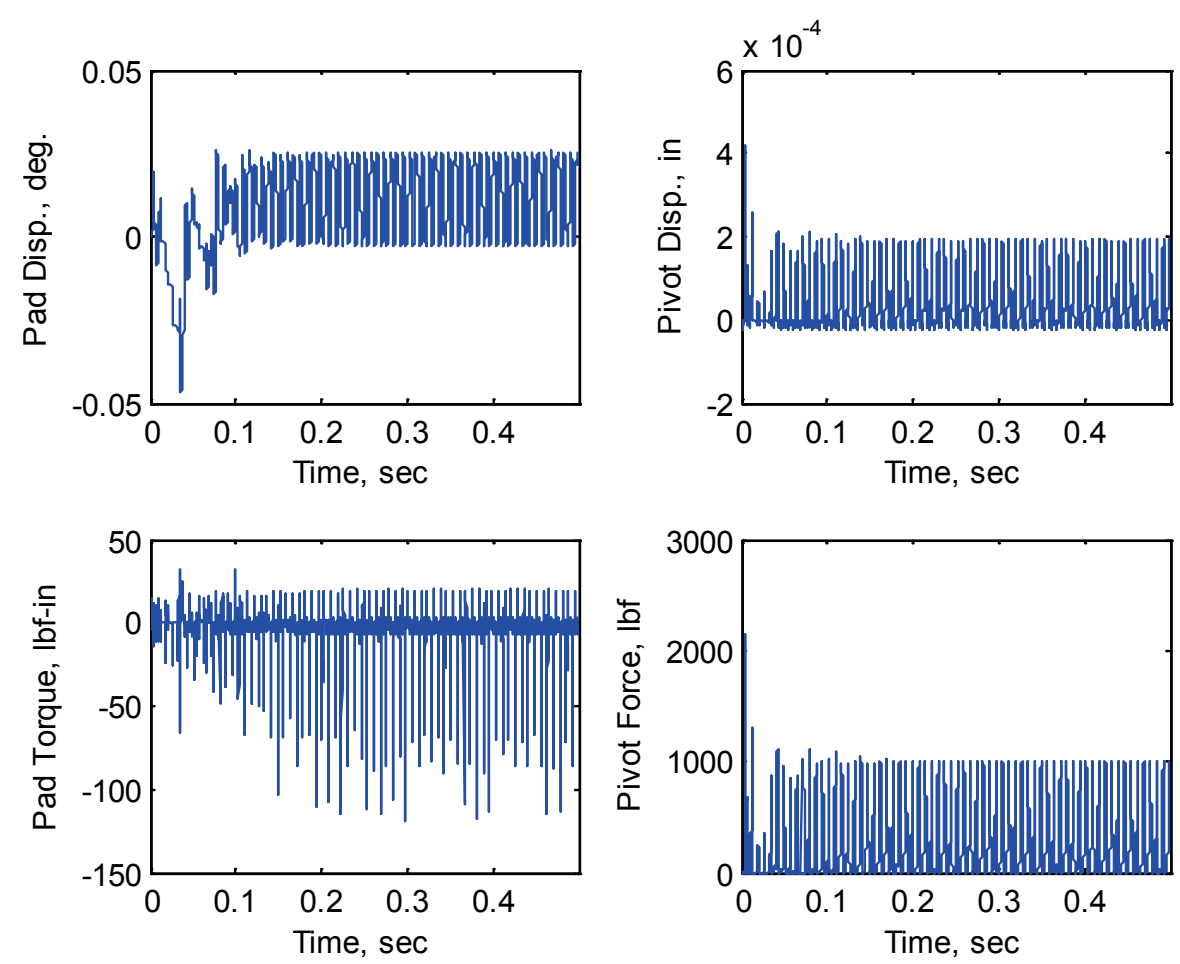

Figure 5.77 Bearing Pad/Pivot \#1 Response at Node 5
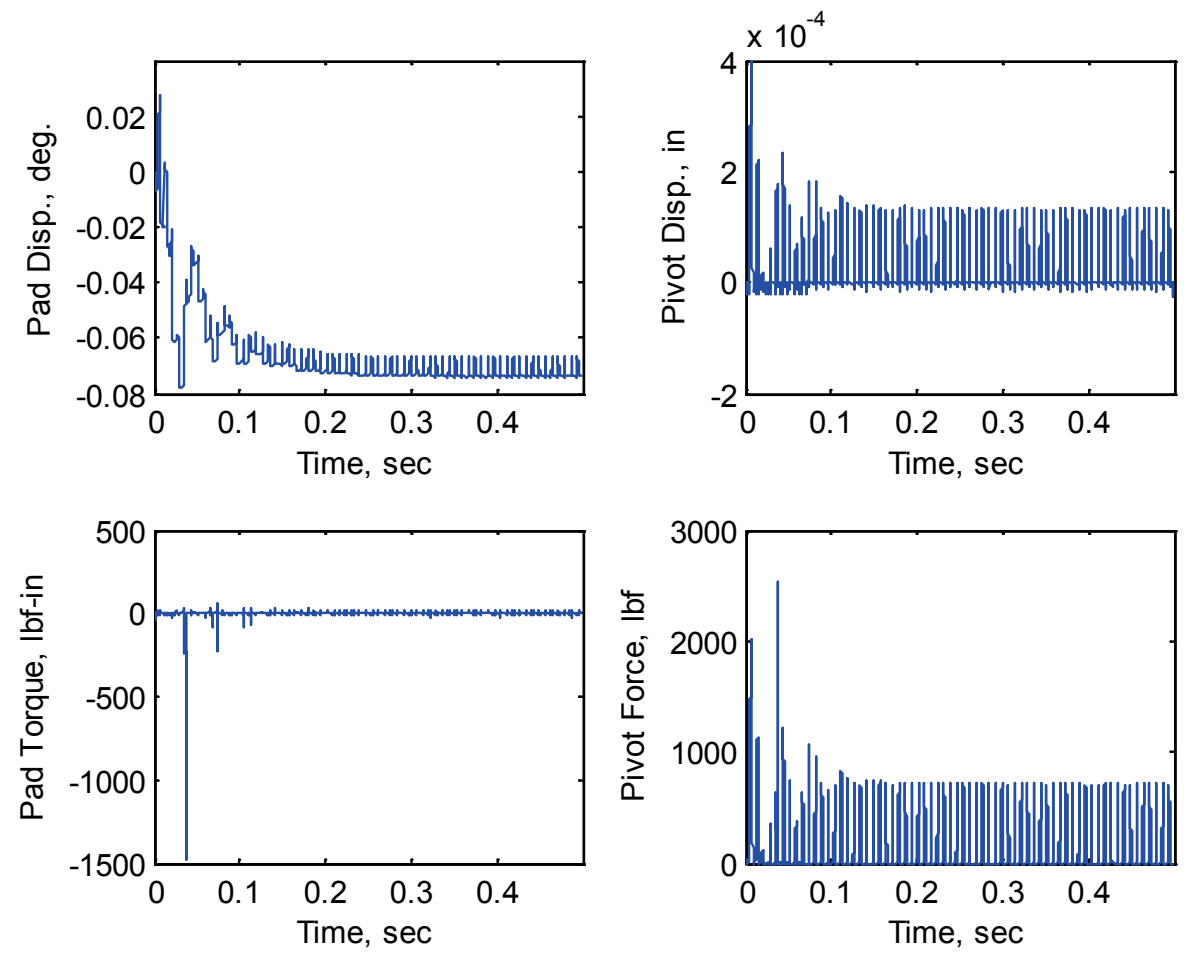

Figure 5.78 Bearing Pad/Pivot \#2 Response at Node 5 

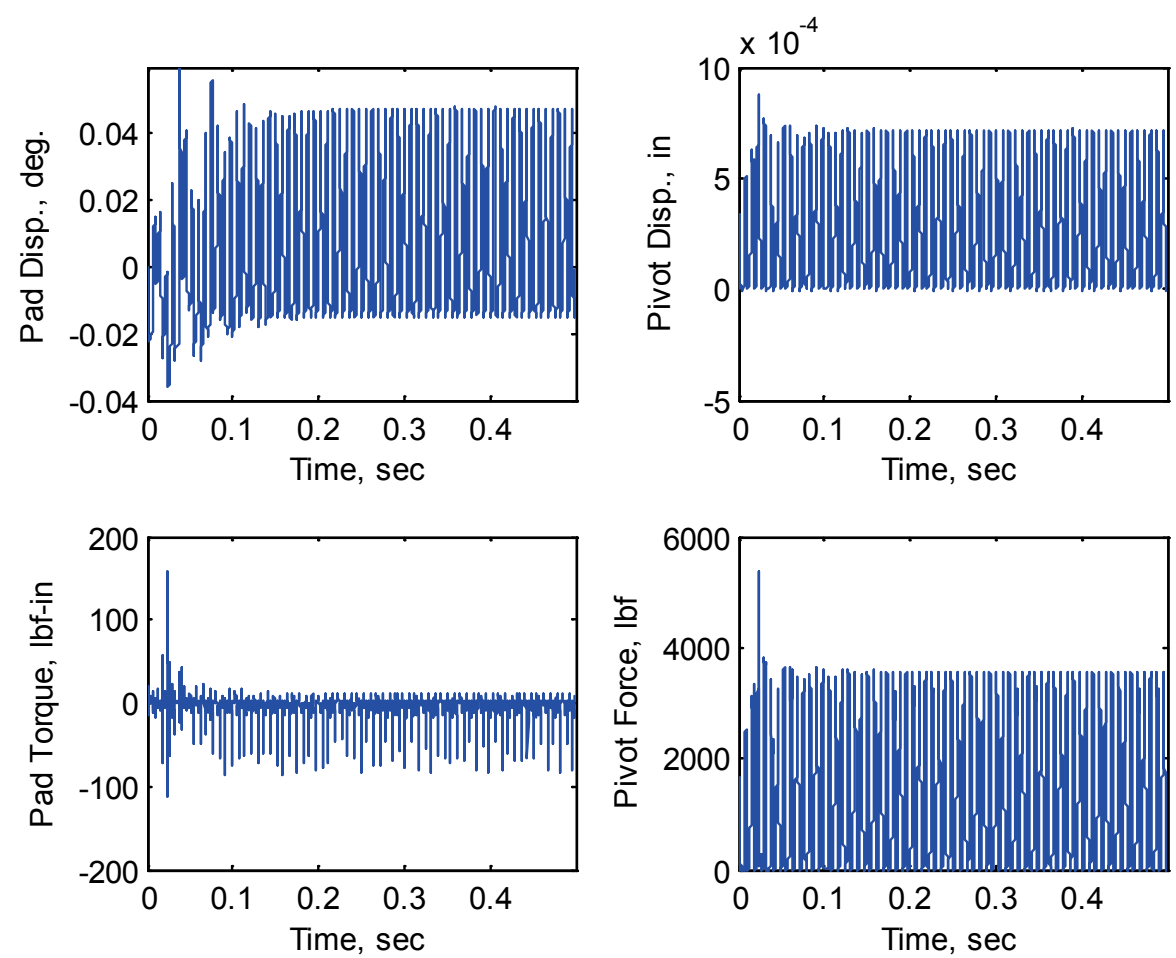

Figure 5.79 Bearing Pad/Pivot \#3 Response at Node 5

\subsection{Summary}

This chapter evaluated the transient response of an eight-stage back to back compressor model supported with nonlinear tilting pad bearings. The dynamic stiffness and damping coefficients based on a rigid rotor model supported with the tilting pad bearings are calculated first to validate the method of the pressure calculation. The verification of calculating nonlinear bearing/damper force was done by comparing the results from THPAD, which calculates the full dynamic bearing stiffness and damping coefficients. The results show a good agreement with the tendency of the coefficients with various running speeds. A maximum percentage difference of $6.67 \%$ in stiffness and $12.08 \%$ in damping are shown between two calculations. The difference is due to constant lubricant density and viscosity used in transient analysis without consideration 
of the thermal heating or different perturbation methods used to calculate the coefficients. The time transient response of a rigid rotor is given at first, and then an 8-stage compressor model supported with tilting pad bearings. The bearing pressure distribution is related not only the shaft, but also the pads and pivots inside the bearings. To obtain the coupled response of the whole system, the bearing pads/pivots must be taken into consideration. In this dissertation, additional nodes are added into the system to describe the bearing pads/pivots effects.

Under the first unbalance force, the transient dies out and the 8-stage compressor model reaches its steady state. A weakly nonlinear response is shown from the response of pad moment. Under a serve unbalance condition (16 times of the first unbalance), the 8-stage compressor reaches the steady state till 9.0 seconds, and the rotor behavior is not synchronous with the running speed but the shaft response is periodic. Also calculated touching between the bearing pads and the shaft occurs when the unbalance increases to 32 times of the first unbalance. To avoid possible touching under the severe condition, the finite squeeze film dampers without centering spring are introduced to the system. The time transient results show that adding the dampers decreases the vibration amplitude and increases the stability of the system. Under the more severe unbalance condition $(32 \mathrm{x}$ of the first unbalance applied), the system reached the steady state after 0.15 second, which is $5 \sim 10$ times faster than the system without the finite length squeeze film dampers; and no touching occurs between the bearing and the shaft. Although the whole shaft shows a synchronous response, strong nonlinear behavior exists at the bearing pad/pivot response due to periodic unloading on the pads. The strong behavior is more obvious from the time and frequency domain response of the pad moments. 
The computing time for different cases is listed in Table 5.7 with the same time step of $8 \times 10^{-6}$ second and same time span of $0 \sim 0.1$ second. If the linear tilting pad bearings are applied to the 8 stage model, the computing time is $44.08 \mathrm{sec}$; if the nonlinear tilting pad bearings with 30 elements per pad are applied, the computing time is $352.01 \mathrm{sec}$; and if the nonlinear tilting pad bearing (30 elements per pad) and the nonlinear squeeze film damper (60 elements per damper) are applied, the computing time is $601.57 \mathrm{sec}$. Because the nonlinear forces are calculated based on the finite element method at each step, most calculating time $(90 \%)$ is used by the nonlinear force calculations.

\section{Table 5.7 Computing Time Comparison}

\begin{tabular}{l|l|l|l}
\hline \multicolumn{3}{l}{ 8-Stage Compressor, $0 \sim 0.1 \mathrm{sec} ., \Delta \mathrm{t}=8 \times 10^{-6}$ sec., Lateral Analyses } \\
\hline & DOF & Time, sec & File Size, KB \\
\hline Linear TPB & 124 & 44.08 & 36,359 \\
\hline Nonlinear TPB & 140 & 352.01 & 39,484 \\
\hline Nonlinear TPB + FLD & 144 & 601.57 & 41,266 \\
\hline
\end{tabular}

The time step is not only depends on DOF of a linear rotor, but also depends on the structure of tilting pad bearing. For the 8-stage compressor with 124 DOF (31 elements) supported with two tilting pad bearings, the time step with stable results is $8 \times 10^{-6}$ second, which depends on the maximum natural frequency of the rotor. However, when the same bearings are applied to the 2 -DOF rigid rotor, the time step with stable results is $1 \times 10^{-5}$ second, which is only $20 \%$ longer than the time step of the 8 -stage compressor with 124 DOF. For the rotor-bearing system with the complicated bearings, the time step is depends on both the structure of the rotor and the bearing. 


\section{Chapter 6}

\section{Transient Analysis of Non-Constant Rotational}

\section{Speed and External Torsional Load Vibration}

\subsection{Overview}

This chapter describes the transient analyses of a flexible rotor under non-constant rotational speed and external torsional torques. In many applications, it is necessary to study the rotor motion during startup, shutdown, going through critical speeds, blade loss, or sudden loading. In those situations, the angular velocity (rotational speed) is no longer a constant, but is function of time or external forces/torques. Under non-constant rotational speed, the unbalance forces and the gyroscopic effects are related not only to the rotational speeds, but also to the angular acceleration of the rotor. When the external torsional torque exists, coupled torsional and lateral responses need to be taken into consideration as well. Beyond that, the numerical integration method has to be modified to solve the implicit problem because the unbalance force and the gyroscopic effects are related to both the rotational speed and the acceleration. The 3-disk linear flexible rotor model is applied to the non-constant rotational speed cases for lateral analyses at first. 
Then the torsional analysis of a three-stage compressor driven by a synchronous rotor is applied. Finally the coupled torsional and lateral response of the 3-disk rotor model with external torque is presented base on the time transient method.

Section 6.2 shows the lateral transient analysis of the 3-disk rotor supported with plain journal bearing and squeeze film dampers under specific non-constant rotational speed. Section 6.3 discusses a startup torsional analysis of a 3-stage compressor driven by a synchronous rotor. Section 6.4 discusses the coupled torsional and lateral transient analysis of the start up of the 3-disk rotor. Section 6.5 discusses the results found through the nonlinear transient analyses.

The analyses performed in this chapter were done using a Dell Precision T3500 computer with an Intel Xeon CPU@2.53 GHz, 12GB of RAM and a 64-bit Windows OS. Matlab version 7.13 (R2011b) was used for the 64-bit OS.

\subsection{Flexible Rotor under Non-Constant Rotational Speed}

The purpose of this section is to study the effect of angular acceleration to the rotor response. For zero angular acceleration or constant rotational speed condition, the steadystate response has been studied based on the linear theory. The nonlinear transient response of the flexible rotors with constant rotational speed (zero angular acceleration) has been approached in Chapter $5 \& 6$.

A transient analysis of the 3-disk rotor-bearing system under with bearings and squeeze film dampers angular acceleration is developed first. The $4^{\text {th }}$ order Runge-Kutta method is then used to solve the problem numerically. However, the gyroscopic effects, 
the bearing stiffness and damping coefficients and the unbalance forces are related to both the rotational speed and the angular acceleration. Those effects have to be calculated at each time step because the rotational speed and the angular acceleration are time dependent. In this dissertation, the lubricant inertia effect is neglected, so the nonlinear bearing/damper forces are not related to the angular acceleration.

\subsubsection{Equations of Motion and Numerical Solver}

If the rotating speed $(\Omega)$ is time dependent (non-constant), the second-order ordinary differential equations of motion are [13]:

$$
M \ddot{u}+\left(C_{s}+C_{b r g}+\Omega G\right) \dot{u}+\left(K_{s}+K_{b}+\dot{\Omega} G\right) u=F(u, \dot{u}, \Omega, \dot{\Omega}, t)
$$

In the equations, the mass matrix $(M)$, the damping and stiffness matrices from the linear shaft and attached disks $\left(C_{s} \& K_{s}\right)$ are constant and do not change with the rotational speed. Normally the linearized bearing damping and stiffness coefficients $\left(C_{b r g} \& K_{b}\right)$ are the rotational speed $(\Omega)$ dependent. The gyroscopic effects are related to both the current rotational speed and the angular acceleration. The unbalance forces and the shaft bow forces are the rotational speed and the acceleration related. The nonlinear bearing/damper forces are functions of the displacement, the velocity and rotational speed. Putting all those rotational speed related terms to the right of the equation (6.2.1), the equations of motion in state space form are:

$$
\begin{aligned}
& {\left[\begin{array}{cc}
M & 0 \\
0 & M
\end{array}\right]\left[\begin{array}{c}
\dot{u} \\
\ddot{u}
\end{array}\right]+\left[\begin{array}{cc}
0 & -M \\
K_{s} & C_{s}
\end{array}\right]\left[\begin{array}{c}
u \\
\dot{u}
\end{array}\right]=} \\
& {\left[\begin{array}{c}
0 \\
F(u, \dot{u}, \Omega, \dot{\Omega}, t)
\end{array}\right]+\left[\begin{array}{c}
0 \\
-\Omega G \dot{u}-\dot{\Omega} G u
\end{array}\right]+\left[\begin{array}{c}
0 \\
-C_{b r g} \dot{u}-K_{b} u
\end{array}\right] }
\end{aligned}
$$


The unbalance and the shaft bow forces, which are related both the rotational speed and the angular acceleration, have the forms:

$$
\begin{aligned}
& \left\{\begin{array}{l}
F_{u, x} \\
F_{u, y}
\end{array}\right\}=\left\{\begin{array}{l}
m e_{u} \Omega^{2} \cos \left(\Omega t+\phi_{u}\right)+m e_{u} \dot{\Omega} \sin \left(\Omega t+\phi_{u}\right) \\
m e_{u} \Omega^{2} \sin \left(\Omega t+\phi_{u}\right)-m e_{u} \dot{\Omega} \cos \left(\Omega t+\phi_{u}\right)
\end{array}\right\} \\
& \left\{\begin{array}{l}
F_{b, x} \\
F_{b, y}
\end{array}\right\}=\left\{\begin{array}{l}
A_{b} \Omega^{2} \cos \left(\Omega t+\phi_{b}\right)+A_{b} \dot{\Omega} \sin \left(\Omega t+\phi_{b}\right) \\
A_{b} \Omega^{2} \sin \left(\Omega t+\phi_{b}\right)-A_{b} \dot{\cos }\left(\Omega t+\phi_{b}\right)
\end{array}\right\}
\end{aligned}
$$

To solve the transient problem numerically using the $4^{\text {th }}$ order Runge-Kutta method, all terms located at the right of Equation (6.2.2) need to be calculated according to instantaneous displacements, velocities, rotational speed and angular acceleration at each time step. The linear interpolation method is used to calculate the linearized bearing coefficients $\left(C_{b r g} \& K_{b}\right)$ at different rotational speeds.

\subsubsection{Response of Flexible Rotor under Non-Constant Rotational Speed}

The rotor model used in this section is the 3-disk flexible rotor with plain journal bearings and squeeze film dampers, which is same as the rotor model introduced in Chapter 4. The 3-disk axially symmetric rotor with 3 lumped disks has a length of 24 inches, a shaft diameter of 2 inches and a total mass of $101.35 \mathrm{lb}$ including the attached disks. The finite element based rotor model, as shown in Fig. 6.1, is defined by 25 nodes and 24 Timoshenko beam elements. The bearing locations are denoted with the red markers across the center of the shaft; the squeeze film damper locations are same as the bearing locations but are installed outside of the bearings. To excite different modes, three unbalances with different phase angles are placed on the three disks. The details of the rotor are described in Chapter 4; here is a brief summary of the rotor model: 
- Three disks at node 10,13 and 16 from left to right on the shaft

- Two journal plain bearings $(\mathrm{L} / \mathrm{D}=0.5)$ at node $4 \& 22$ with radial clearance 3 mils

- Two SFDs located outside bearings with radial clearance 6 mils

- Three unbalances placed on disks $\left(0.5\right.$ oz-in $0^{\circ}, 1$ oz-in $90^{\circ}, 0.5$ oz-in $\left.180^{\circ}\right)$

- Timoshenko beam element, 25 shaft nodes + 2 SFD nodes, 104(100+4) DOF

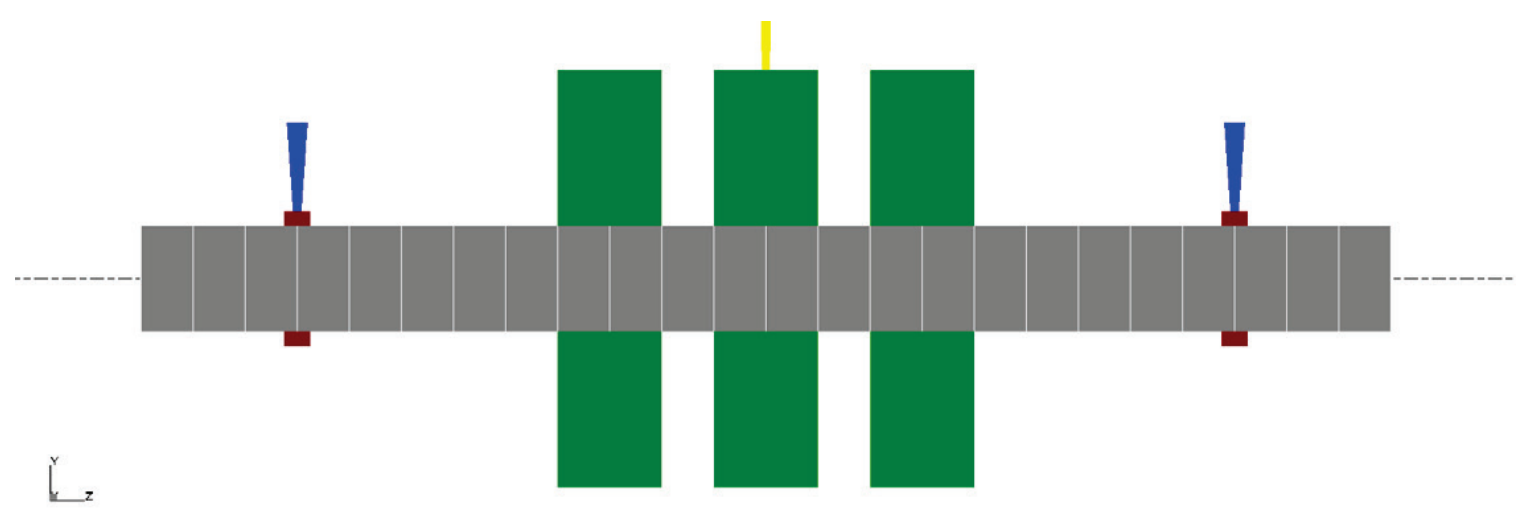

Figure 6.1 3-Disk Flexible Rotor Model with 25 Nodes

To validate the modified numerical method for non-constant rotational speed, a slow angular acceleration case, from $5,950 \mathrm{rpm}$ to $6,000 \mathrm{rpm}$ with a time period of 0.5 second (an acceleration rate of $100 \mathrm{rpm} / \mathrm{sec}$ ), is applied to the linear rotor-bearing system. The unbalance forced response at $\mathrm{t}=0.5$ second is compared with the linear steady state calculation and the transient results at $6,000 \mathrm{rpm}$ to validate the modified numerical solver. The non-constant speed transient solver results from $5,950 \mathrm{rpm}$ to $6,000 \mathrm{rpm}$ within 0.5 second and the constant speed transient solver results at $6,000 \mathrm{rpm}$ are shown in Figs. $6.2 \&$ 6.3. The magnitudes at $\mathrm{t}=0.5$ second are $0.2380 \mathrm{mil}$ in horizontal, 0.2300 mil in vertical for non-constant rotational speed $(5,950 \mathrm{rpm} \sim 6,000 \mathrm{rpm})$. For constant 
speed of $6,000 \mathrm{rpm}$, the results are $0.2381 \mathrm{mil}$ in horizontal, $0.2301 \mathrm{mil}$ in vertical. The steady state analysis has the same results as the constant speed transient analysis, as discussed in Chapter 4, Table 4.3. The initial conditions for the non-constant speed solver and the constant speed solver are different in this case. For the constant rotational speed case, the initial nodal displacements are the static positions calculated from the stiffness of the bearings at $6,000 \mathrm{rpm}$, the stiffness of the shaft and the rotor gravitational force vector. For the non-constant rotational speed case, the linearized bearing stiffness is related to the rotational speed, thus the static nodal position cannot be calculated directly. The initial nodal displacements of the shaft center inside the bearings are set to zero at first, and the other initial nodal displacements are calculated from the stiffness of the shaft and the rotor gravitational force vector. The initial velocities of all nodes are zeros for both cases.
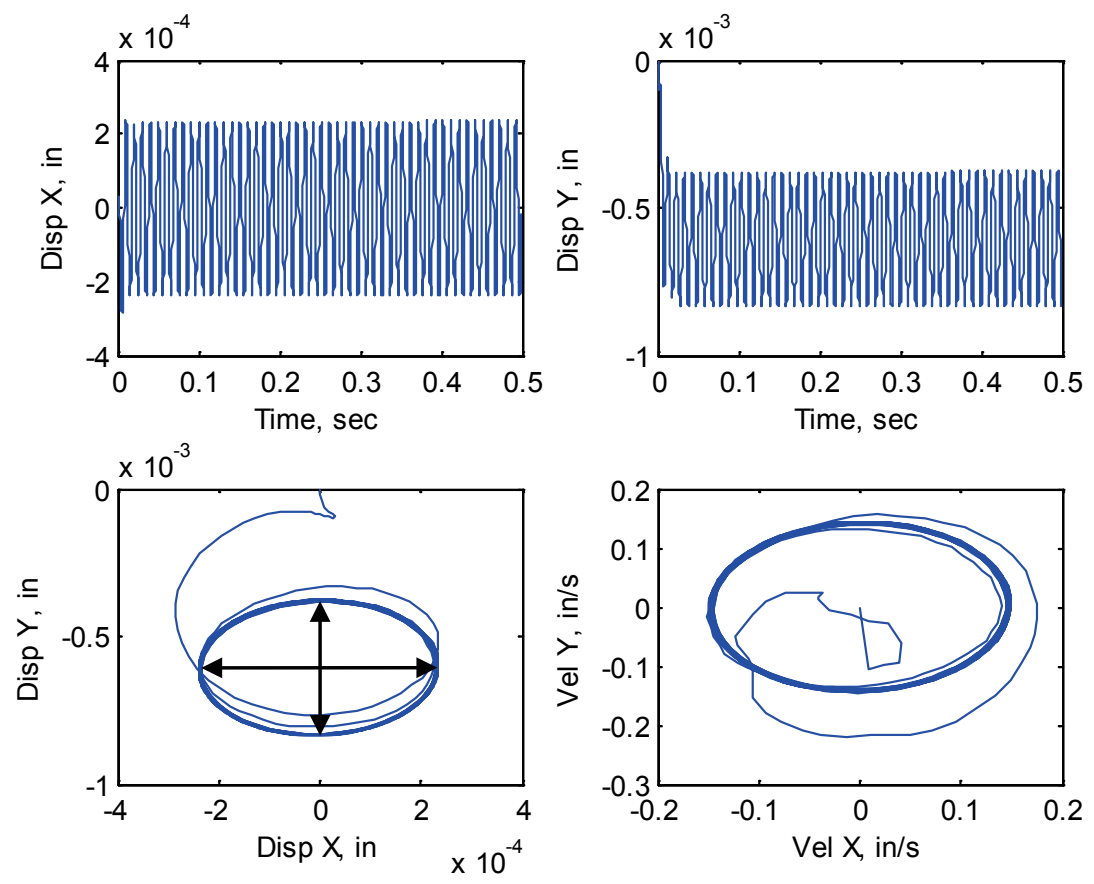

Figure 6.2 Transient Response of 5,950 rpm $6,000 \mathrm{rpm}, 0 \sim 0.5 \mathrm{sec}$. (BRG1, Node4) 

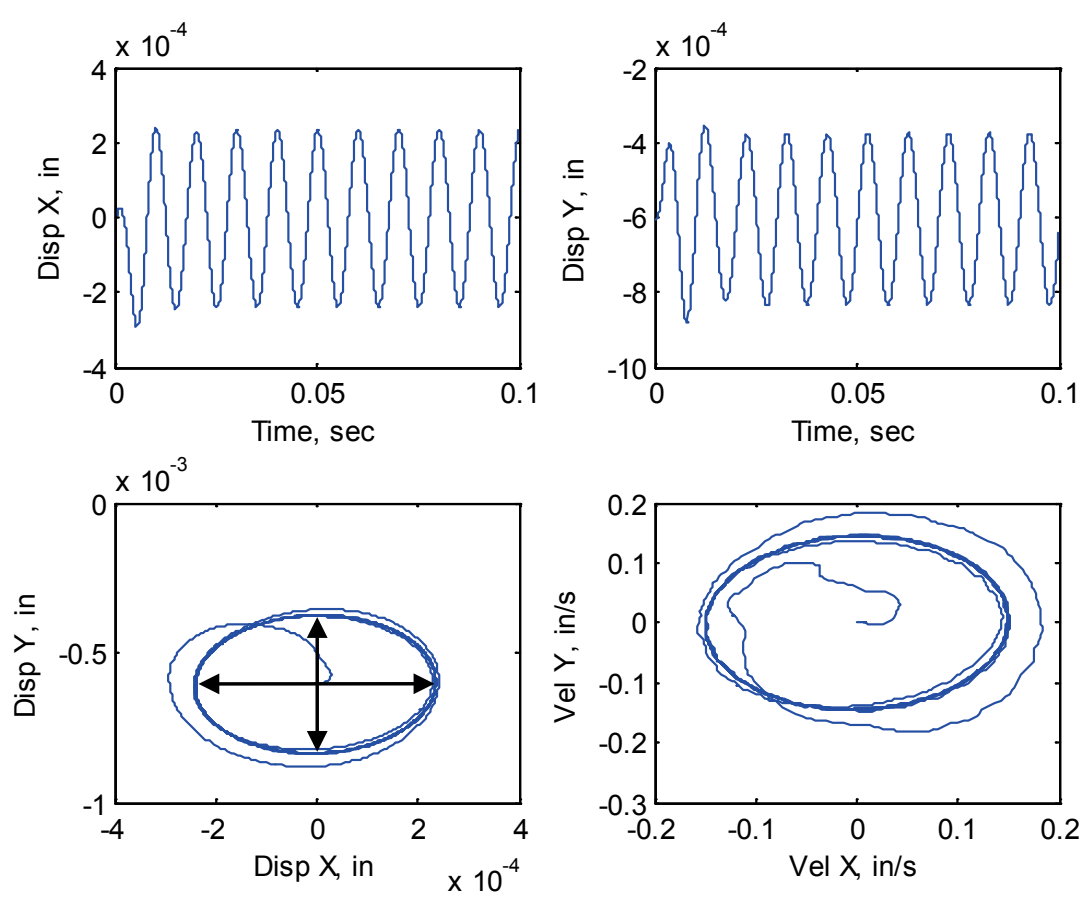

Figure 6.3 Transient Response of 6,000 rpm (BRG1, Node4)

Two types of speed profiles are commonly used for startup and shutdown analyses: the linear speed profile and the exponential speed profile. The two speed profiles in this dissertation are defined by speed vs. time, as illustrated in Fig. 6.4. The speed at each time point during a transient analysis is calculated through a linear interpolation method.

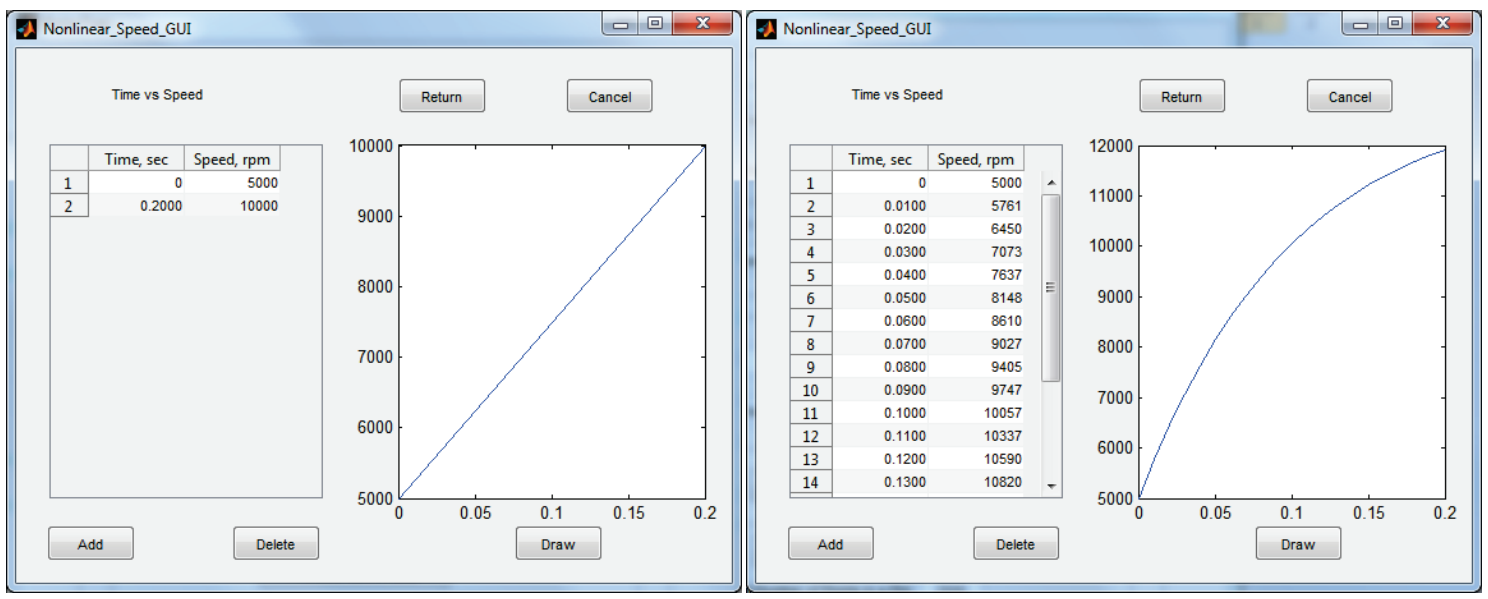

Figure 6.4 User Defined Speed Profile File 
A linear speed profile, which has a constant angular acceleration during startup, is used at first to simulate the system going through critical speeds. The effect of the angular acceleration on the rotor unbalance forced response has been studied by Yamamoto [7] and Chen [13] on a 2-DOF rotor using very slow acceleration $(0.0001 \mathrm{rpm} / \mathrm{sec} \sim 0.01$ $\mathrm{rpm} / \mathrm{sec}$ ) with the system's critical speed at $10 \mathrm{rpm}$. The initial condition is another important issue for the transient analysis, especially for a nonlinear system. In this section, the initial displacement is taken as zero for the node inside the bearings; the other nodal initial displacements are calculated from the static gravity, say the weight of the rotor. The initial velocities for all nodes are zeros $\left(\dot{u}_{0}=0\right)$.

The time transient response of the 3-disk rotor at the center disk (node 13) is shown in Fig. 6.5. The transient simulation is performed for the rotational speed from 5,000 rpm to 12,000 rpm within 10 seconds with a linear acceleration $700 \mathrm{rpm} / \mathrm{sec}$. From the time transient response in Fig. 6.5, the maximum response amplitude occurs at $\mathrm{t}=6.12$ second or 9,284 rpm, which is close to the steady-state result, 9,270 rpm (Fig. 4.3). The amplitudes from different transient analyses at the bearing (node 4) and the center disk (node 13) under various accelerations are plotted in Figs. 6.6\&6.7. In the figures, the rotational speed increases from 5,000 rpm to $12,000 \mathrm{rpm}$ with different acceleration rates. The lines of blue, green, and red indicate acceleration of 70,000 rpm/sec. $(0.1 \mathrm{sec}), 7000$ $\mathrm{rpm} / \mathrm{sec}$. $(1.0 \mathrm{sec}$.) and $700 \mathrm{rpm} / \mathrm{sec}$. (10 sec.) respectively. Also the steady state response under different rotational speeds, the cyan line, is shown in Fig. 6.6 to compare the transient response under different accelerations. From the two figures, it shows that the speed of the maximum amplitude corresponded increases as the acceleration increases. Prior research based on the 2-DOF system shows that the amplitude decreases as 
acceleration increases due to the additional unbalance $\left(m e_{u} \dot{\Omega}\right)$ term. For the complex rotor system with disks attached, however, the additional gyroscopic terms due to acceleration $(\dot{\Omega} G)$ affects the amplitude at the same time. The max amplitude of going though critical speed is related to both the unbalance forces and the gyroscopic effects. With the acceleration increases, the response curve shifts right and are far away from the steady state response gradually, as shown in Figs. 6.6\&6.7.
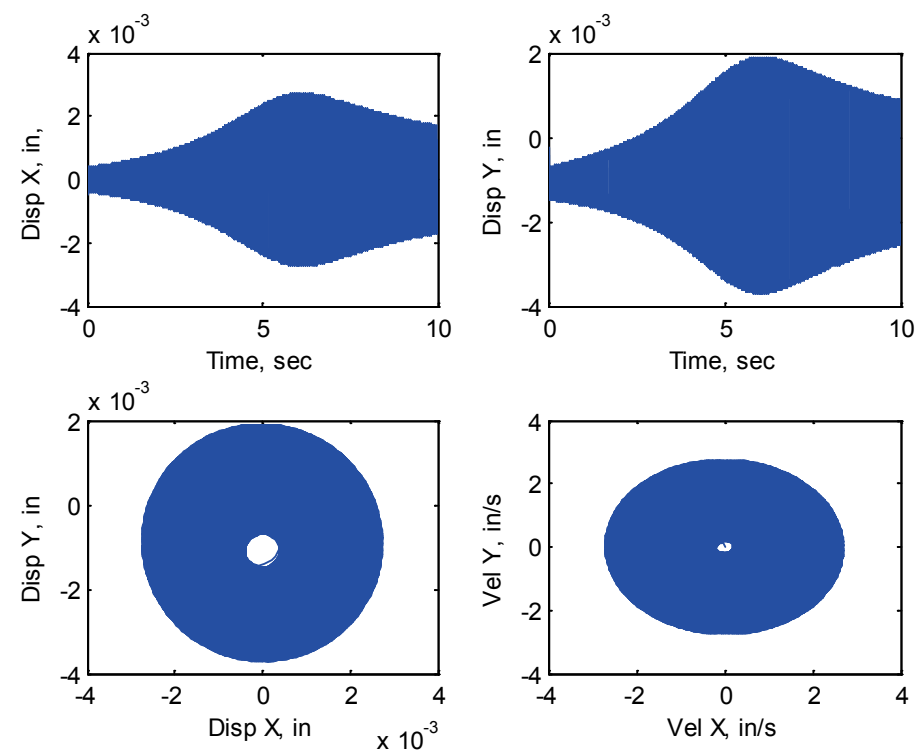

Figure 6.5 Response of Linear System (Node 13) with Linear Acceleration Rate of 700 $\mathrm{rpm} / \mathrm{second}$ 


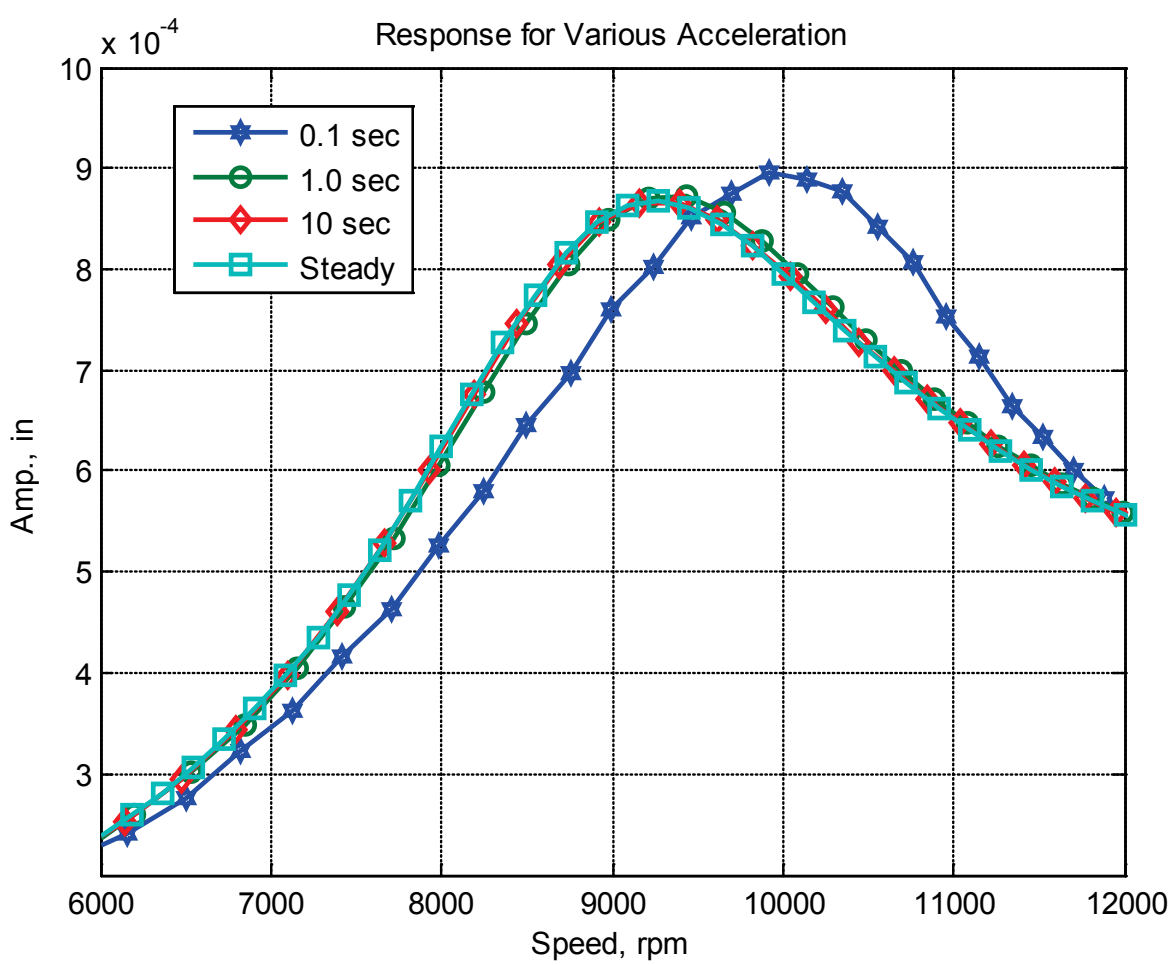

Figure 6.6 Time Transient Response at Bearing with Various Acceleration Rates

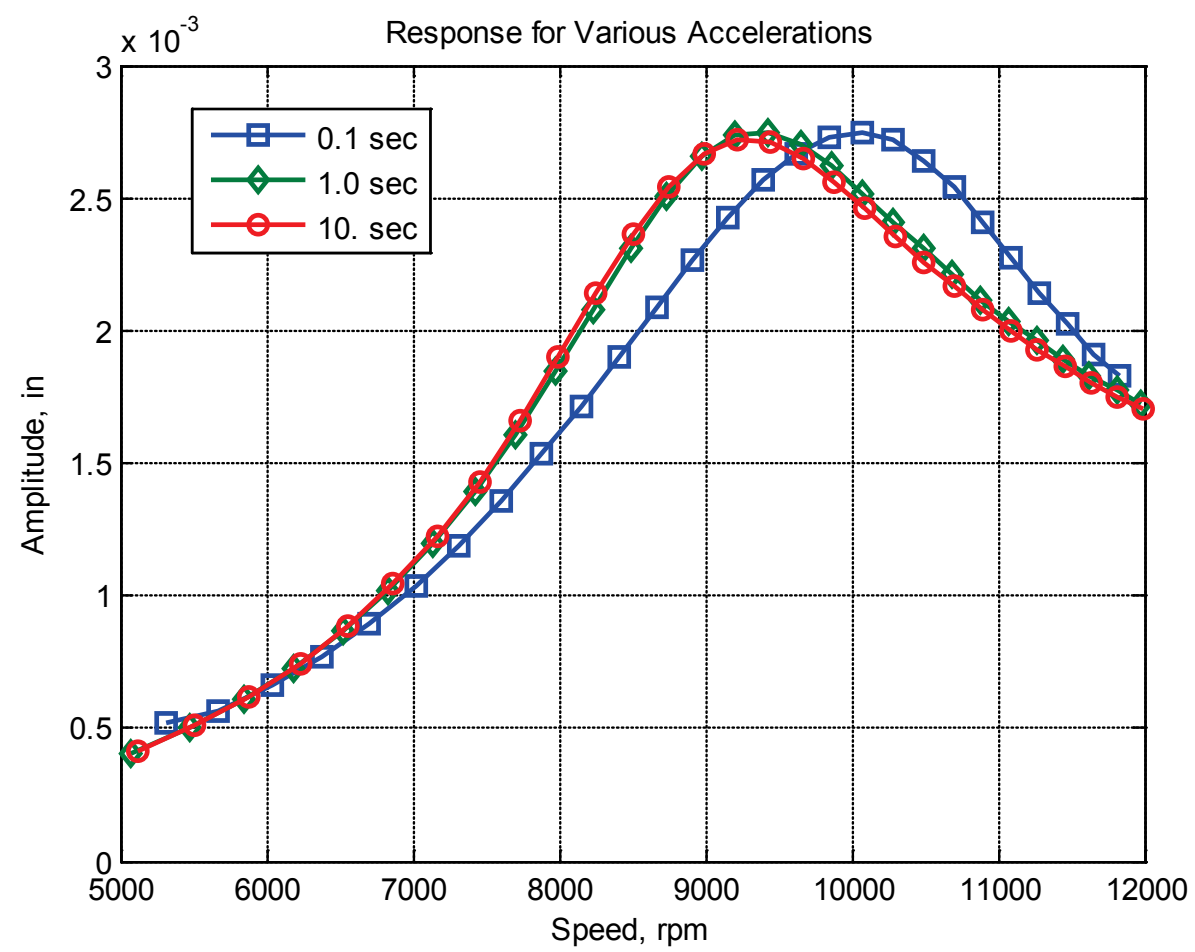

Figure 6.7 Time Transient Response at Center Disk with Various Acceleration Rates 
The time transient response of the 3 disk rotor with two nonlinear bearings and two nonlinear squeeze film dampers under the exponential acceleration (Fig. 6.8), is shown in Figs. 6.9 6.14. The nonlinear bearings and nonlinear squeeze film dampers, which are same as introduced in Chapter 4, are applied here. The transient simulation is performed for the rotor speed from $5,000 \mathrm{rpm}$ to $12,000 \mathrm{rpm}$ within 0.2 second with an exponential acceleration rate $\left(\Omega=12000-7000 e^{-20 t}\right)$, as shown in Fig. 6.8. The time transient response, either from the center disk (node 13, Fig. 6.9), the bearing (node 4, Fig. 6.10), or the damper (node 4, Fig. 6.11) has no obvious peak during the acceleration from 5,000 rpm to $1,2000 \mathrm{rpm}$ due to the additional damping effects from the squeeze film dampers. The frequency domain response of the shaft center inside the bearing at node 4, Fig. 6.12, shows one peak at $11,500 \mathrm{rpm}$ due to the slow acceleration after $11,000 \mathrm{rpm}$. The similar frequency domain response from the bearing inside the squeeze film damper at node 4 is shown in Fig. 6.13. The transient response of the whole shaft during the acceleration is shown in Fig. 6.14.

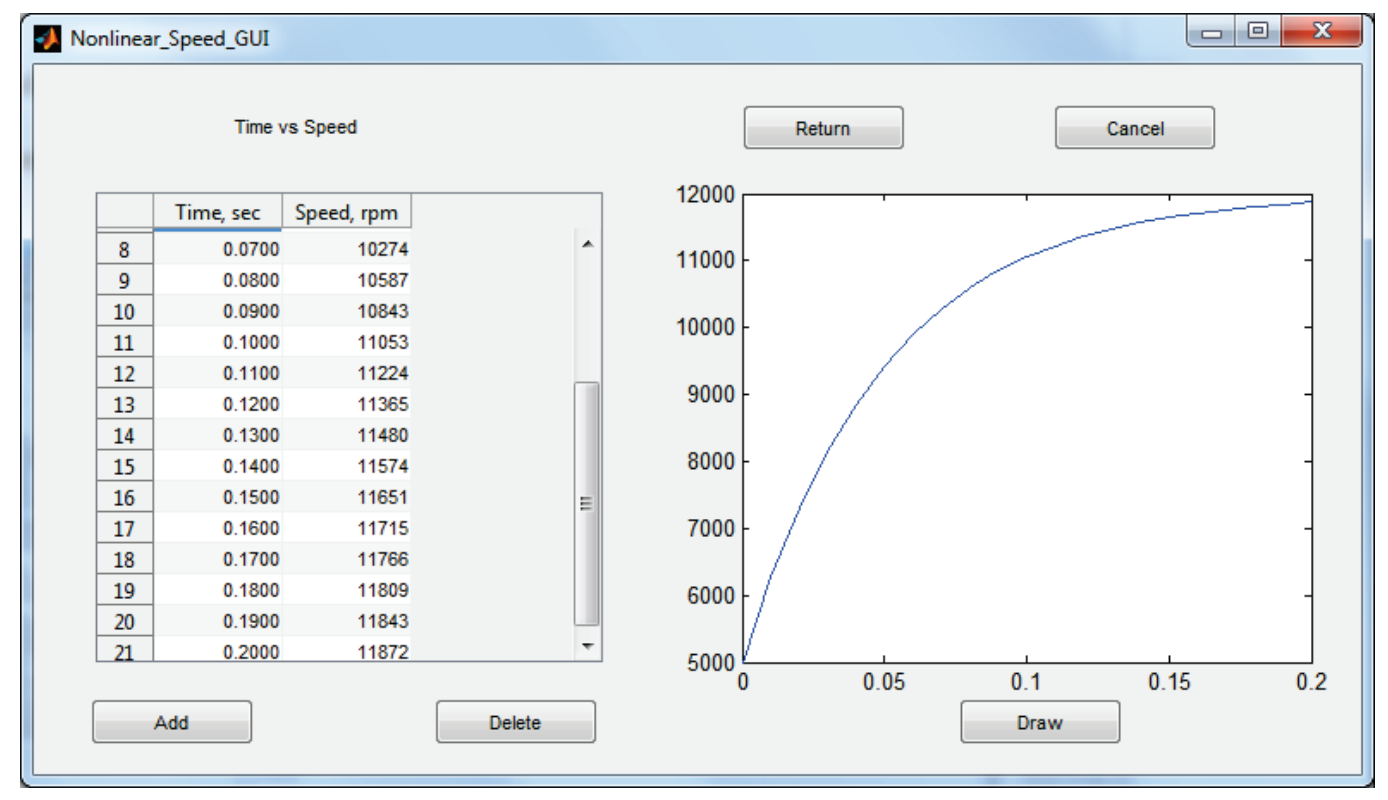

Figure 6.8 Exponential Speed Profile 

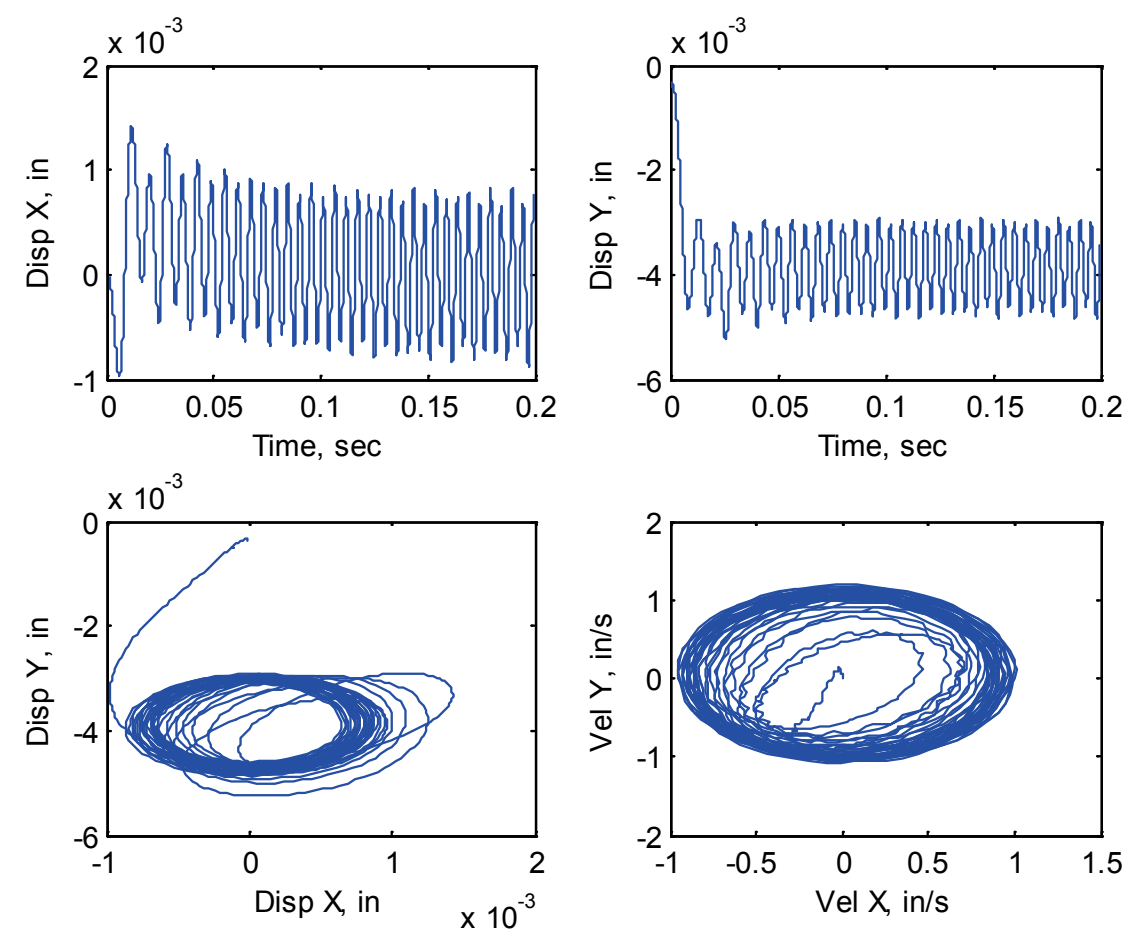

Figure 6.9 Response of Center Disk (Node 13) with an Exponential Acceleration Rate
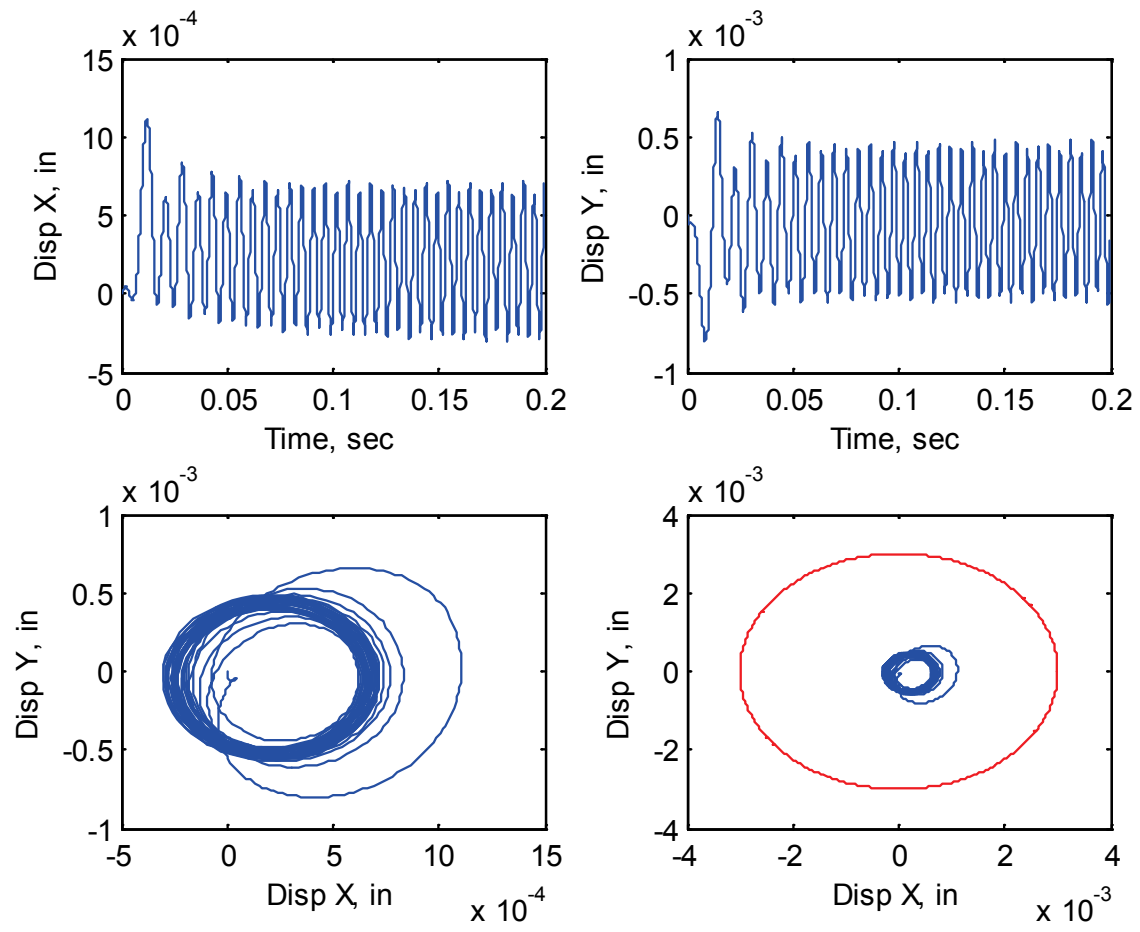

Figure 6.10 Response of the Shaft Center inside Bearing (Node 4) with an Exponential Acceleration Rate 

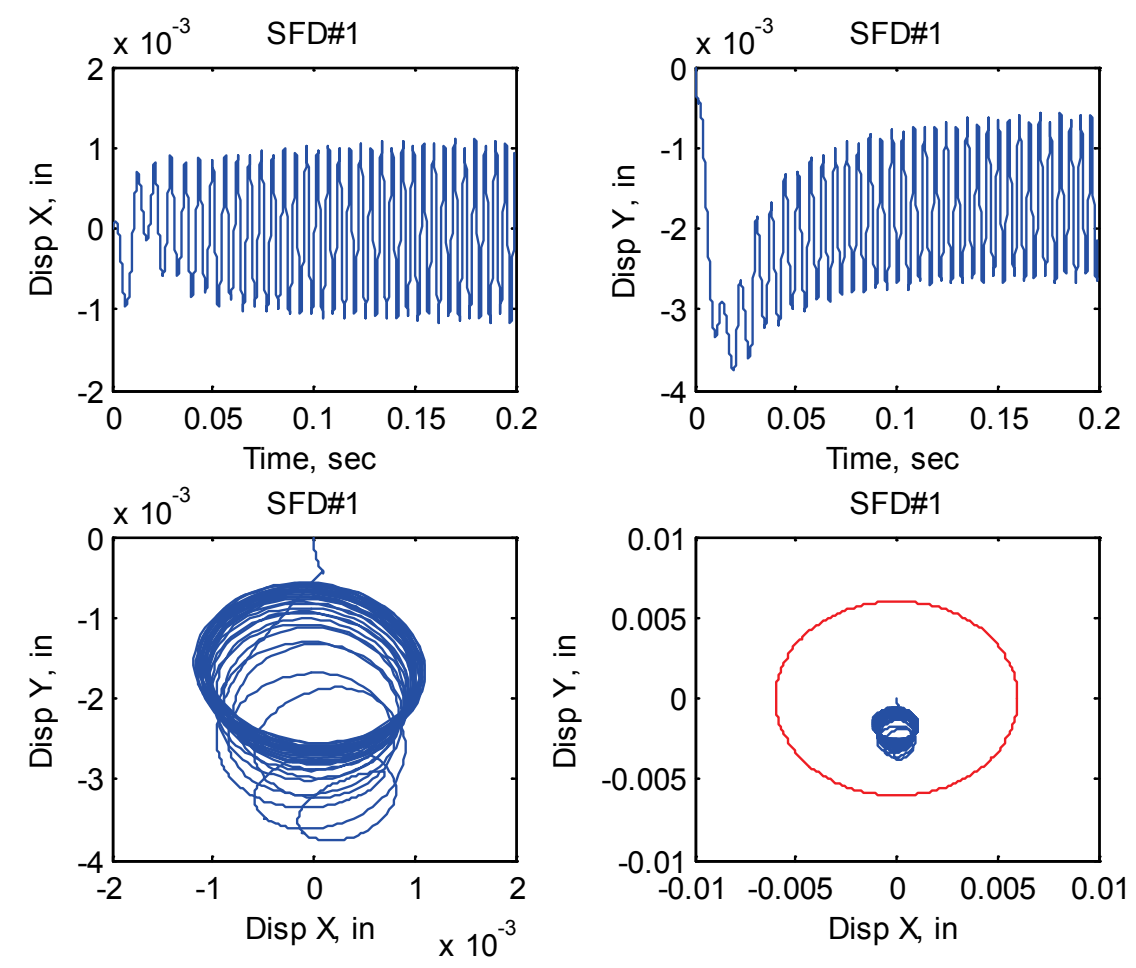

Figure 6.11 Response of the Squeeze Film Damper (Node 4) with an Exponential Acceleration Rate
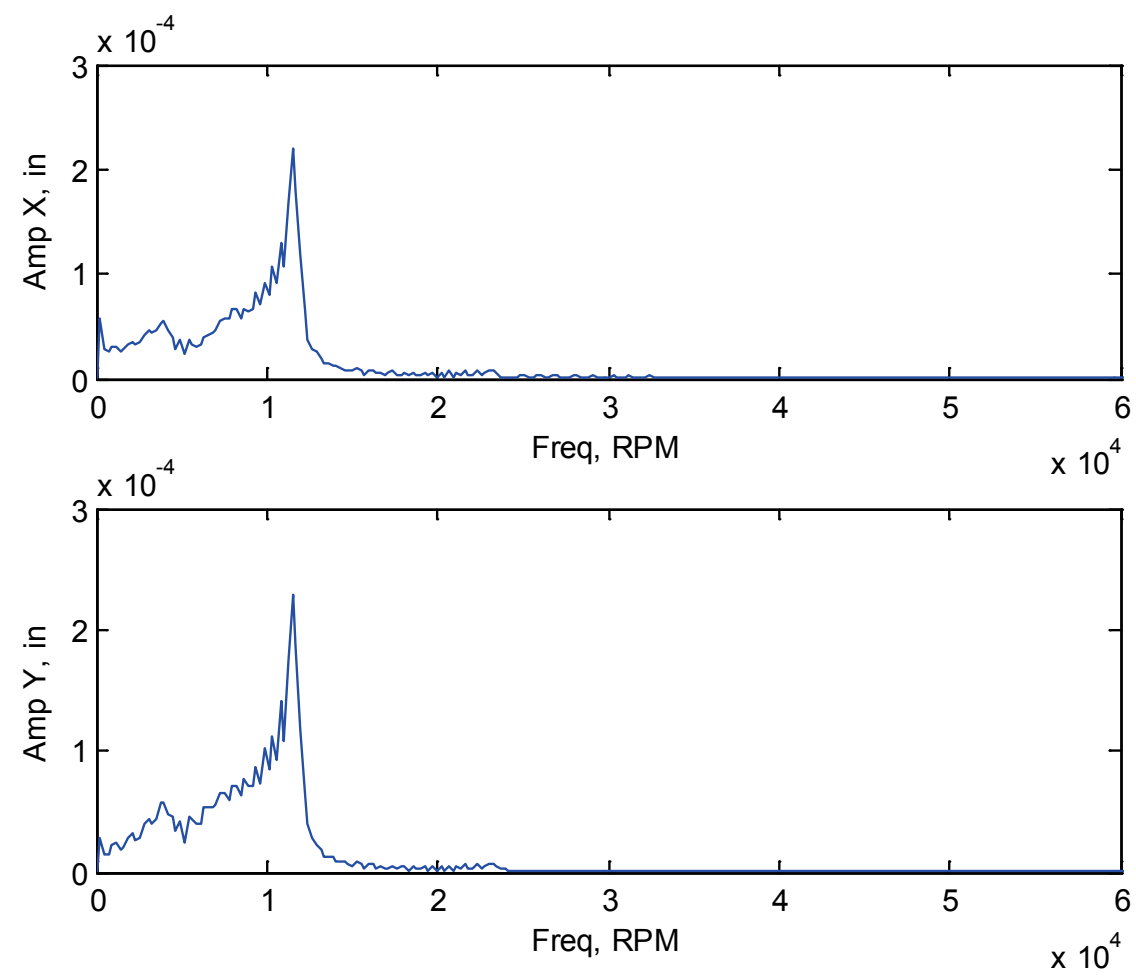

Figure 6.12 FFT of Shaft inside Bearing (Node 4) with an Exponential Acceleration Rate 

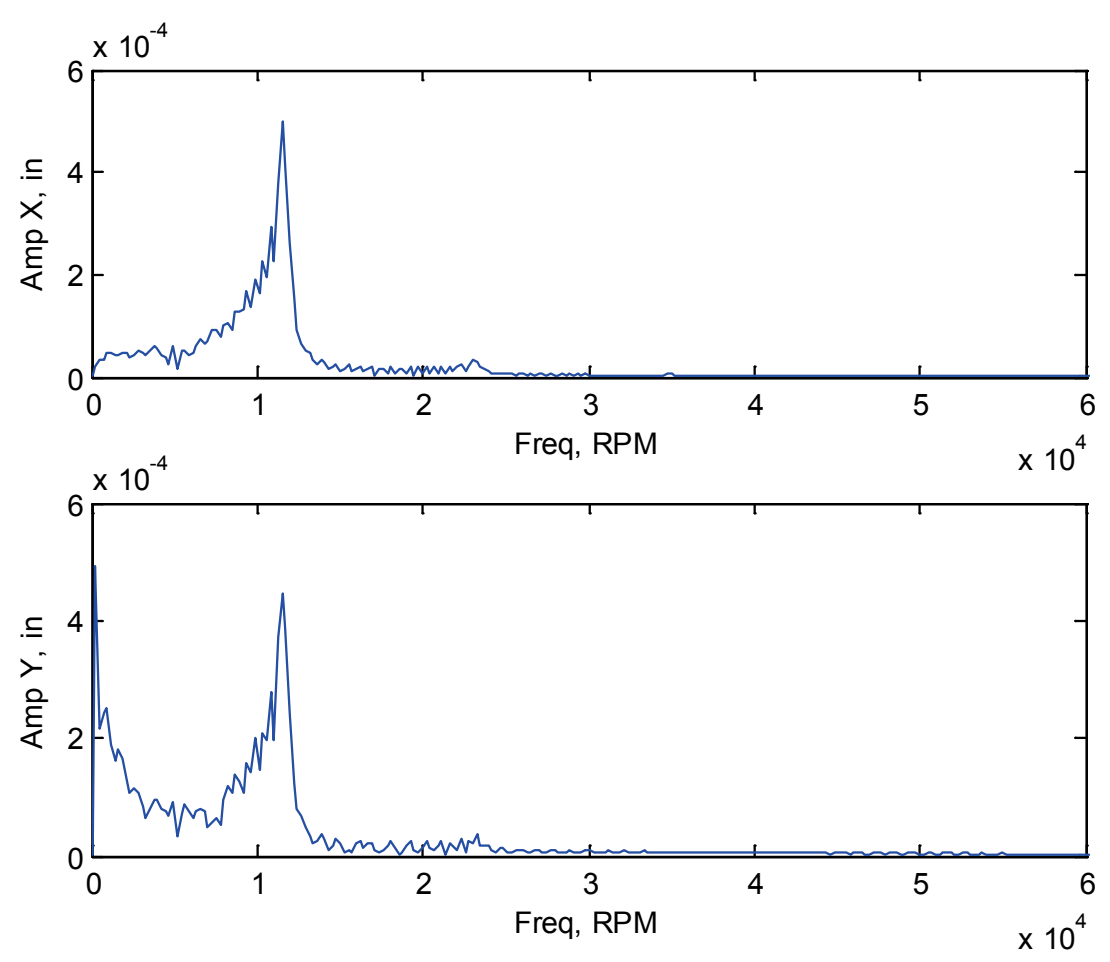

Figure 6.13 FFT of Bearing inside SFD (Node 4) with an Exponential Acceleration Rate

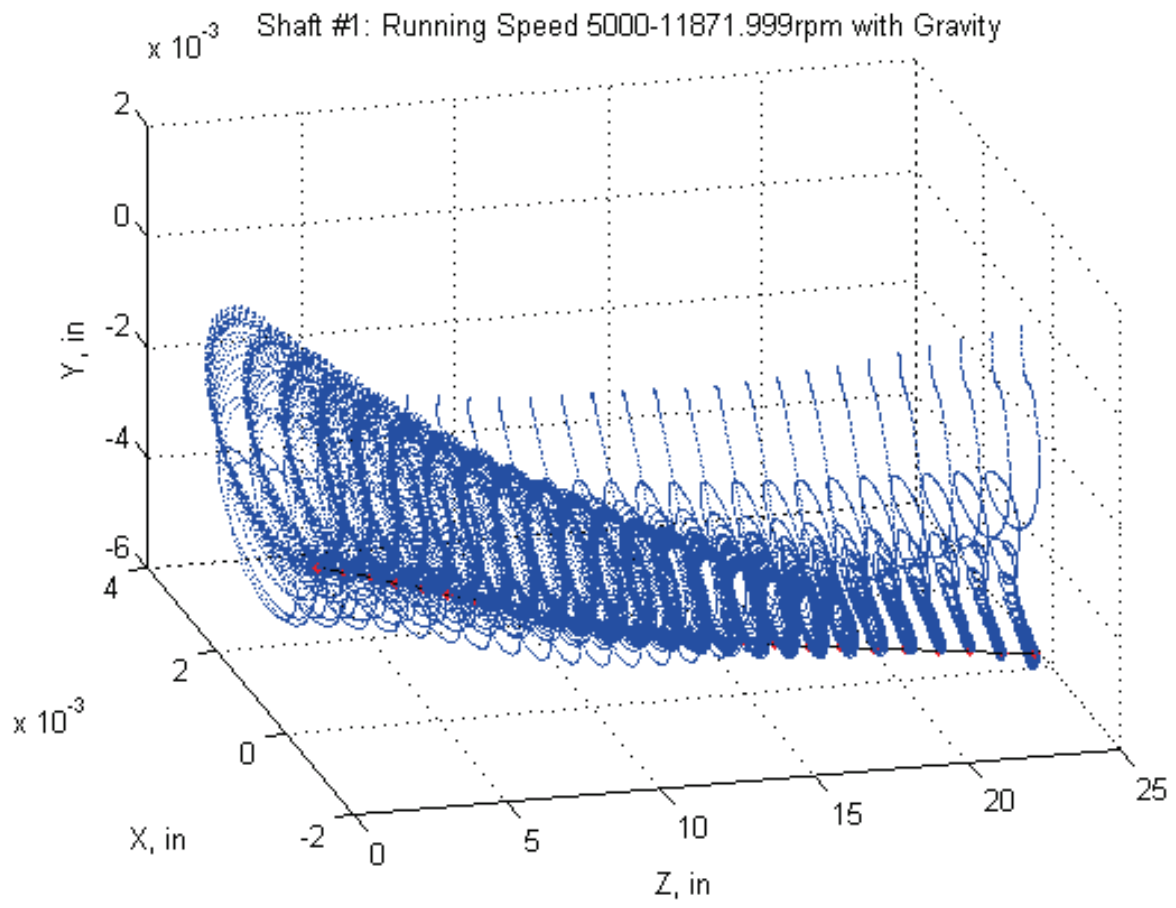

Figure 6.14 Shaft Transient Response during an Exponential Acceleration Rate under Gravity Loading and Unbalances 


\subsection{Torsional Rotor Dynamic Analysis}

Torsional vibrations may cause serious failures in rotating machinery if the complete system is not properly evaluated and designed. Torsional failures of coupling, shafts, and gears may destroy the entire machine. When external torques or torsional forces are applied to the rotating system, the rotational speed of the system often changes with the applied torques.

\subsubsection{Motion Equations and External Torsional Forces}

The equations of motion for torsional analysis only have the form:

$$
M \ddot{\theta}_{z}+C \dot{\theta}_{z}+K \theta_{z}=T\left(\dot{\theta}_{z}, \ddot{\theta}_{z}, t\right)
$$

In general, the driving torque, at any instant during startup, includes a constant average torque and an oscillating torque:

$$
T_{i}=T_{a v g}+T_{o s c} \sin \left(\omega_{e x c} t+\phi_{e x c}\right)
$$

where the magnitude of the torque $\left(T_{i}\right)$ is usually a function of the rotational speed. The excitation frequency of the pulsating torque for a synchronous motor during startup is equal to twice the slip frequency and is a linear function of the rotor speed:

$$
\omega_{e x c}(\mathrm{rad} / \mathrm{sec})=2 \times 2 \pi f_{s}=4 \pi f_{L}\left(1-\frac{N_{m}}{N_{s y n}}\right)
$$

where $N_{m}$ is the current motor speed and $N_{s y n}$ is the designed synchronous speed. 


\subsubsection{Startup Transient Motion and Torsional Analysis}

An example of a three single stage compressors [13] driven by a synchronous rotor is applied at first to validate the nonlinear torque calculation and the torsional transient solver. The schematic of the compressor configuration is shown in Fig. 6.15. A $50 \mathrm{~Hz}$, $4200 \mathrm{~kW}$, 4-pole, 1,500 rpm synchronous motor with a rated torque of $26,716 \mathrm{~N}-\mathrm{m}$ is the prime driver. The synchronous speed is $1,500 \mathrm{rpm}$ and the motor is started with reduced voltage. The driving torque and the load torque vs. the motor speed are shown in Fig. 6.16. Since the loads are quite evenly distributed among three stages, the three compressor load curves are very close. The effective inertias and stiffness coefficients are given in Table 6.1. The bull gear connects to three compressors at node 8, 11, and 14 with speed ratios of $8.43,12.04$, and 16.85 respectively. In this example, the torsional stiffness at the gear mesh is much stiffer than the other stiffness values of the system and thus is considered a rigid connection or infinite torsional stiffness without damping at the gear mesh.

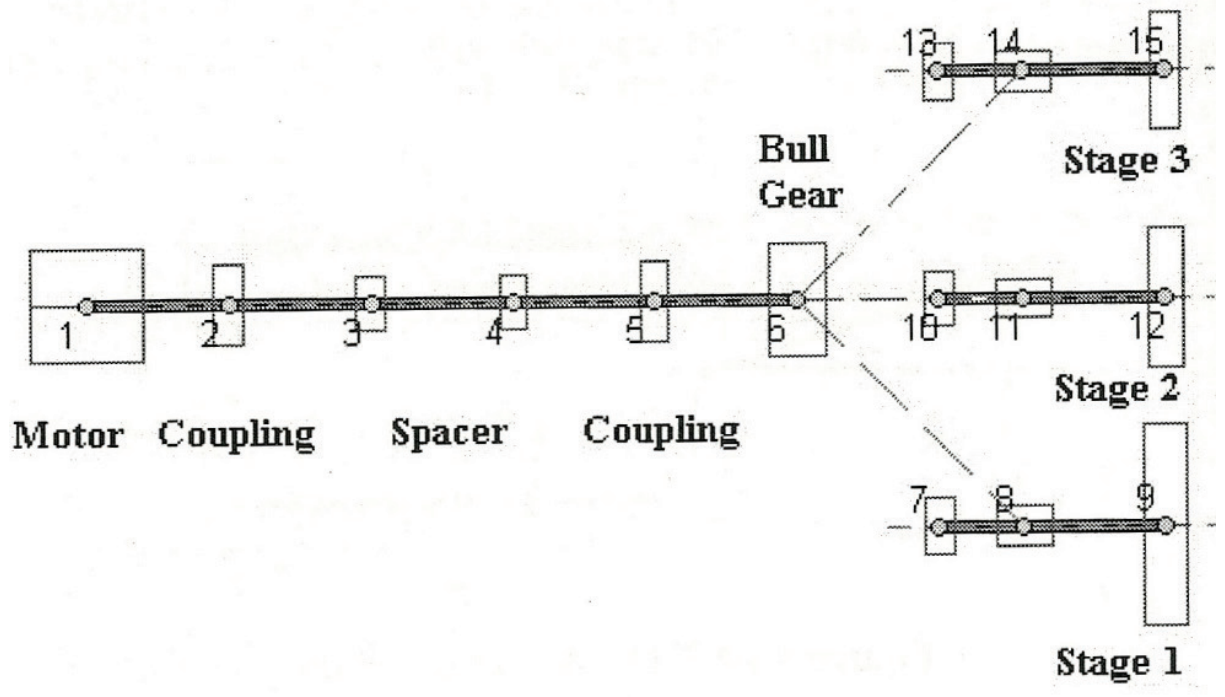

Figure 6.15 Synchronous Motor Driven 3 Compressor System [13] 
Torsional Excitations \& Loads

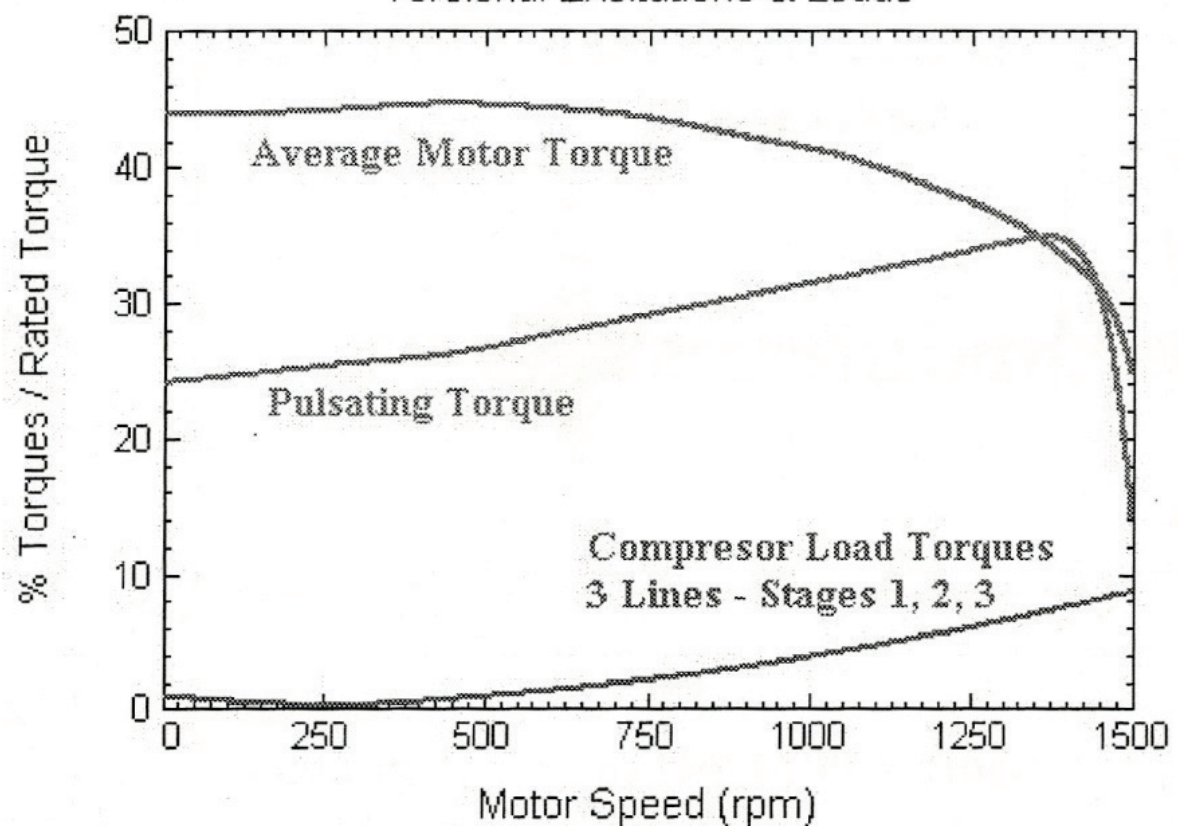

Figure 6.16 Torque Speed Curves [13]

Table 6.1 Model Effective Inertia and Stiffness [13]

\begin{tabular}{|c|c|c|c|c|}
\hline $\begin{array}{c}\text { Node } \\
\text { No. }\end{array}$ & $\begin{array}{c}\text { Effective Inertia } \\
\left(\mathrm{kg}-\mathrm{m}^{2}\right)\end{array}$ & Node I & Node J & $\begin{array}{c}\text { Effective Stiffness } \\
(\mathrm{N}-\mathrm{m} / \mathrm{rad})\end{array}$ \\
\hline 1 & 158.3 & 1 & 2 & $1.4510 \mathrm{E} 7$ \\
\hline 2 & 2.872 & 2 & 3 & $1.4789 \mathrm{E} 6$ \\
\hline 3 & 16.27 & 3 & 4 & $4.8807 \mathrm{E} 8$ \\
\hline 4 & 16.35 & 4 & 5 & $1.4789 \mathrm{E} 6$ \\
\hline 5 & 2.872 & 5 & 6 & $7.5850 \mathrm{E} 6$ \\
\hline 6 & 156.5 & 7 & 8 & $8.9020 \mathrm{E} 7$ \\
\hline 7 & 0.4034 & 8 & 9 & $8.8220 \mathrm{E} 7$ \\
\hline 8 & 3.749 & 10 & 11 & $1.7120 \mathrm{E} 8$ \\
\hline 9 & 248.4 & 11 & 12 & $8.1850 \mathrm{E} 7$ \\
\hline 10 & 0.8233 & 13 & 14 & $1.6040 \mathrm{E} 8$ \\
\hline 11 & 2.067 & 14 & 15 & $1.7180 \mathrm{E} 7$ \\
\hline 12 & 77.5 & & & \\
\hline 13 & 1.614 & & & \\
\hline 14 & 1.68 & & & \\
\hline 15 & 28.29 & & & \\
\hline
\end{tabular}


The natural frequencies of the compressor system are calculated after the system model is assembled. Because the system is a free-free system in torsional direction, the first mode is a free mode with zero frequency. The following two critical speeds with damping ratio of 0.06 are exactly same as Chen's results, as shown in Table 6.2; the fourth natural frequency has a difference of $1.2 \%$. Since the calculated first two nonzero natural frequencies are exactly same, and there is no damping information provided for the $4^{\text {th }}$ model, except the first two modes, the small difference of the $4^{\text {th }}$ mode may arise from the structural damping or the different numerical methods of solving eigenvalue problem.

Table 6.2 Critical Speeds Compare

\begin{tabular}{|c|c|c|c|}
\hline Mode No. & Chen [13], rpm & RotorTran, rpm & Diff. \% \\
\hline 1 & - & $4.36 \mathrm{E}-4$ & \\
\hline 2 & 681 & 680.9 & 0.00 \\
\hline 3 & 2775 & 2775.0 & 0.00 \\
\hline 4 & 6897 & 6979.7 & 1.20 \\
\hline
\end{tabular}

All system natural frequencies less than 2 times of the line frequency, $100 \mathrm{~Hz}$, will be excited by the pulsating torque during synchronous motor startup. The torsional resonant speeds are obtained by setting the exaction frequency to the system natural frequency. The torsional resonance speeds are expressed as following form: 


$$
N_{c r}=N_{s y n}\left(1-\frac{\omega_{i}}{4 \pi f_{L}}\right)
$$

where $\omega_{i}$ is the $\mathrm{i}^{\text {th }}$ mode critical speed, $f_{L}$ is the line frequency, $N_{s y n}$ is the motor synchronous speed and $N_{c r}$ is the torsional resonance speed going through the torsional critical speed. In this example, the synchronous speed is $1,500 \mathrm{rpm}$; the line frequency is $50 \mathrm{~Hz}$; the first two non-zero torsional modes are $681 \mathrm{rpm}$ and 2,775 rpm. So the two transient resonance speeds due to the oscillation calculated from (6.3.4) are $806 \mathrm{rpm}$ and $1,330 \mathrm{rpm}$.

The startup torsional transient analysis is performed in this compressor. The startup time for the system to reach the synchronous speed of $1,500 \mathrm{rpm}$ is around 16 seconds, as shown in Fig. 6.17. The angular displacement $\left(\theta_{z}\right)$ during the startup is shown in Fig. 6.19. The transient vibratory torque, with respect to the steady state torque at the shaft, is shown in Fig. 6.20. It shows that the transient vibratory torque caused by the motor pulsating torque during the startup, can be more than 2 times the steady state value. From the startup figures, Fig. 6.17, the torsional oscillation increases from 11 seconds (around $1,250 \mathrm{rpm})$ and then decreases after 12 seconds $(1,400 \mathrm{rpm})$, this indicates that the system goes through the transient torsional resonant speed around of approximately1,330 rpm. From the shaft torque output, Fig. 6.20, another transient torsional resonant speed is around 5.8 seconds $(820 \mathrm{rpm})$. The results of motor speed, Fig. 6.18, and the results of vibratory torque, Fig. 6.21, by Chen [13] using same model, are copied to here. Since there is no transient data published through Chen's example, the results from same nodes are listed for comparison. The torsional transient response is almost same as the figures of motor speed and vibratory torque by Chen. 


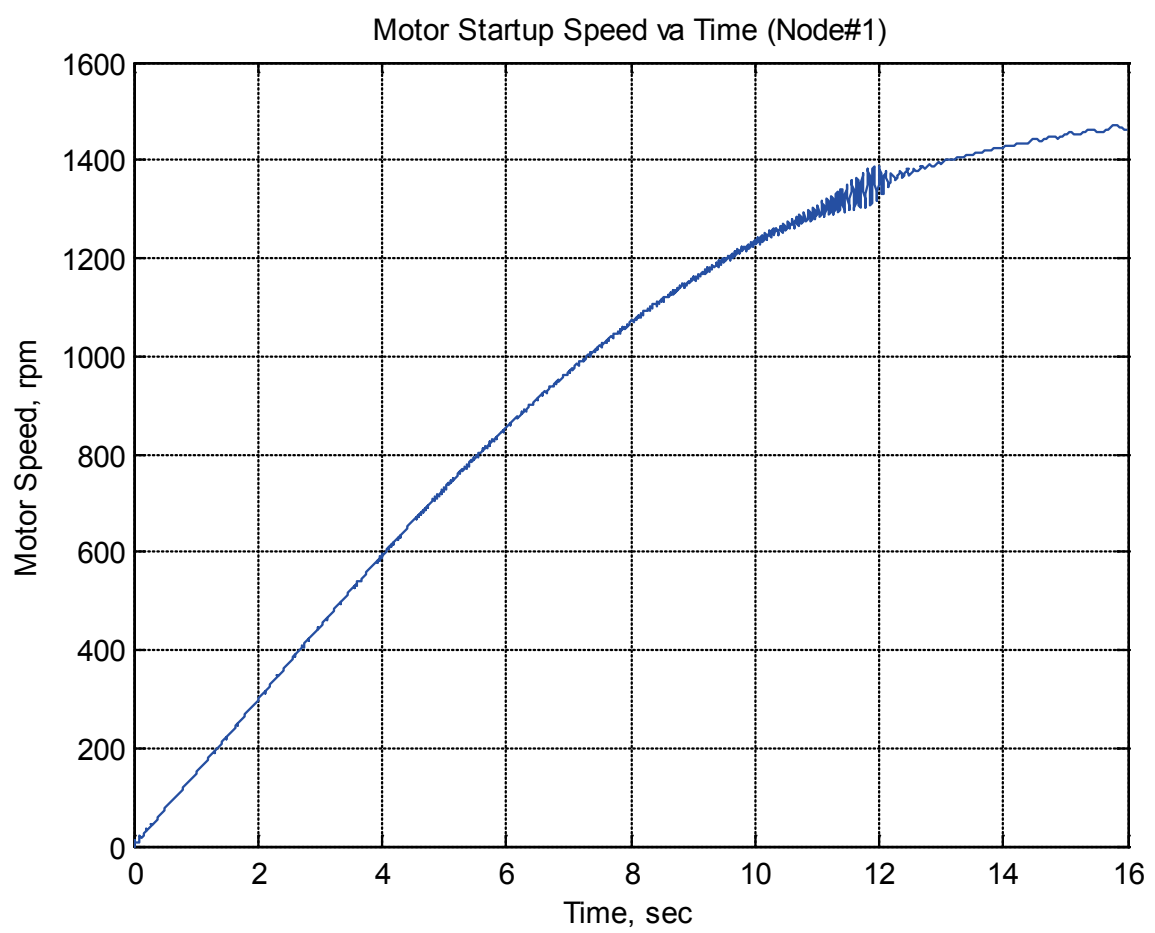

Figure 6.17 Motor Speed vs. Time during Startup

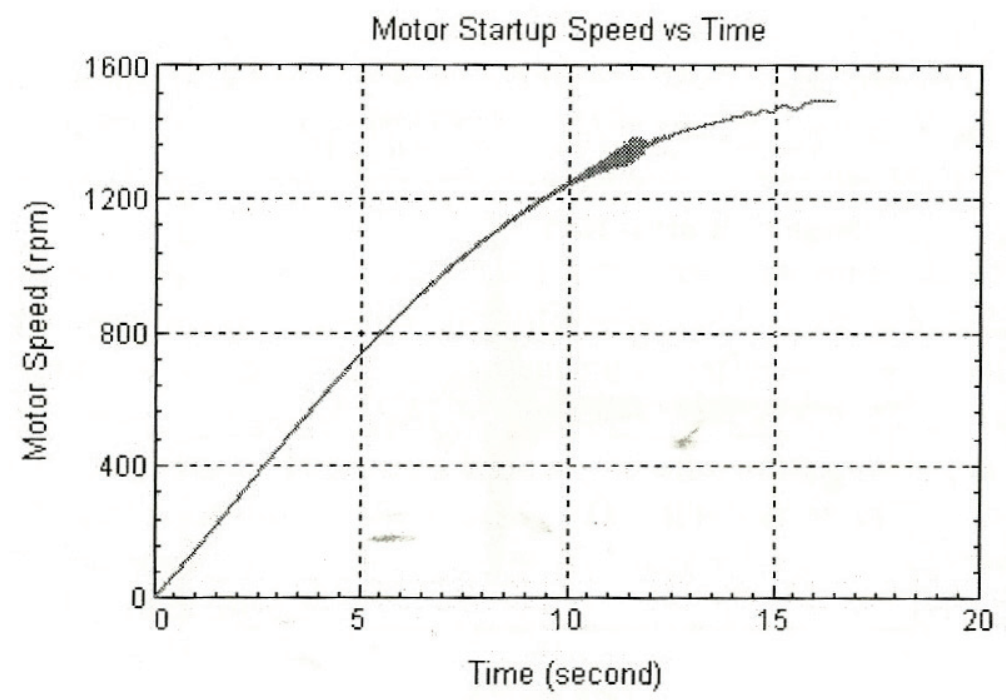

Figure 6.18 Motor Speed vs. Time during Startup from Chen [13] 


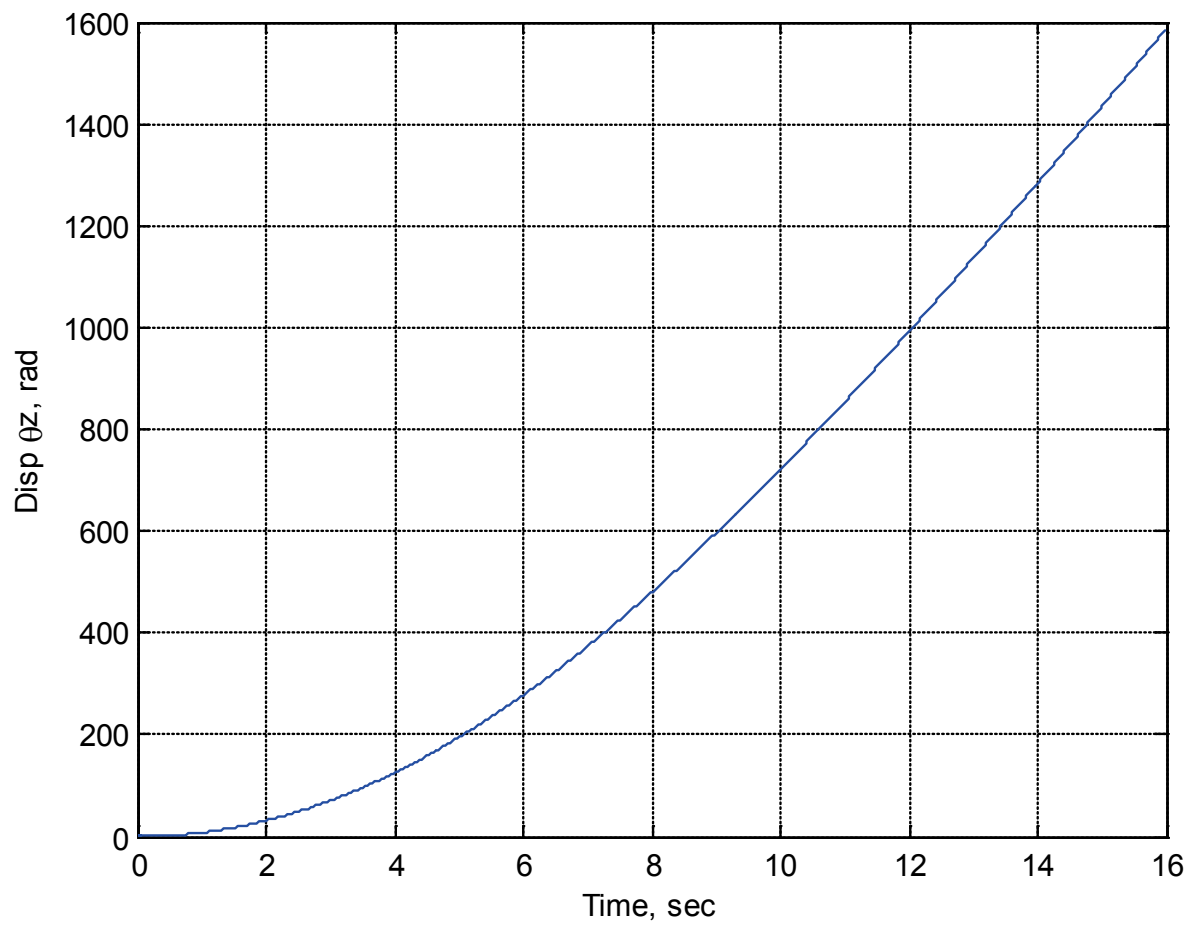

Figure 6.19 Shaft Angular Displacement vs. Time during Startup

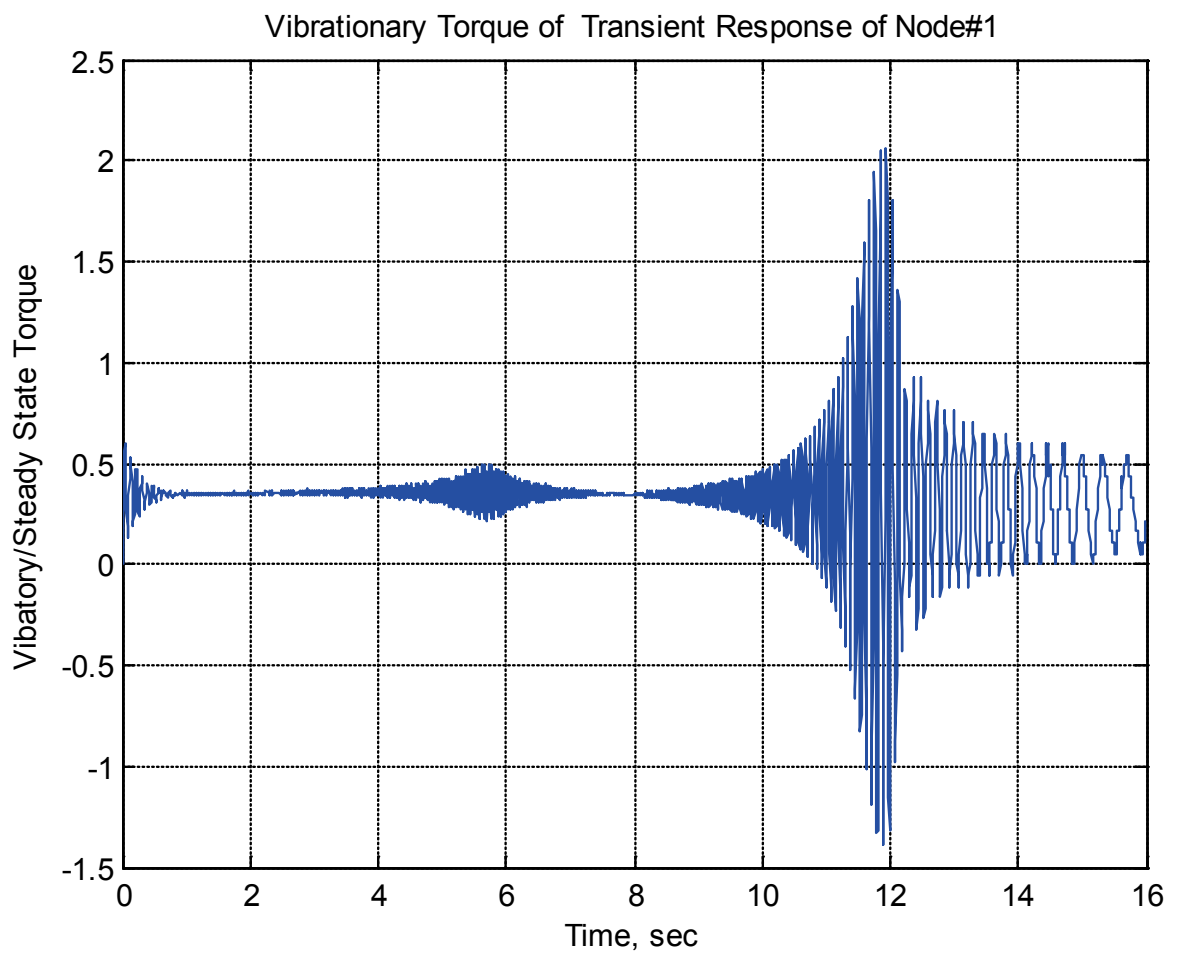

Figure 6.20 Shaft Vibratory Torque vs. Time 


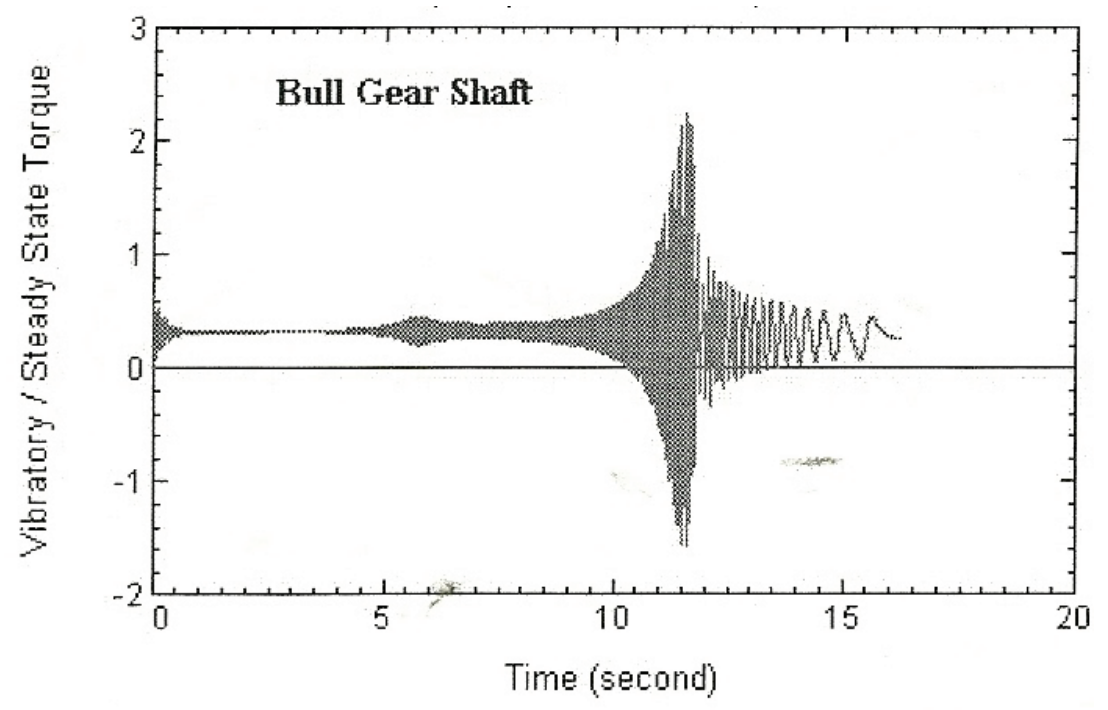

Figure 6.21 Shaft Vibratory Torque vs. Time by Chen [13]

\subsection{Coupled Torsional and Lateral Transient Analysis of the}

\section{3-Disk Rotor}

This section describes the coupled torsional and lateral analyses of the 3-disk rotor model, for which the lateral analyses have been discussed in Chapter 4. The 3-disk rotor is driven by an axial, 4 poles synchronous motor with speed ratio of 1:10, from the left (node 1). The maximum equivalent torque from the motor is $2,336 \mathrm{lbf}-\mathrm{in}$. The load is from the center disks (node 10,13 and 16) on the shaft. Since the loads are quite evenly distributed among three disks, the three loads are concentrated onto the center disk (node 13) in the following analyses to save computational time. For this system, the equivalent synchronous speed is $15,000 \mathrm{rpm}$ with an equivalent line frequency of $500 \mathrm{~Hz}$ and the motor is started with reduced voltage. The same driving torques and the load torques are applied to this rotor, as shown in Fig. 6.16. 
Fig. 6.22 shows the frequency interface diagram for the system driven by an axial synchronous motor. In the figure, the blue and green horizontal lines are $1^{\text {st }}$ and $2^{\text {nd }}$ torsional critical speed of the system; the vertical black line at 15,000 rpm indicates the synchronous speed of the system; the red line indicates the oscillating frequency from the synchronous motor. It shows that the rotor has to go through two torsional critical speeds before reaching the synchronous speed of $15,000 \mathrm{rpm}$. Two torsional natural frequencies below the $2 \mathrm{X}$ lines frequency are $30,342 \mathrm{rpm}$ and $52,860 \mathrm{rpm}$. From Equation (6.3.3), the two torsional resonance speeds due to the oscillation torque are 7,414 rpm and 1,785 rpm.

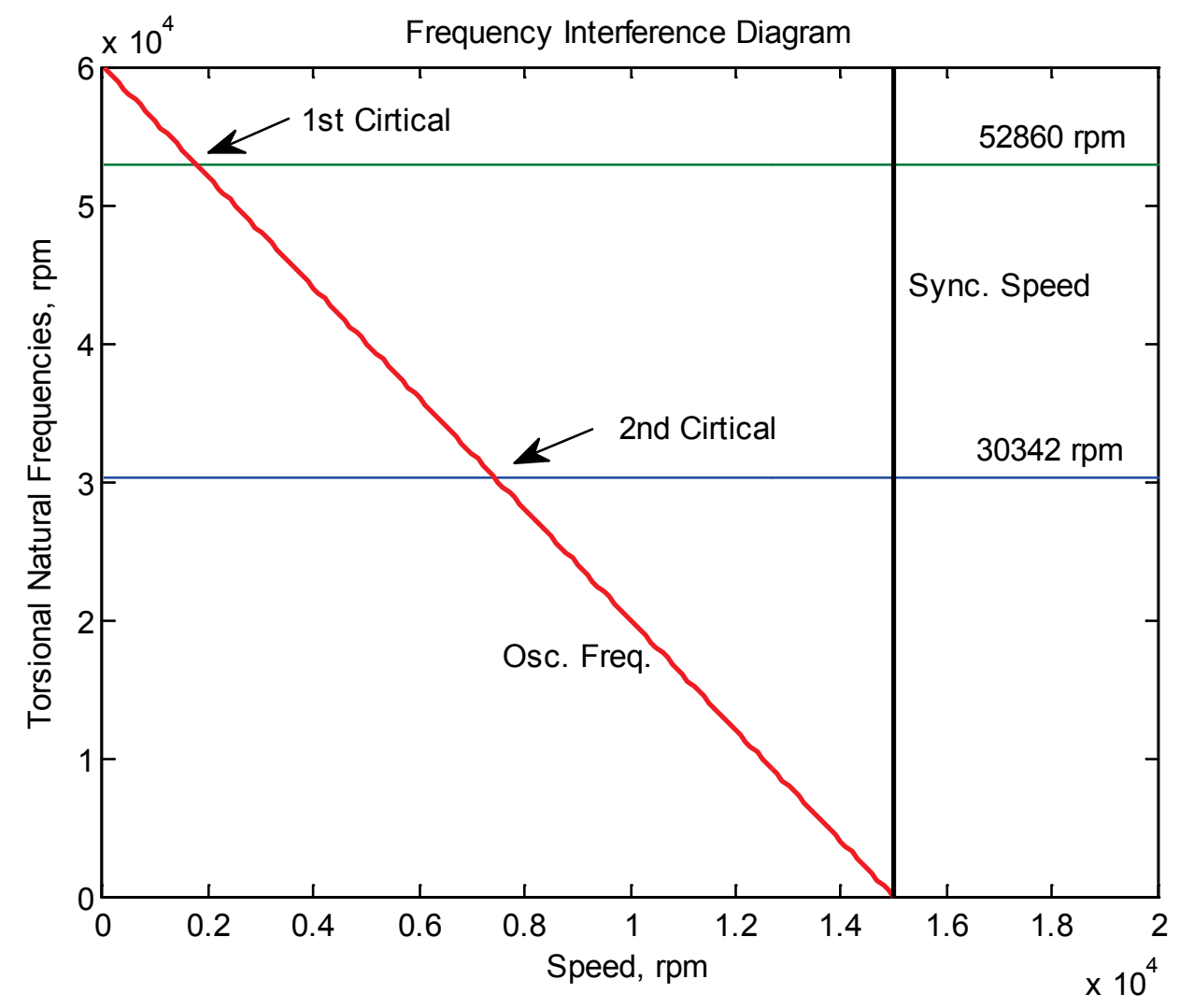

Figure 6.22 Frequency Interface Diagram for a Synchronous Motor and the Three-disk Mass Rotor 
The startup transient analysis is performed on the rotor supported with linearized bearings at first. The startup time for the system to reach the synchronous speed of 15,000 rpm is around 5.0 second, as shown in Figs. 6.23\&6.24. The transient vibratory torque, with respect to the steady state torque at the shaft (node 13), is shown in Fig. 6.25. It shows that the transient vibratory torque caused by the motor pulsating torque during the startup, can more than 12 times the steady state. Due to the acceleration, the system goes through two torsional resonances at 1,970 rpm and 7,625 rpm, which are higher than the speeds of based on the steady state. The center disk nodal displacement during startup is shown in Fig. 6.26. The rotor goes through the bending critical speed at 9,340 rpm, which is slightly higher than the bending critical speed $(9,270 \mathrm{rpm})$ calculated through the steady state due to acceleration. As discussed in Section 6.2, the peak of amplitude and the speed corresponding to the maximum amplitude are related to the angular acceleration rate.

The effect of the angular acceleration rate is calculated by applying different maximum torque outputs from the synchronous rotor. When the same curves of torque vs. speed are used for the different maximum torque outputs, the different angular acceleration rates are obtained. The coupled lateral and torsional transient responses under 10 times $(10 \mathrm{X}$, 23,360 lbf-in) and 50 times $(50 \mathrm{X}, 116,800 \mathrm{lbf}-\mathrm{in})$ of the maximum motor torque are shown in Figs. 6.27 6.29 and Figs. 6.30 6.32. With the angular acceleration rate increases, the maximum amplitude speed shifts right to a higher angular speed, as shown in Table 6.3. 
Table 6.3 Resonance Speed of Different Accelerations

\begin{tabular}{|c|c|c|c|c|}
\hline $\begin{array}{c}\text { Sync. Motor } \\
\text { Torque }\end{array}$ & $\begin{array}{c}\text { Time to } \\
\text { Sync. Speed, } \\
\text { sec }\end{array}$ & $\begin{array}{c}\text { Through } 1^{\text {st }} \\
\text { Torsional Mode }\end{array}$ & $\begin{array}{c}\text { Through } 2^{\text {nd }} \\
\text { Torsional Mode }\end{array}$ & $\begin{array}{c}\text { Through } 1^{\text {st }} \\
\text { Bending Mode }\end{array}$ \\
\hline $50 \mathrm{X}$ & 0.1 & $3,800 \mathrm{rpm}$ & $7,800 \mathrm{rpm}$ & $10,900 \mathrm{rpm}$ \\
\hline $5 \mathrm{X}$ & 1.0 & $2,414 \mathrm{rpm}$ & $7,950 \mathrm{rpm}$ & $9,505 \mathrm{rpm}$ \\
\hline $1 \mathrm{X}$ & 5.0 & $1,970 \mathrm{rpm}$ & $7,625 \mathrm{rpm}$ & $9,340 \mathrm{rpm}$ \\
\hline Steady-State & - & $1,785 \mathrm{rpm}$ & $7,414 \mathrm{rpm}$ & $9,270 \mathrm{rpm}$ \\
\hline
\end{tabular}

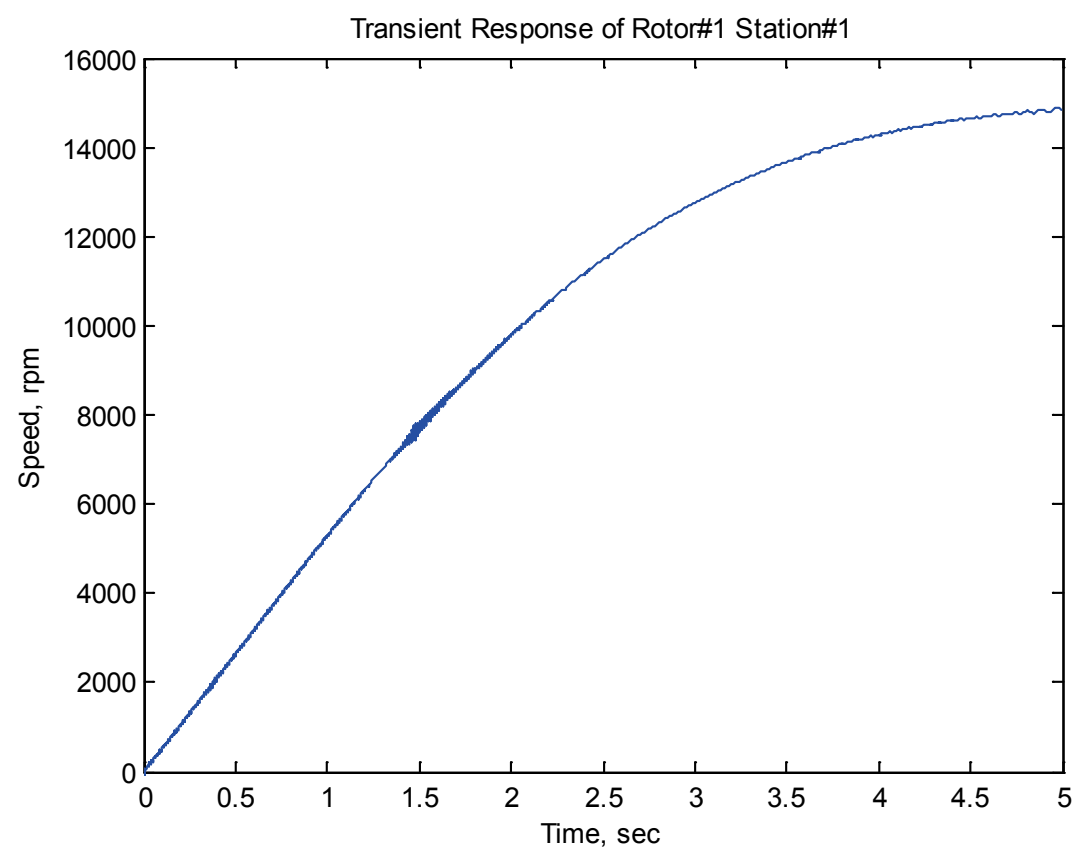

Figure 6.23 Motor Speed vs. Time during Startup, Coupled Analysis of a Synchronous Motor and the Three-disk Mass Rotor, Node\#1 


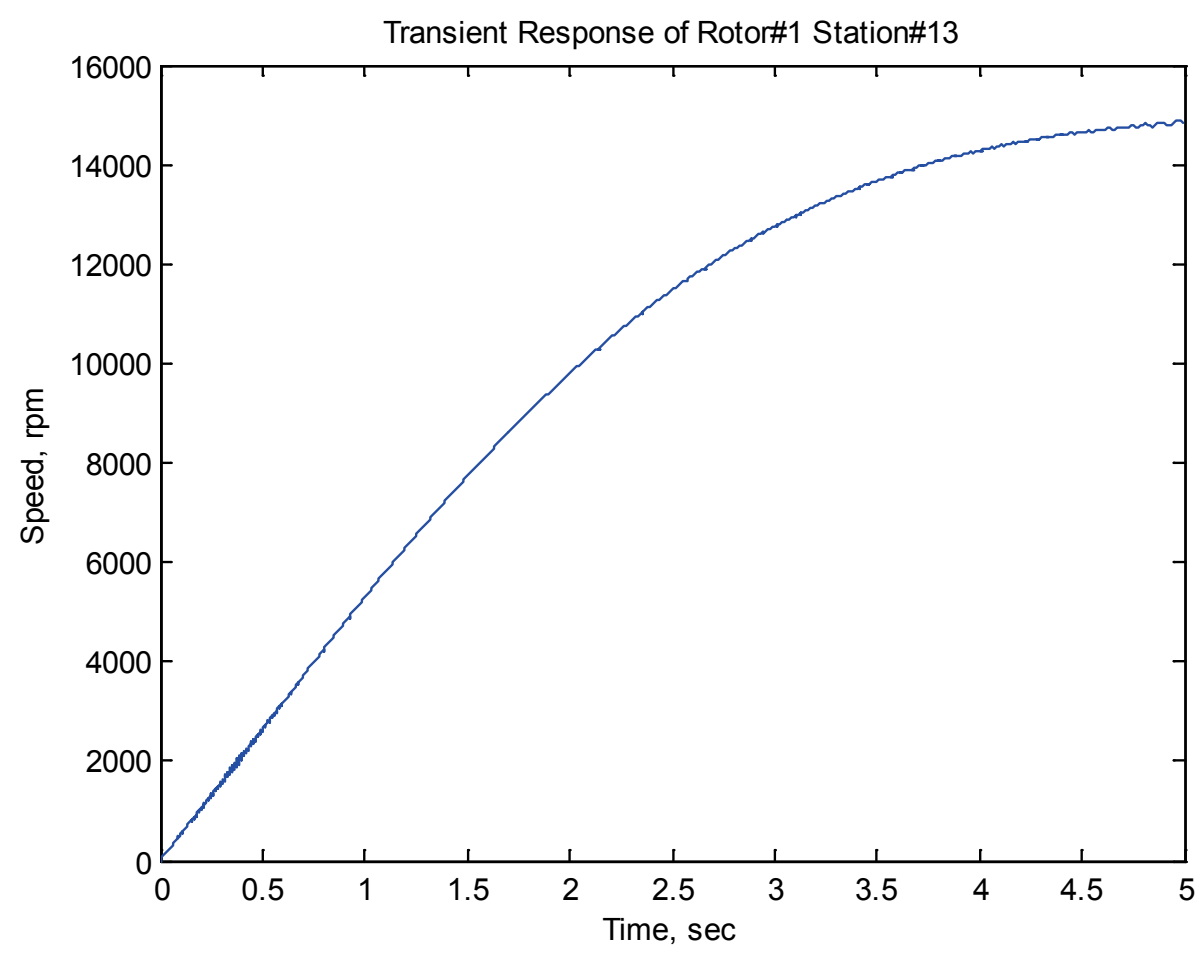

Figure 6.24 Motor Speed vs. Time during Startup, Coupled Analysis of a Synchronous Motor and the Three-disk Mass Rotor, Node\#13

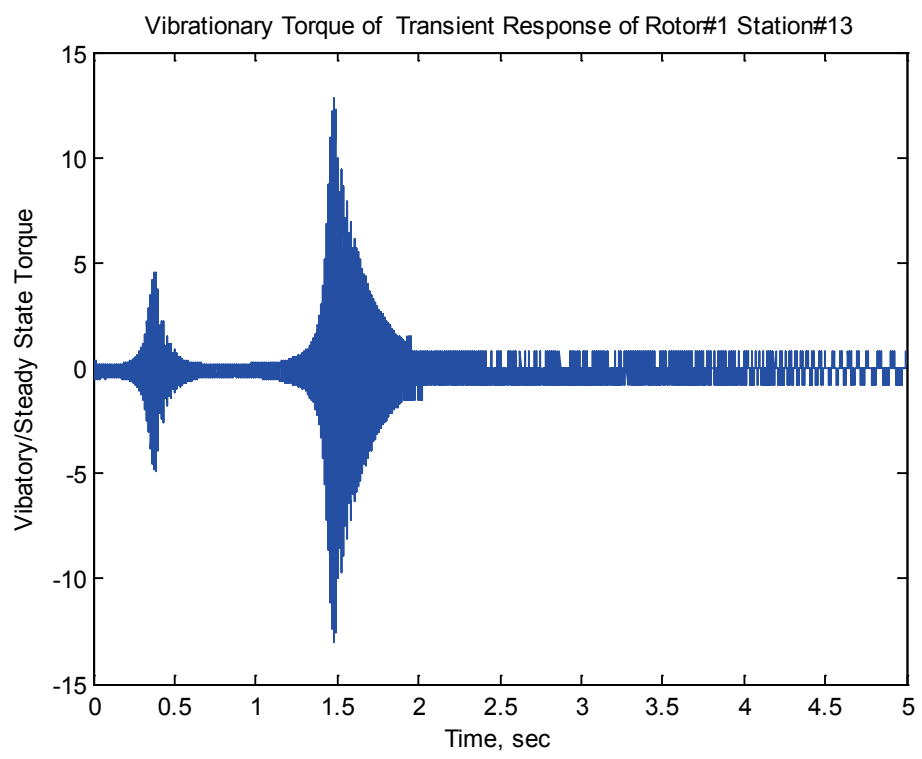

Figure 6.25 Vibratory Torque vs. Time, Coupled Analysis of a Synchronous Motor and the Three-disk Mass Rotor, Element \#13 

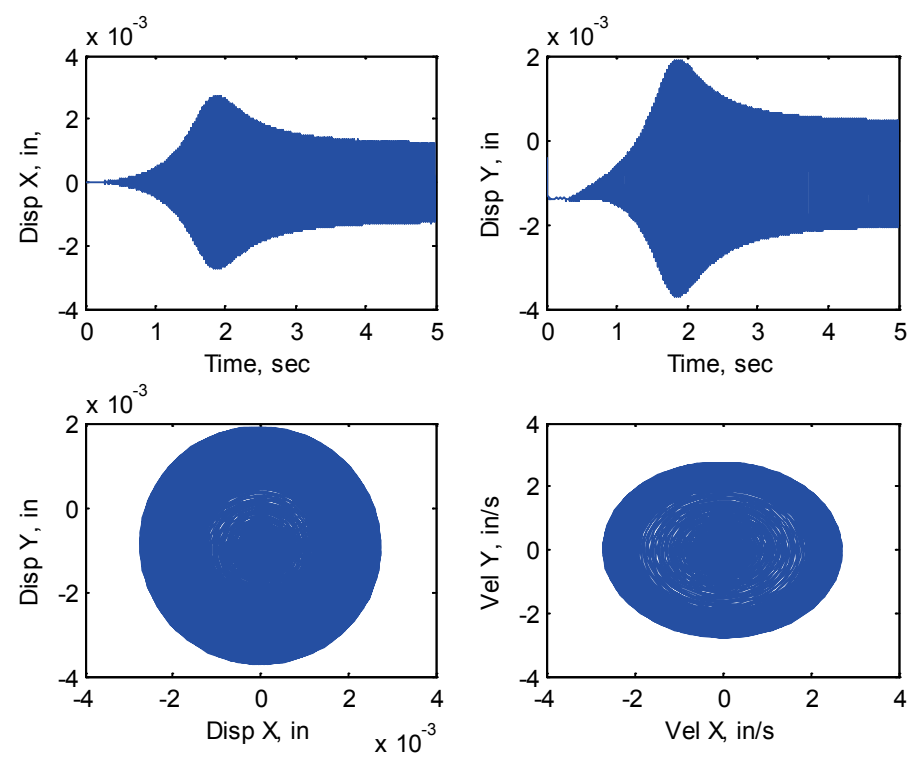

Figure 6.26 Nodal \#13 Displacement, Coupled Analysis of a Synchronous Motor and the Three-disk Mass Rotor

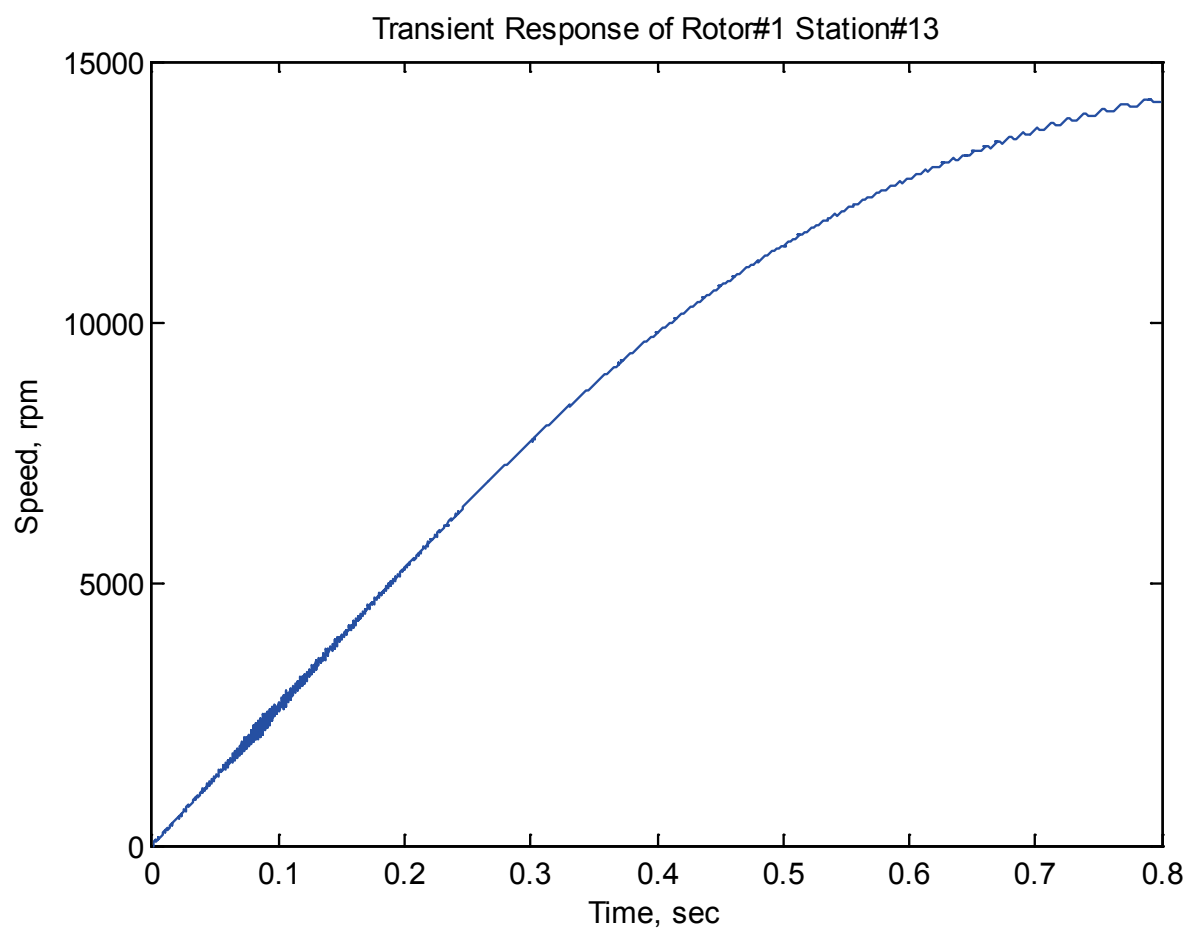

Figure 6.27 Speed vs. Time of 5X Motor Torque, Coupled Analysis of a Synchronous Motor and the Three-disk Mass Rotor, Node 13 


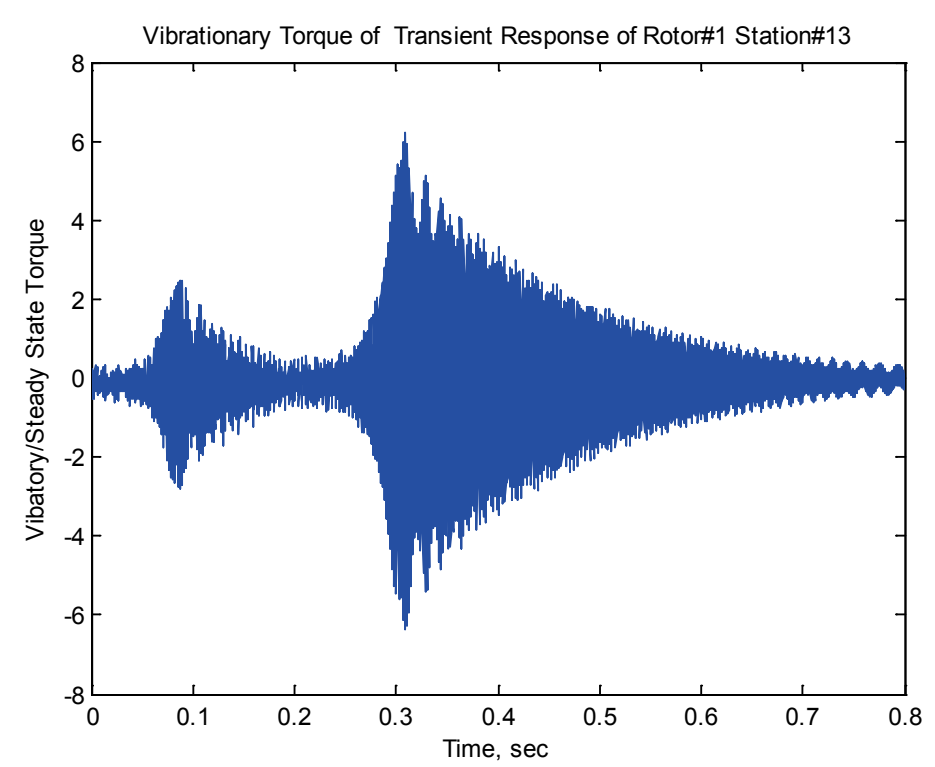

Figure 6.28 Nodal Torque vs. Time of 5X Motor Torque, Coupled Analysis of a Synchronous Motor and the Three-disk Mass Rotor (Element 13)
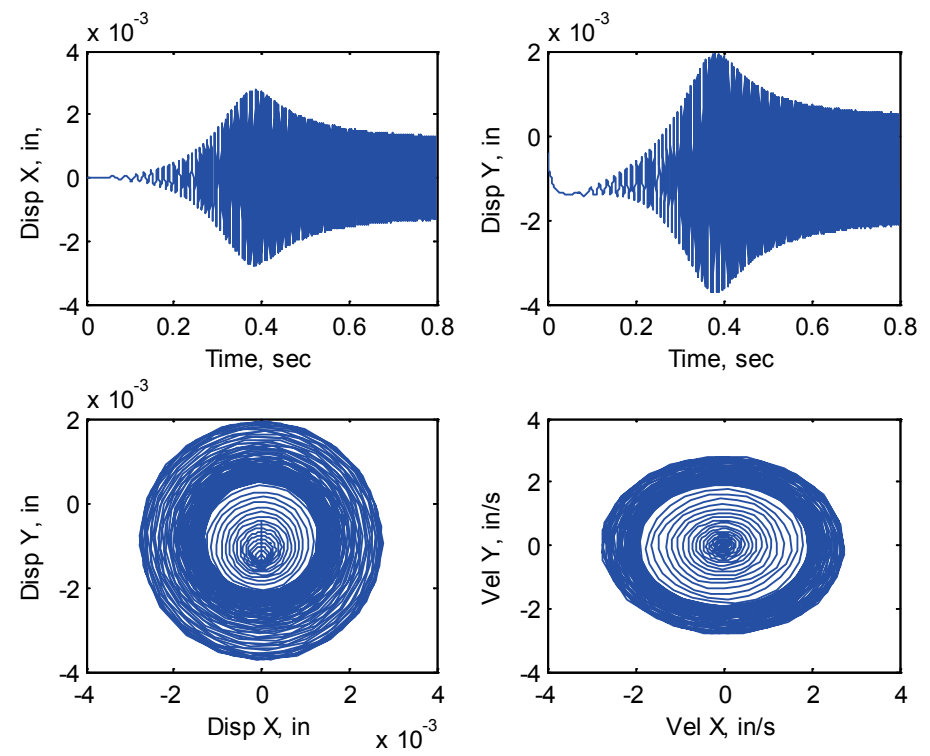

Figure 6.29 Nodal \#13 Displacement, Coupled Analysis of a Synchronous Motor and the 3-disk Mass Rotor, 5X Motor Torque 


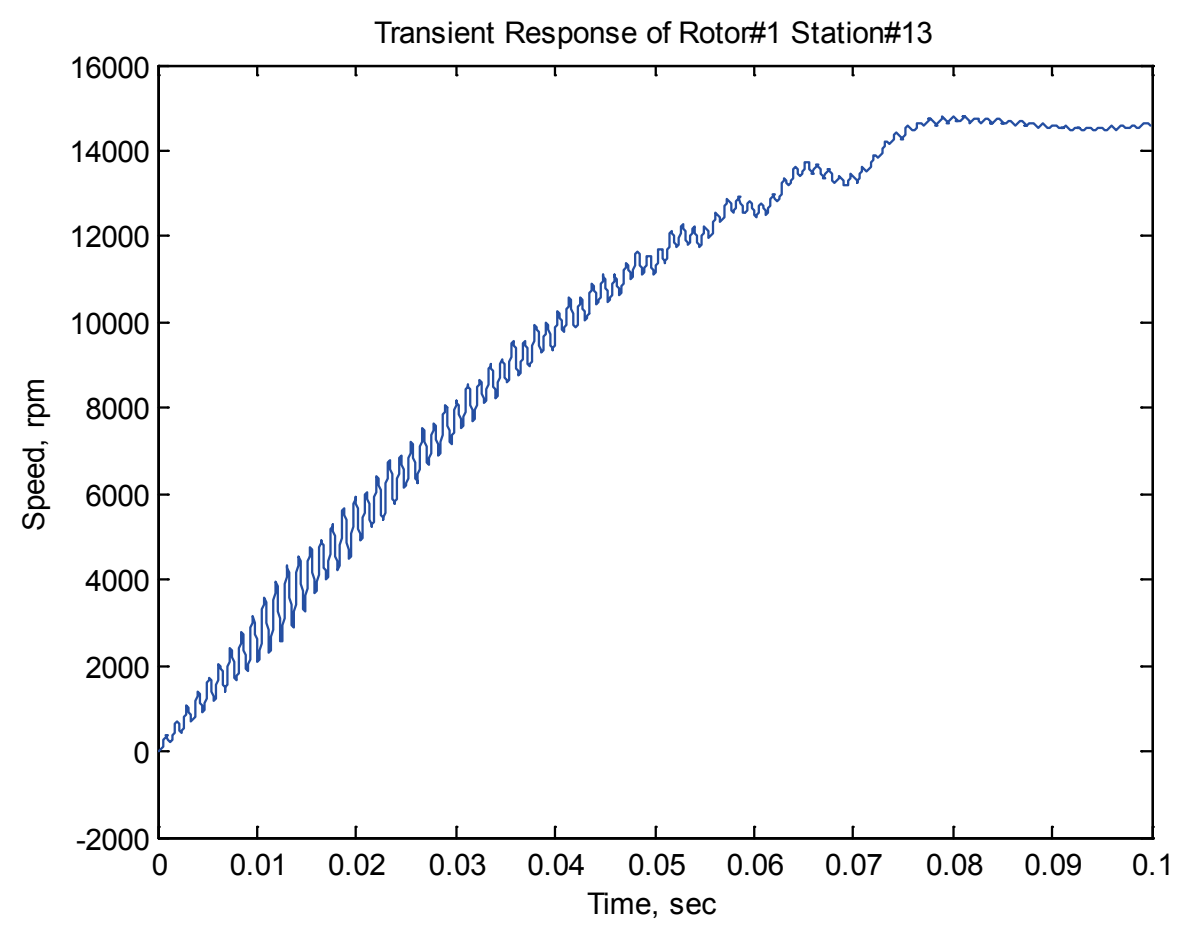

Figure 6.30 Speed vs. Time of 50X Motor Torque, Node 13 of a Synchronous Motor and the Three-disk Mass Rotor

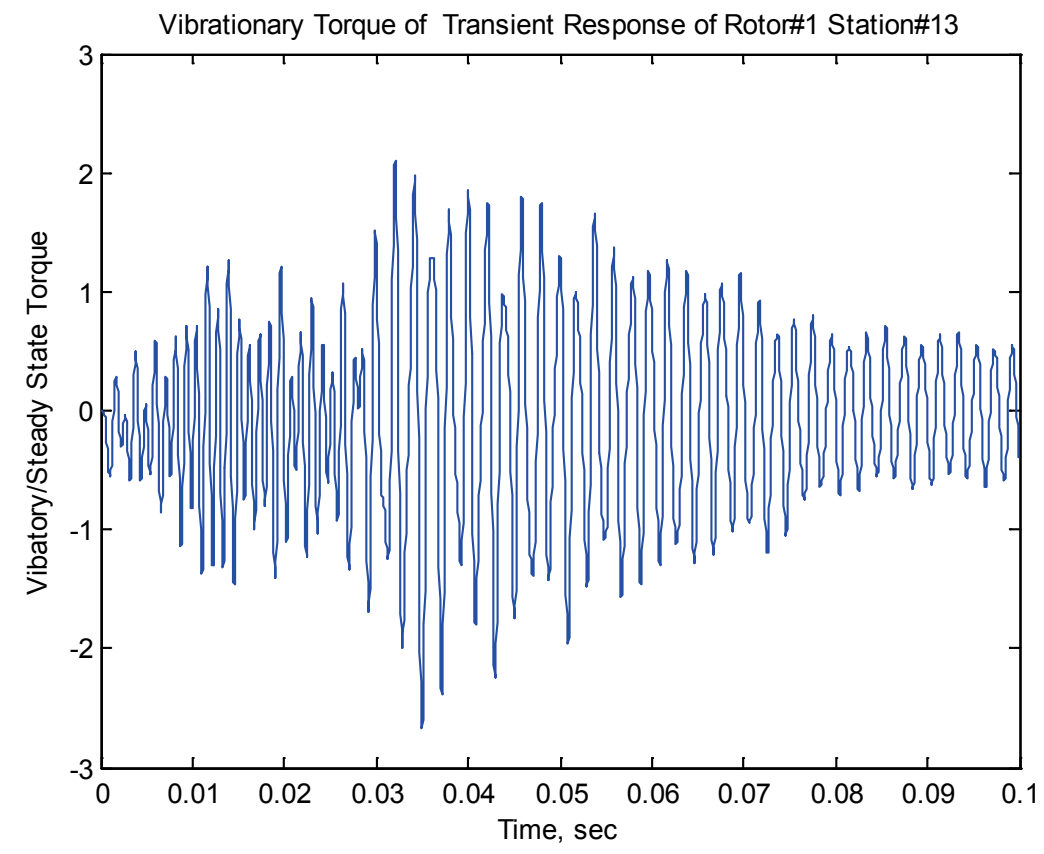

Figure 6.31 Nodal Torque vs. Time of 50X Motor Torque, Element 13 of a Synchronous Motor and the Three-disk Mass Rotor 

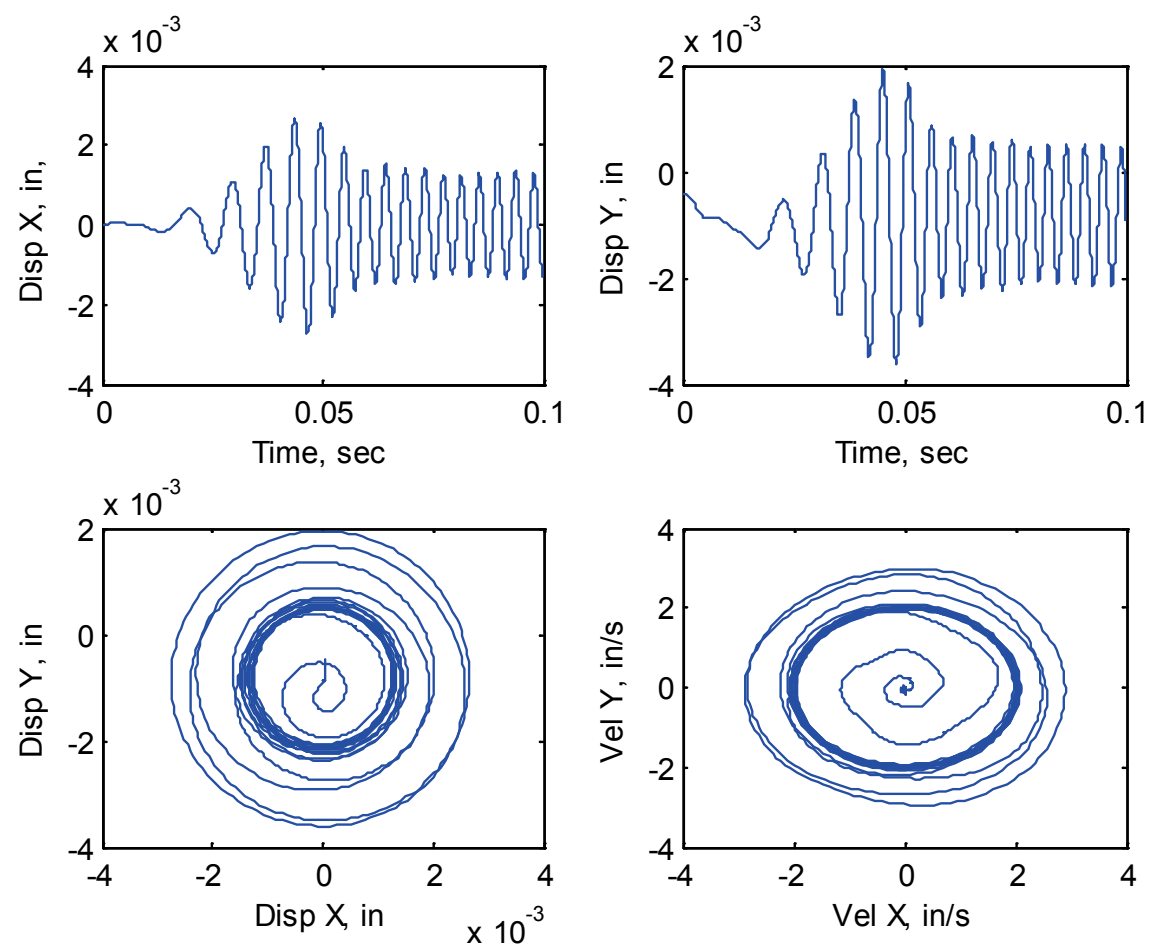

Figure 6.32 Nodal \#13 Displacement, Coupled Analysis of a Synchronous Motor and the Three-disk Mass Rotor, 50X Motor Torque

The coupled torsional and lateral startup transient analysis of the linear 3-disk rotor supported with two nonlinear bearings and two nonlinear dampers is then evaluated. The short plain journal bearings and the short squeeze film dampers used in this section are same as those introduced in Chapter 4, Table 4.4\&4.6. The bearings and damper are located at node 4 and node 22 . For this nonlinear system, the synchronous motor has an equivalent synchronous speed of $15,000 \mathrm{rpm}$ with an equivalent line frequency of $500 \mathrm{~Hz}$ and the motor is started with reduced voltage. The motor drives the rotor from the left (node 1), and the load is concentrated onto the center disk (node 13), as described in the linear rotor-bearing system. The driving torques and load torques are shown in Fig. 6.16. The maximum equivalent torque from the synchronous motor is $2336 \mathrm{lbf}-\mathrm{in}$. The coupled transient analysis result is shown in Fig. 6.33 6.40. According to the transient analysis, 
the startup time for the system to reach the synchronous speed of $15,000 \mathrm{rpm}$ is around 4.5 second, as shown in Fig. 6.33, which as faster than the linear system of 5.0 second. The response of torque from the center element (\#13) is shown in Fig. 6.34, which is same with the linear analysis. From the torque output, there are two peaks at 0.375 second and 1.476 second. The running speeds corresponding to the two peaks are 1,970 rpm and 7,610 rpm from Fig. 6.33. The torque from the center element (\#13) and the rotational speed going through the torsional natural frequencies are almost same as the linear system since the nonlinear bearings and nonlinear dampers have no torsional stiffness or damping effects.

The time transient results from the shaft inside the bearing at node 4 are shown in Fig. 6.35. From the time transient output, when the acceleration rate is slower after the running time $t>3.6$ second, as shown in Fig. 6.33 , the change of amplitudes, including the displacement of the shaft center inside the bearing and the bearing forces, are slower than the values before 3.6 second. Also the amplitude approaches the constant amplitude value gradually under a slow acceleration rate ( $\mathrm{t}>4.0$ second). The bearing forces, Fig. 6.38, keep increasing with the rotational speed increases, but the increasing of the bearing force is slower after $\mathrm{t}>3.6$ second due to the slower angular acceleration rate. A similar result is obtained from the response of the bearing inside the squeeze film damper at node 4 in Fig. 6.36. From the frequency domain results of both the bearing and the squeeze film damper, Figs. 4.37 40, there is a main peak around 15,000 rpm due to the slower acceleration rate after 14,000 rpm. Due to "oil whirl" and "oil whip", there is another main peak at 5,850 rpm which indicates the existence of the sub-harmonic response. Unlike the linear system, there is no obvious peak from the lateral displacements in both horizontal and vertical 
direction. The introduced squeeze film dampers provide additional damping effects to the system, which decreases the maximum vibration amplitude in both the $\mathrm{x}$ and $\mathrm{y}$ directions.

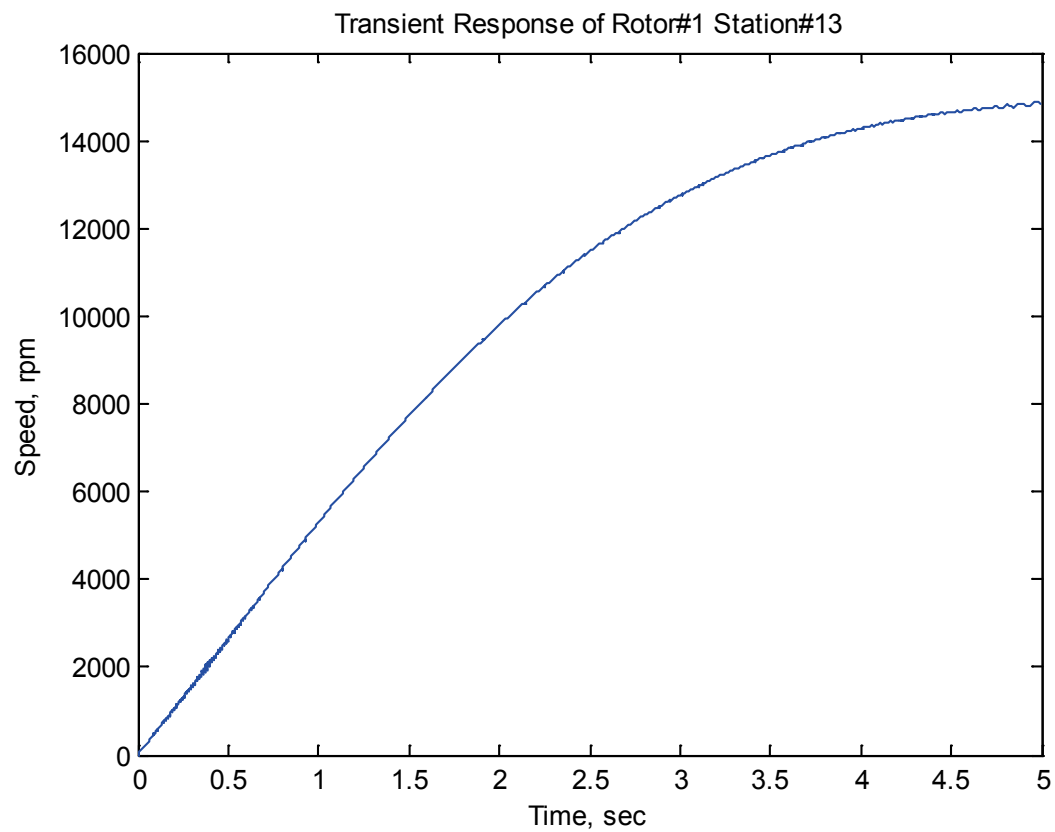

Figure 6.33 Speed vs. Time of Startup for Nonlinear System during Startup of a Synchronous Motor and the Three-disk Mass Rotor, Node 13

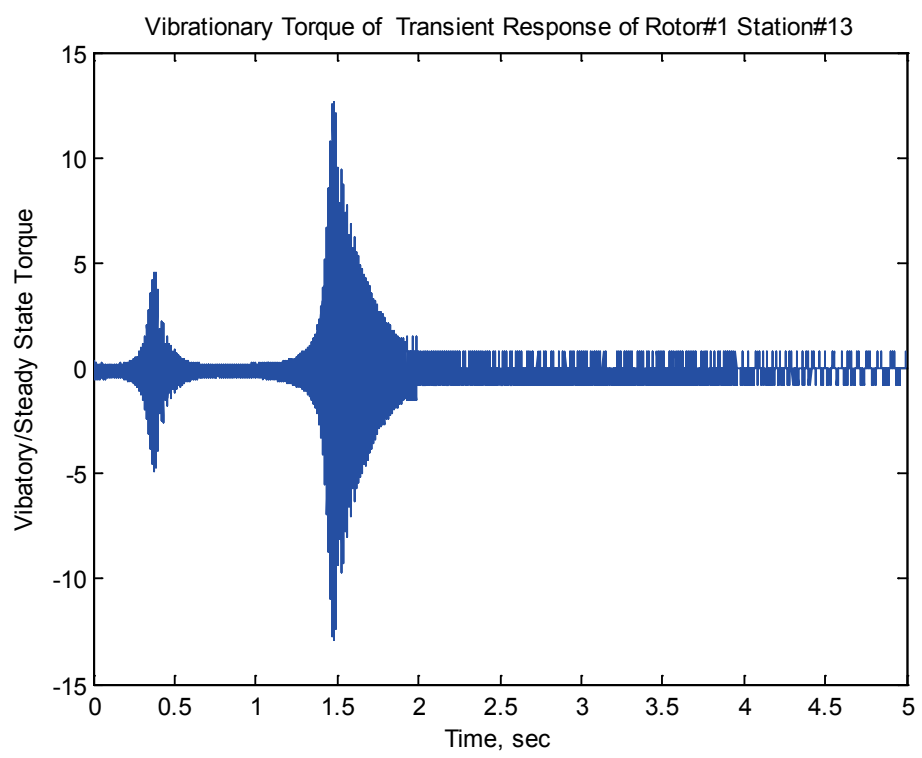

Figure 6.34 Nodal Torque vs. Time at Center Disk (Node 13) during Startup of a Synchronous Motor and the Three-disk Mass Rotor 

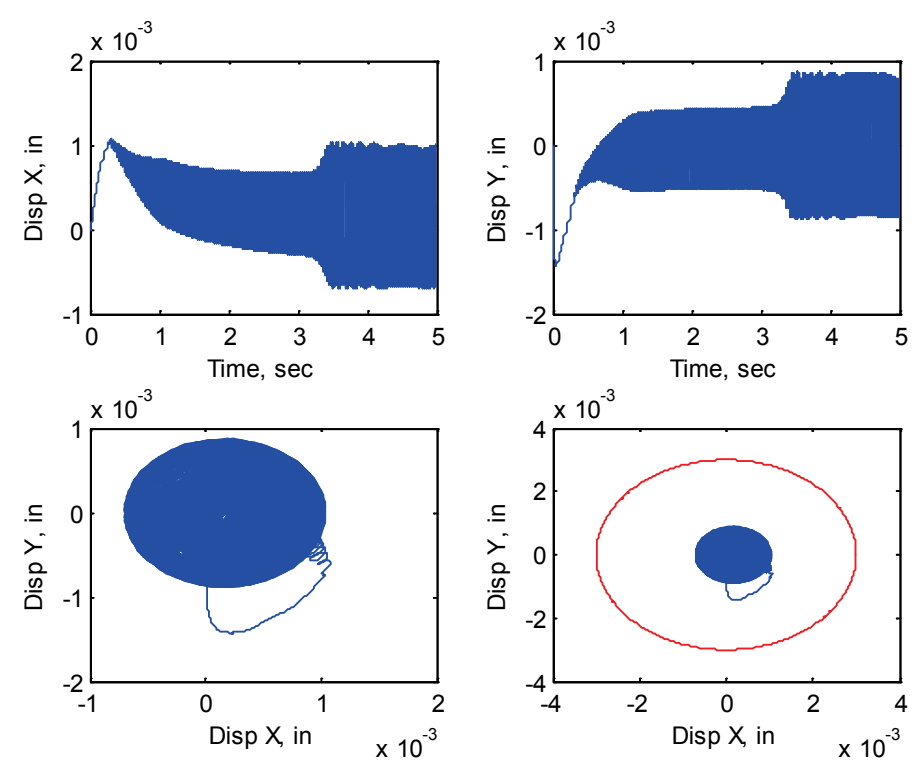

Figure 6.35 Displacement of Shaft Center inside Bearing (at Node 4) during Startup of a Synchronous Motor and the 3-disk Mass Rotor
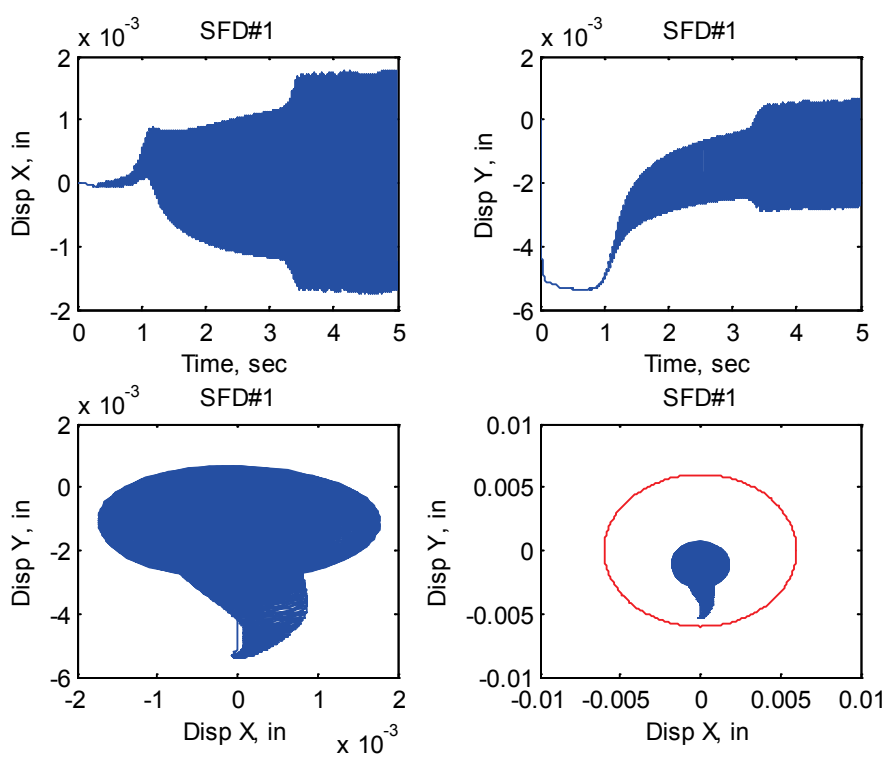

Figure 6.36 Displacement of Bearing inside SFD (at Node 4) during Startup of a Synchronous Motor and the 3-disk Mass Rotor 

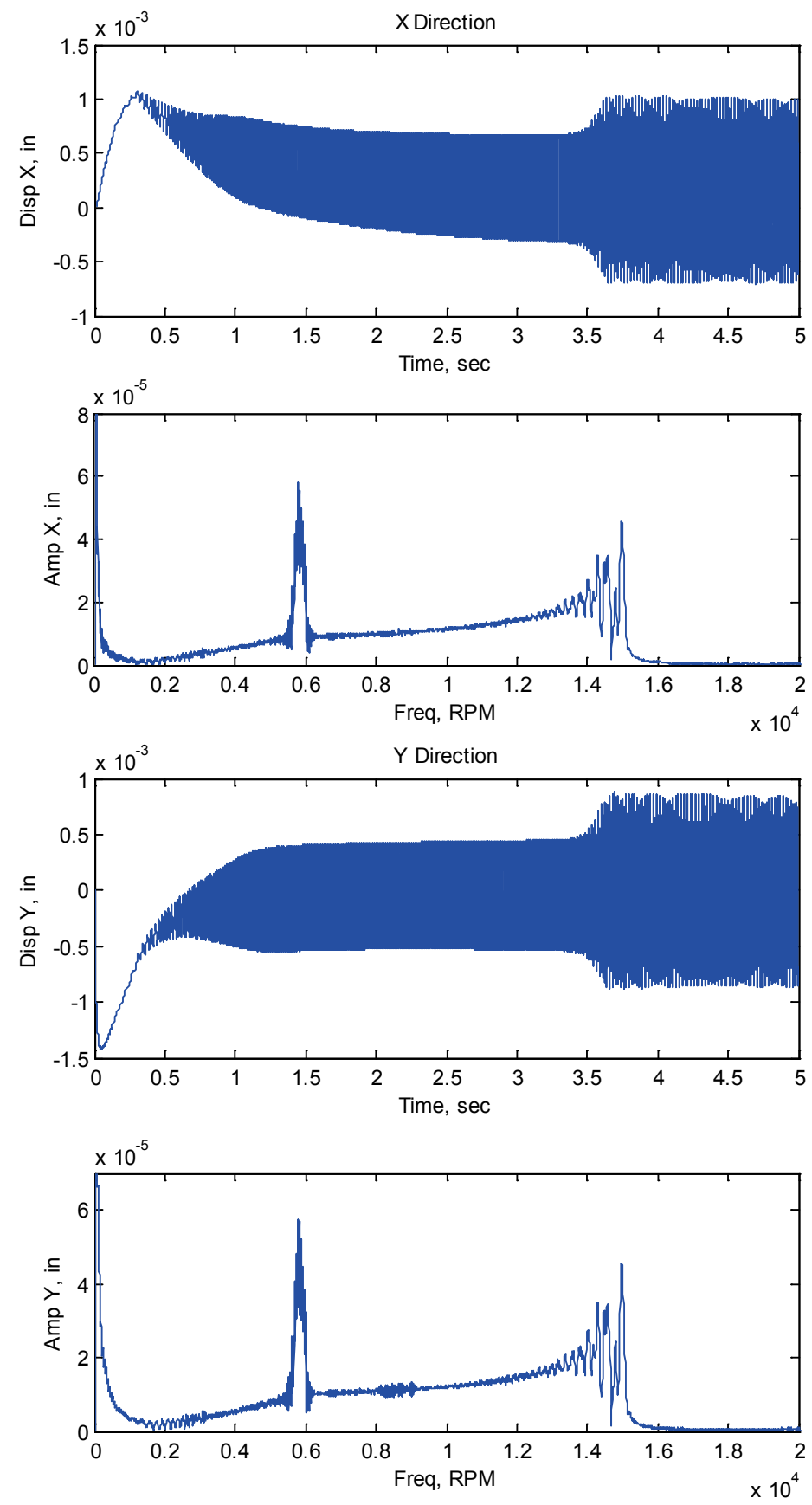

Figure 6.37 Displacement and FFT of Shaft Center inside Bearing (Node 4) during Startup of a Synchronous Motor and the 3-disk Mass Rotor 

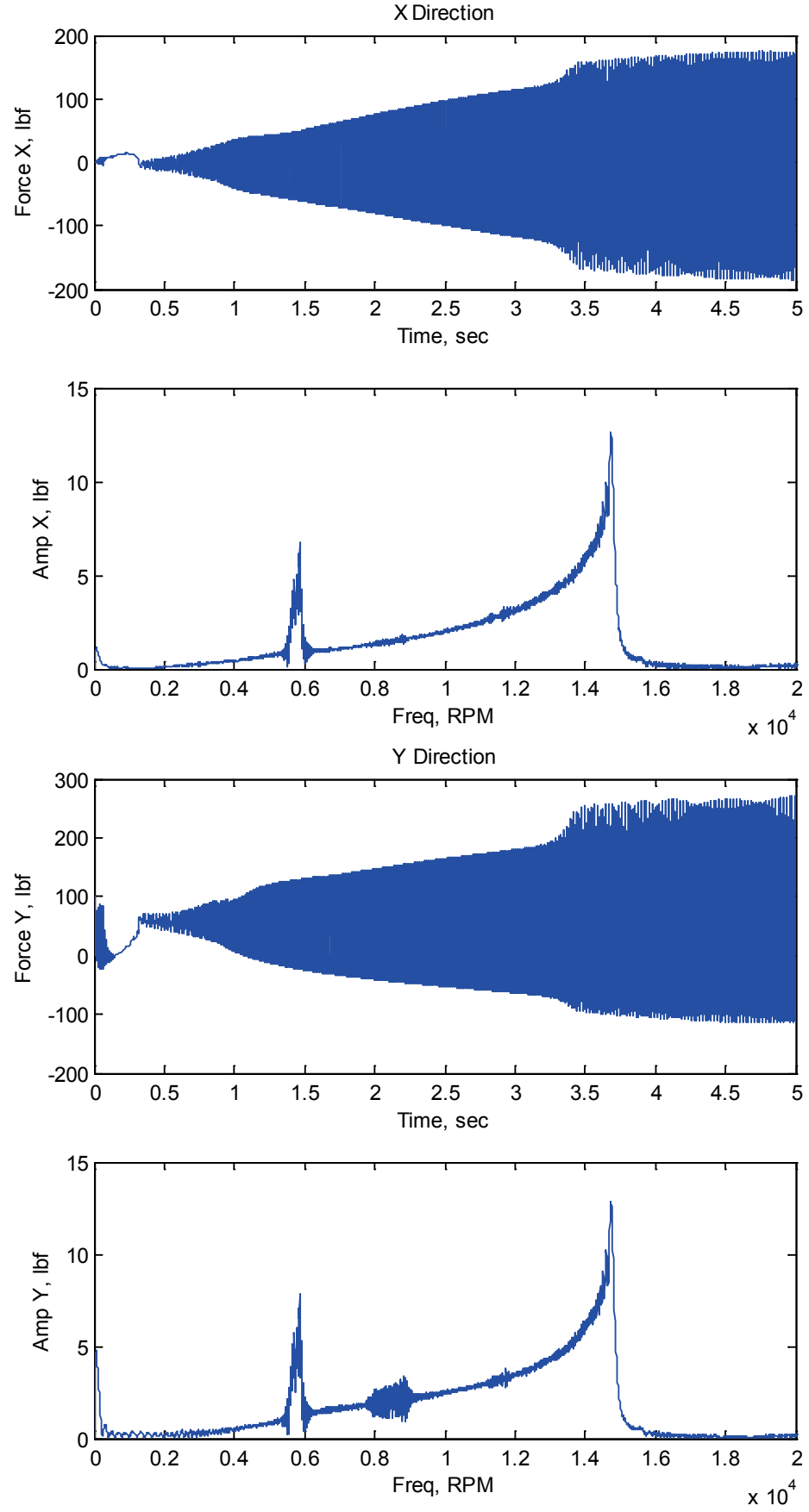

Figure 6.38 Bearing Forces and FFT at Node 4 during Startup of a Synchronous Motor and the 3-disk Mass Rotor 

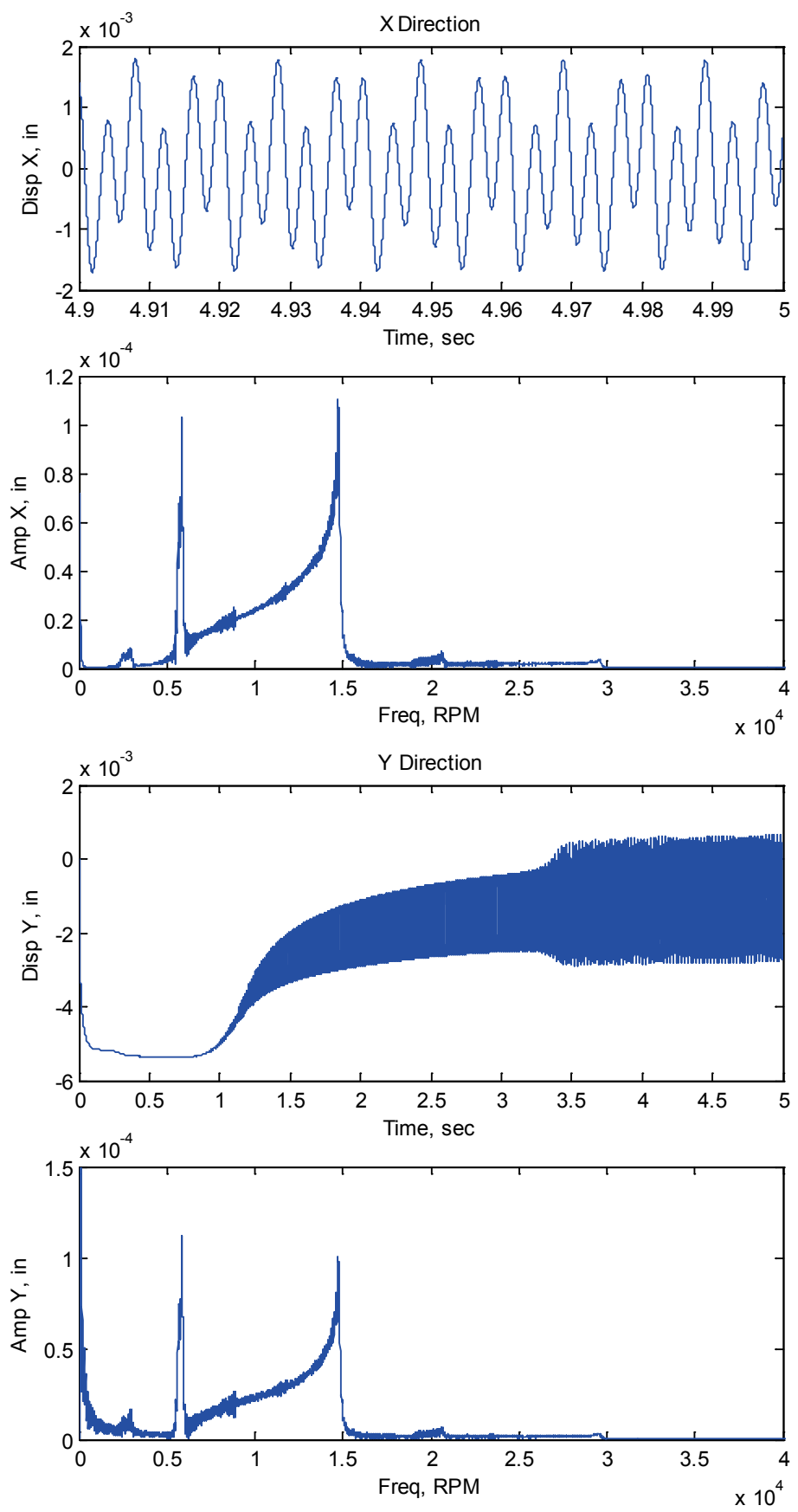

Figure 6.39 Displacement and FFT of Bearing inside SFD (at Node 4) during Startup of a Synchronous Motor and the 3-disk Mass Rotor 

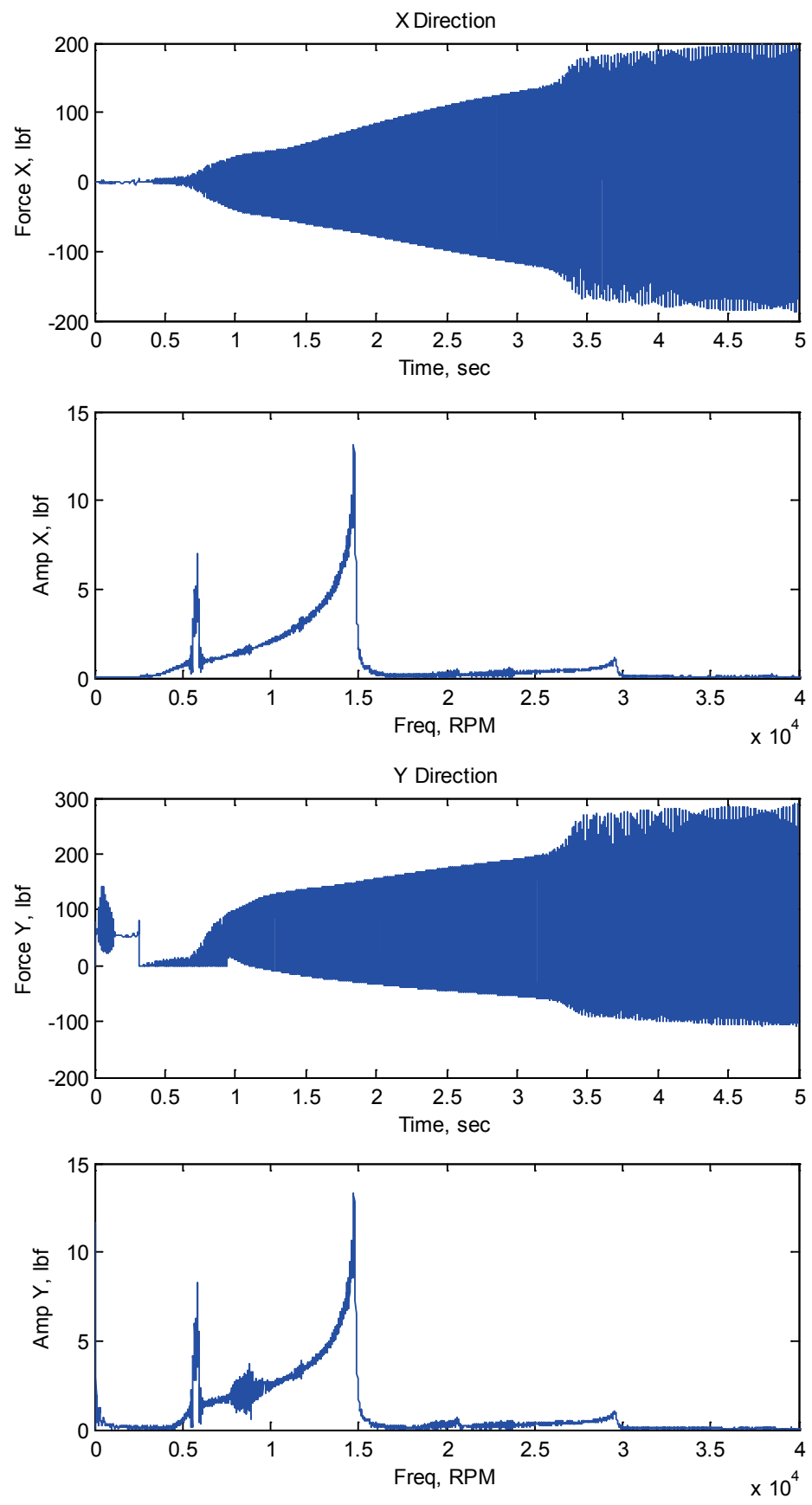

Figure 6.40 SFD (at Node 4) Force and FFT during Startup of a Synchronous Motor and the 3-disk Mass Rotor 


\subsection{Summary}

This chapter presented the transient analyses of the rotor with angular acceleration, or with external nonlinear torque/load. The validation of the non-constant running speed solver is made by comparing the response under different speed acceleration. The response amplitude under a slow acceleration rate $(100 \mathrm{rpm} / \mathrm{sec})$ is very close to the steady-state based analysis at same rotational speed range (error $<0.08 \%$ ). Using the model and the motor torque curves from the published data, an example of a synchronous motor driven 3 compressors is applied to validate the torsional transient solver. The time transient startup speed curve and the vibratory torque are same as the published paper.

The coupled lateral and torsional transient analyses are approached using the modified $4^{\text {th }}$ order Runge-Kutta method. The analyses are applied to a 3-disk rotor driven by a synchronous axial motor. Different accelerations, obtained by setting the maximum torque output from the motor, are applied to the model to evaluate the coupled lateral and torsional transient response during startup. With the angular acceleration rate increases, the speed corresponding to the maximum amplitude increases. During the startup of the 3-disk rotor with nonlinear bearings and nonlinear squeeze film dampers, unlike the linear system, there is no obvious lateral peak in both horizontal and vertical directions. However, the element torque output is same as the linear system when going through the torsional natural frequencies since the nonlinear bearings or the nonlinear squeeze film dampers do not provide additional damping to the torsional or axial direction.

Computing times for different cases are listed in Table 6.4 with same time step of $4 \times 10^{-6}$ second and same time interval of $0 \sim 0.1$ second. For the lateral analyses of the 
linear 3 disk rotor with the linear bearings, the computing time is 181.81 second. If the nonlinear journal bearing and the nonlinear squeeze film damper are applied, the computing time is 185.60 seconds. If the bearings are linear, the linearized stiffness and damping coefficients have to be calculated using the linear interpolation method at each time step. If the bearings and the dampers are nonlinear, the closed-form nonlinear force equation is used at each step. Thus the computational times for the linear and nonlinear systems are almost the same. For the coupled lateral and torsional analyses, the computational time is 449.67 seconds for the linear rotor-bearing system. If the nonlinear journal bearing and the nonlinear squeeze film damper are applied, the computing time is 584.58 seconds. More computational time is needed for the coupled transient analyses of nonlinear system. The reason is that solving implicit equation to obtain the acceleration vector at each step using the fixed-point iteration method takes additional computational time for the nonlinear system.

Table 6.4 Computing Time Comparison

\begin{tabular}{|c|c|c|c|}
\hline \multicolumn{4}{|c|}{$\begin{array}{l}\text { 3-Disk Rotor, } 0 \sim 0.1 \mathrm{sec} ., \Delta \mathrm{t}=4 \times 10^{-6} \mathrm{sec} \text {. } \\
\text { Non-Constant Speed Lateral Analyses }\end{array}$} \\
\hline & DOF & Time, sec & File Size, KB \\
\hline Linear BRG & 100 & 181.81 & 58,645 \\
\hline Nonlinear BRG & 100 & 141.94 & 58,645 \\
\hline Nonlinear BRG + SFD & 104 & 185.60 & 60,208 \\
\hline \multicolumn{4}{|c|}{$\begin{array}{l}\text { 3-Disk Rotor, } 0 \sim 0.1 \mathrm{sec} ., \Delta \mathrm{t}=4 \times 10^{-6} \mathrm{sec} \text {. } \\
\text { Coupled Startup Analyses }\end{array}$} \\
\hline & DOF & Time, sec & File Size, KB \\
\hline Linear BRG & 150 & 449.67 & 60,146 \\
\hline Nonlinear BRG & 150 & 498.26 & 60,146 \\
\hline Nonlinear BRG + SFD & 154 & 584.58 & 61,749 \\
\hline
\end{tabular}




\section{Chapter 7}

\section{Conclusions and Future Work}

\subsection{Dissertation Summary}

The goal of this dissertation is to provide a time transient analysis method for the flexible rotor with nonlinear bearings, nonlinear dampers and nonlinear external forces based on the finite element method. Different analysis algorithms for the flexible rotor including gyroscopic effects, nonlinear components and nonlinear external forces/torques were developed. A new software code, RotorTran, was created to analysis the nonlinear behavior in rotor dynamics. Those analyses include the coupled behavior of the flexible rotor and different types of nonlinear components including bearings and dampers. The coupled lateral and torsional time transient analysis of a nonlinear rotor-bearing system was presented in the work.

Those transient analysis methods were used to study the nonlinear behavior of three different rotor systems: 3 -disk rotor with short journal bearings and squeeze film dampers, 8-stage compressor with tilting pad bearings and finite length squeeze film dampers, and 3-disk rotor with nonlinear journal bearings and squeeze film dampers going through 
critical speed. These rotor systems have been selected based upon their nonlinear components to study specific nonlinear behavior in rotor dynamics. These studies provide several insights into nonlinear transient analysis of rotor dynamics and their handling of coupled motion of rotor and nonlinear components including short journal bearings $(\mathrm{L} / \mathrm{D} \leq 0.5)$, short squeeze film dampers $(\mathrm{L} / \mathrm{D} \leq 0.5)$, tilting pad bearings, finite length squeeze film dampers, and external forces/torques from the synchronous motor and the rotor loads.

Through this research, an in depth analysis of 5 nonlinear components, including coupling of those different components, applied to rotor dynamic system are conducted. Comparison of damping effects of high range running speed, server unbalance condition and going through critical speed provides key insights into the effects of support flexibility in series of the nonlinear bearings and the nonlinear dampers. Different nonlinear behavior, such as sub-harmonic, super-harmonic and torus is presented in those transient analyses.

The proposed work for this dissertation has several novel contributions to the field of transient rotor dynamics.

1. A modeling and assembly method of linear flexible rotor with different types of nonlinear components including tilting pad bearings and squeeze film dampers

2. An evolution of pad and pivot dynamic behaviors of tilting pad bearing to the rotor dynamic system

3. A time transient solver for solving coupled behavior of rotor, nonlinear bearing and nonlinear squeeze film damper 
4. An implicit method for solving coupled lateral and torsional nonlinear transient analysis of nonlinear flexible rotor dynamic system

\subsubsection{Modeling and Assembly Method}

The developed modeling and assembly method was determined by a thorough literature review of methods applied to the nonlinear rotor dynamics analysis and the objectives of this study. In this dissertation, to calculate the coupled lateral, torsional and axial transient motion of the linear rotor with nonlinear components, a 12 DOF Timoshenko beam element was used to model the linear rotor. Built on components were modeled as lumped masses and moments of inertia. Proportional structural damping was considered in this dissertation. Gyroscopic effects, which are function of the rotational speed and the rotational acceleration, were taken into consideration.

The bearings can be treated either linear or nonlinear in this dissertation. If they are treated as linear, the linearized stiffness and damping coefficients are used. If they are treated as nonlinear, the stiffness \& damping effects from the bearings are described by the nonlinear bearing forces.

The motions of the bearing pads/pivots and the bearing housings inside the squeeze film dampers were described by adding additional nodes into the system according to the structure of the nonlinear components. The calculated bearing/damper forces were applied to the force vector at each time step. Assembling of the nonlinear components depends on the structure and the location of the nonlinear components. An additional node with 2 DOF $\left(x_{s d}, y_{s d}\right)$ was added for each short squeeze film damper to describe the motion of the bearings housing inside the damper. For the tilting pad bearing, one node 
with $2 \operatorname{DOF}(\delta, \gamma)$ for each pad/pivot was added. An additional node with 2 DOF $\left(x_{f d}, y_{f d}\right)$ was also added for the finite length squeeze damper. When both the bearing and the damper are nonlinear, the coupled forces of the shaft, the bearings and the dampers are calculated.

\subsubsection{Applied Forces/Torques Calculation}

The force vector includes the linear applied forces and the nonlinear applied forces. The linear applied forces include the unbalance forces, the shaft bow, and the gravitational force due to gravity. The speed acceleration was considered in calculation of the unbalance forces and the shaft bow forces. To calculate the nonlinear forces of the different types of bearings/dampers, the Reynolds equation was solved to obtain the pressure distribution of the fluid film. Then the nonlinear forces were calculated by integrating the pressure profile over the fluid film area.

Closed-form solutions for the short bearing and the short squeeze film damper model $(\mathrm{L} / \mathrm{D} \leq 0.5)$ were used to calculate the pressure profile in this dissertation. A 1-D finite element method was developed by assuming the polynomial pressure distribution along axial direction of the fluid film and by neglecting the energy equation (constant lubricant density and viscosity are used). Similar method was used to calculate the pressure profile and the nonlinear force of the finite length squeeze film damper. For squeeze film damper, the bearing housing inside the damper has zero rotating speed. All nonlinear force calculations were based on the global coordinates to save the computational time of converting displacement and velocity vectors between the global coordinates and the local rotating coordinates, which is often used in the bearing coefficients calculation. 


\subsubsection{Direct Integration Solver}

The $4^{\text {th }}$ order Runge-Kutta method was chosen to solve the equations of motion in this dissertation. For the constant rotational speed problem, a classic explicit $4^{\text {th }}$ order RungeKutta method was used to solve the equations of motion. For the coupled lateral and torsional analysis, a modified Runge-Kutta method was developed to solve the implicit problem. The acceleration was solved by a fixed-point iteration method at each time point according to the current rotor system displacement and velocity, which takes extra computational time.

Because stiffness and damping effects of the nonlinear bearings/damper are the shaft motion dependent, the accurate critical speeds of the nonlinear system can't be calculated directly. The time step of a transient analysis selected in this dissertation for first try was 2.5 times of the maximum natural frequency of the linear system. The initial nodal displacements were calculated from the static displacements due to the gravity loading. The initial displacements of the nodes directly connected to the nonlinear components were set to zeros. In this dissertation, the initial nodal velocity vector was all zeros.

\subsubsection{Validation}

In this dissertation, the modeling and assembly method, the nonlinear force calculation methods and the transient solvers were verified by comparing the published results.

The modeling and assembly method was validated with a transient analysis of a linear 3-disk rotor with linear bearings. The amplitudes under different operating speed show good agreement (a maximum different of $0.2 \%$ ) with those under the steady state 
calculated by MatlabRotor. Pressure profile and nonlinear force calculation method of tilting pad bearing was validated through comparison of equivalent stiffness and damping coefficients with THPAD. The calculated stiffness and damping coefficients (for validating only) based on the method using in this dissertation of different operating speeds have same curves with THPAD's results, but are $6 \%$ of stiffness and $12 \%$ of damping coefficients higher than those of obtained from THPAD due to the neglected thermal equation in this method or different perturbation methods applied.

There is no published result for coupled lateral and torsional analysis in rotor dynamics. The indirect validation was applied to check the coupled lateral and torsional analysis solver. The case of variable speed was validated by comparing constant speed case and slow acceleration case $(100 \mathrm{rpm} / \mathrm{sec})$. The amplitude under slow acceleration shows good match (a maximum different of $0.04 \%$ ) with those under constant speed. The torsional only analysis was validated by comparing of an example of 3 compressors system by Chen [13]. The results of acceleration and element torque show good agreement with Chen's results, which matched the experimental data.

\subsubsection{Conclusions on Application and Performance of Transient}

\section{Analysis}

Different transient analyses for different rotor models with different types of bearings/dampers and/or nonlinear external torques were approached in this dissertation. Time transient analyses of a 3-disk rotor supported with two short plain journal bearings were applied first. Nonlinear behavior due to oil whirl and oil whip, including subharmonic, torus and super-harmonic response was shown from the time transient analyses. 
To increase the system stability under high range of rotational speed, the nonlinear short squeeze film dampers without center spring were introduced into the system. The transient method presented in this dissertation is able to study non-central orbits problems. If the orbital response of the bearing housing is close to the bottom of the squeeze film damper, strongly nonlinear behavior such as sub\&super harmonic response occurs. If the damper forces are able to center the orbital response near to the geometric center of the damper, weakly nonlinear response or synchronous response appears. For the 3-disk rotor supported with plain journal bearings, squeeze film damper is not needed at lower running speed ( $\Omega<11,000 \mathrm{rpm})$; squeeze film damper w/o center spring is recommend for high running speed ( $\Omega>21,000 \mathrm{rpm}$ ); and squeeze film damper with center spring is recommended for mid-range speed $(11,000 \mathrm{rpm}<\Omega<21,000 \mathrm{rpm})$.

Transient analyses of an 8-stage back to back compressor rotor model supported with two tilting pad bearings were approached. To simulate the behavior of the rotor under the severe unbalance condition, the unbalance was suddenly increased from the first unbalance (calculated from API 671) to 16 times of the first unbalance at a specific time. Under the severe unbalance condition, the rotor response is not synchronous with the rotational speed and the rotor won't reach its synchronous steady state. The strongly nonlinear response, especially from the bearing pads/pivot, is shown. Under the more severe unbalance condition, 32 times of the first unbalance, the calculated touch/failure between the shaft and the pad occurs. To avoid that failure, the finite length squeeze film dampers were introduced into the system and were placed outside the bearings. After adding the finite length squeeze film damper, the system under $32 \mathrm{X}$ of the first unbalance was stable and reached its steady state 10 times faster than the case of no dampers under 
the first unbalance. Super-harmonic response from the pad force and strong superharmonic response from the pad moment occur due to instantaneous unloading on the bearing pads under the server unbalance condition.

The coupled lateral and torsional analyses were approached to simulate the startup procedure of a rotor driven by a synchronous motor. The rotor system can be linear or nonlinear. The implicit Runge-Kutta method was used to solve the equation of motion. Different angular accelerations shift the speeds corresponding to the maximum amplitudes in lateral and torsional. The linear rotor-bearing system had obvious amplitude peak in both the horizontal and the vertical displacements during going through the bending critical speed. However, due to additional damping from the squeeze film dampers, there was no obvious amplitude peak from the horizontal or vertical displacements of the linear rotor with nonlinear bearings and nonlinear squeeze film dampers during startup. The torsional response for both the linear system and the nonlinear system were same since the added squeeze film dampers do not provide additional damping effects in torsional or axial direction.

\subsubsection{Computational Time}

The time it takes to calculate the nonlinear forces and to solve the dynamic problem depends on the method of solving the Reynolds equation at each step and the total DOF of the rotor model. For a specific nonlinear rotor dynamic system, the computational time depends on the integration method of solving the equations of motion, especially for the coupled lateral and torsional transient analysis with variable rotational speed. 
More computational time was required for the system including the complicated tilting pad bearings. The finite element method was used to solve the pressure profile of those bearings. The pressure profiles and the nonlinear forces/moments have to be calculated and updated at each time step, which takes up to $90 \%$ of the computational time during the transient analysis of the 8 -stage compressor with two 4-pad tilting pad bearings. The coupled lateral and torsional transient analysis takes a longer time than the lateral or torsional only analysis because 1) the implicit method has to be applied to solve the acceleration vector at each step, and 2) the dimension of those matrices (6 DOF per node) used for the coupled analysis is bigger than those for the lateral analysis only (4 DOF per node). A table of comparing the computational times together with required storage spaces of different conditions is shown in Table 7.1.

Table 7.1 Computational Times of Different Conditions

\begin{tabular}{l|c|c|c|c}
\hline 3-Disk Model, $0 \sim 0.1 \mathrm{sec} ., \Delta \mathrm{t}=4 \times 10^{-6}$ sec., \\
\hline Type of Analysis & & DOF & Time, sec & File Size, KB \\
\hline \multirow{2}{*}{ Constant Speed Lateral } & Linear & 100 & 82.54 & 58,645 \\
\cline { 2 - 5 } & Nonlinear & 104 & 159.66 & 60,208 \\
\hline \multirow{2}{*}{ Non-Constant Speed Lateral } & Linear & 100 & 181.81 & 58,645 \\
\cline { 2 - 5 } & Nonlinear & 104 & 185.60 & 60,208 \\
\hline \multirow{2}{*}{ Coupled Startup Analyses } & Linear & 150 & 449.67 & 60,146 \\
\cline { 2 - 5 } & Nonlinear & 154 & 584.58 & 61,749 \\
\hline
\end{tabular}

\begin{tabular}{l|c|c|c}
\hline \multicolumn{4}{|c}{ 8-Stage Compressor, $0 \sim 0.1$ sec., $\Delta \mathrm{t}=8 \times 10^{-6}$ sec., Lateral Analyses } \\
\hline & DOF & Time, sec & File Size, KB \\
\hline Linear TPB & 124 & 44.08 & 36,359 \\
\hline Nonlinear TPB & 140 & 352.01 & 39,484 \\
\hline Nonlinear TPB + FLD & 144 & 601.57 & 41,266 \\
\hline
\end{tabular}

Post processing of saved data/files was also developed in the code since a transient analysis often takes long time and the generated files during transient analysis are as big 
of hundreds of megabytes or even several gigabytes for the complicated system. To avoid the possible crash due to the error of "out of memory", the transient data were saved every specific numbers of loops. Another advantage of this method is to continue analysis from the end of the prior calculation or from the time point when the crash occurs.

\subsection{Contribution and Innovation}

This work presented different time transient analysis algorithms for the flexible rotor including gyroscopic effects and nonlinear bearings, nonlinear dampers and nonlinear external forces/torques. Unlike most simple nonlinear models considered in the literature examples, this work provides several nonlinear models for complicated bearings and dampers. The nonlinear force calculation is critical in the development of the nonlinear transient analysis. The coupled behavior of the flexible rotor and different types of nonlinear components were applied. The coupled lateral and torsional time transient solution was developed. Various examples of different rotor models with different types of bearings/dampers and different nonlinear external forces/torques were shown in this work to point out the nonlinear behavior due to the nonlinear forces from the supporting system and the synchronous motor. The finished work for this dissertation has several novel contributions to the field of nonlinear transient analysis of rotor dynamics:

1) Nonlinear behavior, including sub-harmonic, torus and super-harmonic response, due to oil whirl and oil whip is shown from the time transient analyses of the 3-disk rotor with short plain journal bearings under different operating speed.

2) The transient method presented in this dissertation is able to study non-central orbits problems of dampers. Strongly nonlinear behavior occurs if the orbital response of the 
rotor is close to the bottom of the squeeze film damper. Transient analysis of the 3-disk showed that adding squeeze film damper increases dynamic stability of the rotor dynamic system, deceases about $50 \%$ of bearing eccentricity and $80 \%$ of bearing force.

3) The transient analyses of a rigid rotor and an 8-stage compressor with same tilting pad bearings indicated that the time step length of an algorithm depends on both the DOF of the rotor and the structure of the bearing.

4) The nonlinear tilting pad bearing model enables the investigation of the coupled behavior of the flexible rotor and the bearing pads/pivots. Strong nonlinear behavior is shown on the bearing pad due to sudden moment and force change on it, especially under server unbalance condition.

5) The effects of the nonlinear dampers may avoid possible touch between the shaft and the bearing under server unbalance condition. With additional damping from the added squeeze film dampers, a synchronous response is shown from the shaft under server unbalance condition. However, the bearing pad/pivot may present strong nonlinear behavior due to temporarily unloaded period (zero pad force and moment).

6) The coupled lateral and torsional transient analyses using implicit method are approached. It was shown that the effect of angular accelerations shifts the maximum amplitudes in both lateral and torsional directions. Additional damping from the squeeze film dampers decreases the lateral amplitude going through the critical speed, but does not change the torsional response.

7) A new software code, RotorTran, was created to approach the transient analyses of the flexible rotor with nonlinear bearings/dampers based on Matlab. 


\subsection{Discussion and Future Work}

There is a significant amount of work that can be extended for the nonlinear transient analysis of rotor dynamic systems:

1. The first is the extension of beam element model to 3-D solid element model to capture dynamic effects which are not possible with the beam based model. However, the nature of these 3D models makes it computationally inefficient or near impossible to perform a rotor dynamic analysis on the system due to high DOF, especially for transient response. An effective model reduction method for large rotor-bearing system, considering accuracy and efficiency, is necessary.

2. The second extension is the nonlinear model. In this dissertation, only the bearings and the dampers are modeled as nonlinear. Further models can be included gearboxes, flexible couplings, seals and thrust bearings. The energy equation, which results various density and viscosity, can be extended to the future model, but additional time consumption needs to be evaluated carefully.

3. The third extension is the effective integration method. The $4^{\text {th }}$ order Runge-Kutta method is used in this dissertation. Some other integration methods using longer time step, such as Newmark- $\beta$ method, which is unconditionally stable by carefully choosing the parameters, may be developed in the future. In the nonlinear rotor dynamics, however, using the Newmark- $\beta$ method has to solve the implicit problem at each step even for the constant rotational speed problem. Solving the implicit problem costs extra computational time than solving the explicit problem. Also the implicit problem is often initial value sensitive, which results in a shorter time step requirement. 
4. The last extension is model reduction. Model reduction is normally used in the linear analysis for the complex rotor. The challenge of nonlinear model reduction is how to obtain an accurately modal basis for the nonlinear rotor dynamic system, especially for variable rotational speed analysis. A result of model truncation method applied to the 3disk rotor model on two nonlinear plan journal bearings at a speed of $12,000 \mathrm{rpm}$ is shown in Fig. 7.1. The response is the displacement of the center disk in horizontal direction. To compare the response of the direct method and the modal method, the time steps of two methods are same $(\Delta \mathrm{t}=4 \mu \mathrm{s})$. The modal truncation method, which used the lower 20 modes of the system, works well for the linear rotor-bearing system. But when this method is applied to the rotor with nonlinear bearings, there are relative bigger errors, as shown in upper diagram of Fig. 7.1.
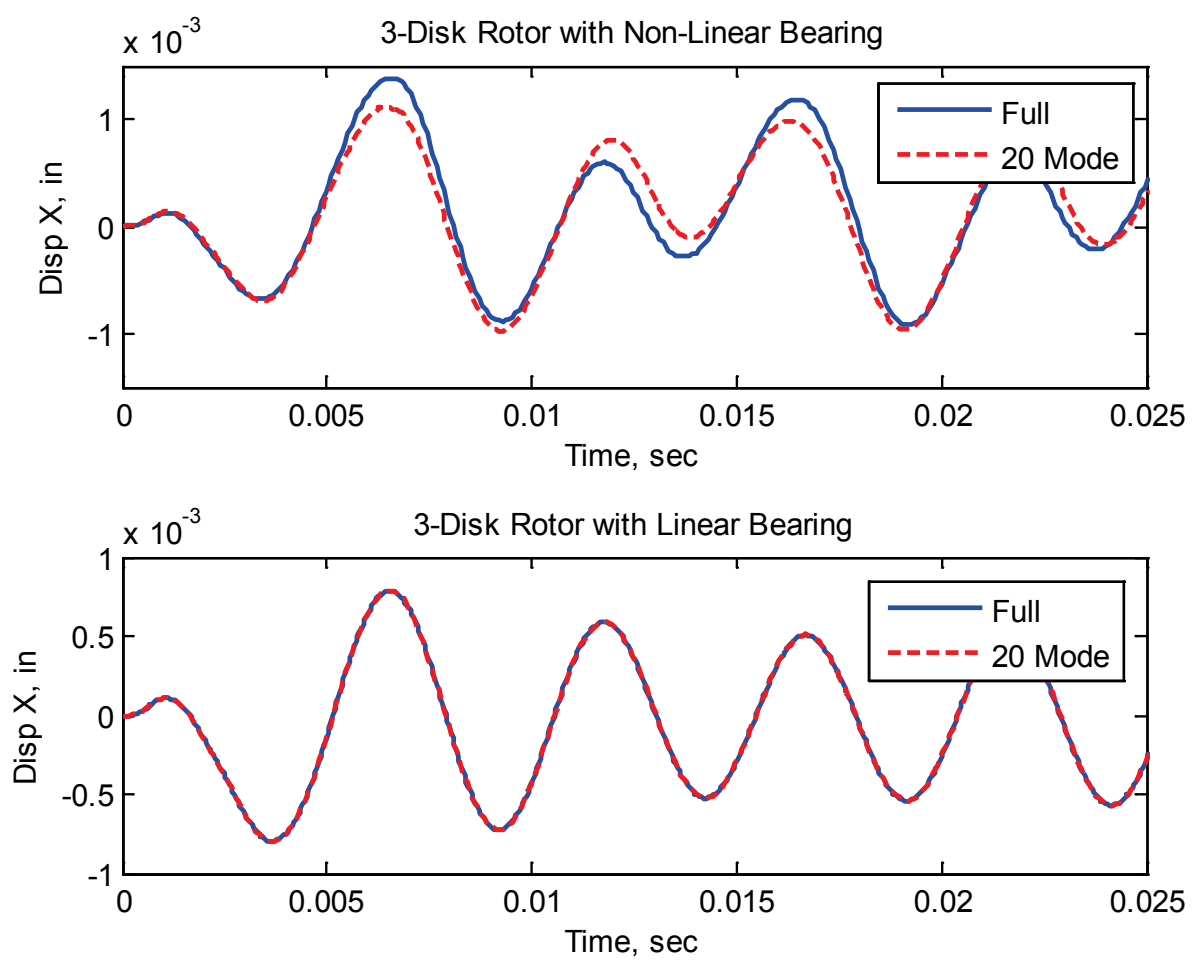

Figure 7.1 Compare of direct solver and linear modal reduction 


\section{References}

[1] T. Yamamoto, "On critical Speeds of a Shaft," Mem. Fac. Eng., Nagoya Univ., vol. 6, no. 2, pp. 106-174, 1954.

[2] D. Bently, "Forced Subrotative Speed Dynamic Action of Rotating Machinery," in Petroleum Mechanical Engineering Conference, Dallas, Texas, 1974.

[3] F. F. Ehrich, "Subharmonic vibration of rotors in bearing clearance," ASME Journal of Engineering for Power, vol. 88, pp. 56-65, 1966.

[4] D. W. Childs, "Rotordynamic Characteristics of HPOPT (High Pressure Oxygen Turbopump) of the SSME (Space Shuttle Main Engine)," Turbomachinery Laboratories Report (Texas A\&M Univ.), 1984.

[5] M. B. Wagner, A. Younan, P. E. Allaire, and R. Cogill, "Model Reduction Methods for Rotor Dynamic Analysis: A Survey andReview," International Journal of RotatingMachinery, pp. 1-17, 2010.

[6] J. W. Lund, "The Stability of an Elastic Rotor in Journal Bearings with Flexible, Damped Supports," ASME, Journal of Applied Mechanics, vol. 32, pp. 911-920, 1965.

[7] T. Yamamoto and Y. Ishida, Linear and Nonlinear Rotordtnamics: A Modern Treatment with Applications. New York, NY, USA: Wiley-Interscience, 2001.

[8] A. Muszynska, "Whirl And Whip - Rotor/Bearing Stability Problems," Journal of SOund and Vibration, vol. 110, no. 3, pp. 443-462, 1986.

[9] A. Muszynska, "Stability of Whirl and WHip in Rotor/Bearing Systems," Journal of Sound and Virbation, vol. 127, no. 1, pp. 49-64, 1988. 
[10] A. Muszynska., Rotordynamics, 1st ed. CRC Press, 2005.

[11] F. F. Ehrich, "Observations of Nonlinear Phenomena in Rotordynamics," Journal of System Design and Dynamics, vol. 2, pp. 641-651, 2008.

[12] D. Bently, S. Zimmer, and G. ,. M. A. Palmetier, "Interpreting Vibration Information from Rotating Machinery," Sound and Vibration magazine, vol. 20, no. 2, pp. 14-23, 1986.

[13] W. J. Chen and E. Gunter, Instruction to Dynamics of Rotor-Bearing System. Victoria, BA, Canada: Trafford Publishing, 2005.

[14] P. E. Allaire, Rotor Dynamics Course Notes. Rotating Machinery and Controls Lab: Univerity of Virginia, , 2009.

[15] F. C. Nelson, "A Brief History of Early Rotordynamics ," Journal of Sound and Vibration, 2003.

[16] H. H. Jeffcott, "The lateral vibration of loaded shafts in the neighbourhood of a whirling speed - The effect of want of balance," Phil. Mag, vol. 6, no. 37, 1919.

[17] S. Yamauchi, "The nonlinear of flexible rotors, 1st report, development of a new analysis technique," Japan Society of Mechanical Engineers, vol. 446, no. 49, pp. 1862-1868, 1983.

[18] J. M. Vance, B. T. Murphy, and H. A. Tripp, "Critical Speeds of Rotating Machinery: Computer Predictions vs. Experimental Measurements, Part II: Effects of Tilting Pad Bearings and Foundation Effcts," ASME Journal of Vibration, Acoustics, Stress and Reliability in Design, vol. 109, pp. 8-14, 1987.

[19] J. W. Zu and Z. Ji, "An Improved Transfer Matrix Method for Steady-State Analysis of Nonlinear Rotor-Bearing Systems," Journal of Engineering for Gas Turbines and Power, vol. 124, no. 2, pp. 303-310, 2002.

[20] R. L. Eshelman and R. A. Eubanks, "On the Critical Speeds of a Continuous Rotor," ASME Journal of Engineering for Industry, vol. 91, pp. 1180-1188, 1969.

[21] D. L. Thomas, J. M. Wilson, and R. R. Wilson, " Timoshenko Beam Finite Elements," Journal of Sound and Vibration, vol. 31, no. 3, pp. 315-330, 1973.

[22] H. D. Nelson, "A Finite Rotating Shaft Element Using Timoshenko Beam Theory," ASME, Journal of Mechanical Design, vol. 102, p. 793-803, 1980. 
[23] R. L. Ruhl and J. F. Booker, "A finite element for distributed parameter turborotor systems," J. Eng. Indust, vol. 94, pp. 116-132, 1972.

[24] H. D. Nelson and J. M. McVaugh, "The dynamics of rotor bearing systems using finite elements," J. Eng. Indust., vol. 98, pp. 593-600, 1976.

[25] T. Zheng and N. Hasebe, "Nonlinear Dynamic Behaviors of a Complex RotorBearing System," Journal of Applied Mechanics, vol. 67, pp. 485-495, 2000.

[26] K. E. Rouch and J. S. Kao, "A Tapered Beam Finite Element for Rotor Dynamics Analysis," Journal of Sound and Vibration, vol. 66, pp. 119-140, 1979.

[27] T. A. Vest and M. S. Darlow, "A Modified Conical Beam Element Based on Finite Element Analysis: Experimental Correlations," Journal of Vibration and Acoustics, vol. 122, no. 3, pp. 350-355, 1990.

[28] A. Nandi and S. Neogy, "Modeling of rotors with three-dimensional solid finite elements," Journal of Strain Analysis, vol. 36, no. 4, pp. 359-371, 2001.

[29] J. S. Rao and R. Sreenivas, "Dynamics of Asymmetric Rotors using Solid Models," in Proceedings of the International Gas Turbine Congress, Tokyo, 2003.

[30] E. Chatlet, D. Lornage, and G. G. Jacquet-Richardet, "A three dimensional modeling of the dynamic behavior of composite rotors," International Journal of Roating Machinery, vol. 8, no. 3, pp. 185-192, 2002.

[31] J. Chaudhry, "3-D Finite Element Analysis of Complex Rotordynamic Systems," Ph.D. Dissertation, University of Virginia, 2011.

[32] B. L. Newkirk and H. D. Taylor, "Shaft whipping due to oil action in journal bearings," General Electric Review, p. 559, 1925.

[33] W. H. Press, B. Flannery, S. Teukolsky, and W. T. Netterling, Numerical Recipes: The Atr of Scientific Computing. New York: Cambridge University Press, 2007.

[34] Y. B. Kim and S. T. Noah, "Response and bifurcation analysis of MDOF rotor system with a strong local nonlinearity," Nonlinear Dynamics, vol. 2, pp. 215-234, 1991.

[35] F. F. Ehrich, "Some Observations of Chaotic Phenomena in High Speed Rorordynamics," ASME Journal of Vibration and Acoustics, vol. 113, pp. 50-57, 1991. 
[36] K. Gjika, L. San Andrés, and G. D. Larue, "Nonlinear Dynamic Behavior of Turbocharger Rotor-Bearing Systems With Hydrodynamic Oil Film and Squeeze Film Damper in Series: Prediction and Experiment," ASME, Journal of Computational and Nonlinear Dynamics, vol. 5, pp. 1-8, 2010.

[37] B. N. Agrawal and R. M. Evan-Iwanowski, "Resonances in nonstationary, nonlinear, multidegree-of-freedom systems," AIAA Journal, vol. 11, no. 7, pp. 907912, 1973.

[38] T. Yamamoto and Y. Ishida, "Theoretical discussions on vibrations of a rotating shaft with nonlinear spring characteristics," Archive of Applied Mechanics, vol. 46, no. 2, pp. 125-135, 1977.

[39] Y. S. Choi and S. T. Noah, "Nonlinear steady state response of a rotor-support system," ASME Journal of Vibratio, Acoustics, Stress and Reliablity in Design, vol. 109, pp. 255-261, 1987.

[40] Y. B. Kim and S. T. Noah, "Stability and bifurcation analysis of oscillators with piecewise-linear characteristics] a general approach," ASME Journal of Applied Mechanics, vol. 58, pp. 545-553, 1991.

[41] D. W. Childs, "Fractional-Frequency Rotor Motion due to Nonsymmetric Clearance Effects," ASME J. Eng. Gas Turbines Power, vol. 104, pp. 533-541, 1982.

[42] D. W. Childs, Tubromachinery Rotordynamics. New York: Wiley, 1993.

[43] F. F. Ehrich, "High Order Subharmonic Response of High Speed Rotors in Bearing Clearnace," Trans. ASME, J. Bib. Acoust. Stress Reliab. Des., vol. 110, no. 9, pp. 9$16,1988$.

[44] F. F. Ehrich, "Observation of subcritical super-harmonic and chaotic response in rotor dynamic," Journal of Vibration and Acoustics, vol. 114, pp. 93-99, 1992.

[45] T. Shiau and A. Jean, "Prediction of periodic response of flexible mechanical systems with nonlinear characteristics," Journal of Vibration and Acoustics, vol. 112, p. 501-507, 1990.

[46] Y. Kim, S. T. Noah, and Y. S. Choi, "Periodic Response of Multi-Disk Rotors with Bearing Clearances," Journal of Sound and Vibration, vol. 144, no. 3, pp. 381-395, 1991. 
[47] P. Sundararajan and S. T. Noah, "An algorithm for response and stability of large order non-linear systems, application to rotor systems - Application to Rotor Systems," Journal of Sound and Vibration, vol. 214, no. 4, pp. 695-723, 1998.

[48] C. Nataraj and H. D. Nelson, "Periodic Solutions in Rotor Dynamic System with Nonlinear Support: A General Approch," Journal of Vibration and Acoustics, vol. 111, pp. 187-193, 1990.

[49] A. N. Jean and H. D. Nelson, "Periodic response investigation of large order nonlinear rotordynamic systems using collocation," Journal of Sound and Vibration, vol. 143 , no. 3 , pp. 473-489, 1990.

[50] M. Kobayashi, S. Saito, and S. Yamauchi, "Nonlinear Steady-State Vibration Analysis of Rotor by Substructure Synthesis," Transactions of the Japan Society of Mechanical Engineers, vol. 53, pp. 1-10, 1991.

[51] B. Moon, J. Kim, and B. Yang, "Non-linear vibration analysis of mechanical structure system using substructure synthesis method," Journal of Mechanical Science and Technology, vol. 13, no. 9, pp. 620-629, 1999.

[52] A. Younan, J. Cao, T. Dimond, and P. E. Allaire, "Nonlinear Analysis of Squeeze Film Damper with Entrained Air in Rotordynamic Systems," Tribology Transactions, vol. 54, pp. 132-144, 2011.

[53] H. L. Hassenpflug, "Transient Analysis of Coupled Rotor-Structure Systems," Ph.D Thesie, University of Virginia, 1988.

[54] W. B. Day, "Asymptotic Expansions in Nonlinear Rotordynamics," Quarterly of Applied Mathematics, vol. 44, pp. 779-792, 1987.

[55] Y. B. Kim and S. T. Noah, "Bifurcation analysis for a modified Jeffcott rotor with bearing clearances," Nonlinear Dynamics, vol. 1, pp. 221-241, 1990.

[56] S. Choi and A. T. Noah, "Mode-Locking and Chaos in a Jeffcott Rotor with Bearing Clearances," Journal of Applied Mechanics, vol. 61, pp. 131-138, 1994.

[57] L. Wang, D. Cao, and W. Huang, "Nonlinear coupled dynamics of flexible bladerotor-bearing systems," Tribology International, vol. 43, p. 759-778, 2010.

[58] J. Jing, G. Meng, Y. Sun, and S. Xia, "On the non-linear dynamic behavior of a rotor-bearing system," Journal of Sound and Vibration, vol. 274, p. 1031-1044, 2004. 
[59] Y. G. Jei and C. W. Lee, "Modal analysis of continuous asymmetrical rotor-bearing systems," Journal of Sound and Vibration, vol. 152, no. 2, p. 245-262, 1992.

[60] Z. Gu, X. Zhi, G. Meng, and T. Fang, "Transient Response Analysis of Large-Scale Rotor-Bearing System with Strong Non-Linear Elements by a Transfer MatrixNewmark Formulation Itegration Method," Journal of Sound and Vibration, vol. 259, pp. 559-570, 2003.

[61] H. F. Castro, K. L. Cavalcaa, and R. Nordmannb, "Whirl and whip instabilities in rotor-bearing system considering a nonlinear force model," Journal of Sound and Vibration, vol. 317, no. 1-2, pp. 273-293, 2008.

[62] C. Chang-Jian, "Non-linear dynamic analysis of dual flexible rotors supported by long journal bearings," Mechanism and Machine Theory, vol. 45, p. 844-866, 2010.

[63] P. E. Allaire, J. K. Parsell, and L. E. Barrett, "A Pad Perturbation Method for the Dynamic Coefficients of Tilting-Pad Journal Bearings," Wear, vol. 79, no. 1, pp. 29-44, 1981.

[64] T. Dimond, A. Younan, and P. E. Allaire, "A Review of Tilting Pad Bearing Theory Part I: Classical Treatments," University of Virginia, ROMAC Report No. 562 UVA/643092/MAE11, 2011.

[65] T. Dimond, A. Younan, and P. E. Allaire, "A Review of Tilting Pad Bearing Theory Part II: Modern Treatments," University of Virginia, ROMAC Report No. 563 UVA/643092/MAE11, 2011.

[66] O. Reynolds, "On the Theory of Lubrication and Its Application to Mr. Beauchamp Tower's Experiments, Including an Experimental Determination of the Viscosity of Olive Oil," Philosophical Transactions of the Royal Society of London, vol. 177, pp. 157-234, 1886.

[67] A. Sommerfeld, "Zur Hydrodynamische Theorie der Schmiermittelreibung," Zeitschrift fur Mathematik und Physik, vol. 50, pp. 97-155, 1904.

[68] W. J. Harrison, "Hydrodynamic theory of lubrication with special reference to air as a lubricant," Cambridge Philosophical Society Transactions, vol. 22, pp. 39-54, 1913.

[69] D. Robertson, "Whirling of a journal in a sleeve bearing," Philosophical Magazine, 
vol. 15, no. 1, pp. 133-137, 1933.

[70] S. E. Cardullo, "Some Practical Deductions From the Theory of Lubrication of Short Cylindrical Bearings," Transactions ASME, vol. 52, pp. 143-153, 1930.

[71] R. H. Badgley and J. F. J.F. Booker, "Turborotor instability, effect of initial transients on plane motion," Journal of Lubrication Technology, vol. 4, p. 625, 1969.

[72] R. G. Kirk and E. J. Gunter, "Transient Response of Rotor Bearing Systems," ASME, Journal of Engineering for Industry, p. 682, 1974.

[73] P. E. Allaire, Basics of Finite Element Method. PWS Pub. Co. , 1999.

[74] R. D. Cook, D. S. Malkus, M. E. Plesha, and R. J. Witt, Concepts and Application of Finite Element Analysis, 4th ed. New York, NY, USA: John Wiley \& Sons, 2011.

[75] L. A. Branagan, L. E. Barrett, and C. H. Cloud, "A Manual for Use with Fixed Geometry Journal Bearing Code: THBRG," University of Virgina, ROMAC Report No. 273 UVA/643092/MAE04/373, 1988.

[76] L. A. Branagan, L. E. Barrett, and C. H. Cloud, "A Manual for Use with the Tilting Pad Bearing Program THPAD," University of Virginia, ROMAC Report No.284 UVA/643092/MAE88/384, 1988.

[77] M. He, "Thermoelastohydrodynamic Analysis of Fluid Film Journal Bearings," Ph.D. Dissertation, University of Virginia, 2003.

[78] L. Branagan, "Thermal Analysis of Fixed and Tilting pad Journal Bearings Including Crossfilm Viscosity Variations and Deformations," Ph.D dissertation, University of Virginia, 1988.

[79] P. Rodriguez and L. Barrett, "Effect of Tilting Pad Thrust Bearings on Rotor Stability and Forced Response," University of Virginia, ROMAC Report No. 453 UVA/643092/MAE01/553, 2000.

[80] B. Ballal and R. S. Rivlin, "Flow of a Newtonian fluid between eccentric rotating cylinders: Inertial effects," Archive for Rational Mechanics and Analysis, vol. 62, no. 3, pp. 237-294, 1976.

[81] L. A. San Andresa and J. M. Vancea, "Force Coefficients for Open-Ended 
Squeeze-Film Dampers Executing Small-Amplitude Motions About an Off-Center Equilibrium Position," Tribology Transactions, vol. 30, no. 1, pp. 69-76, 1987.

[82] J. Tichy and B. Bou-Said, "Hydrodynamic Lubrication and Bearing Behavior with Impulsive Loads," Tribology Transactions, vol. 34, no. 4, pp. 505-512, 1991.

[83] E. Kim and A. Z. Szeri, "On the Combined Effects of Lubricant Inertia and Viscous Dissipation in Long Bearings," Journal of Tribology, vol. 119, no. 1, p. 7684, 1998.

[84] H. Xu and J. Zhu, "Research of Fluid Flow and Flow Transition Criteria from Laminar to Turbulent in a Journal Bearing," Journal Xi'An Jiaotong University, vol. 27, pp. 7-14, 1993.

[85] C. W. Ng, "Fluid dynamic foundation of turbulent lubrication theory," ASLE Trans., vol. 7, pp. 311-321, 1964.

[86] C. W. Ng and C. H. T. Pan, "A linearlized turbulent Lubrication theory," ASME Trans., vol. 87, pp. 675-688, 1965.

[87] A. Younan, "Elastohydrodynamic Lubrication in Rolling Element Bearing: Static and Dynamic Properties," Ph.D. Desseritation, University of Virginia, 2011.

[88] A. Szeri, Fluid Film Lubrication - Theory \& Design. Cambridge University Press, 2005.

[89] G. Adiletta, A. R. Guido, and C. Rossi, "Nonlinear Dynamics of a Rigid Unbalanced Rotor in Journal Bearings Part I: Theoretical Analysis," Nonlinear Dynamics, vol. 14, p. 57-87, 1997.

[90] P. Bonello, M. J. Brennan, and R. Holems, "Non-linear Modeling of Rotor Dynamic Systems with Squeeze Film Dampers - An Efficient Integrated Approach," Journal of Sound and vibration, vol. 249, no. 4, pp. 743-773, 2002.

[91] S. K. Laha and S. K. Kakoty, "Non-linear dynamic analysis of a flexible rotor supported on porous oil journal bearings," Communications in Nonlinear Science and Numerical Simulation, vol. 16, no. 3, pp. 1617-1631, 2011.

[92] C. Chang-Jian, H. Yau, and J. Chen, "Nonlinear dynamic analysis of a hybrid squeeze-film damper-mounted rigid rotor lubricated with couple stress fluid and active control," Applied Mathematical Modelling, vol. 34, p. 2493-2507, 2010. 
[93] R. S. Orsey, "Non-Linear Transient Torsional Analysys of Rotor," Master's Thesie, University of Virginia, 1996.

[94] S. S. Rao, Mechanical Vibrations (4th Edition). Prentice Hall, 2003.

[95] B. C. Moore, "Principal component analysis in linear systems-controllability observability and model reduction," IEEE Transactions on Automatic Control, vol. 26, no. 1, pp. 17-32, 1981.

[96] R. Seydel, Practical Bifurcation and Stability Analysis: From Equilibrium to Chaos. New York: Springer-Verlag, 1994.

[97] T. Iwatsubo, K. Shimbo, and S. Kawamura, "The study of nonlinear vibration analysis of rotor system using component mode synthesis method-(analysis using the harmonic balance method)," JSME International Journal Series C, vol. 45, no. 1, pp. 136-141, 2002.

[98] F. A. Shen, " Transient flexible rotor dynamics analysis PartI: Theory," ASME, Journal of Engineering for Industry, p. 531-538, 1972.

[99] K. C. Choy, E. J. Gunter, L. E. Barrett, and P. E. Allaire, "Transient and Stability Analysis Using the Modal Method," University of Virginia, ROMAC Report No. 61 UVA/643092/MAE81/127, 1976.

[100] M. L. Adams, "Non-linear dynamics of flexible multi-bearing rotors," Journal of Sound and Vibration, vol. 71, no. 1, pp. 129-144, 1980.

[101] W. Li, Y. Yang, D. Sheng, and C. J., "A novel nonlinear model of rotor bearing seal system and numerical analysis," Mechanism and Machine Theory, vol. 46, p. 618-631, 2011.

[102] A. K. Noor, "Recent Advances and Applications of Reduction Methods," Applied Mechanics Reviews, vol. 47, no. 5, pp. 125-146, 1994.

[103] M. B. Wagner, "Model Reduction Methods for Rotordynamic Analysis: An exploration, comparison, and metrics," Ph.D. Dissertation, University of Virginia, 2011.

[104] R. J. Guyan, "Reduction of stiffness andmassmatrices," AIAA Journal, vol. 3, no. 2, p. $380,1965$.

[105] H. D. Jean and A. N. Nelson, "Periodic response investigation of large nonlinear 
rotordynamic systems using collocation," Journal of Sound and Vibration, vol. 143, p. $473-489,1990$.

[106] H. D. Nelson, S. J. Russel, and W. L. Meacham, "Transient response of rotorbearing systems using component mode synthesis, part I -mathematical development," ASME Paper No. 81-GT-10., 1981.

[107] D. A. Nelson and H. D. Glasgow, "Stability analysis of rotor-bearing systems using component mode synthesis," Journal of Mechanical Design, Transactions of the ASME, vol. 102, no. 2, pp. 352-359, 1980.

[108] Z. Qu, Model Order Reduction Techniques. New York, NY, USA: Springer, 2004.

[109] P. Apiwattanalunggarn, S. W. Shaw, and C. Pierre, "Component mode synthesis using nonlinear normal modes," Nonlinear Dynamics, vol. 41, no. 1-3, pp. 17-46, 2005.

[110] D. F. Gunter and J. Li, "A study of the modal truncation error in the component analysis of a dual-rotor system," Tech. Rep., Rotating Machinery and Controls Lab, University of Virginia, 1980.

[111] C. Devulder and M. Marion, "A class of numerical algorithms for large time integration: The nonlinear Galerkin method," SIAM Journal on Numerical Analysis, vol. 29, no. 2, pp. 462-483, 1992.

[112] C. Sansour, P. Wriggers, and J. Sansour, "A finite element post-processed Galerkin method for dimensional reduction in the non-linear dynamics of solids: Applications to shells," Comput Mech, vol. 32, pp. 104-114, 2003.

[113] B. Garcia-Archilla and J. S. Novo, "An approximate inertial manifolds approach to postprocessing the Galerkin method for the Navier-Stokes equations," Math Comput, vol. 68, p. 893-911, 1999.

[114] H. G. Matthies and M. Meyer, "Nonlinear Galerkin methods for the model reduction of nonlinear dynamical systems," Computers \& Structures, vol. 81, no. 12, pp. 1277-1286, 2003.

[115] G. Rega and H. Troger, "Dimension Reduction of Dynamical Systems: Methods, Models, Applications," Nonlinear Dynamics, vol. 41, pp. 1-15, 2005.

[116] G. Fuchs, A. Steindl, and S. Jakubek, "Local Jacobian based Galerkin Order Reduction for the Approximation of Large-Scale Nonlinear Dynamical Systems," 
International Journal of Mathematical Models and Methods in Applied Sciences, vol. 5, no. 3, pp. 567-576, 2011.

[117] H. L. Hassenpflug, "Transient Analysis of Multimass Rotors with Acceleration," Master's Dissertation, University of Virginia, 1981.

[118] S. Singhal and M. Khonsari, "THD Stability and Analysis of Journal Bearings," $J$. Engineering Tribology, vol. 209, pp. 225-234, 2005.

[119] P. Shellya and C. Ettles, "A Tractable Solution for Medium-Length Journal Bearings," Wear, vol. 12, pp. 221-228, 1970.

[120] H. D. Nelson and W. L. Meacham, "Transient analysis of rotor-bearing systems using component mode synthesis," in American Society of Mechanical Engineers, Gas Turbine Conference and Products Show, Houston, Tex, United States, 1981, p. 1981.

[121] I. Christie, K. R. Rajagopal, and A. Z. Szeri, "Flow of a non-Newtonian fluid between eccentric rotating cylinders," International Journal of Engineering Science, vol. 25, no. 8, pp. 1029-1047, 1987.

[122] M. He, P. Allaire, and P. Sheth, "ComboRotor User's Manual," University of Virginia ROMAC Report No. 521, 2007.

[123] J. Chaudhry, "Rotor Dynaminc Analysis in Matlab Framwork," MS Dissertation, University of Virginia, 2008. 


\section{Appendix A: 12 DOF Elemental Matrices}

\section{DOF element}

The full 12 degrees of freedom (DOF) Timoshenko beam element is used for the transient analysis code. The element has 6 DOF at each node: translations in the nodal $\mathrm{x}$, $\mathrm{y}$, and $\mathrm{z}$ directions and rotations about the nodal $\mathrm{x}, \mathrm{y}$, and $\mathrm{z}$ axes. The effects of gyroscopic, rotary inertia and shear deformation effects are also considered in this finite beam element.

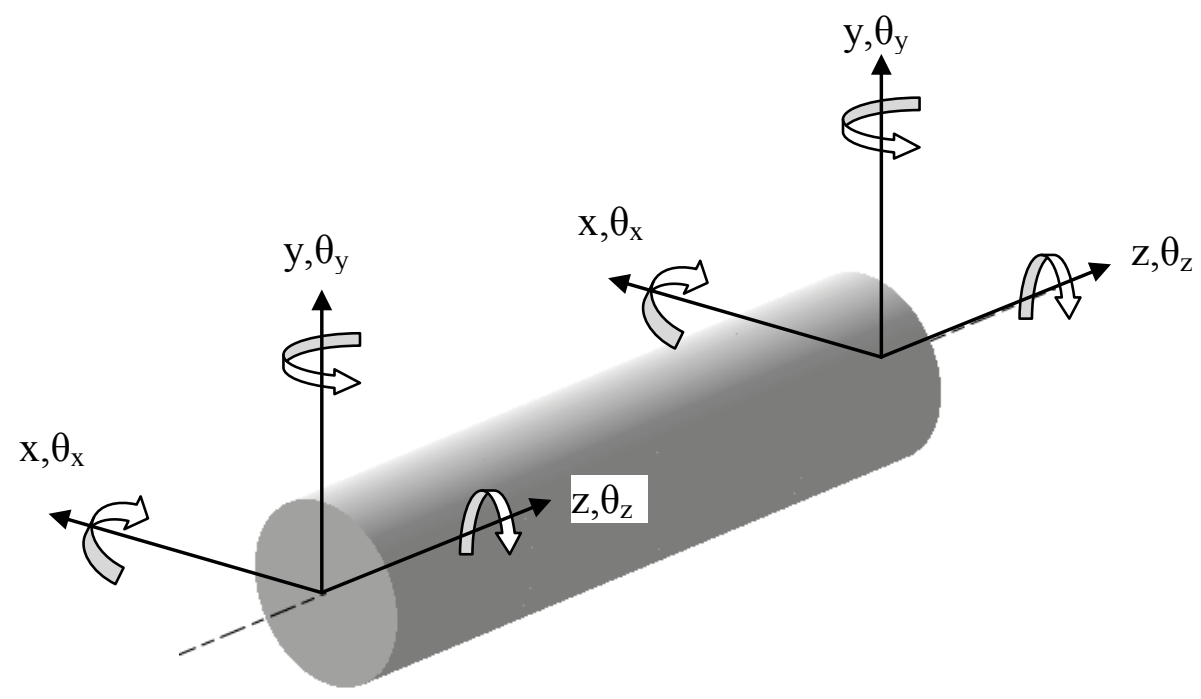

Displacement vector of element:

$$
u=\left[\begin{array}{llllllllllll}
z_{i} & x_{i} & y_{i} & \theta_{z_{i}} & \theta_{x i} & \theta_{y i} & z_{i+1} & x_{i+1} & y_{i+1} & \theta_{z i+1} & \theta_{x i+1} & \theta_{y i+1}
\end{array}\right]^{T}
$$




\section{Shape factor:}

$$
\kappa=\frac{6(1+v)\left(1+\left(D_{i} / D_{o}\right)^{2}\right)^{2}}{(7+6 v)\left(1+\left(D_{i} / D_{o}\right)^{2}\right)^{2}+(20+12 v)\left(D_{i} / D_{o}\right)^{2}}
$$

where $v$ is the Poisson ratio, $D_{i}$ is inner diameter and $D_{0}$ is outer diameter

\section{Transverse shear factor:}

$$
\Phi=\frac{12 E I}{\kappa A G L^{2}}
$$

where $E$ is Young's modulus, $G$ is shear modulus, $I$ is polar moment of inertia, $A$ is crosssection area and $L$ is element length. For Bernoulli-Euler beam element, $\Phi$ is zero.

\section{Elemental mass matrix:}

$$
[M]=\left[\begin{array}{cc}
M_{11} & M_{12} \\
M_{12}^{T} & M_{22}
\end{array}\right]_{12 \times 12}
$$

where

$$
\left[M_{11}\right]=\rho A L\left[\begin{array}{cccccc}
1 / 3 & 0 & 0 & 0 & 0 & 0 \\
0 & m_{A} & 0 & 0 & 0 & m_{C} \\
0 & 0 & m_{A} & 0 & -m_{C} & 0 \\
0 & 0 & 0 & I / 3 A & 0 & 0 \\
0 & 0 & -m_{C} & 0 & m_{E} & 0 \\
0 & m_{C} & 0 & 0 & 0 & m_{E}
\end{array}\right]
$$


$\left[M_{12}\right]=\rho A L\left[\begin{array}{cccccc}1 / 6 & 0 & 0 & 0 & 0 & 0 \\ 0 & m_{B} & 0 & 0 & 0 & -m_{D} \\ 0 & 0 & m_{B} & 0 & m_{D} & 0 \\ 0 & 0 & 0 & I / 6 A & 0 & 0 \\ 0 & 0 & -m_{D} & 0 & m_{F} & 0 \\ 0 & m_{D} & 0 & 0 & 0 & m_{F}\end{array}\right]$

$\left[M_{22}\right]=\rho A L\left[\begin{array}{cccccc}1 / 3 & 0 & 0 & 0 & 0 & 0 \\ 0 & m_{A} & 0 & 0 & 0 & -m_{C} \\ 0 & 0 & m_{A} & 0 & m_{C} & 0 \\ 0 & 0 & 0 & I / 3 A & 0 & 0 \\ 0 & 0 & m_{C} & 0 & m_{E} & 0 \\ 0 & -m_{C} & 0 & 0 & 0 & m_{E}\end{array}\right]$

$m_{A}=\frac{\left(\frac{156+294 \phi+140 \phi^{2}}{420}+\frac{36 I}{30 A L^{2}}\right)}{\left(1+\phi^{2}\right)}$

$m_{B}=\frac{\left(\frac{54+126 \phi+70 \phi^{2}}{420}-\frac{36 I}{30 A L^{2}}\right)}{\left(1+\phi^{2}\right)}$

$m_{C}=\frac{\left(\frac{22+38.5 \phi+17.5 \phi^{2}}{420}+\left(\frac{3-15 \phi}{30}\right) \frac{I}{A L^{2}}\right)}{\left(1+\phi^{2}\right)} L$

$m_{D}=\frac{\left(\frac{13+31.5 \phi+17.5 \phi^{2}}{420}-\left(\frac{3-15 \phi}{30}\right) \frac{I}{A L^{2}}\right)}{\left(1+\phi^{2}\right)} L$ 


$$
\begin{aligned}
& m_{E}=\frac{\left(\frac{4+7 \phi+3.5 \phi^{2}}{420}+\left(\frac{4+5 \phi+10 \phi^{3}}{30}\right) \frac{I}{A L^{2}}\right)}{\left(1+\phi^{2}\right)} L^{2} \\
& m_{F}=\frac{\left(-\frac{-3-7 \phi-3.5 \phi^{2}}{420}+\left(\frac{-1-5 \phi+5 \phi^{2}}{30}\right) \frac{I}{A L^{2}}\right)}{\left(1+\phi^{2}\right)} L^{2}
\end{aligned}
$$

\section{Elemental stiffness matrix:}

$$
[K]=\left[\begin{array}{ll}
K_{11} & K_{12} \\
K_{12}^{T} & K_{22}
\end{array}\right]_{12 \times 12}
$$

where

$$
\left[K_{11}\right]=\frac{E I}{(1+\phi) L^{3}}\left[\begin{array}{cccccc}
\frac{E A}{L}\left(\frac{(1+\phi) L^{3}}{E I}\right) & 0 & 0 & 0 & 0 & 0 \\
0 & 12 & 0 & 0 & 0 & 6 L \\
0 & 0 & 12 & 0 & -6 L & 0 \\
0 & 0 & 0 & \frac{G I}{L}\left(\frac{(1+\phi) L^{3}}{E I}\right) & 0 & 0 \\
0 & 0 & -6 L & 0 & (4+\phi) L^{2} & 0 \\
0 & 6 L & 0 & 0 & 0 & (4+\phi) L^{2}
\end{array}\right] \text { (A5.1) }
$$




$$
\left[K_{22}\right]=\frac{E I}{(1+\phi) L^{3}}\left[\begin{array}{cccccc}
\frac{E A}{L}\left(\frac{(1+\phi) L^{3}}{E I}\right) & 0 & 0 & 0 & 0 & 0 \\
0 & 12 & 0 & 0 & 0 & -6 L \\
0 & 0 & 12 & 0 & 6 L & 0 \\
0 & 0 & 0 & \frac{G I}{L}\left(\frac{(1+\phi) L^{3}}{E I}\right) & 0 & 0 \\
0 & 0 & 6 L & 0 & (4+\phi) L^{2} & 0 \\
0 & -6 L & 0 & 0 & 0 & (4+\phi) L^{2}
\end{array}\right](\mathrm{A} 5.3)
$$

\section{Elemental gyroscopic matrix:}

$$
[G]=\left[\begin{array}{cccccccccccc}
0 & 0 & 0 & 0 & 0 & 0 & 0 & 0 & 0 & 0 & 0 & 0 \\
0 & 0 & g_{A} & 0 & g_{B} & 0 & 0 & 0 & -g_{A} & 0 & g_{B} & 0 \\
0 & -g_{A} & 0 & 0 & 0 & g_{B} & 0 & g_{A} & 0 & 0 & 0 & g_{B} \\
0 & 0 & 0 & 0 & 0 & 0 & 0 & 0 & 0 & 0 & 0 & 0 \\
0 & -g_{B} & 0 & 0 & 0 & g_{C} & 0 & g_{B} & 0 & 0 & 0 & g_{D} \\
0 & 0 & -g_{B} & 0 & -g_{C} & 0 & 0 & 0 & g_{B} & 0 & -g_{D} & 0 \\
0 & 0 & 0 & 0 & 0 & 0 & 0 & 0 & 0 & 0 & 0 & 0 \\
0 & 0 & -g_{A} & 0 & -g_{B} & 0 & 0 & 0 & g_{A} & 0 & -g_{B} & 0 \\
0 & g_{A} & 0 & 0 & 0 & -g_{B} & 0 & -g_{A} & 0 & 0 & 0 & -g_{B} \\
0 & 0 & 0 & 0 & 0 & 0 & 0 & 0 & 0 & 0 & 0 & 0 \\
0 & -g_{B} & 0 & 0 & 0 & g_{D} & 0 & g_{B} & 0 & 0 & 0 & g_{C} \\
0 & 0 & -g_{B} & 0 & -g_{D} & 0 & 0 & 0 & g_{B} & 0 & -g_{C} & 0
\end{array}\right]
$$

where

$$
\begin{aligned}
& g_{A}=\frac{\rho I}{15\left(1+\phi^{2}\right) L}(36) \\
& g_{B}=\frac{-\rho I}{15\left(1+\phi^{2}\right) L}(3-15 \Phi) L \\
& g_{C}=\frac{\rho I}{15\left(1+\phi^{2}\right) L}\left(4+5 \Phi+10 \Phi^{2}\right) L^{2}
\end{aligned}
$$




$$
g_{D}=\frac{-\rho I}{15\left(1+\phi^{2}\right) L}\left(1+5 \Phi-5 \Phi^{2}\right) L^{2}
$$

\section{Lumped mass matrix:}

$$
\left[M_{D}\right]=\left[\begin{array}{cccccc}
m_{d} & 0 & 0 & 0 & 0 & 0 \\
0 & m_{d} & 0 & 0 & 0 & 0 \\
0 & 0 & m_{d} & 0 & 0 & 0 \\
0 & 0 & 0 & J_{p} & 0 & 0 \\
0 & 0 & 0 & 0 & J_{t} & 0 \\
0 & 0 & 0 & 0 & 0 & J_{t}
\end{array}\right]
$$

where $m_{d}$ is lumped mass, $J_{p}$ is polar mass moment of inertia, $J_{t}$ is transverses mass moment of inertia.

\section{Lumped gyroscopic matrix:}

$$
\left[G_{D}\right]=\left[\begin{array}{cccccc}
0 & 0 & 0 & 0 & 0 & 0 \\
0 & 0 & 0 & 0 & 0 & 0 \\
0 & 0 & 0 & 0 & 0 & 0 \\
0 & 0 & 0 & 0 & 0 & 0 \\
0 & 0 & 0 & 0 & 0 & J_{p} \\
0 & 0 & 0 & 0 & -J_{p} & 0
\end{array}\right]
$$

where $J_{p}$ is polar mass moment of inertia. 


\section{Appendix B: Assembling of Linear Rotor and}

\section{Nonlinear Bearings/Dampers}

To be easier handled in Matlab coding, assembly of the rotor-bearing system is layer by layer, which means the linear rotor with built on disks is assembled first, and then the linear supporting components such as bearing, gearbox, seal and coupling are assembled. After that, nonlinear bearings and dampers are added into the global matrices and the force vector.

Assembly method of the rotor and the linear supporting system into global mass, damping, gyroscopic, and stiffness matrices is explained in details by Chen [13] and Allaire [14]. Here only the method of assembling the nonlinear components is introduced.

Four nonlinear components, including short journal bearing (L/D $\leq 0.5)$, short squeeze film damper ( $\mathrm{L} / \mathrm{D} \leq 0.5)$, tilting pad bearing, and finite length squeeze film damper, are taken into consideration. The method of adding nonlinear components is discussed case by case. Because linear bearings have been added into the system in prior layer, it is possible that a linear bearing has been added to the position of a nonlinear bearing. If this condition occurs, the linear bearing will be removed from the global matrices and then the nonlinear bearing will be added. The changing of the global damping is similar with 
the changing of the global stiffness matrix. The following equations only show the changing of the global mass and stiffness matrices.

\section{Case I: Nonlinear short journal bearing only}

Changing of the global stiffness matrix $(K)$ for bearing connects to the ground directly:

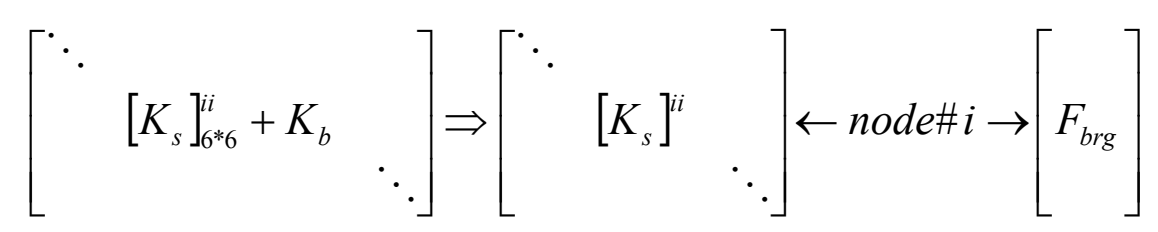

where $\left[K_{s}\right]^{i i}$ is the linear shaft stiffness of node $i, K_{b}$ is the linear bearing stiffness coefficients, and $F_{b r g}$ is the nonlinear bearing forces.

Changing of the global stiffness matrix $(K)$ for bearing connects between nodes:

$$
\begin{aligned}
& {\left[\begin{array}{ccccc}
\ddots & & & & \\
& {\left[K_{s}\right]^{i i}+K_{b}} & & {\left[K_{s}\right]^{j j}-K_{b}} & \\
& \ddots & & \\
& {\left[K_{s}\right]^{j i}-K_{b}^{T}} & & {\left[K_{s}\right]^{j j}+K_{b}} & \\
& & & & \ddots
\end{array}\right] \Rightarrow} \\
& {\left[\begin{array}{ccccc}
\ddots & & & & \\
& {\left[K_{s}\right]^{i i}} & & {\left[K_{s}\right]^{j j}} & \\
& \ddots & & \\
& {\left[K_{s}\right]^{j i}} & & {\left[K_{s}\right]^{j j}} & \\
& & & & \ddots
\end{array}\right] \leftarrow \text { node\# } i \rightarrow\left[\begin{array}{c}
F_{\text {brg }} \\
-F_{\text {brg }}
\end{array}\right]}
\end{aligned}
$$

where $\left[K_{s}\right]^{i i},\left[K_{s}\right]^{j i},\left[K_{s}\right]^{j i},\left[K_{s}\right]^{j j}$ are the linear shaft stiffness coefficients of node $i \& j$. 


\section{Case II: Linear bearing + nonlinear short squeeze film damper}

Changing of the global mass matrix $(M)$ :

$$
[M]_{6 N^{*} 6 N} \Rightarrow\left[\begin{array}{cccc}
{[M]_{6 N^{*} 6 N}} & & & {[0]} \\
& {[0]} & {\left[\begin{array}{ccc}
M_{s d}^{1} & & \\
& \ddots & \\
& & M_{s d}^{s}
\end{array}\right]_{2 S^{* 2 S}}}
\end{array}\right]
$$

where $[M]$ is the linear shaft mass matrix, $M_{s d}^{1} \cdots M_{s d}^{1}$ are masses inside nonlinear squeeze film dampers, $N$ is total number of shaft element, and $S$ is total number of squeeze film dampers.

Changing of the global stiffness matrix $(K)$ :

$$
\left[\begin{array}{lll}
\ddots & & \\
& {\left[K_{s}\right]^{i i}+\left[K_{b}\right]} & \\
& & \ddots
\end{array}\right]_{6 N^{*} 6 N} \Rightarrow
$$

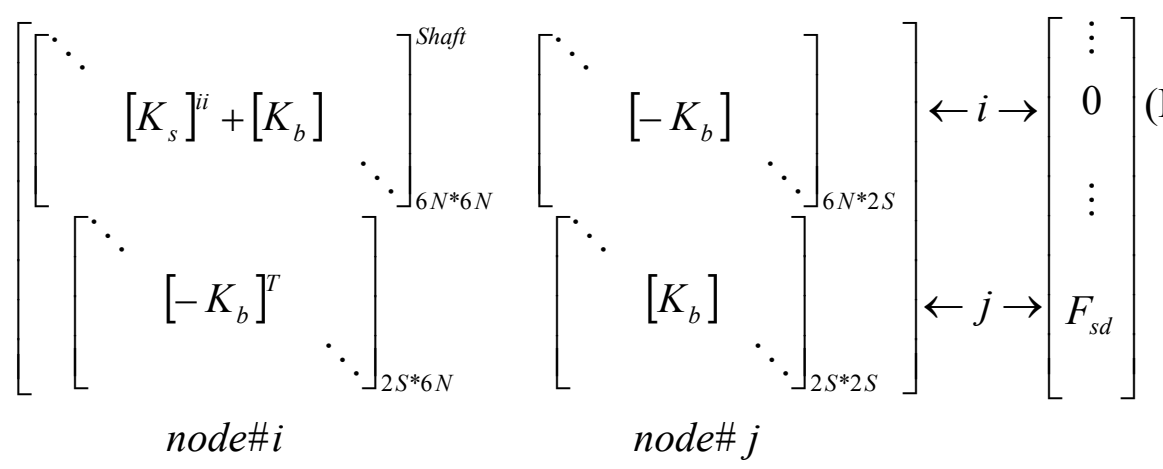

where $F_{s d}$ is the nonlinear squeeze film damper force. The bearing is on $\mathrm{i}^{\text {th }}$ node, the added damper node is on $\mathrm{j}^{\text {th }}$ node. 


\section{Case III: Nonlinear short journal bearing + nonlinear short squeeze film damper}

Changing of the global mass matrix (M): same as (B.3).

Changing of the global stiffness matrix $(K)$ :

$$
\begin{aligned}
& {\left[\begin{array}{lll}
\ddots & & \\
& {\left[K_{s}\right]^{i i}+K_{b}} & \\
& & \ddots
\end{array}\right]_{6 N^{*} 6 N} \Rightarrow}
\end{aligned}
$$

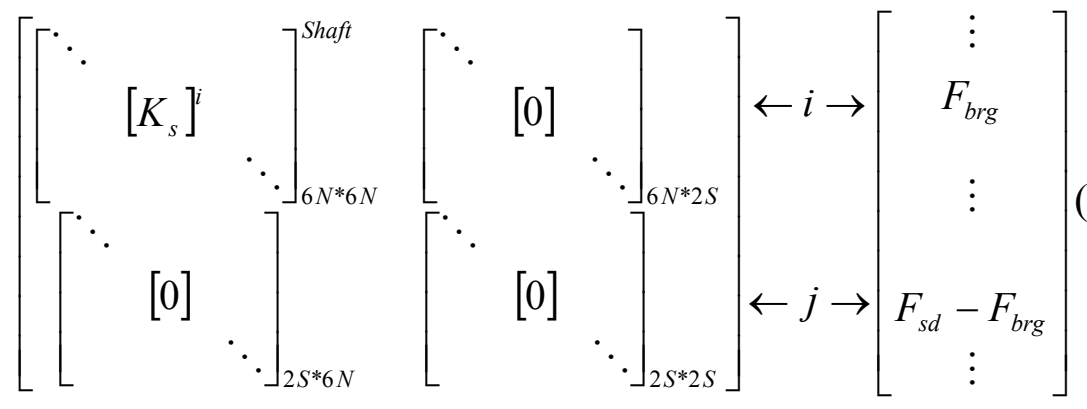

$$
\begin{aligned}
& \text { node\# } i \quad \text { node\# } j
\end{aligned}
$$

\section{Case IV: Nonlinear tilting pad bearing only}

Changing of the global mass matrix $(M)$ :

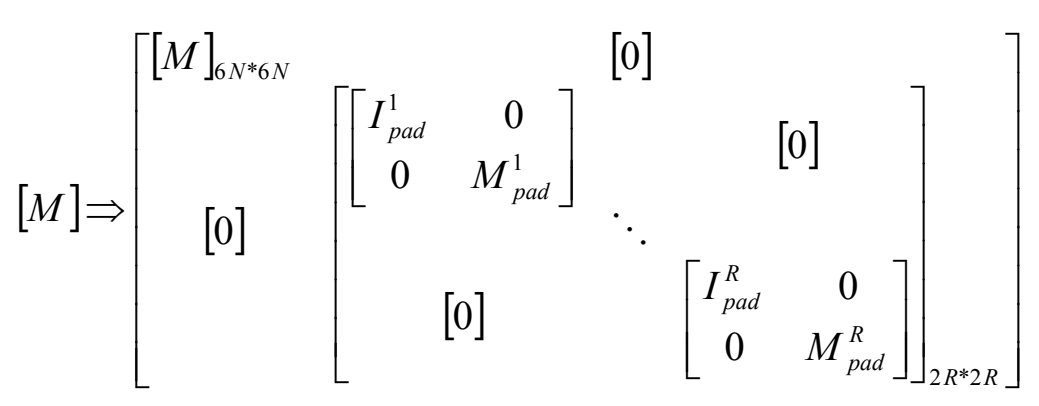

where $M_{p a d}^{1} \cdots M_{p a d}^{R}$ and $I_{p a d}^{1} \cdots I_{p a d}^{R}$ are the masses and moments of inertia of all pads. 
Changing of the global stiffness matrix ( $K$, bearing connects to ground):

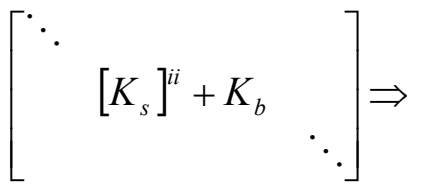

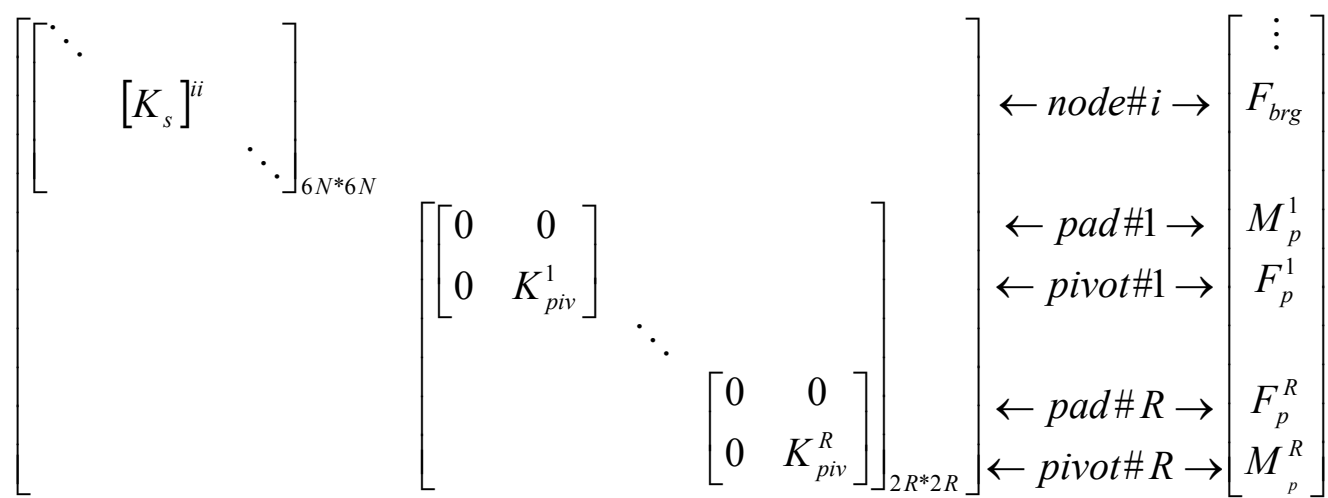

Changing of the global stiffness matrix ( $K$, bearing connects between nodes):

$$
\begin{aligned}
& {\left[\begin{array}{ccccc}
\ddots & & & & \\
& {\left[K_{s}\right]^{i i}+K_{b}} & & {\left[K_{s}\right]^{i j}-K_{b}} & \\
& {\left[K_{s}\right]^{j i}-K_{b}} & & {\left[K_{s}\right]^{j j}+K_{b}} & \\
& & & & \ddots
\end{array}\right] \Rightarrow}
\end{aligned}
$$

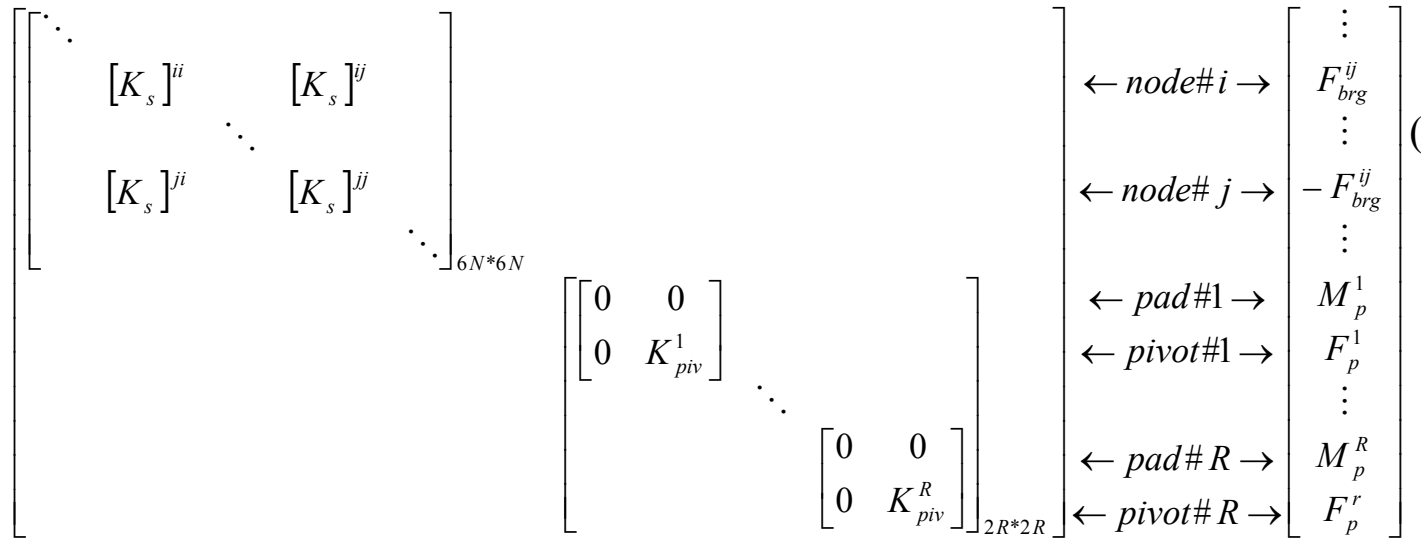

where $K_{p i v}^{1} \cdots K_{p i v}^{R}$ are the pivot stiff nesses, $M_{p}^{1} \cdots M_{p}^{R}$ are the pad moments, $F_{p}^{1} \cdots F_{p}^{R}$ are the pad forces. 


\section{Case VI: Nonlinear tilting pad bearing + nonlinear finite length squeeze film}

\section{damper}

Changing of the global mass matrix $(M)$ :

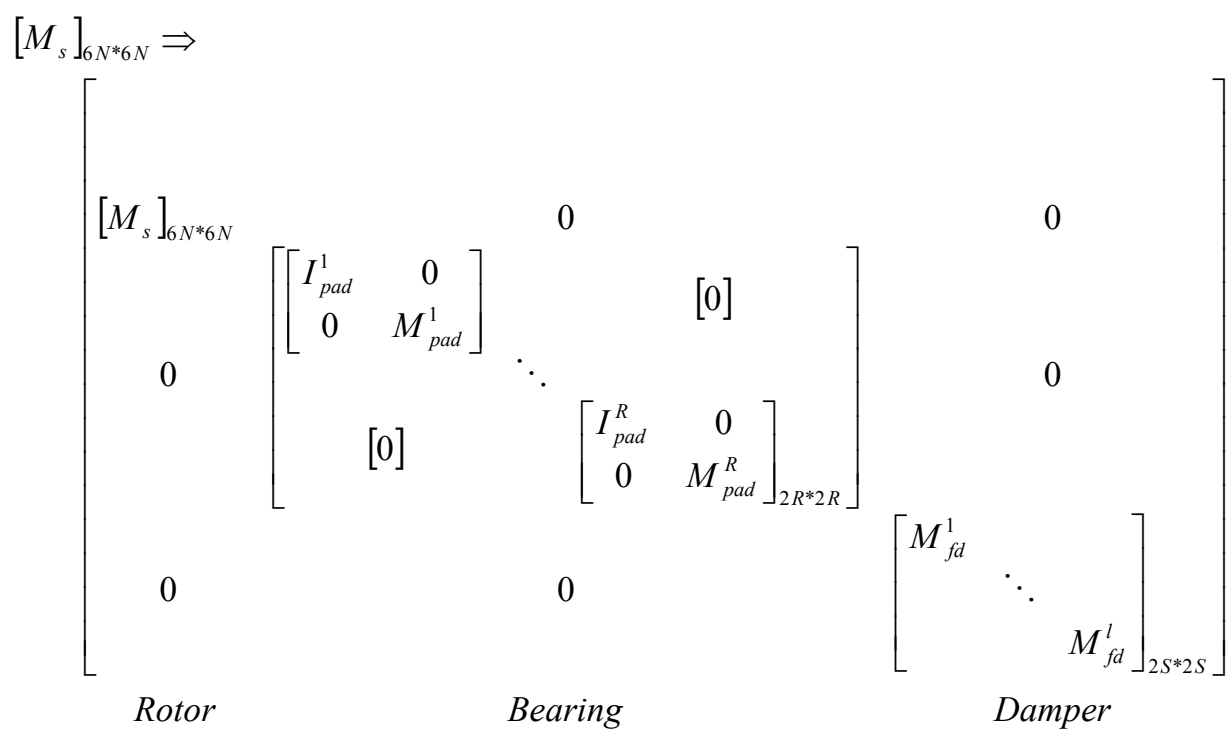

where $M_{f d}^{1} \cdots M_{f d}^{l}$ are the masses of all finite length squeeze film dampers.

Changing of the global stiffness matrix $(K)$ :

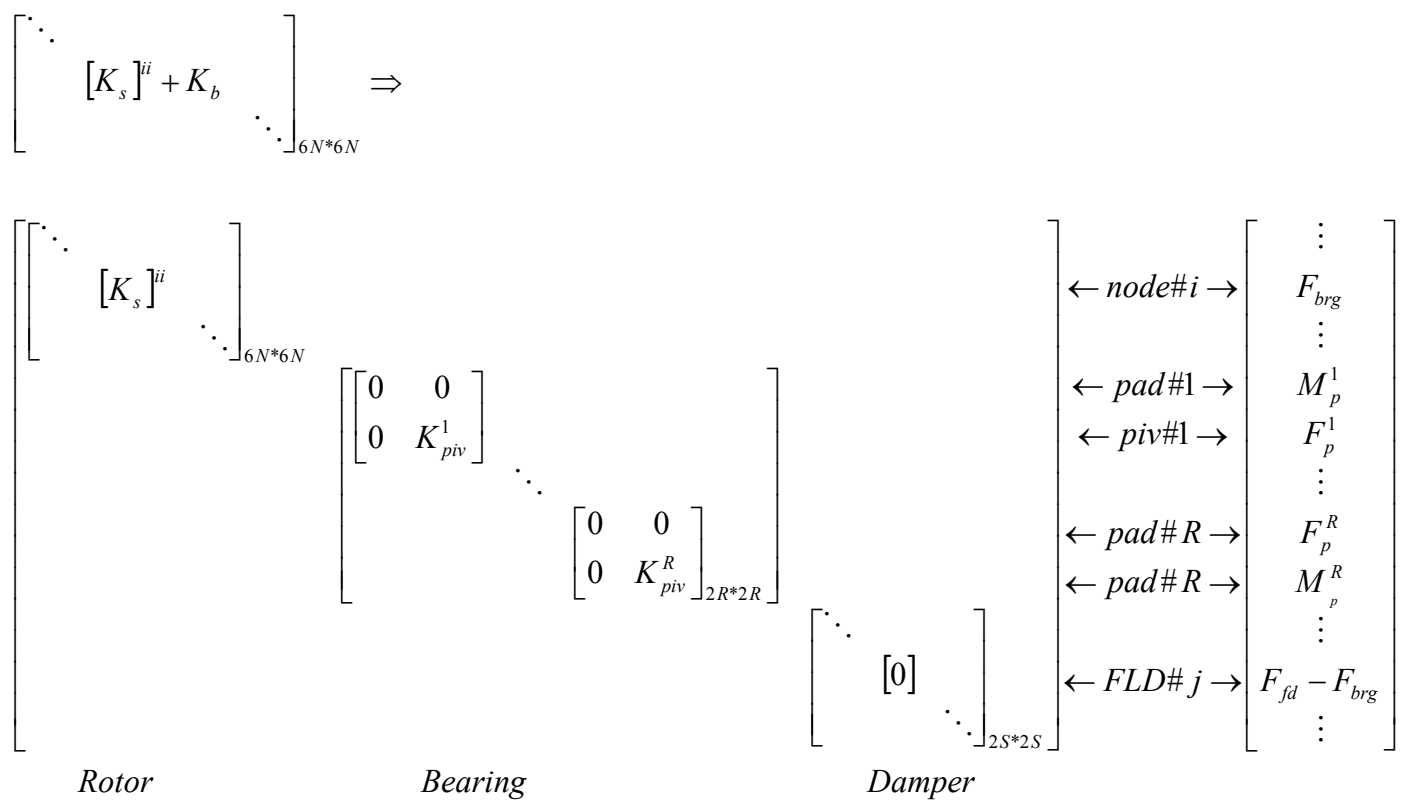

